\title{
IMPROVED BIOMASS UTILIZATION THROUGH REMOTE FLOW SENSING
}

Identification Number: DE-FC36-01G011054

Final Report

March 2007 


\section{IMPROVED BIOMASS UTILIZATION THROUGH REMOTE FLOW SENSING}

\section{Identification Number: DE-FC36-01G011054 \\ Final Report \\ March 2007}

From:

Prof. Muthanna Al-Dahhan

Principal Investigator

Department of Energy, Environment and Chemical Engineering

Washington University

One Brookings Dr.

St. Louis, MO 63130

Tel: (314) - 935-7187

Fax: (314) - 935-4832

E-mail: muthanna@wustl.edu

Submitted To:

Dr. James J. Spaeth

U.S. Department of Energy

Golden Field Office

1617 Cole Boulevard

Golden, CO 80401-3393

Tel: (303) - 275-4771

E-mail: jim_spaeth@nrel.gov 


\title{
IMPROVED BIOMASS UTILIZATION THROUGH REMOTE FLOW SENSING
}

\author{
Identification Number: DE-FC36-01G011054 \\ Final Report \\ March 2007
}

Project Teams:

Washington University- St. Louis

Muthanna Al-Dahhan (Principal Investigator)

E-mail: muthanna@wuche.che.wustl.edu

Rajneesh Varma

Khursheed Karim

Mehul Vesvikar

Rebecca Hoffman

Oakridge National Laboratory

David Depaoli, (Co-principal investigator)

Email: depaolidw@ornl.gov

Thomas Klasson

Alan L. Wintenberg

Charles W Alexander

Lloyd Clonts

Iowa Energy Center

Norm Olson

Email: nolson@energy.iastate.edu 


\section{Executive Summary}

The growth of the livestock industry provides a valuable source of affordable, sustainable, and renewable bioenergy, while also requiring the safe disposal of the large quantities of animal wastes (manure) generated at dairy, swine, and poultry farms. For example, the USA produces 1.8 billion tons of cow manure annually (USDA 2002/2003). If these biomass resources are mishandled and underutilized, major environmental problems will be created, such as surface and ground water contamination, odors, dust, ammonia leaching, and methane emission. Methane emission notably results in a greenhouse gas effect that is considered 22 times worse than carbon dioxide (Sheffield, 2002). Anaerobic digestion of animal wastes, in which microorganisms break down organic materials in the absence of oxygen, is one of the most promising waste treatment technologies. This process produces biogas typically containing $~ 65 \%$ methane and $\sim 35 \%$ carbon dioxide. The production of biogas through anaerobic digestion from animal wastes, landfills, and municipal waste water treatment plants represents a large source of renewable and sustainable bio-fuel. Such bio-fuel can be combusted directly, used in internal combustion engines, converted into methanol, or partially oxidized to produce synthesis gas (a mixture of hydrogen and carbon monoxide) that can be converted to clean liquid fuels and chemicals via Fischer-Tropsch synthesis.

Different design and mixing configurations of anaerobic digesters for treating cow manure have been utilized commercially and/or tested on a laboratory scale. These digesters include mechanically mixed, gas recirculation mixed, and slurry recirculation mixed designs, as well as covered lagoon digesters. Mixing is an important parameter for successful performance of anaerobic digesters. It enhances substrate contact with the microbial community; improves $\mathrm{pH}$, temperature and substrate/microorganism uniformity; prevents stratification and scum accumulation; facilitates the removal of biogas from the digester; reduces or eliminates the formation of inactive zones (dead zones); prevents settling of biomass and inert solids; and aids in particle size reduction. Unfortunately, information and findings in the literature on the effect of mixing on anaerobic digestion are contradictory. All the published studies on cow manure anaerobic digestion have been performed using small laboratory scale digesters ( $\sim 4$ liter). No reported investigations have been conducted in pilot plant and/or commercial scale digesters. Hence, the impact of mixing intensity, operating conditions, and design variables on the performance of large digesters are unknown.

One reason is the lack of measurement techniques for opaque systems such as digesters. Better understanding of the mixing and hydrodynamics of digesters will result in appropriate design, configuration selection, scale-up, and performance, which will ultimately enable avoiding digester failures. Anaerobic digesters are highly opaque systems and contain a large volume fraction of dispersed solids as well as produced gas. Non-invasive advanced techniques such as computed tomography (CT) and computer automated radioactive particle tracking (CARPT), used together, can provide valuable information about the digester hydrodynamics and mixing intensity and their impact on performance. 
Accordingly, this project sought to advance the fundamental knowledge and understanding of the design, scale up, operation, and performance of cow manure anaerobic digesters with high solids loading. It is hoped that the findings can be applied to promote bioenergy production and eliminate major environmental pollution problems. The project systematically studied parameters affecting cow manure anaerobic digestion performance, in different configurations and sizes by implementing computer automated radioactive particle tracking (CARPT), computed tomography (CT), and computational fluid dynamics (CFD), and by developing novel multiple-particle CARPT (MP-CARPT) and dual source CT (DSCT) techniques. The accomplishments of the project were achieved in a collaborative effort among Washington University, the Oak Ridge National Laboratory, and the Iowa Energy Center teams.

The following investigations and achievements were accomplished:

- Systematic studies of anaerobic digesters performance and kinetics using various configurations, modes of mixing, and scales (laboratory, pilot plant, and commercial sizes) were conducted and are discussed in Chapter 2.

It was found that in laboratory scale ( 3.78 liter) digesters using low solids loading, mixing does not affect the digester performance when adequate mixing is provided by evolution of biogas bubbles and addition of feed and removal of effluent. However, it was found that mixing significantly affected the performance of the pilot plant scale digester ( 97 liter). It also had a considerable effect when high solids loading was used in both laboratory and pilot plant scale digesters. It was also found that in a pilot plant digester, as the energy input (in terms of the gas recirculation rate) increased, the energy output also increased (in terms of the biogas production) up to an asymptote. This finding indicates that the power input that maximizes energy output can be determined for large scale digesters.

- The detailed mixing and hydrodynamics were investigated using computer automated radioactive particle tracking (CARPT) techniques, and are discussed in Chapter 3.

In this work, single particle CARPT was used for the first time to study the hydrodynamics and mixing of anaerobic cow manure digesters of different sizes (lab and pilot plant scales), configuration, and modes of mixing. A novel multiple particle tracking technique (MP-CARPT) technique that can track simultaneously up to 8 particles was developed, tested, validated, and implemented.

- $\quad$ Phase distribution was investigated using gamma ray computer tomography (CT) techniques, which are discussed in Chapter 4.

For the first time, single source CT was used to study the phase distribution in anaerobic digesters. This CT was also used to investigate the impact of the 
sparger design of a gas recirculation digester on the gas holdup distribution in the draft tube and its effect on the slurry circulation and reduction of the dead zones. A novel dual source CT (DSCT) technique was developed to measure the phase distribution of dynamic three phase system such as digesters with high solids loading and other types of gas-liquid-solid fluidization systems. A new image reconstruction algorithm was developed which is suitable for DSCT and for a wide range of isotope combinations.

- Evaluation and validation of the computational fluid dynamics (CFD) models and closures were conducted to model and simulate the hydrodynamics and mixing intensity of the anaerobic digesters (Chapter 5). The validated CFD models and closures were used to investigate various designs and operating conditions, as well as configurations that can enhance slurry recirculation, phase distribution, and reduction or elimination of the inactive volume (dead zones).

All the objectives and tasks set for the project have been successfully accomplished. The results and findings advance the fundamental understanding of the hydrodynamics and mixing of the studied anaerobic digesters and their impact on the digester performance, design, and scale-up.

It is strongly recommended that additional studies be conducted, both on hydrodynamics and performance, in large scale digesters. The studies should use advanced non-invasive measurement techniques, including the developed novel measurement techniques, to further understand their design, scale-up, performance, and operation to avoid any digester failure. The final goal is a system ready to be used by farmers on site for bioenergy production and for animal/farm waste treatment. 


\section{TABLE OF CONTENTS}

CHAPTER 1 : INTRODUCTION, MOTIVATION AND OBJECTIVES

$\begin{array}{lll}1.1 & \text { Introduction and Motivation } & 1-4\end{array}$

$\begin{array}{llr}1.2 & \text { Objectives } & 1-11\end{array}$

1.3 Tasks Accomplished: $\quad 1-12$

1.4 Structure of the final Report: $1-13$

$\begin{array}{ll}1.5 & \text { Published Manuscripts and Theses: }\end{array}$

CHAPTER 2 : PERFORMANCE STUDIES OF ANAEROBIC DIGESTERS $\quad$ 2-16

$\begin{array}{lr}\text { 2. Performance Studies of Anaerobic Digesters } & 2-17\end{array}$

2.1 Effect of mixing intensity, operating conditions and design parameters on the anaerobic digestion of animal waste (cow manure) using laboratory scale ( $\sim 3.78 \mathrm{I})$ digesters $2-19$

$\begin{array}{lll}2.3 & \text { Effects of mixing and digester scale on the performance of anaerobic digesters } & 2-27\end{array}$

$2.4 \quad$ Kinetics of animal waste (cow manure) anaerobic digestion.

2.5 Commercial scale digester for biogas production and animal waste treatment at Iowa Energy

Center $\quad$ 2-34

CHAPTER 3 : INVESTIGATION OF THE DETAILED MIXING AND HYDRODYNAMICS USING COMPUTER AUTOMATED RADIOACTIVE PARTICLE $\begin{array}{lr}\text { TRACKING (CARPT) TECHNIQUES } & 3-45\end{array}$

3.1 Investigation of the hydrodynamics and mixing of anaerobic digesters using computer automated radioactive particle tracking (CARPT) technique 3-47

3.2. The development of a novel multiple-particle CARPT (MP-CARPT) and its implementation. 3-52

CHAPTER 4 : INVESTIGATION OF THE PHASES' DISTRIBUTION USING GAMMA RAY COMPUTED TOMOGRAPHY (CT) TECHNIQUES $\quad 4-56$

The accomplished work consists of the following:

4.1. Gas holdup distribution using single source CT 4-58

4.1.1. Gas holdup distribution in a mimicked flat bottom anaerobic digester. 4-58

4.1.2. Gas distribution in a 6 inch anaerobic digester using a single point sparger and a single source CT. 
4.1.4. Effect of sparger design and the degree of uniformity of the gas holdup in the draft tube on the hydrodynamics and dead zones of the anaerobic digesters mixed by gas recirculation 4-58

4.1 Gas holdup distribution using single source CT

4.2 Development of the novel dual source (energy) gamma ray computed tomography (DSCT) 4-63

CHAPTER 5 : COMPUTATION FLUID DYNAMICS (CFD) OF ANAEROBIC DIGESTERS

REFERENCES

\section{Appendix 1}

Hoffmann RA. 2005. Effect of shear on the performance and microbial ecology of anaerobic digesters treating cow manure from dairy farms. [Masters thesis]. St. Louis USA: Washington University.

\section{Appendix 2}

Vesvikar M. (2006) Understanding the hydrodynamics of anaerobic digester for bioenergy production.[D.Sc. Thesis], St. Louis -USA, Washington University.

\section{Appendix 3}

Investigation of the phases' distribution using gamma ray computed tomography (CT) techniques 


\section{Chapter 1 : Introduction, Motivation and Objectives}




\subsection{Introduction and Motivation}

The, growth of the livestock industry means a corresponding growth in animal wastes at dairy, swine, and poultry farms. While their disposal creates large scale environmental problems, their potential for conversion into sustainable and renewable bio energy offers substantial benefits. For example US produces 1.8 billion tons of cow manure annually (USDA 2002/2003). Sheffield (2002) reported that, in general about 230 million tons (dry basis) of animal wastes are generated annually in the USA. These valuable biomass resources are often mishandled and underutilized, which results in numerous environmental threats. Unsafe and improper disposal of decomposable livestock wastes may result problems like surface and groundwater contamination, odors, dust, and ammonia leaching. There is also threat from methane emission, which results in a greenhouse gas effect that is considered 22 times worse than carbon dioxide (Sheffield 2002).

Anaerobic digestion (AD) of animal wastes is one of the most promising waste management options. It is a waste treatment alternative for both industrial and agricultural wastes and has several advantages over aerobic treatment, including higher organic removal rates, lower sludge production, and lower energy requirements. Anaerobic digestion is among the oldest biological wastewater treatment processes, having first been studied more than a century ago (McCarty and Mosey 1991; Persson et al. 1979). During World War II approximately 30 farmscale biogas plants for the anaerobic treatment of manure were developed in Germany for energy production. Anaerobic digestion is the bacterial fermentation of organic material in an oxygen-free environment. It produces biogas typically containing 65\% methane and 35\% carbon dioxide, with traces of nitrogen, sulphur compounds, volatile organic compounds and ammonia. Anaerobic digestion occurs in a series of steps, employing different types of bacteria to break down complex polymers into simple carbon compounds (e.g., straight chain fatty acids) that can then be utilized by archea to produce methane. Conceptually, anaerobic digestion can be represented as the three step process (Hill 1984; Parkin et al. 1986) shown in Figure 1.1, which involves: (1) hydrolysis, liquidation, and fermentation, (2) hydrogenation and acetic acid fermentation, and (3) methane formation.

The production of biogas represents a large source of renewable and sustainable bioenergy, which can be combusted directly in modified gas boilers, used to run an internal combustion engine, or converted into methanol. It also can be partially oxidized to produce synthesis gas (a mixture of hydrogen and carbon monoxide) which can be converted to clean liquid fuels and chemicals via Fischer-Tropsch synthesis. The caloric value of this biogas is typically 17 to 25 $\mathrm{MJ} / \mathrm{m}^{3}$. Typically, between $40 \%$ and $60 \%$ of the organic matter present is converted to biogas. The remainder consists of odor-free residues rich in nutrients, which can be used as fertilizers. It is also important to note that the effluents from anaerobic digesters are rich in nitrogen content, and thus could be used to grow microalgae. The algae can be converted to bioenergy, or used to produce high value products such as single cell proteins which could be used as animal feed or fish feed, or as a raw material for the production of other valuable pharmaceutical compounds (Rulkens et al. 1998). 


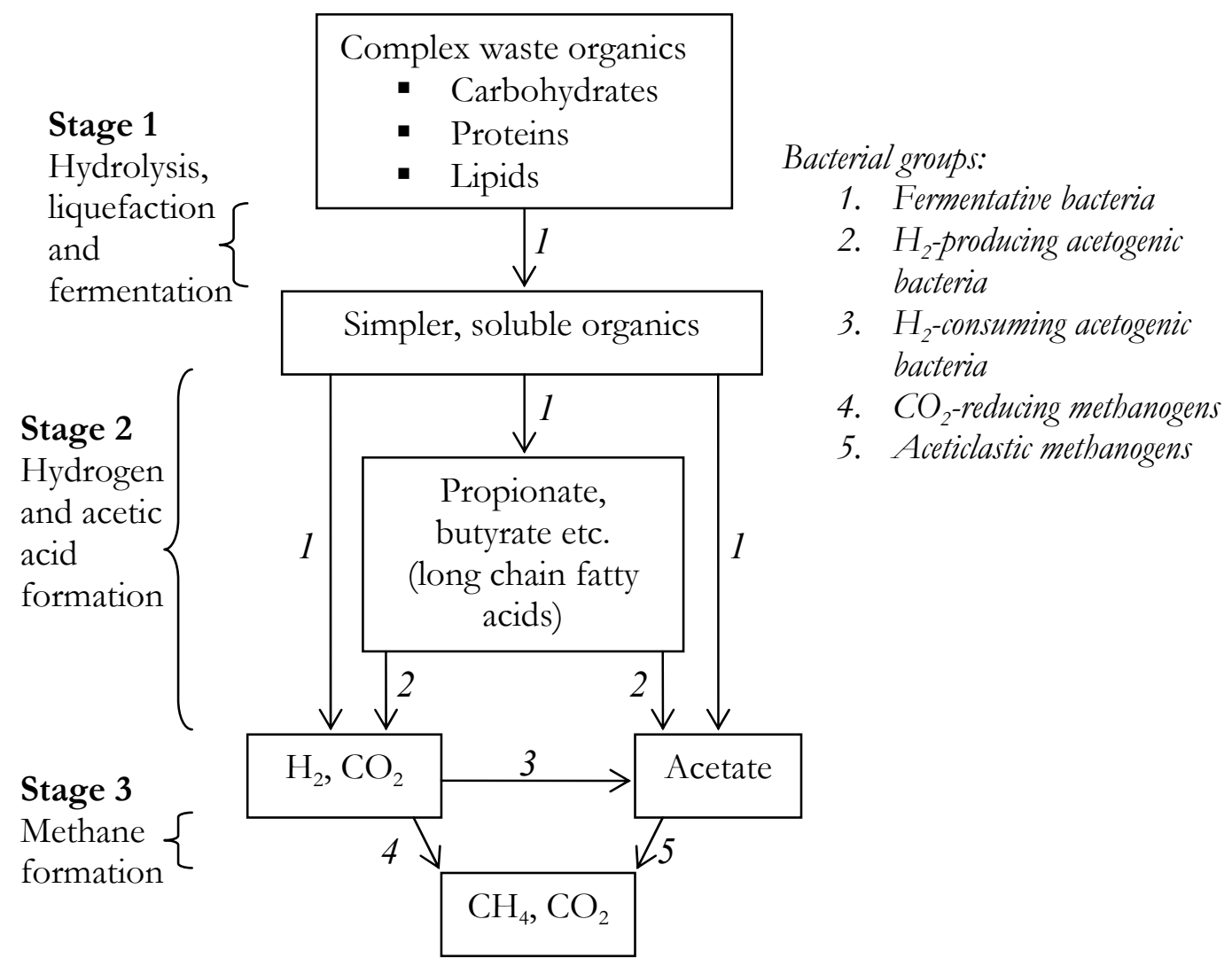

Figure 1.1: A three-step anaerobic digestion mechanism

A survey of dairy and swine farms in the country reaffirmed that anaerobic digestion is a technology with considerable potential (Lusk 1998). Deploying digesters in just three key states (North Carolina, California, and Illinois) could potentially recover $79 \%$ of these methane emissions. This deployment level could basically meet the AgSTAR program's goal as defined in the President's Climate Change Action Plan. AgSTAR is a voluntary federal program that encourages the use of effective technologies to capture methane gas, generated from the decomposition of animal manure, for use as an energy resource. Assuming that all recoverable methane emissions are converted into electricity, even today's low efficiency rates, suggests that slightly more than 165 megawatts (MW) could be produced.

Over the past 25 years, anaerobic digestion processes have been developed and applied to a wide array of industrial and agricultural wastes (Gosh 1997; Speece 1996). However, different aspects of these digesters, design, scale-up, and operation have not been fully understood.

In order to achieve the benefits of anaerobic digestion, the anaerobic facility must be designed to meet the individual characteristics of each animal waste. Conventional anaerobic digesters 
were designed as anaerobic CSTRs (continuous stirred tank reactor) and have been used for many decades in sewage treatment plants for stabilizing activated sludge and sewage solids. Interest in energy-saving waste treatment systems has led to the development of high rate anaerobic digesters for low solids concentrations. These high-rate digesters are also known as retained biomass reactors, since they are based on the concept of retaining viable biomass by sludge immobilization. Examples of high-rate anaerobic digesters (for low solids concentration) are the upflow anaerobic sludge blanket (UASB), anaerobic fluidized bed (AFB), anaerobic filter (AF), expanded granular sludge bed reactor (EGSBR), and the anaerobic sequencing batch reactor (ASBR).

The digestion of animal wastes is complicated by operational problems such as the necessity of homogeneous slurry preparation and pretreatment, and floating of the light material and scum formation, which, in turn, affect biogas production. Many different design and mixing configurations of anaerobic digesters have been utilized commercially and/or tested on a laboratory scale, is such as plug flow digesters, completely mixed digesters, slurry digesters, covered lagoon digesters, up-flow sludge blanket reactors, and sequence batch reactors (Lusk, 1998) (Table 1.1). Among the types of farm-based digesters actually built, the failure rates for complete-mix and plug-flow technologies are staggering, $70 \%$ and $63 \%$, respectively. For covered lagoon digesters, the failure rate is $22 \%$. Because there are fewer operating slurry digesters, their reported $100 \%$ success rate is certainly inconclusive. In general, such high failure rates have been attributed to poor design, insufficient mixing, and an insufficient understanding of hydrodynamics.

It has been shown that mixing affects several essential parameters that determine digester performance. These functions include enhancing substrate and microorganism distribution, ensuring uniform $\mathrm{pH}$ and temperature, preventing stratification and scum formation in dilute waste slurries, facilitating biogas removal from the liquid, aiding in particle size reduction, minimizing or eliminating dead zones (inactive digester volumes), and preventing settling of biomass and inert solids (Smith et al. 1996; Stafford et al. 1980). It is noteworthy that the effects of mixing and hydrodynamics on the investigated digesters performance have not been quantified and characterized. Better understanding of mixing and hydrodynamics of digesters will result in appropriate design and configuration, which will ultimately enable avoiding digester failures.

The performance of anaerobic digesters is affected primarily by the retention time of the substrate in the reactor and the degree of contact between the incoming substrate and the viable bacterial population. These parameters are primarily a function of the hydraulic regime (mixing) in the reactors. For cow manure anaerobic digesters, mixing can be accomplished through various methods, including mechanical mixers, recirculation of digester contents, or by recirculating the produced biogas (Figure 1.2). Gas recirculation anaerobic digesters (also called gas-lift digesters) have several advantages compared to other types, such as simple design, no moving parts, ease of cleaning and sterilization, low shear, low energy consumption, and good heat and mass transfer. 
Table 1.1 Numerical status of farm based anaerobic digesters in the United States (Lusk, 1998).

\section{Status of Farm-Based Digesters in the United States*}

\begin{tabular}{l|l|l|l|l|l|l|}
\hline & Slurry & Plug & Mix & Lagoon & Other & TOTAL \\
\hline Operating & 7 & 8 & 6 & 7 & 0 & 28 \\
Not Operating & 0 & 18 & 10 & 1 & 0 & 29 \\
Farm Closed & 0 & 11 & 5 & 1 & 0 & 17 \\
Under Construction/Planning Phase & 0 & 2 & 4 & 0 & 4 & 10 \\
Planned but Never Built & 0 & 8 & 1 & 1 & 0 & 10 \\
TOTAL & 7 & 47 & 26 & 10 & 4 & 94 \\
\hline
\end{tabular}

*The data presented include digesters that are installed on or were planned for working dairy, swine, and cagedlayer poultry farms. It excludes 65 to 70 digesters that are installed on or were planned for beef farms, and digesters that are primarily university research oriented.
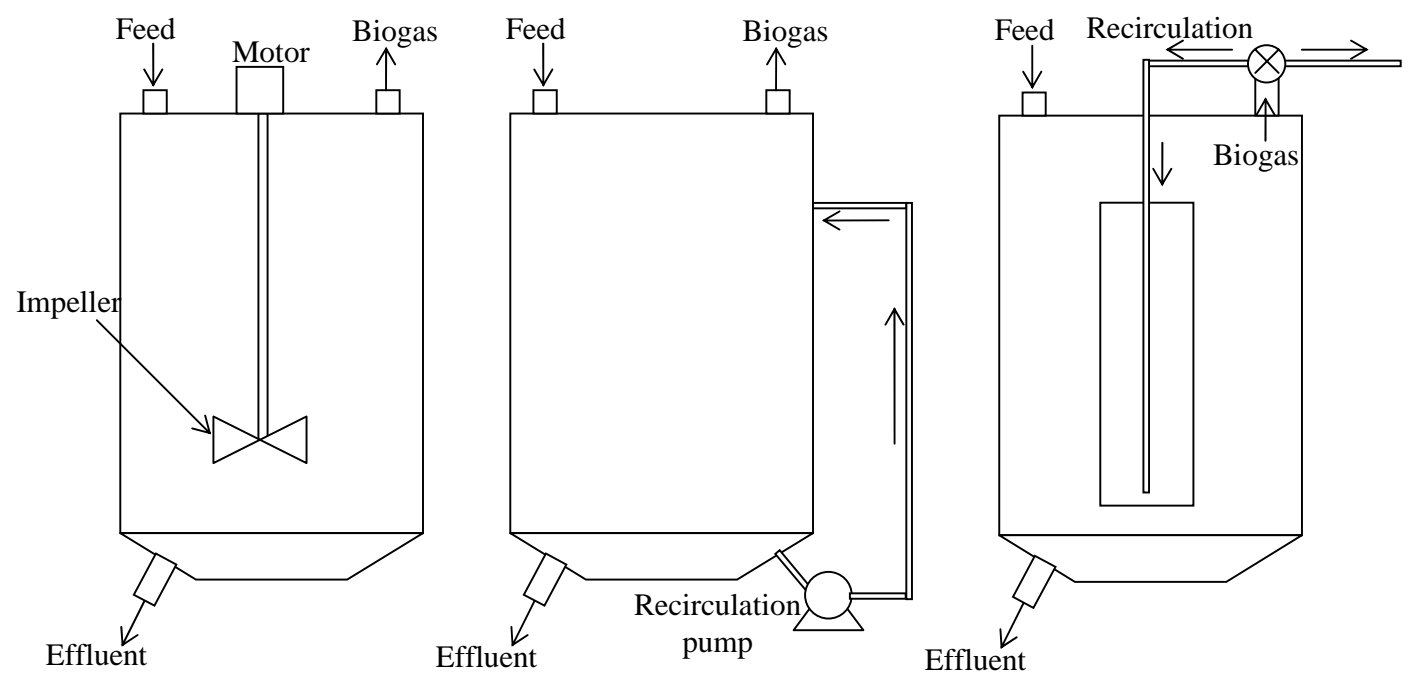

Figure 1.2 Modes of mixing in mixed digesters

However, the information in the literature on the role of mixing in anaerobic digesters is contradictory. Most of the literature on anaerobic digestion, for both low and high solids applications, emphasizes the importance of adequate mixing to improve the distribution of microorganisms throughout the digester (Chapman 1989. ; Lema et al. 1991; Parkin et al. 1986; Strenstrom et al. 1983). Several studies have indicated that a lack of sufficient mixing in low solids digesters dealing with municipal waste resulted in a floating layer of solids (Diaz and Trezek 1977; James et al. 1980.; Strenstrom et al. 1983). These literature sources reported that the mixing level was increased to prevent formation of the solids layer. Chen et al. (1990) observed the development of a floating layer of solids in a $4.5 \mathrm{~m}^{3}$ nonmixed digester treating a mixture of refuse derived fuel and primary sludge. They compared the performance of a nonmixed (downward flow) and a continuously impeller mixed digester at mesophilic 
conditions (30 to $40^{\circ} \mathrm{C}$ ). The nonmixed digester exhibited a higher methane yield than the continuously mixed digester. Ho and Tan (1985) reported greater gas production from palm oil mill effluents for a continuously mixed digester than for an unmixed digester. Mixing can be provided continuously or intermittently. Dague et al. (1970) also observed that shifting from continuous mixing to intermittent mixing ( 2 min of mixing/h) resulted in significantly higher gas production during the anaerobic treatment of a liquid municipal waste stream. Similar controversies and uncertainties have been reported in the case of livestock waste digestion. Intermittent mixing in the anaerobic digestion of livestock waste under mesophilic temperature conditions has been recommended by Mills (1979). Hashimoto (1982) found higher biogas production from beef cattle wastes under both continuous mixing and in vacuum than under intermittent mixing and normal pressure conditions. On the contrary, Ben-Hasson et al. (1985) observed $75 \%$ lower methane production rates from a continuously mixed reactor than from an unmixed reactor when treating dairy cattle manure anaerobically. Whitmore et al. (1987) suggested that very rapid mixing disrupts the structure of flocs in completely mixed reactors, thereby disturbing the syntrophic relationships between organisms. Dolfing (1992) provided a similar argument within the context of high-rate treatment systems. Biofilms and granules represent ideal conditions for close physical associations between electron-producing and electron-consuming organisms (Dolfing, 1992). Appropriate spatial juxtaposition (i.e., the spatial distribution of syntrophic bacteria and their methanogenic partners) allows for high hydrogen fluxes at relatively low hydrogen concentrations by minimizing the development of electron gradients. In vigorously mixed systems, spatial associations are likely continuously disrupted, leading to a state of instability. The results observed by Stroot et al. (2001). suggest that vigorous, continuous mixing may prevent good performance of high solids anaerobic digesters. In this case, minimal mixing was provided to distribute the feed adequately and may have allowed the formation of new spatial associations. Minimal mixing may improve high solids anaerobic digestion by providing a quiescent environment for bacteria. The US EPA has recommended a power input of $0.20-0.30 \mathrm{HP} / 1000 \mathrm{cu} \mathrm{ft}\left(5.26-7.91 \mathrm{~W} / \mathrm{m}^{3}\right)$ for proper digester mixing (EPA 1979).

The contradictory mixing findings reported in the literature indicate the urgent need to conduct systematic performance and hydrodynamics investigation using various sizes of digesters. One reason for the controversies and uncertainties about the effect of mixing is that mixing in the digesters has not been quantified and characterized appropriately. In other words, none of the literature has focused on the hydrodynamic characteristics of the digesters for a particular mixing condition.

In this work, gas recirculation anaerobic digesters are selected to be investigated in detail due to their earlier mentioned advantages. In these digesters, gas holdup and its distribution are among the important hydrodynamic parameters, since the difference in gas holdup between the riser (draft tube) and the downcomer (the rest of the digester volume) generates the driving force for liquid/slurry circulation and the flow pattern. These affect the distribution of substrate and organisms, the uniformity of $\mathrm{pH}$ and temperature, the volume and formation of the dead zones, the extent of biomass and inert solids settling, the mean and local residence times, and the mass and heat transfer. Hence, quantification and investigation of these parameters are important for proper design, scale-up, and performance of gas recirculation digesters, as well as other types. Advanced non-invasive measurement techniques offer great promise in studying, the effects of various design and operating variables on gas 
holdup and its distribution, liquid/slurry velocity and its distribution in the 3D domain, flow pattern, turbulent parameters, dead zones, and local and mean residence time distributions.

In general, for multiphase flow systems, various types of non-invasive hydrodynamics measurement techniques have been developed and implemented (Dudukovic et. al. 1997). These include: tomographic techniques for the measurement of holdup distribution of phases, and particle tracking techniques for the measurement of flow pattern, velocity, and turbulent parameters.

Most of the tomographic techniques developed so far are single-modal and used for investigating dynamic two phase flow systems. Such techniques are not readily applicable to dynamic three-phase systems, such as anaerobic digesters of high solids concentration and gasliquid-solid fluidized bed reactors. Warsito et al. (1999) have proposed three strategies to perform dynamic three-phase imaging using a tomographic technique: (1) by combination of two different single-modal sensing systems, (2) by using an inherently multi-modal sensing system, and (3) by means of a single-modal sensing system with sound assumptions and/or a reconstruction technique capable of differentiating between three phases in the object space.

Examples of the first approach are the uses of electrical capacitance tomography (ECT) combined with gamma ray tomography for imaging multiple-components of gas, oil, and water in a pipeline (Johansen et al. 1996) and electrical resistance tomography (ERT) combined with gamma densitometry tomography (GDT) to measure the gas and solid concentration (holdup) profiles in a three-phase slurry bubble column (George et al. 2001). However, the crucial problem in using this approach is that the measurements are not conducted simultaneously in the same object domain. Hence, the reconstructed profiles may be severely distorted. In addition, this approach is complex to implement.

The second approach uses a single sensing technique, which is an inherently multimodal system capable of differentiating between two or more species in the object space. One advantage of this approach is that all the information required is available using the same measuring technique and image reconstruction. Examples of this approach are a dualfrequency ultrasonic method implemented by Warsito et al ( 1995) to measure gas and solid concentration distributions in a three-phase slurry bubble column and a multi-modal ultrasonic tomography with two-parameter sensing (ultrasonic attenuation and time-of-flight) implemented by Warsito et al $(1999,1995)$ to measure the cross-sectional distributions of gas and solid concentrations in a gas-liquid-solid slurry bubble column. However, the technique is limited to relatively low concentrations of gas or solids, lower than $20 \%$ for both.

The third approach uses a single-modal sensing system with sound assumptions and/or a reconstruction technique capable of differentiating between three phases in the object domain. An example is the use of electrical capacitance tomography with a neural networkbased image reconstruction technique proposed by Nooralahiyan and Hoyle (1997). To enable the identification of gas bubbles and water drops in an oil environment, they used a singlelayer feed forward neural network with a double-step sigmoid function to replace the one-step sigmoid function in the neural network computing. However, one problem is that the feed forward neural network needs prior knowledge of the flow pattern for the training before any measurement is made. This makes the technique impractical for real application when training is not possible, particularly for real time imaging of a complex flow when the pattern is highly fluctuating and unknown before an exact image is obtained. Recently, Rados (2003) used a single source gamma ray computed tomography (CT) to estimate the distribution of the dynamic three phases in a slurry bubble column. He used two assumptions (cross-sectionally 
uniform solids loading and constant cross-sectional time averaged gas hold up) at certain operating conditions within the fully developed flow region where these assumptions should not cause significant errors. However, these assumptions cannot be generalized for all operating conditions and for other gas-liquid-solids fluidized systems. Therefore, an advanced non-invasive measurement technique based on dual source (energy) that can measure the phase distribution in dynamic three phase systems needs to be developed.

Various velocity measurement methods have been developed and used for multiphase flow systems. These can be classified into two categories, based on the type of the system: for non-opaque (optical based methods) and for opaque systems. Most optical based methods (e.g., Pulsed Laser Velocimetry (PLV) and Laser Doppler Velocimetry (LDV)) cannot be applied to two-phase and three-phase flows with large volume of the dispersed phase. For the case of opaque systems, Magnetic Resonance Imaging (MRI) can be used, but its three-dimensional intensity distribution, using a transverse-phase encoding gradient, is based on the assumption that the motion of the flow is steady in the statistical sense along the flow direction within the test section. Apart from this, the MRI method needs knowledge of a general flow direction to get accurate information. Positron emission particle tracking (PEPT) and computer-automated radioactive particle tracking (CARPT) techniques are suitable for opaque media, but they involve single particle measurements (Rammohan et al. 2001). Kantaz et al. (2001) developed a multiple radioactive particle tracking (MRPT) technique using gamma-ray cameras. They conducted MRPT experiments on polyethylene-fluidized beds with up to five particles present in the column. However the technique failed to give information about the entire 3D domain which CARPT does give. Hence, there is a need to advance CARPT into tracking of multiple particles, which can allow simultaneously investigating the hydrodynamics of various phases and solid types (size, shape, density) of the system.

In our Chemical Reaction Engineering Laboratory (CREL), gamma ray computed tomography (CT) using a single sealed source and single particle computer automated radioactive particle tracking (CARPT) have been used extensively. CT has been used to measure and study the time averaged cross-sectional phase distributions and their radial profiles along the reactor height in two phase systems and in three phase flow systems when the solids phase is stationary (i.e. packed beds, monoliths, etc.) (Luo 2005; Rados 2003; Rammohan 2002; Roy 2006). CARPT has been used to measure and investigate the liquid's or solid's 3D velocity distribution, flow pattern, mixing intensity, turbulent parameters, dead zones, and residence time distribution in various multiphase flow systems (Luo 2005; Rados 2003; Rammohan 2002; and many others). In addition, the knowledge and findings obtained by these techniques can be used as benchmark data to evaluate the computational fluid dynamics (CFD) models and their closures for reactor design, scale-up, and optimization.

Anaerobic digesters and dynamic three phase gas-liquid-solid systems for various industrial practices in general, are highly opaque systems and contain a large volume fraction of the dispersed solids as well as gas phase. Thus, CARPT and CT, in addition to the techniques such as dual source computed tomography (DSCT) and multiple particle computer automated radioactive particle tracking, (MP-CARPT) are required. These techniques will provide valuable information about the hydrodynamic behavior of anaerobic digesters and other multiphase systems. However, both DSCT and MP-CARPT techniques are novel and need to be developed, tested, and implemented. 


\subsection{Objectives}

The overall objective of this project is to advance the fundamental knowledge and understanding of the design, scale up, operation and performance of high solids loading cow manure anaerobic digesters to promote bio-energy production and to eliminate major environmental pollution problems. This can be achieved by systematically studying the cow manure anaerobic digestion performance, mixing and hydrodynamics and their impact on the digesters performance in different configurations and sizes via implementing CARPT, CT and CFD and by developing novel MP-CARPT and DSCT techniques. The accomplishments of the project were achieved in a collaborative effort among Washington University, Oak Ridge National Laboratory and Iowa Energy Center teams. Figure 1.3 summarizes the accomplished objectives and tasks of this study.

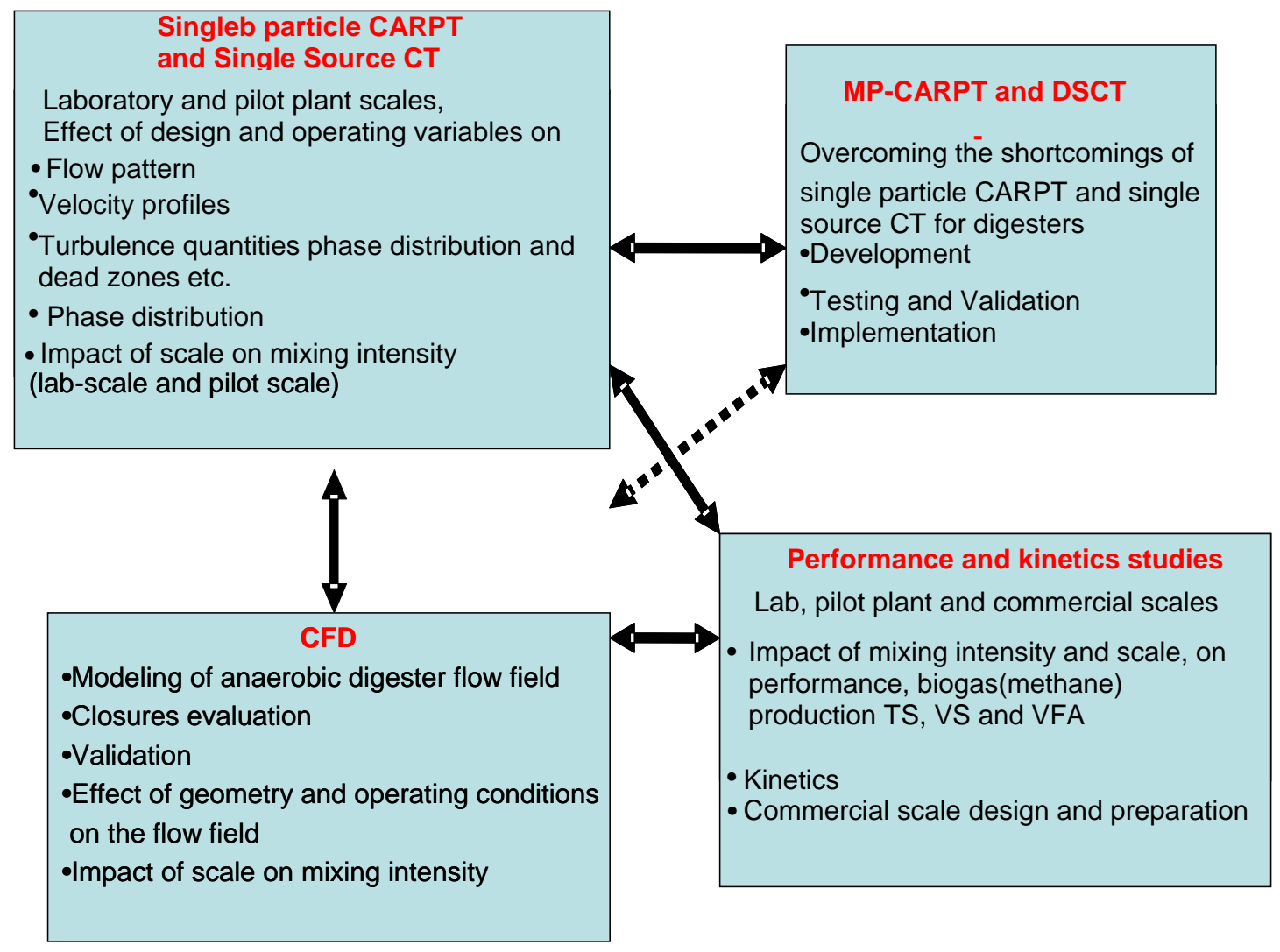

Figure 1.3: Integration of the accomplished objectives and tasks of this study 


\subsection{Tasks Accomplished:}

The following tasks were accomplished through a close collaborative effort among Washington University (WU), Oak Ridge National Laboratory (ORNL), and Iowa Energy Center teams.

\section{Tasks For The First Year:}

1. Design and development of laboratory scale digesters with different means of mixing and operating conditions.

2. Development a single particle CARPT technique and related structure that is suitable for the digester application. Initiate the development for multiple-particle tracking technique (MP-CARPT).

3. Initiation of CARPT experimental investigations on the laboratory scale digester.

4. Review of the tomography techniques, designing the dual source computed tomography (DSCT) technique, and initiating its development and construction.

\section{Tasks For The Second Year}

1. Experimental investigations of the mixing and hydrodynamic parameters of the digester, using single particle CARPT and single source CT.

2. Anaerobic digester performance studies on laboratory scale digesters to evaluate the effect of mixing and hydrodynamics on the digesters' performance.

3. Development of multiple-particle CARPT (MP-CARPT) and dual source CT (DSCT) techniques.

4. Initiation of models development (kinetics model; computational fluid dynamics (CFD) based model; evaluation and validation of CFD with CARPT and CT data)

5. Design and construction of a pilot plant scale anaerobic digester to evaluate its performance and hydrodynamics.

\section{Tasks For The Third Year}

1. Experimental investigation of the mixing and hydrodynamic parameters and their effects on the digester's performance using single particle CARPT and single source CT.

2. Anaerobic performance study on the laboratory scale to evaluate the effect of mixing and hydrodynamics on the digester's performance.

3. Development of multiple-particle CARPT (MP-CARPT) and dual source CT (DSCT) techniques and their implementation on the laboratory scale anaerobic digesters. 
4. Further kinetics models evaluation and development.

5. Further development of computational fluid dynamics (CFD) model for the anaerobic digester, and evaluation and validation of its closures with CARPT.

6. Design, construction and operation at ORNL of a pilot plant scale anaerobic digester to evaluate its performance and its flow pattern and hydrodynamics, using the developed techniques.

7. Redesign of the large scale anaerobic digester unit at the Iowa Energy Center (BECON) and test operation with air-water.

8. Evaluation of the reactor scale and the operating conditions on the mixing and reactor performance by comparing the experimental results obtained with laboratory and pilot plant units.

9. Evaluation of the hydrodynamics of the studied anaerobic digesters.

\section{$\underline{\text { Tasks For The Fourth Year (no-cost extension) }}$}

1. Further laboratory anaerobic digester performance studies and initiation of pilot plant performance study at Washington University.

2. Further development and testing of MP-CARPT and DSCT.

3. Evaluation of the current algorithm for DSCT image reconstruction.

4. Initiation of the development of a new algorithm for DSCT image reconstruction.

5. Further experimental investigations of the hydrodynamics of laboratory and pilot plant anaerobic digesters, using CARPT and CT.

6. Further development, evaluation, and validation of the CFD model and its closures for anaerobic digesters' design and scale-up

\section{Tasks For The Fifth Year (no-cost extension)}

1. Validation, testing and implementation of MP-CARPT

2. Completion of the data processing and analyses of CARPT and MP-CARPT results.

3. Completion of the performance studies of the pilot plant digester and their analyses at Washington University.

4. Completion of the algorithm development for DSCT image reconstruction.

5. Final report preparation.

\subsection{Structure of the final Report:}

The final report structure consists of chapters and appendices which outline the investigations and the development made during the duration of this grant. All the details are reported in the quarterly reports, theses, and published manuscripts which are attached. Appendices 1 and 2 represent the completed theses (where the authors (graduate students) who have the copyright have given permission to be used by DOE and in the final report). Appendix 3 represents part of another doctoral thesis under preparation. To avoid copyright issues, for the details reported in published manuscripts, these manuscripts are cited only, and are not attached as appendices. 


\subsection{Published Manuscripts and Theses:}

- Karim, K., Klasson, K.T., Drescher, S.R., Ridenour, W., Borole, A.P., and Al-Dahhan, M.H. (2007) "Mesophilic Digestion Kinetics of Manure Slurry", Applied Biochemistry and Biotechnology (in press).

- Karim, K., Hoffmann, R., and Al-Dahhan, M.H. (2007) "Digestion of sand-laden Manure Slurry in an Upflow Anaerobic Solids Removal (UASR) Digester", Biodegradation (in press).

- Borole, A. P., Klasson, K. T., Ridenour, W., Holland, J., Karim, K., and Al-Dahhan, M. H. (2006) "Methane Production in a 100-L Upflow Bioreactor by Anaerobic Digestion of Farm Waste”, Applied Biochemistry and Biotechnology, 129-132 (1), 887-896.

- Mehul S. V. and Al-Dahhan M.H. 2005. Flow pattern visualization in a mimic anaerobic digester using CFD. Biotechnology and Bioengineering, 89 (6), 719-732.

- Mehul S. Vesvikar, Varma R., Karim K., Al-Dahhan M.H. 2005. Flow pattern visualization in a mimic anaerobic digester: Experimental and Computational Studies. Water Science and Technology, 52 (1-2), 537-543.

- Mehul S. Vesvikar, Varma R., Karim K., Al-Dahhan M.H. 2005. Flow pattern visualization in a mimic anaerobic digester: Experimental and computational studies. Anaerobic Digestion 2004 Proceedings, Vol 1, 123-128.

- Karim, K., Hoffmann, R., Klasson, K.T., and Al-Dahhan, M.H. (2005) "Anaerobic digestion of animal waste: Effect of mode of mixing”, Water Research, 39 (15), 35973606.

- Vesvikar, M.S., Varma, R., Karim, K., Al-Dahhan, M.H. (2005) "Flow Pattern Visualization in a Mimic Anaerobic Digester: Experimental and Computational Studies”, Water Science and Technology, 52 (1-2), 537-543.

- Karim, K., Hoffmann, R., Klasson, K.T., and Al-Dahhan, M.H. (2005) "Anaerobic digestion of animal waste: Waste strength versus impact of mixing", Bioresource Technology, 96 (16), 1771-1781.

- Karim, K., Klasson, K.T., Hoffmann, R., Dresher, S.R., Depaoli, D.W. and Al-Dahhan, M.H. (2005) "Anaerobic digestion of animal waste: Effect of mixing”, Bioresource Technology, 96 (14), 1607-1612.

- Hoffman R., Varma R., Vesvikar M., Karim K., Al-Dahhan M.H., Angenent L.T. 2004. Effect of shear rate on hydrodynamics, performance, and microbial community dynamics for an anaerobic digester treating cow waste. Anaerobic Digestion 2004 Proceedings, Vol 3, 1755. 
- Karim K., Varma R., Vesvikar M. and Al-Dahhan M. H. 2004. Flow pattern visualization of a simulated digester. Water Research, 38 (17), 3659-3670.

\section{Manuscripts under review:}

- Varma R, and Al-Dahhan MH. (2007) Effect of sparger design on hydrodynamics of a gas recirculation anaerobic bioreactor. In review by Biotechnology Bioengineering.

- Varma R, Bhusarapu S, O'Sullivan J A and Al-Dahhan M H. (2007). Comparison of alternating minimization and expectation maximization algorithms for single source gamma ray tomography. In review by Measurement Science and Technology.

\section{Theses:}

- Vesvikar M. (2006) Understanding the hydrodynamics of anaerobic digester for bioenergy production.[D.Sc. Thesis], St. Louis -USA, Washington University.

- Hoffmann RA. 2005. Effect of shear on the performance and microbial ecology of anaerobic digesters treating cow manure from dairy farms. [Masters thesis]. St. Louis USA: Washington University.

- Varma R. (2007). Phase distribution using tomography and performance of anaerobic digesters for bioenergy generation [Ph.D.Thesis in preperation] Washington University -St. Louis MO USA 


\section{Chapter 2 : Performance Studies of Anaerobic Digesters}




\section{Performance Studies of Anaerobic Digesters}

Although industrial wastes have successfully utilized anaerobic digestion to reduce organic pollutants in waste streams for over 30 years, implementation of anaerobic digesters for the purpose of treating animal and farm wastes has had high failure rates as mentioned earlier. Such high failure rates are believed to be mainly due to poor design, construction and mixing, and absence of a well acclimated microbial community. Mixing is an important parameter in the operation of anaerobic digesters which enhances substrate contact with the microbial community, improves $\mathrm{pH}$ and temperature uniformity, prevents stratification and scum accumulation, facilitates the removal of biogas from the digestant, reduces the inactive zones or volumes (dead zones) and aids in particle size reduction. Information in the literature on the effect of mixing on anaerobic digestion is contradictory. A better understanding of the role of mixing in anaerobic digestion will result in better design and operation, leading to a reduction in failure rate and increased utilization of anaerobic technology on the farm. The United States produce 1.8 billion metric tons of cow manure annually (EPA; USDA 2002/2003). This waste is often improperly stored and handled, leading to several environmental problems, such as surface and groundwater contamination and emissions of atmospheric pollutants. Anaerobic digestion is a waste treatment technology by which microorganisms break down organic materials in the absence of oxygen to create biogas. Biogas consists of approximately 65\% methane and 35\% carbon dioxide, with traces of dinitrogen gas and gaseous sulfur compounds. With such a high methane content, biogas can be used as an energy source. Besides reducing greenhouse gas emissions and producing a renewable energy source, anaerobic digestion systems also reduce odor, protect water quality, control ammonia release, and produce a fertilizer.

Anaerobic digestion occurs in a series of steps as mentioned earlier employing different types of Bacteria to break down complex polymers into simple carbon compounds that can subsequently be utilized by Archaea to produce methane (Figure 2.1). Five physiologically different groups of microorganisms are involved: three groups of Bacteria (fermentative bacteria, obligatory $\mathrm{H}_{2}$ producing acetogens, and homoacetogens) and two groups of Archaea (hydrogenotrophic methanogens and acetoclastic methanogens).

The extent of mutual reliance among these microorganisms varies substantially. While the lower members of the food chain depend on the higher members for substrate, the lower members also positively impact higher members by removing metabolic products. An important example of this type of mutual reliance is the syntrophic relationship which is a special case of symbiotic relationship in which two organisms with different metabolic capabilities work together to degrade a compound. This type of relationship usually develops due to energy limitations (Madigan et al. 2003; Schink 1997). In anaerobic systems, syntrophic relationships exist between hydrogen producing acetogenic bacteria and hydrogenotrophic methanogens. Organisms involved in syntrophic relationships generally develop in close, physical associations, such as flocs, to decrease diffusion limitations of metabolic products.

Regarding the role of mixing, all the studies in the literature on cow manure anaerobic digestion have been performed on laboratory scale digesters ( $\sim 4$ lit). No investigations have been reported in the literature that were conducted in pilot plant and/or commercial scales to characterize the impact of mixing intensity, operating conditions and design parameters on the performance of the digesters. In this work, the following studies were systematically 
performed using cow manure and are outlined in this chapter. However, the details of them are reported in the quarterly reports, published manuscripts, theses and appendices supported by this grant.

These studies include:

2.1 Effect of mixing intensity, operating conditions and design parameters on the anaerobic digestion of animal waste (cow manure) using laboratory scale ( 3.78 l) digesters.

This includes the following investigations:

2.1.1 Effect of hopper bottom and method of mixing using $50 \mathrm{~g} / \mathrm{L}$ TS loading of cow manure.

2.1.2 Mixed vs unmixed digesters using $50 \mathrm{~g} / \mathrm{L}$ TS loading of cow manure.

2.1.3 Effect of method of mixing and TS loading on the performance of anaerobic digesters.

2.1.4 Effect of mixing on anaerobic digestion of animal waste (cow manure) using laboratory scale gas recirculation digesters.

2.1.5 Effect of anaerobic digester design and mode of mixing on the performance of laboratory scale digesters using animal waste (cow manure).

2.1.6 Digestion of and-laden manure slurry in an up flow anaerobic solids removal digester.

2.2 Effect of shear on the performance and microbial ecology of anaerobic digesters.

2.3 Effect of mixing and digester scale on the performance of anaerobic digesters: Pilot plant scale digesters studies.

2.3.1. Effects of mixing and scale on the biogas production in a pilot plant scale anaerobic digester by comparing lab scale and pilot plant scale digesters performance.

2.3.2. Effect of mixing intensity on the biogas production in a pilot plant scale anaerobic digesters.

2.4 Kinetics of animal waste (cow manure) anaerobic digestion.

2.5 Commercial scale animal waste anaerobic digester at Iowa Energy Center (IEC)

2.5.1. Operating Conditions and initial suggested modifications.

2.5.2. Reconstruction of the IEC large scale anaerobic digester and its flow test using air-water. 


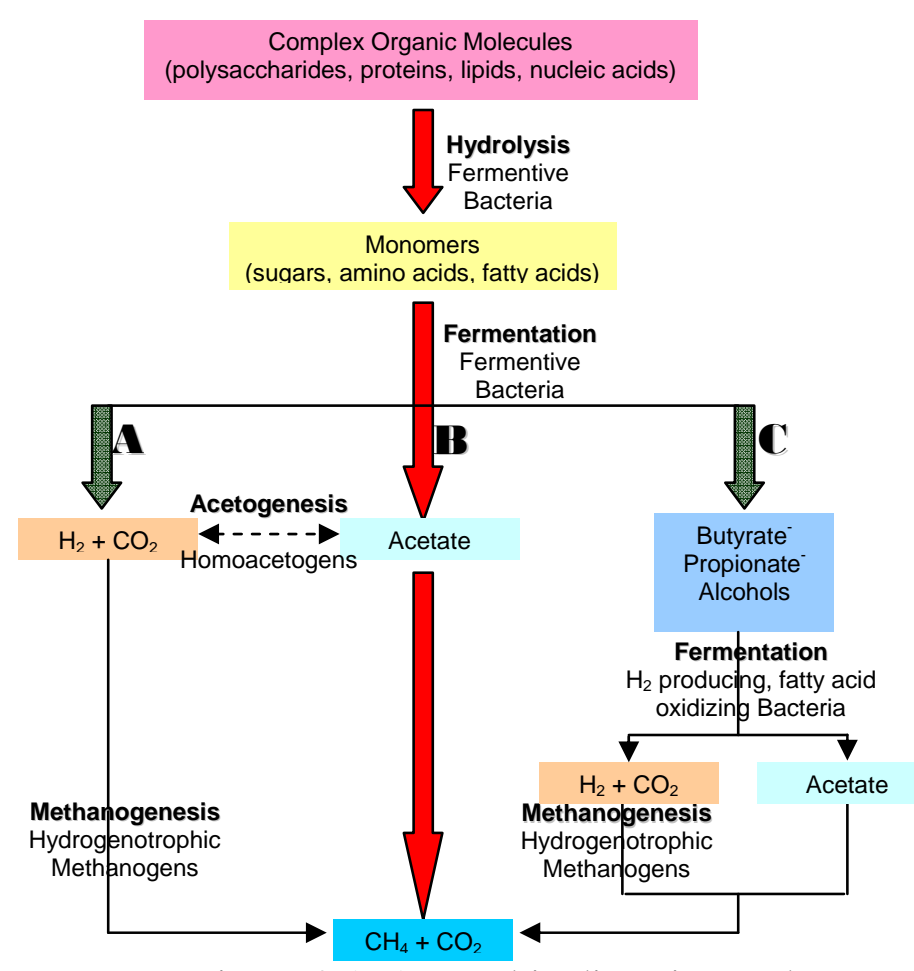

Figure 2.1: Anaerobic digestion pathway.

\subsection{Effect of mixing intensity, operating conditions and design parameters on the anaerobic digestion of animal waste (cow manure) using laboratory scale ( $\sim 3.78 \mathrm{l})$ digesters}

Studies were conducted in our laboratory (WU) and in ORNL to systematically investigate and characterize the impact of mixing intensity, operating conditions and design parameters on the digester performance. These systematic studies were performed at the same conditions in order to evaluate properly the contradictory findings in the literature about the effect of mixing on the anaerobic digester performance. It was found, that neither mixing nor method of mixing affects digester performance in the laboratory scale when treating feed manure containing 50 g/L TS (Total Solids). In this case, the local generated gas bubbles are enough to promote the needed agitation and mixing. However, this was not found to be the case when treating a higher concentration feed manure of $100 \mathrm{~g} / \mathrm{L} \mathrm{TS}$. At such TS loading rate or higher, the performance of the digesters was affected by both the presence of mixing and the method of mixing. The unmixed digester was found to produce the least amount of biogas, while the slurry recirculation was found to produce the most. Thus, the overall findings suggest that mixing plays more of a role in its effect on digester performance when treating thicker manure feeds and using large scale digesters. This has been confirmed by the results and findings obtained from the pilot-plant scale performance studies. This work includes the following investigations: 


\subsubsection{Effect of hopper bottom and method of mixing using $50 \mathrm{~g} / \mathrm{L}$ TS loading of cow manure}

This study was performed using cow manure with a total solids (TS) concentration of 50 $\mathrm{g} / \mathrm{L}$. Four digesters $\left(3.78 \mathrm{l}\right.$ ) were used: biogas recirculation (hopper bottom slope of $60^{\circ}$ ), biogas recirculation (hopper bottom slope of $25^{\circ}$ ), slurry recirculation (hopper bottom slope of $25^{\circ}$ ), and an impeller mixer (hopper bottom slope of $25^{\circ}$ ). The effects of hopper bottom slope and mixing method were investigated. All digesters were operated with a power input per unit volume of $8 \mathrm{~W} / \mathrm{m}^{3}$, corresponding to a gas recirculation rate of $1 \mathrm{~L} / \mathrm{min}$, an impeller speed of $275 \mathrm{RPM}$, and a slurry recirculation rate of $0.82 \mathrm{~L} / \mathrm{min}$.

Results showed no differences in performance between all four digesters after reaching steady-state. No statistically significant difference in chemical oxygen demand (COD) removal, biogas production rate, and other operational parameters were found. The methane yield, calculated based on the volatile solids (VS) loading rate of $2 \mathrm{~g} / \mathrm{L}$-day, was found to be between 0.21 and $0.27 \mathrm{~L} \mathrm{CH}_{4} / \mathrm{g}$ VS fed.

The details of the results are reported in Appendix 1 (Appendix B), $8^{\text {th }}$ and $9^{\text {th }}$ quarterly reports and the following published manuscript:

Karim, K., Hoffmann, R., Klasson, T. and Al-Dahhan, M.H. (2005) "Anaerobic digestion of animal waste: Effect of mode of mixing”, Water Res., 39 (15): 3597-606.

\subsubsection{Mixed vs. unmixed digesters using $50 \mathrm{~g} / \mathrm{L}$ TS loading of cow manure}

This study was also performed using cow manure with $50 \mathrm{~g} / \mathrm{L}$ TS concentration. Two digesters with $25^{\circ}$ sloped hopper bottoms, one unmixed (i.e. no agitation was provided by the impeller) and one mixed by an impeller at 275 RPM, were operated at the same time to compare the performance of mixed and unmixed digesters. This study also served as a verification of the reproducibility for the impeller mixed digester used in the study mentioned in section 2.1.1.

The methane yield found for these digesters, based on the VS loading rate of $2 \mathrm{~g} / \mathrm{L}$-day, was 0.27 and $0.31 \mathrm{~L} \mathrm{CH}_{4} / \mathrm{g}$ VS fed for the unmixed and impeller mixed digesters, respectively. The reproducibility of the study discussed in section 2.1.1. was verified with the performance of the impeller mixed digester used in this work. No statistical difference in biogas production was found between the impeller mixed digesters.

The details of the results are reported in Appendix 1 (Appendix B), quarterly reports and the following published paper:

Karim, K., Hoffmann, R., Klasson, T. and Al-Dahhan, M.H. (2005) "Anaerobic digestion of animal waste: Effect of mode of mixing”, Water Res., 39 (15): 3597-606. 


\subsubsection{Effect of method of mixing and TS loading on the performance of anaerobic digesters.}

This study was performed to determine if the method of mixing became important at a higher TS loading rate of $100 \mathrm{~g} / \mathrm{L}$ TS. Four laboratory scale (3.78 l) digesters with $25^{\circ}$ sloped hopper bottoms were used. These digesters were: unmixed, biogas recirculation, impeller, and slurry recirculation digesters. The three mixed digesters were again operated at a power input per unit volume of $8 \mathrm{~W} / \mathrm{m}^{3}$, corresponding to a gas recirculation rate of $1 \mathrm{~L} / \mathrm{min}$, an impeller speed of $275 \mathrm{RPM}$, and a slurry recirculation rate of $0.82 \mathrm{~L} / \mathrm{min}$.

Results showed that there was statistically significant difference in the biogas production rates of the digesters. The digester mixed by slurry recirculation was found to produce $22 \%$ more gas than the unmixed digester during steady-state conditions. No significant difference in biogas production was found between the slurry recirculation digester and the impeller mixed digester. However, a statistically significant difference in biogas production was found between the slurry recirculation digester and the gas recirculation digester, revealing that the slurry recirculation digester produced $10 \%$ more biogas than the biogas recirculation digester. The methane yields for the unmixed, biogas recirculation, impeller, and slurry recirculation digesters were $0.19,0.21,0.23$, and $0.24 \mathrm{~L} \mathrm{CH}_{4} / \mathrm{g}$ VS fed, based on a VS loading rate of $3.2 \mathrm{~g}$ VS/L-d.

The details of this work are reported in Appendix 1, quarterly reports and the following published paper:

Karim, K., Hoffmann, R., Klasson, T. and Al-Dahhan, M.H. (2005) "Anaerobic digestion of animal waste: Effect of mode of mixing”, Water Res., 39 (15): 3597-606.

\subsubsection{Effect of mixing on anaerobic digestion of animal waste (cow manure) using laboratory scale gas recirculation digesters}

Six laboratory scale (3.78 l) biogas mixed anaerobic digesters were operated to study the effect of biogas recycling rates and draft tube height on their performance. The digesters produced methane at 0.40 to 0.45 liter per liter of digester volume per day. A higher methane production rate was observed in unmixed digesters, while increased biogas circulation rate reduced methane production. However, different draft tube heights caused no difference in the methane production rate. The similar performance of the six mixed and unmixed digesters is the result of the low solids concentration (50 g dry solids per liter of slurry) in the fed animal slurry, which could be sufficiently mixed by the naturally produced biogas

Figures 2.2 to 2.6 show a schematic diagram and the photos of these digesters.

Further details are discussed in quarterly reports and the following published manuscript:

Karim, K., Thomas Klasson, K. Hoffmann, R. Drescher, S. R. Depaoli, D. W. Al-Dahhan, M. H. 2005. Anaerobic digestion of animal waste: effect of mixing. Bioresour Technol 96(14):1607-12. 


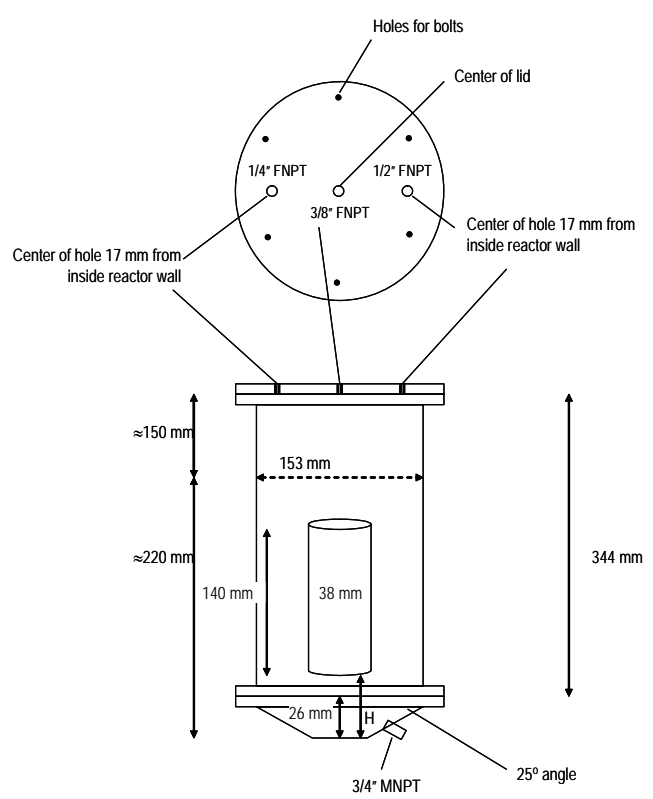

Figure 2.2. Schematic of the anaerobic digesters. Circular body in the center of the digester is a hollow draft tube suspended from the top of the digester.

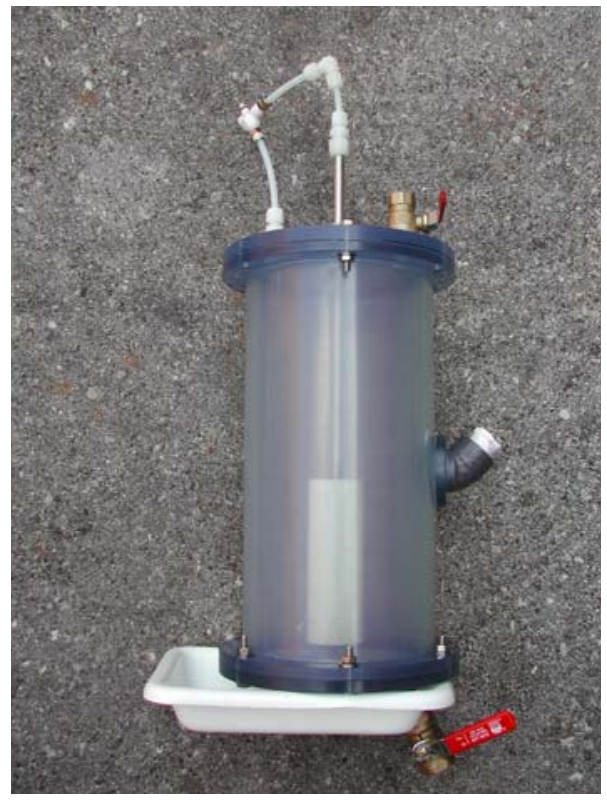

Figure 2.3. A Photograph of one of the anaerobic digesters used. From left to right the connections on top are gas outlet, gas inlet, and feed addition.

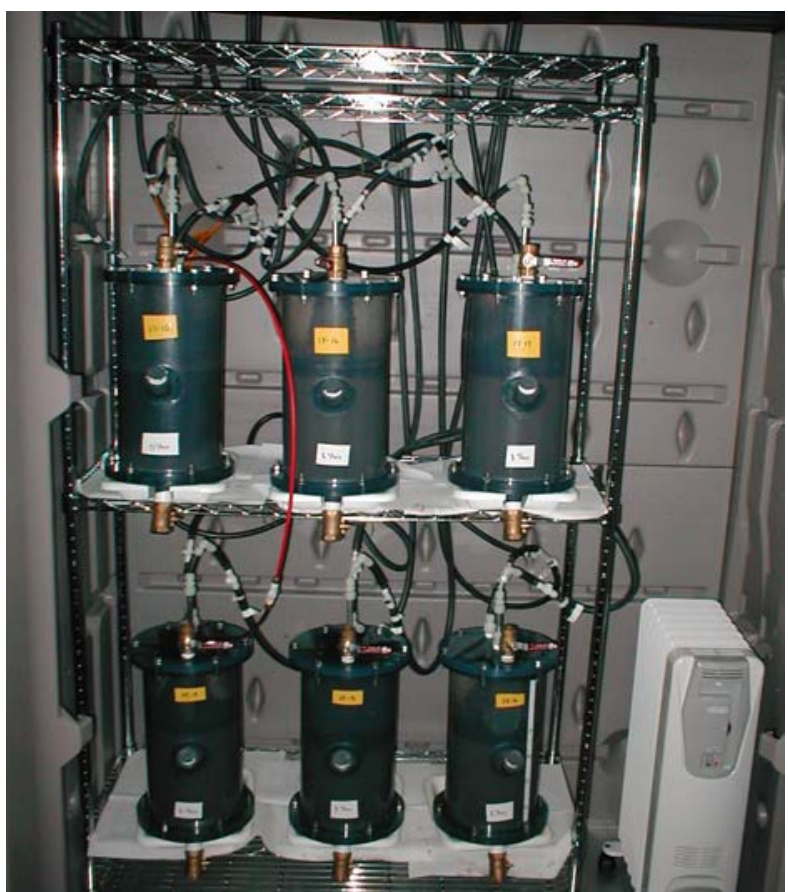

Figure 2.4. Six digesters housed in a temperature-controlled environment.

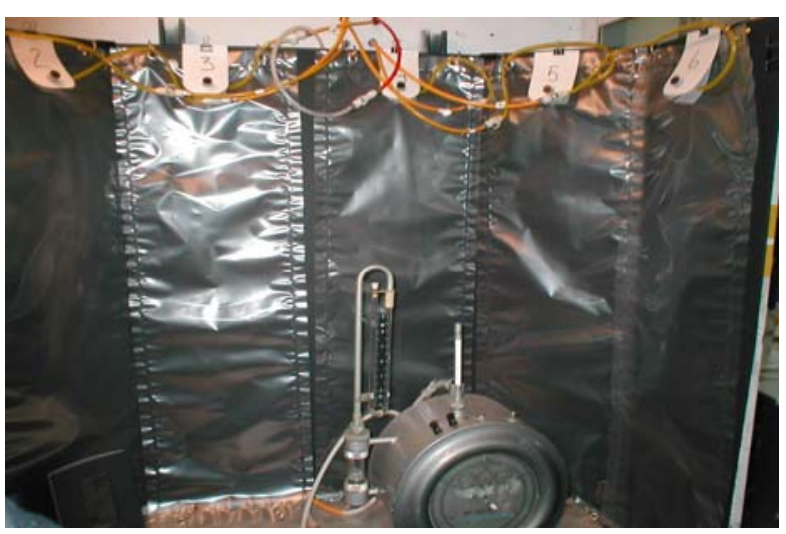

Figure 2.5. Gas bags for collecting biogas and wet gas test meter for measuring amount of gas generated. 


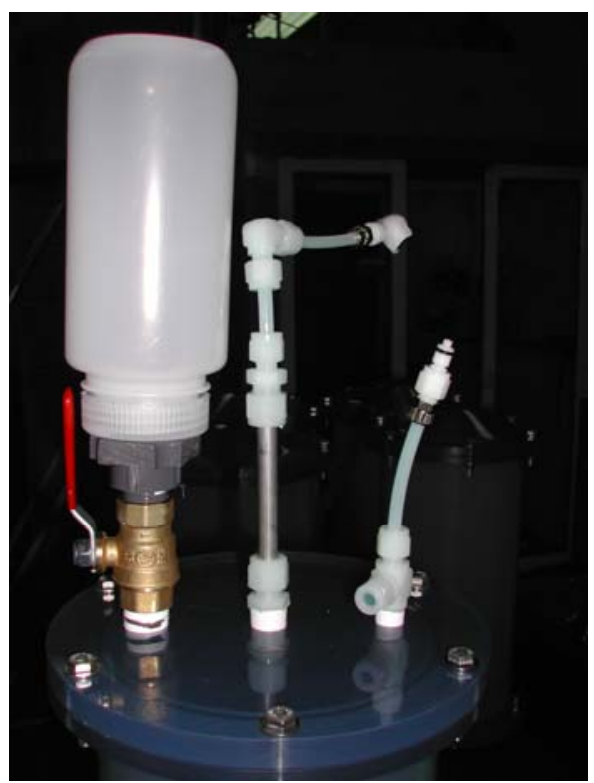

Figure 2.6. The slurry was fed through the top of the digester via a ball-valve.

\subsubsection{Effect of anaerobic digester design and mode of mixing on the performance of laboratory scale digesters using animal waste (cow manure)}

Laboratory-scale (3.78 l) digesters were operated to study the effect of mixing (via biogas recirculation, impeller mixing, and slurry recirculation) on biogas production. Three sets of experiments were performed using cow manure slurry feed with either 50,100 or 150 g/L total solids (TS) concentrations (referred as 5, 10, and 15\% manure slurry). The experiments were conducted at a controlled temperature of $35^{\circ} \mathrm{C}$ and a hydraulic retention time of 16.2 days, resulting in TS loadings of 3.1, 6.2 and $9.3 \mathrm{~g} / \mathrm{L}-\mathrm{d}$ for 5,10 and 15\% manure slurry feeds, respectively. Results showed that the unmixed and mixed digesters performed quite similarly when fed with 5\% manure slurry and produced biogas at a rate of 0.84-0.94 $\mathrm{L} / \mathrm{L}-\mathrm{d}$. The methane yield was found to be $0.26-0.28 \mathrm{~L} \mathrm{CH} / \mathrm{g}$ volatile solids loaded. However, the effect of mixing and the mode of mixing became important when the digesters were fed with thick manure slurry feeds (10\% and 15\%). Digesters fed with 10 and 15\% manure slurry and equipped with external mixing produced about $10 \%-30 \%$ more biogas than the unmixed digester. While the mixed digesters produced more biogas than unmixed digesters, digester mixing during start-up was not beneficial, as it resulted in lower $\mathrm{pH}$, performance instability and prolonged start-up time. Mixing using biogas recirculation system was found not to be effective in the case of $15 \%$ manure slurry feed under the experimental conditions studied.

Deposition of solids inside the digesters was not observed in the case of 5\% manure slurry, but it became significant in the case of $10 \%$ and $15 \%$ manure slurry. Therefore, mixing issue becomes more critical with thicker manure slurry.

The schematic diagrams of these digesters are shown in Figure 2.7. 
The detailed results are discussed in Appendix 1 (Appendix B), quarterly reports and in the following published paper:

Karim, K., Hoffmann, R. Thomas Klasson, K. Al-Dahhan, M. H.(2005). Anaerobic digestion of animal waste: effect of mode of mixing. Water Res 39 (15):3597-606.

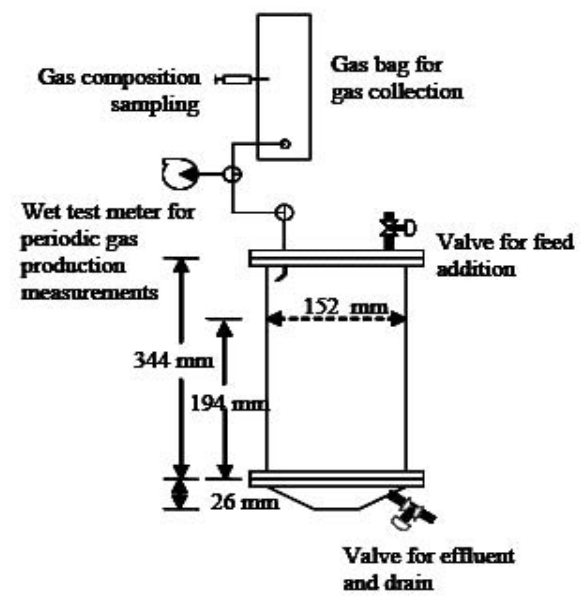

(a)

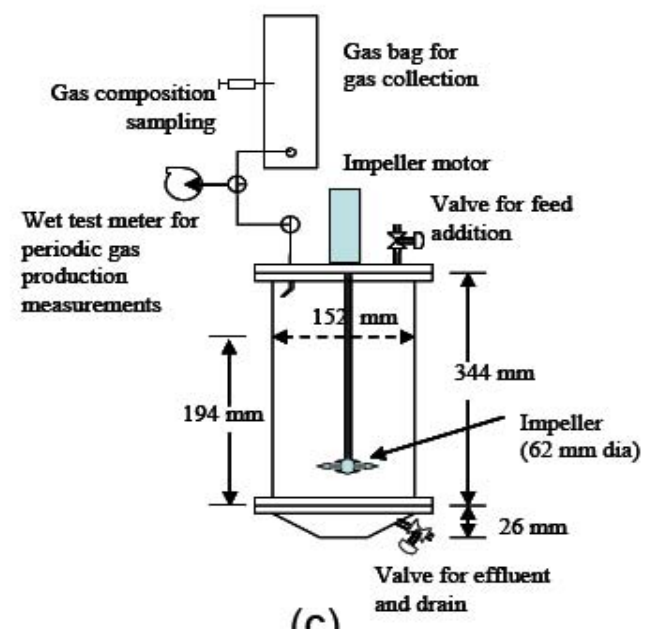

(c)

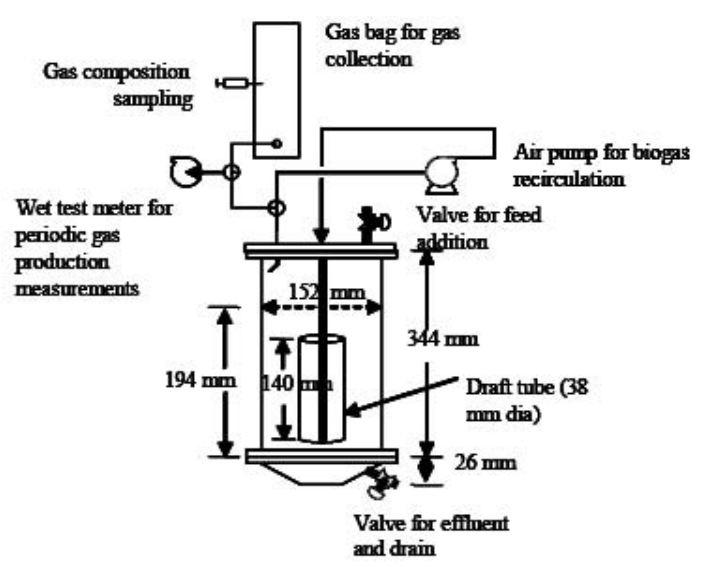

(b)

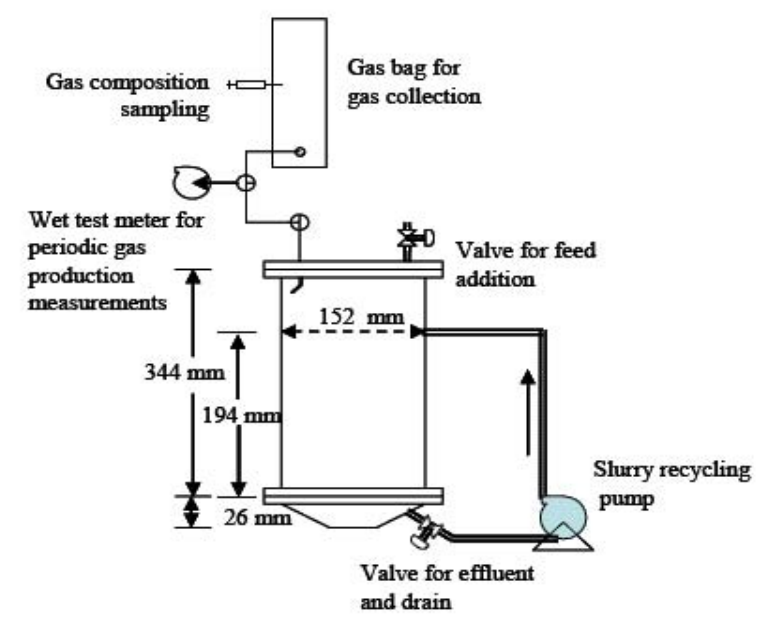

(d)

Figure 2.7: Schematic diagrams of the anaerobic digesters used

\subsubsection{Digestion of sand-laden manure slurry in an upflow anaerobic solids removal digester}

Studies on the performance of a laboratory scale up-flow anaerobic solids removal (UASR) digester (shown in Figure 2.8) were carried out using sand-laden cow manure slurries having total solids (TS) concentrations of 50 and $100 \mathrm{~g} / \mathrm{l}$. Hydraulic retention time (HRT) was maintained as 32.4 days, which resulted in the volatile solids loading rates of 1 and $1.64 \mathrm{~g} / \mathrm{l}-\mathrm{d}$. 
The UASR system was designed to remove sand from the manure slurry, while digesting biodegradable solids inside a single reactor. To enhance the contact of microorganisms and substrate, the liquor from the top of the digester was recirculated through the bed of settled solids at its bottom. Volatile solids (VS) reduction through this process was observed to be $62 \%$ and $68 \%$ in the case of feed slurries having total solids (TS) concentrations of 50 and 100 $\mathrm{g} / \mathrm{l}$ (referred as $5 \%$ and $10 \%$ feed slurries), respectively. The methane production rates were observed to be 0.22 and $0.38 \mathrm{l} / \mathrm{l}-\mathrm{d}$, while methane yield was 0.21 and $0.27 \mathrm{l} \mathrm{CH} / \mathrm{g}$ VS loaded, for $5 \%$ and $10 \%$ feed slurries, respectively.

This indicates that the increase in the volatile solids loading had a positive impact on methane production rate and methane yield. It would be of interest to study the performance of a UASR digester at higher solids loadings and with longer solids retention times. Nonetheless, the presented study showed that sand-laden manure slurries can be successfully digested in a UASR digester producing methane energy equivalent to $19.4 \mathrm{kWh}$ per $\mathrm{m}^{3}$ of digester volume per day.

The reported study demonstrates a new approach to sand-laden manure slurry digestion in an upflow anaerobic solids removal (UASR) digester. Since the studied system works both as a settling unit as well as a high rate digester, the approach was found especially promising.

The details and results of this work are discussed in the quarterly reports and in the following published manuscript:

Karim, K., Hoffmann, R., and Al-Dahhan, M.H. (2007) "Digestion of sand-laden Manure Slurry in an Up flow Anaerobic Solids Removal (UASR) Digester”, Biodegradation (In press).

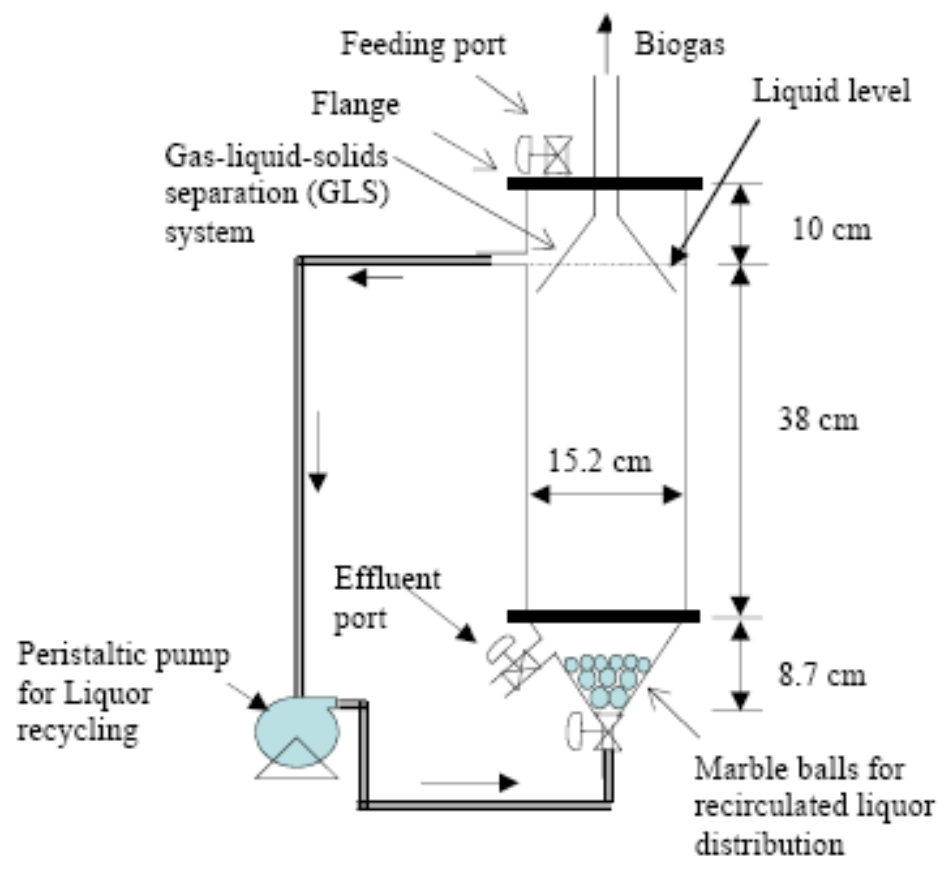

Figure 2.8: Schematic of the experimental UASR setup 


\subsection{Effects of shear on the performance and microbial ecology of anaerobic digesters treating cow manure}

The objective of this work was to study the effects of mixing intensity (i.e. applied shear) on the digester performance, microbial ecology, and syntrophic relationships in anaerobic digesters treating cow manure. Stirred tank digesters (laboratory scale of 4.5 liters volume (Figure 2.9) running at four different mixing intensities, 1500, 500, 250, and 50 RPM, were operated over a 160 day period with a series of five different organic loading rates between 0.6 and $3.5 \mathrm{~g} \mathrm{VS/L-d.}$

Results of this study demonstrated that using small scale digesters, different mixing intensities produced no effect on the biogas production of the continuously-stirred digesters during steady-state periods. A methane yield of $0.241 \pm 0.007 \mathrm{~L} \mathrm{CH}_{4} / \mathrm{g}$ VS fed was obtained by pooling the data of all four digesters during steady-state periods. However, digester performance was affected by mixing intensity during startup of the digesters. The 1500 and 500-RPM digesters were negatively impacted with lower biogas production rates and higher volatile fatty acids concentrations.

Anaerobic digestion utilizes many different types of bacteria and archaea to break down organic molecules as mentioned earlier. Thus, performance of anaerobic digesters is closely tied with the composition of the microbial community. Therefore, the following two techniques were used in this work which is based on ribosomal RNA (rRNA) targeting oligonucleotide probes that hybridize to their complimentary sequences within environmental samples.

Fluorescent in situ hybridization (FISH): It is based on fluorescently- labeled probes hybridization in whole, fixed cells. This allows for visualization of individuals or groups of individual population members in complex communities through the use of fluorescence microscopy. As a qualitative technique, FISH is mostly utilized to determine spatial associations of organisms in environmental samples.

Membrane hybridization: it is based on radioactively-labeled probes hybridizing to immobilize RNA extracted from an environmental sample. The degree of hybridization can be used to estimate the abundance of a target population. A qualitative technique, membrane hybridization is a powerful tool that can be used to detect population shifts over time, or the development of different microbial communities within systems of interest.

In this study, fluorescent in situ hybridization (FISH) revealed decreasing floc sizes beginning at week 4 and continued through week 26, at which time almost no flocs remained. This decrease in size, and subsequent loss of microbial flocs did not, however, produce any visible upsets in digester performance. A statistical difference was seen between the digesters in terms of VS removal, while no statistical difference was seen between the digesters in terms of VS removal efficiencies.

The details of the results of this work are discussed in following masters thesis attached as Appendix 1 
Hoffman RA.(2005). Effect of shear on the performance and microbial ecology of anaerobic digesters treating cow manure from dairy farms.[Masters thesis] St. Louis USA; Washington University-St. Louis).

In addition to the work of Hoffman (2005), membrane hybridiation analyses were conducted. Also to integrate the findings of this study with the mixing intensity, shear stresses and velocity field in 3D domain of the above mentioned digesters were measured using computer automated radioactive particle tracking (CARPT) technique and computational fluid dynamics (CFD). The details of this work will be submitted for publication shortly.

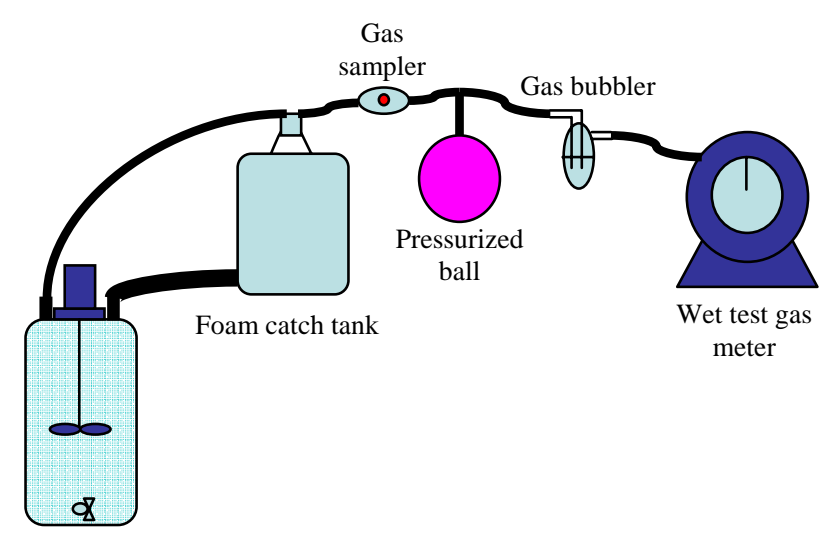

A

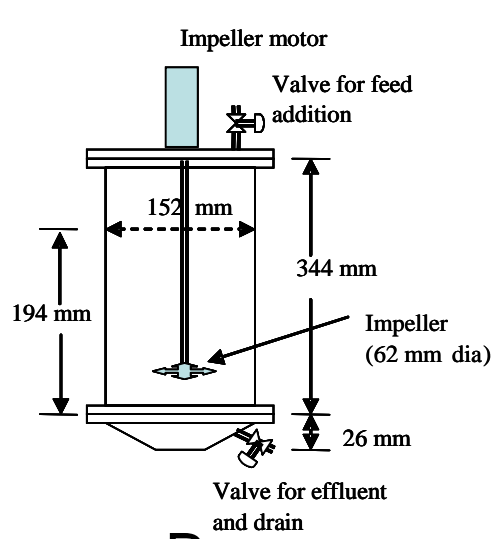

$\mathrm{B}$

Figure 2.9. Continuously-stirred anaerobic digesters setup (A) and diagram (B).

\subsection{Effects of mixing and digester scale on the performance of anaerobic digesters}

This study consists of the following investigations:

\subsubsection{Effects of mixing and scale on the biogas production in a pilot plant scale anaerobic digester by comparing lab scale and pilot plant scale digesters performance}

In spite of the crucial role of mixing in digester operation, contradictory findings are reported in the literature as mentioned earlier, about the necessity of mixing and the required mixing intensity to enhance the digester performance. There are many reasons for these controversies and uncertainties. One of them is, mixing is not adequately quantified and characterized in these systems. Another important reason is, most of these digester performance studies were performed in small laboratory-scale digesters and/or using low solids concentration. Our systematic investigations showed that with laboratory scale digesters and low solids loading, 
mixing does not affect the digester performance. Therefore, investigation of anaerobic digester in small laboratory scales do not contribute greatly in understanding influence of mixing on digester performance or in providing criteria for full scale digester design. However, laboratoryscale digesters are valuable in estimating kinetic parameters, in estimation of nutrient and alkalinity requirements and discovering potential problems like toxicity, because they are easy to control, efficient mixing and uniform environment can be guaranteed. On the other hand, experimentation on a large scale digester is necessary to elucidate the operational problems and difficulties like effects of improper mixing, clogging of feed and outlet ports, solids accumulation, foaming and so on.

Accordingly, in this work the following investigations were performed:

-Studying the effects of mixing on the performance of a pilot plant scale anaerobic digester.

-Demonstrating the effect of digester size on the role of mixing by comparing the labscale and pilot- scale digester performance.

The pilot-plant scale digester developed at the Oakridge National Laboratory (ORNL) had working volume of 97 liters (18 inches in diameter) and was geometrically similar to the laboratory-scale digester (Figure 2.10). This digester was first operated at ORNL then transferred and operated at Washington University (WU).

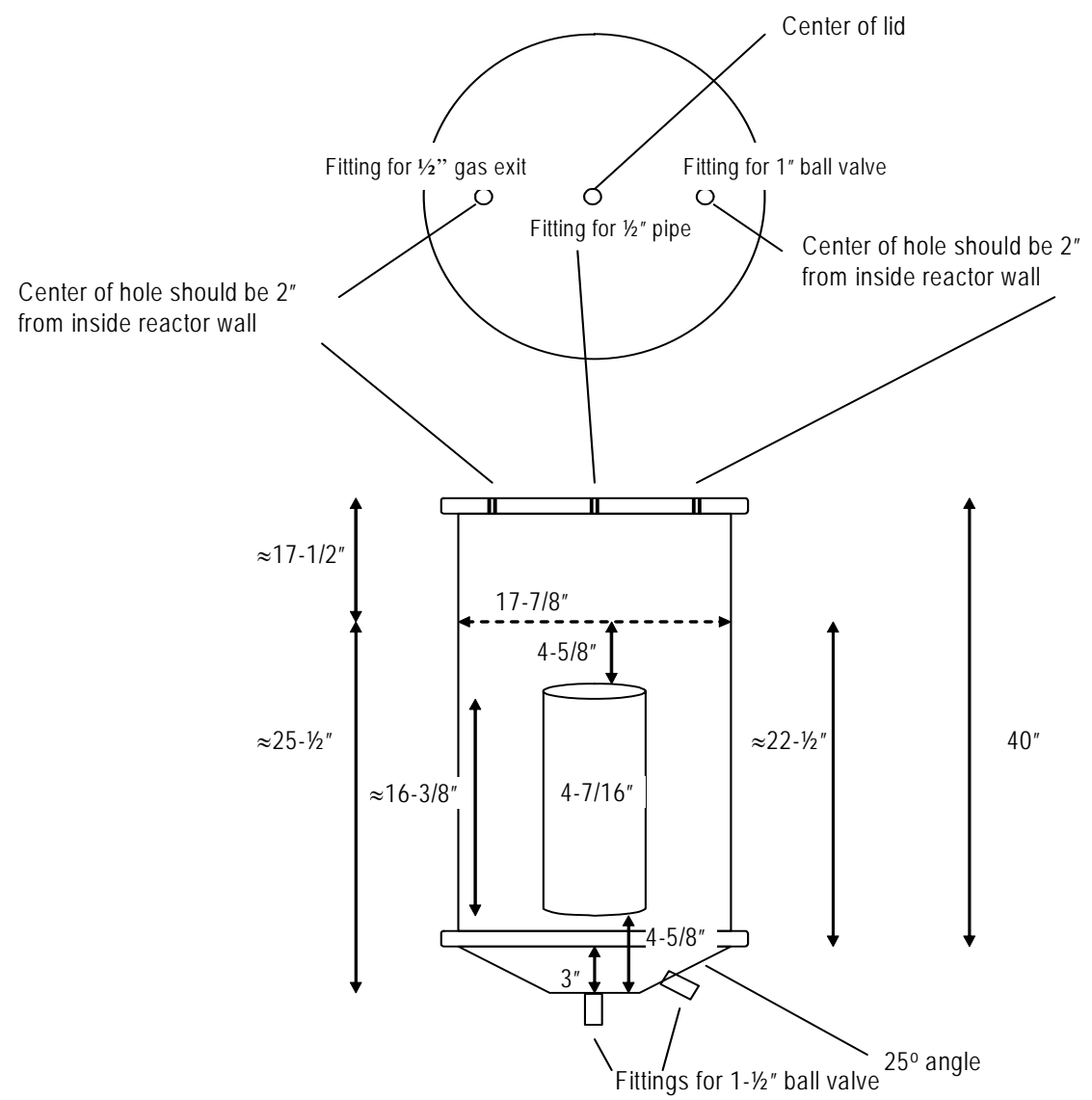

Figure 2.10: Schematic of the pilot-plant scale digester. 
The pilot-plant scale digester operation was started with biogas recirculation. After 70 days of operation of the pilot-scale digester in mixed condition, biogas recirculation was stopped and it was operated in unmixed condition for more than 70 days. Again the biogas recirculation was started and the digester was operated in mixed condition for more than 12 days, this was done to check the reproducibility of the results obtained. The biogas recirculation rate in pilot-scale digester was $9.07 \mathrm{l} / \mathrm{min}$, resulting in an input power density of $8 \mathrm{~W} / \mathrm{m}^{3}$, which corresponds to 1 $\mathrm{l} / \mathrm{min}$ biogas recirculation rate in the 6-inch laboratory scale unit.

In order to compare this pilot scale digester performance with that of laboratory scale, two identical laboratory-scale digesters with working volume of 3.87 liters (6 inches in diameter) using similar cow manure were used at Washington University. One was mixed by gas recirculation at a rate of $1 \mathrm{l} / \mathrm{min}$; digester was equipped with draft tube of diameter one fourth of digester diameter and a multipoint sparger to facilitate mixing. Another digester was unmixed; unmixed condition implies that no mixing is provided by external means, but digester is naturally mixed due to the evolution of biogas bubbles and addition of feed and effluent removal.

Both the digesters were operated in same manner using same cow manure collected from a local dairy farm in the Oak Ridge, TN area. The raw sludge was processed and diluted with water to obtain $6.6 \%$ total volatile solids (total solids of about 12-13\%) concentration. This feeding rate was maintained corresponding to a hydraulic retention time of 16 days. Gas samples were analyzed for methane and carbon dioxide content. Slurry samples were analyzed for total solids (TS), total volatile solids (TVS), Volatile Fatty acids (VFA), and total alkalinity (TA).

Table 2-1 shows the performance results of two scales of digesters, whereas Figure 2.11 compares their cumulative methane production rates. Laboratory-scale digester produced more biogas with higher methane content than the pilot-scale digester. The TS, TVS and VFA content in the effluent of laboratory-scale was also lower than the pilot-scale digester. The laboratoryscale digester in mixed and unmixed condition showed same performance in terms of methane production. Pilot-scale digester in mixed condition performed significantly better than in unmixed condition with approximately 100\% higher methane production. Increase in VFA in the effluent reaching the values of feed VFA indicated that unmixed pilot-scale digester was failing.

Since the rate of bioreaction is low, anaerobic digesters are kinetically controlled. But, still sufficient amount of mixing is required to maintain a uniform environment inside the digester to guarantee efficient distribution of substrate, $\mathrm{pH}$ and temperature. Even the small amount of mixing produced by the motion of evolving gas bubbles and the addition of feed in the unmixed digester could be sufficient for efficient operation of the laboratory scale digester. Since the reaction is kinetically controlled, any additional amount of mixing does not further improve the performance of the mixed laboratory-scale digester over an unmixed digester. As the size of the reactor increases, difficulty in achieving complete mixing increases, and additional mixing is required. Since, no additional mixing was provided in pilot-scale unmixed reactor, it showed poorer performance than the pilot-scale mixed reactor. 
In conclusion significant differences between the results obtained for mixed and unmixed conditions in the pilot-scale digester were observed. Mixing provided in the digester results in its efficient operation and avoids its failure. Mixing played no significant role in the performance of the laboratory-scale digesters. At the smaller scale the mixing created by the evolution of gas bubbles is sufficient for proper operation of the unit. Any additional amount of mixing does not benefit the digesters to create more gas, necessarily because the digestion process is kinetically controlled. Excessive amount of mixing is also not recommended as mixing needs energy and spending more energy will not be profitable. This concludes that large scale operation of digester is necessary to obtain meaningful results and findings that can be used for proper design of commercial scale units.

Details of the results and findings of this work are discussed in Appendix 2 (Doctoral thesis) and in the following published paper:

Borole P., Klasson T. K., Ridenour W., Holland J., Karim K., Al-Dahhan M. H.(2005). Methane production in a 100-L upflow bioreactor by anaerobic digestion of farm waste. Applied Biochemistry and Biotechnology (129-132):887-896.

Table 2.1 Effect of mixing on performance of laboratory-scale and pilot-scale anaerobic digester

\begin{tabular}{|l|l|l|l|l|}
\hline Scale & \multicolumn{2}{|l|}{$\begin{array}{l}\text { Laboratory-scale } \\
\text { (6-inch, 3.78 L) }\end{array}$} & \multicolumn{2}{l|}{$\begin{array}{l}\text { Pilot-scale } \\
\text { (18-inch, 97 L) }\end{array}$} \\
\hline Condition & Mixed & Unmixed & Mixed & Unmixed \\
\hline Gas recirculation rate $($ L/min) & 1 & - & 9 & - \\
\hline Feed/effluent rate $(L / 2$ days $)$ & 0.470 & 0.470 & 12 & 12 \\
\hline Biogas production rate $(L / L /$ day $)$ & 1.2 & 1.1 & 0.55 & 0.3 \\
\hline Methane content $(\%)$ & 76 & 73 & 65 & 52 \\
\hline $\begin{array}{l}\text { Cumulative methane production } \\
\text { rate }(L / \text { day) }\end{array}$ & 3.3 & 3.1 & 40 & 20 \\
\hline $\begin{array}{l}\text { Cumulative methane production } \\
\text { rate per unit volume }(L / L / \text { day })\end{array}$ & 0.87 & 0.82 & 0.41 & 0.2 \\
\hline
\end{tabular}




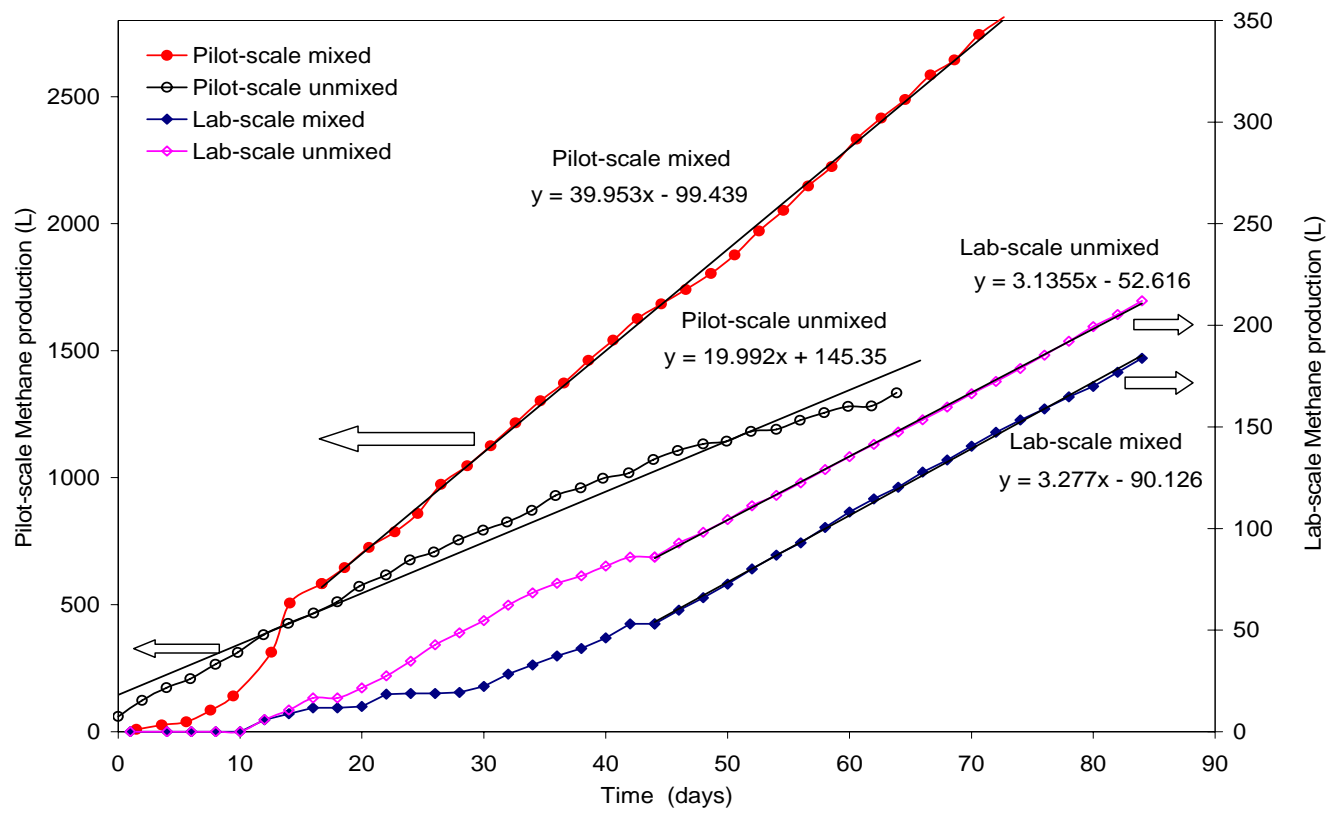

Figure 2.11: Comparison of cumulative methane production rates for laboratory-scale and pilot scale digesters

\subsubsection{Effect of mixing intensity on the biogas production in a pilot plant scale anaerobic digesters}

Based on the study reported in section 2.3.1 the following essential question arises: what is the best or suitable mixing intensity to ensure efficient digester performance or what is least energy input to maximize the energy output as biogas obtained from the digester. To answer this question further investigation was conducted as outlined below.

The findings in the pilot scale digester and their comparison with those obtained with 6-inch digester suggest that laboratory scale digesters are of no use to determine the best mixing intensity needed for efficient digester performance. Hence, the studies of this section were performed using the pilot plant anaerobic digester (18 inch diameter) at Washington University (WU) to determine the effect of energy input in the form of gas recirculation rate on the obtained energy in the form of biogas $\left(\mathrm{CH}_{4}\right)$ production while maintaining the proper performance of the digester. The pilot plane set up used at the Oak Ridge National Lab described in section 2.3.1 (Figure 2.10) was transferred and operated at WU for this work. The best gas recirculation rate occurs when the difference between the energy output and energy input is at a maximum.

The gas recirculation rate has been kept at $9.7 \mathrm{~L} / \mathrm{min}, 19.4 \mathrm{l} / \mathrm{min}$, and $29 \mathrm{lit} / \mathrm{min}$. For each of these flow rates the pilot plant was operated till a steady volume of gas was produced. Once steady state was reached, the digester was maintained at the same condition for a duration of two hydraulic retention times (32 days). The cumulative biogas generated for this period was used as a basis for comparison. Hence three different sizes of pumps were used for such investigation operation. 
Every other day $12 \mathrm{~L}$ of effluent was extracted from the bottom of the digester and $12 \mathrm{~L}$ of fresh feed slurry was added to the top of the digester. To keep conditions similar for each feeding and extraction separate bottles were used for each process. On these days gas meter readings were taken, as well as samples were withdrawn for analysis of volatile fatty acids (VFA), determining total solid (TS), volatile solid (VS) concentrations, and pH levels.

The digester was operated with manure obtained from a dairy farm with concrete bedding in Pevely, MO. The manure was obtained fresh and stored at a temperature of $4{ }^{\circ} \mathrm{C}$ until use. When needed the manure was thawed, sieved, and mixed with water to obtain a volatile solid concentration of $6.6 \mathrm{wt} \%$. Preparation of the manure involved a number of steps. Wet manure was blended with tap water (in 1:3 ratio, to adjust total volatile solids content) for two minutes with an impeller mixer and placed into a large bucket for the heavy solids (sand, etc.) to settle out. Then triplicate samples were taken to determine total solids by drying at $105^{\circ} \mathrm{C}$, after which the samples were toasted in an oven for 60 minutes to determine total volatile solids. Then the slurry was passed through a sieve with 9.5-mm openings. (In practice, a very small fraction failed to pass through the sieve). Finally the slurry was diluted as needed with water to obtain $6.6 \%$ total volatile solids concentration (total solids of about $12-13 \%$ with very low sand content). Solids occur in the waste due to different sources like husk, straw and fiber coming from the feed and sand particles, saw dust, wood chips/shavings, rice hulks, etc. depending on the type of the bedding. Since the waste was collected from concrete bedding, it had very low sand content. Every second day, gas composition and cumulative gas production volume were determined. Feeding rate (or effluent removal rate) was adjusted to maintain a hydraulic retention time of 16 days. Gas samples were collected using a gas-tight syringe from a sampling port in the gas production line to analyze the methane and carbon dioxide content with a GOW-Mac gas chromatography unit. Liquid samples were analyzed for total solids (TS) and the total volatile solids (TVS).

The results of methane production are shown in Figure 2.12. For three biogas recirculation rates used in this work there is an increase in methane generation.

The average cumulative production of methane per day (Figure 2.13) shows that a plateau is reached after a certain gas recirculation rate. Hence, there is a range where for a given gas input the production of biogas would not further increase. With energy evaluation, one can determine the best gas recirculation rate range (as energy input) that can be used to maximize the energy obtained in biogas production.

The details of this work will be reported in the following doctoral thesis:

Varma R. (2007). Phase distribution using tomography and performance of anaerobic digesters for bioenergy generation [Ph.D.Thesis in preperation] Washington University -St. Louis MO USA 


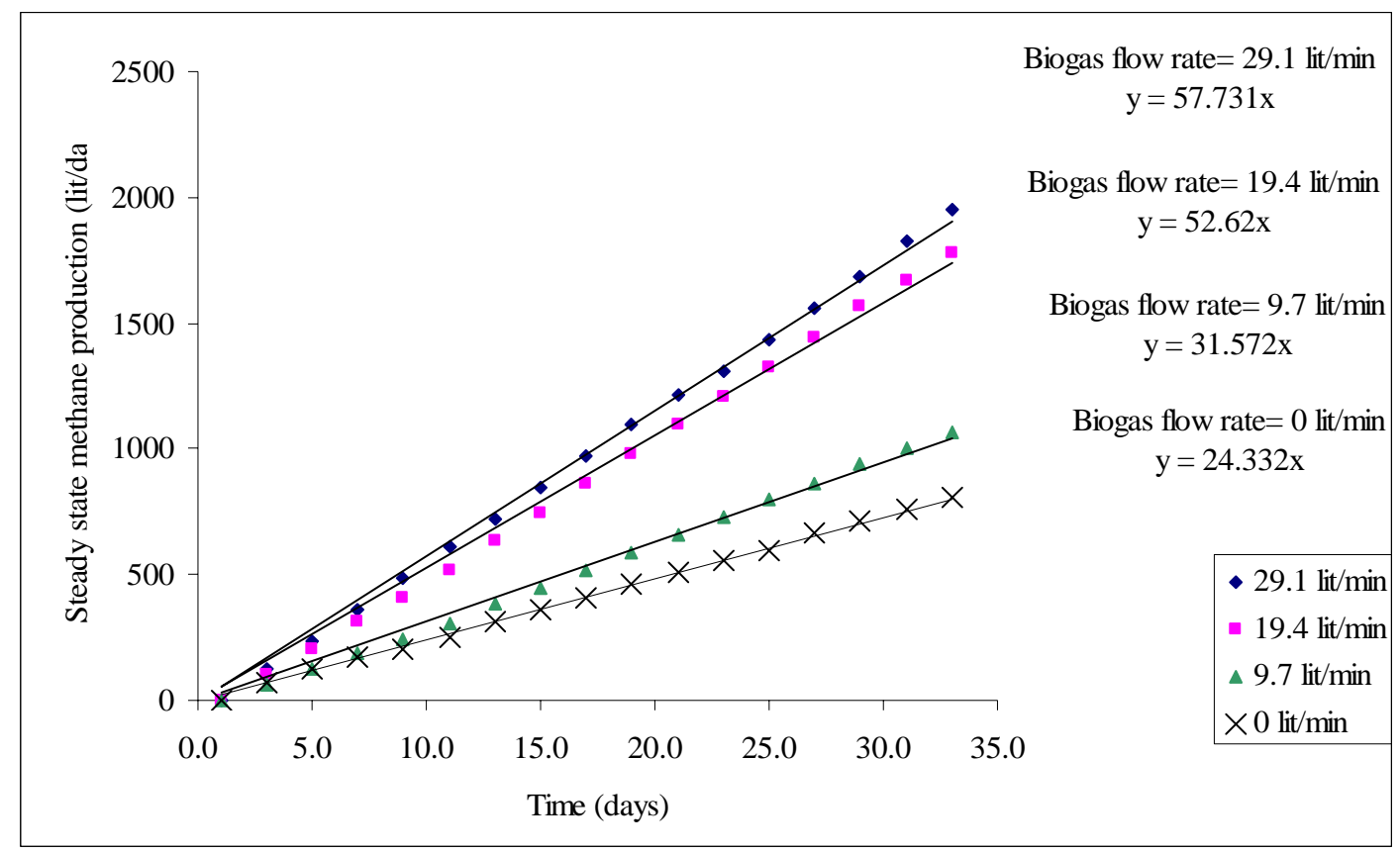

Figure2.12: Cumulative steady state methane (bio gas) production for different biogas circulation rates circulation

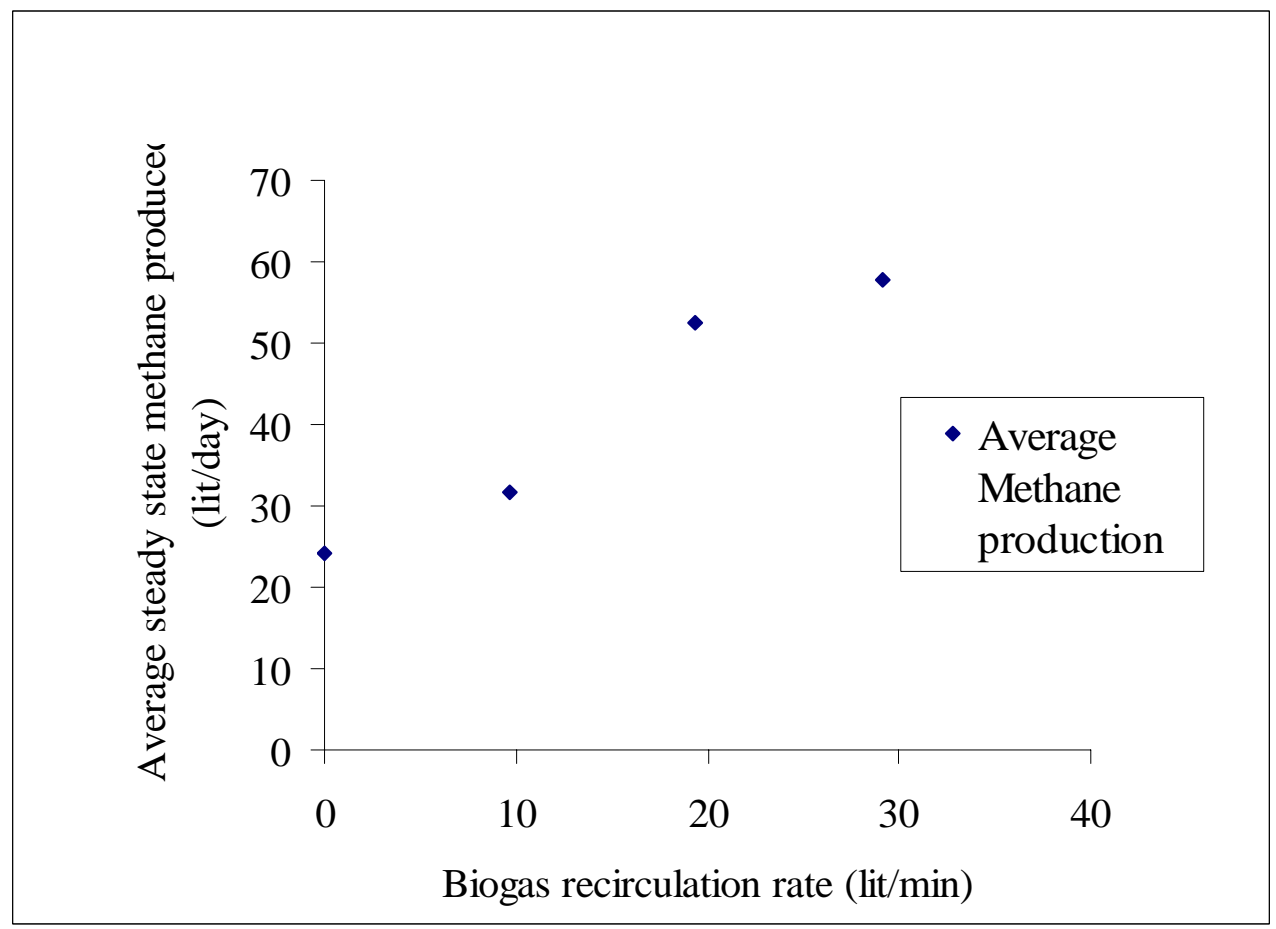

Figure 2.13 : Slope of cumulative methane production versus biogas recirculation rate. 


\subsection{Kinetics of animal waste (cow manure) anaerobic digestion.}

Anaerobic digestion kinetics study of cow manure was performed at $35^{\circ} \mathrm{C}$ in continuous bench-scale gas-lift digesters (3.78 L working volume) at eight different volatile solids (VS) loading rates in the range of $1.11-5.87 \mathrm{~g} / \mathrm{L}-\mathrm{d}$. The digesters produced methane at the rates of 0.44-1.18 L/L-d, and the methane content of the biogas was found to increase with longer hydraulic retention time (HRT). Based on the experimental observations the ultimate methane yield and the specific methane productivity were estimated to be $0.42 \mathrm{~L} \mathrm{CH} / \mathrm{g}$ VS loaded and $0.45 \mathrm{~L} \mathrm{CH}_{4} / \mathrm{g}$ VS consumed, respectively. Total and dissolved COD consumptions were calculated to be $59-17 \%$ and $78-43 \%$ at $24.4-4.6$ days HRTs, respectively. Maximum concentration of volatile fatty acids in the effluent was observed as $0.7 \mathrm{~g} / \mathrm{L}$ at 4.6 days HRT, while it was below detection limit at HRTs longer than 11 days. The observed methane production rate did not compare well with the predictions of Chen and Hashimoto's and Hill's models using their recommended kinetic parameters. However, under the studied experimental conditions, the predictions of Chen and Hashimoto's model fitted better to the observed data than that of Hill's model. The nonlinear regression analysis of the experimental data was performed using a derived methane production rate model, for a completely mixed anaerobic digester, involving Contois kinetics with endogenous decay. The best fit values for the maximum specific growth rate $(\mu m)$ and dimensionless kinetic parameter $(K)$ were estimated as 0.43 per day and 0.89 , respectively. The experimental data were found to be within $95 \%$ confidence interval of the prediction of the derived methane production rate model with the sum of residual squared error as 0.02. The findings of this study suggest that rather indiscriminate use of the manure digestion models and the recommended kinetic parameters may lead to significant error in the methane production rate prediction. Therefore, it is strongly recommended that each manure digestion system should be individually analyzed for its kinetics and designed to efficiently serve its purpose.

The details of this work are reported in the following manuscript:

Karim, K., Klasson, K.T., Drescher, S.R., Ridenour, W., Borole, A.P., and Al-Dahhan, M.H. (2007) "Mesophilic Digestion Kinetics of Manure Slurry", Applied Biochemistry and Biotechnology (in press)

\subsection{Commercial scale digester for biogas production and animal waste treatment at Iowa Energy Center}

Washington University and Iowa Energy Center (IEC) team cooperated to conduct performance and hydrodynamics investigation using the large scale anaerobic digester unit (1500 gallon volume) at the Iowa energy center. The planned study was to investigate the effects of mixing intensity on the biogas production using the commercial scale cow manure anaerobic digester and to characterize the hydrodynamcis of such digester at the operating conditions studied. Washington University has evaluated the IEC commercial unit and assessed its suitability for the planned study. We found that the IEC large scale anaerobic digester needs significant modifications, redesign and reconstruction based on the findings 
obtained at Washington University related to the performance of laboratory and pilot plant scale digesters and their hydrodynamics obtained by using advanced measurement techniques such as Computer Automated Radioactive Particle Tracking (CARPT) and Computed Tomography (CT) and using computational fluid dynamics (CFD).

Washington University team suggested the needed modifications and how to redesign IEC digester. IEC implemented all the needed modifications and reconstructed the unit to fulfill as well their cost sharing for the project. The redeveloped unit was tested using air-water system and based on the results the sparger design was further modified. After the unit was ready for operation to perform the planed study, the $3^{\text {rd }}$ year budget of this grant received a cut which prevented the execution of such important investigation. However, in this section, the modifications and the preparation of the commercial scale digester are discussed.

It was decided to initially study two conditions, unmixed and biogas mixed digestion. The modifications suggested by Washington-University for the existing IEC unit and the final reconstructed digester process are discussed as follows.

\subsubsection{Operating conditions and initial suggested modifications}

Total volume of the digester $=1500$ gallon Working volume of the digester $=1200$ gallon (considering 20\% head space)

Hydraulic retention time $=16$ days

Temperature $=35$ Degree Celsius

Feed rate $=1200$ gallon $/ 16$ days $=75$ gallon $=284.25$ Liter per day

Total solids concentration in manure slurry $=100 \mathrm{~g}$ dry solids per liter slurry $(10 \%$ slurry $)$

Total solid loading rate,

$=100=\frac{100 g / L \times 284.25 L / d a y}{4548 L}=6.25 g / L-d$

Assuming 70\% volatile solids (VS),

VS loading rate $=6.25 \mathrm{~g} / \mathrm{L}-\mathrm{d}$ X $0.7=4.375 \mathrm{~g} / \mathrm{L}-\mathrm{d}$

Assuming 1.43 g COD per g VS and 60\% VS conversion to biogas (Note: one g COD give 0.35 L methane gas),

Daily biogas production $=0.35 \times 1.43 \times(4.375$ X 280.74) $\mathrm{X} 0.6=368.84$ L methane per day at STP.

Assuming biogas contains 65\% methane, total biogas production $=368.84 / 0.65=567.44 \mathrm{~L}$ per day at STP. 
Initial suggested modifications:

- $\quad$ A 500 Gallon settling tank needs to be added (Note: If the collected manure does not have sand then this unit will not be needed).

- $\quad$ The settling tank should have three outlet ports (one at the bottom, one at one third height of the tank and another on two third height of the tank) as shown in Figure 2.14, and a pump to pump the slurry to the feed tank. Pipe, fittings and valves will be of the same size and diameter as the existing ones.

- $\quad$ There should be one water line close by so that animal waste can be diluted to make slurry of required concentration.

- $\quad$ Feed tank (500 gallon) already exists which has many ports. It needs a sampling valve close to mid height of the tank.

- $\quad$ Digester tank (1500 gallon) is well equipped with slurry recirculation arrangements. It needs to be redesigned for gas recirculation mixing mode. In addition, there is a need to put three sampling valves (same as that exists in the feed tank), one at bottom, one close to working height and one in between.

- $\quad$ There could be about $0.5 \mathrm{~m}^{3}$ biogas production every day. Therefore, there should be proper venting facility. Biogas venting facility, biogas recirculation pump and fittings already exist there. However, there is a need of gas meter or flow measuring system on the biogas line, which can be used to measure biogas production rate.

- $\quad$ A gas collection bag or similar storage is needed for biogas recirculation. Since biogas recirculation is at a higher rate than its natural production rate. Thus, there is a need to have a biogas storage bag in the biogas recirculation line. Apart from that, a sampling nozzle (for collecting biogas samples) on the biogas collection line is required.

- $\quad$ There is also a need of a flow measuring and control device on the biogas recirculation line, to facilitate gas recirculation at a certain rate. Capacity of the biogas recirculation pump should be sufficient enough to pump $1000 \mathrm{~L} / \mathrm{hr}$. A flow control valve and a flowmeter/rotameter are required to be fixed on the biogas recirculation main line. The method of measurement will be discussed later.

- $\quad$ Design of draft tube, gas sparger and the digester bottom are designed based on WU findings (laboratory and pilot plant performance and hydrodynamics studies).

The digester needs to be operated under each condition for about three months starting with unmixed condition first.

\subsubsection{Reconstruction of the IEC large scale anaerobic digester and its flow test using air water.}

The biogas recirculation arrangements were made using a draft tube of 0.25 times diameter of the digester, similar as the one used in the case of laboratory and pilot scale digesters. The biogas recirculation rate for the commercial scale is decided as 124.5 liter per minute, resulting in an input power density of $8 \mathrm{~W} / \mathrm{m}^{3}$, same as that of used for the 6-inch laboratory scale and for the pilot plant scale digesters. The 64 inch tank bottom is decided to be shaped with a $25^{\circ}$ hopper bottom, as it was observed from the CFD simulations that a $25^{\circ}$ hopper bottom gives 
less stagnancy as compare to flat bottom and $60^{\circ}$ hopper bottom. The schematic of the commercial scale digester and the draft tube is shown in Figure 2.15.

Sparger was designed to have at least a reasonable distribution of biogas inside the draft tube and the needed pressure drop to have the gas uniformly distributed from all its holes. It was decided to have a six arm sparger with one hole of $3.56 \mathrm{~mm}$ diameter on each arm as shown in Figure 2.16, and one in the center (which was added after the first operational test with air-water). The schematic diagram of the modified commercial scale digester unit, heating loop and gas recirculation system is shown in Figures 2.17 - 2.22.

The commercial scale biomass digestion facility at Iowa consists of a 300 gallon premix tank and a 1500 gallon methane tank (digester). The plastic tanks used as premix and methane tanks are made of polyethylene and have maximum temperature ratings of $140^{\circ} \mathrm{F}$. The 300 gallon premix tank has a diameter of 45 inches and stands 62 inches tall. The 1500 gallon Methane tank is 64 inches in diameter and 115 inches tall, while the 600 gallon Gas Collection and Water Storage tanks are 46 inches in diameter and 88 inches tall. Each tank is provided with a 16 inch diameter manhole. Plexiglas manhole covers was constructed and installed to allow visual examination of the tank contents during operation. The 300 gallon polyethylene Premix tank is mounted directly above a Moyno Industries open cavity pump (Model \# C2E CDQ3SPA) which feeds the Biomass solution into the Methane Tank at flowrates up to 16 gpm. The Moyno Industries open cavity pump is controlled with a Toshiba Variable Frequency Drive (VFD, Model \# VFS7-4022UPL). Mixing in the Premix tank will be accomplished using a Neptune tank mixer (Model \# JG-2.1) mounted to the top of the tank. The Biomass solution (animal waste slurry) can be preheated in the Premix tank using a Propylene Glycol heating coil constructed of 3/4 inch polyethylene tubing, approximately 30 feet long. Digestion will take place in the 1500 gallon, polyethylene Methane Tank (Digester). Mixing in the Methane tank is accomplished by recirculating Biogas from the outlet of the tank back to the bottom of the tank through seven, $3.56 \mathrm{~mm}$ diameter orifices. A 16 inch diameter sparging tube, 58 inches long, is mounted vertically in the center of the tank, 17 inches above the tank bottom. The orifices are located 6 inches above the bottom of the sparging tube with one orifice in the center (which was added after the first operational test with water) and the other six located radially about the center axis, $14.3 \mathrm{~cm}$ from the axis and $60^{\circ}$ apart. Arrangement is made to mixing the methane tank by slurry recirculation, if needed, from the bottom to the top of the tank at flowrates up to $4.1 \mathrm{gpm}$ using a Moyno Industries open cavity pump (Model \# B1C CDQ3SAA), which is controlled by a Toshiba VFD (Model \# VFS7- 4007UPL). In addition to recirculation, the Moyno Recirculation pump can also be used to transfer liquid slurry from the Methane tank to either the Premix Tank, or to the drain system.

Temperature in the Methane tank is maintained using a Propylene Glycol heating coil constructed of 3/4 inch polyethylene tubing, approximately 150 feet long. Three sampling ports are provided in the Methane tank at heights of 6, 39, and 74 inches above the bottom of the tank. The floor of the Methane tank was sloped to an angle of $25^{\circ}$ through the installation of high-density Polyurethane insulating foam. The foam was applied by spraying until the desired slope was achieved.

Biogas recirculation in the Methane tank is accomplished using the Gas Recirculation System, which consists of two, $9.3 \mathrm{ft}^{3}$ cylinders constructed from 12 inch PVC pipe and fittings. The cylinders are connected with electrically actuated three-way ball valves such that a centrifugal pump (Grundfos, Model \# TP32-80) can be used to continuously pump water from one cylinder to the other. This allows Biogas from the Methane tank outlet to be drawn into 
one cylinder while Biogas in the other cylinder is pumped back to the Methane tank for recirculation. The Gas Recirculation System is capable of flowrates up to 3.1 CFM. A new pump (Teel Mfg., Model \# 4RJ63) was installed, which increases the Gas Recirculation capability to 5 CFM.

Biogas generated in the methane tank flows to the Gas Collection Tank. On startup, the 600 gallon, polyethylene Gas Collection Tank is initially filled to the high level point (approximately 500 gallons) with water from the 600 gallon Water Storage Tank. As Biogas is generated, the gas pressure in the tank is maintained at approximately 12 inches of water column by pumping water as necessary from the Gas Collection Tank to the Water Storage Tank using a Grundfos centrifugal pump (Model \# TP32-80). Once the water level in the Gas Collection Tank reaches the low level point (approximately 150 gallons), the Gas Collection Tank inlet isolation valve is shut and the outlet isolation valve opened. Water from the Water Storage Tank is then pumped back to the Gas Collection Tank at flowrates up to 100 gpm using a Grundfos centrifugal pump (Model \# UPS40-80/4). Pressure in the Gas Collection Tank is measured with a Rosemount Pressure Transmitter (Model \# 1151DP3E12B1E6) while the flowrate of the expelled Biogas is measured using an Eldridge Flowmeter (Model \# 8659MPSSS- 133-DC24- $\mathrm{CH}_{4} / \mathrm{CO}_{2}$ ). Biogas discharged from the Gas Collection Tank is vented to atmosphere. A Varec Waste Gas flare was purchased to burn off the biogas as it is discharged from the facilities

The Premix and Methane tank bulk temperatures and the Propylene Glycol heating coil inlet and outlet temperatures of both tanks are measured with Weed Instrument RTD's (Model \# 201-01B-C-3-010.0-A2-Z006). The pH of the Methane tank is measured using an Innovative Sensors Model $40 \mathrm{pH}$ probe. The exiting facility provides a Propylene Glycol heat loop, which runs throughout the building and is capable of delivering Propylene Glycol at $250^{\circ} \mathrm{Ffor}$ process loads. Propylene Glycol is pumped through the heating coils in the Premix and Methane tanks using Grunfos recirculating pumps (Model \# UP 26-64F in Premix and Model \# UP 26-116F in Methane Tank). Belimo 3-way control valves (Model \# B323 + LR24-SR-2.0 US) installed on the suction side of the recirculation pumps is used to control the temperature of the fluid supplied to the heating coils. The valves accomplish this by mixing the hot fluid supplied by the heat loop with the relatively cooler fluid returning from the heating coils. This allows a temperature in the Premix and Methane tanks to be controlled while limiting the Propylene Glycol temperature supplied to the heating coils to $160^{\circ} \mathrm{F}$, which is the maximum temperature of the polyethylene tubing (Endot Part \# PBJ07541010004) used in the heating coils.

A steel draft tube was affixed in the center that is supported by a small table such that the table doesn't obstruct the flow (not shown in the image of Figure 2.20). On the upper level three tie roads are used to support the system, as shown in Figure 2.20. Figure 2.21 shows the image of thesparger.

The design modifications were found to be satisfactory. However two additional changes were recommended and made based on the first operational tests with air-water.

1. An additional pore to be added in the sparger that is in the center and facing upwards. This was recommended to improve the gas distribution and was feasible as the available gas pressure drop was sufficient. 
2. The suggested gas flow rate was 4.2 CFM, however the current pumping capacity is about half of this. A change in the gas pump was recommended to increase the pumping capacity to match the required rate.

The first change was implemented right away after performing the first operational tests with air-water as mentioned earlier. Figure 2.22 shows the operation of the reactor filled with water with a gas (air) flow rate of 60 liter per minute. Clearly all the pores in the sparger seem to have opened successfully and the liquid appears to circulate. All the process components and units of the commercial scale anaerobic digester were tested successfully and the process is ready to be tested for biogas production and animal waste (cow manure) treatment. Unfortunately as mentioned earlier due to the $3^{\text {rd }}$ year budget cut such test and the mentioned planned investigation were not performed.
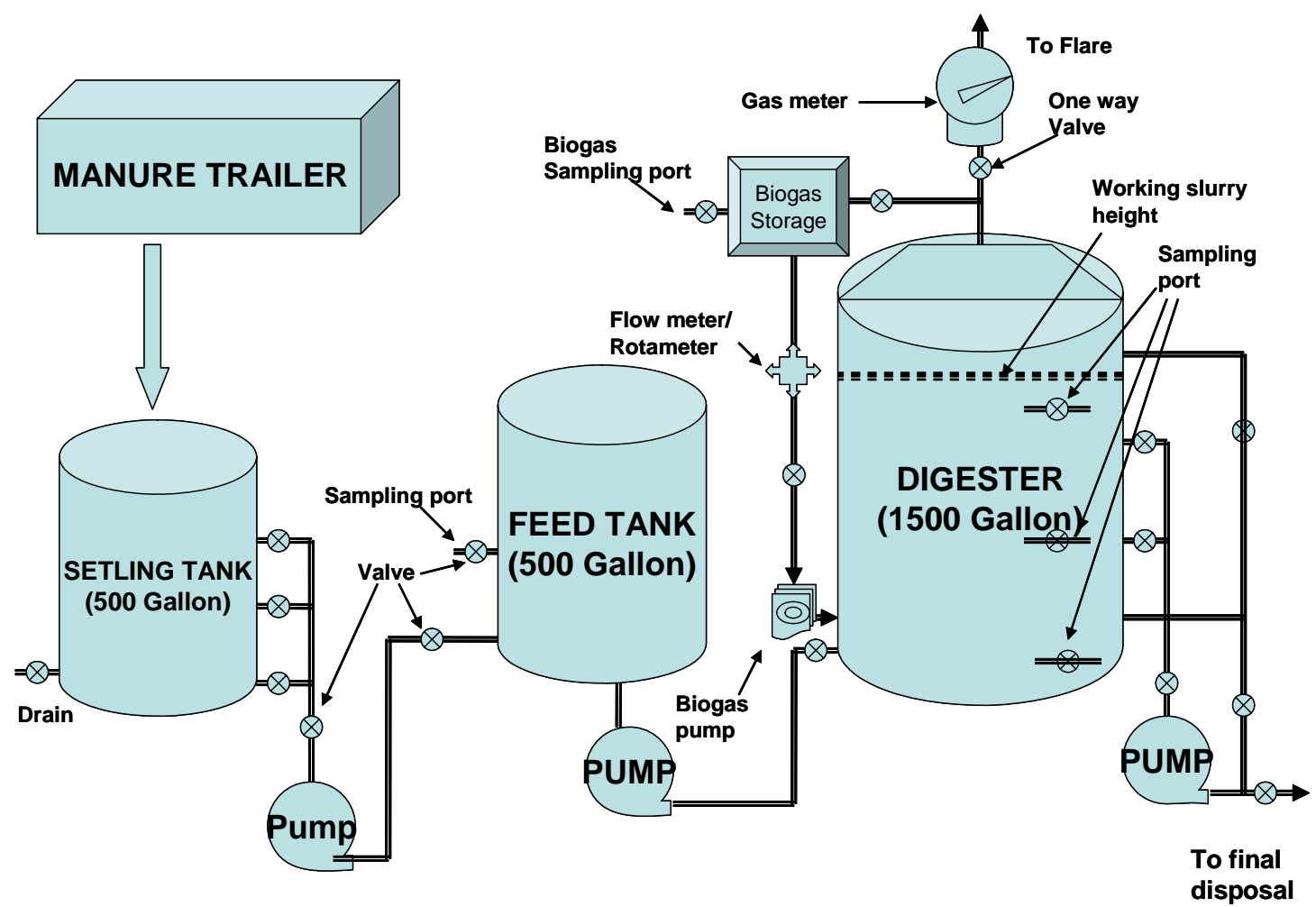

Figure 2.14: Commercial scale suggested digester process set-up at Iowa Energy Center 


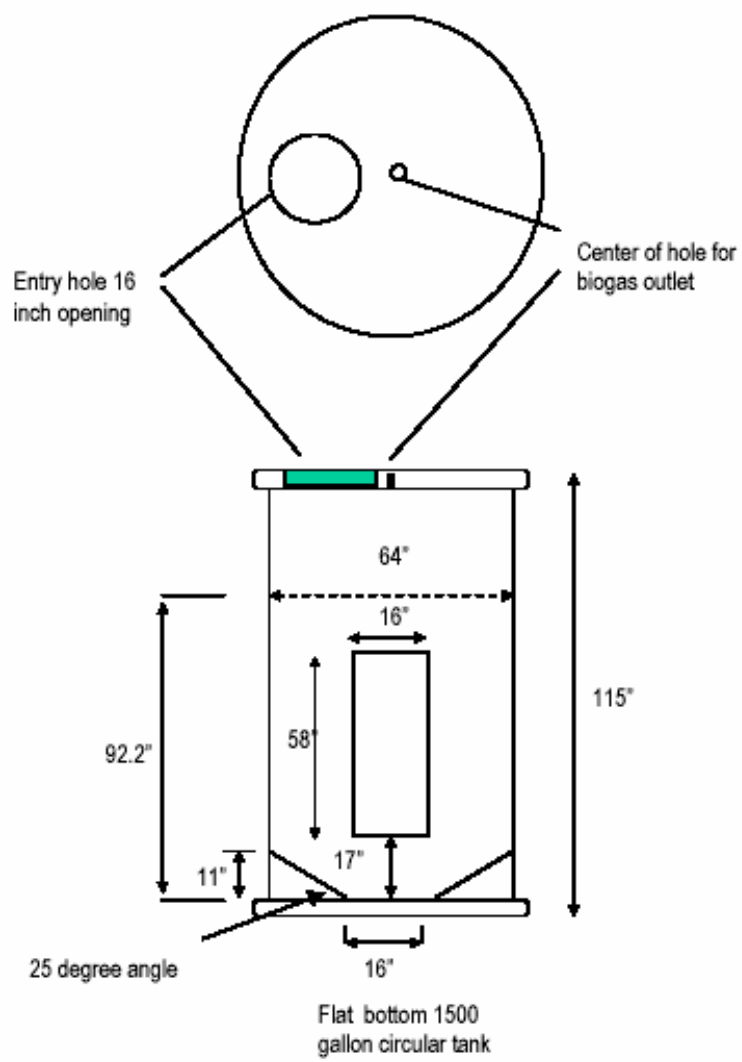

All dimensions in inch

Drawing is not to scale

Figure 2.15. Schematic of the commercial scale digester 


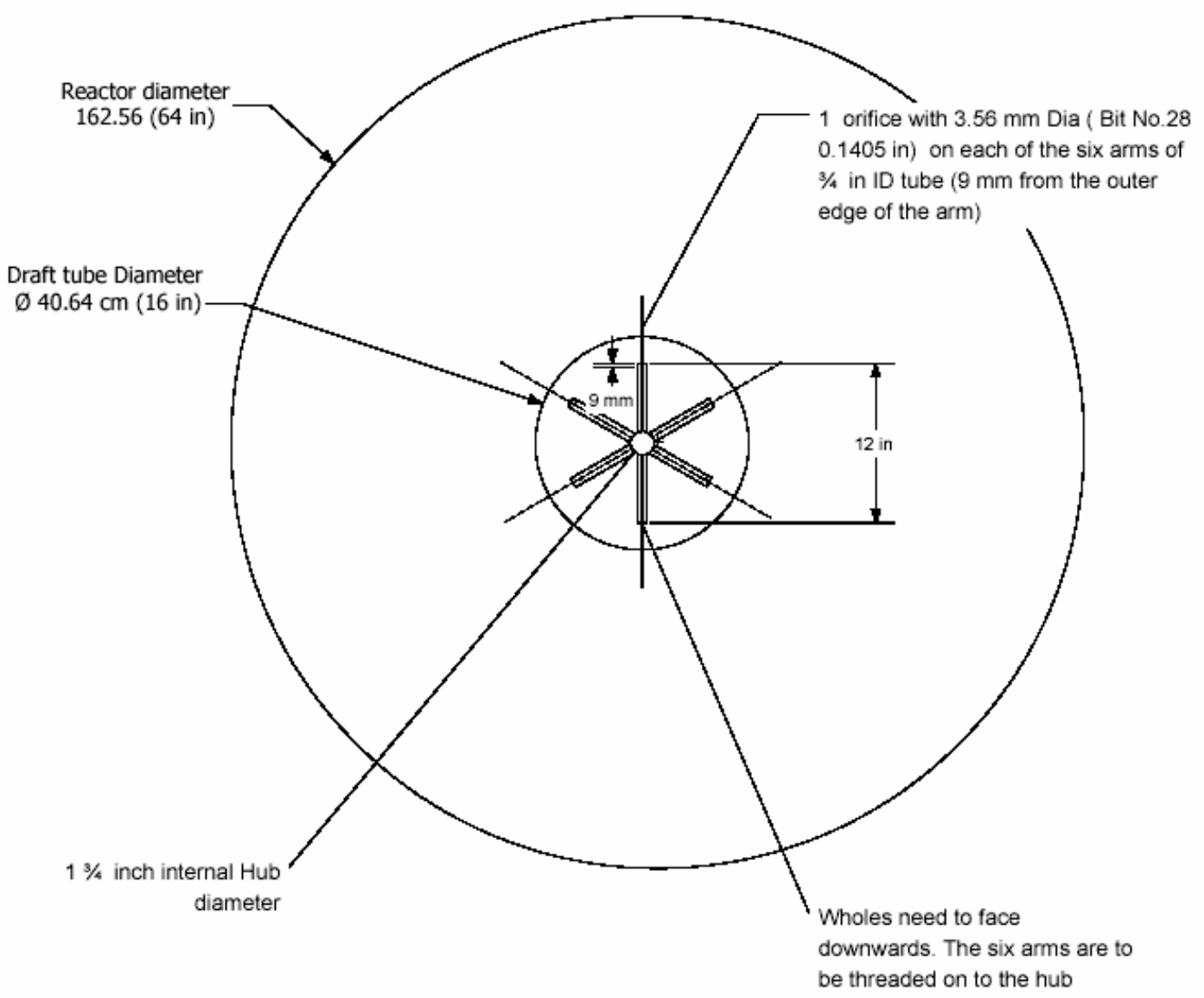

Figure 2.16: Schematic of the sparger for commercial scale digester. 


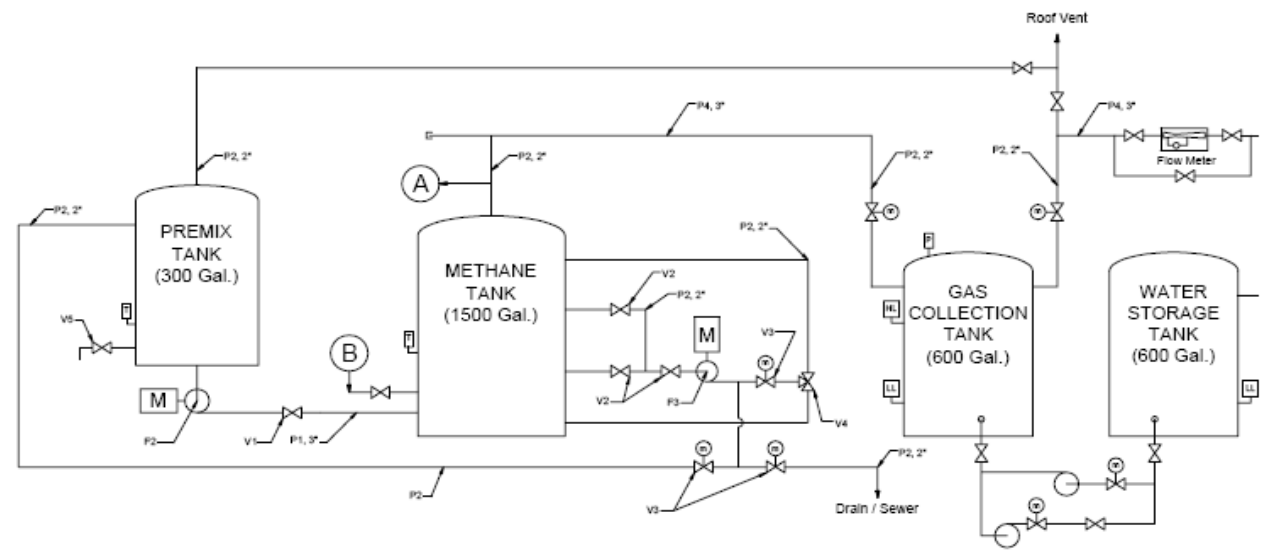

Figure 2.17: Schematic of the digester facility at IEC, Ames Iowa.

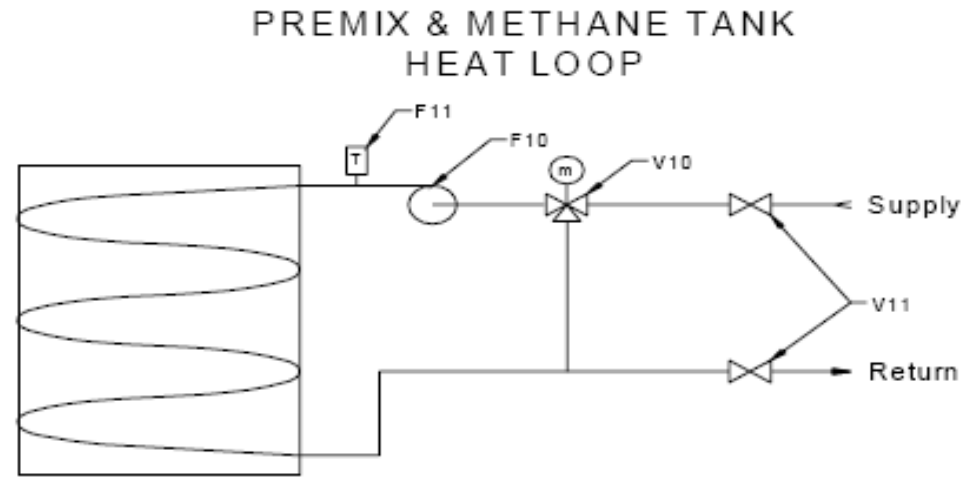

Figure 2.18: Schematic of the heating loop in the digester unit 


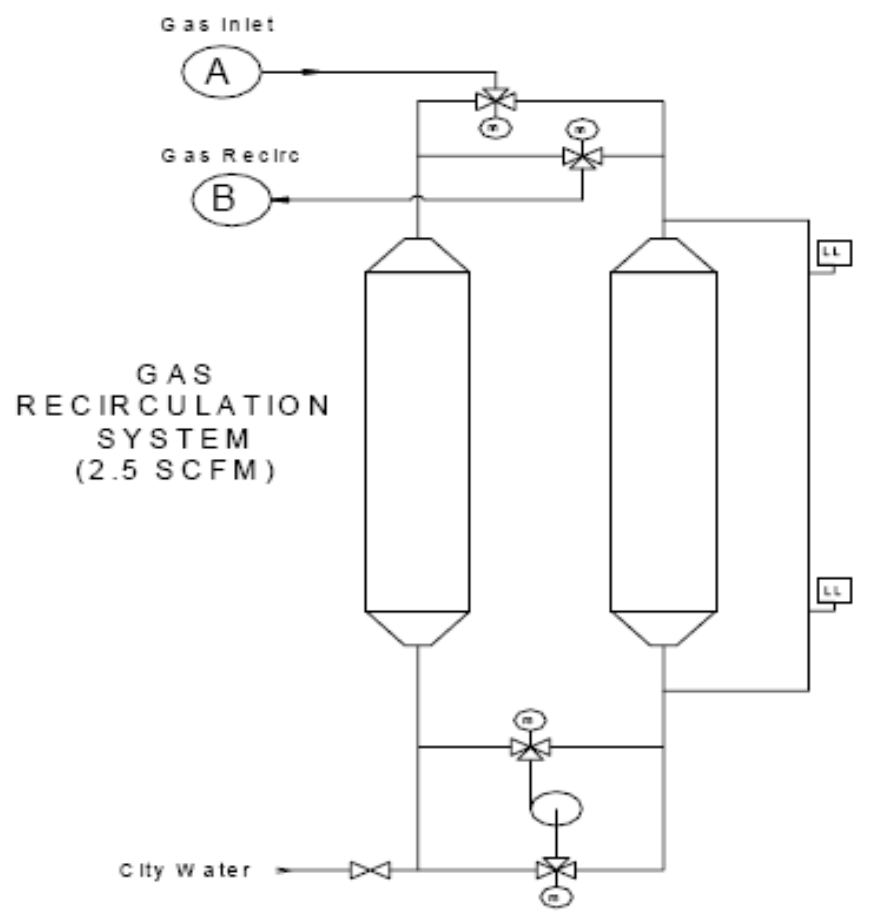

Figure 2.19: Schematic of the biogas recirculation system

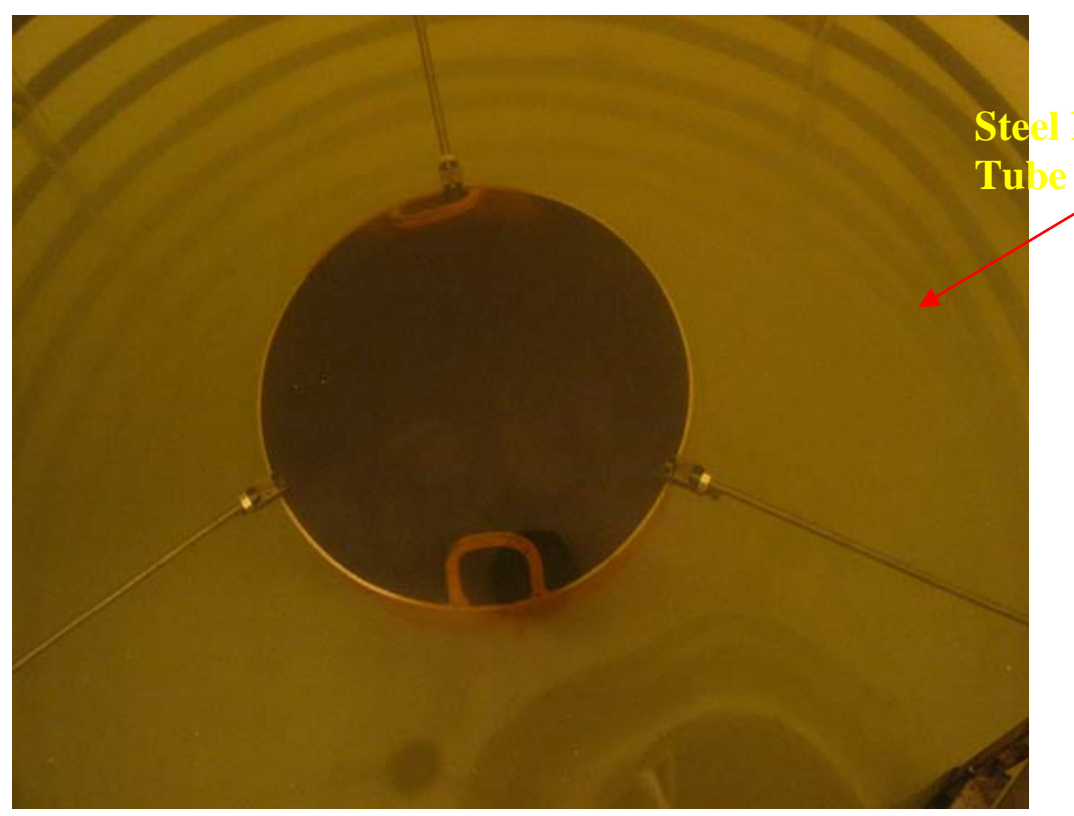

Figure 2.20: The top view of the reactor as seen through a man hole. The steel draft tube is visible in the center; it is supported by three tie rods. 


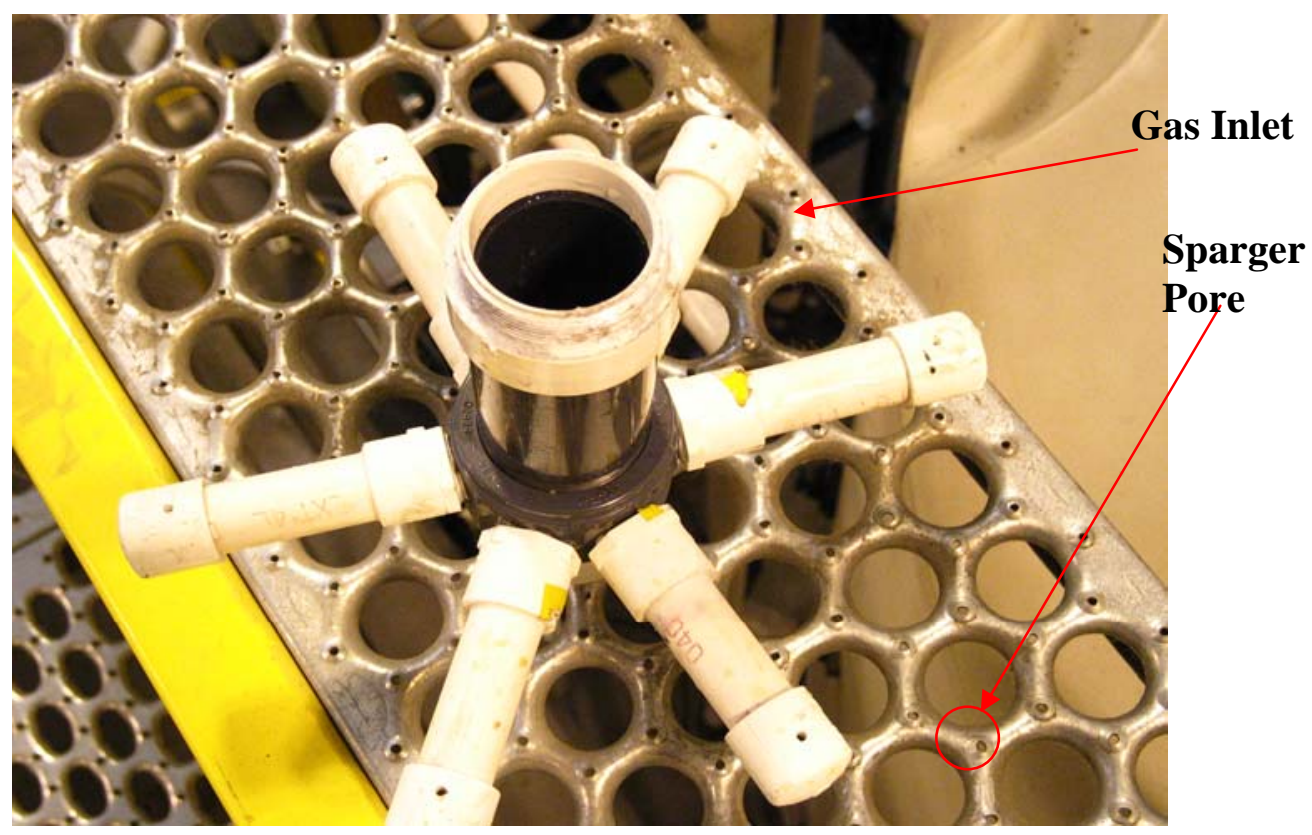

Figure 2.21: Image of sparger inverted. Six pores have been made as per the design. $7^{\text {th }}$ whole added at the center of the sparger on the upper surface (not visible in photograph).

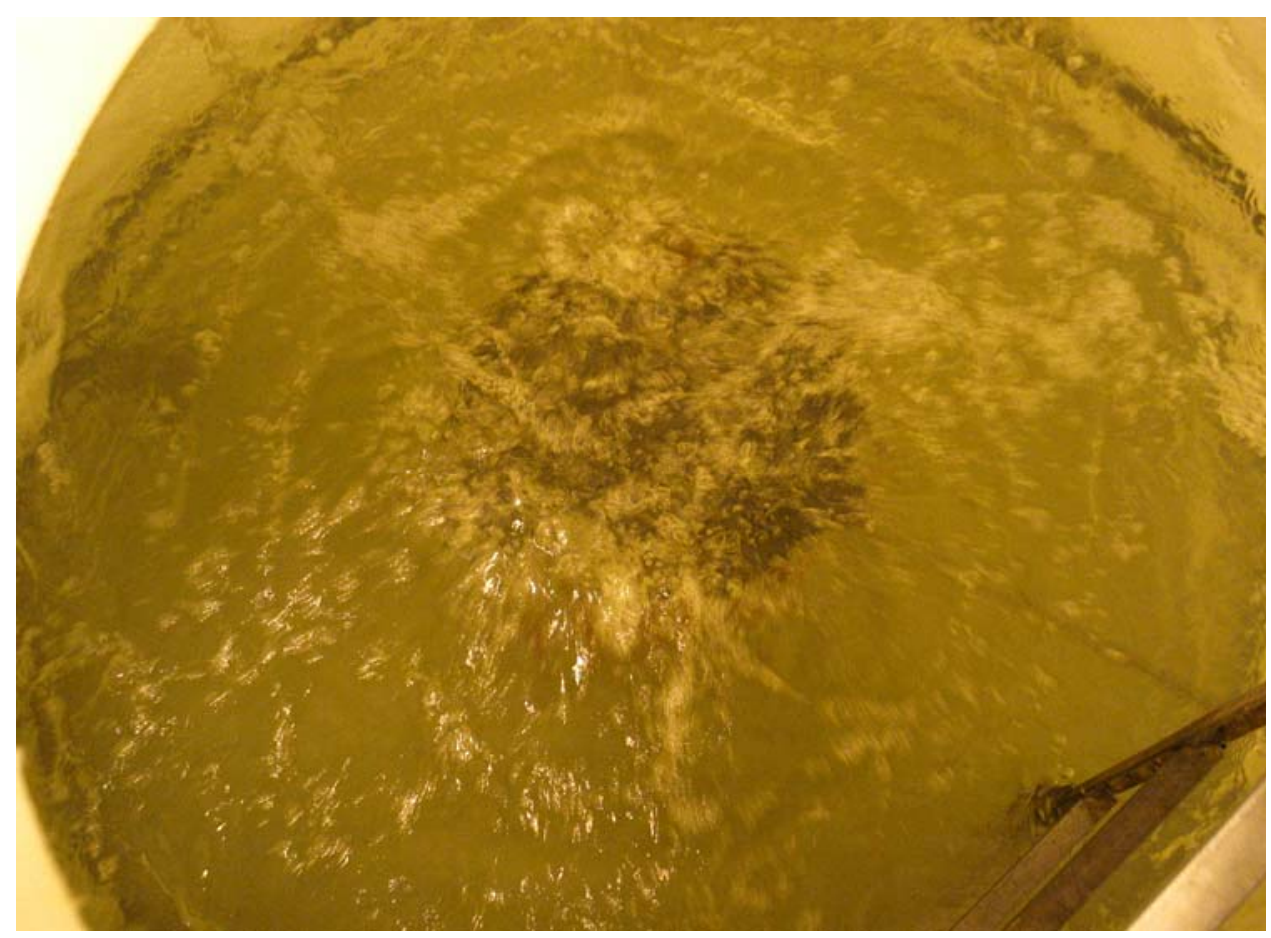

Figure 2.22: View of the reactor from the man whole with the gas flow on. 
Chapter 3 : Investigation of the detailed mixing and hydrodynamics using computer automated radioactive particle tracking (CARPT) techniques 


\section{Investigation of the detailed mixing and hydrodynamics using computer automated radioactive particle tracking (CARPT) techniques}

The mixing and hydrodynamics of gas lift anaerobic digesters were investigated for the first time using advanced non-invasive radioactive particle tracking techniques suitable for opaque systems like digesters. As a part of this work a novel multiple-particle computer automated radioactive particle tracking (MP-CARPT) technique was designed, developed, tested and implemented. In addition, the single particle computer automated radioactive particle tracking (CARPT) technique was used to systematically investigate the detailed mixing and hydrodynamics of different configurations, sizes, mode of mixing (gas-lift and impeller mixer) of anaerobic digesters. These techniques measure in a non invasive and in a 3D domain the flow pattern, velocity field, turbulent parameters, dead zones, residence time, and many other parameters of any multiphase flow opaque systems. The knowledge obtained helps advancing the fundamental understanding of the digesters and their design, scale-up and operation. In addition, the obtained data and findings are used as benchmark to evaluate and validate the computational flow dynamics (CFD) models and their closures as will be discussed in chapter 5 . The accomplished work consists of the following:

3.1 Investigation of the hydrodynamics and mixing of anaerobic digesters using computer automated radioactive particle tracking (CARPT) technique

3.1.1 Hydrodynamics of a simulated gas recirculation digester with a flat bottom

3.1.2 Investigation of the hydrodynamics of different configurations and sizes of gas recirculated anaerobic digesters.

3.1.3 The impact of sparger design and the degree of uniformity of gas holdup distribution in the draft tube on the mixing intensity and hydrodynamics of gas recirculation mixed digester

3.2 The development of a novel multiple-particle CARPT (MP-CARPT) and its implementation.

The details of this work are reported in following doctoral thesis and published manuscripts:

- Vesvikar M.(2006). Understanding the hydrodynamics of anaerobic digesters for bioenergy production [D.Sc. Thesis] St. Louis USA, Washington University. (Appendix-2)

-Karim K, Varma R, Vesvikar M, Al-Dahhan MH.(2004) Flow pattern visualization of a simulated digester. Water research 38(17):3659-3670

-Varma R., Al-Dahhan M.H.(2007) Effect Of sparger design on hydrodynamics of a gas recirculation anaerobic bioreactor. in review Biotechnology bioengineering. (Appendix-3.3)

-Vesvikar MS., Al-Dahhan MH. (2005) Flow pattern visualization in a mimic anaerobic digester using CFD. Biotechnology Bioengineering 89(6):719-732. 
-Vesvikar M. S., Varma R., Karim K., Al-Dahhan M H.(2005). Flow pattern visualization in a mimic anaerobic digester: experimental and computational studies. Water Science and Technology 52(1-2):537-43

\subsection{Investigation of the hydrodynamics and mixing of anaerobic digesters using computer automated radioactive particle tracking (CARPT) technique}

\subsubsection{Hydrodynamics of a simulated gas recirculation digester with a flat bottom.}

Mixing patterns inside a simulated flat bottom digester were investigated using the noninvasive techniques of Computer Automated Radioactive Particle Tracking (CARPT) and Computed Tomography (CT). Mixing was provided using air recirculation at three different flow rates of $28.32 \mathrm{l} / \mathrm{h}, 56.64 \mathrm{l} / \mathrm{h}$, and $84.96 \mathrm{l} / \mathrm{h}$.

Better mixing was observed in the upper zone near the top of the draft tube. However, at the bottom of the digester there was a stagnancy at all the three gas flow rates. The maximum value of the time averaged axial velocity inside the draft tube, at a gas flow rate of $84.96 \mathrm{l} / \mathrm{h}$, was observed as $34.4 \mathrm{~cm} / \mathrm{sec}$. The turbulent kinetic energy was observed to be maximum (724 dyne $/ \mathrm{cm}^{2}$ ) inside the draft tube, and to decrease radially towards the wall of the digester. The study showed that the CARPT and CT techniques could be successfully used to identify the flow pattern in the digester and to calculate velocity and turbulent parameters quantitatively. On the other hand, the increase in gas circulation rate from $28.32 \mathrm{l} / \mathrm{h}$ to $84.96 \mathrm{l} / \mathrm{h}$ did not significantly reduce the dead zones inside the flat bottom digester. To achieve the desired mixing and reactor performance, the operating conditions and reactor configuration need to be optimized.

The detailed results are reported in the following published manuscript:

-Karim K, Varma R, Vesvikar M,Al-Dahhan MH.(2004) Flow pattern visualization of a simulated digester. Water research 38(17):3659-3670

\subsubsection{Investigation of the hydrodynamics of different configurations and sizes of gas recirculated anaerobic digesters.}

The results of the performance studies (chapter 2) showed that the scale of operation has a significant effect on the performance of digesters. Mixing affects the performance of the large-scale digesters but not of laboratory-scale digesters. Performance of digesters is partly governed by the mixing characteristics/hydrodynamics inside the digester, which in turn are affected by the scale of operation. In addition, the systematic performance investigations in laboratory and pilot plant digesters treating cow manure showed that large- scale experiments are required to obtain information for design and scale up of digesters. The hydrodynamics of 
the low $L / D$ ratio gas-lift (gas recirculating) reported in the literature digesters are lacking. Thus, there is a need to investigate the hydrodynamics of these gas lift digesters in detail. In this work the following studies were performed:

- Investigation of the flow pattern and detailed hydrodynamics of laboratory and pilotplant scale anaerobic digesters using cow manure: The investigated digesters were equipped with a draft tube and a sparger to allow mixing by gas recirculation. The CARPT technique was used for such investigation and the obtained data were used as a benchmark for evaluation and validation of CFD models.

- Investigation of the effect of gas flow rate, shape of tank bottom, draft tube diameter to tank diameter ratio, type of sparger, solids content of the slurry, and scale (digester size) on the mixing pattern and hydrodynamics of the digester: The digester geometric parameters and operating conditions were varied to evaluate their effect on flow patterns, liquid velocity profiles, turbulence parameters such as shear stress, turbulent kinetic energy, and eddy diffusivities using CARPT. These investigations were conducted to understand and compare between the nature of the flow in the digesters of two scales. Mixing intensity was quantified in terms of dead space volume and turbulent diffusivities to understand the effect of scale (i.e. size) on the formation of dead zones. The increase in gas flow rate increased the liquid velocity and decreased mean circulation time for all configurations at both scales. However increased gas flow rate did not offer any advantage of lowering significantly the dead zone volume. The larger draft tube diameters (with $D / T$ of 0.5 or 0.75 ) reduced the dead zones and produced relatively homogenous mixing throughout the digester volume. Digester with $D / T$ of 0.5 had minimum percentage of dead volume for laboratory-scale digester. For pilot-scale digester lowest percentage of dead volume was obtained for $D / T$ ratio of 0.75 at highest gas flow rate. Mean circulation time increased with increase in $D / T$ ratio. Decreasing solids content in the slurry decreased the mean circulation time. Figures 3.1 and 3.2 show sample of the obtained results using CARPT technique.

If different laboratory-scale configurations of digester are compared on the basis of flow pattern, liquid velocities and dead zone volume, the configuration with $D / T$ ratio of 0.5 at low gas flow rate seems to be the best configuration for anaerobic digester operation for the studied conditions. This is because, it provides good liquid circulation throughout the volume of the digester, lowest volume of dead zones and low mean circulation time were obtained. Higher gas flow rates can provide more circulation but they are not desirable from the energy consumption consideration. Flow pattern, liquid velocity digester profile, dead zone volume and diffusivities were used to evaluate the effect of scale (i.e. size) on the hydrodynamics. Geometric similarity and same power input density to identify the superficial gas velocity were used as scale-up criteria in this study. Scale of operation affected the flow pattern and liquid velocity profile significantly for $D / T$ of 0.75 . The liquid velocities were significantly higher in pilot-scale in the riser region but the change was less significant in the downcomer section. Comparing the values of dead zone volume and axial diffusivities to quantify mixing, proved that the mixing performance is better in small-scale digesters as compared to large-scale units in spite of liquid velocities being higher for pilot-scale units. Sparger geometry affected the hydrodynamics performance significantly in pilot-scale but not in laboratory-scale digesters. Thus, it can be concluded that only geometric similarity and same energy input per unit volume does not guarantee similar mixing intensity or hydrodynamic performance at different scales of operation. 


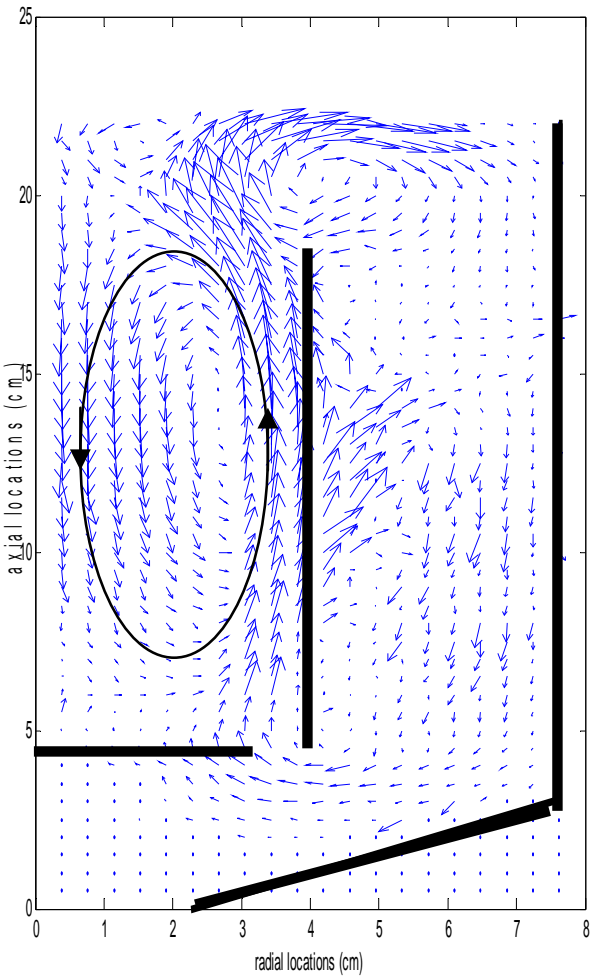

(a)

Figure 3.1: $\quad$ Effect of draft tube diameter on the flow pattern

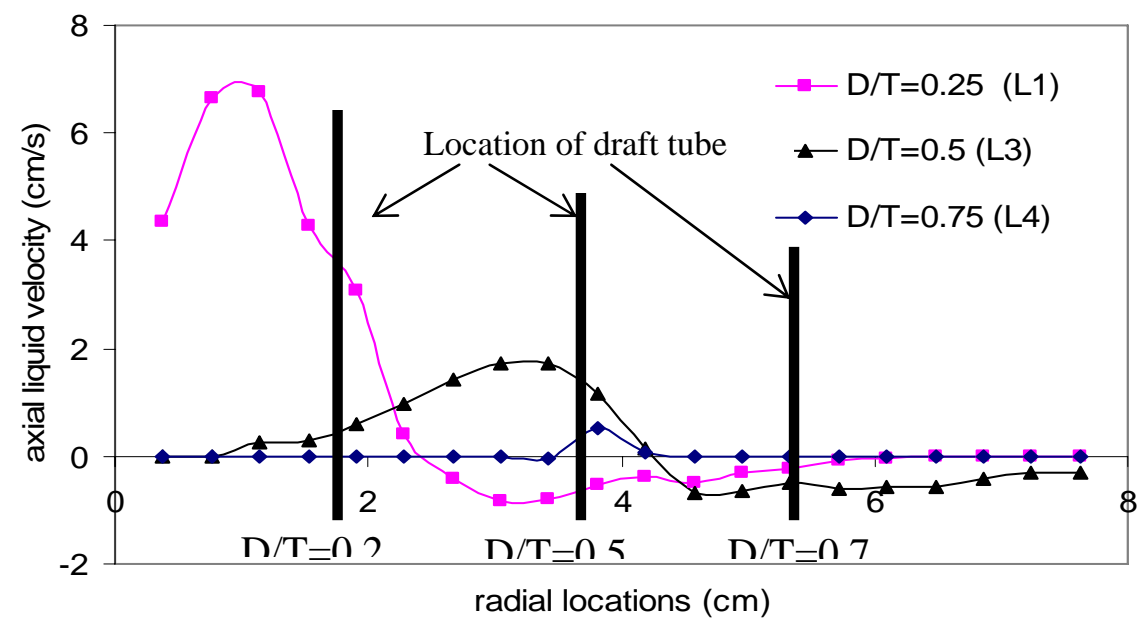

Figure 3.2 : Effect of draft tube diameter on the axial liquid velocity at the bottom of the draft tube, $\mathrm{z}=4 \mathrm{~cm}$, (experiment no L1, L3 and L4) (Vesvikar, 2006), Appendix 2). 
Only two $D / T$ ratios were tested in pilot-scale experiments. Hence, the best $D / T$ ratio for pilot-scale configuration to provide improved circulation and improved mixing performance was not examined or identified. However, CFD studies were used for this purpose. The CFD predictions were first evaluated with CARPT results and then the validated CFD code was used to understand the hydrodynamics of gas-lift digesters in detail and to compare additional laboratory-scale and pilot-scale configurations. The CFD study is outlined in Chapter 5.

The details of this work are reported in Appendix 2, which represents the following doctoral thesis supported by this grant:

Vesvikar M.(2006). Understanding the hydrodynamics of anaerobic digesters for bioenergy production [D.Sc. Thesis] St. Louis USA, Washington University.

\subsubsection{The impact of sparger design and the degree of uniformity of gas holdup distribution in the draft tube on the mixing intensity and hydrodynamics of gas recirculation mixed digester}

The effects of sparger design and gas flow rate on, gas holdup distribution and liquid (slurry) recirculation velocity were studied in a surrogate anaerobic laboratory digester (6- inch in diameter) used for treating bovine waste with a conical bottom mixed by gas recirculation. A single orifice sparger (SOS) and a multi-orifice (25 holes) ring sparger (MORS) with the same orifice open area and gas flow rates (hence the same process power input) were used in this study. The advanced non-invasive techniques of Computer Automated Tomography (CT) and Computer Automated Radioactive Particle Tracking (CARPT) were employed to determine the distribution of the gas holdup, liquid recirculation velocity and the poorly mixed zones. Gas flows $\left(Q_{g}\right)$ ranging of $0.017 \times 10^{-3} \mathrm{~m}^{3} / \mathrm{s}$ to $0.083 \times 10^{-3} \mathrm{~m}^{3} / \mathrm{s}$ were used which correspond to draft tube superficial gas velocities ranging from $1.46 \times 10^{-2} \mathrm{~m} / \mathrm{s}$ to $7.35 \times 10^{-2} \mathrm{~m} / \mathrm{s}$ (based on draft tube diameter). Air was used for the gas, as the molecular weights of air and biogas (consisting mainly of $\mathrm{CH}_{4}$ and $\mathrm{CO}_{2}$ ) are in the same range (biogas: $28.32-26.08 \mathrm{~kg} / \mathrm{kmol}$ and air: $28.58 \mathrm{~kg} / \mathrm{kmol}$ ). For a given gas flow rate, the MORS gave better gas holdup distribution in the draft tube and hence, enhanced liquid (slurry) recirculation and reduced the fraction of the poorly mixed zones compared to the SOS. The improved gas holdup distribution in the draft tube was found to have increased the overall liquid velocity. Hence, for the same process power input the MORS system performed better by enhancing the liquid recirculation and reducing the poorly mixed zones.

For a given power input, fixed by the gas flow rate, the Multi Orifice Ring Sparger (MORS) was found to gives better gas phase distribution and higher mean gas holdup in the draft tube when compared to Single Orifice Sparger (SOS). All pores in the MORS did not open. In order to conduct this study at gas flow rates that ensure all the MORS pores are open, would need a power input range that would exceed the recommended range for anaerobic systems ( 8 $\mathrm{W} / \mathrm{m}^{3}$, US EPA, 1979). The normalized standard deviation, $N_{\text {Mal }}$, for the gas holdup distribution was put in perspective as a parameter for characterizing the uniformity of gas 
holdup distribution in the draft tube. Lower values of $N_{\text {Mal }}$ show better performance of the MORS in terms of gas holdup characteristics.

There were two loops in the circulation patterns observed in the system. It was also determined by CARPT that the there are poorly mixed zones in the downcomer region of the reactor. Higher liquid velocity values were observed in the draft tube region for the digester with MORS for a fixed gas flow rate. The poorly mixed zones are drastically reduced in the digester when a MORS system is used as the lower values of gas holdup $N_{M a l}$ ensures better liquid circulation. The same trend was observed with the root mean square liquid fluctuation velocity (RMS) in the entire reactor. Hence the MORS system is considerably more efficient for mixing the reactor than the SOS system. The reduction in the poorly mixed zones would make a larger impact when the digesters based on the configurations discussed here are scaled up for pilot plant operations.

Recirculation of the liquid is facilitated by the density gradient between the material in the draft tube and periphery area of the draft tube. This difference triggers the buoyancy forces that enable the liquid to circulate. High gas holdup and better distribution are therefore desirable in the draft tube region to create an effective density gradient for better mixing in the reactor by reducing the fraction of the poorly mixed zones. This objective could be easily achieved with an increase in the superficial gas velocity. However, the energy constraints in such systems give limited flexibility in manipulating the superficial gas velocity as operating parameter. Hence, this necessitates the careful consideration of the sparger design for introducing gas into the system. The impact of increased uniform gas holdup distribution in the draft tube, and the reduction in the poor mixing zones in the digester on the production of methane from bovine waste could be the subject of a performance study. This paper confirms that for a given power input, efficiency in mixing can be obtained by appropriate sparger design. Figure 3.3 show a sample of the obtained results.

The details of this work are reported in the following manuscript (Appendix 3.3):

-Varma R., Al-dahhan M.H.(2007) Effect Of sparger design on hydrodynamics of a gas recirculation anaerobic bioreactor. Biotechnology bioengineering. under review. 


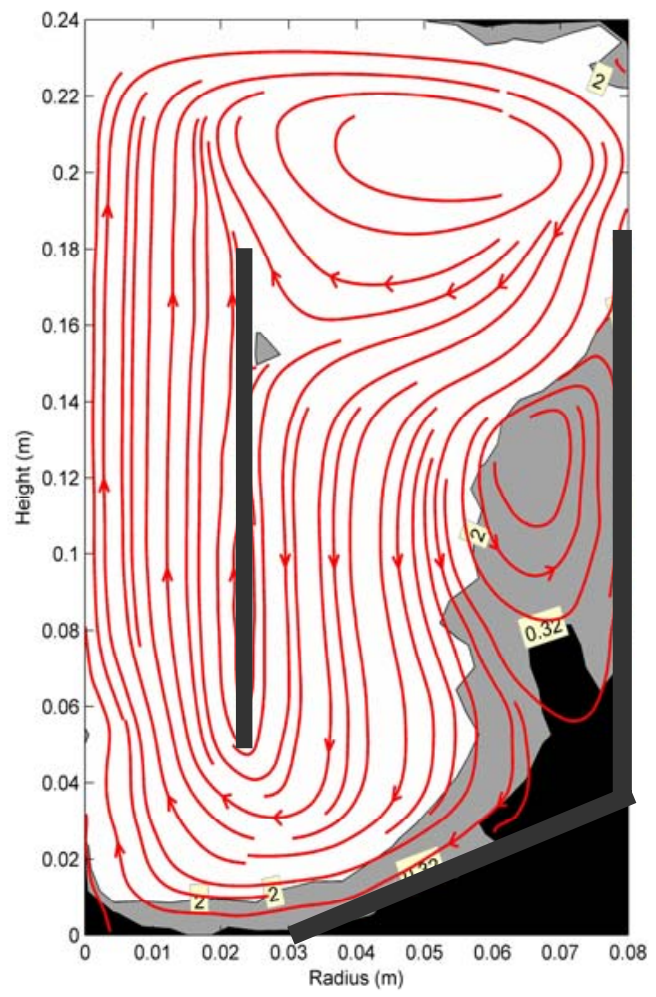

(a)

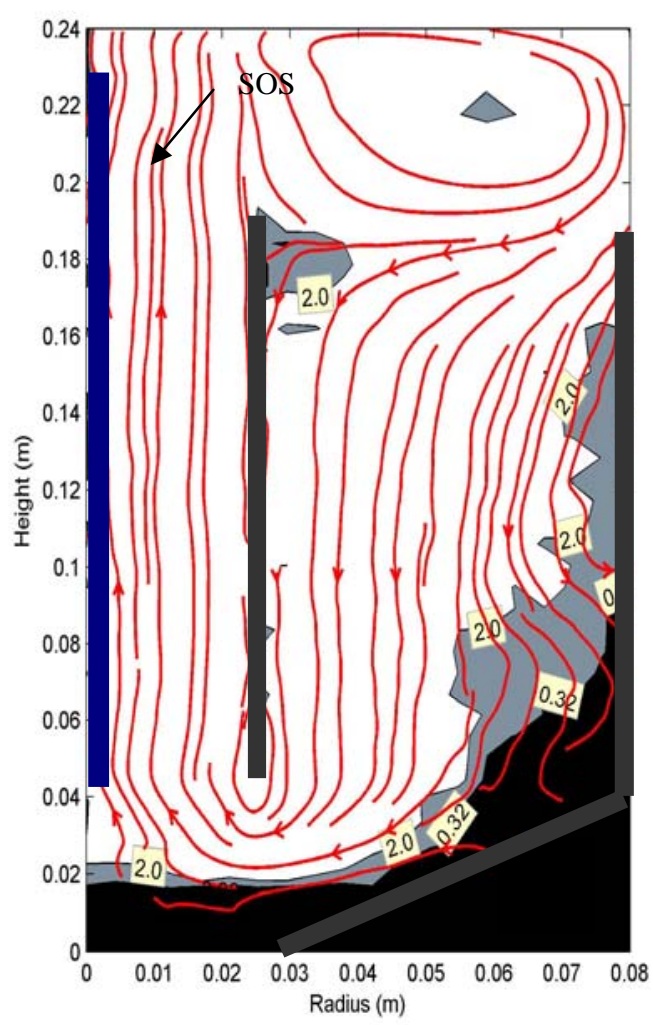

(b)

Figure3.3: Contour plots showing the time averaged values of the magnitude of the liquid circulation velocity for the bioreactor with $5 \%$ (TS) solid loading slurry at for gas flow rate $\mathrm{Q}_{\mathrm{g}}=0.05 \times 10^{-3} \mathrm{~m}^{3} / \mathrm{s}\left(\mathrm{V}_{\mathrm{g}}=4.4 \times 10^{-2} \mathrm{~m} / \mathrm{s}\right)$ : (a) MORS, (b) SOS. The dark lines indicate the location of the wall of the reactor and the draft tube; the fine lines with arrows indicate the stream lines. Numeric values in the box indicate velocity $\times 10^{2}(\mathrm{~m} / \mathrm{s})$ values the contour represents. The black colored zones indicate poorly mixed regions (Reproduced from Varma Al-Dahhan (2007))..

\subsection{The development of a novel multiple-particle CARPT (MP- CARPT) and its implementation.}

CARPT is an advance non invasive measurement technique suitable for opaque multiphase flow systems like anaerobic digesters. However, the nature of the slurry and the flow in the digester present some technical challenges that were not encountered in the previous applications of CARPT in multiphase reactors, including:

1. With gas-lift digester designs studied, we observed very slow flows in some portions of the digesters. These slow flows may have caused possible solids settling. This caused two difficulties: (a) data collection was very slow in certain portions of the reactor and/or under certain operating conditions, and (b) the tracer radioactive particle would settle in more than 
one instances and thus will cause a halt to data collection. These problems are enhanced with increase in the scale of operation.

2. The solids phase of the anaerobic digester and in many fluidization processes (gas -solid systems and gas-liquid-solid systems) consists of particles having different properties (size, shape, and density), while the current CARPT technique used only single-particle tracking.

Thus the data collection process was slow and all the required information such as the hydrodynamics behavior of the solids of different physical properties, and the segregation and interaction of the solid particles could not be obtained by tracking a single radioactive particle.

Furthermore, the current CARPT data-acquisition assembly has many components, which is bulky and also expensive. The assembly and the synchronization of the components of single particle CARPT unit is very time consuming and laborious. Since the CARPT was introduced at our Chemical Reaction Engineering Laboratory in 1990, very few changes or modifications have been made with the CARPT hardware. Thus, a development of a new system was required, which will not only extend the capabilities and overcome the limitations of the current CARPT system, but will also improve the current CARPT assembly, in terms of accuracy and cost.

The data collection rate and the capability to deal with the settling of the tracer can be greatly improved by the introduction of multiple tracers that can be tracked simultaneously. This is a challenging task. Multiple-particle tracking (MP-CARPT) can be pursued by introduction of particles containing different isotopes emitting gamma radiation of different energies that can be discriminated. In addition to speeding up the data collection rate for slow flows and reducing the impact of the particle settling, multiple-particle tracking will offer other important advantages, such as the capability to simultaneously track the motion of particles of different size, shape, and density, determining segregation of particles, and probing particle interactions. Use of the advanced technology in designing a new system will also provide an opportunity to make the new assembly compact, cheaper, faster, and easy to operate and understand.

The developed novel multiple-particle tracking technique is a valuable tool for characterization of a number of multiphase processes/reactor systems of industrial interests, which use a range of particles with different properties. For example, gas-solid fluidized beds are widely used in process industries for large-scale applications like coal gasification and small scale operation such as polymer and pharmaceutical, production (Lee et al. 2005.). These reactors contain a large amount of solids with a wide range of sizes and some times different densities. Characterization of flow of these solids of different physical properties can provide valuable information for designing and understanding these systems. Similarly MP-CARPT can be very useful in the evaluation of multiphase processes in gas-liquid-solid (GLS) and liquid-solid (LS) fluidized beds, stirred tanks, slurry bubble columns, etc.

To accomplish the objective of this work, a new data acquisition system for tracking multiple radioactive particles was designed and manufactured. Because of its ability to track more than one radioactive particle, it was named as Multiple-Particle Tracking Technique and abbreviated as MP-CARPT after CARPT. The development of MP-CARPT is a challenging 
task and faced many technical difficulties which all were overcome. The system was developed with the help of the team from the Oak Ridge National Laboratory (ORNL) consisting of electronic engineers, software engineers and nuclear engineers. Dr. Alan Winterberg (or r)berg designed the hardware and electronics, Dr. Lloyd Clonts helped with the development of the data acquisition software, and Dr. Chuck Alexander provided the input on the radiation and radioactive particles. Dr. David Depaoli oversaw the activities at the ORNL as a Co-PI with Prof. Muthanna Al-Dahhan as project PI. The hardware was assembled at Washington University and the necessary modifications to hardware and software were also made at the Washington University. Also at Washington University a new particle reconstruction algorithm and methodology were developed.

The developed new MP-CARPT unit offers number of advantages over the old single particle CARPT unit. The new unit is compact, cheaper, faster, and easy to use and operate. It provides ability to track eight different radioactive sources simultaneously.

The MP-CARPT electronics and technique was validated to track two stationary particles simultaneously. A new reconstruction algorithm was developed at Washington University which showed small error (less than 10\%) in reconstruction of Co-60 and Sc-46 particles. The validation was taken further to next step to track two moving particles representing the same liquid phase. The MP-CARPT was successful in tracking two particles in motion as well. Next, two radioactive particles of different densities, one mimicked the liquid phase $\left({ }^{60} \mathrm{Co}\right.$ inside 1 mm polypropylene ball with density similar to the water) and the other mimicked the solid phase (300 $\mu \mathrm{m}$, paralyene coated ${ }^{46}$ Sc with overall density similar to the glass beads density), were tracked in a 6-in. slurry bubble column reactor (SBCR). Particles representing different phases could also be tracked simultaneously using MP-CARPT unit.

The solids fraction in SBCR was kept low to $1 \%$ to obtain true hydrodynamic information of liquid phase. When tracking two different phases, ex. solids and liquid, care should be taken to design the experiment in such a way that the tracer follows the represented phase as closely as possible. Collisions of tracer representing liquid phase with the solid particles in the system can be minimized by using very low solids fraction.

MP-CARPT can be used conveniently to track two or more solids phases in a system with different properties (for example size, shape or density). However, how much difference in size or density of tracers is required so that the tracers can provide true hydrodynamics of phase being tracked needs to be evaluated. This issue can be addressed by tracking tracers of same size and different densities or same density and different sizes and observing the difference in hydrodynamics. Rammohan (2002) evaluated some of these issues.

Having validated the dual particle tracking, this technique can be easily extended to track more than two radioactive sources simultaneously. The current unit is capable of tracking maximum of eight tracers at a time, but it is limited due to availability and suitability of radioactive sources for this technique.

The error in the reconstruction of the MP-CARPT can be further reduced by some modifications of the experimental set-up, procedures, and the reconstruction algorithms. If the 
number of detectors for tracking are increased, such that the detector are packed closely together, then the error in the reconstruction will be reduced due to increased spatial resolution (CARPT manual, 2007). The current MP-CARPT reconstruction algorithm is based on the principle of addition of the calibration counts of Co-60 and Sc-46 obtained separately to represent the counts obtained together. Instead, if the calibration is performed with the Co-60 and Sc-46 particles present together, keeping one particle fixed at one location and placing other particle at all the calibration locations one by one and thus covering all the possible permutations, then more accurate calibration region can be obtained. This calibration technique will take into consideration the effect of presence of two particles together on their total counts. In addition, if the number of calibration points is increased, it will also help to increase the accuracy of reconstruction. The reconstruction method developed by Bhusarapu (Bhusarapu 2005) based on Monte Carlo simulations to generate fine CARPT calibration points can also be evaluated for the increased accuracy.

Since the technique is validated and the protocols for operation of MP-CARPT unit are understood, a manual for MP-CARPT is also prepared. This manual will help future users to understand and operate properly the MP-CARPT electronics and also provide guidelines to process the raw data obtained from tracking experiments using the new electronics. Figure 3.4 shows a sample of the results of the calibration step.

The details of this achievement and its results are discussed in appendix 2 which represents the following doctoral thesis:

- Vesvikar M.(2006). Understanding the hydrodynamics of anaerobic digesters for bioenergy production [D.Sc. Thesis] St. Louis USA, Washington University.

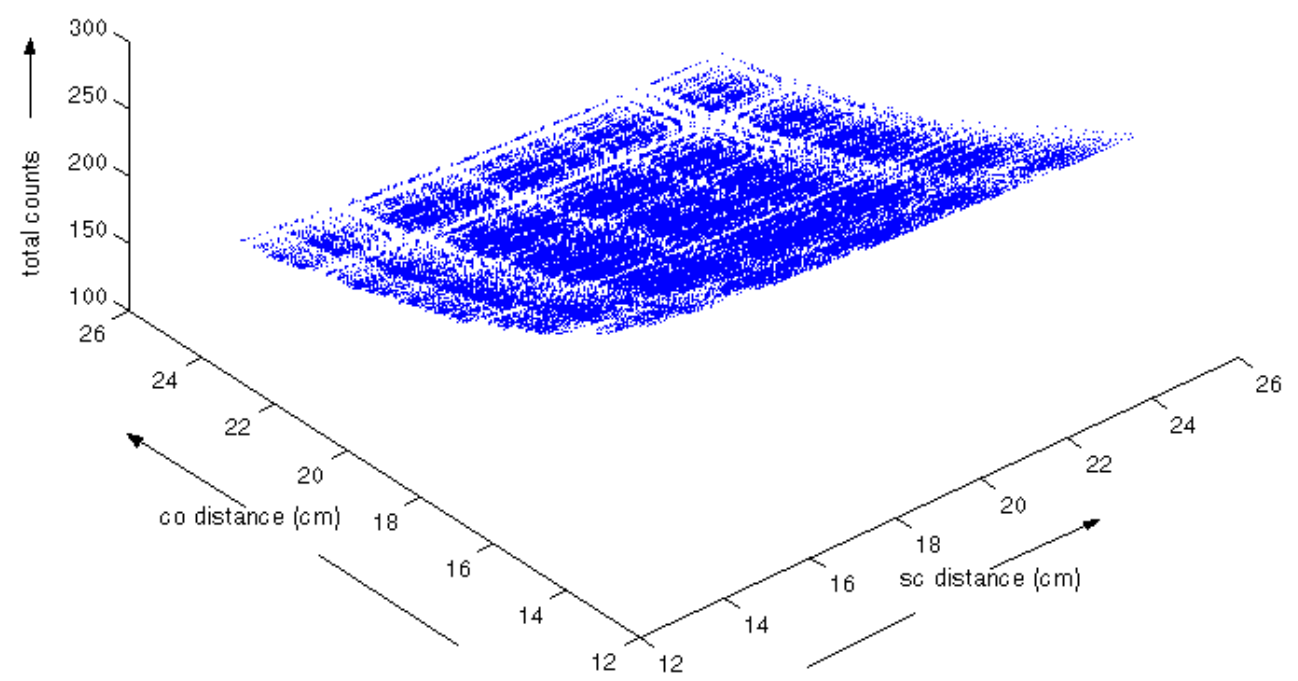

Figure 3.4 Calibration plane for detector 1 for low energy window and total counts of Co60 and Sc-46 (Vesvikar, 2006). 


\section{Chapter 4 : Investigation of the phases' distribution using gamma ray computed tomography (CT) techniques}




\section{Investigation of the Three Phases Distribution Using Gamma Ray Computed Tomography (CT) Techniques}

Quantification of the Phases distribution in anaerobic digesters is important for proper understanding of their hydrodynamics, mixing intensity and the formation, location of and volume of the dead zones. This will help reducing or eliminating the failure of the anaerobic digesters. Fundamental knowledge and understanding of the effects of various design and operating variables on the phases' distribution will help avoiding or minimizing the dead zones (i.e. inactive reactor volumes). Therefore, studying the gas holdup distribution particularly in the draft tube of the gas-lift anaerobic digesters (gas recirculation mixed digesters) is important to help ensuring desired slurry recirculation and reduced dead zones. This is because in gas-lift digesters, the slurry recirculation between the draft tube and the region outside the draft tube is caused by the gradient in the overall density between these regions (imbalance in the buoyancy forces). This means that better gas distribution in the draft tube would cause enhanced slurry recirculation and reduction in dead zones. However, no study in the literature has investigated the effect of gas distribution in the draft tube and sparger design on the mixing intensity, flow field and the dead zones.

As mentioned earlier, single source gamma ray computed tomography (CT) can measure the phase distribution of two phase systems. However, it can be used for three phase systems with one of the following: i) assumptions are used in case of dynamic three phases, ii) the third phase (solids) is stationary, and iii) the solids loading of the third phase is low so that the system can be considered as a pseudo two phase flow system. For dynamic three phase systems encountered in high solids loading anaerobic digesters and gas-liquid-solid fluidization in general, dual source (energy) tomography technique is needed to measure the phase distribution with desirable accuracy. Such technique is not yet available where its development is a challenging task. Accordingly, to advance the application of gamma ray computed tomography (CT) which is the technique of choice for opaque large scale systems, a novel dual source (energy) gamma ray computed tomography (DSCT) was developed with the cooperation of Oakridge National Laboratory (ORNL, Dr. Alan Wintenberg and Dr. Charles Alexander). This development was not an easy task. Many challenging technical problems were encountered and all were resolved in a systematic manner. In addition, many radiation safety measures and calculations were required. Fortunately all these have been successfully achieved and tested. Unfortunately, however, these caused delay in finalizing the development of DSCT. Furthermore, the delay in placing the yearly funds (particularly for the second and third year) and the cut in the budget of the third year further caused such delay. Despite of all these, the DSCT technique was fully developed. Due to the radiation safety issues associated with the radioactive particles, and the need to develop safer protocols for the use of CARPT and MP-CARPT, the new administration (Dean and Department Chair) took additional step in August 2006 of removing the approved DSCT sealed radioactive sources. We hope to validate and implement DSCT technique in the near future where the results will be published in manuscripts and in a doctoral thesis in preparation.

Accordingly, we report here the work that has been done using single source CT and the development of the novel DSCT technique.

The accomplished work consists of the following: 
4.1. Gas holdup distribution using single source CT

4.1.1. Gas holdup distribution in a mimicked flat bottom anaerobic digester.

4.1.2. Gas distribution in a 6 inch anaerobic digester using a single point sparger and a single source CT.

4.1.3. Gas distribution with a multipoint gas sparger in a 6 inch anaerobic digester

4.1.4. Effect of sparger design and the degree of uniformity of the gas holdup in the draft tube on the hydrodynamics and dead zones of the anaerobic digesters mixed by gas recirculation

4.2 Development of the novel dual source (energy) gamma ray computed tomography (DSCT)

4.2.1 Design, construction and test of the DSCT hard ware.

4.2.2. Development of the data acquisition system and control for the automation of the DSCT hardware

4.2.3. Design and development of the electronics and the needed data acquisition for measuring the gamma ray counts

4.2.4. Selection of the gamma ray sources and design and manufacture of their collimators

4.2.5. Validation of the DSCT operation as a single source CT with a two phase phantom.

4.2.7. Evaluation of CREL estimation maximization (EM) algorithm for image reconstruction of dynamic three phases system using DSCT

4.2.8. Development of a new image reconstruction algorithm and programs for dynamic three phase system using DSCT

4.2.9. Validation of DSCT for determining holdup distribution in a three phase system using AM algorithm

The details are available in the quarterly reports, Appendix 3, doctoral thesis in preparation and the following manuscripts:

-Karim K, Varma R, Vesvikar M,Al-Dahhan MH.(2004) Flow pattern visualization of a simulated digester. Water research 38(17):3659-3670

-Vesvikar MS., Al-Dahhan MH. (2005) Flow pattern visualization in a mimic anaerobic digester using CFD. Biotechnology Bioengineering 89(6):719-732.

-Vesvikar M. S., Varma R., Karim K., Al-Dahhan M H.(2005). Flow pattern visualization in a mimic anaerobic digester: experimental and computational studies. Water Science and Technology 52(1-2):537-43

-Varma R. (2007). Phase distribution using tomography and performance of anaerobic digesters for bioenergy generation [Ph.D.Thesis in preperation] Washington University -St. Louis MO USA 
-Varma R, and Al-Dahhan MH. (2007) Effect of sparger design on hydrodynamics of a gas recirculation anaerobic bioreactor. In review by Biotechnology Bioengineering.

-Varma R, Bhusarapu S, O’Sullivan J A and Al-Dahhan M H. (2007). Comparison of alternating minimization and expectation maximization algorithms for single source gamma ray tomography. In review by Measurement Science and Technology.

\subsection{Gas holdup distribution using single source $C T$}

\subsubsection{Gas holdup distribution in a mimicked flat bottom anaerobic digester.}

The effects of gas recirculation flow rates on the gas holdup distribution in a mimicked 8 inch flat bottom digester equipped with a single orifice sparger were investigated using single source CT. In this work manual sampling was conducted to measure the solids loading where these manual measurements were used to reconstruct the distribution of gas and liquid phases. The measured information along with the data obtained by CARPT in the system were used to preliminarily evaluate the computational fluid dynamics models and closure.

The detailed results of this work are reported in the following published manuscripts:

-Karim K, Varma R, Vesvikar M,Al-Dahhan MH.(2004) Flow pattern visualization of a simulated digester. Water research 38(17):3659-3670

-Vesvikar MS., Al-Dahhan MH. (2005) Flow pattern visualization in a mimic anaerobic digester using CFD. Biotechnology Bioengineering 89(6):719-732.

-Vesvikar M. S., Varma R., Karim K., Al-Dahhan M H.(2005). Flow pattern visualization in a mimic anaerobic digester: experimental and computational studies. Water Science and Technology 52(1-2):537-43

\subsubsection{Gas distribution in a 6 inch anaerobic digester using a single point sparger and a single source $C T$.}

The objective of this work is to visualize the gas phase holdup distribution in the digesters on which performance studies were carried out (chapter 2). The 6 inch $(153 \mathrm{~mm})$ anaerobic digester mixed by gas recirculation at rates of 1 liter/min and 3 liter/min were selected. These biogas recirculation rates represent the lowest and the highest flow rates that were used for the performance studies. CT measurements were conducted for the above conditions at 5 levels (Figure 4.1). The gas phase is introduce at the bottom of the draft tube through a $1 / 4$ inch tubing inserted from the top of the digester with a single hole of $5 \mathrm{~mm}$ diameter. A sample of the results is shown in Figure 4.2 at level3 (middle of the draft tube). It is obvious that the gas phase in the draft tube is confined to a very small region. This finding 
suggests that proper sparger design is needed to ensure uniform gas phase distribution in the draft tube.

The detailed results of this work is reported in the quarterly reports and summarized in Appendix 3.1.

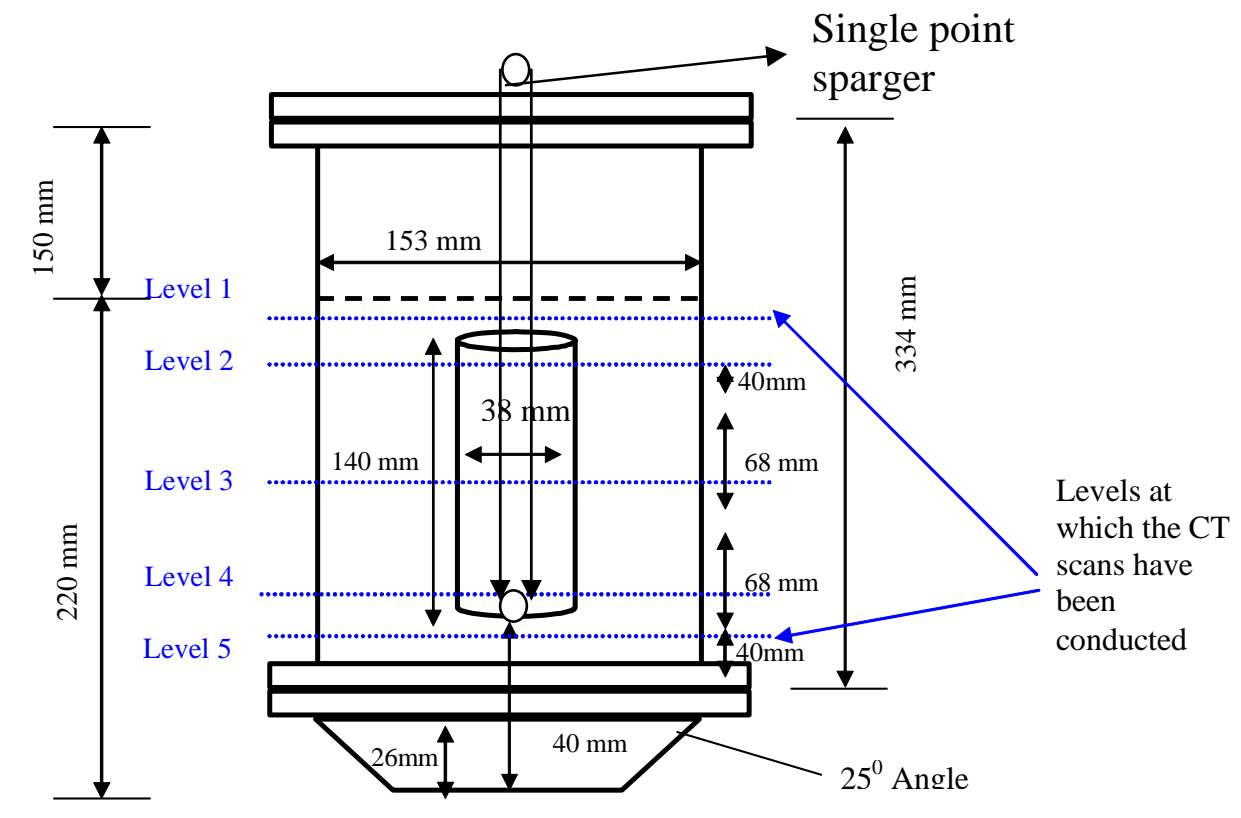

Figure 4.1. Schematic (not to scale) of the biodigester used with indications of the positions of the CT scans carried out.

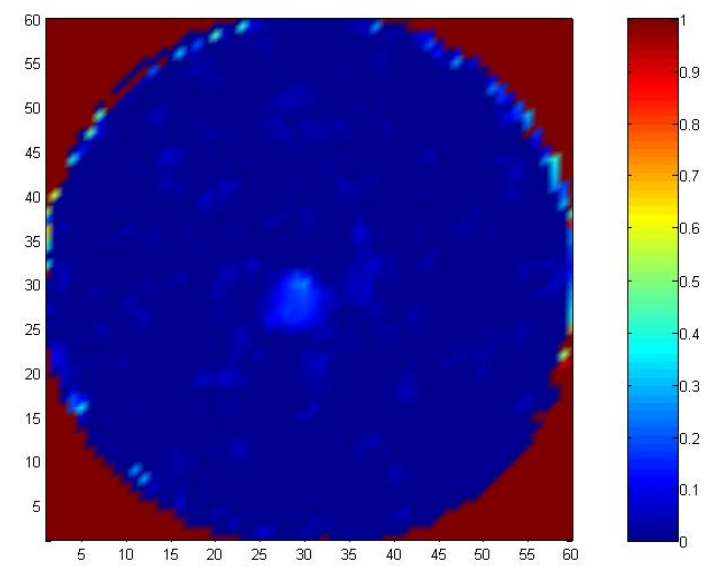

Figure 4.2: $\quad$ Image of the cross-sectional time averaged gas phase distribution at level- 3 with Biogas recirculation rate 1 lit/min (Pixel size 153 mm x 153 mm). Color bar to the right indicates the magnitude of gas holdup 


\subsubsection{Gas distribution with a multipoint gas sparger in a 6 inch anaerobic digester}

The objective of this study was to asses how the gas phase distribution inside the draft tube can be enhanced by using a ring sparger with multiples holes ( 25 holes). To achieve this, a ring sparger was designed and shown in Figure 4.3. The details of the ring sparger are shown in Figure 4.4. It was expected that the improvement of the gas phase distribution and its holdup in the draft tube region or the riser region of the reactor will reduce the apparent density of the mixture. This would create a larger density gradient between the draft tube and the region outside it that shall cause enhanced circulation of the liquid/slurry. Hence, this causes improved mixing and reduced dead zones (in active volumes). The results showed improvement in the gas distribution and increased in the gas holdup inside the draft tube as shown in Figure 4.5. The details of the results are reported in the quarterly reports and in the Appendix 3.2.

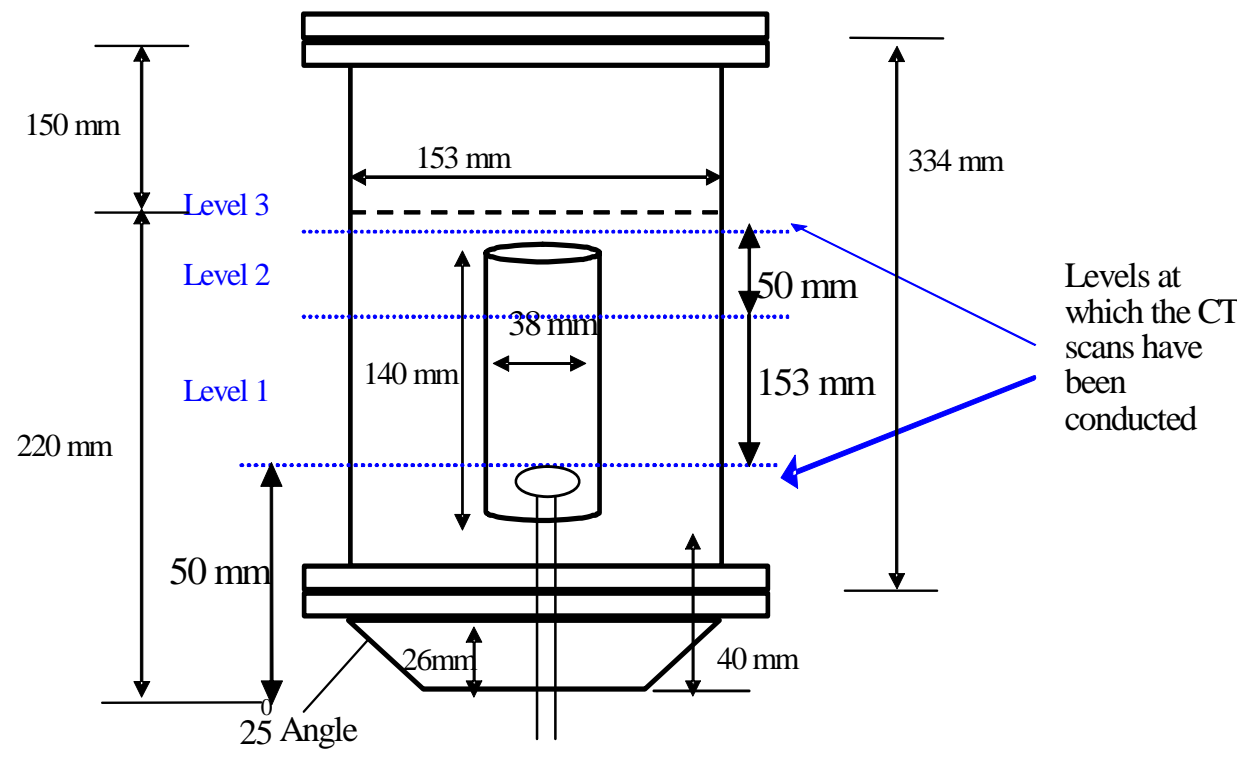

Figure 4.3: Schematic diagrams of 6 inch digester with ring sparger . 


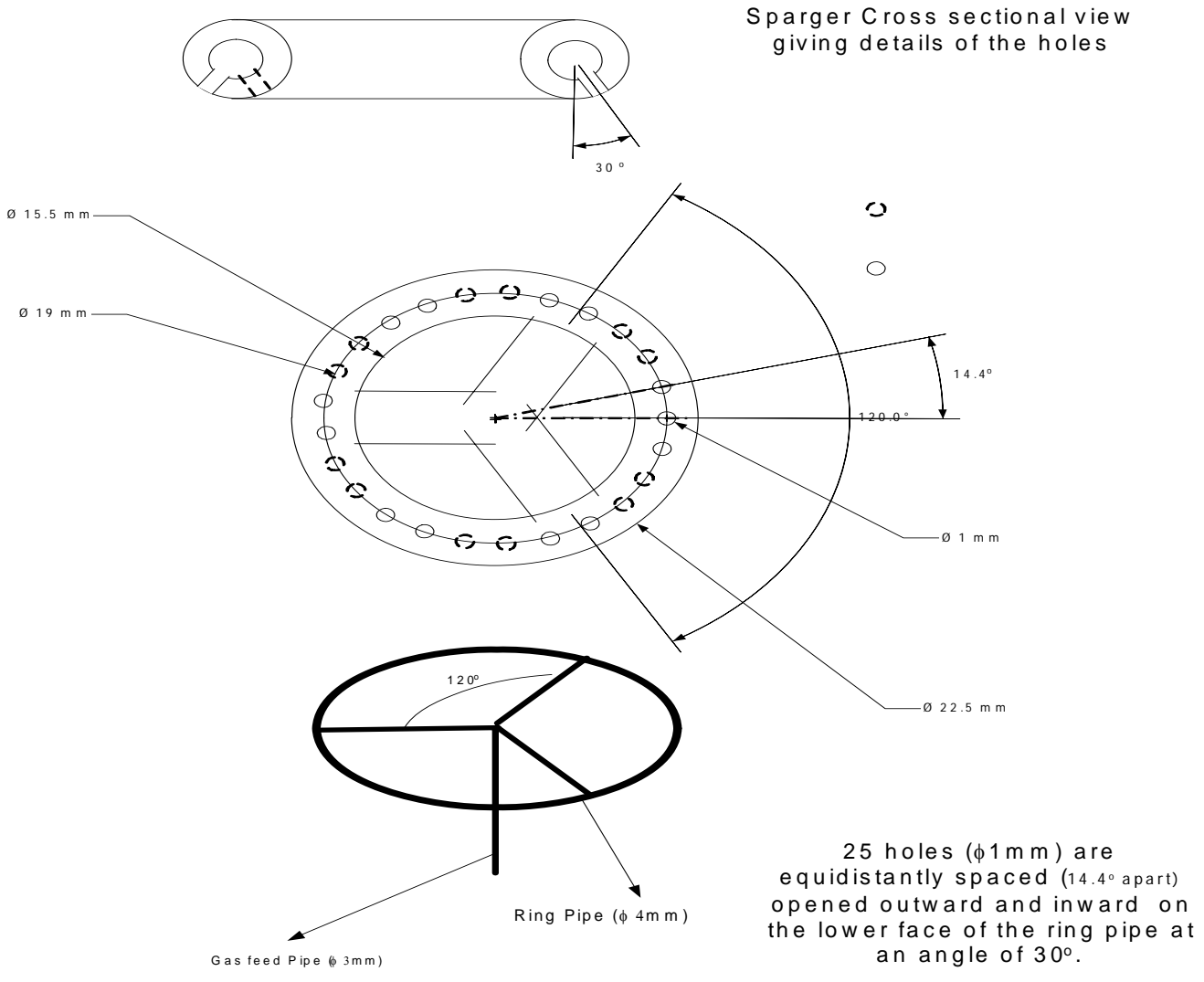

Figure 4.4: Schematic diagram of the sparger with the same open area as the single point injection system studied earlier

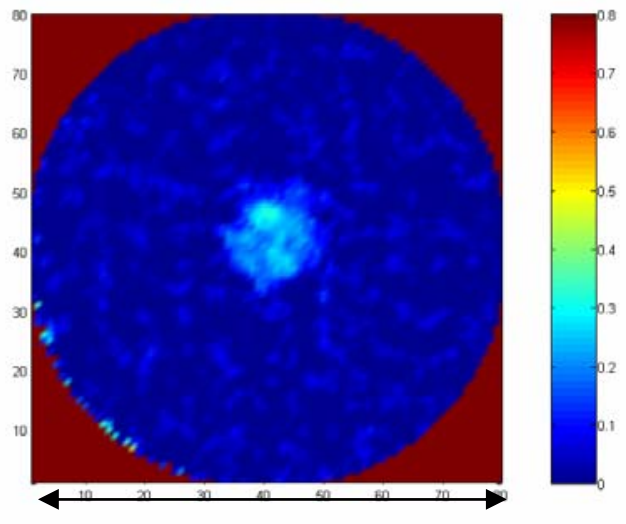

$153 \mathrm{~mm}$ diameter

Figure 4.5: Images of the cross-sectional time averaged gas phase distribution in the digester with ring system with an gas recirculation rate of 5 l/min (80x80 pixels) at level-3. 


\subsubsection{Effects of sparger design and the degree of uniformity of the gas holdup in the draft tube on the hydrodynamics and dead zones of the anaerobic digesters mixed by gas recirculation}

The effects of sparger design and gas flow rate on, gas holdup distribution and hence, on the liquid (slurry) recirculation and dead zones were studied in a surrogate anaerobic digester used for treating cow manure with a conical bottom mixed by gas recirculation. The hydrodynamics obtained by a single orifice sparger (SOS) and a multi-orifice ring sparger (MORS) with the same orifice open area and gas flow rates (hence the same process power input) are compared in this study. The advanced noninvasive techniques of Computer Tomography (CT) and Computer Automated Radioactive Particle Tracking (CARPT) were employed to determine gas holdup, liquid recirculation velocity and the poorly mixed zones. Gas flows $\left(Q_{g}\right)$ ranging of $0.017 \times 10^{-3} \mathrm{~m}^{3} / \mathrm{s}$ to $0.083 \times 10^{-3} \mathrm{~m}^{3} / \mathrm{s}$ were used which correspond to draft tube superficial gas velocities ranging from $1.46 \times 10^{-2} \mathrm{~m} / \mathrm{s}$ to $7.35 \times 10^{-2} \mathrm{~m} / \mathrm{s}$ (based on draft tube diameter). Air was used for the gas, as the molecular weights of air and biogas (consisting mainly of $\mathrm{CH}_{4}$ and $\mathrm{CO}_{2}$ ) are in the same range (biogas: $28.32-26.08 \mathrm{~kg} / \mathrm{kmol}$ and air: $28.58 \mathrm{~kg} / \mathrm{kmol}$ ). For a given gas flow rate, the MORS gave better gas holdup distribution in the draft tube and hence, enhanced liquid (slurry) recirculation and reduced the fraction of the poorly mixed zones compared to the SOS. The improved gas holdup distribution in the draft tube was found to have increased the overall liquid velocity. Hence, for the same process power input the MORS system performed better by enhancing the liquid recirculation and reducing the poorly mixed zones.

The details of the results and analysis are presented in the following manuscript attached in Appendix-3.3.

-Varma R, and Al-Dahhan MH,. (2007).Effect of sparger design on hydrodynamics of a gas recirculation anaerobic bioreactor. In review Biotechnology Bioengineering.

\subsection{Development of the novel dual source (energy) gamma ray computed tomography (DSCT)}

As mentioned earlier the development steps of a novel dual source (energy) gamma ray computed tomography (DSCT) encountered many challenging technical problems and required radiation safety measures which were all resolved successfully in a collaborative effort between Washington University (WU) and Oakridge National Laboratory (ORNL). The accomplishments consists of the following:

\subsubsection{Design, construction and test of the DSCT hard ware.}

The Dual Source Tomography Set up consists of two gamma ray sources emitting gamma ray photon of two different energies. A fan beam geometry is employed in this system as shown in Figure 4.6(a). The detectors array consists of 15 detectors totally subtending an angle of $38.4^{\circ}$ with the source as the center. The photograph of the system is shown in figure 4.6(b). These detectors are then moved in increments of $0.12^{\circ}$ subtended to the gamma ray source. An object (multi-phase flow system) of 24 inch diameter can be covered within the 
angle span of $38.4^{\circ}$ of the detectors. Each detector is moved 21 times by moving the plate on which the detectors rest to create 21 projections. This is achieved with the aid of a stepper motors that are connected to the computer through controllers. This motion of detectors simulates a fan beam with 315 detectors hence 315 projections per source position. After this, the source plate is reoriented along the horizontal plane and the process of motion of the detector plate is repeated. A total of 198 source positions are used to gather projections data. Hence, date for a total of 315 x $198=62,370$ projections are gathered for a domain represented by a circle of 24 inch diameter. The horizontal plate is attached to four ball screws along the side of the frame. These ball screws are connected with a chain in the bottom portion of the system. A motor with a gear is attached which can be made to rotate counter clockwise or clockwise. This enables the plate to move vertically and cover a height of nine feet. Thus any vertical location of any multiphase system can be scanned in this range. This setup was designed by the team at Washington University (WU) and fabricated by John Ramming Machine Shop- St. Louis USA.

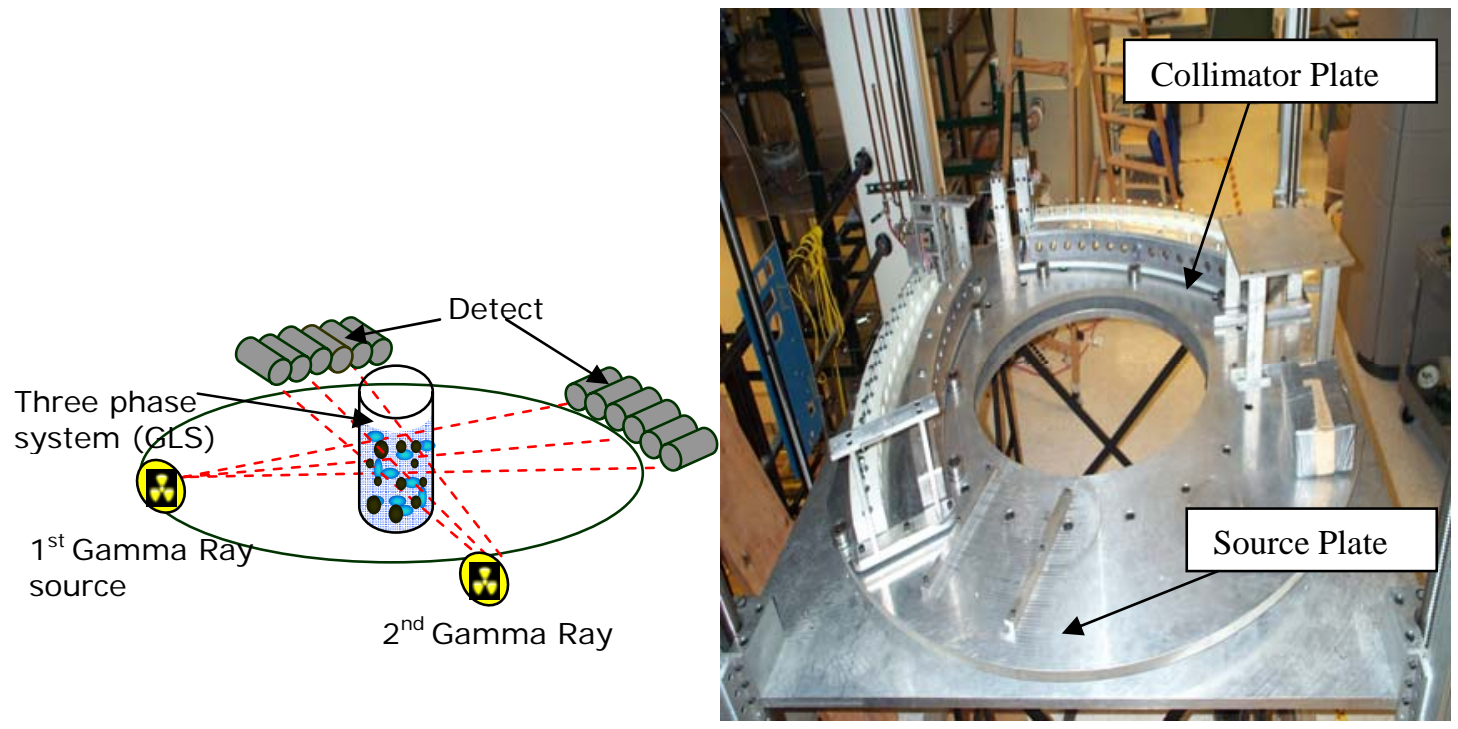

(a)

(b)

Figure 4.6: Details of the Dual Source tomography set up (a) Schematic of the set up, (b) a top view of the system with out the sealed source collimators

\subsubsection{Development of the data acquisition system and control for the automation of the DSCT hardware}

The DSCT setup requires various motions to rotate the detectors array and the source positions and to move the set-up vertically at the desired plane for collecting the gamma ray counts data. These are achieved with the aid of stepper motors. The stepper motors (manufactured by oriental motors) are connected to controllers. The controllers in turn are connected to the computer. The controller upon receiving commands from the computer sends pulses to the stepper motors that cause the desired motions. A computer program was developed at Washington University that was integrated with the $\mathrm{C}++$ program for the data 
acquisition system consisting of the electronic components developed with the help of Oak Ridge National Laboratory (ORNL) team. This way the DSCT setup motions were synchronized with the data acquisition system for the electronic components. Figure 4.7 shows a photo of the electronics developed for DSCT.

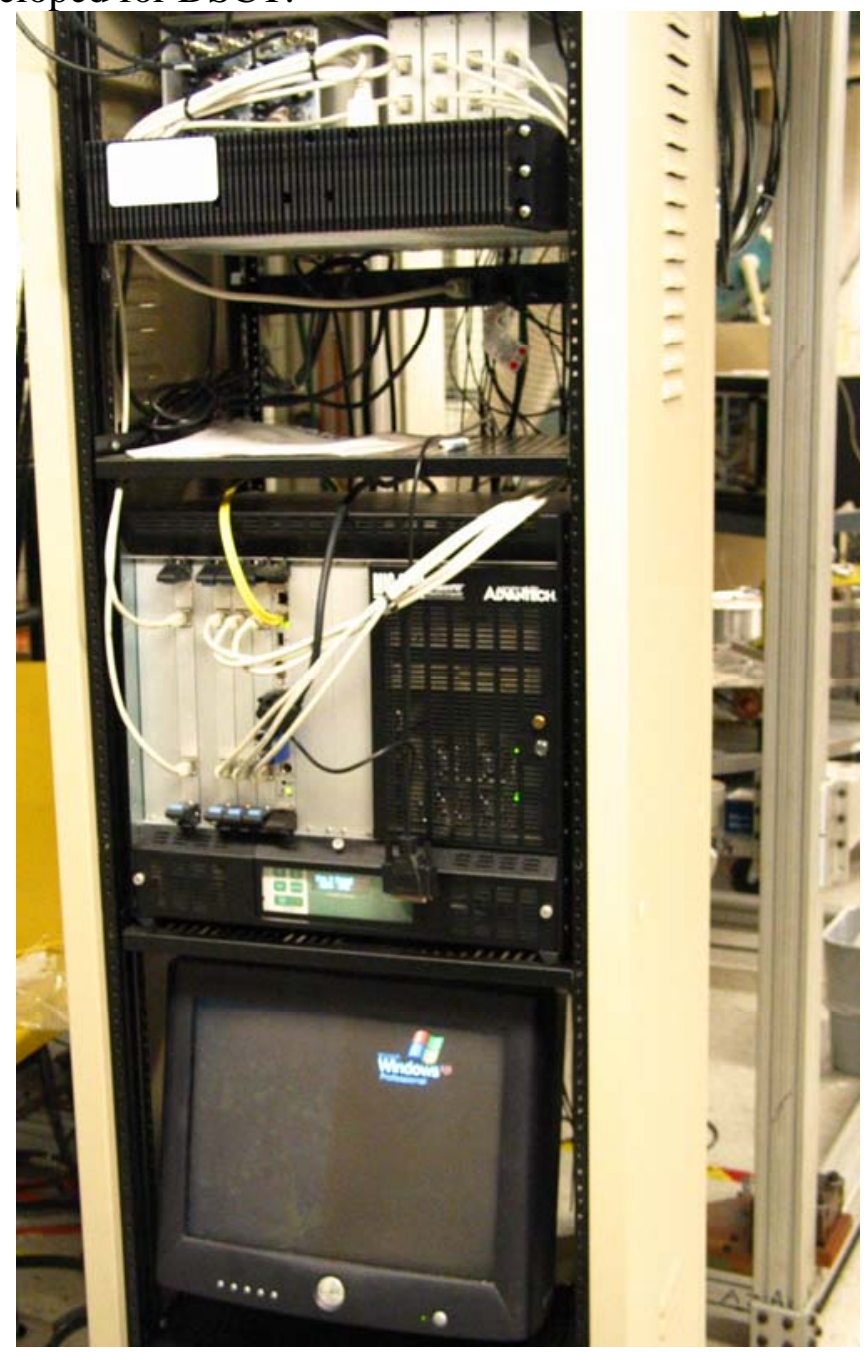

Figure 4.7: $\quad$ Picture of the electronics and computer control system developed for DSCT.

\subsubsection{Design and development of the electronics and the needed data acquisition system for the measurement of the gamma ray counts}

The data acquisition system for measuring gamma ray counts was developed by the Oak Ridge National Laboratory (ORNL) team. This system is similar to the one used for MPCARPT described in Appendix -2. Figure 4.8 below shows the schematic of the data acquisition electronics. The assembled system is shown in Figure 4.7. The unit essentially consists of detectors, formed by a photomultiplier tube (PMT) connected to the base amplifier. This base amplifier is powered by a power supply unit and the output signal from the base amplifier goes to timing filter amplifier (TFA) input for amplification. Both power supply unit 
and timing amplifier sit in a NIM bin. Each timing amplifier has 8 channels (one for each detector). The timing amplifier is connected to the pulse processor card (one card is required for one timing amplifier, thus 8 detectors need only one card). The pulse processor card functions as a discriminator, scaler and an interface to the PC. This pulse processor card sits in a compact PCI box and it is connected to the back plane of compact PCI which also holds a PC on a card.

A single $\mathrm{C}++$ program compiled and run by the user performs the data acquisition according to the needs of the user. Further details of this system is available in Appendix 2 (Appendix B of Appendix-2) of the following doctoral thesis:

Vesvikar M. (2006) Understanding the hydrodynamics of anaerobic digester for bioenergy production.[D.Sc. Thesis], St. Louis -USA, Washington University.

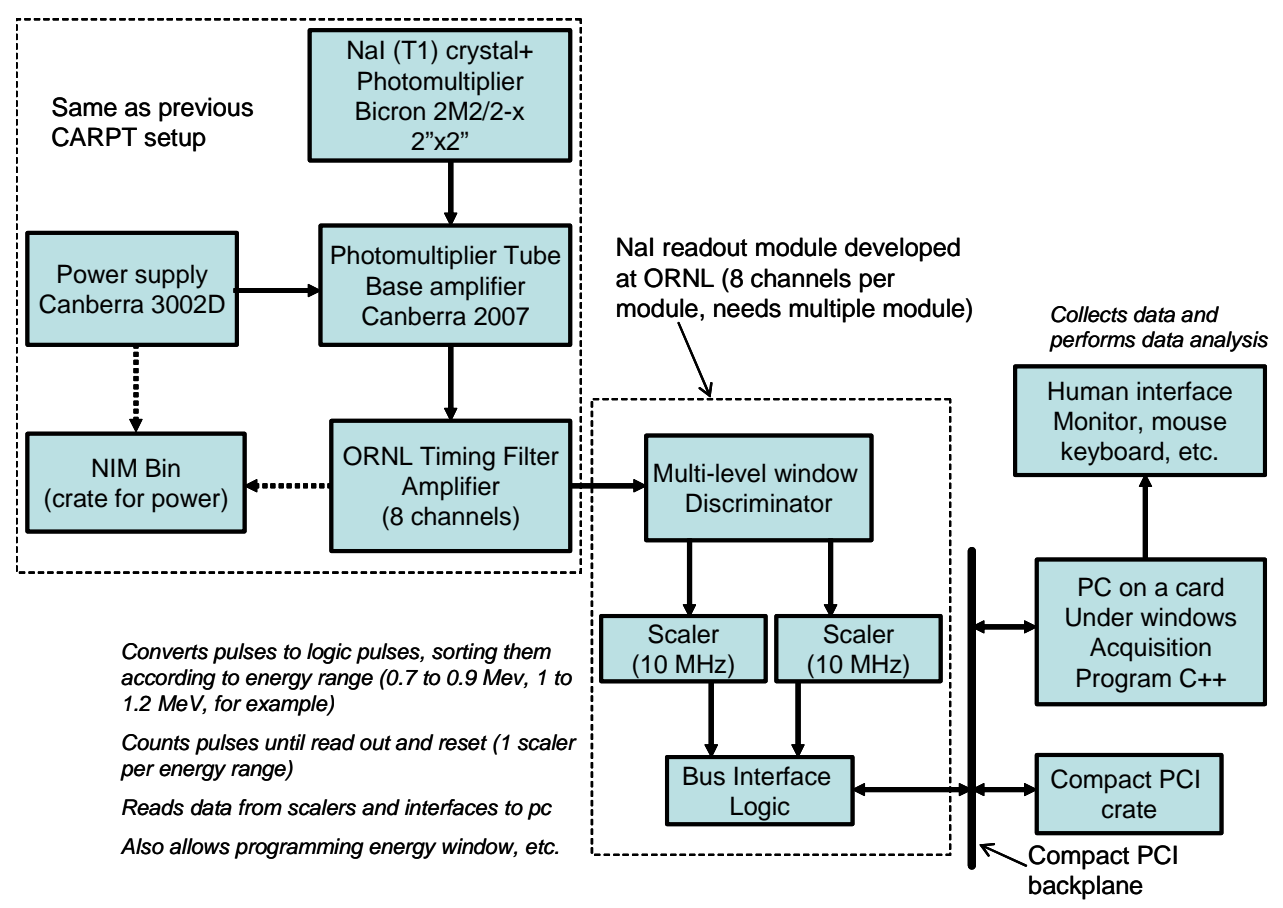

Figure 4.8: DSCT data acquisition system electronics

\subsubsection{Selection of the gamma ray sources and design and manufacture of their collimators}

Out of the multiple options available of radioactive isotopes, ${ }^{137} \mathrm{Cs}$ and ${ }^{60} \mathrm{Co}$ sources were selected as they have photo peaks at $661 \mathrm{keV}$ and $1330 \mathrm{keV}$, respectively. These peaks have high enough energy to be able to penetrate a lager diameter column. Also these sources have a reasonable half life (about 30 years for ${ }^{137} \mathrm{Cs}$ and about 5.5 years for ${ }^{60} \mathrm{CO}$ ) which enables their use for a long period of time. A simple set of densitometry based experiments 
were carried out to select the source for dual source gamma ray tomography purposes. A small 6 innch phantom was sued for this study. The details of this work are given in section 4.2.6. A $250 \mathrm{mCi}{ }^{137} \mathrm{Cs}$ source and $50 \mathrm{mCi}{ }^{60} \mathrm{Co}$ sources were used in the sealed form. These sources are place in collimators made of lead for ${ }^{137} \mathrm{Cs}$ and Tungsten for ${ }^{60} \mathrm{Co}$. The collimators are shown in Figure 4.9. The source was procured from AEA Technology, LA 70809. The collimators we re design by Dr. Charles Alexander at ORNL and were manufactured at the ORNL machine shop. As per the requirements of radiation safety, to secure these collimators special straps were designed that bolt the collimators to the DSCT set up. These straps are visible in yellow color as shown in Figure 4.10. The special security bolts are indicated in circles in the same figure. More information on the experiments done and the methodology for the source selection is available in Appendix 3.4
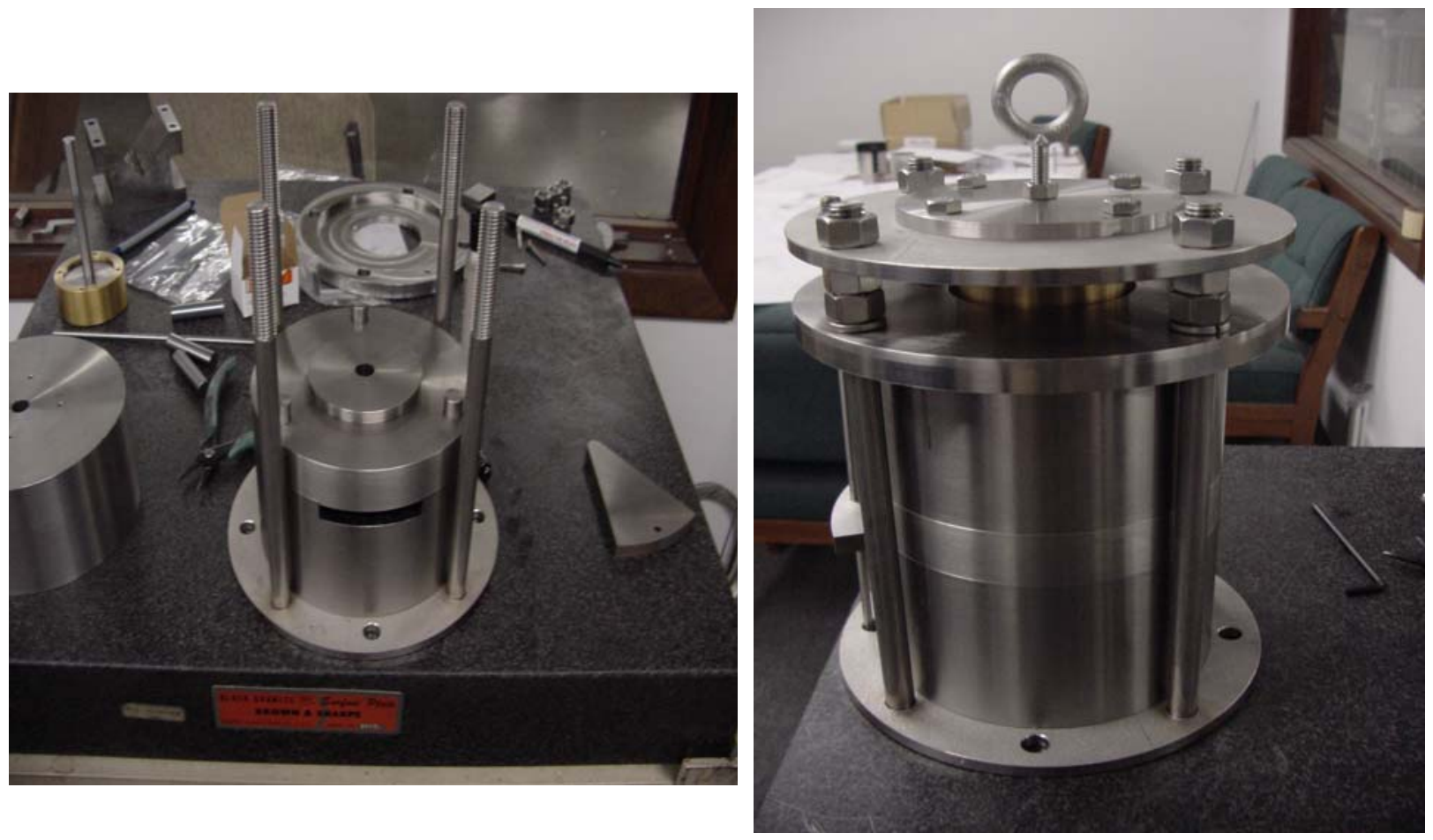

Figure 4.9: Tungsten collimator to house up to $100 \mathrm{mCi} \mathrm{Co}^{60}$ source 


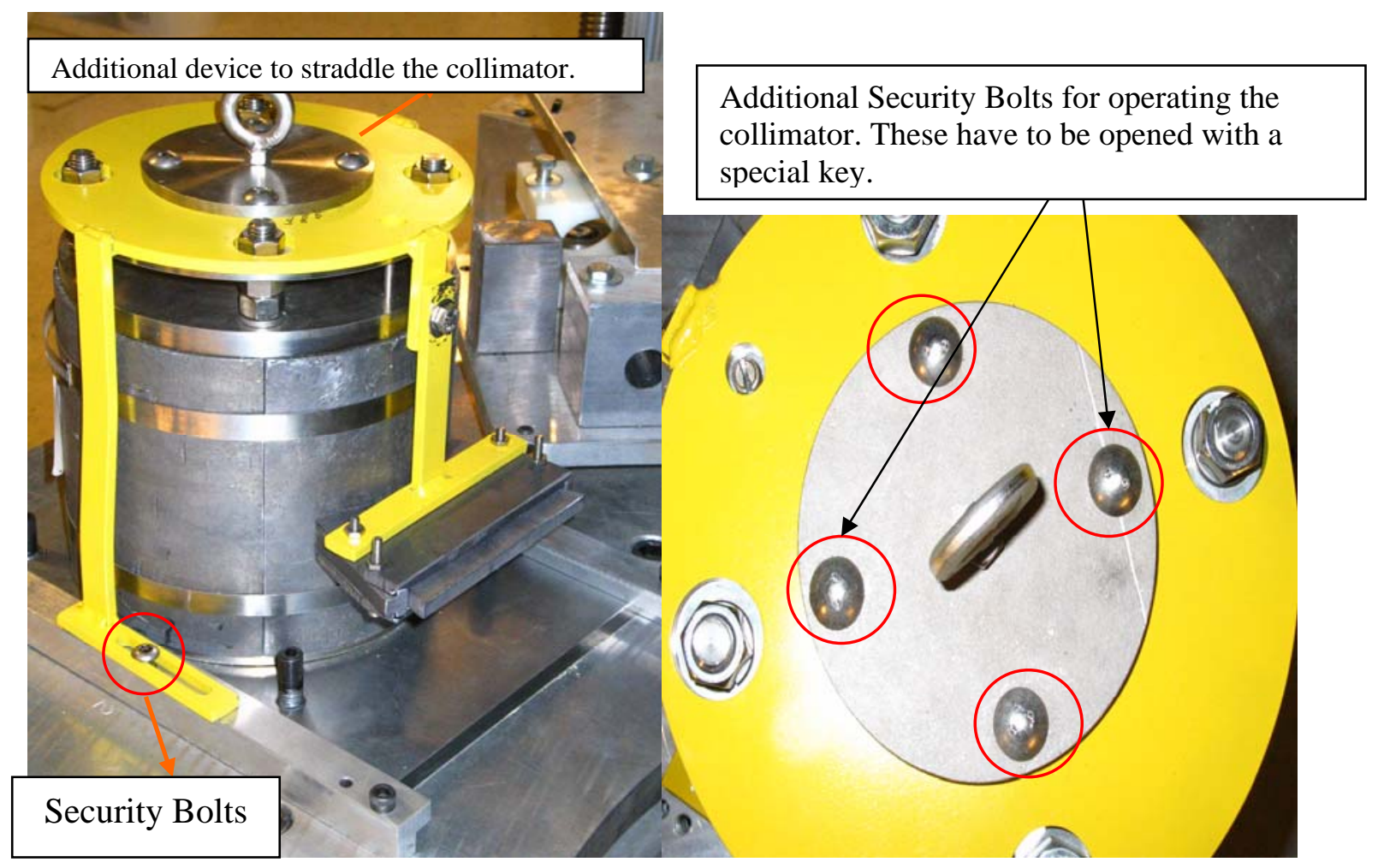

(a)

(b)

Figure 4.10: (a) Shows the additional device fabricated to hold the collimators in a secured place. The security bolts that hold the device on the set up and can only be opened with a special key. (b) Shows the other set of security bolts that control the operation of the source. A special key is required to open the upper flap and turn on the source.

\subsubsection{Validation of the DSCT operation as a single source CT with a two phase phantom.}

To confirm if the Dual Source Computer Tomography system is able to first accomplish what the single source tomography scanner are able to, a two phase phantom was scanned. The idea is to have the two phases static such that the hold up and phase distribution can be calculated on the basis of the tomography scan and can be compared to the actual hold up distribution based on the geometry of the system.

This phantom consisted of two concentric cylinders as shown in Figure 4.11. The inner cylinder was left empty (hence consisted of air), and the outer annular region was filled with water. This way the system consisted of two phases and the hold up of the gaseous phase in the center of the system is one (100\%) and the hold up of the liquid phase in the annular region is one (100\%).The image reconstruction was carried out with our EM algorithm. The details of experiment and the results are reported in $7^{\text {th }}$ and the $8^{\text {th }}$ quarterly reports. 
The results of the scans as seen in Figures 4.12 and 4.13 clearly show that both the ${ }^{137} \mathrm{Cs}$ and the ${ }^{60}$ Co source are able to characterize the two phases successfully. Hence, the DSCT setup can be used to image two phase systems with the Cobalt $\left({ }^{60} \mathrm{Co}\right)$ or with the Cesium $\left({ }^{137} \mathrm{Cs}\right)$ sources. The liquid and gas hold up distributions matches those based on the geometry shown in Figure 4.11.

Further details on the methodology involved and details of the base scans used to arrive at the hold up distribution of the phases as shown in Figures 4.12 and 4.13 are available in Appendix 3.5.

Water filled annular region (Liquid Hold up=1)

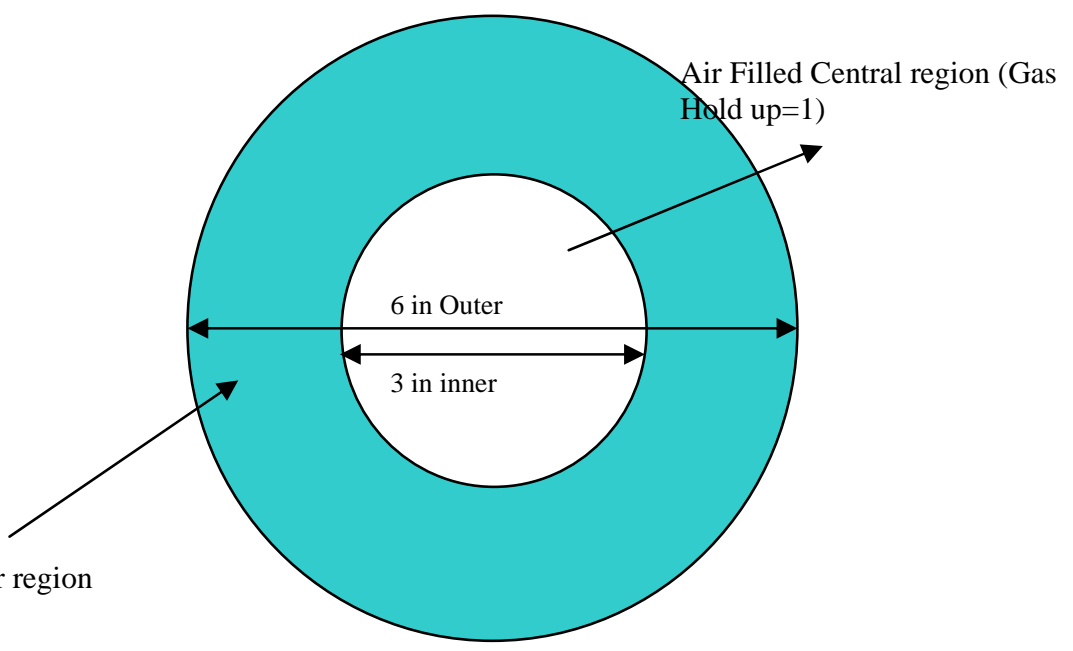

Figure 4.11: Cross sectional schematic of the phantom. The Annular region is filled with water.
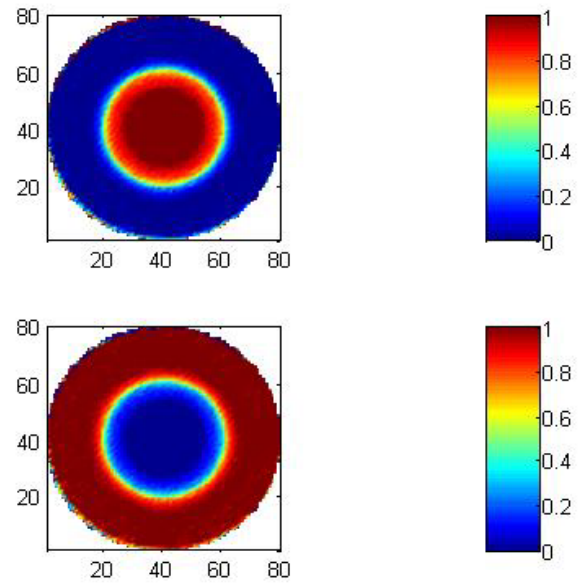

Figure 4.12: Hold up profile of gas phase (air) above and Hold up profile of liquid phase (below) based on data from ${ }^{137} \mathrm{Cs}$. 

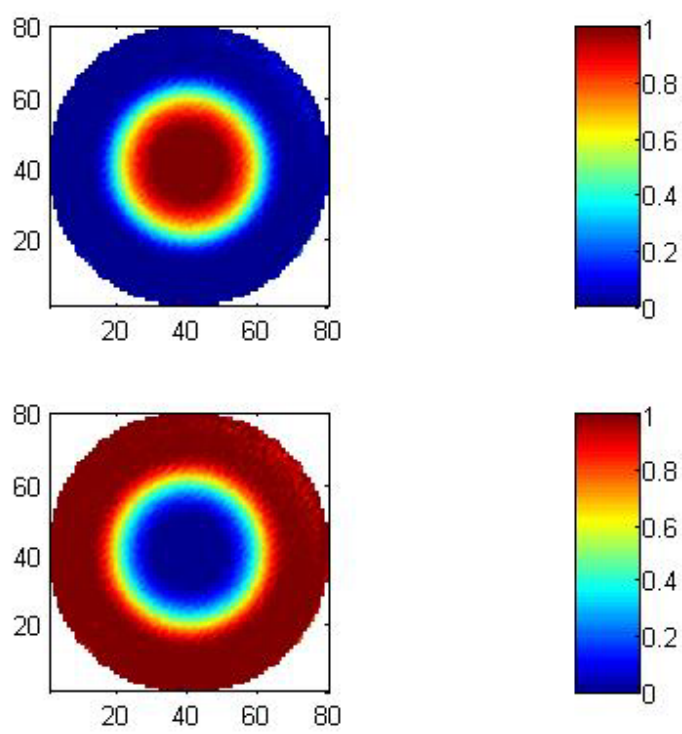

Figure 4.13: Hold up profile of gas phase (air) above and Hold up profile of liquid phase (below) based on data from ${ }^{60} \mathrm{Co}$.

\subsubsection{Evaluation of CREL estimation maximization (EM) algorithm for image reconstruction of dynamic three phases system using DSCT}

The principle behind the Chemical Reaction engineering Laboratory (CREL) EM algorithm for CT image reconstruction used for determining phase holdup distribution in two phase systems (as used in section 4.2.6.), was extended to determine the solids phase hold up in a three phase system with DSCT. In this method the gamma ray projections data from the two different sources is collected and then processed by the Estimation Maximization algorithm separately to determine the attenuation coefficient distribution (images). This is then combined, post data processing, with the data from calibration scans to determine the holdups distribution. To evaluate this methodology the phantom as shown in Figure 4.14 was considered.

The gamma ray counts data for the schematic of the phantom (Figure 4.14) were generated by simulation based on the geometry of DSCT setup. Schaffer (1970) algorithm was used to generate passion random number that represents gamma ray counts detected by the detectors. The attenuation data of the materials in the phantom were based on the energies of the gamma ray photons from ${ }^{60} \mathrm{Co}$ and ${ }^{137} \mathrm{Cs}$. These simulated data were processed to determine the solids holdup based on the CREL EM algorithm. The details of this procedure have been out lined in section 3.0 of the manuscript attached as Appendix 3.6. A schematic of the CREL EM method followed is shown in Figure 4.15.

Image reconstruction is first carried out based on the data for each of the radio isotopes individually. This gives the attenuation coefficient distribution as indicated by Equation (1) in Figure 4.15. This is then combined with calibration scans if the system consisting of each of the phase purely. This gives Equation 5 (in Figure 4.15) which represents the basis for 
determining the solids hold up distribution. Figure 4.16 shows the result obtained for the solids holdup distribution.

The result, as shown in Figure 4.16 is not clear and is noisy. The neat circular solid domain in the center (Figure 4.14) doesn't appear in a clear manner with a holdup value of unity. This shows that the CREL EM based image reconstruction which is used for two phase systems doesn't work for reconstructing hold up profile for a three phase system. The image appears to be noisy and grainy and no clear pattern can be seen. This can be attributed to the short coming of the EM algorithm as proposed by Lange and Carson (1984) which defined the image reconstruction for tomography as a maximum likelihood estimation problem. However, in the maximization step or M-step of the EM algorithm, an approximation is made in the solution which can affect the image quality, particularly in the case of domains with high attenuation material. Hence, there is a need to evolve a different methodology for determining the phase holdup distributions using DSCT.

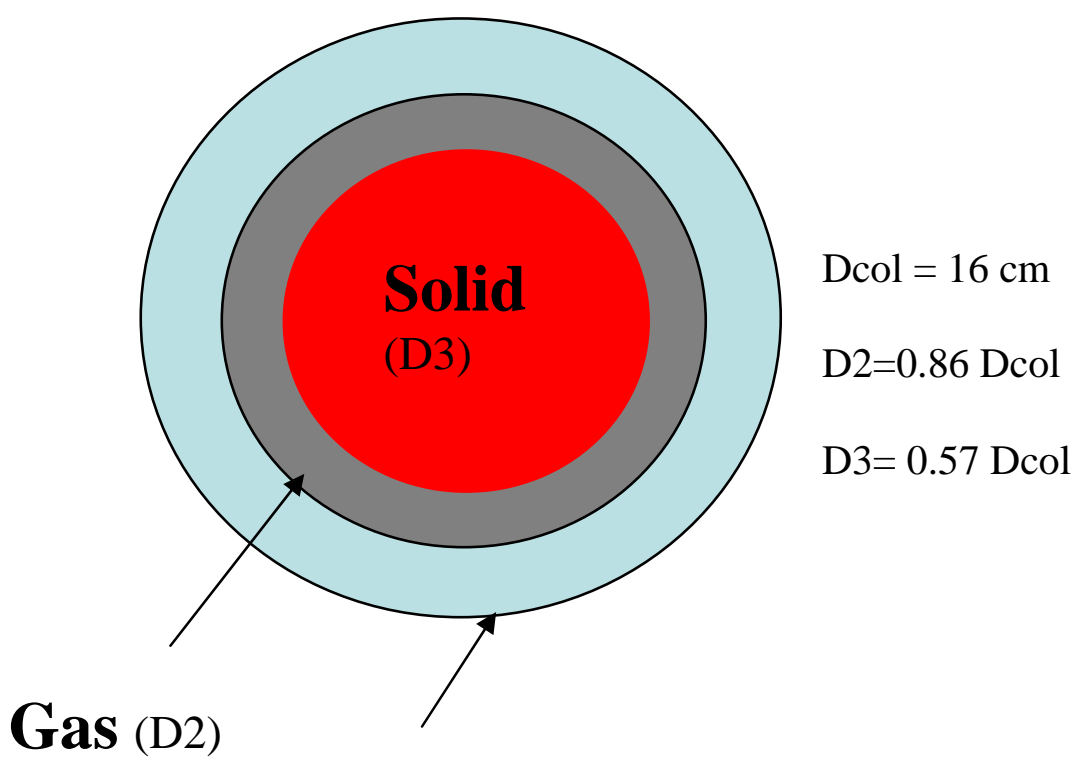

Liquid (Dcol)

Area of Solid $=$ Area of Liquid $=$ Area of Gas

Figure 4.14: Schematic of the three phase phantom used to test CREL EM algorithm. 
DSCT Transmission data

\section{Image reconstruction (attenuation distribution profile) using Estimation- Maximization}

\section{Dual Energy Computer Tomography}

For determining solids holdup

Equations 2, 3 and 4 give Equation 5.

Subscript $g$ represents gas, $l$ represents liquid and $s$ represents solid.

$\varepsilon_{S}(x)=\frac{\left[\mu_{A l}(x, 2)-\mu_{A l}(x, 1)\left(\frac{\mu_{l}(x, 2)}{\mu_{l}(x, 1)}\right)\right]}{\left[\mu_{S}(x, 2)-\mu_{S}(x, 1)\left(\frac{\mu_{l}(x, 2)}{\mu_{l}(x, 1)}\right)\right]}$

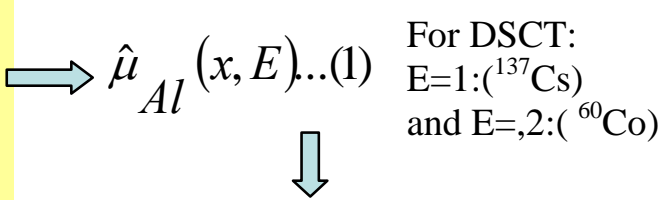

Post attenuation image reconstruction of phase holdup distribution is determined as follows.

$$
{ }_{4 l}(x, E)=\mu_{g}(E) \varepsilon_{g}(x)+\mu_{l}(E) \varepsilon_{l}(x)+\mu_{s}(E) \varepsilon_{s}(x) . .
$$

$$
\begin{gathered}
\varepsilon_{g}(x)+\varepsilon_{l}(x)+\varepsilon_{s}(x)=1 \ldots .(3) \\
\mu_{g}(E), \mu_{l}(E) \text { and } \mu_{s}(E) \ldots . . .(4) \\
\text { Calibration scans are made } \\
\text { to determine the } \\
\text { attenuation values of phase } \\
\text { for both the energies from } \\
\text { the two radio isotopes } \\
\left({ }^{137} \mathrm{Cs} \text { and }{ }^{60} \mathrm{Co}\right)
\end{gathered}
$$

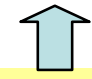

Figure 4.15: Schematic of the steps for using the CREL EM algorithm for image reconstruction using DSCT .

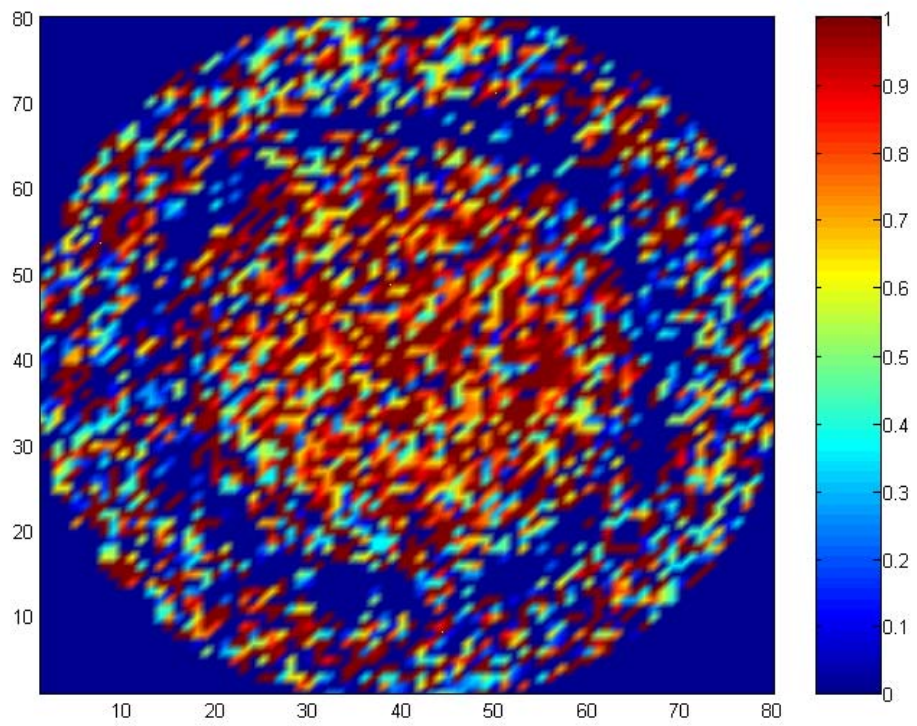

Figure 4.16: Solid holdup distribution obtained using the CREL EM algorithm method as shown in Figure 4.15. 


\subsubsection{Development of a new image reconstruction algorithm and programs for dynamic three phase system using DSCT}

O'Sullivan and Benac (2007) reformulated the maximum likelihood problem as a double minimization of an I-divergence to obtain a family of image reconstruction algorithms, called the alternating minimization algorithm (AM). The AM algorithm increases the log-likelihood function while minimizing the I-divergence. In this work, the AM algorithm for gamma ray tomography image reconstruction was implemented for industrial applications. The Alternating -Minimization algorithm developed by O’Sullivan and Benac (2007) was implemented for single source CT using a two phase phantom with the objective of computing the holdup of the individual phases. It was found that for this case the AM algorithm gives far improved results as compared to the CREL EM algorithm.

The details of this work is available in the manuscript attached as Appendix 3.6.

-Varma R, Bhusarapu S, O’Sullivan J A and Al-Dahhan M H. (2007). Comparison of alternating minimization and expectation maximization algorithms for single source gamma ray tomography. In review Measurement Science and Technology.

The AM algorithm as proposed by O'Sullivan and Benac (2007) was applied to DSCT. In this case the gamma ray counts data from both of the isotopes are processed simultaneously to directly compute the holdup distribution. The intermediate step of determining the attenuation distribution first, as indicated in Figure 4.15, is eliminated. A schematic of this algorithm's approach is described in Figure 4.. This algorithm was originally proposed for Xray tomography and has been modified for DSCT here. Further mathematical details of this algorithm are available in Benac (2005) and O'Sullivan and Benac (2007).

Simulated projections were generated using Scahfler's algorithm (1970) for the ${ }^{137} \mathrm{Cs}$ isotope with $661 \mathrm{keV}$ energy gamma ray photon and ${ }^{60} \mathrm{Co}$ isotope with $1330 \mathrm{keV}$ energy gamma ray photon. Details of this procedure are available in section 3.0 of Appendix-3.6. The phantom used for this study comprised of circular domain with a uniform mixture of $30 \%$ glass, $50 \%$ water and $20 \%$ gas. This could be imagined as the three phases are well mixed and completely dispersed in the domain. Figure 4.17(a), (b) and (c) shows the ideal values of the holdup distribution of the three phases as it appears in the phantom.

For the purpose of comparing the results that one would obtain if the CREL based EM algorithm approach was used, the projections data was processed as per the schematic show in Figure 4.15. The method followed for obtaining solids hold up profile by this procedure (Equation 5 in Figure 4.15) was applied to gas and the liquid phases also. The values of hold up in any given pixel, that were greater than unity for any phase were set and unity and the values below zero were set as zero. Figure 4.19 shows hold up profiles obtained for the three phases using CREL EM algorithm. These results show that the holds up profiles are highly erroneous and the images appear noisy. Ideally these should resemble the corresponding images in Figure 4.18 which is not the case here. The mean error, based on difference from the ideal values, was found to be $436 \%$ for the liquid holdup values, $631 \%$ for the solids hold up values and $117.7 \%$ for the gas. Clearly these errors are very high and unacceptable by any standard. 
In comparison, the AM algorithm directly computes the data from both the energies and computes the phase holdup of the phases involved directly. Calibration scans need to be conducted for this approach just as it was done for the CREL based EM approach (Figure 4.15). The results obtained by this algorithm for the same data set are shown in Figure 4.20. These images are far clearer than those seen in Figure 4.19. The holdup values obtained match those of the phantom as shown in Figure 4.18. These results show tremendous improvement in the images obtained using the AM algorithm as compared to the EM algorithm approach. The error for hold up estimate of liquid, solid and gas was found to be $2.6 \%, 1.18 \%$ and $7.3 \%$ respectively. Clearly there are a couple of orders of magnitude of difference in the error between the two algorithms.

Hence, it can be concluded that the A- $\mathrm{M}$ algorithm helps reduce the error drastically and is suitable for ${ }^{60} \mathrm{Co}_{-}{ }^{137} \mathrm{Cs}$ radioisotope combination used in DSCT to accurately determine the phase holdup distribution in dynamic three phase system.

Transmission data for both radio isotopes $\left({ }^{137} \mathrm{Cs}\right.$ and $\left.{ }^{60} \mathrm{CO}\right)$

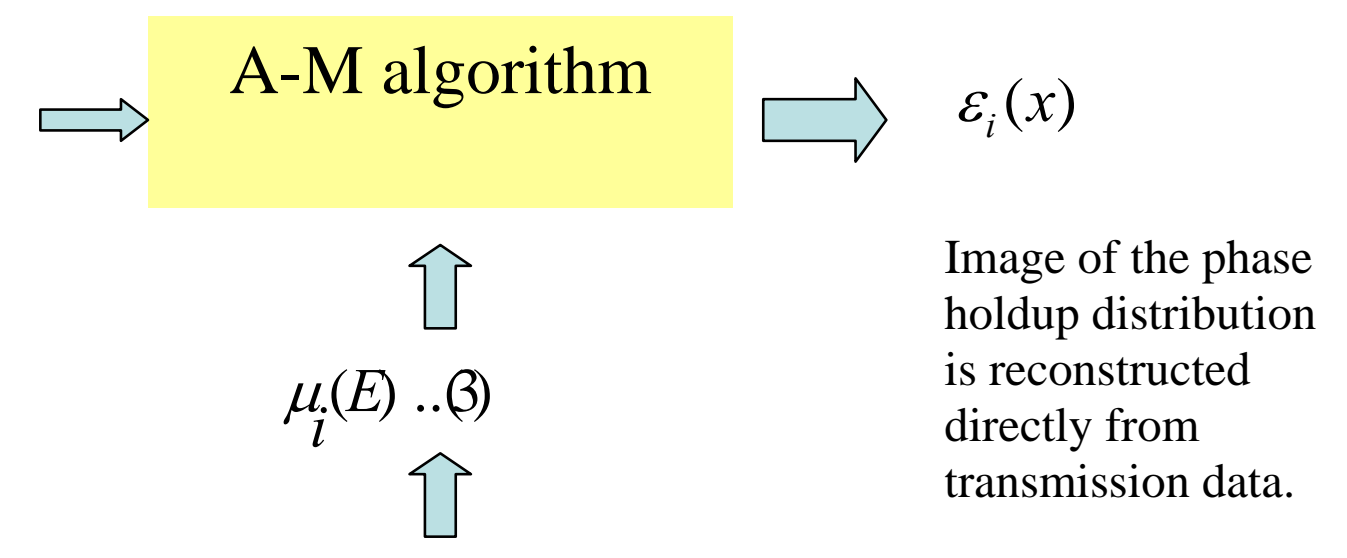

\section{A-M algorithm}

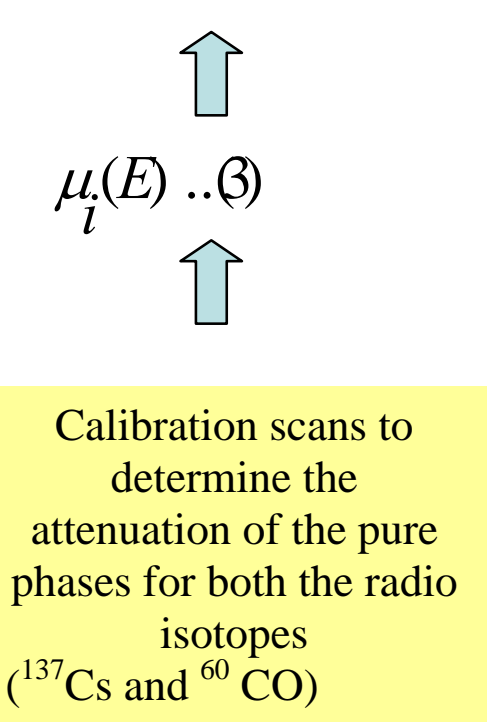

Figure 4.17.: Shows the schematic of the AM algorithm's approach for processing the data.

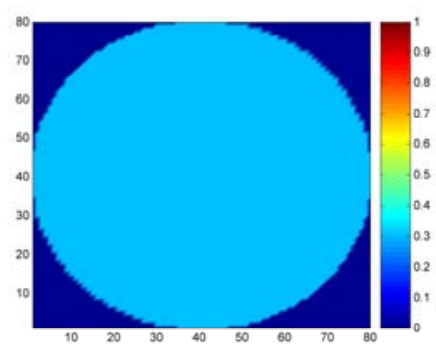

(a) Glass

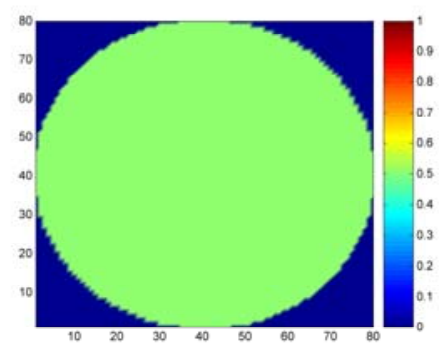

(b) Water

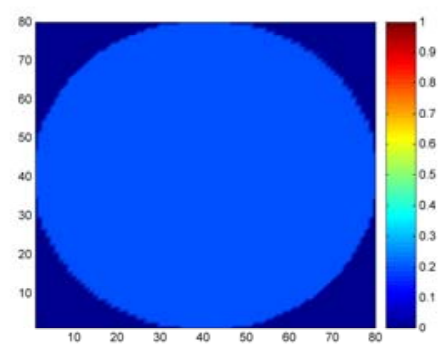

(c) Air 
Figure 4.18: Ideal holdup values for (a) solid phase (glass) and (b) liquid phase (water) (a) air, for which the simulate projections data was generated.

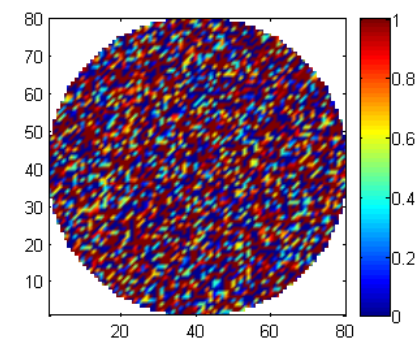

(a) Water

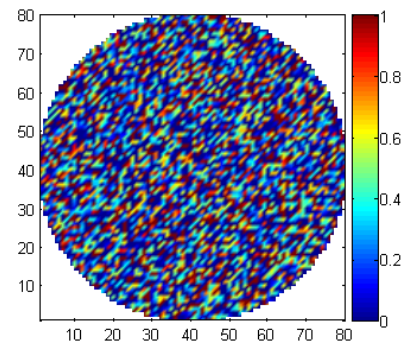

(b)Solid

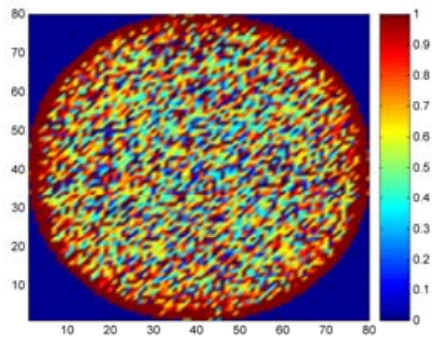

(c) Air

Figure 4.19: These images show the holdup profiles obtained by the CREL method based on EM algorithm:(a) Liquid (water) holdup profile obtained, (b) solid (glass) holdup profile obtained, (c) gas (air) holdup profile obtained,

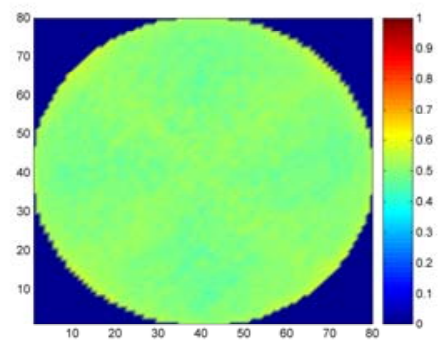

(a)

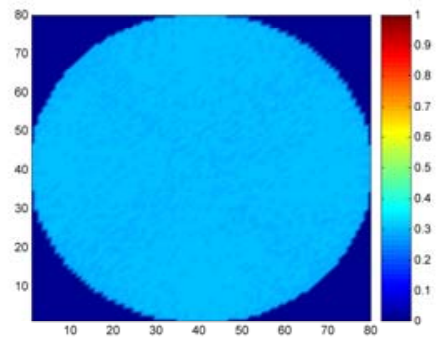

(b)

Figure 4.20: These images show the holdup profiles obtained by the AM algorithm:(a) Liquid (water) holdup profile obtained, (b) solid (glass) holdup profile obtained, (c) gas (air) holdup profile obtained

\subsubsection{Validation of DSCT for determining holdup distribution in dynamic three phases using AM algorithm}

Before the DSCT is used along with the AM algorithm to determine the phase hold distribution in any three phase system, it should be first validated experimentally based on a three phase phantom. Figure 4.21 shows the schematic of a three phase phantom made of Perspex for validating the DSCT and the developed AM algorithm. This phantom was constructed for the purpose of conducting such validation experiments. Once the DSCT with the AM algorithm are validated they could be used to study the phase holdup distribution in any dynamic three phase system.

However, as mentioned earlier, due to the removal of the sealed sources from the DSCT unit in August 2006 this process was not accomplished at this time. In the future such work will be performed and the results will be published in manuscripts and a doctoral thesis in preparation (Varma 2007). It is note worthy that in addition to the, too many challenging technical problems and issues that were encountered, the development of the image 
reconstruction algorithm suitable for DSCT that can cover a wide range of isotopes combinations was one of the hardest hurdles. A new reconstruction algorithm and programs based on alternating minimization (AM) algorithm were developed and implemented, in collaboration with Prof. O'Sullivan from the Electrical systems and signals research laboratory (ESSRL) in the Electrical engineering department at Washington University (WU).

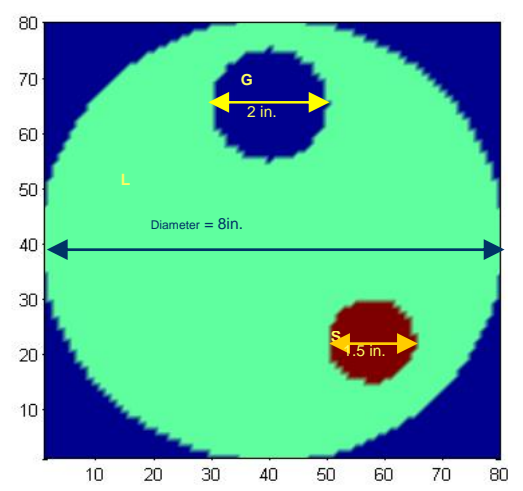

Figure 4.21 Three phase phantom consisting of liquid domain(L) of 8 inch diameter, solid region(s) of 1.5 inch diameter and gas(G) if 2 inch diameter. 


\section{Chapter 5 : Computation Fluid Dynamics (CFD) Of Anaerobic Digesters}




\section{Computational Fluid Dynamics (CFD) of Anaerobic Digesters}

Advanced non-invasive experimental techniques like computer automated radioactive particle tracking (CARPT) and computed tomography (CT) are essential to fundamentally understand the hydrodynamics of any opaque multiphase flow systems. However, their application is limited by the time and resource constraints. Thus these techniques cannot be used to evaluate the effect of every design and operating parameter on the hydrodynamics. This is where Computational Fluid Dynamics (CFD) code can help in such evaluation and for design and scale-up once it is validated. CFD proves to be a valuable and efficient tool to understand and evaluate hydrodynamics of a flow system. For single-phase systems, CFD models and closures are well established and validated with benchmark experimental data, such that CFD can be used with high level of confidence for simulating single-phase systems. However, this is not the case with multiphase systems. The complex flow structure and interactions within different phases in addition to the turbulence make it very difficult to develop models for multiphase systems that can mimic reality. The closures (i.e. the models that are needed for the parameters and the interfacial forces to close (or to solve) the CFD equations) are modeled hypothetically or correlated empirically from experimental data at different conditions thus cannot be universally applied to all cases. Therefore, multiphase CFD simulations need to be developed for each different case and validated against experimental data. Once the CFD results are validated for a particular system and mechanistic closures based on physics are developed, CFD can be used to optimize the system by varying parameters and operating conditions to achieve proper design and scale-up.

Accordingly, the accomplished work here consists of the following:

i) A 3D two phase CFD model of CFX was used,

ii) Various closures for interfacial forces and turbulent viscosity were evaluated by comparing the simulation predictions with CARPT data,

iii) The suitable combination of closures were selected based on the best representation of both trends and quantitative values,

iv) The ability of CFD to account for the effect of geometry, operating conditions and size was evaluated and confirmed, and

v) The evaluated CFD code and its closures were used to study the effects of various design and operating variables on the hydrodynamics, flow pattern, mixing intensity and the size of dead zones.

Karim et al. (2004) performed CARPT studies on 8-inch diameter gas-lift digester. Thus, preliminary CFD studies were performed by Vesvikar and Al-Dahhan (2005) to simulate the digester used by Karim et al. (2004) in order to evaluate the predictability of CFD simulations. Vesvikar (2006) in his doctoral thesis (Appendix 2) further validated CFD model and its closures and studied the effects of various design and operating variables on the mixing, hydrodynamics of gas recirculation digesters. He also applied CFD in stirred tank digesters to account for the effect of shear stress on the digester performance outlined in chapter 2 (Appendix 1). Karim et. al.(2006) further utilized CARPT data of Karim et.al.(2004) to simplify the CFD for viable use of closures to study the impact of different designs on the reduction of the dead zones. They proposed a new design for the animal waste anaerobic digester that significantly reduces the dead zones and hence, such design would significantly improve the biogas production and the digester performance. 
Vesvikar (2006) (Appendix 2) used 3D CFD simulations to predict the flow in gas-lift digesters. The CFD predictions showed good qualitative comparison with the experimental data but gave only reasonable quantitative agreement (Example of results are shown in Figure 5.1). The CFD results in terms of overall flow pattern, location of circulation cells and stagnant zones, trends of liquid velocity profiles and other parameters agree reasonably well with the experimental data. Different closures and interfacial forces for the CFD model did not improve the CFD predictions. CFD simulations were performed using different digester configurations and sizes by changing draft tube size, clearance, shape of the tank bottom, sparger type, etc. to evaluate the effects of digester design on its flow pattern. CFD was able to account for the effect of geometry and operating variables at both laboratory and pilot-plant scales. The geometrical variations and operating conditions that were not used in experiments to evaluate their effects on hydrodynamics of gas-lift digesters were evaluated with CFD. The increase in the gas flow rate for all configurations of gas-lift digesters increased the axial liquid velocities in the riser but showed no added advantage in decreasing the dead zone volumes effectively in the downcomer. The flow pattern was affected appreciably by changing the draft tube diameter. The configuration with $D / T$ ratio of 0.5 showed lowest volume of dead zones. From the conclusions of CARPT experiments and the results of CFD simulations at the studied conditions, it becomes clear that the $D / T$ ratio of 0.5 would offer better liquid circulation as compared to other $D / T$ of 0.25 and 0.75 . However, such large size of draft tube would require larger energy input for the gas to be well distributed in the draft tube. Sparger design affects flow pattern for higher $D / T$ ratios of 0.5 and 0.75 , but showed no effect for smaller $D / T$ ratio of 0.25. Better gas dispersion created by cross sparger is advantageous in increasing the circulation and decreasing the dead zone volume over the local dispersion created by the single point sparger. This effect is due to the increased difference in density of the medium between the draft tube and downcomer zones which represents the driving force for circulation. The conical bottom of the digester helped in reducing the volume of the dead zones as compared to flat bottom digester. However, changing the draft tube clearance and height did not provide practical influence on the flow pattern and dead zones.

CFD simulations were able to account for the effects of scale. The scale affects the flow pattern and as a result, liquid velocities are also affected. At same power input, the liquid velocities are about 2-3 times higher in pilot-scale as compared to laboratory-scale. Such results raise the following questions: does it imply that the large-scale reactors are better mixed than the small reactors at same power input? Is 2-3 times increase in liquid velocity for a scale-up ratio of 25 enough to achieve same intensity of mixing at two scales? If the dead zone volumes and liquid circulation are treated as parameters that quantify mixing, then the answer to above questions is 'No'. Same power input per unit volume did not provide same mixing intensities in the two scales of reactor. Thus related superficial gas velocity cannot be used as a scale-up criterion.

The details of the results and the evaluation conducted are discussed in the following doctoral thesis (Appendix 2) and manuscripts:

- Vesvikar M.(2006). Understanding the hydrodynamics of anaerobic digesters for bioenergy production [D.Sc. Thesis] St. louis USA, Washington University. 
- Vesvikar, M S, Varma R., Karim K., Al-Dahhan M.H. 2005. Flow pattern visualization in a mimic anaerobic digester: Experimental and Computational Studies. Water Science and Technology, 52(1-2), 537-543.

-Vesvikar MS., Al-Dahhan MH. (2005) Flow pattern visualization in a mimic anaerobic digester using CFD. Biotechnology Bioengineering 89(6):719-732.

-Karim k. Thoma G.J., Al-Dahhan MH (2006) Gas-Lift digester configuration effects on mixing effectiveness Water Science and Technology 52(1-2)537-43.

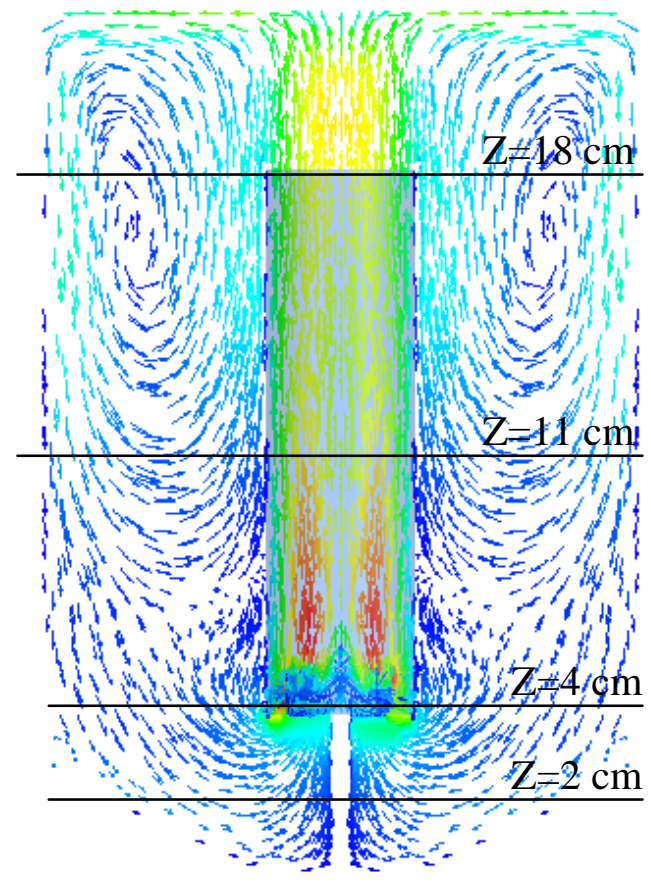

(a)

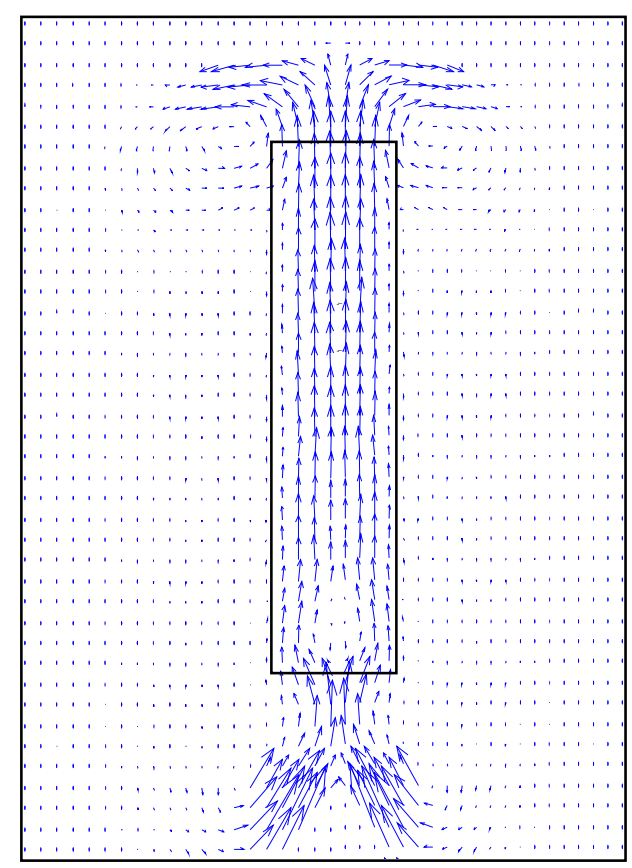

(b)

Figure 5.1: $\quad$ Comparison of (a) simulated flow pattern (LS1) and (b) flow pattern obtained from CARPT (L1) for 6-inch digester (Vesvikar, 2006) 


\section{References}

Ben-Hasson R, Ghaly A, Singh R. Design and evaluation of no-mix energy efficient anaerobic digester 1985; Charlottetown, P.E.I. .

Benac J. 2005. Alternating minimization algorithms for X-ray computed tomography : multigrid acceleration and dual energy application [D.Sc.]: Washington University- St. Louis.

Chapman D. 1989. . Mixing in anaerobic digesters: state of the art. . Chermisinoff P, editor: Houston: Gulf Publishing Company. 325-54 p.

Chen T, Chynoweth D, Biljetina R. 1990. Anaerobic digestion of municipal solid waste in a nonmixed solids concentrating digester. Applied Biochemistry and Biotechnology 24/25:533-44.

Dague R, McKinney R, Pfeffer J, . 1970. Solids retention in anaerobic waste treatment systems. Journal of the Water Pollution Control Federation 42(2 Part 2):R29-R46.

Diaz L, Trezek G. 1977. Biogasification of a selected fraction of municipal solid wastes. Compost Science:8-13.

EPA. Animal Waste Management. EPA.

EPA. Global Warming -- Emissions. EPA.

EPA. 2003. Concentrated Animal Feeding Operations (CAFO) - Final Rule. EPA.

EPA USA, editor. 1979. Process Design Manual for Sludge Treatment and Disposal Cincinnati, OH USA. 79-011 p.

George DL, Shollenberger KA, Torczynski JR, O'Hern TJ, Ceccio SL. 2001. Three-phase material distribution measurements in a vertical flow using gamma-densitometry tomography and electrical-impedance tomography. International Journal of Multiphase Flow 27(11):1903-1930. 
Gosh S. Anaerobic digestion for renewable energy and environmental restoration. ; 1997; Japan, Sendai International Center, Sendai.

Hashimoto AG. 1982. Effect of mixing duration and vacuum on methane production rate from beef cattle waste. Biotechnology and Bioengineering 24(1):9-23.

Hill DT. 1984. Methane Productivity of the Major Animal Waste Types. Transactions of the ASAE 27(2):530-534.

Ho C, Tan Y. 1985. Anaerobic treatment of palm oil mill effluent by tank digesters. Journal of Chemical Technology and Biotechnology 35B:155-164.

James S, Wiles C, Swartzbaugh J, Smith R. 1980. Mixing in large-scale municipal solid wastesewage sludge anaerobic Biotechnology and Bioengineering Symposium digesters 10(259-72.).

Johansen GA, Froeystein T, Hjertaker BT, Olsen O. 1996. A dual sensor flow imaging tomographic system. Measurement Science \& Technology 7(3):297-307.

Kantzas A, Wright I, Bhargava A, Li F, Hamilton K. 2001. Measurement of hydrodynamic data of gas-phase polymerization reactors using non-intrusive methods. Catalysis Today 64(3-4):189-203.

Karim K, Varma R, Vesvikar M, Al-Dahhan MH. 2004. Flow pattern visualization of a simulated digester. Water Research 38(17):3659-3670.

Karim K, Thoma GJ, Al-Dahhan MH. 2006. Gas-Lift digester configuration effects on mixing effectiveness. Submitted to Water Research.

Lange K, Carson R. 1984. EM Reconstruction Algorithims for Emission and Transmission Tomography. Journal of Computer Assisted Tomography 8(2):306-316.

Lee L, Chandrasekaran B, Hulme I, Kantzas A. 2005. A Non-Invasive Hydrodynamic Study of Gas-Solid Fluidised Bed of Linear Low Density Polyethylene. Canadian Journal of Chemical Engineering 83(1):119-126.

Lema J, Mendez R, J I, Garcia P, Fernandez-Polonco F. 1991. Chemical reactor engineering concepts in design and operation of anaerobic treatment processes. Water Science and Technology 24(8):79-86.

Luo H-P. 2005. Analyzing and modeling of airlift photobioreactors for microalgal and cyanobacteria cultures [Ph.D. thesis]: Washington University, St. Louis, MO, USA.

Lusk P. 1998. Methane Recovery from Animal Manures: A Current Opportunities Casebook. 3rd Edition. 
Madigan MT, Martinko JM, Parker J. 2003. Brock Biology of Microorganisms. 10, editor: Prentice Hall.

McCarty P, Mosey F. 1991. Modelling of anaerobic digestion processes (A discussion of concepts). Water Science and Technology 24:17-33.

Mills P. 1979. Minimization of energy input requirements of an anaerobic digester. Agricultureal Wastes 1:57-59.

Nooralahiyan AY, Hoyle BS. 1997. Three-component tomographic flow imaging using artificial neural network reconstruction. Chemical Engineering Science 52(13):21392148.

O'Sullivan JA, Benac J. 2007. Alternating Minimization Algorithms for Transmission Tomography. Medical Imaging, IEEE Transactions on 26(3):283-297.

Parkin G, Owen W, . 1986. Fundementals of anaerobic digestion of wastewater sludges. Journal of Environmental Engineering 112:869-920.

Persson SPE, Bartlett HD, Branding AE, Regan RW. 1979 Agricultural anaerobic digesters: Design and operation. The Pennsylvania State University, Pennsylvania, USA.

Rados N. 2003. Slurry bubble column hydrodynamics [Ph.D. thesis]: Washington University St. Louis MO USA. .

Rammohan A. 2002. Characterization of single and multiphase flows in stirred tank reactors [Doctoral ]: Washington Univ., St. Louis, MO, USA.

Rammohan AR, Kemoun A, Al-Dahhan MH, Dudukovic MP. 2001. Characterization of single phase flows in stirred tanks via computer automated radioactive particle tracking (CARPT). Chemical Engineering Research and Design 79(A8):831-844.

Roy S. 2006. Phase distribution and performance studies of gas-liquid monolith reactor. [D.Sc. Thesis]: Washington University - St. Louis. 203 p.

Rulkens WH, Klapwijk A, Willers HC. 1998. Recovery of valuable nitrogen compounds from agricultural liquid wastes: potential possibilities, bottlenecks and future technological challenges. Environmental Pollution 102(1):727-735

Schink B. 1997. Energetics of syntrophic cooperation in methanogenic degradation. Microbiology and Molecular Microbiology Reviews 61:262-280.

Schaffer HE. 1970. Algorithms 369: Generation of random numbers satisfying the poission distribution Communication of the assocations for computing machinery 13(1). 
Sheffield J. Financial approaches to animal manure managment; 2002; Joint Institute for Energy and Environment. 314 UT Conference Center Building Knoxville, TN 379964138. Report Number: JIEE 2002-04.

Smith LC, Elliot DJ, James A. 1996. Mixing in upflow anaerobic filters and its influence on performance and scale-up. Water Research 30(12):3061-3073.

Speece RE. 1996. Anaerobic Biotechnology for Industrial Wastewaters.: Vanderbilt University, Arachae Press.

Stafford D, Hawkes D, Horton R. 1980. Methane Production from Waste Organic Matter. Raton B, editor: CRC Press.

Strenstrom M, Ng A, Bhunia P, Abramson S. 1983. Anaerobic digestion of municipal solid waste. Journal of Environmental Engineering 109:1148-1158.

Stroot PG, McMahon KD, Mackie RI, Raskin L. 2001. Anaerobic codigestion of municipal solid waste and biosolids under various mixing conditions--I. digester performance. Water Research 35(7):1804-1816.

Vesvikar MS, Muthanna A-DH. 2005. Flow pattern visualization in a mimic anaerobic digester using CFD. Biotechnology and Bioengineering 89(6):719-732.

Vesvikar M.(2006). Understanding the hydrodynamics of anaerobic digesters for bioenergy production [D.Sc. Thesis] St. louis USA, Washington University.

USDA, http://www.scorecard.org/env-releases/aw/us.td, 2003.

USDA. 2002/2003. Statistical Highlights of United States Agriculture 2002/2003. Washington, D.C: National Agricultural Statistics Service.

Warsito, Ohkawa M, Kawata N, Uchida S. 1999. Cross-sectional distributions of gas and solid holdups in slurry bubble column investigated by ultrasonic computed tomography. Chemical Engineering Science 54(21):4711-4728.

Warsito W, Maezawa A, Uchida S, Okamura S. 1995. A model for simultaneous measurement of gas and solid holdups in a bubble column using ultrasonic method.

. Canadian Journal of Chemical Engineering 73 (5):734-43.

Whitmore T, Lloyd D, Jones G, Williams T. 1987. Hydrogen-dependent control of the continuous anaerobic digestion process. Applied Microbiology and Biotechnology 26:383-388. 


\section{Appendix 1}

Thesis: Hoffmann RA. 2005. Effect of shear on the performance and microbial ecology of anaerobic digesters treating cow manure from dairy farms. [Masters thesis]. St. Louis USA: Washington University. 


\section{WASHINGTON UNIVERSITY \\ THE HENRY EDWIN SEVER GRADUATE SCHOOL \\ ENVIRONMENTAL ENGINEERING SCIENCE PROGRAM}

The Effect of Shear on the Performance and Microbial Ecology of Anaerobic Digesters Treating Cow Manure from Dairy Farms

By

Rebecca A. Hoffmann

Under the direction of

Prof. Muthanna Al-Dahhan, Prof. Largus Angenent, And Dr. Thomas Klasson

Thesis presented to the Henry Edwin Sever Graduate School of Washington University in partial fulfillment of the requirements of the degree of

MASTER OF SCIENCE

August 2005

Saint Louis, Missouri 


\title{
WASHINGTON UNIVERSITY \\ THE HENRY EDWIN SEVER GRADUATE SCHOOL ENVIRONMENTAL ENGINEERING SCIENCE PROGRAM
}

\author{
ABSTRACT \\ ADVISORS: Prof. Muthanna Al-Dahhan and \\ Prof. Largus Angenent, Dr. Thomas Klasson \\ August 2005 \\ Saint Louis, Missouri
}

The interest in more environmentally-sound waste management practices has grown over the last several years due to recent EPA rulings on concentrated animal feeding operations. Anaerobic digestion is an attractive waste treatment option for animal manures because it produced energy in the form of methane. This study sought to determine the effect of different mixing intensities on digester performance and syntrophic relationships in anaerobic digesters treating cow manure. Continuouslystirred tank reactors running at four different mixing intensities, 1500, 500, 250, and 50 RPM, were operated over a 160 day period with a series of five different organic loading rates between 0.6 and $3.5 \mathrm{~g} \mathrm{VS} / \mathrm{L}$-d. Results of this experiment demonstrated that different mixing intensities produced no effect on the biogas production of continuouslystirred digesters during steady-state periods. A methane yield of $0.241 \pm 0.007 \mathrm{~L} \mathrm{CH}_{4} / \mathrm{g}$ VS fed was obtained by pooling the data of all four digesters during steady-state periods. However, digester performance was affected by mixing intensity during startup of the digesters. The 1500 and 500-RPM digesters were negatively impacted with lower biogas production rates and higher volatile fatty acids concentrations. Fluorescent in situ hybridization revealed decreasing floc sizes beginning at week 4 and continued through week 26, at which time almost no flocs remained. This decrease in size, and subsequent loss of microbial flocs did not, however, produce any visible upsets in digester performance. A statistical difference was seen between the digesters in terms of VS removal, while no statistical difference was seen between the digesters in terms of VS removal efficiencies. 


\section{DEDICATION}

This work is dedicated to:

My Mother and Father: Thank you for giving me the opportunity, strength, and encouragement to realize my dreams. I love you both. 


\section{Contents}

List of Tables .................................................................................................................... vii

List of Figures........................................................................................................................ viii

Chapter 1 Overview .......................................................................................................... 1

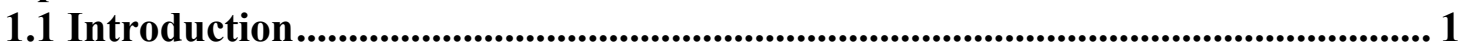

1.2 Abbreviated Background ............................................................................... 1

1.3 The Effect of Mixing Method.............................................................................. 4

1.3.1 $50 \mathrm{~g} / \mathrm{L}$ TS loading: Effect of hopper bottom and method of mixing ........... 4

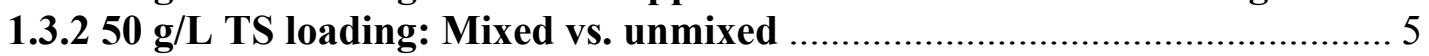

$1.3 .3100 \mathrm{~g} / \mathrm{L}$ TS loading: Effect of method of mixing and TS loading ................ 5

1.3.4 Conclusions from the preliminary studies.................................................... 6

1.4 The Effect of Mixing Intensity ................................................................................. 6

Chapter 2 Background and Literature Review.................................................................... 9

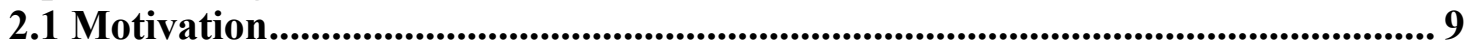

2.2 Development of Anaerobic Digestion ............................................................................ 12

2.3 The Anaerobic Digestion Food Web ......................................................................... 16

2.4 Factors Affecting Anaerobic Digestion ..................................................................... 18

2.5 The Role of Mixing in Anaerobic Digestion ............................................................. 21

2.6 Microbial Ecology in Anaerobic Digesters ............................................................. 27

Chapter 3 Effect of Mixing Intensity on Performance and Biomass Characteristics in Continuously-Stirred Anaerobic Digesters Treating Dairy Cow Manure............ 29

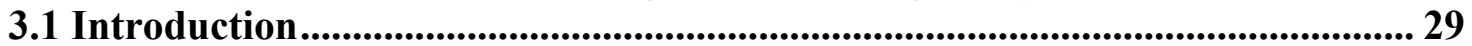

3.2 Materials and Methods........................................................................................ 31

3.3 Results ............................................................................................................................ 33

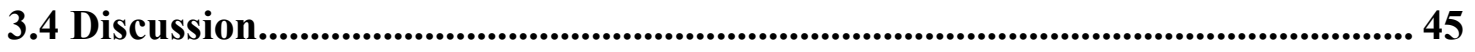

Chapter 4 Summary and Recommendations ................................................................. 51 Appendix A Anaerobic Digestion of Animal Waste: Waste Strength vs. Impact of

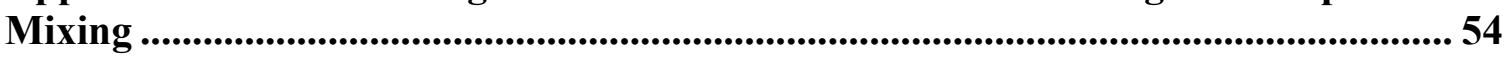

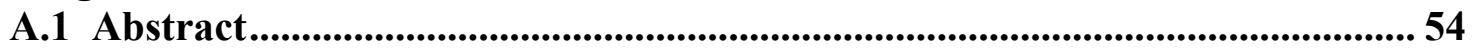

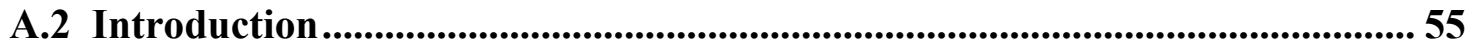

A.3 Materials and Methods........................................................................................ 57

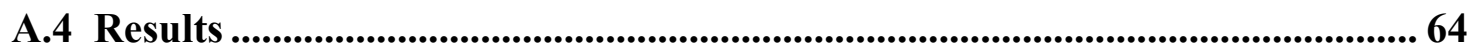

A.5 Discussion.................................................................................................................... 70

A.6 Conclusions ................................................................................................................. 76

Appendix B Standard Operating Procedures ............................................................ 77

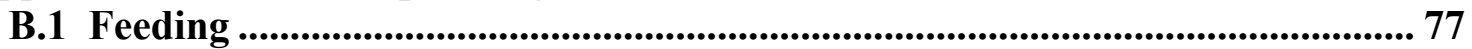

B.2 Soluble Chemical Oxygen Demand ............................................................................... 778

B.3 Volatile Fatty Acids - The Distillation Method ...................................................... 81

B.4 Ammonia - Probe Method ........................................................................... 83

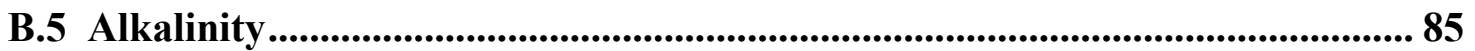

B.6 Fluorescent in situ Hybridization............................................................................. 87

References................................................................................................................................. 97

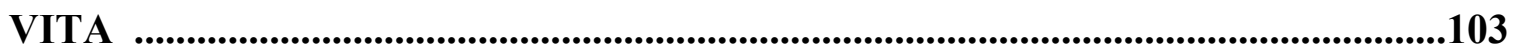




\section{List of Tables}

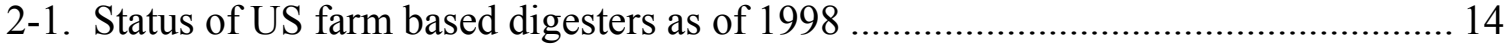

3-1. VS removal efficiencies for steady-state periods. ................................................ 45

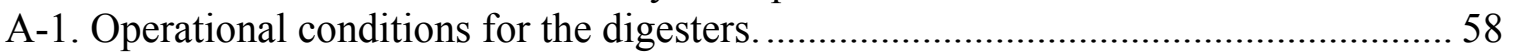

A-2. Characteristics of the prepared feed, 5\% and 10\% manure slurry........................... 61

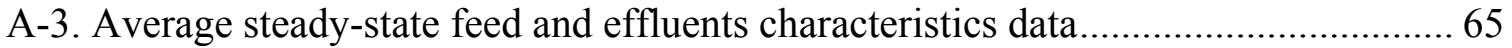

A-4. Average steady-state feed and effluents characteristics data, averaged .................. 68

A-5. Biogas production rate, methane yield and methane productivity .......................... 70 


\section{List of Figures}

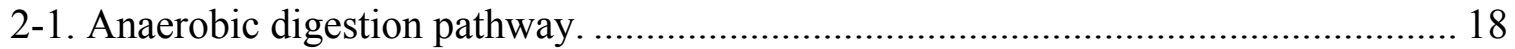

3-1. Continuously-stirred anaerobic digester setup and diagram...................................... 31

3-2. Performance data: daily biogas production and volatile fatty acid levels ............... 39

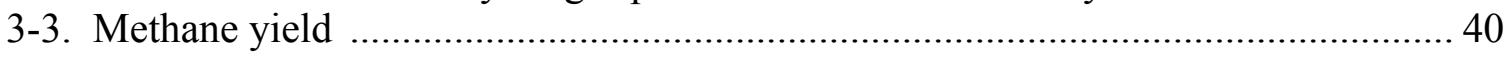

3-4. Performance data from shock load on day 260 .................................................... 41

3-5. Performance data: volatile solids concentration and SCOD concentrations ........... 42

3-6. FISH views from weeks 1, 7, and 14 .............................................................. 43

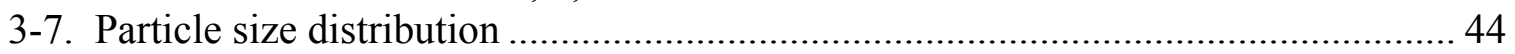

A-1. Schematic diagram of the experimental set-up ....................................................... 59

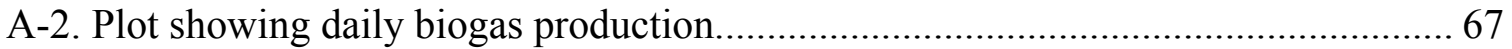

A-3. Plot showing daily biogas production, and solids concentrations in the feed. ......... 69 


\section{Chapter 1 Overview}

\subsection{Introduction}

Anaerobic digestion is a waste treatment alternative for both industrial and agricultural wastes and has several advantages over aerobic treatment, including higher organic removal rates, lower sludge production, lower energy requirements, and the production of bioenergy. Although industrial wastes have successfully utilized anaerobic digestion to reduce organic pollutants in waste streams for over 30 years, implementation of anaerobic digesters on farms for the purpose of treating animal manure and farm wastes has had high failure rates. Such high failure rates are believed to be mainly due to poor design, construction, and absence of a well acclimated microbial community. Mixing is an important parameter in the operation of anaerobic digesters and has several functions, including enhancing substrate contact with the microbial community, improving $\mathrm{pH}$ and temperature uniformity, preventing stratification and scum accumulation, facilitating the removal of biogas from the digestant, and aiding in particle size reduction. Information in the literature is contradictory on the effect of mixing on anaerobic digestion, resulting in a need for further study on the subject. A better understanding of the role of mixing in anaerobic digestion will result in better design and operation, leading to a reduction in failure rate and increased utilization of anaerobic technology on the farm. The objective of the proposed research is to study the effect of mixing intensity (i.e., applied shear) on digester performance, microbial ecology, and syntrophic relationships in anaerobic digesters treating cow manure.

\subsection{Abbreviated Background}

The United States produces 1.8 billion metric tons of cow manure annually (EPA; USDA 2002/2003). This waste is often improperly stored and handled, leading to several environmental problems, such as surface and groundwater contamination and emissions of atmospheric pollutants. Anaerobic digestion is a waste treatment 
technology by which microorganisms break down organic material in the absence of oxygen to create biogas. Biogas consists of approximately $65 \%$ methane and $35 \%$ carbon dioxide, with traces of dinitrogen gas and gaseous sulfur compounds. With such a high methane content, biogas can be used as an energy source. Besides reducing greenhouse gas emissions and producing a renewable energy source, anaerobic digestion systems also reduce odor, protect water quality, control ammonia release, and produce a fertilizer.

Anaerobic digestion occurs in a series of steps employing different types of Bacteria to break down complex polymers into simple carbon compounds that can subsequently be utilized by Archaea to produce methane. Five physiologically different groups of microorganisms are involved: three groups of Bacteria (fermentative bacteria, obligatory $\mathrm{H}_{2}$ producing acetogens, and homoacetogens) and two groups of Archaea (hydrogenotrophic methanogens and acetoclastic methanogens). The extent of mutual reliance among these microorganisms varies substantially. While the lower members of the food chain depend on the higher members for substrate, the lower members also positively impact higher members by removing metabolic products. An important example of this type of mutual reliance is the syntrophic relationship. Syntrophic relationships are a special case of symbiotic relationship in which two organisms with different metabolic capabilities work together to degrade a compound. This type of relationship usually develops due to energy limitations (Madigan et al. 2003; Schink 1997). In anaerobic systems, syntrophic relationships exist between hydrogen producing acetogenic bacteria and hydrogenotrophic methanogens. Organisms involved in syntrophic relationships generally develop in close, physical associations, such as flocs, to decrease diffusion limitations of metabolic products.

Farm-based anaerobic digesters have been used in the United States since the 1970s. The failure rates for farm based anaerobic technologies in 1998 were as high as 70\% (Lusk 1998). Mixing is an important design parameter impacting the performance of anaerobic digesters, primarily functioning to distribute enzymes and 
microorganisms throughout the digester ( Lema et al. 1991; Chapman 1989; Parkin and Owen 1986; Strenstrom et al. 1983). However, the effects of mixing on the anaerobic process are not well understood, and the information available in the literature is contradictory. While some research has shown that continuous mixing in digesters results in better performance than unmixed digesters (Ho and Tan 1985; Finny and Evans 1975) and intermittently mixed digesters (Hashimoto 1982), other research has produced data that disagrees with these findings (Stroot et al. 2001; Rivard et al. 1995; Diaz et al. 1974). The intensity of mixing also has been found to have an effect on digester performance, but again much discrepancy is found in the literature. While some studies have shown increasing mixing results in better performance (Lanting 2003), other studies have found that different mixing intensities have no effect on performance (Karim et al. 2005b; Lin and Pearce 1991). In addition, others have found that high mixing intensities negatively affect digester performance (Angenent et al. 2001; McMahon et al. 2001; Stroot et al. 2001; Dague et al. 1970).

In fact, some researchers believe that vigorous mixing may disrupt the spatial associations of syntrophic microorganisms (Dolfing 1992; Whitmore et al. 1987; Conrad et al. 1985). Close physical associations between the organisms involved ensures high rates of hydrogen transfer at relatively low concentrations of hydrogen. In vigorously mixed systems, continuously disrupted spatial associations is thought to lead to a state of instability (Stroot et al. 2001). This rationale has led some researchers to believe that minimal mixing may improve anaerobic digestion by providing a quiescent environment for bacteria (Lettinga 1981). However, it is argued that some mixing is required to distribute substrate and allow new spatial associations to form between different microorganism populations; otherwise, due to their growth, cells will be surrounded by their own offspring, causing reduced kinetic effectiveness (Schink 1992).

The contradictory findings reported in the literature about the role of mixing in anaerobic digesters, and a lack of research to determine the effect on performance and microbial ecology, justify further research in this area. Therefore, the present work 
was designed to focus on determining the effect of mixing on both digester performance and the microbial community.

\subsection{The Effect of Mixing Method}

Several different ways of creating mixing in anaerobic digesters exist and have been reported in the literature. For continuously-stirred applications, these include biogas recirculation, slurry recirculation, and impeller mixing. These three mixing methods were investigated as part of this work in a series of preliminary studies where the power per unit volume used for mixing was kept at $8 \mathrm{~W} / \mathrm{m}^{3}$ as recommended by the U.S. EPA. The objectives of these studies were to determine the effects of the angle of the hopper bottom used, the method of mixing, and the effect of total solids loading rate. Each of these studies is summarized below, but more detailed information can be found in Appendix A.

\subsection{1 $50 \mathrm{~g} / \mathrm{L}$ TS loading: Effect of hopper bottom and method of mixing}

This study was performed using feed manure with a total solids (TS) concentration of $50 \mathrm{~g} / \mathrm{L}$. Four digesters, biogas recirculation (hopper bottom slope of $60^{\circ}$ ), biogas recirculation (hopper bottom slope of $25^{\circ}$ ), slurry recirculation (hopper bottom slope of $25^{\circ}$ ), and an impeller mixed (hopper bottom slope of $25^{\circ}$ ), were used to determine the effect of hopper bottom slope and mixing method. All digesters were operated with a power input per unit volume of $8 \mathrm{~W} / \mathrm{m}^{3}$, corresponding to a gas recirculation rate of $1 \mathrm{~L} / \mathrm{min}$, an impeller speed of $275 \mathrm{RPM}$, and a slurry recirculation rate of 0.82 $\mathrm{L} / \mathrm{min}$.

Results showed no differences in performance between all four digesters after reaching steady-state. No statistically significant difference in chemical oxygen demand (COD) removal, biogas production rate, and other operational parameters 
were found. The methane yield, calculated based on the volatile solids (VS) loading rate of $2 \mathrm{~g} / \mathrm{L}$-day, was found to be between 0.21 and $0.27 \mathrm{~L} \mathrm{CH}_{4} / \mathrm{g} \mathrm{VS}$ fed.

\subsection{2 $50 \mathrm{~g} / \mathrm{L}$ TS loading: Mixed vs. unmixed}

This study was also performed using a feed manure with $50 \mathrm{~g} / \mathrm{L}$ TS concentration. Two digesters with $25^{\circ}$ sloped hopper bottoms, one unmixed (i.e. no agitation was provided by the impeller) and one mixed by an impeller at 275 RPM, were operated to compare the performance of a mixed and unmixed reactor. This study also served as a verification of reproducibility for the impeller mixed digester used in the first study described in section 1.3.1.

Results showed that the impeller mixed digester produced $10 \%$ more biogas than the unmixed digester. However, analysis of variance (ANOVA) performed on the data showed that while the biogas production rates were statistically different, the probability of the difference arising from random error was high at 3.6\%. The methane yield found for these digesters, based on the VS loading rate of $2 \mathrm{~g} / \mathrm{L}$-day, was 0.27 and $0.31 \mathrm{~L} \mathrm{CH}_{4} / \mathrm{g}$ VS fed for the unmixed and impeller mixed digesters, respectively. The reproducibility of the first study discussed in section 1.3.1 was verified with the performance of the impeller mixed digester used in this study. No statistical difference in biogas production was found between the impeller mixed digesters.

\subsection{3 $100 \mathrm{~g} / \mathrm{L}$ TS loading: Effect of method of mixing and TS loading}

This study was performed to determine if the method of mixing became important at a higher TS loading rate of $100 \mathrm{~g} / \mathrm{L}$ TS. Four digesters with $25^{\circ}$ sloped hopper bottoms were used, these being an unmixed, biogas recirculation, impeller, and slurry recirculation digester. The three mixed digesters were again operated at a power input per unit volume of $8 \mathrm{~W} / \mathrm{m}^{3}$, corresponding to a gas recirculation rate of $1 \mathrm{~L} / \mathrm{min}$, an impeller speed of 275 RPM, and a slurry recirculation rate of $0.82 \mathrm{~L} / \mathrm{min}$. 
Results showed that there was statistically significant difference in the biogas production rates of the digesters. The digester mixed by slurry recirculation was found to produce $22 \%$ more gas than the unmixed digester during steady-state conditions. No significant difference in biogas production was found between the slurry recirculation digester and the impeller mixed digester. However, a statistically significant difference in biogas production was found between the slurry recirculation digester and the gas recirculation digester, revealing that the slurry recirculation digester produced $10 \%$ more biogas than the biogas recirculation digester. The methane yields for the unmixed, biogas recirculation, impeller, and slurry recirculation digesters were $0.19,0.21,0.23$, and $0.24 \mathrm{~L} \mathrm{CH}_{4} / \mathrm{g}$ VS fed, based on a VS loading rate of $3.2 \mathrm{~g} \mathrm{VS} / \mathrm{L}-\mathrm{d}$.

\subsubsection{Conclusions from the preliminary studies}

From the results of the three preliminary studies outlined above, it can be concluded that neither mixing nor method of mixing affects digester performance when treating dilute feed manure containing $50 \mathrm{~g} / \mathrm{L}$ TS. However, this was not found to be the case when treating a higher concentration feed manure of $100 \mathrm{~g} / \mathrm{L} \mathrm{TS}$. At this higher TS loading rate, the performance of the digesters was affected by both the presence of mixing and the method of mixing. The unmixed digester was found to produce the least amount of biogas, while the slurry recirculation was found to produce the most. Thus, the overall findings suggest that mixing plays more of a role in its effect on digester performance when treating thicker manure feeds.

\subsection{The Effect of Mixing Intensity}

Since the effect of mixing was shown to be significant when feeding a $100 \mathrm{~g} / \mathrm{L} \mathrm{TS}$ feed manure, the question arose as to whether or not the intensity of mixing would become important as well. Thus, this study was designed to experimentally investigate the effect of mixing intensity on digester performance. However, in addition to investigating the effect on performance, the effects on the microbial 
community was also investigated. Due to concerns of sand and other non-biological material interfering with the molecular biology analysis, a manure different from that in the previous studies was used, which contained only the solids that were present in the manure as it was excreted. In order to reproduce the conditions of the $100 \mathrm{~g} / \mathrm{L}$ TS study discussed in section 1.3.3, the VS concentration in the feed manure, and the VS loading rate, were designed to be similar. Since mechanical mixers are reported to be the most efficient in terms of power consumed per gallon mixed (Brade and Noone 1981), mechanical mixing was the mixing method chosen.

Three hypotheses were developed and investigated in accordance with the overall goals of this study:

\section{Hypothesis 1: Higher mixing intensities have a detrimental effect upon}

reactor performance. This observation has been seen by Stroot et al. (2001) and Angenent et al. (2001). Reactor instability may be due to rapid hydrolysis and fermentation, which leads to higher production of volatile fatty acids. Acid concentrations in excess of $2000 \mathrm{mg}$ as acetic acid/L may cause inhibitory effects on methanogenesis, further increasing acid build-up. Instability may also be a result of susceptibility of flocs to break apart at increasing applied shear.

\section{Hypothesis 2: Different mixing intensities create different microbial}

communities. Due to the stresses and different environmental conditions (differing levels of intermediate products) created by different mixing intensities, it is expected that different microbial communities will develop. Two lines of speculation are behind this hypothesis. One is that decreasing methanogen populations may be found as mixing speed and shear increase. The decreases may be due to higher sensitivities to perturbations, as observed by Angenent et al. (2001). If this is the case, gas production, COD removal, and VS removal would all be lower in the most intensely mixed reactors. The second speculation argues that stressed systems, such as those receiving high shear, develop a more diversified microbial community due to 
increases in concentrations of intermediate products. If this hypothesis is true, performance of digesters receiving high shear will handle shocks to the system better.

Hypothesis 3: Higher mixing intensities break up and/or prevent the formation of larger flocs of syntrophic microorganisms. As previously discussed, many researchers believe that high shear created by intense mixing may disrupt flocs of syntrophic microorganisms (Dolfing 1992; Whitmore et al. 1987; Conrad et al. 1985). This disruption in juxtaposition then results in a lower hydrogen transfer efficiency, and hence, a lower removal efficiency as compared to undisrupted flocs. 


\section{Chapter 2 Background and Literature Review}

\subsection{Motivation}

The United States is home to 100 million head of cattle (USDA 2002/2003). Each cow produces $54.4 \mathrm{~kg}$ of wet manure a day (the equivalent to the waste produced by 20-40 people), and thus the bovine population produces approximately 1.8 million metric tons of wet waste annually (EPA). If properly handled, this waste can be a valuable resource to produce bioenergy and fertilizer. Cattle waste is often improperly stored and handled, leading to several environmental problems. These problems include:

- Ground water contamination - Contaminated ground water can lead to high nitrate levels in drinking water, which can cause a fatal blood disorder in infants known as the Blue Baby Syndrome. Ground water contaminated with cow manure can also carry deadly pathogens. In 1993, an outbreak of cryptosporidium in Milwaukee's water supply, after severe rainstorms, led to 403,000 cases of illness, 69 deaths, and between 75 and 118 million dollars in medical costs and productivity losses (Corso et al. 2003; MacKenzie et al. 1994).

- Surface water contamination - Ammonia in surface waters due to contamination from manure can result in fish kills. Surface waters contaminated with manure may also experience eutrophication due to increased amounts of nitrogen and phosphorous. Eutrophication is the excessive growth of algae due to an increased amount of nutrients, which causes a sharp decrease in dissolved oxygen. Low levels of dissolved oxygen result in fish kills and can devastate entire food chains (Combs et al. 19811982). 
- Emission of greenhouse gasses - Methane emission from decomposing wastes is also a concern, as methane traps more than 21 times the amount of heat per molecule that carbon dioxide does, thus contributing to global warming (EPA).

- Emission of other atmospheric pollutants - Atmospheric pollutants such as ammonia, and reactive organic compounds that are emitted from the decomposition of animal waste impact air quality through the formation of ozone and particulate matter (EPA).

- Odors - For people who live near concentrated feeding operations, i.e., livestock farms containing 700 or more head of cattle, the stench can be unbearable. Waste stored in open-air lagoons can spread odors for miles (Kaye 2004).

Rapid growth in the livestock industries over the past 40 years has resulted in the formulation of new laws and regulations governing safe handling and disposal of animal wastes, as well as increased support to federal programs aimed at minimizing the effects of animal wastes on the environment. The EPA passed the Concentrated Animal Feeding Operations (CAFOs) Rule in December of 2003, requiring the Nation's largest CAFOs to aquire Clean Water Act permits. This forces CAFOs to develop manure management plans that ensure proper management and land application of manure (EPA). Created under the Climate Change Action Plan (Clinton and Gore 1993), AgSTAR is a voluntary federal program led by a cooperative effort by the U.S. Environmental Protection Agency (EPA), U.S. Department of Agriculture (USDA), and U.S. Department of Energy (DOE), which encourages the use of effective technologies to capture methane gas generated from decomposing animal manure for use as a renewable energy source.

Several biological conversion methods to treat animal wastes exist, including composting, aerobic treatment, and anaerobic digestion. Composting can only be accomplished with high solids concentration wastes, and is likely not applicable to livestock operations that use flush systems. Also, composting does not reduce the 
amount of ammonia released from the manure, and still presents threats to surface and ground water contamination if the composting area is not properly lined. Aerobic treatment entails the breakdown of organic material by oxygen-utilizing microorganisms that produce new cells, carbon dioxide, and water. Although aerobic treatment decreases odors and organic matter, it is not generally used due to high land, energy, and maintenance requirements. Anaerobic digestion is the process by which microorganisms breakdown organic material to create biogas in the absence of oxygen. Biogas consists typically of $65-70 \%$ methane and $30-35 \%$ carbon dioxide, with traces of nitrogen and sulfur gases. Biogas created from anaerobic digestion can be captured and used as an energy source. A survey conducted under the AgSTAR program found that installing anaerobic digesters on farms in just three top livestock producing states, North Carolina, California, and Illinois, could virtually meet AgSTAR's goals for decreasing methane emissions, dropping the amount of methane emissions from animal waste by $79 \%$. This survey also reported that the amount of economically recoverable emissions from 3,000 dairy and swine farms within 19 states totals 0.426 teragrams of methane, corresponding to approximately 165 megawatts of electricity (Lusk 1998).

Besides reducing greenhouse gas emissions and producing a renewable energy source, anaerobic digestion also reduces odor, protects water quality, controls ammonia release, and produces a fertilizer. Effluent that arises from anaerobic digestion generates considerably less odor than from conventional manure management system. In addition, phosphorous and metal loadings to surface waters are reduced, and many disease-causing organisms that can be carried by surface waters are destroyed in the process of anaerobic digestion. The effluent from anaerobic digesters is high in nutrients and can be used as fertilizer. During the anaerobic digestion process, some of the nitrogen that is bound up in proteins is converted to ammonia during hydrolysis (Sung and Liu 2003). Although ammonia emissions can lead to the formation of particulate matter in the atmosphere, proper land application of digester effluents as fertilizer can reduce the volatilization of ammonia, allowing for 
bacteria to convert more ammonia into nitrate, which is the form of nitrogen usable by plants. Ammonia losses to the atmosphere through land application of digester effluents are much less than that associated with raw manure application (EPA; Lusk 1998). Thus, anaerobic digestion is a promising waste management technology that not only protects air and water quality, but also produces two valuable products, bioenergy and fertilizer.

\subsection{Development of Anaerobic Digestion}

Decaying organic matter was first recognized to produce a flammable gas by Von Helmont in 1630. In 1776, Volta concluded that a direct correlation existed between the amount of decaying matter and the amount of flammable gas produced.

Laboratory experiments on anaerobic digestion of manure in 1808 by Humphrey Davy revealed that the gas produced contained methane (Lusk 1998). A fair amount of knowledge of the process was gained in the following years, by Bunsen in 1856, Hoppe-Seyler in 1886, Omelianskii in 1900, and Sohngen in 1906. Even as early as 1884, Louis Pasteur presented data to the Academy that his pupil Gayon had obtained $100 \mathrm{~L}$ of methane per $\mathrm{m}^{3}$ of manure, and suggested fermentation could be used as a fuel source for heating and lighting. In 1885, Cameron did just that, and lit the streets of Exeter with digester gas from sewage treatment. Another famous early application of anaerobic digestion was at the Matinga Leper Asylum in Bombay, India, in 1897. Gas produced from the digester was used for lighting and, after 1907, also for power. Most work on anaerobic digestion between 1900 and 1960 was in France, Germany, and India (van Brakel 1980). In the United States, A. M. Bruswell carried out extensive studies on anaerobic digestion between 1928 and 1965 (Buswell and Hatfield 1936; Buswell and Boruff 1933; Buswell 1930).

Given that anaerobic digestion has been in use since the end of the $19^{\text {th }}$ century, it is considered as one of the oldest technologies for stabilizing wastes and wastewaters. Its main application is for treatment of household wastewaters through the use of septic tanks and in municipal treatment plants for treatment of sewage sludge. In 
addition, over the past 30 years, anaerobic digestion processes have been developed and applied to a wide array of industrial and agricultural wastes. Industrial wastes in which anaerobic digestion has been applied include food processing wastes, pharmaceutical wastes, and distillery wastes (Banerjee and Biswas 2004; Karim and Gupta 2001; Hawkes et al. 1995). Agricultural applications include cattle, swine, and poultry wastes, as well as slaughterhouse wastes (Salminen and Rintala 2002; Misi and Forster 2001).

Farm-based anaerobic digesters have been used in the United States since the 1970s. The most commonly used types of farm based anaerobic digesters are as follows:

- Covered Lagoons - These digesters are simply manure lagoons with a flexible, impermeable cover to trap the produced biogas. The digester usually operates at ambient temperatures with liquid manures of less than $2 \%$ solids, being most suitable for livestock operations using flush systems. Usually used in southern regions with warmer climates, these digesters are not cost-effective for creating biogas for energy when constructed in northern areas. Anaerobic lagoons may have hydraulic retention times (HRT) greater than 60 days (Lusk 1999).

- Plug Flow - These digesters are usually utilized when the manure wastes to be treated contain between 11 and 14\% solids. This type of waste is usually generated on farms that use scraping systems to collect waste. Organic material is digested as it moves from one end of the digester to the other, because new manure entering the system provides the driving force for movement. Typical solid retention times are between 20 and 30 days. Biogas is collected in the same fashion as in anaerobic lagoons. Mixing pits with the capacity to store one days worth of manure production are often needed (Lusk 1999).

- Continuously Mixed - Usually constructed as a large vertical cylinder made from either concrete or steel, mixed digesters typically handle between $3 \%$ and $10 \%$ total solids. Mixing pits may also be needed for use with these digesters 
to dilute and preheat the manure before it enters the reactor. The digester is mixed to create a homogeneous substrate, keeping solids in suspension. Mixed systems range in size from $3,500 \mathrm{ft}^{3}$ to $70,000 \mathrm{ft}^{3}$, and have a typical HRT between 15 and 20 days. These digesters are generally more expensive to install, operate, and maintain (Lusk 1999; Pillars).

Even though farm-based anaerobic digestion has been applied for over 30 years in the United States, it has been implemented only on a small percentage of farms. Such low rates of implementation are due to several reasons: 1) over the past 25 years, lowcost fossil fuels have been readily available, making it difficult to sell biogas at prices that justify the investment, 2) anaerobic digesters are more complex and require more capital, both to install and operate, than alternative waste management options (Miner et al. 2000), and 3) failure rates are generally high in the U.S. Table 2-1 shows the status of U.S. farm based digesters as of 1998. Mixed and plug flow digesters have failure rates as high as 70\% and 63\%, respectively. Lagoon digesters have a lower failure rate than complete mix or plug flow, yet are still as high as $22 \%$. Such high failure rates can be attributed mostly to poor design (Lusk 1998), but also because of a non-adapted microbial community (Angenent et al. 2002).

Table 2-1 Status of US farm based digesters as of 1998 (adapted from Lusk (1998)).

\begin{tabular}{|l|c|c|c|c|c|}
\hline & Lagoon & Plug & Mix & Other & Totals \\
\hline Operating & 7 & 8 & 6 & 7 & 28 \\
\hline Not operating & 1 & 18 & 10 & 0 & 29 \\
\hline Farm closed & 1 & 11 & 5 & 0 & 17 \\
\hline Under construction & 0 & 2 & 4 & 4 & 10 \\
\hline TOTALS & 9 & 39 & 25 & 11 & 84 \\
\hline
\end{tabular}


Over the last few decades, several other types of digesters have been developed based on the concept of retaining viable biomass by sludge immobilization. Examples of these types of high-rate digesters include:

- Anaerobic sequencing batch reactor (ASBR) - This is a batch fed system continuously sequencing through four steps; feed, react, settle, decant (Sung and Dague 1995). Angenent et al. (2002) demonstrated the applicability of the ASBR by describing a $600-\mathrm{m}^{3}$ ASBR on a 3000 -head swine farm.

- Anaerobic migrating blanket reactor (AMBR) - The AMBR was designed to overcome the problems associated with both the upflow anaerobic sludge blanket reactor (see below) and the ASBR. The AMBR is a compartmentalized reactor with influent flowing horizontally into one end, and out the other. The final compartment serves as an internal clarifier, thus making gas-solids separation equipment unnecessary. The horizontal flow in the reactor is periodically reversed to prevent biomass from building up in the final compartment.

Most of these newly designed systems, however, have mainly been used on industrial wastes and have not been utilized for the treatment of manures. Several of these types of high-rate digesters are as follows:

- Upflow anaerobic sludge blanket (UASB) - The upflow anaerobic sludge blanket reactor (Lettinga et al. 1980) is based on dense granular development. Influent enters the reactor through the bottom, flowing through a dense sludge bed as it passes upward at velocities between 0.5 and $1 \mathrm{~m} / \mathrm{hr}$, creating an upward circulation of liquid and gasborne granules. After degasification, the sludge particles sink back to the bottom, creating continuous convection and ensuring good microorganism to substrate contact. The hydraulic retention times for UASB reactors are usually less than 48 hours, and are mainly used on high strength wastewaters. Solids buildup inside the reactors is one of the major downfalls of the UASB reactor when treating wastewaters with a high solids content (Nicolella et al. 2000). 
- Anaerobic fluidized bed (AFB) - Mainly used on brewery, food-processing, and paper industry wastewaters, the AFB (Heijnen et al. 1989) utilizes a bed of sand or other small media $(0.2-0.8 \mathrm{~mm}$ in diameter) to provide a large surface for attached biological growth. Influent enters through the bottom of the reactor with a velocity in the range of $10-30 \mathrm{~m} / \mathrm{h}$ to create fluidization (Nicolella et al. 2000). Because such high liquid velocities are needed to create fluidization for the biolayer-covered particles, inert sediments do not accumulate in the reactor (Heijnen et al. 1989).

- Anaerobic filter (AF) - Young and McCarty (1967) developed the AF, which is typically used in the treatment of soluble industrial wastewaters. AF reactors rely on packing material made of ceramic, glass, plastic or wood to retain biomass. Hydraulic retention time is usually between 1 and 3 days. The main limitation of this reactor design is accumulation of solids in the packing media, leading to plugging of the reactor (Lusk 1998; Van den Berg 1986).

- Expanded granular sludge bed reactor (EGSB) - The expanded granular sludge bed reactor (Franklin et al. 1992) is a combination of both the UASB and the AFB. Biomass is present as granules, however the upflow liquid velocity is more like that in the AFB, at $10-15 \mathrm{~m} / \mathrm{hr}$, while the gas velocity is around 7 $\mathrm{m} / \mathrm{hr}$. This technology is mainly used on high strength wastewaters such as those from the food, pharmaceutical, and chemical industries (Nicolella et al. 2000).

Several other types of high rate digester designs exist. However, utilization of highrate anaerobic technologies at the farm scale requires more capital and operating costs because of greater digester complexity (Lusk 1998).

\subsection{The Anaerobic Digestion Food Web}

Anaerobic digestion occurs in a series of steps employing different types of Bacteria to break down complex polymers into simple carbon compounds (e.g., straight chained fatty acids) that can then be utilized by Archaea to produce methane. 
At least five different groups of microorganisms are involved, three groups of Bacteria (fermentative bacteria, homoacetogens, and obligatory hydrogen-producing acetogens) and two groups of Archaea (hydrogen-consuming methanogens and acetateconsuming methanogens). The extent of mutual reliance among these microorganisms varies substantially. While the lower members of the food web depend on the higher members for substrate, the higher members rely on the lower members to remove metabolic products.

The pathway in which anaerobic digestion takes place is shown in Figure 2-1. Polysaccharides, proteins, lipids, and other insoluble organic matter are hydrolyzed in the first step, typically through the excretion of extracellular hydrolytic enzymes by primary fermentive bacteria. The primary fermentive bacteria will then convert the soluble elements (e.g., sugars, fatty acids, glycerol, and amino acids) into a mixture of acetate, other organic acids, such as butyrate and propionate, alcohols, hydrogen, and carbon dioxide. Hydrogen-producing acetogens then convert organic acids and alcohols to acetate, hydrogen, and carbon dioxide. The hydrogen and carbon dioxide produced by the fermentive bacteria are usually converted directly to methane and carbon dioxide by methanogens, but can also be converted to acetate by homoacetogenic bacteria. However, in conventional anaerobic digesters conversion of hydrogen and carbon dioxide to acetate accounts for only approximately $1-4 \%$ of acetate synthesis (Zinder 1988).

The pathway in which primary fermenting bacteria break down monomers, either to organic acids and alcohols (pathway $\mathrm{C}$ in Figure 2-1), or to acetate, carbon dioxide, and hydrogen (pathways B and A, respectively), is determined mainly by environmental conditions. The environmental condition that governs which of these pathways is predominant is the partial pressure of $\mathrm{H}_{2}$. If hydrogen-consuming methanogens maintain a $\mathrm{H}_{2}$ partial pressure lower than $10 \mathrm{~Pa}$, pathways $\mathrm{A}$ and $\mathrm{B}$ are favored. However, if hydrogen consuming methanogens fail to keep the partial pressure less than $10 \mathrm{~Pa}$, pathway C will be favored (Schink 1997; Zinder 1988; Novaes 1986). The pathways A and B in this process are preferred because more 
energy is available in these reactions to form adenosine triphosphate (ATP). In a well developed and stable digestion process the $\mathrm{H}_{2}$ partial pressure is maintained at low values, and digestion proceeds mainly through the pathway in which more energy is available. However, due to production of fatty acids through the fermentation of lipids and amino acids, the carbon flux through intermediate organic acids and alcohols (pathway C) typically accounts for $30 \%$ of the carbon flow (Speece 1996). It is in this pathway that syntrophic relationships exist. Syntrophic relationships are a special case of symbiotic relationship in which two organisms with different metabolic capabilities work together to degrade a compound, usually occurring due to energy limitations.

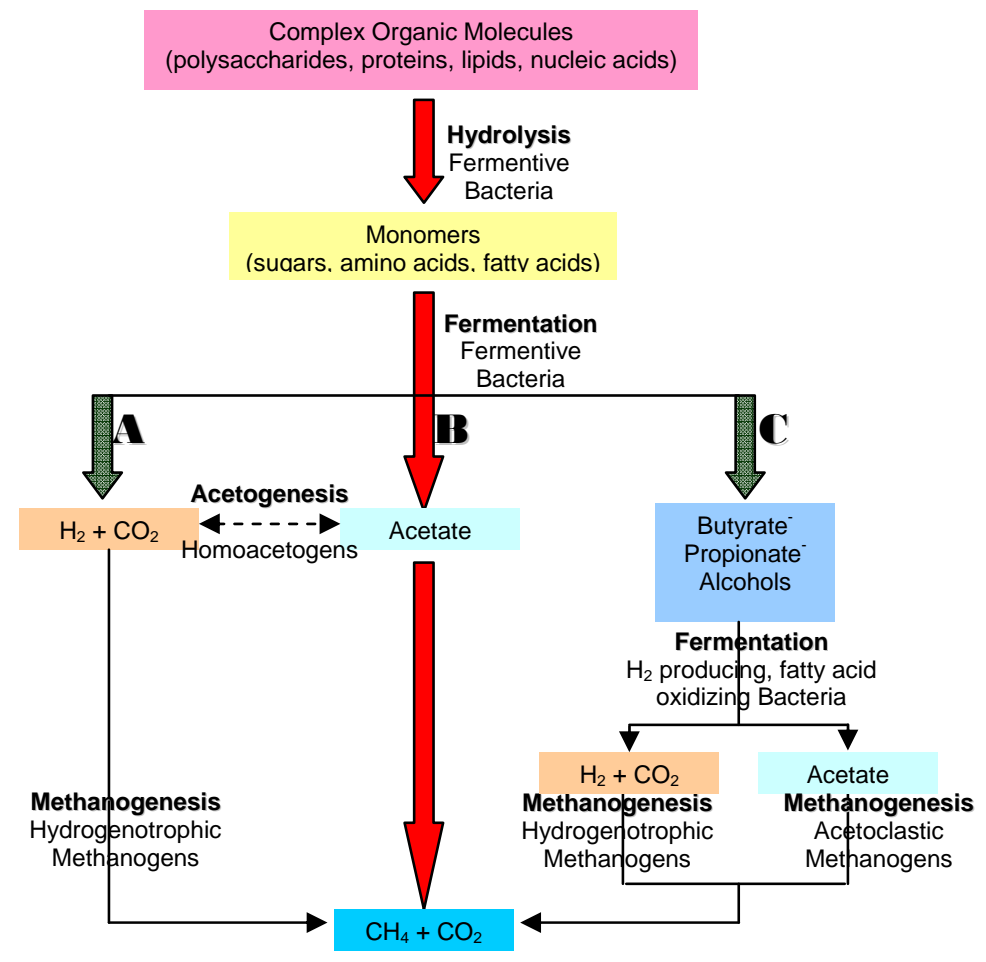

Figure 2-1 Anaerobic digestion pathway.

\subsection{Factors Affecting Anaerobic Digestion}

Successful implementation of anaerobic digestion depends on the function of a complex community of microorganisms. Several environmental conditions need to be taken into consideration to maintain optimum performance. These conditions are: 
Nutrients. An adequate supply of nutrients, particularly nitrogen and phosphorous, is needed for the growth of the anaerobic community of microorganisms. In addition, trace elements such as iron, nickel, cobalt, magnesium, barium and molybdenum are also essential for increasing the microbial activity and biomass concentrations. Livestock manure generally contains a well balanced and ample amount of nutrients to support microbial growth during anaerobic digestion (Miner et al. 2000).

Temperature. Temperature determines the speed of microbial activity. Anaerobic digestion usually takes place in one of three temperature ranges: psychrophilic (less than $20^{\circ} \mathrm{C}$ ), mesophilic (between $20^{\circ} \mathrm{C}$ and $40^{\circ} \mathrm{C}$ ), and thermophilic (between $40^{\circ} \mathrm{C}$ and $70^{\circ} \mathrm{C}$ ). Traditionally, anaerobic digesters are operated in the mesophilic range, particularly between $35-37^{\circ} \mathrm{C}$. Temperature fluctuations also affect the stability of anaerobic systems, making it desirable to operate in a narrow temperature range (Fannin 1987).

pH, Organic Acids, and Alkalinity. Optimum pH levels for anaerobic digestion are between 7 to 8 . A build-up in organic acids, which are the intermediates in the digestion process, can cause decreases in digester $\mathrm{pH}$. Organic acid build-up occurs when methanogens are inhibited after, for example, temperature fluctuations and excessive organic loading. If $\mathrm{pH}$ levels are allowed to fall below 6 , acidic conditions may completely inhibit methanogens. Alkalinity provides a buffering capacity within the digester and is a function of the concentrations of carbonate, bicarbonate, and hydroxide. If enough buffering capacity is available to maintain a stable $\mathrm{pH}$ in the optimal range during periods of increased organic acids, growth of the methanogenic population may occur. However, if the buffering capacity is insufficient, and the $\mathrm{pH}$ decreases below that tolerable by methanogens, preventative actions must taken to increase $\mathrm{pH}$ and avoid further deterioration (Miner et al. 2000; Fannin 1987). 
Ammonia Toxicity. Equilibrium exists in the aqueous phase between the ammonium ion $\left(\mathrm{NH}_{4(a q)}^{+}\right)$, free ammonia $\left(\mathrm{NH}_{3(a q)}\right)$, the hydronium ion $\left(\mathrm{H}_{3} \mathrm{O}^{+}\right)$, the hydroxyl ion $\left(\mathrm{OH}^{-}\right)$, and the ammonia in the gas phase $\left(\mathrm{NH}_{3(g)}\right)$. Free ammonia has

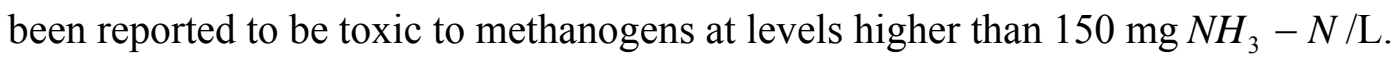
Since the $\left[\mathrm{NH}_{4(a q)}^{+}\right] /\left[\mathrm{NH}_{3(a q)}\right]$ ratio is $\mathrm{pH}$ dependent, as long as $\mathrm{pH}$ values are less than 8 , greater than $90 \%$ of the ammonia present is in the less toxic form of ammonium. However, it has also been reported that total ammonia concentrations of 1000 $\mathrm{mg} \mathrm{NH}_{3}-\mathrm{N} / \mathrm{L}$ are also detrimental to digester performance regardless of the $\mathrm{pH}$ level (Miner et al. 2000).

Hydraulic Retention Time (HRT). The HRT is the average number of days a molecule in the liquid phase is retained within the digester. It is equal to the working volume of the digester divided by the daily amount of influent. The HRT is an important operational parameter because it determines the amount of time available for treatment of dissolved organic material. Different HRTs are required for different types of digesters; high-rate digesters, which retain large amounts of biomass, can operate at shorter HRTs than low-rate systems, such as CSTRs, which do not retain biomass.

Solid Retention Time (SRT). The SRT is the amount of solids retained in the digester divided by the amount of solids taken out in the effluent per day. It is important to note that in conventional digesters, such as CSTRs, the HRT and SRT are the same. However, in high-rate systems, such as ASBRs, SRTs are much longer than HRTs, resulting in solids conversion to gas ratios that are comparable to conventional digesters in a much smaller area. 
Total Solids. The total solids concentration present in the feed is critical to mechanical handling, digester design, and operation. Feeds with low solids concentration, for instance, require larger volume reactors and higher heating requirements for CSTR systems. However, as total solids in the feed increases, the available net energy also increases. Typical total solids concentrations for anaerobic digesters are in the range of $3-10 \%$ (Stafford et al. 1980).

Volatile Solids. The amount of volatile solids within a digester is a key operating parameter that must be controlled. Volatile solids are composed of organic material, a portion which is utilized as substrate for microorganisms. Volatile solids are an important parameter used to estimate gas production, and should be kept fairly uniform to prevent digester upset due to over-loading. Loading rates for reactors fed high-solids wastewater are often based on the amount of volatile solids in the feed.

Loading Rate. The loading rate is one of the most important parameters in digester design. Loading rate is expressed as the amount of biodegradable solids per unit volume introduced into the digester. Typical loading rates for anaerobic digesters range from $0.7-5 \mathrm{~kg} \mathrm{VS} / \mathrm{m}^{3}-\mathrm{d}$ (Lapp et al. 1975). If the loading rate is too low, metabolic activity of the microorganisms will be low, resulting in reduced gas production. If the loading rate is too high, the balance between the acid forming bacteria and acid consuming methanogens may be disrupted, resulting in VFA build up within the digester.

\subsection{The Role of Mixing in Anaerobic Digestion}

Mixing in anaerobic digesters has several functions in maintaining efficient digester performance. These functions include (Stafford et al. 1980):

- Enhancing substrate and microorganism distribution

- Ensuring uniform $\mathrm{pH}$ and temperature 
- Preventing stratification and scum accumulation in dilute waste slurries

- Facilitating biogas removal from the liquid

- Aiding in particle size reduction

Mixing can be performed by a range of different methods, including mechanical mixers, recirculation of digester contents, and recirculation of produced biogas. While mechanical mixing with an impeller is effective, fibrous material can entangle the shaft and blades, digester contents can be abrasive to internal parts, and a high energy input is needed (Stafford et al. 1980). Digester recirculation is not as efficient at creating agitation, but several other advantages to this method exist including: all moving parts are outside the digester, allowing for easy maintenance, heat exchangers can be used to maintain temperature instead of separate heating units, and lower capital and operational cost because less equipment is needed (Stafford et al. 1980). Several studies have shown that mixing by gas recirculation improves performance (Konstandt and Roediger 1977; Sen and Baskaran 1962). However, the gas distribution system lies within the interior of the reactor and can be prone to clogging when used in high solids applications.

The effects of mixing on the anaerobic process are not well understood, and the information available in the literature is contradictory. Mixing can either be continuous or intermittent, as well as vigorous or gentle. The primary function of mixing, for both low and high solids applications, is stated in the literature to be the distribution of enzymes and microorganisms throughout the digester (Lema et al. 1991; Chapman 1989; Parkin and Owen 1986; Strenstrom et al. 1983). However, many studies have revealed other functions of mixing. For example, insufficient mixing in low solids digesters has been shown to produce an undesirable floating layer of solids, while insufficient mixing in high solids digesters has been shown to have solids accumulate at the bottom of the digester, leading to a decrease of up to $70 \%$ in the effective volume in full-scale digesters (Monteith and Stephenson 1981; James et al. 1980; Strenstrom et al. 1983; Diaz and Trezek 1977; Torpey 1955). 
Research has shown evidence that continuous mixing in digesters results in better performance. In fact, continuous mixing has been found to increase methane production rates over both unmixed digesters and intermittently-mixed digesters. Proposing that the phase transfer of gaseous products was rate limiting in anaerobic digesters, Finney and Evans (1975) suggested that at high substrate concentrations, microorganisms could become surrounded by self-produced gas bubbles, leading to interference with substrate diffusion. By using vigorous agitation in digesters treating sewage sludge, they saw as much as a six-fold increase in methane formation under low pressure. Studies by Ho and Tan (1985) found continuously mixed digesters treating palm oil mill effluents had higher gas production and growth constants than those of unmixed digesters. Hashimoto (1982) reported 8-11\% higher methane production rates from a continuously-mixed digester treating beef cattle wastes than intermittent mixing for 2 hours per day. However, in other instances, intermittent mixing has been shown to be superior. Stroot et al. (2001) compared the startup and performance of continuously and minimally-mixed digesters treating organic fraction of municipal solid waste operating with three different organic loading rates. They found that continuously mixed digesters performed poorly with respect to the minimally mixed digesters at higher organic loading rates. While the higher loaded continuously-mixed digesters accumulated very high levels of acetate, which deter performance, the minimally-mixed digesters showed better performance with respect to VFA turnover and gas production rates. Intermittent mixing has also been found to help develop well-settling biomass in ASBRs (Zhang et al. 1997; Sung and Dague 1995).

Moreover, shifts from continuous to intermittent mixing have resulted in increased digester performance, while shifts from intermittent to continuous mixing have resulted in decreased performance (Stroot et al. 2001). Stroot et al. (2001) examined the effect of mixing on stable (low loading, minimal mixing) and unstable (high loading, continuous mixing) digesters operating on a mixture of organic fraction municipal solid waste, primary sludge, and activated sludge. They showed that 
changing from continuous to minimal mixing on an unstable digester operated with high solids concentration resulted in attaining better digester stability through reduction of propionate and acetate levels. However, a change from minimal mixing to continuous mixing in a stable digester operated with low loading resulted in the accumulation of acetate and propionate. This increase in levels of propionate and acetate, when switched to continuous mixing, was unexpected due to the good performance seen for continuously-mixed digesters at low loading rates. Dague et al. (1970) observed appreciably higher gas production, and increased chemical oxygen demand (COD) and solids reduction after shifting a continuously-mixed digester treating a synthetic liquid waste stream to an intermittently-mixed regime of 2 minutes per hour. Dague et al. (1970) theorized that better bioflocculation was attained in the intermittently mixed digester as compared to the continuously mixed digester, resulting in better digester performance. A study by Angenent et al. (2001) observed similar results when changing mixing levels in an ASBR. They found that a change from gentle, intermittent mixing to gentle, continuous mixing resulted in a slightly negative impact on performance. In addition to decreased performance, a decrease in the population levels of most methanogens was also observed. This finding may also be used to explain the findings of Stroot et al. (2001).

The intensity of mixing also has been found to have an effect on digester performance. Recent work by Lanting (2003) aimed at investigating the ability to increase processing capacity of municipal sludge digesters through increasing the rate of substrate utilization, investigated the impact of shear on biomass activity. Mixing and shear was applied by slurry recirculation from the bottom of the digester to the top through a downward-facing nozzle and draft-tube configuration by a variable speed circulation pump. Different levels of shear power were imparted to the digester contents by altering the circulation flow, changing the nozzle discharge velocity and nozzle pressure drop. Lanting (2003) found that the higher the mixing, the better the performance, increasing the activity of anaerobic sludge digesters to $2.5 \mathrm{~kg}$ VS destroyed per kg biomass VS per day as compared to conventional high-rate digesters, 
which have activities on the average of $0.6 \mathrm{~kg}$ VS destroyed per $\mathrm{kg}$ biomass VS per day. A study by Angenent et al. (2001) observed opposite results when changing mixing levels in an ASBR. They found that a shift from gentle, continuous mixing to a more vigorous, continuous mixing resulted in rising VFA levels in the effluent and severe impairment of performance. In addition to decreased performance, a decrease in the population levels of most methanogens was also observed. Different findings between the results of Lanting (Lanting 2003) and Angenent et al. (Angenent et al. 2001) may be explained by the different types of digester configuration and method of mixing. While Lanting (2003) used a digester continuously stirred by recirculation of digester contents, Angenent (2001) studied a high-rate digester that relies heavily on the high amounts of settled biomass. However, both studies show that differences in mixing intensities have affected digester performance.

Some researchers believe that vigorous mixing may disrupt the spatial juxtaposition of syntrophic microorganisms (Dolfing 1992; Whitmore et al. 1987; Conrad et al. 1985). Close physical associations between electron-producing and electron-consuming syntrophs allows for high rates of hydrogen transfer at relatively low concentrations of hydrogen. In vigorously-mixed systems, continuously disrupted spatial associations can lead to a state of instability (Stroot et al. 2001). This rationale has led some researchers to believe that minimal mixing may improve anaerobic digestion by providing a quiescent environment for bacteria and archaea (Lettinga 1981). However, it is argued that some mixing is required to distribute substrate and allow new spatial associations to form between different microorganism populations; otherwise, due to their growth, cells will be surrounded by their own offspring, causing reduced kinetic effectiveness (Schink 1992).

In spite of the mounting evidence that mixing effects digester performance, some evidence has been found to suggest otherwise. Lin and Pearce (1991) studied the effects of both mixing intensity and duration on the anaerobic treatment of potatoprocessing wastewater, using impeller speeds of 20, 50, and $100 \mathrm{RPM}$, and two mixing durations of 15 and 45 min per hour. They found that reactor start-up was 
accelerated by increasing mixing intensities. However, no significant difference in biogas production was seen between the different digesters, although higher production rates were found at the 15 minute mixing duration as compared to the 45 minute duration. A study by Karim et al. (Karim et al. 2005b) also found that differing levels of mixing did not have an effect on digester performance. Karim et al. (Karim et al. 2005b) studied different levels of continuous biogas recirculation (1,2 and $3 \mathrm{~L} / \mathrm{min}$ ) on anaerobic digesters treating dairy cow manure having a TS concentration of $50 \mathrm{~g} / \mathrm{L}$. The authors attribute their findings possibly to the low solids concentration in the manure feed. Coppinger et al. (1979) saw no decrease in gas production when mixing was halted in a full-scale digester treating dairy manure. In contrast, Ben-Hasson et al. (1985) observed that a continuously-mixed digester treating dairy cattle waste had a $75 \%$ lower methane production rate than that of an unmixed digester. Similarly, Chen et al. (1990) observed a higher methane yield from an unmixed digester than that of a continuously-mixed reactor treating a mixture of refuse-derived fuel and primary sludge at low solids levels. The authors attribute this finding to longer effective solids retention time in the unmixed digester. Longer effective solids retention time allows biomass to accumulate within the digester, therefore, operation resembles that of high-rate digesters, which can not be compared with completely mixed systems in terms of performance. In addition, it has been shown that mixing does not significantly affect the performance of both high solids (Rivard et al. 1995), and low solids digestion (Stroot et al. 2001; Diaz et al. 1974).

The contradictory findings reported in the literature about the role of mixing in anaerobic digesters, and a lack of research to determine the effect on performance and microbial ecology, justify further research in this area. Therefore, the present study is designed to focus on determining the effect of mixing on both digester performance and microbial community. 


\subsection{Microbial Ecology in Anaerobic Digesters}

Anaerobic digesters utilize many different types of Bacteria and Archaea to break down organic molecules as described in section 2.3. Thus, performance of anaerobic digesters is closely tied with the composition of the microbial community. In particular, the methanogenic community is of great importance (Zinder 1984). The organisms which play the main roles in methanogenesis in anaerobic digesters belong to the following four out of the five orders of methanogens: Methanomicrobiales, Methanobacteriales, Methanococcales, and Methanosarcinales (Griffin et al. 1998; Zheng and Raskin 2000).

Nucleic acid-based techniques have been used to characterize microbial communities in environmental samples for close to fifteen years. The use of these techniques can give valuable insight into the structure and composition of environmental microbial communities. McMahon et al. (2001) showed that in digesters with high acid concentrations, Methanosarcina spp. was the dominating aceticlastic methanogen, while digesters with low acid concentrations were dominated by Methanosarsina concilii. McMahon et al. (2001) also showed that an increase in mixing level resulted in dramatic increases of Methanosarcina spp. and Methanobacteriace. Angenent et al. (2001) studied the methanogenic population dynamics in an ASBR, showing that an increase in mixing intensity resulted in a decrease of most methanogens. Only relative levels of Methanococcaceae and Methanobacteriaceae increased, but levels of these methanogens only increased by $1 \%$ of the total $16 \mathrm{~S}$ RNA.

The effects of shear on the microbial community within an anaerobic digester can be elucidated through the utilization of two molecular biology techniques, fluorescent in situ hybridization (FISH) and membrane hybridization. These two techniques are based on ribosomal RNA (rRNA) targeting oligonucleotide probes, which hybridize to their complimentary sequence within environmental samples. rRNAs are more widely used than other nucleic acids for comparative analysis due to their universal distribution, lack of interspecies transfer of the rRNA gene, and high level of 
conservation (Zheng et al. 1996). The 16S rRNA is more often utilized as a target than the 23S rRNA, because a more extensive collection of data exists for this region of RNA. The 16S rRNA contains sections that vary in sequence conservation, allowing for the design and utilization of these oligonucleotide probes. Universal probes, which are complimentary to universally conserved sections of 16S rRNA, will hybridize to all $16 \mathrm{~S}$ rRNA in the sample, while probes which are complimentary to sections containing variability are more selective (species-, genus-, or phylogeneticgroup-spcecific probes). The hybridization of these rRNA probes can either occur in nucleic acid extracts, or in fixed cells (whole cell hybridization).

FISH is based on fluorescently-labeled probes hybridizing in whole, fixed cells. This allows for visualization of individuals or groups of individual population members in complex communities through the use of fluorescence microscopy. Mainly a qualitative technique, FISH is mostly utilized to determine spatial associations of organisms in environmental samples.

Membrane hybridization is based on radioactively-labeled probes hybridizing to immobilized RNA extracted from an environmental sample. The degree of hybridization can be used to estimate the abundance of a target population. A qualitative technique, membrane hybridization is a powerful tool that can be used to detect population shifts over time, or the development of different microbial communities within systems of interest. 


\section{Chapter 3 Effect of Mixing Intensity on Performance and Biomass Characteristics in Continuously-Stirred Anaerobic Digesters Treating Dairy Cow Manure}

\subsection{Introduction}

The interest in more environmentally-sound waste management practices in the livestock industry has intensified while the traditional waste management practices of storage in open-air lagoons and unrestricted land application is being phased out. In 2003, the U.S. Environmental Protection Agency (EPA) passed the Concentrated Animal Feeding Operations (CAFOs) Rule, requiring the largest CAFOs in the U.S. to acquire Clean Water Act permits, forcing them to develop manure management plans that ensure proper management and land application of manure.

Anaerobic digestion is a waste treatment technology that utilizes microorganisms to break down organic material in the absence of oxygen to create biogas (i.e. a mixture of methane and carbon dioxide). Besides reducing greenhouse gas emissions from open-air lagoons and producing a renewable energy source, anaerobic digestion also reduces odor, protects water quality, controls ammonia release, and produces a fertilizer. For these reasons, anaerobic digestion is seen as an environmentally-sound waste management practice by the U.S. EPA, Department of Agriculture (USDA), and the Department of Energy (DOE) under the Climate Change Action Plan (Clinton and Gore 1993). Anaerobic digestion has several advantages over aerobic treatment, including achieving higher organic removal rates, producing less sludge, and net gaining of energy rather than requiring energy. Although industrial wastes have successfully utilized anaerobic digestion to reduce organic pollutants in waste streams for over 30 years, implementation of anaerobic digesters on farms for the purpose of treating animal manure and farm wastes has had high failure rates (Lusk 1998). 
Mixing plays several essential roles during anaerobic digestion of sludges (e.g., animal waste and waste activated sludge). These roles include enhancing substrate contact with the microbial community, improving $\mathrm{pH}$ and temperature uniformity, preventing stratification and scum accumulation, facilitating the removal of biogas from the digestant, and aiding in particle size reduction (Stafford et al. 1980). The intensity at which mixing occurs, and thus the shear rate, has been shown to have an effect on performance of anaerobic digestion (Angenent et al. 2001; Lanting 2003). Research has shown that high mixing intensities resulted in particle size reduction and diffusion limitation reduction, which increased processing capacity for a digester treating waste activated sludge (Lanting 2003). In contrast, several studies have shown that high mixing intensity and duration had a detrimental effect on digester performance (McMahon et al. 2001; Stroot et al. 2001; Angenent et al. 2001; Dague et al. 1970). It has been hypothesized that a high shear rate may be harmful to anaerobic digestion because it disrupts spatial associations between syntrophic microorganisms (Dolfing 1992; Whitmore et al. 1987; Conrad et al. 1985). This has not been experimentally verified, however.

A better understanding of the role of mixing in anaerobic digestion will result in better design and operation, leading to a reduction in failure rate and increased utilization of anaerobic digestion on the farm. Therefore, the objective of this work was to study the effect of mixing intensity (i.e., applied shear) on digester performance, microbial ecology, and syntrophic relationships in continuously-stirred anaerobic digesters treating dairy cow manure. Impeller speeds of 50, 250, 500, and 1,500 rotations per minute (RPM), were applied to four identical 4.5-L digesters. Performance of each digester was monitored with parameters indicating stability and performance, while molecular techniques, such as fluorescent in situ hybridization (FISH) and membrane hybridization, were used to track changes in flocs of syntrophic organisms and the microbial community, respectively. Results on membrane hybridization, however, will not be presented in this thesis, but will be presented in a future publication. 


\subsection{Materials and Methods}

Reactor Operation. The experiments were conducted in four, 4.5-L laboratory scale reactors made from clear $\mathrm{PVC}$, with a $25^{\circ}$ slope angle hopper bottom. The digesters were kept in a temperature-controlled environment in which the liquid inside the digesters was at a constant $35 \pm 2{ }^{\circ} \mathrm{C}$. The gas collection system of each digester setup was equipped with a foam separation bottle, a pressurized ball used to eliminate outside air from being suctioned into the digesters during the decanting of effluent, a bubbler to allow visual determination of gas production, a biogas sampler, and a gas meter (Actaris Meterfabriek BV, Delft, The Netherlands). Each digester was continuously mixed by an impeller motor (Model 5vb, EMI Inc., Clinton, Connecticut, USA) with a 62-mm diameter axial flow impeller (Lightnin A-310, Rochester, New York, USA). The applied RPM of the impellers was determined by a tachometer (Bex-O-Meter, Model 38, The Bex Company, San Fransisco, California, USA). Baffles were not used in the digesters to allow for comparison to previous work performed at a mixing level of 250 RPM, in which baffles were not used.

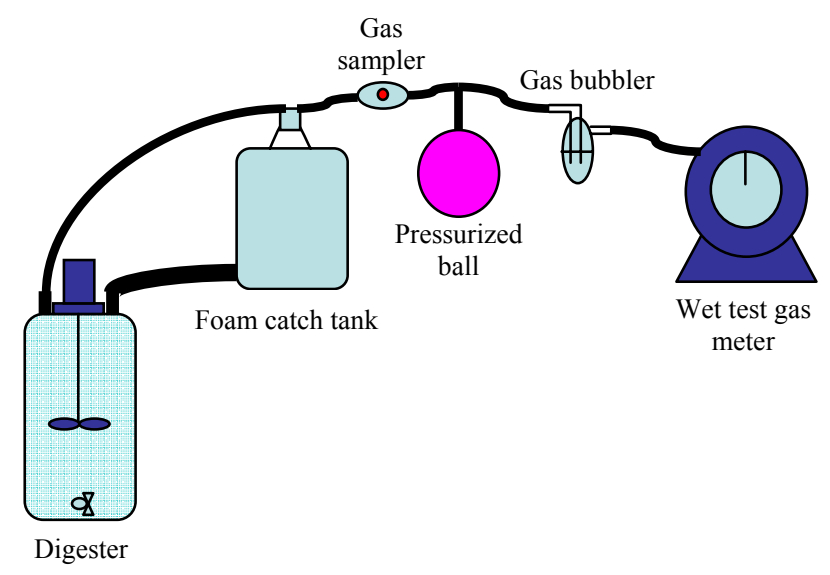

A

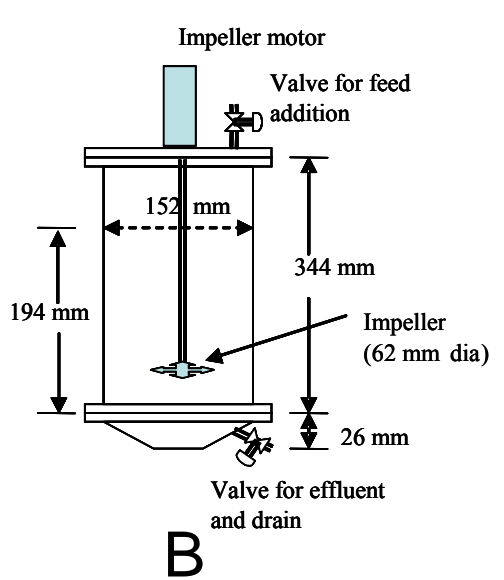

Figure 3-1. Continuously-stirred anaerobic digester setup (A) and diagram (B). 
The digesters were inoculated with 4.5-L anaerobic sludge collected from the Metropolitan Sewer District's Coldwater Creek facility, St. Louis, MO. After inoculation of the digesters, a 24 hour acclimation period was allowed before the commencement of mixing. Raw dairy cow manure was collected fresh (less than 6 hours after excretion) from the Martin Dairy Farm, Pevely, MO, twice throughout the study and stored in a freezer. The feed slurry was prepared from the collected raw manure by dilution and screening through a 2-mm sieve. After total solids (TS) and volatile solids (VS) for the prepared slurry were determined, it was then diluted with tap water to achieve the desired VS concentration of $50 \mathrm{~g} \mathrm{VS}$ per liter (5\% solids content based on VS). The reactors were fed manually every $24 \pm 1$ hour by first removing an appropriate amount of reactor effluent and then adding the same volume of prepared manure feed (mixers were continuously operating during decanting and feeding). To avoid overloading of the reactors at startup, the initial loading rate was $0.6 \mathrm{~g} \mathrm{VS} / \mathrm{L}-\mathrm{d}$, which was $16 \%$ of the target loading rate of $3.5 \mathrm{~g} \mathrm{VS} / \mathrm{L}-\mathrm{d}$. The loading rate was periodically increased in a step-wise manner after steady-state biogas production levels had been reached, with a minimum time period of 1 hydraulic and/or solid retention time (HRT/SRT), except during the initial $0.6 \mathrm{~g} \mathrm{VS} / \mathrm{L}-\mathrm{d}$ loading period. The loading rates used for the different loading steps throughout the study were 0.6, 1.0, 1.7, 2.5, and $3.5 \mathrm{~g} \mathrm{VS} / \mathrm{L}-\mathrm{d}$, corresponding to a HRT (and SRT since they are the same in CSTRs) of 76, 50, 30, 20, and 15 days, respectively.

Physical and Chemical Analysis. Feed and effluent samples were analyzed for pH, TS, VS, volatile fatty acids (VFA), soluble chemical oxygen demand (SCOD), alkalinity, and total reactive phosphorous according to procedures in Standard Methods (APHA 1998). The amount of TS and VS in the inoculum was also determined in order to calculate the VS removal efficiencies. Methane content of the biogas was determined weekly using a gas chromatograph (Series 350, Gow-Mac Instruments, Co., Bridgewater, NJ, USA) with a thermal conductivity detector. The temperature of the injection port was $20^{\circ} \mathrm{C}$, and that of the detector was $40^{\circ} \mathrm{C}$. Helium 
at a flow rate of $60 \mathrm{ml} / \mathrm{min}$ was used as the carrier gas. Inductively Coupled Plasma Atomic Emissions Spectroscopy (ICP-AES) was used to determine metal concentrations on samples taken at the end of the study with a Varian Liberty RL Sequential ICP-AES (Walnut Creek, CA, USA). The elements measured at the wavelengths used were Al: 396.152, Ca: 317.933, Cu: 324.754, Fe; 238.204, Hg: 253.652, Mg: 285.213, Mn: 257.610, Si: 212.412, Ti; 336.121, Zn: 206.200. The detection limit was 5 ppm for all metals except for $\mathrm{Mg}$ and $\mathrm{Zn}$, which had detection limits of 1ppm). In addition, the distribution of particle size was measured at the end of the digester operational period using a series of six sieves with sizes of 1.4, 1.0, 0.6, $0.3,0.15$, and $0.045 \mathrm{~mm}$. Samples were screened through these sieves and then collected into pre-weighed crucibles, which were then analyzed for TS and VS. Each sample was analyzed three separate times. Ammonia was measured using an ammonia electrode (Model 95-12, Thermo Electron Corporation, Beverly, MA, USA)

FISH. Digester samples were fixed with $4 \%$ paraformaldehyde for $2 \mathrm{~h}$ at $20^{\circ} \mathrm{C}$ and stored with phosphate buffer saline solution and ice-cold ethanol at $-20^{\circ} \mathrm{C}$. Hybridization was performed with 16S rRNA-targeting oligonucleotide DNA probes specific for Archaea (ARC 915) (Stahl and Amann 1991) and Bacteria (EUB 338) (Amann et al. 1990) according to de los Reyes et al. (de los Reyes et al. 1998). Specimens were viewed with an epifluorescence microscope (BX41, Olympus, Melville, NY) and digital images were taken with a CCD camera (QImaging, Burnaby, Canada.) and saved in Openlab 3.5 software (Improvision Inc., Lexington, MA). Finally, images were overlaid in Photoshop 7 (Adobe System, Seattle, WA).

\subsection{Results}

The mixing intensity levels chosen were 1500, 500, 250 and 50 RPM. The 1500 and 50-RPM intensities were close to the highest and lowest operational RPM achievable by the type of impeller motor that we used. The 500 and 250-RPM 
intensities were the highest and lowest RPM, respectively, which can be used to achieve accurate results from computer automated radioactive particle tracking (CARPT) (results are part of another thesis in the Department of Chemical Engineering at Washington University in St. Louis (not yet published)). With such a broad range of applied shear, significantly different mixing conditions existed within each digester. Vortices in the 1500 and 500-RPM digesters created a 35\% and 10\% increase, respectively, in maximum height of the liquid inside the digester, while only a small vortex developed in the 250-RPM digester, and no vortex was noticed in the 50-RPM digester. The spatially-averaged velocity gradient for the 1500, 500, 250, and 50-RPM digesters were found to be $3500,630,210$, and $17 \mathrm{~s}^{-1}$, respectively. Despite such a large range of applied RPMs, the biogas production of all four digesters was found to be similar during steady-state periods (Figure 3-2 (A)). The methane yield obtained by graphing the standard methane production rate over the VS loading rate for each digester during the steady-state periods was statistically not different between treatments (analysis of variance (ANOVA): $n=20, p=0.73$ ). The overall methane yield obtained by pooling the data for each digester was $0.241 \mathrm{~L} \mathrm{CH}_{4} / \mathrm{g} \mathrm{VS}$ fed with a standard error of \pm 0.007 (Figure 3-3). A second methane yield was calculated similarly, based on the amount of VS consumed (as compared to the amount of VS fed), and was found to be $0.541 \mathrm{~L} \mathrm{CH}_{4} / \mathrm{g}$ VS consumed with a standard error of \pm 0.01 (Figure 3-3). Only four data points were used in the calculation of the methane yield based on VS removed, because the VS concentration in the digesters were still increasing because of solids accumulation during the first loading period. The percentage of methane in the biogas for each digester over the entire operational period was similar with a pooled average of $67.4 \pm 5.0 \%$.

Differences in performance were seen during the initial start-up period though, when the most intensely mixed digester (1500 RPM) produced little or no biogas between day 10 and 25 (Figure 3-2 (A)), and accumulated VFAs greater than 4000 $\mathrm{mg} / \mathrm{L}$ as acetic acid (Figure 3-2 (B)), while the other digesters showed a constant biogas production rate between approximately 0.6 and $1.0 \mathrm{~L} / \mathrm{d}$ with low VFA 
concentrations in the digesters. The 1500-RPM digester began to recover between day 29 and day 45, showing peaks in biogas production, which corresponded with drops in levels of VFAs (Figures 3-1 (A) and (B)). Similar observations were made in the 500RPM digester after the first increase in loading rates from 0.6 to $1.0 \mathrm{~g} \mathrm{VS} / \mathrm{L}-\mathrm{d}$.

Between day 52 and day 78, the biogas production for the 500-RPM digester remained lower than that of the 250 and 50-RPM digesters (Figures 3-1 (A) and (B)). On day 78 , the VFA levels in the 500-RPM digester reached a peak and then began to rapidly decrease, causing the biogas production to rise to a level that was similar to the other digesters (Figures 3-1 (A) and (B)). This phenomenon indicates that intense mixing during the start-up period had detrimental effects on reactor performance.

Accidental addition of 3.3 times the normal volume of $50 \mathrm{~g} \mathrm{VS} / \mathrm{L}$ cow manure feed occurred on day 150 for the 500-RPM and 250-RPM digesters, yielding information regarding the differences in the ability of the digesters to handle a transient hydraulic and organic shock load. It is clear from Figures 3-1 (A) and (B) that the 500-RPM digester was able to handle the shock better than the 250-RPM digester, consuming almost all of the excess substrate in four days with only a small increase in VFA concentration. In contrast, the 250-RPM digester required almost thirty days to stabilize, and showed a much larger VFA accumulation. A planned transient shock load was performed at the end of the operational period by decanting and feeding twice the normal amount, corresponding to a doubling of the VS loading rate to $7.0 \mathrm{~g}$ VS/L-d for one day (day 260). The digesters continued to be operated for four days after this shock load occurred, at a VS loading rate of $3.5 \mathrm{~g} \mathrm{VS} / \mathrm{L}-\mathrm{d}$, while biogas production and VFA concentrations were monitored (Figure 3-4). After this transient shock load, biogas production was seen to spike, however, differences between the digesters were seen. The 500-RPM digester showed the largest spike in biogas production with an increase of $48 \%$ the day after the shock load as compared to the previous day, followed by the 250 and 50-RPM digesters with $47 \%$ and $28 \%$ increase, respectively. The 1500-RPM digester showed the lowest spike in biogas production with an increase of only $22 \%$. This spike in biogas production was accompanied by a 
spike in VFAs for all reactors, showing an increase in VFAs from the sample analyzed only two days before the shock load occurred of $67 \%, 60 \%, 131 \%$, and $200 \%$ for the 1500, 500, 250, and 50-RPM mixing intensities, respectively. This data indicates that, with exception of the 1500-RPM digester during the transient shock load, the capability of the digesters to handle this shock load increased with increasing mixing intensity. Levels of VFAs in the 1500-RPM digester were already high at the time of the transient shock load, after the shock load VFA concentrations spiked even higher.

The inoculum for each digester was obtained from a conventional full-scale primary anaerobic digester treating waste activated sludge having TS and VS concentrations of $18.5 \mathrm{~g} / \mathrm{L}$ and $10.2 \mathrm{~g} / \mathrm{L}$, respectively. The dairy cow manure feed had an average TS and VS concentrations of $59.7 \mathrm{~g} / \mathrm{L}$ and $50.9 \mathrm{~g} / \mathrm{L}$, respectively, during the operational period. Because of the solids being fed to the digesters, the initial VS concentration in the digesters increased from $10.2 \mathrm{~g} / \mathrm{L}$, up to an average for all digesters of $22.3 \pm 0.9 \mathrm{~g} / \mathrm{L}$ on day 89 , where it began to level off (Figure 3-5 (A)). This increase in VS in the digesters was due to gradual replacement of the initial, low VS concentration inoculum with incoming cow manure feed. In addition, some of the increase was because of biomass growth. The increase in VS concentration that would have been seen during this replacement period if no degradation had occurred is shown in Figure 3-5. The effect of VS loading rate was seen to be statistically significant on the VS removal efficiencies in all digesters (ANOVA; $n=16 ; p=0.0013$ ). After each increase in VS loading rate by feeding an increased volume of cow manure feed, the VS concentration in the digesters increased, thus increasing the VS concentration in the digester effluent. This is anticipated because as the VS loading rate increased, the HRT decreased, allowing less time for organic substrate to be degraded. The steady-state VS removal efficiencies for all digesters during the loading rates of 1.0, 1.7, 2.5, and 3.5 g VS/L-d were between 52 and 58\% (Table 3-1). During steady-state conditions, no statistically significant differences in VS removal efficiencies were found between the digesters (ANOVA: $n=16 ; p=0.84$ ). This result 
verifies the statistically similar methane yields from all digesters during steady-state periods.

FISH analysis showed that the microbial flocs in all digesters were similar in size between weeks 1 and 4 . However, the average floc size decreased between week 4 and 7, with very few large flocs left by week 7, but with numerous smaller flocs present. By week 26, no flocs were documented in all digesters, only single cells and small clusters of cells up to $10 \mu \mathrm{m}$ were visualized by FISH. FISH images from week 1, 7, and 14 can be seen in Figure 3-6. This result indicates that continuous mixing in digesters treating animal manure may prevent the formation of microbial flocs.

The distribution of particle size for each digester was measured at the end of the operational period to determine if different mixing levels had an effect on particle size. Results showed that shear did have some effect on the organic material inside the digester, showing larger concentrations (by weight) of particles between the 0.3 and $0.045 \mathrm{~mm}$ size range in the 1500-RPM digester, while larger concentrations (by weight) of particles in the 1.4 and $0.6 \mathrm{~mm}$ size range were found in the 50-RPM digester (Figure 3-7). The weight concentrations of the 500 and 250-RPM digester's particles fell between that of the 1500 and 50-RPM digesters. The particles collected between the size range of 1.4 and $0.045 \mathrm{~mm}$, however, only account for approximately $34 \%$ of the total VS present in the samples analyzed, the remaining $56 \%$ of VS was less than $0.045 \mathrm{~mm}$ in diameter. The results suggest that small differences exist in particle size distribution between the reactors with a negative correlation to mixing intensity.

Concentrations of soluble COD within each digester were also seen to be affected by mixing intensity (Figure 3-6 (B)). Throughout the course of the study, the levels of soluble COD generally increased with increasing RPMs. The levels of soluble COD were considerably higher for the 1500-RPM than that for the other three digesters. SCOD concentrations in all the reactors rose gradually with every increase in loading rate. The rise in SCOD concentrations seen between the increase in loading from 1.7 
to $2.5 \mathrm{~g} \mathrm{VS} / \mathrm{L}$-d, and again from 2.5 to $3.5 \mathrm{~g} \mathrm{VD} / \mathrm{L}$-d are much higher than the increases seen during the increase in loading rates from 0.6 to $1.0 \mathrm{~g} \mathrm{VS} / \mathrm{L}-\mathrm{d}$.

Aluminum, calcium, copper, iron, mercury, magnesium, manganese, silicon, titanium, and zinc were all measured in samples from the effluent of the 500 and 250RPM digesters at the end of the study. Aluminum, copper, iron, mercury, and titanium were all below the detection limits for these elements ( $5 \mathrm{ppm}$ for $\mathrm{Al}, \mathrm{Cu}, \mathrm{Fe}, \mathrm{Hg}$, and Ti). The average concentrations of magnesium was the highest with $219 \mathrm{ppm}$, followed by calcium at $104 \mathrm{ppm}$, silicon at $53 \mathrm{ppm}$, manganese at $1.6 \mathrm{ppm}$, and zinc at $1.5 \mathrm{ppm}$. Total reactive phosphorous levels were also measured, with an average of $15.9 \mathrm{mg} / \mathrm{L} \mathrm{PO}_{4}^{3-}-\mathrm{P}$. Ammonia concentrations in the effluent of all the reactors were similar over the entire operational period with an average of $1.24 \pm 0.04 \mathrm{~g} \mathrm{NH}_{4}-\mathrm{N} / \mathrm{L}$. 

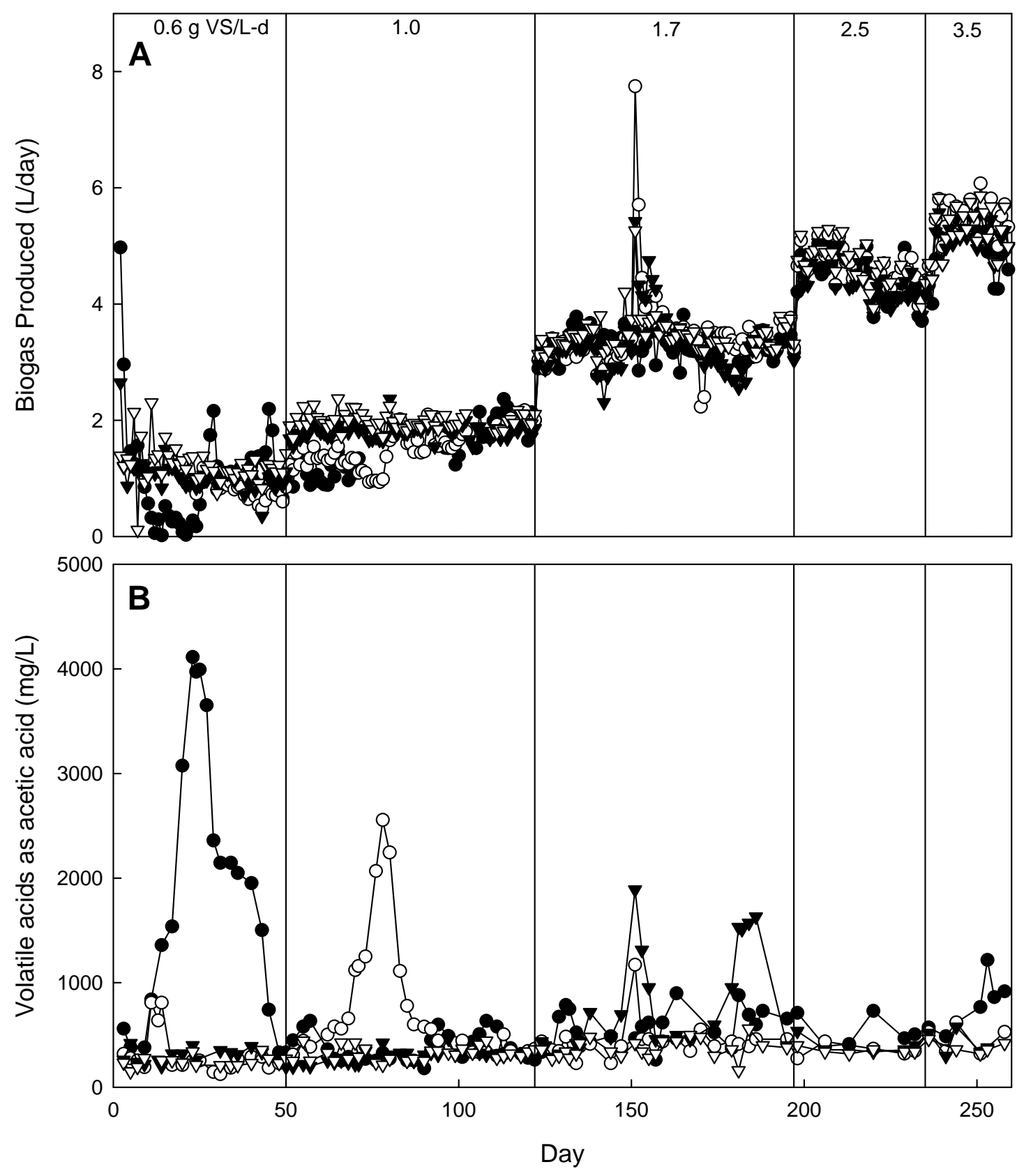

Figure 3-2. Performance data for the digesters throughout the entire operational period: (A) daily biogas production. (B) volatile fatty acid levels. Digesters: (•) 1500 RPM, ( $)$ 500 RPM, ( $) 250$ RPM, ( $\nabla) 50$ RPM. 


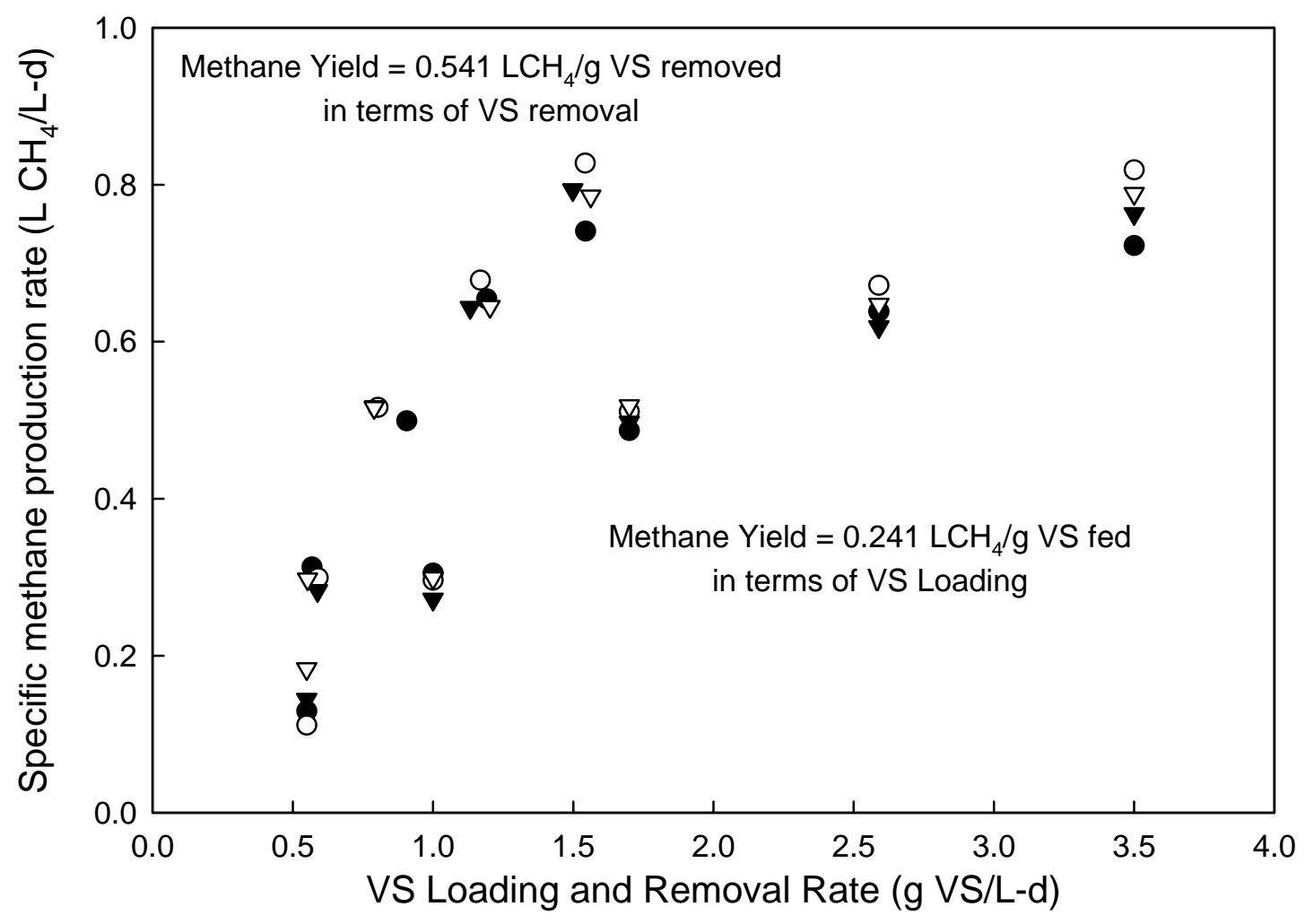

Figure 3-3. Methane yields obtained by regression analysis on data pooled from all digesters during each steady-state operational period. Digesters: (•) 1500 RPM, ( $)$ 500 RPM, $(\nabla) 250$ RPM, $(\nabla) 50$ RPM. 

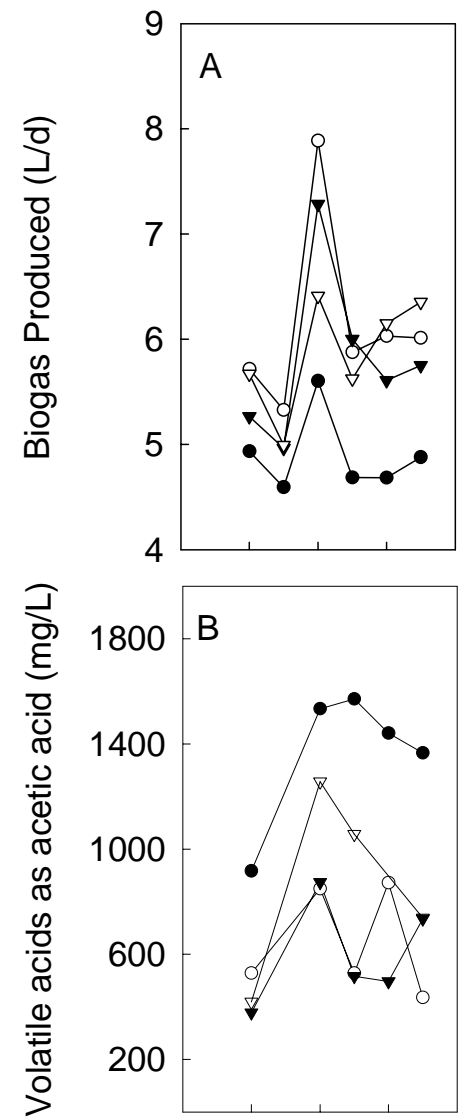

256258260262264

Days

Figure 3-4. Performance data for the digesters between two days prior to shock load and four days after shock load occurring on day 260: (A) daily biogas production. (B) volatile fatty acid levels. Digesters: (•) 1500 RPM, (०) 500 RPM,

( $) 250$ RPM, $(\nabla) 50$ RPM. 

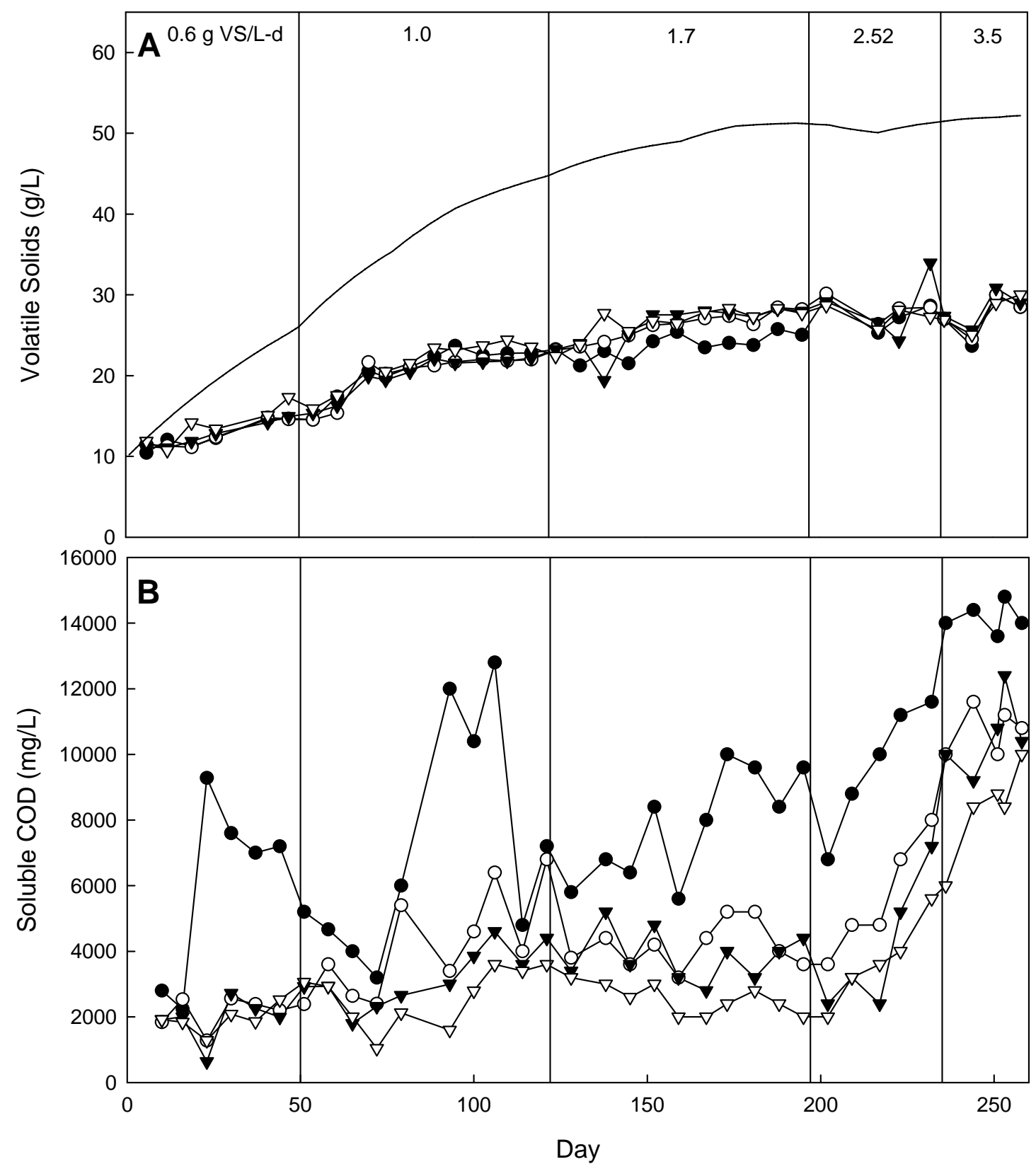

Figure 3-5. Performance data for the digesters over the entire operational period: (A) volatile solids concentration in the digester effluent. Solid like depicts the VS concentration in the effluent if no degradation was taking place. (B) SCOD concentrations. Digesters: (•) 1500 RPM, ( ) 500 RPM, ( $\nabla) 250$ RPM, ( $\nabla) 50$ RPM. 
Week

1

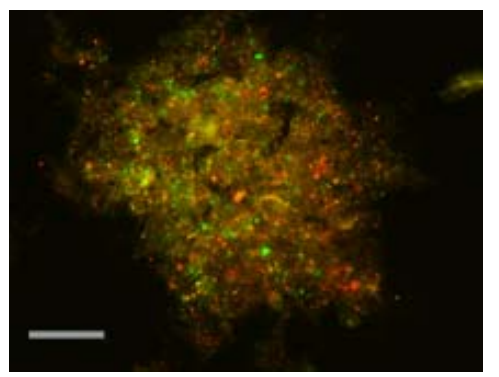

B

C
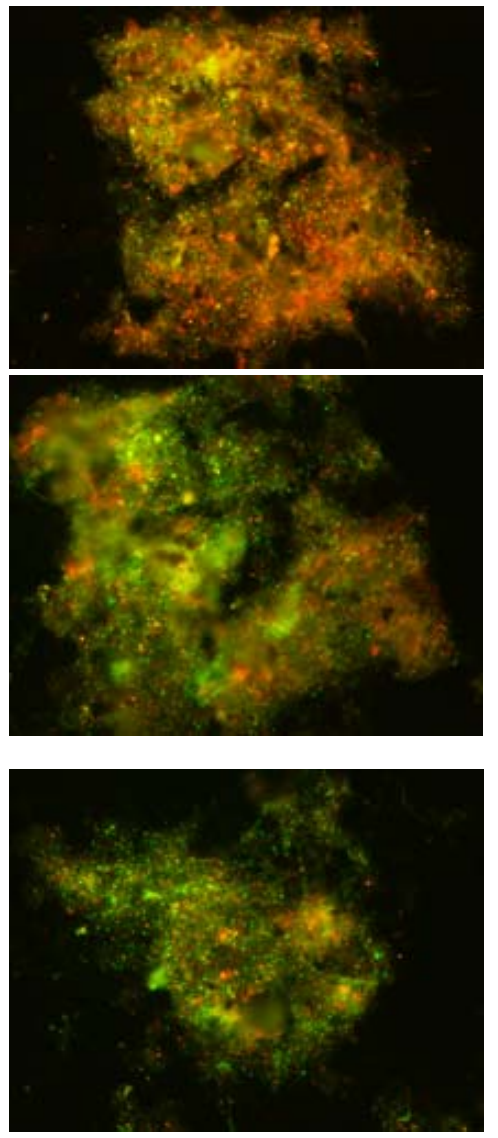

7
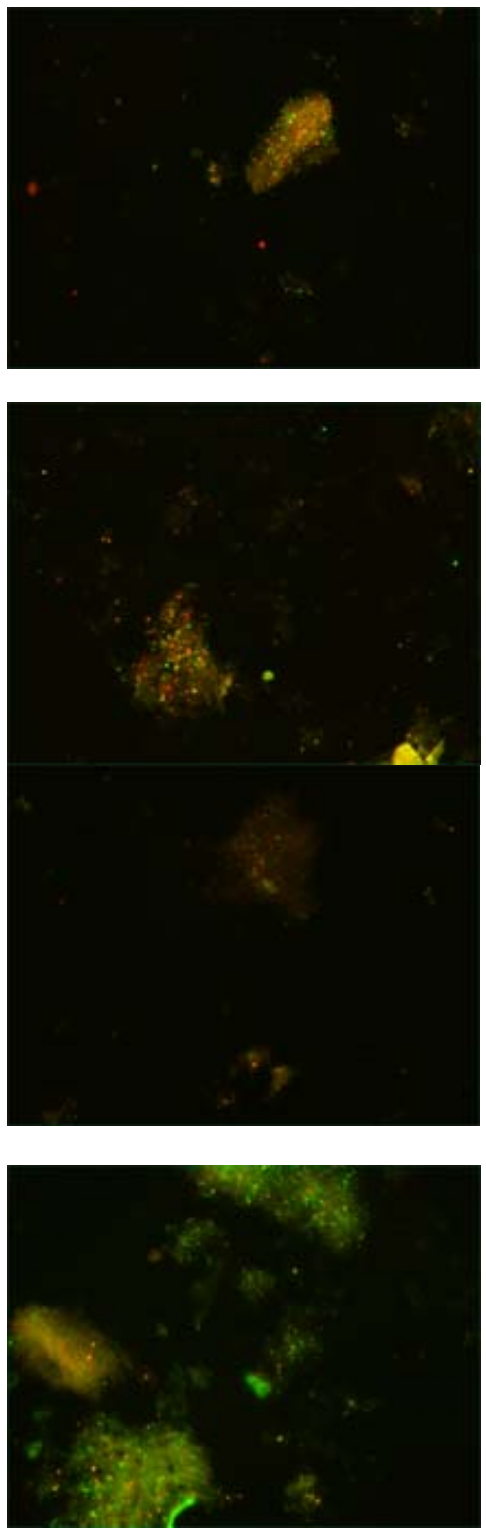

14
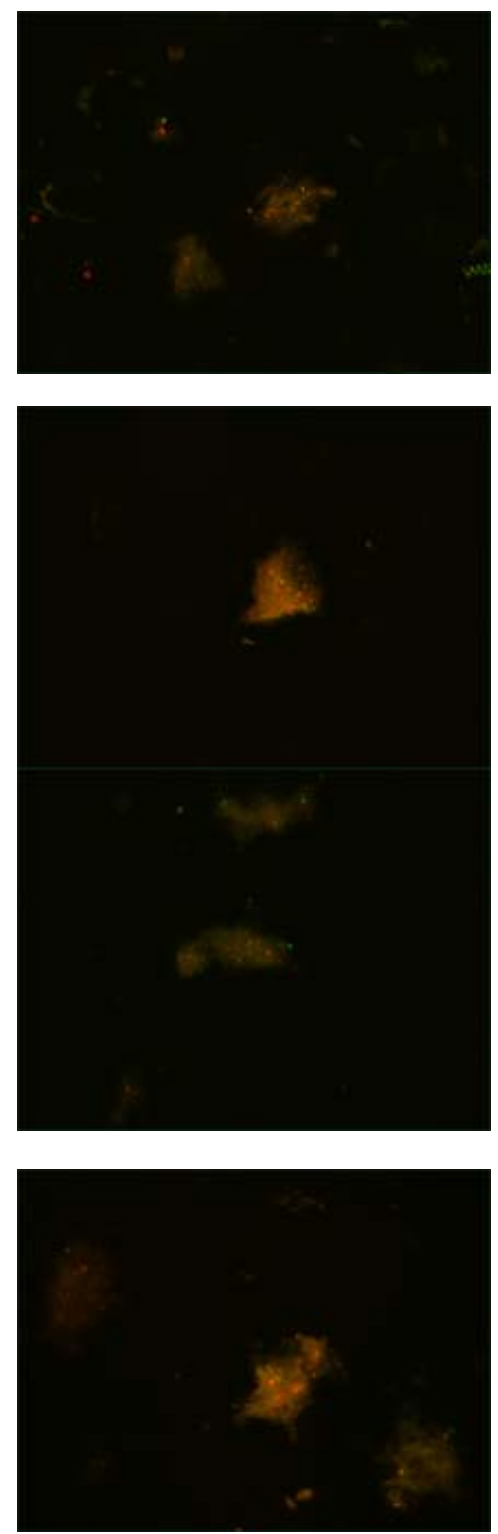

Figure 3-6 FISH views from weeks 1, 7, and 14 at a 400X magnification. Probes used were Arch915 labeled with FITC (shown in green), and Bact338 labeled with CY3 (shown in red). Biomass shown from digesters: (A) 1500 RPM, (B) 500 RPM, (C) 250 RPM, (D) 50 RPM. The gray bar represents 50 micrometers. 


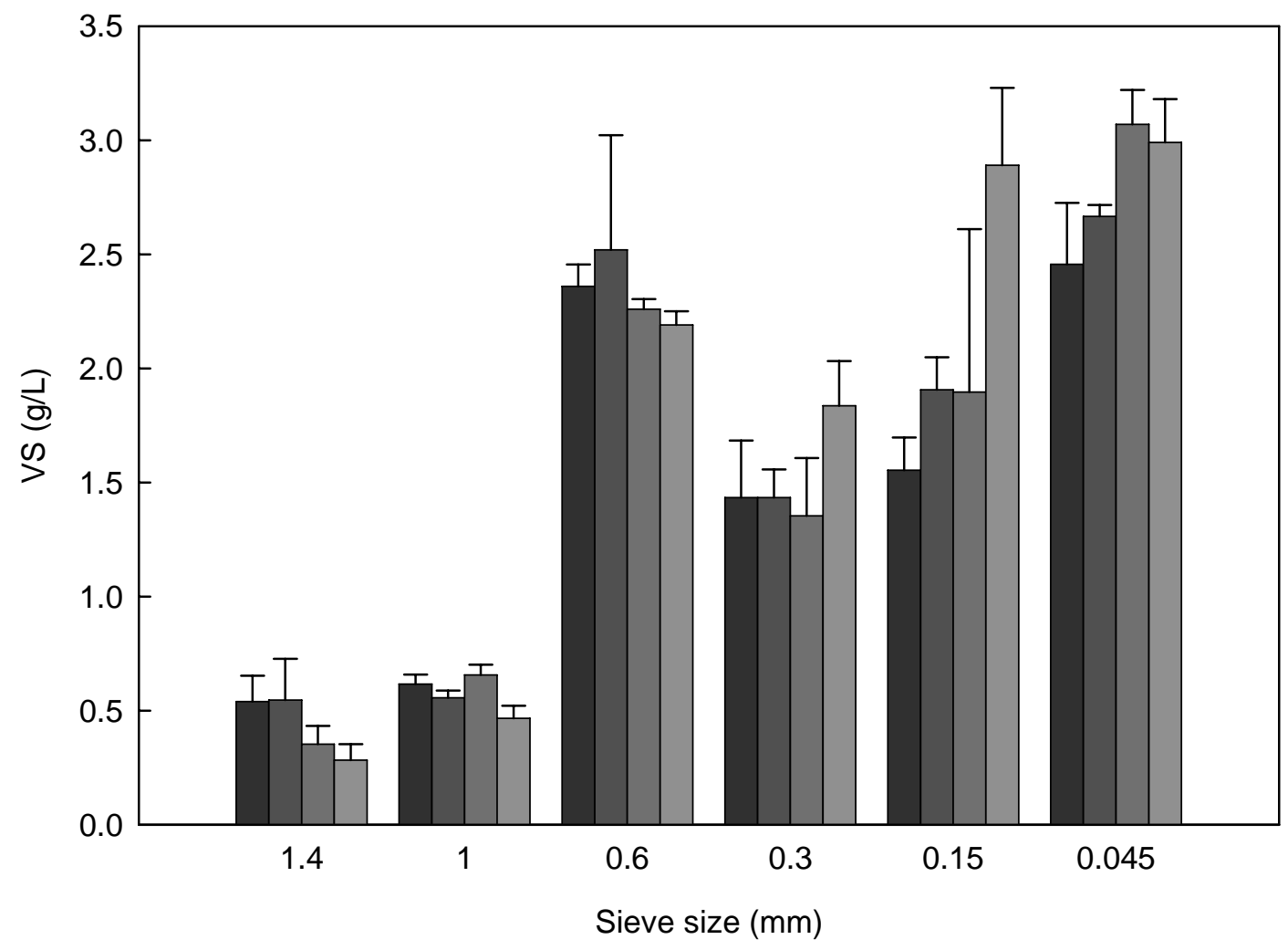

Figure 3-7. Particle size distribution in digester effluent at the end of the operational period. Error bars represent standard deviation based triplicate sample measurement. Bars shown

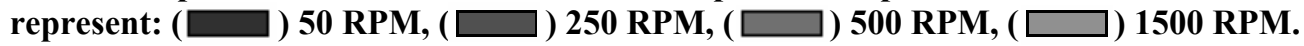


Table 3-1. VS removal efficiencies for steady-state periods during the last four loading rates for all digesters. Standard deviation of the points used are shown as well as the number of data points considered.

\begin{tabular}{|c|c|c|c|c|}
\hline Loading Rate & 1500 RPM & 500 RPM & 250 RPM & 50 RPM \\
\hline $1.0 \mathrm{~g} \mathrm{VS} / \mathrm{L}-\mathrm{d}$ & $\begin{array}{c}55.5 \pm 1.2 \% \\
n=5\end{array}$ & $\begin{array}{c}57.6 \pm 1.6 \% \\
n=5\end{array}$ & $\begin{array}{c}57.4 \pm 1.2 \% \\
n=5\end{array}$ & $\begin{array}{c}53.9 \pm 1.9 \%, \\
n=5\end{array}$ \\
\hline $1.7 \mathrm{~g} \mathrm{VS} / \mathrm{L}-\mathrm{d}$ & $\begin{array}{c}52.7 \pm 2.2 \% \\
\mathrm{n}=5\end{array}$ & $\begin{array}{c}46.7 \pm 2.2 \% \\
n=5\end{array}$ & $\begin{array}{c}46.0 \pm 1.6 \% \\
n=5\end{array}$ & $\begin{array}{c}46.0 \pm 1.1 \%, \\
n=5\end{array}$ \\
\hline $2.6 \mathrm{~g} \mathrm{VS} / \mathrm{L}-\mathrm{d}$ & $\begin{array}{c}46.7 \pm 1.9 \% \\
\mathrm{n}=2\end{array}$ & $\begin{array}{c}45.8 \pm 0.1 \% \\
\mathrm{n}=2\end{array}$ & $44.4 \pm 13 \%, \mathrm{n}=2$ & $\begin{array}{c}47.1 \pm 1.2 \%, \\
n=2\end{array}$ \\
\hline $3.5 \mathrm{~g} \mathrm{VS} / \mathrm{L}-\mathrm{d}$ & $\begin{array}{c}44.2 \pm 2.2 \% \\
n=2\end{array}$ & $\begin{array}{c}44.1 \pm 2.2 \% \\
n=2\end{array}$ & $\begin{array}{c}42.9 \pm 2.7 \% \\
n=2\end{array}$ & $44.6 \%, n=1$ \\
\hline
\end{tabular}

\subsection{Discussion}

In this study, we monitored the performance and biomass characteristics of four laboratory-scale digesters fed with cow manure. Our goal was to determine if different mixing intensities had an affect on digester performance and the syntrophic relationships between bacteria and archaea within the microbial consortia. To determine this, each of the four reactors were operated identically, except for impeller speeds of 1500, 500, 250, and 50 RPM. Performance of the reactors was monitored using biogas production rates, VS removal rates, VFA, and SCOD levels in the digesters. Microbial floc sizes and syntrophic relationships were monitored with epifluorescent microscopy by using a non-specific fluorescent DNA stain and specific DNA probes labeled with a fluorescent dye, respectively.

\section{Different mixing levels had no effect on CSTR performance during steady-state periods, but high mixing intensities had negative effects during startup}

During steady-state periods, all digesters performed similarly by producing equal amounts of biogas. We had anticipated that different mixing intensities in digesters would show visible differences in performance since this has been reported in the literature (Angenent et al. 2001; Lanting 2003). For example, Lanting (2003) found that the processing capacity of municipal sludge digesters could be increased through increasing levels of shear imparted to the digester contents through discharge velocity 
and pressure drop (Lanting and Murphy 2002). On the other hand, a study by Angenent et al. (2001) revealed that the performance of an ASBR treating swine waste declined after mixing intensity was increased, resulting in the concentration of VFAs in the reactor doubling within a 13 day period. Different findings between the results of Lanting (Lanting Annual Conference of the Water Environment Federation) and Angenent et al. (Angenent et al. 2001) may be explained by the different types of digester configuration, mixing methods, and feed substrates used in these studies. However, both studies show that differences in mixing intensities have affected digester performance. In addition, differences in mixing duration have also been shown to result in differences in digester performance (Angenent et al. 2001; Dague et al. 1970; Stroot et al. 2001).

Our results are similar to Karim et al. (2005b), who studied different levels of continuous biogas recirculation $(1,2$, and $3 \mathrm{~L} / \mathrm{min}$ ) on anaerobic digesters treating dairy cow manure. The feed manure used by Karim et al. (2005b) had a TS concentration of $50 \mathrm{~g} / \mathrm{L}$, as compared to the current work, which utilized a manure manure containing $60 \mathrm{~g} \mathrm{TS} / \mathrm{L}$. Although the TS concentrations are comparable, the VS concentration in the current work is double that in the work by Karim et al. (2005b), resulting in a much thicker manure feed. The authors attribute their findings possibly to the low solids concentration in the manure feed.

Lin and Pearce (Lin and Pearce 1991) also showed similar findings when operating digesters with two different mixing duration regimes (45 minutes per hour and 15 minutes per hour) and four different impeller speeds (0, 20, 50, and 100 RPM) on potato-processing wastewater. They found that there was no significant difference in biogas production between the 20,50, and 100-RPM mixing intensities, although higher productions rates were found at the 15-min mixing duration compared to the 45-min mixing duration for all digesters. While Lin and Pearce (Lin and Pearce 1991) found that at both mixing durations the SCOD concentration in the effluent decreased with higher mixing intensity, this study found that SCOD concentrations in the effluent increased with increasing mixing intensities. A difference in outcome 
between our study and Lin and Pearce's study (1991) may be explained because of the intermittent mixing regime used in the later study, while our study operated continuously-stirred digesters. To our knowledge, no study has been previously published in which mixing levels as high as 1500 RPM have been used in continuously-stirred tank anaerobic digesters.

Two methane yields, one based on VS fed and one based on VS removed, were presented in this study as each yield gives different information. The yield based on VS fed is useful in estimating the amount of biogas produced per $\mathrm{g}$ VS fed for future purposes design purposes. However, since the amount of biogas produced is based upon the amount of VS removed, the methane yield based on VS destroyed is a much better way to compare the performance data from this work to that of others. Also, it is important to note that the data used to calculate the methane yield based on VS fed is not linear, possibly due to gradual build up in solids over the course of the study as the initial low solids inoculum was replaced with manure feed as previously discussed.

Although the digesters all performed the same during steady-state operation, performance during start up was severely retarded in the highest mixed digester, showing a high build up in VFA levels. In addition, after the first increase in VS loading, the 500-RPM digester behaved similarly to that of the 1500-RPM digester during the first loading period. Similar findings have been reported by Griffin et al. (1998), who studied an aggressive startup on continuously-mixed digesters treating a mixture of organic fraction municipal solid waste, primary sludge, and waste activated sludge at mixing intensities between 400 and 600 RPM. Using an organic loading rate of $3.1 \mathrm{~g} \mathrm{VS} / \mathrm{L}-\mathrm{d}$, Griffin et al. (1998) found that at both mesophilic and thermophilic conditions digesters performed poorly during startup. The startup is considered by most to be the critical step in anaerobic digester operation. The findings presented in this study suggest that high mixing intensities should be avoided during start-up periods to acclimate microorganisms properly without upsets in digester performance.

While the data presented in this study indicates that high levels of mixing disrupt syntrophic flocs, while no effect on performance occurs, it is expected that this would 
not hold for high-rate systems, such as ASBRs. ASBRs are different from CSTRs because they utilize solid settling to maintain high levels of biomass within the digesters. Such high levels of retained biomass allow the digester to operate at lower HRTs and higher VS loading rates. As earlier discussed, increases in mixing intensity in an ASBR operating on swine waste was found to severely decrease digester performance (Angenent et al. 2001). This study also found that when mixing intensity was increased, a decrease in the levels of most methanogens occurred (Angenent et al. 2001).

\section{Shear affected the microbial flocs, particle size, and SCOD levels in the digesters}

Analysis using FISH showed that during weeks 1 through 4 all digesters contained similar sized flocs. However, the average floc size began decreasing around week 5 . Others had speculated that vigorous mixing disrupts the spatial juxtaposition of microorganisms (Dolfing 1992; Whitmore et al. 1987; Conrad et al. 1985). Not only have we found this to be true, we also found that it can completely break syntrophic relationships, even at low mixing intensities when digesters are completely mixed. This result was observed in all digesters by week 26 of operation, and verified during particle size analysis performed at the end of the study, in which no flocs were visually identified on any of the screens. However, we did not find that this disruption of flocs directly caused digester instability, VFA build-up, or digester failure at the conditions studied. Syntrophic organisms present within this biologically diverse and interdependent system are usually viewed as utilizing close, physical associations to transfer metabolic products. However, it is possible that the continuous mixing applied to the digesters studied here was sufficient enough to overcome diffusion limitations, and thus remove the need for close spatial associations. Although microbial floc size was not found to vary with mixing intensity, particle size analysis on samples from each digester suggests that increased mixing increased the concentrations of smaller particles. SCOD concentrations were also affected by the intensity of mixing, showing that when mixing intensity increased, SCOD 
concentrations in the effluent increased. This result is most likely due to higher concentrations of non-degradable material below $1.2 \mu \mathrm{m}$ in size, being produced as mixing intensity increased.

\section{Digesters which have been upset once are more capable of handling future upsets}

The shock load that occurred on the 500 and 250-RPM digesters in the middle of the study revealed that the 500-RPM digester, which had survived an upset during the increase in VS loading rate from 0.6 to $1.0 \mathrm{~g} \mathrm{VS} / \mathrm{L}-\mathrm{d}$, was more capable of handling the shock load than the 250-RPM digester, which had yet to see an upset, maintaining steady levels of volatile fatty acids up to that point. It is expected, however, that due to the increased shear in the 500-RPM digester, the extra substrate from the shock load would have been hydrolyzed and fermented much quicker, causing a spike in VFA concentrations as compared to the 250-RPM digester. The response seen from the 500-RPM digester was much different though, with a large spike in biogas production, and a small increase in VFA concentrations, showing that the methanogenic population was able to effectively handle higher substrate concentrations. On the other hand, the 250-RPM digester behaved much like the 500-RPM digester was expected to behave, showing a large spike in VFA concentrations, and a decrease in biogas production, suggesting that the methanogenic population was not as capable of handling the shock load as that of the 500-RPM digester. This phenomenon is possibly due to the development of a more diversified microbial community in the 500-RPM digester stemming from its past history of high VFA concentrations (McCarty and Mosey 1991). Work by McMahon (2001) supports this speculation by showing that generalist organisms were present in the highest concentrations within digesters having high concentrations of VFAs in the past, whereas digesters with no past VFA build-up showed high concentrations of specialist organisms which degrade acetate.

The results from the transient shock load performed at the end of the study gave results similar to the first shock load, with the exception of the 1500-RPM digester. 
Levels of VFAs in the 1500-RPM digester were already high at the time of the transient shock load, after the shock load VFA concentrations spiked even higher, but only a $22 \%$ increase in biogas production occurred, while the 500-RPM digester saw up to a $48 \%$ increase in biogas production.

The data presented in the current study does not give enough evidence to support the expectation that a more diversified microbial community develops in digesters with past histories of high VFA concentration. However, further investigation through the use of membrane hybridization will be performed to allow greater insight. A metabolically diverse microbial community is a key factor in the success of both lab and full-scale digesters. An adequate seed consortium, and possible engineered upsets during digester operation, may yield much healthier digesters which would be able to handle unexpected upsets without jeopardizing digester performance and overall health. 


\section{Chapter 4 Summary and Recommendations}

The results of this study show that increasing energy inputs to create mixing in anaerobic digesters does not increase performance. We tested the hypotheses given below with the presented study and have concluded the following:

\section{Hypothesis 1: Higher mixing intensities have a detrimental effect upon} reactor performance. This observation was made by Stroot et al. (2001) and Angenent et al. (2001). Reactor instability is thought to be due to rapid hydrolysis and fermentation, which lead to a higher production of VFAs than the methanogens can metabolize. This was seen in both the 1500-RPM digester at startup, and in the 500RPM digester during the first loading rate increase from 0.6 and $1.0 \mathrm{~g} \mathrm{VS} / \mathrm{L}-\mathrm{d}$. This stems from higher mixing levels physically producing smaller particles, which was seen in the particle size distribution data (see Figure 3-5), and thus more soluble organic material and organic acids. Methanogen populations were unable to handle such high concentrations of organic acids and possible inhibition occurred. However, after these initial disturbances in performance, reactor stability was regained and all the digesters performed the same during steady-state periods. The shock load that the 500 and 250-RPM digester received showed that the 500 RPM digester was better able to handle the extra organic substrate without compromising reactor stability, while the 250-RPM digester, coping with its first build-up of fatty acids, showed a marked decrease in biogas production for close to 30 days. This may be due to a more diversified microbial community developing in the 500-RPM digester after the initial build up in VFAs during the $1.0 \mathrm{~g}$ VS/L-d loading rate as compared to the 250-RPM digester. This is discussed in more detail under hypothesis 2.

The second shock load produced similar results, showing that the higher and more frequent build-up in VFA concentrations in the past, the better able to handle future shocks to the system, with the exception of the 1500-RPM digester. Directly 
before the second shock load, the 1500-RPM digester was shown to contain higher concentrations of VFAs than the other digesters.

\section{Hypothesis 2: Different mixing intensities create different microbial}

communities. Due to the stresses and different environmental conditions (differing levels of intermediate products) created by different mixing intensities, it is expected that different microbial communities will develop. Two lines of speculation are behind this hypothesis. One is that decreasing methanogen populations may be found as mixing speed and shear increase. The decreases may be due to higher sensitivities to perturbations, as observed by Angenent et al. (2001). The second speculation is that stressed systems, such as those receiving high shear, develop a more diversified microbial community, possibly due to higher concentrations of intermediate products. The observation of the 500-RPM digester during this study supports this, and was discussed in connection with hypothesis 1. After the recovery from the initial stressor, the 500-RPM digester handled the shock load in one-third the time it to for the 250RPM digester to recover. However, specific data on the methanogenic populations of the different anaerobic digesters was not able to be obtained within the time constraints of this project.

\section{Hypothesis 3: Higher mixing intensities break up and/or prevent the} formation of larger flocs of syntrophic microorganisms. As previously discussed, many researchers believe that high shear created by intense mixing may disrupt flocs of syntrophic microorganisms (Dolfing 1992; Whitmore et al. 1987; Conrad et al. 1985). This disruption in juxtaposition then results in a lower hydrogen transfer efficiency, and hence, a lower removal efficiency as compared to undisrupted flocs. Here, we not only found that mixing disrupts the flocs, but broke them altogether. However, this seemed to have no observable effect on digester performance. 
In light of the information collected from this study, several recommendations for future work have been made, including the following:

Pilot-Scale Study. It is expected that the results found in this study are not applicable to full-scale digesters due to issues related to scale up. In order to determine the effects of mixing on full-scale digesters, a pilot-scale study, which duplicates the mixing conditions used in the lab-scale study, would need to be performed. However, since the use of high energy inputs would not be economically justified for use at the full-scale level, the 500 RPM mixing intensity is recommended as the highest mixing intensity used in the pilot-scale study. In addition to the 500 , 250, and 50 RPM mixing intensities, it is advisable to add a fourth digester as a duplicate of one of the three mixing intensities. This study would be beneficial since few studies on anaerobic digestion have focused on pilot-scale studies, especially using continuously-stirred digesters.

Microbial Population Study. Although we are able to use techniques like membrane hybridization to elucidate information about the microbial populations inside anaerobic digesters, normally this only gives us a feeling for specific order, family, or genus populations. Rarely are individual species targeted by this technique due to the lack of information on what species exist in these digesters, since many environmental anaerobic samples have been found to contain previously unknown species of organisms. By extracting DNA from stored samples taken from each digester in this study, and amplifying the DNA by polymerase chain reaction (PCR) using universal primers, the DNA would be able to be sequenced. Once these sequences are obtained, previously unidentified organisms can be phylogenetically examined, and information on microbial populations with respect to specific species could be determined. 


\section{Appendix A Anaerobic Digestion of Animal Waste: Waste Strength vs. Impact of Mixing Published as: (Karim et al. 2005a)}

\section{A.1 Abstract}

The effects of the method of mixing (biogas recirculation, impeller mixing, and slurry recirculation) and concentration of solids in the feed on the performance of laboratory scale digesters have been investigated. The digesters were fed with $5 \%$ and $10 \%$ manure slurry, at a constant energy supply per unit volume $\left(8 \mathrm{~W} / \mathrm{m}^{3}\right)$. The experiments were conducted in eight laboratory scale digesters, each having a working volume of $3.73 \mathrm{~L}$, at a controlled temperature of $35 \pm 2^{\circ} \mathrm{C}$. Hydraulic retention time (HRT) was kept constant at 16.2 days, resulting in a total solids (TS) loading rate of $3.08 \mathrm{~g} / \mathrm{L}-\mathrm{d}$ and $6.2 \mathrm{~g} / \mathrm{L}-\mathrm{d}$ for 5\% and 10\% manure slurry feeds, respectively. Results showed that the unmixed and mixed digesters performed quite similarly when fed with $5 \%$ manure slurry and produced biogas at a rate of 0.84-0.94 L/L-d with a methane yield of $0.26-0.31 \mathrm{~L} \mathrm{CH}_{4} / \mathrm{g}$ volatile solids (VS) loaded. This was possibly because of the low solids concentration in the case of $5 \%$ manure slurry, where mixing created by the naturally produced biogas might be sufficient to provide adequate mixing. However, the effect of mixing and the mode of mixing became prominent in the case of the digesters fed with thicker manure slurry (10\%). Digesters fed with 10\% manure slurry and mixed by slurry recirculation, impeller, and biogas recirculation produced approximately $29 \%, 22 \%$ and $15 \%$ more biogas than unmixed digester, respectively. Deposition of solids inside the digesters was not observed in the case of 5\% manure slurry, but it became significant in the case of $10 \%$ manure slurry. Therefore, mixing issue becomes more critical with thicker manure slurry. 


\section{A.2 Introduction}

Growth in livestock industries has resulted in large amounts of animal waste (cow manure) generation. In the United States over 100 million tons of dry matter is produced every year (Fontenot and Ross 1980). This has brought in the requirement of safe waste management. Different types of waste management options may include technologies based on physical, chemical, or biological conversions. Examples are combustion/incineration (gasification), chemical conversion (methanol) and biological conversion (anaerobic digestion). Combustion/incineration efficiently recovers the greatest amount of energy from manure, but the practicality of using the ash as a recycled material has yet to be proven. Moreover, self-sustaining incineration requires a waste of about 30 percent solids. Wetter manure with lower solids content requires supplemental fuel to sustain incineration. The possibility of producing methanol production from animal wastes is promising, but there is no specific technology or research is available yet. Anaerobic Digestion is biological means of decomposition of manure in an oxygen-free environment, and has the advantage of producing a fuel gas (methane) and odor free residues rich in nutrients, which can be used as fertilizers.

The performance of anaerobic digesters is affected primarily by the retention time of substrate in the reactor and the degree of contact between incoming substrate and a viable bacterial population. These parameters are primarily a function of the hydraulic regime (mixing) in the reactors. The importance of mixing in achieving efficient substrate conversion has been noted by many researchers, although the optimum mixing pattern is a subject of much debate. Mixing of the substrate in the digester helps to distribute organisms uniformly throughout the mixture and to transfer heat. Furthermore, agitation aids in particle size reduction as digestion progresses and in removal of gas from the mixture. Mixing can be accomplished through various methods, including mechanical mixers, recirculation of digester contents, or by recirculating the produced biogas using pumps.

The two very important aspects of digester mixing are the intensity and duration of mixing. Most of the literature on anaerobic digestion emphasizes the importance of 
adequate mixing to improve the distribution of substrates, enzymes and microorganisms throughout the digester (Chapman 1989; Lema et al. 1991; Parkin and Owen 1986). However, the information available in the literature about the effect of the intensity and duration of mixing on the performance of anaerobic digesters are contradictory. Several studies indicated that a lack of sufficient mixing in low solids digesters dealing with municipal waste resulted in a floating layer of solids (Diaz and Trezek 1977; James et al. 1980; Strenstrom et al. 1983). Chen et al. (1990) observed higher methane yield in the case of a $4.5 \mathrm{~m}^{3}$ digester under unmixed conditions than continuously mixed conditions. In another study, Ben-Hasson et al. (1985) observed $75 \%$ lower methane production rate from dairy cattle manure under continuously mixed conditions than unmixed conditions. On the contrary, Ho and Tan (1985) reported greater gas production for a continuously mixed digester than for an unmixed digester fed with palm oil mill effluents, and Hashimoto (1982) found higher biogas production from beef cattle wastes under continuously mixed conditions than under intermittent mixing conditions. At the same time, Dague et al. (1970), Mills (Mills 1979) and Smith et al. (1979) recommended intermittent mixing of anaerobic digesters over continuous mixing. It has been observed that very rapid mixing disrupts the structure of flocs inside a biological reactor which disturbs the syntrophic relationships between organisms, thereby adversely affecting the reactor performance (Dolfing 1992; Stroot et al. 2001; Whitmore et al. 1987). However, there is no clear information available in the literature about the threshold limits of digester mixing, other than a power input of $0.20-0.30 \mathrm{HP} / 1000 \mathrm{cu} \mathrm{ft}\left(5.26-7.91 \mathrm{~W} / \mathrm{m}^{3}\right)$ is recommended by the US EPA for proper digester mixing (EPA 1979).

The contradictory findings reported in the literature about the effect of mixing on the performance of anaerobic digesters bring the need of extensive research in this direction. Therefore, the present study was designed to focus on the performance of digesters having three different modes of mixing, — biogas recirculation, impeller mixing, and slurry recirculation - keeping same amount of energy applied per unit volume of the waste digested. 


\section{A.3 Materials and Methods}

The reported study was performed in the three sets of experiments. The first set of experiments was performed with four laboratory scale digesters, Digesters 1-4, each having a working volume of $3.73 \mathrm{~L}$, were operated at a controlled temperature of $35 \pm$ $2{ }^{\circ} \mathrm{C}$. Schematics of the digesters are shown in Figure A-1. Digesters 1 consisted of a hopper bottom with a $60^{\circ}$ slope angle, because Choi et al., (1996) reported that a $60^{\circ}$ double slopped bottom helped in reducing the sedimentation of solids. Digester 2 consisted of a hopper bottom with a $25^{\circ}$ slope angles, as this lesser slope angle is easy to construct in the field and requires less earth work. Biogas generated in the digesters was collected in tedlar bags and was recirculated from the top of the digesters by an air pump and draft tube arrangement. The draft tubes were located at mid-height of the hopper bottoms (Table A-1). The biogas recirculation rate was kept as $1 \mathrm{~L} / \mathrm{min}$, as no significant change in the digester performance was observed with increased biogas recirculation rate up to $3 \mathrm{~L} / \mathrm{min}$ rate (Karim et al. 2003). Digester 3 had a hopper bottom with a $25^{\circ}$ slope angles and was mixed by $62 \mathrm{~mm}$ diameter axial flow impeller (Lightnin A-310, Rochester, New York, USA), and the impeller motor was Model 5vb, EMI Inc. (Clinton, Connecticut, USA). Digester 4 had a hopper bottom with a $25^{\circ}$ slope angles and was mixed by slurry recirculation. The pump used for slurry recirculation was, a Masterflex pump from Cole Parmer Instrument Co. (Chicago, Illinois, USA). 
Table A-1. Operational conditions for the digesters.

\begin{tabular}{|c|c|c|c|c|c|}
\hline Expt. set & Digester & Mode of mixing & $\begin{array}{c}\text { Hopper } \\
\text { bottom } \\
\text { angle }\end{array}$ & $\begin{array}{l}\text { Draft tube* } \\
\text { position from } \\
\text { bottom }(\mathrm{mm})\end{array}$ & $\begin{array}{c}\text { Feed } \\
\text { manure } \\
\text { slurry }\end{array}$ \\
\hline \multirow{4}{*}{1} & 1 & $\begin{array}{c}\text { Biogas recirculation } \\
(1 \mathrm{~L} / \mathrm{min})\end{array}$ & $60^{\circ}$ & 48 & $5 \%$ \\
\hline & 2 & $\begin{array}{c}\text { Biogas recirculation } \\
(1 \mathrm{~L} / \mathrm{min})\end{array}$ & $25^{\circ}$ & 13 & $5 \%$ \\
\hline & 3 & $\begin{array}{l}\text { Impeller } \\
(275 \mathrm{rpm})\end{array}$ & $25^{\circ}$ & NA & $5 \%$ \\
\hline & 4 & $\begin{array}{c}\text { Slurry recirculation } \\
(0.82 \mathrm{~L} / \mathrm{min})\end{array}$ & $25^{\circ}$ & NA & $5 \%$ \\
\hline \multirow{2}{*}{2} & 5 & Unmixed & $25^{\circ}$ & NA & $5 \%$ \\
\hline & 6 & $\begin{array}{l}\text { Impeller } \\
(275 \mathrm{rpm})\end{array}$ & $25^{\circ}$ & NA & $5 \%$ \\
\hline \multirow{4}{*}{3} & 7 & Unmixed & $25^{\circ}$ & NA & $10 \%$ \\
\hline & 8 & $\begin{array}{c}\text { Biogas recirculation } \\
(1 \mathrm{~L} / \mathrm{min})\end{array}$ & $25^{\circ}$ & 13 & $10 \%$ \\
\hline & 9 & $\begin{array}{c}\text { Impeller } \\
(275 \mathrm{rpm})\end{array}$ & $25^{\circ}$ & NA & $10 \%$ \\
\hline & 10 & $\begin{array}{l}\text { Slurry recirculation } \\
(0.82 \mathrm{~L} / \mathrm{min})\end{array}$ & $25^{\circ}$ & NA & $10 \%$ \\
\hline
\end{tabular}

*NA: Not applicable 


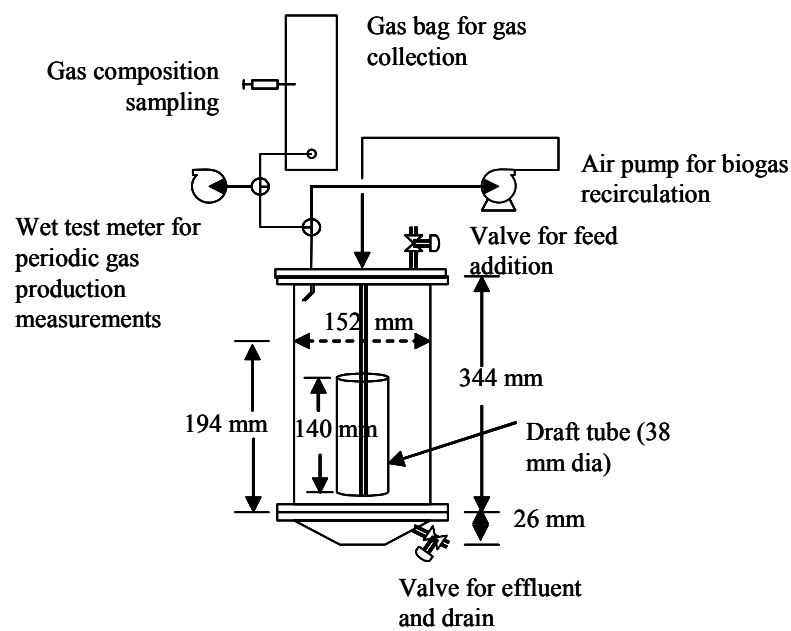

(a)

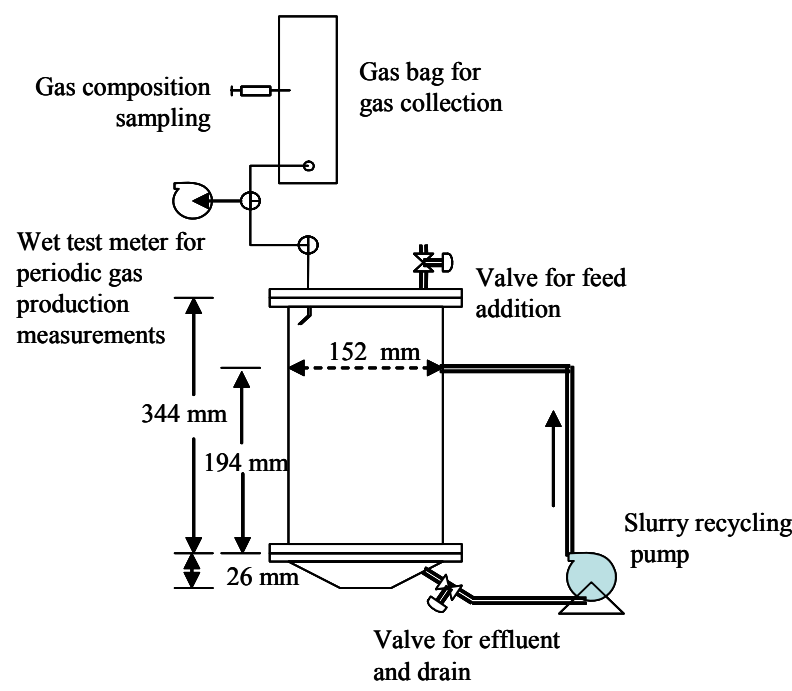

(c)

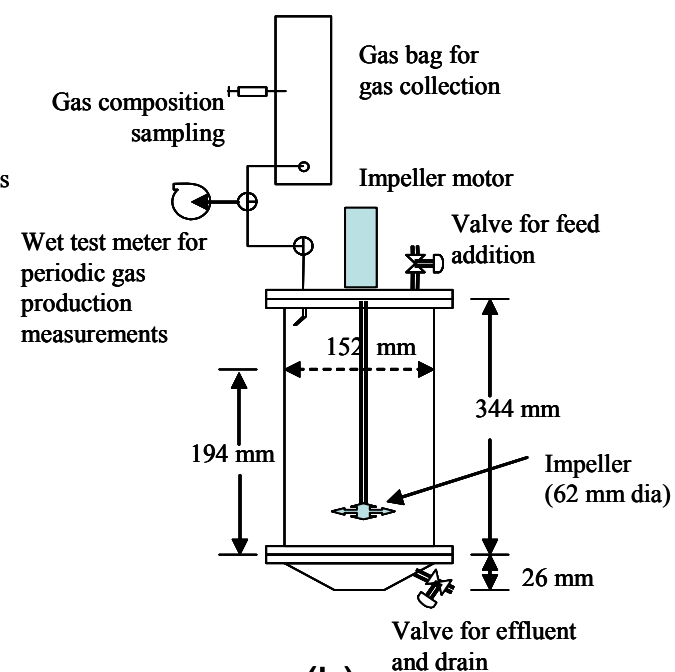

(b)

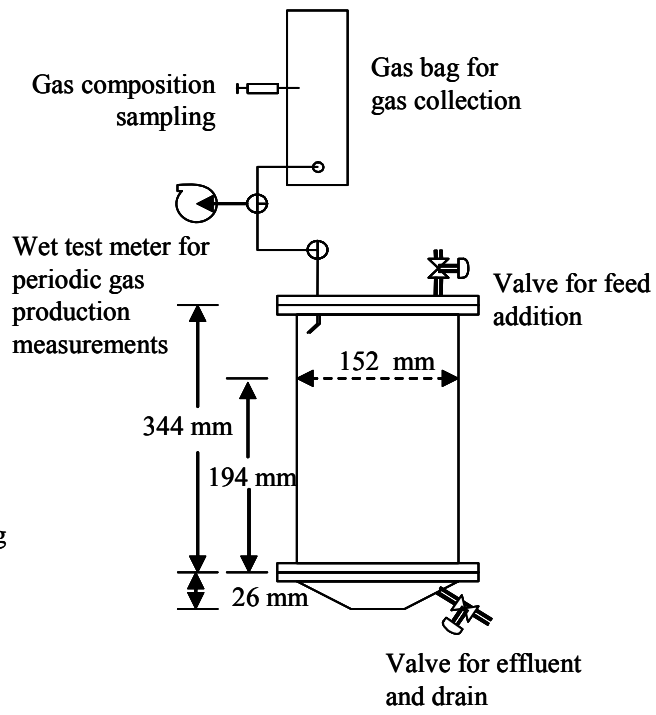

(d)

Figure A-1. Schematic diagram of the experimental set-up. (a) Digesters 1, 2 and 8, (b) Digesters 3, 6 and 9 (c) Digesters 4 and 10, (d) Digesters 5 and 7. 
All four digesters were mixed while keeping a constant energy supply per unit volume of slurry treated $\left(8 \mathrm{~W} / \mathrm{m}^{3}\right)$. In the case of digesters mixed by biogas recirculation, power per unit volume was calculated per Equation A-1 (Casey 1986).

$$
\frac{P}{V}=\frac{\lambda G_{r} P_{2}}{(\lambda-1)}\left[\left(\frac{P_{1}}{P_{2}}\right)^{(\lambda-1) / \lambda}-1\right]
$$

Where $P$ is power, $V$ is the volume of the slurry mixed, $G_{r}$ is specific biogas recirculation rate $\left(\mathrm{m}^{3} / \mathrm{d}-\mathrm{m}^{3}\right), \mathrm{P}_{2}$ is the head space pressure (equal to $101416.83 \mathrm{~N} / \mathrm{m}^{2}$ $($ atmospheric $)=101325$ Pascal), $\mathrm{P}_{1}$ is the pressure at the injection point (i.e., $\mathrm{P}_{2}+$ Static head of slurry), and $\lambda$ is the polytropic exponent. Under isothermal conditions the value of $\lambda$ approaches unity, while under adiabatic conditions its value for biogas is about 1.3. Since the digesters in this study were operated at a controlled temperature of $35 \pm 2{ }^{\circ} \mathrm{C}$. The value of $\lambda$ was taken as 1.01 , as suggested by Casey (1986).

Keeping the same power input per unit volume of the slurry treated $\left(8 \mathrm{~W} / \mathrm{m}^{3}\right)$, the impeller speed for Digester 3 was calculated as 275 RPM, using Equation A-2. The torque applied was determined by a rotating torque meter (Bex-O-Meter, Model 38, The Bex Company, San Francisco, California, USA).

$$
\mathrm{P}=\text { Torque }(\mathrm{N}-\mathrm{m}) \times \text { Angular velocity }(\mathrm{RPM})
$$

Similarly, the slurry recirculation rate $(0.82 \mathrm{~L} / \mathrm{min})$ was decided based on Equation A3.

$$
P=\rho g H ~ Q
$$

Where, $Q=$ discharge $\left(\mathrm{m}^{3} / \mathrm{sec}\right), \mathrm{H}=$ head of the slurry $(\mathrm{m}), \rho=$ density of the slurry pumped $\left(\mathrm{kg} / \mathrm{m}^{3}\right)$.

The digesters were inoculated with $373 \mathrm{ml}$ (10\% of the total working volume) anaerobic seed sludge collected from a dairy farm operated by the University of 
Tennessee, Knoxville, TN. The seed sludge had total suspended solid (TSS) and volatile suspended solid (VSS) of $66.13 \mathrm{~g} / \mathrm{L}$ and $35.63 \mathrm{~g} / \mathrm{L}$, respectively. The remaining $90 \%$ of the working volume was filled with fresh prepared $5 \%$ manure slurry (i.e., having $50 \mathrm{~g}$ dry solid per liter of slurry). Manure slurry, having $50 \mathrm{~g}$ dry solid per liter, was considered for this study knowing the fact that dairy manure "as excreted" has approximately 12\% total solids (TS) and 10.5\% volatile solids (VS), while most of the treatment systems operate at a lower solids concentration than the "as excreted" values (Burke, 2001). The raw cow manure was collected fresh (less than 2 days old) from University of Tennessee Institute of Agriculture, Tennessee and stored in a freezer. It was verified that the cows were not receiving any antibiotic treatment, as some of the antibiotic treatments limit the viability of methane generating microorganisms in their manure (Masse et al., 2002). The waste slurry was prepared from the collected raw manure after blending, screening, settling and dilution. The blending of the manure was done at 10,500 rpm for 2 minutes in a household blender to break big pieces of wood, straw and hay, and to create the slurry. Later on, an equal volume of water was added to the blended slurry to dilute it and then it was screened through a $2 \mathrm{~mm}$ sieve, followed by settling for one hour to remove sands. After total solids for the prepared slurry were determined, it was then diluted with tap water to achieve the required solid concentration (50 g TS/L). The characteristics of the prepared feed slurry are given in Table A-2.

Table A-2. Characteristics of the prepared feed, $5 \%$ and $10 \%$ manure slurry.

\begin{tabular}{|c|c|c|c|c|c|c|}
\hline $\begin{array}{c}\text { Feed } \\
\text { slurry }\end{array}$ & TS (g/L) & VS (g/L) & TSS (g/L) & VSS (g/L) & TCOD (g/L) & SCOD (g/L) \\
\hline $5 \%$ & $51 \pm 1$ & $34 \pm 2$ & $37 \pm 5$ & $25 \pm 3$ & $58.7 \pm 4$ & $19.6 \pm 1$ \\
\hline $10 \%$ & 100 & $52.6 \pm 3$ & $40 \pm 8$ & $36 \pm 7$ & $61 \pm 10$ & $15 \pm 2$ \\
\hline
\end{tabular}

*TS $=$ total solids, $\mathrm{VS}=$ volatile solids, $\mathrm{TSS}=$ total suspended solids, $\mathrm{VSS}=$ volatile suspended solids, $\mathrm{TCOD}=$ total chemical oxygen demand, $\mathrm{DCOD}=$ Dissolved chemical oxygen demand, \pm shows the standard error. 
Digesters 1 and 2 were started simultaneously, whereas Digesters 3 and 4 were started after 48 days due to late procurement of fittings. Hydraulic retention time (HRT) was kept constant at 16.2 days, resulting in a total solids loading rate of 3.08 g/L-d (2 g volatile solids/L-d) for all four digesters. Effluent $(460 \mathrm{ml})$ was taken out from the bottom of the digesters on alternate days and fed with same amount of freshly prepared cow manure slurry.

Since, there were no replications, digesters were operated under steady-state conditions for a long period (approximately three to four weeks) for statistical comparison. However, a second set of experiments, as explained in the following paragraph, was conducted to check the reproducibility of the digester performance. Steady-state conditions were considered achieved when the variation in biogas production and total COD (chemical oxygen demand) concentration in the effluent was within 15\% of the average value (Haghighi-Podeh et al. 1995).

The second set of performance experiments was conducted to compare the performance of mixed and an unmixed digesters, as well as to check the reproducibility of the performance data obtained in the first set of experiments. Two 3.73 L working volume digesters, Digester 5 and Digester 6, with $25^{\circ}$ hopper bottom (Table A-1), were operated for approximately two months. One of the digesters was unmixed and the other was mixed by impeller at 275 RPM. All other conditions were kept the same, as described in the first set of experiments.

Later, a third set of experiments was conducted to evaluate whether mixing becomes more important with an increase in the TS concentration in the animal waste slurry. To evaluate this, four digesters, Digesters 7 (unmixed), Digester 8 (biogas mixed), Digester 9 (impeller-mixed) and Digester 10 (slurry recirculation), of $3.73 \mathrm{~L}$ working volume and with $25^{\circ}$ hopper bottom, as mentioned in Table 1, were operated for approximately 120 days. The digesters were fed with 10\% (i.e., $100 \mathrm{~g} \mathrm{TS} / \mathrm{L}$ ) manure slurry, resulting in TS and VS loading of $6.2 \mathrm{~g} / \mathrm{L}-\mathrm{d}$ and $3.2 \mathrm{~g} / \mathrm{L}-\mathrm{d}$, respectively, until the $71^{\text {st }}$ day. Thereafter a more dilute manure slurry $(3.5 \%)$ was fed for four feeding days to destabilize the digesters, followed by continuation of $10 \%$ manure 
slurry feed till the end of the study. The digesters were destabilized to study the recovery process and to check the reproducibility of their performance. The feed slurry was prepared per the procedure described for the first set of experiments. Input power density for the mixed digesters was kept the same as used in the first set of experiments $\left(8 \mathrm{~W} / \mathrm{m}^{3}\right)$.

Feed and effluent samples were analyzed for total solids (TS), volatile solids (VS), total suspended solids (TSS), volatile suspended solids (VSS), volatile fatty acids (VFA), total chemical oxygen demand (TCOD), dissolved chemical oxygen demand (DCOD), and total nitrogen (TN). Total volume of the biogas generated was measured, and the composition of the biogas was analyzed three times a week. All analyses were performed per standard procedures (APHA 1998), unless otherwise mentioned.

Volatile fatty acids (formic, acetic, propionic, butyric, and valeric acids) were determined by centrifuging a small sample at greater than $10,000 \mathrm{rpm}$ for $5 \mathrm{~min}$, filtering the liquid through a $0.2-\mu \mathrm{m}$-pore-size filter, and injecting a $10 \mu \mathrm{L}$ sample into a high pressure liquid chromatograph (HPLC). In the HPLC, the mobile phase (filtered $\left.5 \mathrm{mM} \mathrm{H}_{2} \mathrm{SO}_{4}\right)$ was pumped at $0.6 \mathrm{~mL} / \mathrm{min}$ through a $300 \mathrm{~mm} \times 7.8 \mathrm{~mm}(8 \mu \mathrm{m}$ particle size) RHM Monosaccharide column (Phenomenex, Torrance, CA), held at a temperature of $65^{\circ} \mathrm{C}$, to a refractive index detector (Model 2410, Waters Corporation, Miltford, MA) held at a temperature of $40^{\circ} \mathrm{C}$.

Biogas volume was measured using wet gas test meters (GSA/Precision Scientific, Chicago, Ill), and the samples ( $1 \mathrm{~mL})$ for biogas composition were collected using a gas-tight syringe. The samples were injected in duplicate into a Gow-Mac (Model 69350 Series, Lehigh Valley, PA) gas chromatograph (GC) equipped with a $6 \mathrm{ft} \times 1 / 8$ inch, 80x100 Hayesep Q, S.S packed column (Supelco, USA). The oven, injector and thermal conductivity detector (TCD) temperatures were kept as 45,90 and $110^{\circ} \mathrm{C}$, respectively. The carrier gas (helium) flow rate through the column was maintained as $30 \mathrm{~mL} / \mathrm{min}$. Initially, the $\mathrm{GC}$ was calibrated with $99.9 \%$ pure methane $\left(\mathrm{CH}_{4}\right)$ and nitrogen standards. 
Average steady-state data and the standard error presented in the paper have been calculated as a mean value over 20-30 days of observations. Statistical significance $(\mathrm{P}=0.05)$ of the experimental data was tested using one way ANOVA statistical program (Microsoft Excel 2002).

\section{A.4 Results}

\section{5\% Manure Slurry Study 1:}

Four laboratory scale digesters, fed with cow manure slurry, were continuously operated over a period of approximately 108 days. Initially there was variation in the performance of the four digesters, however it decreased with time. All four digesters behaved quite similarly as shown in Table A-3. Total solids and volatile solids reduction was approximately $37-40 \%$ and $50-63 \%$, respectively, in all four digesters. Total COD in the feed was approximately $58.7 \mathrm{~g} / \mathrm{L}$, approximately $33 \%$ of which was present in the form of dissolved COD. The reduction of TCOD was observed as $56 \%$, $58 \%, 57 \%$, and $56 \%$ for Digesters $1-4$, respectively. The effluent DCOD concentration from the digesters was observed at 3.7-4.2 $\mathrm{g} / \mathrm{L}$, showing approximately $79-81 \%$ reduction in the DCOD in the digesters under steady-state conditions. Better reduction of DCOD is quite obvious as the dissolved substrate would be more readily available for bacterial attack. The nitrogen component of the influent waste slurry under anaerobic conditions remained unchanged. Volatile fatty acids concentrations in the effluents from the digesters were observed as less than $250 \mathrm{mg} / \mathrm{L}$ with $\mathrm{pH}$ in between 7 and 7.8 . 
Table A-3. Average steady-state feed and effluents characteristics data, averaged over last 30 days, for $5 \%$ feed slurry study.

\begin{tabular}{|c|c|c|c|c|c|c|c|}
\hline & $\begin{array}{c}\text { TS } \\
(\mathrm{g} / \mathrm{L})\end{array}$ & $\begin{array}{c}\text { VS } \\
(\mathrm{g} / \mathrm{L})\end{array}$ & $\begin{array}{c}\text { TSS } \\
(\mathrm{g} / \mathrm{L})\end{array}$ & $\begin{array}{c}\text { VSS } \\
(\mathrm{g} / \mathrm{L})\end{array}$ & $\begin{array}{c}\text { TCOD } \\
(\mathrm{g} / \mathrm{L})\end{array}$ & $\begin{array}{c}\text { DCOD } \\
(\mathrm{g} / \mathrm{L})\end{array}$ & $\begin{array}{c}\text { TN* } \\
(\mathrm{g} / \mathrm{L})\end{array}$ \\
\hline Feed & $51 \pm 1$ & $34 \pm 2$ & $37 \pm 5$ & $25 \pm 3$ & $58.7 \pm 4$ & $19.6 \pm 1$ & $1.3 \pm 0.3$ \\
\hline Digester 1 & $31 \pm 3$ & $18 \pm 1.8$ & $23.8 \pm 2.7$ & $15.5 \pm 1.3$ & $24.7 \pm 3.4$ & $3.7 \pm 0.5$ & $1.1 \pm 0.2$ \\
\hline Digester 2 & $30 \pm 4$ & $14 \pm 1.7$ & $23.6 \pm 2.9$ & $16.2 \pm 1.4$ & $24.4 \pm 3$ & $4 \pm 0.67$ & $1.2 \pm 0.3$ \\
\hline Digester 3 & $32 \pm 2.5$ & $14 \pm 0.8$ & $23 \pm 3.5$ & $16.6 \pm 1.6$ & $25 \pm 2$ & $4.2 \pm 0.57$ & $1.3 \pm 0.4$ \\
\hline Digester 4 & $31 \pm 3.8$ & $13 \pm 1.3$ & $23.3 \pm 3.8$ & $14.8 \pm 1.7$ & $25.6 \pm 2.7$ & $4.1 \pm 0.65$ & $1.2 \pm 0.3$ \\
\hline
\end{tabular}

$* \mathrm{TN}=$ Total nitrogen, \pm shows the standard error.

The average biogas production rates for Digesters $1-4$ were $0.84 \pm 0.1,0.94 \pm 0.07$, $0.88 \pm 0.09$, and $0.85 \pm 0.09 \mathrm{~L} / \mathrm{L}-\mathrm{d}$ with methane content as $62 \pm 3,56 \pm 3,61 \pm 3$, and $67 \pm 2 \%$, respectively. The biogas production rate was calculated as volume of biogas produced per liter of digester volume per day and averaged over a period of more than 30 days ( $86^{\text {th }}$ day onward). The biogas production rate data shows that Digester 2 produced slightly more biogas than the other digesters, but the corresponding methane content was found to be lower in comparison, probably due to infiltration of air, which was observed to be up to $18 \%$ in the case of Digester 2. It is worthwhile mentioning that biogas circulation in laboratory digesters increases the chances for 'infiltration' of air into the system (due to slight air permeability of tubing, leakage on the vacuum side of the air pump, etc.). Average steady-state performance data of the four digesters were found to be quite similar. However, to elucidate further, the data were subjected to analysis of variance (ANOVA). There was no significant difference for TCOD reduction at the $5 \%$ level $\left(\mathrm{P}=0.68, \mathrm{~F}=0.5, \mathrm{~F}_{\text {crit }}=2.75, \mathrm{df}=3,60\right)$ for the four digesters. The ANOVA test was also performed for biogas production rate for the four digesters, and it was observed that the value does not differ significantly at the $5 \%$ level $(\mathrm{P}=$ $\left.0.05, \mathrm{~F}=2.6, \mathrm{~F}_{\text {crit }}=2.77, \mathrm{df}=3,56\right)$. Similar results were observed for other parameters.

Volume of biogas produced per unit weight of VS removed was calculated as 0.68-0.84 liters. This value compares well with the reported value of $0.7 \mathrm{~L} / \mathrm{g}$ VS removed, reported by Persson et al., 1979. Methane yield was calculated based on the mass of the VS added every day; it was observed to be $0.21-0.27 \mathrm{~L} / \mathrm{g}$ VS added. It is 
important to note that the VS loading in the present study was $2 \mathrm{~g} / \mathrm{L}-\mathrm{d}$. The observed methane yield is in accordance with a reported methane yield of $0.376 \mathrm{~L} / \mathrm{g}$ VS added, observed at a loading of $2.86 \mathrm{~g} \mathrm{VS} / \mathrm{L}-\mathrm{d}$ (Linke, 1997).

\section{5\% Manure Slurry Study 2:}

Results of the second set of performance studies, including stagnant and impellermixed digesters, showed no significant difference in their start-ups and performance. Unmixed and impeller-mixed digesters produced biogas at a rate of $0.84 \pm 0.07$ and $0.93 \pm 0.09 \mathrm{~L} / \mathrm{L}-\mathrm{d}$. Their methane contents were $64 \pm 3 \%$ and $66 \pm 2 \%$, respectively. The impeller-mixed digester produced slightly more biogas (approximately 10\%) than the unmixed digester. To elucidate further, steady-state biogas production rates of the two digesters were subjected to analysis of variance (ANOVA). At the 5\% level, the two sets of data varied significantly $\left(\mathrm{P}=0.036, \mathrm{~F}=5.04, \mathrm{~F}_{\text {crit }}=4.35, \mathrm{df}=1,20\right)$. However, the probability of the difference occurring due to random error in the measurement was $3.6 \%$. Thus the difference recorded in the biogas production rate of the two digesters was more probably due to random error than the effect of mixing. The methane yield, calculated based on the weight of the VS added every day, was 0.27 and $0.31 \mathrm{~L} / \mathrm{g} \mathrm{VS}$ added for the unmixed and impeller-mixed digester, respectively.

To show the reproducibility of the laboratory scale digester performance, the daily biogas production data of Digester 6 (impeller-mixed) was plotted with one operated during first set of experiments (Digester 3) in Figure A-2. The figure shows that the second set of experimental data matches very well with the first set of experiments. ANOVA of the daily biogas production data for whole operational period at the $5 \%$ level showed no significant difference in the two cases $\left(\mathrm{P}=0.95, \mathrm{~F}=0.003, \mathrm{~F}_{\text {crit }}=4.04\right.$, $\mathrm{df}=1,48)$. The average steady-state biogas production rates for the impeller-mixed digester in the first and second sets of experiments were $0.88 \mathrm{~L} / \mathrm{L}-\mathrm{d}$ and $0.93 \mathrm{~L} / \mathrm{L}-\mathrm{d}$, which is within $6 \%$ error. In sum, the performance of the impeller-mixed digester was successfully reproduced, and thus the data observed during the ongoing study is reproducible. 


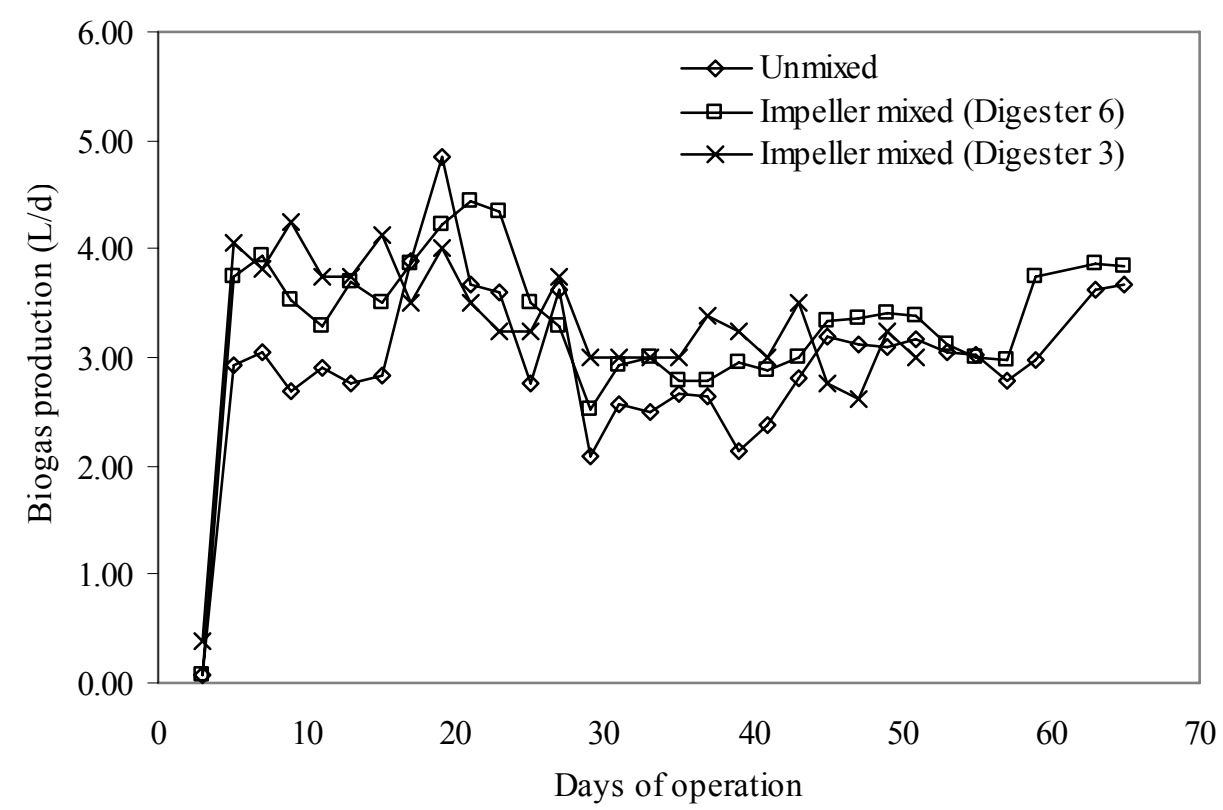

Figure A-2. Plot showing daily biogas production for Digester 5 (unmixed), Digester 3 (impeller-mixed), and Digester 6 (impeller-mixed).

\section{0\% Manure Slurry Study:}

The third set of experiments was conducted with four digesters, Digesters 7 (unmixed), Digester 8 (biogas mixed), Digester 9 (impeller-mixed) and Digester 10 (slurry recirculation). The goal was to study whether the role of mixing becomes more important with increase in the total solid concentration in the animal waste slurry. The average steady-state data calculated over a period of 30 days (from Day 41 to Day 71) of TS, VS, TSS, VSS, TCOD, and DCOD in the feed and effluents are given in Table 4. The data show that during steady-state period, Digesters $7-10$ had a VS removal efficiency of $35 \%, 39 \%, 41 \%$ and $35 \%$, respectively, while TS removal was in between $41-49 \%$. However, the data presented in Table A-4 do not clearly show the superiority of any of the digesters. 
Table A-4. Average steady-state feed and effluents characteristics data, averaged over 30 days (from Day 41 to Day 71 ), for $10 \%$ feed slurry study.

\begin{tabular}{|c|c|c|c|c|c|c|}
\hline & $\begin{array}{c}\text { TS } \\
(\mathrm{g} / \mathrm{L})\end{array}$ & $\begin{array}{c}\text { VS } \\
(\mathrm{g} / \mathrm{L})\end{array}$ & $\begin{array}{c}\text { TSS } \\
(\mathrm{g} / \mathrm{L})\end{array}$ & $\begin{array}{c}\text { VSS } \\
(\mathrm{g} / \mathrm{L})\end{array}$ & $\begin{array}{c}\text { TCOD } \\
(\mathrm{g} / \mathrm{L})\end{array}$ & $\begin{array}{c}\text { DCOD } \\
(\mathrm{g} / \mathrm{L})\end{array}$ \\
\hline Feed (10\% slurry) & 100 & $52.6 \pm 3$ & $40 \pm 8$ & $36 \pm 7$ & $61 \pm 10$ & $15 \pm 2$ \\
\hline Digester 7 & $59 \pm 3.5$ & $34 \pm 3$ & $43 \pm 4$ & $28 \pm 3$ & $44 \pm 4$ & $8 \pm 2$ \\
\hline Digester 8 & $51 \pm 5$ & $32 \pm 5$ & $40 \pm 11$ & $27 \pm 7$ & $41 \pm 3$ & $8 \pm 1$ \\
\hline Digester 9 & $53 \pm 4$ & $31 \pm 2$ & $40 \pm 5$ & $26 \pm 3$ & $41 \pm 3$ & $7 \pm 2$ \\
\hline Digester 10 & $55 \pm 1$ & $34 \pm 1$ & $43 \pm 4$ & $30 \pm 3$ & $44 \pm 5$ & $9 \pm 2$ \\
\hline
\end{tabular}

\pm shows the standard error.

Daily biogas production from Digesters 7-10 along with the TS and VS concentrations in the used feed slurry have been shown in Figure A-3. Digester 10, equipped with slurry recirculation, seems to have produced more gas than any of the other digesters, while the unmixed digester (Digester 7) produced the least. Average steady-state data were calculated over a period of 30 days (from Day 41 to Day 71). ANOVA of the daily biogas production data for the steady-state period at the $5 \%$ level showed significant difference among the digesters $\left(\mathrm{P}=4.41 \times 10^{-7}, \mathrm{~F}=14.4, \mathrm{~F}_{\text {crit }}=2.76\right.$, $\mathrm{df}=3,56$ ). The steady-state biogas production rates for Digesters 7-10 were calculated as $0.93 \pm 0.1,1.07 \pm 0.08,1.14 \pm 0.13$ and $1.2 \pm 0.14 \mathrm{~L} / \mathrm{L}$-d. The methane contents were $66 \pm 3,65 \pm 4,65 \pm 3$ and $66 \pm 4$, respectively. The above data show that the slurry recirculation digester (Digester 10) had the highest biogas production rate, and the unmixed digester produced biogas at a rate almost 22\% less than Digester 10 (Figure A-3). Digester 8 (mixed by biogas recirculation) produced biogas approximately $10 \%$ less than Digester 10 (slurry recirculation). However, ANOVA shows that there was no significant difference $\left(\mathrm{P}=0.26, \mathrm{~F}=1.31, \mathrm{~F}_{\text {crit }}=4.22, \mathrm{df}=1,26\right)$ between the biogas production rates of Digester 9 (impeller-mixed) and Digester 10 (slurry recirculation). Methane yield was observed to be $0.19,0.21,0.23$ and $0.24 \mathrm{~L} / \mathrm{g}$ VS added for Digesters 7-10, respectively (Table A-5). 


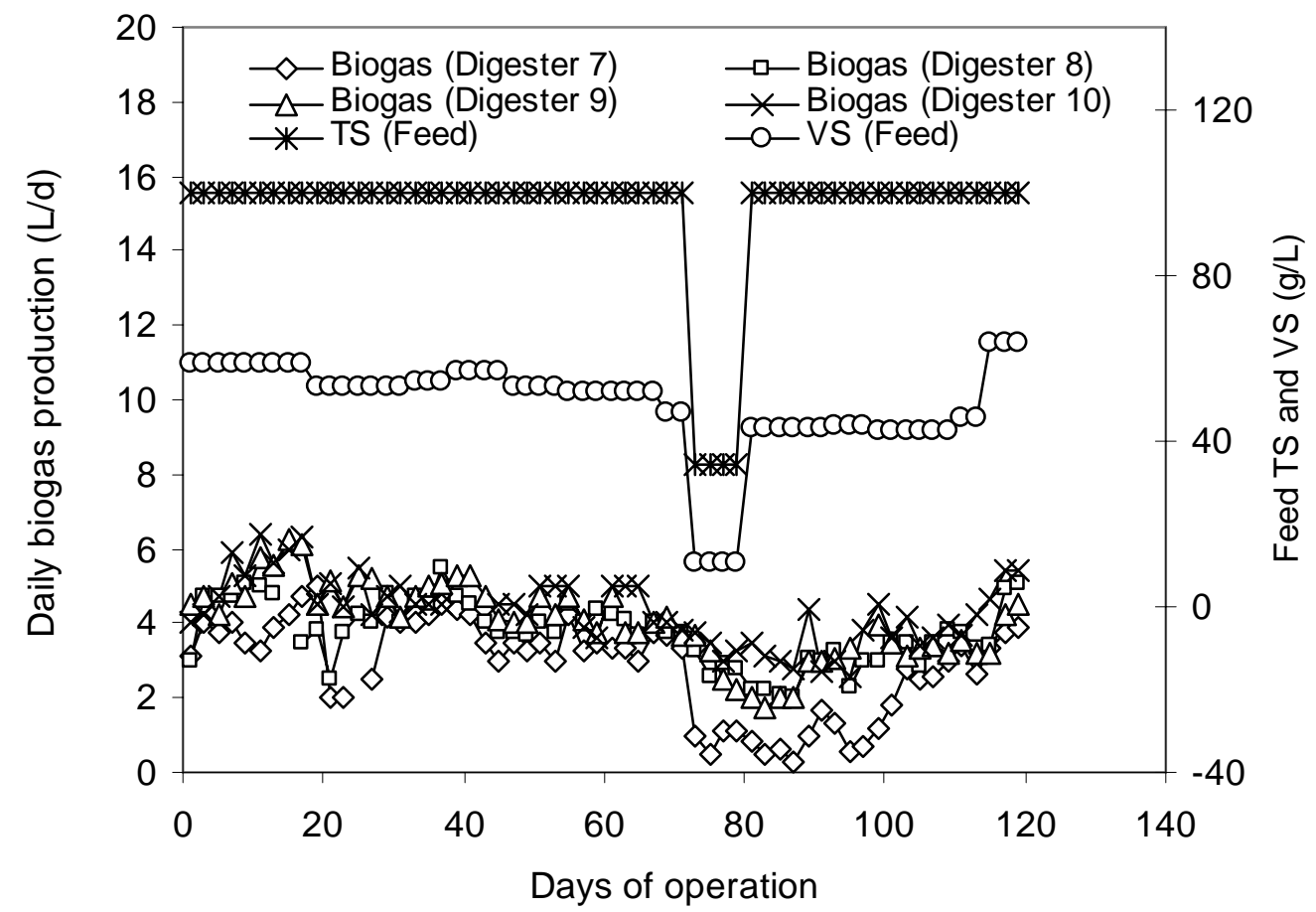

Figure A-3. Plot showing daily biogas production from Digesters 7-10 along with the TS and VS concentrations in the used feed slurry.

After steady-state data had been collected for 30 days, the digesters were fed with a more dilute manure slurry $(3.5 \%)$ in between the $71^{\text {st }}$ and $79^{\text {th }}$ days to perturb the digesters. Thereafter, the digesters were fed with normal 10\% manure slurry feed till the end of the study. With the change in feed slurry concentration, all four digesters became unstable and produced less biogas. However, the effect of perturbation was greater in the case of unmixed digester in comparison to mixed digesters, as the biogas production for the unmixed digester dropped severely as shown in Figure A-3. Upon continuation of normal 10\% feed slurry, the mixed digesters started recovering, although it took almost 10 days longer for unmixed digester to return to the earlier performance level. These results show that the mixed digesters were better able to handle a sudden change in the influent slurry than the unmixed digester. However, 
after recovery, all four digesters reached their earlier methane yield level of 0.18 $0.251 \mathrm{CH}_{4} / \mathrm{g}$ VS loaded (averaged over the $103^{\text {rd }}-120^{\text {th }}$ day), showing the consistency of the reported performance data.

Table A-5. Biogas production rate, methane yield and methane productivity for the digesters under steady-state conditions

\begin{tabular}{|c|c|c|c|c|c|c|}
\hline $\begin{array}{l}\text { Set of } \\
\text { Expt. }\end{array}$ & Digester & Mode of mixing & $\begin{array}{c}\text { VS } \\
\text { loading } \\
\text { (g/L-d) }\end{array}$ & $\begin{array}{c}\text { Biogas } \\
\text { production } \\
\text { Rate }(\mathrm{L} / \mathrm{L}-\mathrm{d})\end{array}$ & $\begin{array}{c}\text { Methane } \\
\text { yield } \\
\text { ( } \mathrm{L} \mathrm{CH}_{4} / \mathrm{g} \\
\text { VS loaded) }\end{array}$ & $\begin{array}{c}\text { Methane } \\
\text { productivity } \\
\left(\mathrm{L} \mathrm{CH}_{4} / \mathrm{g} \mathrm{VS}\right. \\
\text { consumed) }\end{array}$ \\
\hline \multirow{4}{*}{1} & 1 & $\begin{array}{c}\text { Biogas } \\
\text { recirculation, } \\
60^{\circ} \text { hopper bottom }\end{array}$ & 2 & $0.84 \pm 0.1$ & $0.26 \pm 0.03$ & $0.53 \pm 0.06$ \\
\hline & 2 & $\begin{array}{c}\text { Biogas } \\
\text { recirculation } \\
25^{\circ} \text { hopper bottom }\end{array}$ & 2 & $0.94 \pm 0.07$ & $0.26 \pm 0.02$ & $0.43 \pm 0.03$ \\
\hline & 3 & Impeller & 2 & $0.88 \pm 0.09$ & $0.27 \pm 0.03$ & $0.44 \pm 0.04$ \\
\hline & 4 & $\begin{array}{c}\text { Slurry } \\
\text { recirculation }\end{array}$ & 2 & $0.85 \pm 0.09$ & $0.28 \pm 0.03$ & $0.44 \pm 0.05$ \\
\hline \multirow{2}{*}{2} & 5 & Unmixed & 2 & $0.84 \pm 0.07$ & $0.27 \pm 0.02$ & $0.73 \pm 0.06$ \\
\hline & 6 & Impeller-mixed & 2 & $0.93 \pm 0.09$ & $0.31 \pm 0.03$ & $0.77 \pm 0.07$ \\
\hline \multirow{4}{*}{3} & 7 & Unmixed & 3.24 & $0.92 \pm 0.1$ & $0.19 \pm 0.02$ & $0.53 \pm 0.06$ \\
\hline & 8 & $\begin{array}{c}\text { Biogas } \\
\text { recirculation }\end{array}$ & 3.24 & $1.07 \pm 0.08$ & $0.21 \pm 0.02$ & $0.55 \pm 0.04$ \\
\hline & 9 & Impeller & 3.24 & $1.14 \pm 0.13$ & $0.23 \pm 0.03$ & $0.55 \pm 0.06$ \\
\hline & 10 & $\begin{array}{c}\text { Slurry } \\
\text { recirculation }\end{array}$ & 3.24 & $1.20 \pm 0.14$ & $0.24 \pm 0.03$ & $0.69 \pm 0.08$ \\
\hline
\end{tabular}

\pm shows the standard error

\section{A.5 Discussion}

In this investigation of different modes of mixing with 5\% feed slurry (loading $=2$ $\mathrm{g} \mathrm{VS/L-d),} \mathrm{the} \mathrm{two} \mathrm{different} \mathrm{bottoms} \mathrm{and} \mathrm{three} \mathrm{different} \mathrm{modes} \mathrm{of} \mathrm{mixing} \mathrm{did} \mathrm{not}$ significantly affect the digesters performance. Mechanical mixers are reported to be 
most efficient in terms of power consumed per gallon mixed (Brade and Noone 1981). Obviously the digester mixed by an impeller would have had better mixing than the others, although they all behaved the same. In the case of $10 \%$ feed slurry the impeller-mixed digester produced approximately $10 \%$ more biogas than the unmixed digester. However, this difference was more probably due to random error than the effect of mixing, as ANOVA showed a probable random error difference in the measurement of $3.6 \%$. Moreover, the $10 \%$ difference in biogas production was not very significant, especially when the steady-state is considered as $15 \%$ variation from the mean daily biogas production. Therefore, mixing had almost negligible effect on the digester performance in the case of digesters fed with 5\% manure slurry. A similar finding was observed in a previous study conducted with 5\% manure slurry in unmixed and biogas mixed digesters (Karim et al. 2003).

The above findings raise questions of whether the 16.2 days HRT was long enough for the microbes to assimilate whatever organics were readily available or if the mixing intensity was not high enough to play a role. To answer the first question, one should conduct a similar study at different HRTs. Linke (1997) conducted studies with cattle and pig waste slurries in a $2.5 \mathrm{~L}$ mechanically stirred digester (working volume $2.3 \mathrm{~L})$ at different HRTs, and observed that the methane production rate (L/L-d) increased with reduced HRT, but methane yield (L/g VS added) decreased almost linearly. Since energy production and disintegration of organic matter have priority, Linke (1997) suggested an HRT range of 10-15 days. The answer to the second question is no. Stafford (1981) conducted an extensive study on a laboratory scale digester (3 L volume) to see the effect of eight different stirring rates (140-1000 rpm) on biogas production in an anaerobic digester fed with primary sewage sludge. The digester was fed with primary sewage sludge, keeping the HRT at 10 days. He concluded that as the stirring rate was increased from 140-1000 rpm, the average gas production decreased by approximately $12 \%$. Further, Ghaly and Ben-Hasson (1989) observed higher biogas production rates in a $25 \mathrm{~L}$ unmixed digester fed with dairy manure than in a completely mixed digester. However, only further study can reveal if 
the role of mixing becomes favorable with the increase in TS concentration in the feed slurry.

Another reason for conducting the second set of experiments was to check the reproducibility of the laboratory scale digester performance. The daily biogas production data of impeller-mixed digesters from the first and second set of experiments (Digester 4 and Digester 6) show that statistically (at the 5\% level) there was no significant difference for the whole operational period $(\mathrm{P}=0.95, \mathrm{~F}=0.003$, $\left.\mathrm{F}_{\text {crit }}=4.04, \mathrm{df}=1,48\right)$. Similarly, the biogas production rate and methane yield observed for the unmixed digester (Digester 5) in this study $(0.8 \mathrm{~L} / \mathrm{L}-\mathrm{d}$ and $0.271 / \mathrm{g}$ VS loaded, respectively) are comparable to the earlier observed biogas production rate and methane yield ( $0.7 \mathrm{~L} / \mathrm{L}-\mathrm{d}$ and $0.29 \mathrm{~L} / \mathrm{g}$ VS loaded, respectively) for an unmixed digester fed with manure 5\% manure slurry at 16.2 days HRT (Karim et al. 2003). Therefore, the performance of the digesters reported in this paper is consistent and reproducible.

The results obtained from the first and second set of experiments did not show a significant effect of mixing or mode of mixing under the studied experimental conditions with 5\% manure slurry. However, the role of mixing becomes more significant with an increase in TS concentration in the feed slurry, as observed from the third set of experiments. Statistical analysis (ANOVA) of the biogas production rate for the steady-state period (from day 41 to day 71) showed significant difference among the digesters at the $5 \%$ level $\left(\mathrm{P}=1.26 \times 10^{-7}, \mathrm{~F}=15.8, \mathrm{~F}_{\text {crit }}=2.76, \mathrm{df}=3,58\right)$, with 0.08 as the least significant difference value. Thus the unmixed digester biogas production rate was significantly different from all other digesters. The above data further show that the slurry recirculation digester (Digester 10) had the highest biogas production rate. The slurry recirculation digester (Digester 10) produced approximately 29\% more biogas than the unmixed digester (Digester 7). The impellermixed digester (Digester 9) produced approximately 22\% more biogas than the unmixed digester (Digester 7), but there was no significant difference in the biogas production for the impeller-mixed digester and the slurry recirculation digester (at the 
$5 \%$ level, $\mathrm{P}=0.26, \mathrm{~F}=1.31, \mathrm{~F}_{\text {crit }}=4.22, \mathrm{df}=1,26$ ). The biogas mixed digester (Digester 8) produced approximately 15\% more biogas than the unmixed digester (Digester 7). Therefore, the results show that when thicker manure slurry $(10 \%)$ was fed, mixing improved the biogas production. We conclude that the role of mixing becomes more important with an increase in TS concentration in the feed slurry.

So far as the mode of mixing is concerned, at the 5\% level significant difference was observed $\left(\mathrm{P}=0.03, \mathrm{~F}=3.77, \mathrm{~F}_{\text {crit }}=3.22, \mathrm{df}=2,41\right)$. Statistically, there was no significant difference in the biogas production for the slurry recirculation digester and the impeller-mixed digester, as mentioned earlier. However, the biogas production for the slurry recirculation was significantly higher than that of the biogas mixed digester. The above mentioned statistical comparison of the biogas production for the digesters mixed by slurry recirculation, impeller and biogas shows that the probability of the difference occurring due to random error in the measurement is $3.6 \%$. Thus the difference recorded in the biogas production rate of the three cases was more probably due to random error than the effect of mixing. The high biogas production in the case of the slurry recirculation digester can also be attributed to the fact that the particles, chunks and flocs were exposed to higher shear and were crushed while passing through the hub of the recycling pump used. However, to provide quantitative information about the differences in degree of mixing, level of stagnancy, shear level inside the digesters, etc., one needs to conduct hydrodynamic studies as explained elsewhere (Karim et al. 2004).

The biogas production rates, methane yields and methane productivities observed during the studies reported in this paper are summarized in Table A-5. Methane yield is defined as the volume of methane produced per unit weight of VS loaded, while methane productivity is defined as the volume of methane produced per unit weight of the VS consumed. Biogas production rate increased with an increase in the TS concentration in the feed slurry, while the methane yield decreased (Table A-5). These results are as expected as with the increase in solid concentration, the slurry loading increased, and thus the microorganisms had less time to degrade per unit waste. 
Similar observations were also reported by Linke (1997). It is further evident from the data given in Table A-5 that the methane productivity for the digesters varied between $0.43-0.77$ (i.e., $0.37-0.66$ at standard temperature and pressure) without a clear trend. It is reported that dairy cattle manure should theoretically give a methane productivity of $0.469 \mathrm{~L} / \mathrm{g}$ VS destruction (Moller et al. 2004). In another study, Harikishan and Sung (2003) observed 36-41\% VS reduction in cattle waste in a temperature phased anaerobic digester, with a methane productivity of 0.52-0.62 1 methane /g of VS destroyed, at a loading rate of 1.87 to $5.82 \mathrm{~g} \mathrm{VS} / \mathrm{L}-\mathrm{d}$. It is important to note that methane productivity will differ with the type of animal and type of fodder used, and thus will vary with the manure collected from different farms. For the present study the manure was collected from the same farm but at different times; however, all different digesters used for a particular set of experiments received the same manure slurry, though in some cases their values differ significantly from others. For example, the methane productivity of Digester 1 is different from Digesters 2-4, and the methane productivity for Digester 10 is different from that of Digesters 7-9 (Table A-5). This difference seems to be because of different degrees of mixing (or level of settling/stagnancy) inside the digesters. Since the effluents were taken from the bottom of the digesters, settled volatile solids came out with the effluent giving a higher VS value than were actually present inside the digester, and giving lower VS removal efficiency and higher methane productivity values than the actual ones. As in the first set of experiments, the $60^{\circ}$ hopper bottom (Digester 1) provided better settling of solids than did the $25^{\circ}$ hopper bottom (Digesters 2-4). Therefore, Digester 1 gave significantly low VS reduction (47\%) than Digesters 3-4 (59-61\%), though the biogas production rate does not vary much (Table A-5). Similarly, Digester 10 in the third set of experiments showed less VS reduction (32\%) than Digesters 7 (35\%), though the biogas production rate of Digester 10 was approximately $22 \%$ more than Digester 7 (Table 5). However, a flow imaging technique needs to be used to characterize the flow patterns inside these digesters, as explained elsewhere (Karim et al. 2004). 
Therefore, methane productivity is not a very reliable parameter for comparing the performance of digesters other than CSTR.

One of the roles of mixing inside digesters is to avoid stratification and accumulation of inert solids, especially if the feed manure has a high concentration of inert solids, such as sand (from bedding). Solids accumulation inside any digester can be judged from the mass balance of TS and VS. From Tables A-3 and A-4 it can be seen that the amount of TS removed is very much close to the amount of VS removed, and thus there was insignificant accumulation of solids inside the digesters. However, in the third set of experiments (with 10\% feed slurry), the amount of TS reduced is almost twice the amount of VS reduced (Table A-4). Clearly inert solids accumulated inside the digesters. For confirmation, the digesters were opened after completion of the study, and the bulk liquid was gently poured out, and the deposits were analyzed for TS and VS. It was observed that the Digesters 7-10 was having approximately 337, 205, 260 and $190 \mathrm{~g}$ deposits (dry weight), which had approximately 23, 9, 5 and 6\% VS, respectively. However, the bulk liquid had a TS concentration of 71, 59, 64 and $57 \mathrm{~g} / \mathrm{L}$ TS with 52, 57, 53 and 59\% VS for Digesters 7-10, respectively. These results show that the unmixed digester was had more deposits and with a high percentage of VS than the mixed digesters. The deposits in the mixed digesters were mostly inert sand. This suggests that stratification and deposition were not problems when more dilute feed slurry (5\%) was used, but mixing does become important to avoid stratification/deposition with an increase in the TS concentration in the feed slurry. Stratification will become more critical with an increase in scale of the digester, and will ultimately reduce the effective volume of the digester and lead its failure. This brings to the attention the need of inert solids removal prior to the slurry being fed to the digester. Alternatively, there should be a proper arrangement such as scraper and a properly designed effluent port for settled solids removal at the bottom of the digesters. Of the three modes of mixing used, impeller-mixed and slurry recirculation gave better biogas production rate and methane yield. However, biogas recirculation seems to be a promising option, considering the fact that pumping of thick slurry is not 
an easy task and the energy requirement for impeller mixing increases in orders of magnitude with the size of the digester.

\section{A.6 Conclusions}

Mixing did not improve the performance of the digester fed with more dilute (5\%) manure, as both unmixed and mixed digesters (energy input of $8 \mathrm{~W}$ per $\mathrm{m}^{3}$ volume) performed the same under the studied conditions. Thus, there was no difference in the performance of digesters fed with 5\% manure slurry and mixed by different modes of mixing, including biogas recirculation, impeller mixing, and slurry recirculation. However, the effect of mixing and the mode of mixing (at constant energy input of 8 $\mathrm{W}$ per $\mathrm{m}^{3}$ volume) became prominent when digesters were fed with thicker manure slurry $(10 \%)$. With this feed the unmixed digester produced the least biogas. The digesters fed with $10 \%$ manure slurry and mixed by slurry recirculation, impeller mixing and biogas recirculation produced approximately $29 \%, 22 \%$ and $15 \%$ more biogas than the unmixed digester. Solids deposition and stratification were not observed to be problems with more dilute manure slurry (5\%), but became significant when thicker manure slurry $(10 \%)$ was fed to both unmixed and mixed digesters. However, mixing seems help segregate volatile solids from inert solids, which would help to keep light weight biodegradable deposits at the top of the heavier inert deposits, furthering biodegradation. Based on the findings of this study, it can be concluded that mixing becomes more critical with thicker manure slurries. 


\section{Appendix B Standard Operating Procedures}

\section{B.1 Feeding}

Purpose: The anaerobic digesters used to treat cow manure were fed everyday according to the following procedure.

\section{Recording}

- Record the date, time, temperature, and pressure, as well as the gas meter readings on the worksheet.

\section{Removing Effluent}

- $\quad$ Take $250 \mathrm{~mL}$ beaker and funnel labeled 1-4, and collect appropriate amount of effluent from the bottom valve of each reactor, matching the beaker and funnel to the digester number. If more than effluent is collected than is needed, it must be put back into the reactor by one of two ways.

o If only a 1-2 mL needs to be put back, carefully pore some effluent from the cylinder into the clear tubing on the valve on the top of the reactor. This must be done carefully and slowly to avoid overfilling the available area in the tubing, and creating a stinky mess.

o If more than $3 \mathrm{~mL}$ needs to be put back, insert syringe into tubing on the valve on the top of the reactor and poor the needed amount into the syringe. Then feed the reactor as described in steps 10-16.

- After taking effluent from each reactor, place the sample in plastic sample cups found in the drawer below the $\mathrm{pH}$ meter. These cups will have lids in which the date and reactor number needs to be marked.

\section{Feeding}

- Remove a bottle of feed from the fridge which is located on the third shelf from the top on the door.

- Vigorously shake the feed bottle to ensure it is well mixed. Measure appropriate amount of feed, allowing foam to decrease for several seconds, and then adjust the volume to make sure the right amount is obtained.

- Remove plunger from the syringe and connect syringe to the hose connected to the top valve on the reactor.

- Place approximately $60 \mathrm{~mL}$ of feed into the syringe and open the valve slowly allowing approximately $30 \mathrm{~mL}$ to enter the reactor. Close valve. DO NOT 


\section{ALLOW ALL OF THE CONTENTS OF THE SYRINGE TO ENTER THE REACTOR AT THIS POINT.}

- Add the rest of the feed to the syringe, making sure to mix the remaining feed contained in the cylinder to ensure that most solids are transferred to the syringe.

- Wet the tip of the plunger with feed (this only needs to be done the first time it is used each day). Place the plunger at the end of the syringe without depressing. Open the valve and depress the plunger until all feed has entered the reactor.

- Close valve and remove syringe. VALVE MUST BE CLOSED BEFORE SYRINGE CAN BE TAKEN OUT.

- Remove plunger from syringe and repeat the feeding steps for each reactor.

- Rinse measuring cylinder and syringe when finished.

\section{$\mathrm{pH}$ Measurement}

- Take cover from $\mathrm{pH}$ meter and rinse with DI water. Dry with Chimwipe.

- Place probe in $\mathrm{pH} 4$ buffer and allow reading to stabilize, press the standardize button.

- Repeat step 2 with pH 7 and 10 buffer, rinsing and drying the probe between buffers. When finished standardizing, rinse and leave the probe wet hanging over the waste cup.

- Add a magnetic stir bar and place on the stirring plate, slowly increase the stirring speed until the knob is a little over half way turned (about the 2 o'clock position). Dry the $\mathrm{pH}$ probe and insert into the sample. Allow several minutes for the reading to stabilize.

- Repeat step 6 for all digester samples, rinsing and drying both the $\mathrm{pH}$ probe and the stir bar in between samples. Record $\mathrm{pH}$ readings for each digester on the digester worksheet.

- Put lids on the samples (which have been labeled with date and reactor number) and place in the fridge.

\section{B.2 Soluble Chemical Oxygen Demand}

Purpose: COD is the amount of a specified oxidant that reacts with the sample under controlled conditions. The quantity of oxidant consumed is expressed in terms of its oxygen equivalence. COD is used as a measure of pollutants in water. In cases where samples are not very homogeneous, as in the case of animal manure slurry, total COD 
does not give a representative picture because samples may not be completely homogeneous. Thus, soluble COD gives a better representation.

\section{Preparation of Reagents}

\section{Standard potassium dichromate digestion solution, $0.01667 \mathrm{M}-1 \mathrm{~L}$}

$4.903 \mathrm{~g} \mathrm{~K}_{2} \mathrm{Cr}_{2} \mathrm{O}_{7}$, previously dried at $150 \mathrm{C}$ for $2 \mathrm{hr}$

$167 \mathrm{~mL}$ of $\mathrm{H}_{2} \mathrm{SO}_{4}$

$33.3 \mathrm{~g} \mathrm{HgSO}_{4}$

Add $\mathrm{K}_{2} \mathrm{Cr}_{2} \mathrm{O}_{7}$ to about $500 \mathrm{~mL}$ of DI water. Add $\mathrm{H}_{2} \mathrm{SO}_{4}$ and $\mathrm{HgSO}_{4}$. Dissolve, cool to room temp and dilute to $1 \mathrm{~L}$.

Sulfuric acid reagent $-2.5 \mathrm{~L}$

$2.5 \mathrm{~L}$ bottle of concentrated $\mathrm{H}_{2} \mathrm{SO}_{4}$

$25 \mathrm{~g} \mathrm{Ag}_{2} \mathrm{SO}_{4}$

Add $\mathrm{Ag}_{2} \mathrm{SO}_{4}$ crystals or powder to concentrated $\mathrm{H}_{2} \mathrm{SO}_{4}$. Dissolution takes 1 to 2 days.

\section{Ferroin indicator solution}

Usually is purchased ready made

\section{Standard ferrous ammonium sulfate titrant (FAS), 0.1 M - 1 L}

\section{$39.2 \mathrm{~g} \mathrm{Fe}\left(\mathrm{NH}_{4}\right)_{2}\left(\mathrm{SO}_{4}\right)_{2} * 6 \mathrm{H}_{2} \mathrm{O}$}

$20 \mathrm{~mL}$ concentrated $\mathrm{H}_{2} \mathrm{SO}_{4}$

Dilute to $1 \mathrm{~L}$ with distilled water

Dissolve $\mathrm{Fe}\left(\mathrm{NH}_{4}\right)_{2}\left(\mathrm{SO}_{4}\right)_{2}$ in $500 \mathrm{~mL}$ of distilled water and add sulfuric acid. Cool and dilute to $1 \mathrm{~L}$. Standardize against $2 \mathrm{~mL}$ of potassium dichromate solution.

$$
\text { Calculation of Molarity of FAS: }
$$

$$
\text { MolarityofFAS }=\frac{\text { volume0.01667Mpotassiumdichromatesolutiontitrated }(\mathrm{mL})}{\text { VolumeofFASused int itration }(\mathrm{mL})} \times 0.10
$$

\section{Potassium hydrogen phthalate (KHP) standard}

$500 \mathrm{mg}$ KHP

Dilute to $1 \mathrm{~L}$ with distilled water

*Solution is stable when refrigerated, but not indefinitely. Weekly preparation is satisfactory. 
Crush and then dry KHP to a constant weight at $110 \mathrm{C}$. Dissolve $425 \mathrm{mg}$ in DI water and dilute to $1 \mathrm{~L}$. KHP has a theoretical COD of $1.176 \mathrm{mg} \mathrm{O}_{2} / \mathrm{mg}$, this solution has a theoretical COD of 500 micrograms $\mathrm{O}_{2} / \mathrm{mL}$.

\section{Preparation of COD Vials}

- Add 1.5 mL of digestion solution

- Add $3.5 \mathrm{~mL}$ of Sulfuric acid solution

- Cap tightly, vials can be stored for future use.

Disappointed

\section{Preparation of Sample and Vial}

- Place $2 \mathrm{~mL}$ of thoroughly mixed sample in each of $16,2 \mathrm{~mL}$ centrifuge tubes and centrifuge at $10000 \mathrm{rpm}$ for 5 minutes.

- Place a $2 \mu \mathrm{m}$ glass fiber filter in the cartridge filter and assemble the filter holder. Attach a $10 \mathrm{~mL}$ plastic syringe without the plunger.

- Empty the supernatant from each centrifuge vial inside the syringe, insert the plunger, and slowly force the liquid through. Only about $2-5 \mathrm{~mL}$ of filtered sample needs to be collected.

- Dilute sample. Usually a dilution factor of 25 is used for SCOD samples of digester effluent, and of 100 for feed samples.

o For effluent samples add $1 \mathrm{~mL}$ of filtered sample to $25 \mathrm{~mL}$ volumetric flask and dilute with distilled water.

o For feed samples add $1 \mathrm{~mL}$ of filtered sample to $100 \mathrm{~mL}$ volumetric flask and dilute with distilled water.

- Add $2.5 \mathrm{~mL}$ of sample to a prepared COD vial. Samples are generally run in duplicate.

- At least 2 blanks are needed for each batch of samples. Blanks are made by adding $2.5 \mathrm{~mL}$ of deionized water to a prepared COD vial.

\section{Digestion of COD vials}

- Turn digestion block on by flipping the switch to the infinity sign and allow to warm up.

- Slowly invert sample vials after caps are firmly on to mix the contents

- Once all vials are ready, and temperature of heating block has reached $150{ }^{\circ} \mathrm{C}$, place vials into heating block.

- Flip the switch from the infinity sign to the timer, and turn the timing dial all the way to the right.

- Digest at $150{ }^{\circ} \mathrm{C}$ for 2 hours. 


\section{Titration}

- Allow vials to cool to room temperature. Some mercuric sulfate may have precipitated out, however this will not effect analysis

- Transfer contents to a $50 \mathrm{~mL}$ Erlenmeyer flask for titration. Rinse vial 3 times with DI water and add to flask containing sample (addition of water does not alter the test at this point).

- Add 1 drop of ferroin indicator and a magnetic stirrer

- Titrate with 0.1 M FAS until sharp color change from blueish-green to redishbrown.

\section{Calculation}

$$
\text { CODasmgO2 } / L=\frac{(A-B) \times M \times 8000}{m L s a m p l e} \times \text { dilutionfactor }
$$

Where $\mathrm{A}=\mathrm{mL}$ FAS used for blank

$\mathrm{B}=\mathrm{mL}$ FAS used for sample

$\mathrm{M}=$ molarity of FAS

The term 8000 comes from the milliequivalent weight of oxygen $* 1000 \mathrm{~mL} / \mathrm{L}$

\section{B.3 Volatile Fatty Acids - The Distillation Method}

Purpose: Acids up to six carbon atoms can be recovered using this method. The larger the molecular weight of the acid, the higher the fractional recovery achieved. Calculations and reporting are on the basis of acetic acid.

\section{Preparation of Reagents}

$1+1$ Sulfuric acid $-1 \mathrm{~L}$

$500 \mathrm{~mL}$ of concentrated $\mathrm{H}_{2} \mathrm{SO}_{4}$

Dilute to $1 \mathrm{~L}$ with distilled water

Potassium hydrogen phthalate solution (aprox. 0.05N) - 1 L

$15-20 \mathrm{~g} \mathrm{KHC}_{8} \mathrm{H}_{4} \mathrm{O}_{4}$

Dilute to $1 \mathrm{~L}$ with distilled water 
Crush 15 to $20 \mathrm{~g}$ primary standard $\mathrm{KHC}_{8} \mathrm{H}_{4} \mathrm{O}_{4}$ and dry at $120^{\circ} \mathrm{C}$ for $2 \mathrm{hr}$. Cool in a desiccator. Weigh $10.0+/-0.5 \mathrm{~g}$ and transfer to a $1 \mathrm{~L}$ volumetric flask and dilute with distilled water.

\section{Standard sodium hydroxide titrant, $0.1 \mathrm{~N}-1 \mathrm{~L}$}

$4 \mathrm{~g} \mathrm{NaOH}$

Dilute to $1 \mathrm{~L}$ with distilled water

Standardize by titrating $40 \mathrm{~mL}$ of potassium hydrogen phthalate solution. Titrate to the inflection point (point on titration curve where curve switches from convex to concave or vise versa) near 8.7

Calculation of normality of $\mathrm{NaOH}$ is:

$$
\text { Normality }=\frac{A \times B}{204.2 \times C}
$$

Where $\mathrm{A}=\mathrm{g} \mathrm{KHC}_{8} \mathrm{H}_{4} \mathrm{O}_{4}$ weighed into 1-L flask, $\mathrm{B}=\mathrm{mL} \mathrm{KHC}_{8} \mathrm{H}_{4} \mathrm{O}_{4}$ taken for titration, $\mathrm{C}=\mathrm{mL} \mathrm{NaOH}$ solution used

\section{Phenolphthalein indicator solution (alcoholic) - 1 L}

$5 \mathrm{~g}$ phenolphthalein

$500 \mathrm{~mL}$ of $95 \%$ ethyl or isopropyl alcohol

Dilute to $1 \mathrm{~L}$ with distilled water

Dissolve $5 \mathrm{~g}$ phenolphthalein in $500 \mathrm{~mL}$ 95\% ethyl or isopropyl alcohol and add 500 $\mathrm{mL}$ distilled water.

\section{Acetic acid stock solution $(2000 \mathrm{mg} / \mathrm{L})-1 \mathrm{~L}$}

$1.9 \mathrm{~mL}$ conc $\mathrm{CH}_{3} \mathrm{COOH}$

Dilute to $1 \mathrm{~L}$ with distilled water

Standardize by titrating $40 \mathrm{~mL} 0.1 \mathrm{~N} \mathrm{NaOH}$. Titrate to the inflection point.

Calculation of normality of acetic acid solution is:

$$
\text { Normality }=\frac{A \times B}{C}
$$

Where $\mathrm{A}=$ Normality of $\mathrm{NaOH}, \mathrm{B}=\mathrm{mL} \mathrm{NaOH}$ taken for titration $\mathrm{C}=\mathrm{mL}$ of acetic acid solution used 


\section{Determination of Recovery Factor}

This procedure only needs to be done approximately once a year, or when a change in the equipment set-up occurs.

- Dilute the appropriate volume of acetic acid stock solution $(200 \mathrm{~mL})$ to 250 $\mathrm{mL}$ in a volumetric flask to approximate the expected sample concentration and distill using procedure steps 2.

- Calculation

$$
f=\frac{a}{b}
$$

Where $\mathrm{a}=$ volatile acid concentration recovered in distillate (see Calculation section), $\mathrm{mg} / \mathrm{L}$,

And $b=$ volatile acid concentration in standard solution used, $\mathrm{mg} / \mathrm{L}$

Sample Analysis

- Place $100 \mathrm{~mL}$ of sample, or smaller portion diluted to $100 \mathrm{~mL}$, in a $500 \mathrm{~mL}$ distillation flask.

- Add $100 \mathrm{~mL}$ of distilled water, four to five clay chips or glass beads and $5 \mathrm{~mL}$ $\mathrm{H}_{2} \mathrm{SO}_{4}$ and mix

- Distill at the rate of about $5 \mathrm{~mL} / \mathrm{min}$ until exactly $150 \mathrm{~mL}$ distillate has been collected in a $200 \mathrm{~mL}$ graduated cylinder.

- Titrate with $0.1 \mathrm{~N} \mathrm{NaOH}$, using phenolphthalein indicator and/or a $\mathrm{pH}$ meter (end point is $\mathrm{pH}=8.3$ ))

- Distill and analyze a blank and reference standard with each sample batch to insure system performance or perform regularly for daily reoccurring events.

Calculation

mgvolatileacidsasaceticacid

$$
/ L=\frac{m L N a O H \quad \times N \times 60000}{\text { mLsample } \times f}
$$

Where $\mathrm{N}=$ normality of $\mathrm{NaOH}$ and $\mathrm{f}=$ recovery factor

\section{B.4 Ammonia - Probe Method}

Purpose: Ammonia is monitored in anaerobic digesters because it can be inhibitory to some methanogenic organisms in high concentrations. 
Preparation of Reagents

\section{Standard ammonium chloride, $0.1 \mathrm{M}-100 \mathrm{~mL}$}

$0.535 \mathrm{~g} \mathrm{NH}_{4} \mathrm{Cl}$

Dilute to $100 \mathrm{~mL}$ with distilled water

\section{Series of Dilutions for preparation of standard curve}

- $10^{-6} \mathrm{M} \mathrm{NH}_{4} \mathrm{Cl}-0.01 \mathrm{~mL}(10 \mu \mathrm{L})$ of standard diluted to $1 \mathrm{~L}$

- $10^{-5} \mathrm{M} \mathrm{NH}_{4} \mathrm{Cl}-0.1 \mathrm{~mL}(100 \mu \mathrm{L})$ of standard diluted to $1 \mathrm{~L}$

- $10^{-4} \mathrm{M} \mathrm{NH}_{4} \mathrm{Cl}-1 \mathrm{~mL}$ of standard diluted to $1 \mathrm{~L}$

- $10^{-3} \mathrm{M} \mathrm{NH}_{4} \mathrm{Cl}-1 \mathrm{~mL}$ of standard diluted to $100 \mathrm{~mL}$

- $10^{-2} \mathrm{M} \mathrm{NH}_{4} \mathrm{Cl}-10 \mathrm{~mL}$ of standard diluted to $100 \mathrm{~mL}$

Sample Preparation

- Dilute $10 \mathrm{~mL}$ of each of the standard dilutions to $100 \mathrm{~mL}$ and place in a 150 $\mathrm{mL}$ plastic sample cup. Add $2 \mathrm{~mL}$ of ISO $\mathrm{pH}$ stabilizing solution.

- Dilute $10 \mathrm{~mL}$ of each digester sample to $100 \mathrm{~mL}$ and place in a $150 \mathrm{~mL}$ plastic sample cup. Add $2 \mathrm{~mL}$ of ISO stabilizing solution.

\section{Using the Probe}

- Connect ammonia probe the $\mathrm{pH}$ meter

- Make sure that the meter is set to read in $\mathrm{mV}$

- Rinse the electrode and pat dry before putting it into the sample and between each sample.

- Place a stir bar in the sample and set the stir rate to an appropriate level (do not change stirring rate throughout the analysis as this will cause interference in the results.

- Analyze the standards by placing the probe in the liquid being careful not to allow bubbles to rest on the membrane. Once the reading stabilized (about 5 minutes) the value can be recorded.

- After all standards have been completed, samples can be analyzed in the same manner. 
- Prepare a calibration curve by plotting the change in the electrode potential in $\mathrm{mV}$ vs. the concentration of $\mathrm{NH}_{4} \mathrm{Cl}$ in the standard as demonstrated in Figure 1 with the $\mathrm{x}$-axis being a log scale.

- Fit a line to the data and display the equation

- Use calibration curve equation to determine equivalent $\mathrm{NH}_{4} \mathrm{Cl}$ concentration (mol/L) in digester samples. Multiply this number by the factor of $14 \mathrm{~g} \mathrm{~N} / \mathrm{mol}$ $\mathrm{N}$ and report answer as $\mathrm{g} \mathrm{NH}_{4}-\mathrm{N} / \mathrm{L}$

\section{B.5 Alkalinity}

Purpose: Alkalinity is the sum of all titratable bases and is used as a measure of an aggregate property of water. Alkalinity can be interpreted in terms of specific substances only when the chemical composition of the sample is known. Properly operating anaerobic digesters typically have supernatant alkalinities in the range of 2000 to $4000 \mathrm{mg}$ calcium carbonate per liter.

\section{Preparation of Reagents}

\section{Sodium carbonate solution (aprox. 0.05N) - 1 L}

3-5 $\mathrm{g} \mathrm{Na}_{2} \mathrm{CO}_{3}$

Dilute to $1 \mathrm{~L}$ with distilled water

*This solution can not be used longer than one week.

Dry 3-5 g primary standard $\mathrm{Na}_{2} \mathrm{CO}_{3}$ at $250^{\circ} \mathrm{C}$ for $4 \mathrm{hrs}$ and cool in a desiccator. Weigh $2.5+/-0.2 \mathrm{~g}$ and transfer to a $1 \mathrm{~L}$ volumetric flask and fill with distilled water

\section{Standard sulfuric acid $(0.1 \mathrm{~N})-1 \mathrm{~L}$}

$2.8 \mathrm{~mL}$ of $36 \mathrm{~N} \mathrm{H} 2 \mathrm{SO} 4$

Dilute to $1 \mathrm{~L}$ with distilled water

Add $2.8 \mathrm{~mL}$ of concentrate sulfuric acid $(36 \mathrm{~N})$ to a volumetric flask and dilute to $1 \mathrm{~L}$. Standardize by adding $40 \mathrm{~mL} 0.05 \mathrm{~N} \mathrm{Na}_{2} \mathrm{CO}_{3}$ solution in a volumetric flask and diluting to $100 \mathrm{~mL}$ and titrate potentiometrically to $\mathrm{pH}$ of 5 . Lift electrode and rinse into the same beaker, and boil gently 3 to 5 minutes under a watch glass cover. Cool to room temp, rinse cover glass into beaker, and finish titrating to the $\mathrm{pH}$ inflection point. 
Calculation normality of sulfuric acid:

$$
\text { Normality }=\frac{A \times B}{53.00 \times C}
$$

Where $\mathrm{A}=\mathrm{g} \mathrm{Na}_{2} \mathrm{CO}_{3}$ weighed into 1-L flask, $\mathrm{B}=\mathrm{mL} \mathrm{Na} \mathrm{CO}_{3}$ taken for titration,

$\mathrm{C}=\mathrm{mL}$ acid solution used

\section{Standard sulfuric acid $(0.02 \mathrm{~N})-\mathbf{1} \mathrm{L}$}

$200 \mathrm{~mL}$ of $0.1 \mathrm{~N}$ sulfuric acid (or $0.56 \mathrm{~mL}$ of concentrated $\mathrm{H}_{2} \mathrm{SO}_{4}$ )

Dilute to $1 \mathrm{~L}$ with distilled water

Standardize by potentiometric titration of $15 \mathrm{~mL} 0.05 \mathrm{~N} \mathrm{Na}_{2} \mathrm{CO}_{3}$ according to the same procedure presented above.

Mixed bromcresol green-methyl red indicator solution (alcoholic solution) - 100

$\underline{\mathbf{m L}}$

$\overline{100} \mathrm{mg}$ bromcresol green

20 mg methyl red

Dilute to $100 \mathrm{~mL}$ with ethyl alcohol

\section{Sample Analysis}

Sample should be analyzed within 6 hours of sampling.

- Rinse electrodes and titration vessel with distilled water and drain.

- Take $100 \mathrm{~mL}$ of sample and adjust to room temperature if necessary

- Measure sample pH. Add .02 N sulfuric acid in increments of $0.5 \mathrm{~mL}$ or less such that the change of less than $0.2 \mathrm{pH}$ units occurs per increment

- After each addition mix thoroughly but gently with a magnetic stirrer

- Record $\mathrm{pH}$ when a constant reading is obtained

- Continue adding titrant and measure $\mathrm{pH}$ until $\mathrm{pH} 4.5$ is reached

- Construct the titration curve by plotting observed $\mathrm{pH}$ versus cumulative milliliters of titrant added. A smooth curve showing one or more inflections should be obtained

\section{Calculation}

$$
\text { Alkalinity, } \mathrm{mgCaCO}_{3} / L=\frac{A \times N \times 50000}{\text { mlsample }}
$$

Where $\mathrm{A}=\mathrm{ml}$ standard acid used, $\mathrm{N}=$ normality of standard acid 
Report as follows:

The alkalinity to $\mathrm{pH}$

Indicate clearly if this $\mathrm{pH}$ corresponds to an inflection point of the titration curve

\section{B.6 Fluorescent in situ Hybridization}

Purpose: FISH allows for the visualization of the target organisms with an epifluorescent microscope. This is the procedure used to store biological samples for later use, dilute probes to create a working stock, and prepare slides by drying, hybridization, and washing. Also included is basic operation of the epifluorescent microscope, camera, and software.

\section{Special equipment needed to perform this protocol:}

Autoclave ( $\mathrm{Rm} 108)$ : The autoclave is used to sterilize pipette tips, water, and any other items necessary for solution preparation. Please see autoclave protocol for operating this piece of equipment.

\section{Epifluorescent Microscope with Camera attached to a Computer with Openlab}

Software and Photoshop (Rm 108): The epifluorescent microscope is needed to visualize the probes hybridized within the samples contained on the slides. The camera and computer software are used to capture, store, and manipulate the pictures to achieve the desired end-product. Directions on how to use this equipment and software are given within this protocol.

\section{Solution Preparation for Fixation}

All solutions must be prepared by using sterile pipette tips and autoclaved, filtered, deionized water and stored at $4{ }^{\circ} \mathrm{C}$. Usually $15 \mathrm{~mL}$ polystyrene vials are used in these solution preparations. If a higher volume of solution is needed, recipes may be multiplied by the correct factor.

\section{Solution 1: $5 \mathrm{M} \mathrm{NaCl}-15 \mathrm{~mL}$}


Solution 2: $0.5 \mathrm{M} \mathrm{NaH}_{2}{\underline{\mathrm{PO}_{4}}}_{4}-15 \mathrm{~mL}$ $1.035 \mathrm{~g} \mathrm{NaH}_{2} \mathrm{PO}_{4}$

Dilute to $15 \mathrm{~mL}$ with sterile water

Solution 3: $0.5 \mathrm{M} \mathrm{Na}_{2} \underline{\mathrm{H}}_{2} \underline{\mathrm{PO}}_{4} \underline{-15 \mathrm{~mL}}$ $1.065 \mathrm{~g} \mathrm{Na}_{2} \mathrm{H}_{2} \mathrm{PO}_{4}$

Dilute to $15 \mathrm{~mL}$ with sterile water

Solution 4: 0.5 M NPO 4 buffer $-15 \mathrm{~mL}$

$4.2 \mathrm{~mL}$ of Solution 2

$10.8 \mathrm{~mL}$ of Solution 3

Solution 5: 10M NaOH - $15 \mathrm{~mL}$

$6 \mathrm{~g} \mathrm{NaOH}$

Dilute to $15 \mathrm{~mL}$ with sterile water

Solution 6: 10\% HCl - $15 \mathrm{~mL}$

$4 \mathrm{~mL}$ of $37.5 \% \mathrm{HCl}$

$11 \mathrm{~mL}$ sterilized water

Solution 7: 3 x PBS - $15 \mathrm{~mL}$

$1.17 \mathrm{~mL}$ of Solution 1

$1.08 \mathrm{~mL}$ of Solution 4

Dilute to $15 \mathrm{~mL}$ with sterile water

Solution 8: 1 x PBS - 15 mL

$5 \mathrm{~mL}$ of Solution 7

Dilute to $15 \mathrm{~mL}$ with sterile water

Solution 9: $200 \mathrm{mM}$ Tris - $15 \mathrm{~mL}$

$0.363 \mathrm{~g}$ Tris

Dilute to $15 \mathrm{~mL}$ with sterile water

Solution 10: 3\% Tgepal - $15 \mathrm{~mL}$

2-3 drops of Tgepal

Dilute to $15 \mathrm{~mL}$ with sterile water

Solution 11: 2 x Storage Buffer - $15 \mathrm{~mL}$

$3 \mathrm{~mL}$ of Solution 9

$1 \mathrm{~mL}$ of Solution 10

Dilute to $15 \mathrm{~mL}$ with sterile water

Solution 12: 100\% Ethanol 


\section{Solution 13: 4\% PFA - $15 \mathrm{~mL}$}

0.6 g paraformaldahyde

1 drop of Solution 5

$4.95 \mathrm{~mL}$ of Solution 7

Solution 6

*This solution can only be used for up to 2 weeks.

Dissolve paraformaldahyde in $9.9 \mathrm{~mL}$ of sterile water warmed in the microwave for 10 seconds. Dissolve as much as possible using vortexer. Then add Solution 5 and Solution 7. Adjust $\mathrm{pH}$ using drops of Solution 6 with $\mathrm{pH}$ strips to 7.2. Filter through $0.2 \mu \mathrm{m}$ filter.

\section{Fixation Procedure}

For each sample desired, 4 separate vials of the same sample must be stored; 2 samples fixed with 4\% PFA and 2 samples fixed with 100\% ethanol.

- Mix sample vigorously and add $1 \mathrm{~mL}$ to centrifuge vial with pipette and large pipette tips cut with a straight razor

- Centrifuge at 1000 RPM for 5 min@ @ ${ }^{\circ} \mathrm{C}$

- Remove supernatant with pipette

- Fill centrifuge tube with either:

o 4\% PFA (Solution 13) (must use on 2 out of 4 samples)

o $100 \%$ ethanol (Solution 12) (must use on 2 out of 4 samples)

- Store overnight at $4{ }^{\circ} \mathrm{C}$ or for $2 \mathrm{hr}$ at room temp

- Centrifuge at 1000 RPM for 5 min@ @ ${ }^{\circ} \mathrm{C}$

- Remover supernatant and wash by adding $1 \mathrm{~mL}$ of 1 x PBS (Solution 8), mix and let sit at room temp for 15 min

- Centrifuge again as before and repeat step 6

- Centrifuge for the last time, remove supernatant

- Add equal volumes ( $\sim 0.5 \mathrm{~mL})$ of first $2 \times$ storage buffer (Solution 11$)$, then $100 \%$ ethanol (Solution 12)

- Store in $-20^{\circ} \mathrm{C}$ fridge

- Samples may be stored for up to 1 year.

\section{Preparation of Probes from Stock}

Once a working solution of probes is made from the stock solution, the stock solution should not be removed from the freezer or exposed to light until more working solution needs to be prepared. All dilutions should be made with sterile pipette tips, sterile centrifuge tubes, and LAL reagent water. 


\section{Dilution of Bacterial Probe:}

Stock solution contains $1 \mu \mathrm{g} / \mu \mathrm{L}$ of probe. Working solution should contain $50 \mathrm{ng} / \mu \mathrm{L}$. Thus, add $10 \mu \mathrm{L}$ of stock probe to $190 \mu \mathrm{L}$ of LAL Reagent water.

Dilution of Archaea Probe:

Stock solution contains $1 \mu \mathrm{g} / \mu \mathrm{L}$ of probe. Working solution should contain $50 \mathrm{ng} / \mu \mathrm{L}$. Thus, add $10 \mu \mathrm{L}$ of stock probe to $190 \mu \mathrm{L}$ of LAL Reagent water.

\section{Solution Preparation for Slide Preparation}

Some chemicals may be made and stored, while other chemicals should only be made a day or two in advance if desired. All solutions should be made using sterile pipette tips with autoclaved, filtered, deionized water. Usually $50 \mathrm{~mL}$ polystyrene vials are used in these solution preparations.

Solution $14-0.5 \mathrm{M}$ EDTA $(\mathrm{pH}=8.0)-50 \mathrm{~mL}$

$7.31 \mathrm{~g}$ of disodium ethylenediaminetraacetate

Dilute to $50 \mathrm{~mL}$ with sterile water

* This solution may be used for up to one year as long as no solid precipitation is visible.

Prepare solution and adjust $\mathrm{pH}$ to 8.0 with $\mathrm{NaOH}$ and $\mathrm{pH}$ strips.

\section{Solution $15-20 \%$ SDS (Sodium dodecyl sulfate) - $50 \mathrm{~mL}$}

$10 \mathrm{~g}$ of electrophoresis grade SDS

Concentrated $\mathrm{HCl}$

Dilute to $50 \mathrm{~mL}$ of sterile water

*This solution should be stored at room temperature.

Add SDS to $45 \mathrm{~mL}$ of sterile water using a mask for respiratory protection. Heat to 68 ${ }^{\circ} \mathrm{C}$ and adjust $\mathrm{pH}$ to 7.2 by adding a few drops of concentrated $\mathrm{HCl}$. Adjust volume to $50 \mathrm{~mL}$ using sterile water.

\section{Solution 16 - $1 \mathrm{mg} / \mathrm{mL}$ DAPI stock solution - $1 \mathrm{~mL}$}

$1 \mathrm{~g}$ DAPI

$1 \mathrm{~mL}$ sterilized water

*This solution should be stored at $4{ }^{\circ} \mathrm{C}$ wrapped in aluminum foil.

Weigh $1.0 \mathrm{~g}$ of DAPI using RNase/DNase free microspatula in a $2 \mathrm{~mL}$ sterile centrifuge tube. Add $1 \mathrm{~mL}$ of sterile water.

\section{Solution 17 - $1 \mathrm{ug} / \mathrm{mL}$ DAPI solution $-\mathbf{5 0} \mathbf{~ m L}$}


Dilute to $50 \mathrm{~mL}$ with sterilized water

*This solution should be stored at $4{ }^{\circ} \mathrm{C}$ wrapped in aluminum foil.

Solution $18-1 \mathrm{M}$ Tris-HCI Solution $-15 \mathrm{~mL}$

$1.815 \mathrm{~g}$ Tris- $\mathrm{HCl}$

Dilute to $15 \mathrm{~mL}$ with sterile water

\section{Solution 19 - In Situ Hybridization Buffer - 2 mL}

$360 \mu \mathrm{L}$ Solution 1

$40 \mu \mathrm{L}$ Solution 18

$400 \mu \mathrm{L}$ formamide (stored in $4{ }^{\circ} \mathrm{C}$ fridge)

$1200 \mu \mathrm{L}$ sterile water

$1 \mu \mathrm{L}$ of $20 \%$ SDS $(2 \mu \mathrm{L}$ of $10 \%$ SDS $)$

*This solution should only be stored for up to a week before use. $2 \mathrm{~mL}$ of buffer is used for the preparation of 1 slide.

Add Solution 1, Solution 18, formamide, and water into a $2 \mathrm{~mL}$ sterile centrifuge tube. Then add SDS. *Note*: This is for $20 \%$ formamide, if a different $\%$ is needed, please review the chart below.

\section{Solution 20 - Washing Buffer $-50 \mathrm{~mL}$}

$1 \mathrm{~mL}$ Solution 18

$2150 \mu \mathrm{L}$ Solution 1

$500 \mu \mathrm{L}$ Solution 14

Dilute to $50 \mathrm{~mL}$ with sterile water

$25 \mu \mathrm{L}$ of $20 \%$ SDS $(50 \mu \mathrm{L}$ of $10 \%$ SDS $)$

*This solution should only be stored for up to a week before use. $50 \mathrm{~mL}$ of buffer is used for the preparation of 1 slide.

Add Solution 18, Solution 1, Solution 14, and water into a $50 \mathrm{~mL}$ polypropylene vial. Then add SDS. *Note*: This is for $20 \%$ formamide, if a different $\%$ is needed, please review the chart below. 


\begin{tabular}{|c|c|c|c|}
\hline $\begin{array}{c}\% \text { formamide } \\
\text { (bybridization buffer) }\end{array}$ & $\begin{array}{l}\mathrm{NaCl} \\
\mathrm{mM}\end{array}$ & $\begin{array}{c}5 \mathrm{M} \mathrm{NaCl}(=\mathrm{Y}) \\
\mu \mathrm{l}\end{array}$ & $\begin{array}{c}\text { 0.5M EDTA } \\
-\mathrm{Tt}\end{array}$ \\
\hline 0 & 900 ats & 9000 & 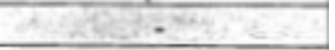 \\
\hline 5 & 636 & 6300 & - \\
\hline 10 & 450 & $\because 3500$ & 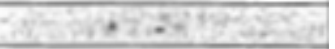 \\
\hline 15 & 318 & 3180 & 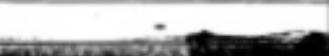 \\
\hline 25 & $159^{-}$, $\rightarrow$ a & 1490 & 500 \\
\hline 30 & 112 제 & 1020 & $5 \times 500$ \\
\hline 35 & 80 & 700 & 500 \\
\hline 40 & 56 & 460 brite? & 5.500 \\
\hline 45 & 40 & 300 & 500 \\
\hline $50 \div \geq=$ & $28 ? 2$ & $=6250180$ & सX:500 500 \\
\hline 55 & 20 & 100 & 500 \\
\hline 60 & $14 \div \div 12$ & 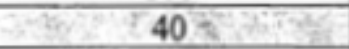 & WES 500 ? \\
\hline 65 & 10 & $\therefore$ & 500 \\
\hline 70 & 7 & 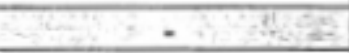 & 350 \\
\hline 75 & 5 & 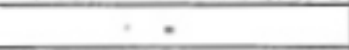 & 250 \\
\hline 80 & 3.5 & 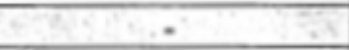 & $175 \quad 5+28$ \\
\hline 85 & 2.5 & - & 125 \\
\hline 90 & 1.75 & +6 & 88 \\
\hline 95 & 1.24 &. & 62 \\
\hline
\end{tabular}

Preparation of Fixed Samples for Slide Preparation

Obtain desired fixed samples from $-20{ }^{\circ} \mathrm{C}$ storage and place on ice while performing the dilution procedures. Shake samples before removing desired amount for dilution.

\section{Digester samples - Diluted 50 times $-10 \mathrm{~mL}$}

$0.2 \mathrm{~mL}$ of fixed digester sample

Dilute to $10 \mathrm{~mL}$ with sterile water

E.coli standard - Diluted 50 times $-10 \mathrm{~mL}$

$0.2 \mathrm{~mL}$ of fixed E.coli sample

Dilute to $10 \mathrm{~mL}$ with sterile water

\section{Methanogen standard}

No dilution is required. Use straight from fixed sample centrifuge tube.

Preparation of Slides - Deposition of Samples, Hybridization, and Washing

Each step of slide preparation should occur consecutively. Preparation is time consuming, as hybridization requires 3 hours. Each slide must contain 3 wells 
containing standards in addition to those wells containing digester samples. These three wells are as follows: Methanogens only, E.coli only, and a mixture of Methanogens and E.Coli. All wells receive both probes. All work should be done using sterile pipette tips.

- Add a sample or standard to the desired well, amount varies depending on which. Allow to dry in the airstream provided by a hood.

o $10 \mu \mathrm{L}$ of Methanogens

o $5 \mu \mathrm{L}$ of diluted E.coli

o $10 \mu \mathrm{L}$ of diluted digester sample

- Dehydrate each slide by placing it for three minutes in each of a $50 \%, 80 \%$, $100 \%$ ethanol dilution series. Let dry.

- Add $8 \mu \mathrm{L}$ hybridization buffer (Solution 19) to each well

- Add $1 \mu \mathrm{L}$ of each probe to each well

o Add one probe to a well and mix, disposing of pipet tip. Add the same probe to all wells and then place back in $-20{ }^{\circ} \mathrm{C}$ fridge and take out other probe.

o Repeat this procedure for the second probe. Make sure that all probes are hidden from exposure to light as much as possible and sterile methods are used. Place back in $-20{ }^{\circ} \mathrm{C}$ fridge as soon as additions are complete.

$0 \quad * * * *$ Once probes are added, slides must be hidden from exposures to light as much as possible.

- Place the slide in an empty $50 \mathrm{~mL}$ vial and add a small amount of paper towel underneath the slide. Pour hybridization buffer onto this paper towel and seal vial.

- Place in a $46{ }^{\circ} \mathrm{C}$ oven for 3 hours. Make sure vial will not roll around, and that foil is placed over the glass portion of the door to protect slides from light.

- Remove slides from the vial and rinse with washing buffer pre-heated in $48{ }^{\circ} \mathrm{C}$ water-bath.

- Add slide in $50 \mathrm{~mL}$ vial of washing buffer and leave in $48{ }^{\circ} \mathrm{C}$ water-bath for 15 minutes.

- Pour out buffer and rinse with sterile water. Dry without exposing to light.

- Add 1 drop of DAPI from a $0.2 \mu \mathrm{m}$ syringe filter to each well. Wait 1 to 5 minutes without exposing slide to light, and then rinse with sterile water. Allow to dry.

- Put 5 drops (from a $100 \mu \mathrm{L}$ pipette) of citiflour oil on the black portion of the slide, in a line right down the middle of the slide.

- Put slip cover on and spread oil out so as to remove all air bubbles. Seal edges with fingernail polish and allow to dry.

- Slides can now be viewed or stored at $-20^{\circ} \mathrm{C}$. 


\section{Basic Operation of the Epifluorescent Microscope}

- Turn on the Mercury bulb and record the reading in the display along with your name and date on the log-sheet.

- Turn on the camera positioned on top of the microscope by pushing the toggle switch down toward the left.

- Place slide on the microscope stage and position the 40X objective in the viewing position. Using the stage-movement controls, position the slide so one of the wells that contains a sample is positioned under the $40 \mathrm{X}$ objective.

- Open the shutter using the slide toggle underneath and to the right of the viewer. Place the circular sliding filter switch (also under the viewer) to position 3, containing the FITC filter.

- Look through the viewer, using the course focus knob on the right back portion of the microscope, slowly bring the sample into focus. If nothing can be seen through the viewer, check two settings on the microscope:

0 The sliding filter contained on a strip located between the camera and the optical viewing piece must be in the third position to the right.

o The sliding bar located underneath the camera must be fully pushed in to allow all light to go to the eye viewing piece.

- Using the stage movement controls the slide can be moved around under the objective to allow visualization of the entire well. Once a desired area has been found the circular sliding filter switch can be moved between position 3, FITC, and position 5, CY3, to visualize both bacteria (CY3 filter), and archaea (FITC filter).

- When higher magnification is desired, use the course focus knob to back the slide away from the objective. Position the $100 \mathrm{X}$ objective over the slide. Place a drop of immersion oil on the well and use the fine focus knob to bring the sample into focus. *NOTE*: Immersion oil is only needed when using the $100 \mathrm{X}$ objective, after use, wipe using the ocular cleansing wipes.

\section{Taking Pictures and Saving Using the Openlab Software}

- Open the software by double clicking on the hard drive icon on the PC adjacent to the microscope. Find Openlab version 3.1 in the list and double click to open the program.

o Always make sure that the camera has been turned on before opening the software. If it is not, the software will not recognize the camera. To solve this problem, turn the camera off, close the software, turn the camera back on, and re-open the software.

- Once an object of interest has been found, slide the bar located underneath the camera all the way out to allow all light to go to the camera. 
- Click the movie camera icon on the tool bar on the left side of the screen and the live view from the camera should be shown.

- To get the best picture, the exposure level must be set. This can be accomplished by selecting Video Manager from the Special drop-down menu. In the Video Manager screen, douple click in the exposure time box and adjust the setting. A preview of the image will be shown in the window above after a few seconds.

o Generally, for digester samples different exposure times are needed for the FITC filter and the CY3 filter.

- FITC $-700 \mathrm{~s}$

- CY3 - $500 \mathrm{~s}$

o This exposure time will vary with the amount of probe hybridized and the sample properties.

- To take the picture, click the still picture camera icon on the tool bar on the left side of the screen.

- The picture should be shown as a small thumbnail in a window on the right hand side of the screen. If this thumbnail does not appear, go to the Window drop-down menu, select Pallates, and then select the last option on the Pallates menu.

- Two pictures of each object must always be taken, one using the FITC filter, and one using the CY3 filter. If desired, a third picture may be taken under the DAPI filter which hybridizes to all biological material.

- After a set of pictures has been taken, each picture-layer can be named by clicking on the upside-down triangle in the lower right-hand corner of the picture thumbnail to show the dropdown menu. Select Set Name from the drop-down menu. Name the picture making sure to include detailed information such as floc number, filter, and magnification.

o It is advisable to take pictures of one well in the same file, label them by floc number, filter and magnification. When saving, the file can be named by the slide name and well number. This eliminates excessive labeling.

- Once all the desired pictures are taken from one well, click File, and then click Save As from the drop-down menu. Name the file, and switch file type to TIFF.

- Once the file is named, it can be closed. To get a new file click on the Openlab drop-down menu and select New, and then select Image Document.

\section{Manipulating Pictures}

Pictures can either be manipulated in Openlab and then saved in a suitable format within Photoshop, or they can be copied from Openlab and manipulated in Photoshop. This procedure describes these methods. However, the same procedure should be used on all pictures. 
- Manipulating pictures in Openlab and then saving in a suitable format within photoshop

o Add color to a picture by double clicking on the desired picture, and then click on the color button icon on the tool bar to the left of the picture. Choose either DAPI, FITC, or CY3.

o To merge two colored pictures together, click on the first picture and then click on the second picture while holding down the shift key. Go to the Layers drop-down menu and select Merge RGB Plane. This will create a new layer. This layer can then be named, and the file can be resaved.

o To get a merged picture to Photoshop, double click on the thumbnail and then click on the full size picture and press Apple A to select all, then Apple $\mathrm{C}$ to copy. Open a new file in Photoshop and press Apple $\mathrm{V}$ to paste. Under the File menu select Save As. Name the file, and change format to BMP.

- Copying pictures from Openlab and manipulating in Photoshop

o To get a picture into Photoshop, double click on the thumbnail and then click on the full size picture and press Apple A to select all, then Apple $\mathrm{C}$ to copy. Open a new file in Photoshop and press Apple V to paste.

o Paste Both pictures in the same document and in the Layers window, select either Difference or Overlay from the drop-down menu. Under the File menu select Save As. Name the file, and change format to BMP. 


\section{References}

Amann RI, Krumholz L, Stahl DA. 1990. Fluorescent-oligonucleotide probing of whole cells for determinative, phylogenetic and environmental studies in microbiology. Journal of Bacteriology 172(2):762-770.

Angenent LT, Sung S, Raskin L. Mixing intensity in anaerobic sequencing batch reactors affects reactor performance and microbial community structure; 2001 September 2-6; Antwerp, Belgium. International Water Association. p 267-274.

Angenent LT, Sung S, Raskin L. 2002. Methanogenic population dynamics during startup of a full-scale anaerobic sequencing batch reactor treating swine waste. Water Research 36(18):4648-4654.

APHA. 1998. Standard Methods for the Examination of Water and Wastewater. Washington D.C.: American Public Health Association.

Banerjee S, Biswas GK. 2004. Studies on biomethanation of distillery wastes and its mathematical analysis. Chemical Engineering Journal 102(2):193-201.

Ben-Hasson RM, Ghaly AE, Singh RK. Design and evaluation of no-mix energy efficient anaerobic digester; 1985; Charlottetown, P.E.I.

Brade CE, Noone GP. 1981. Anaerobic Digestion - need it be expensive? Water Pollution Control 80:70-76.

Buswell AM. 1930. Production of fuel gas by anaerobic fermentations. Ind. Eng. Chem. 22:1168-1172.

Buswell AM, Boruff CS. 1933. Mechanical equipment for continuous fermentation of fibrous materials. Ind. Eng. Chem. 25:147-49.

Buswell AM, Hatfield WD. 1936. Anaerobic Fermentations. Urbana, IL: State of Illiniois Department of Registration and Education.

Casey TJ. 1986. Requirements and methods for mixing in anaerobic digesters. Anaerobic digestion of sewage sludge and organic agricultureal wastes: Elsevier, Applied Science Publication. p 90-103.

Chapman D. 1989. Mixing in anaerobic digesters: state of the art. In: Chermisinoff P, editor. Encyclopedia of Environmental Control Technology. Houston: Gulf Publishing Company. p 325-54.

Chen T, Chynoweth DP, Biljetina R. 1990. Anaerobic digestion of municipal solid waste in a nonmixed solids concentrating digester. Applied Biochemistry and Biotechnology 24/25:533-44.

Choi KH, Christi Y, Moo-Young M. 1996. Comparative evaluation of hydrodynamics and gas-liquid mass transfer characteristics in bubble column and air lift slurry reactors. Chemical Engineering Journal 62:223229.

Clinton WJ, Gore AC. 1993. Climate Change Action Plan.

Combs JJ, Ward GM, Miller WC, Ely LO. 1981-1982. Cattle as competitors for biomass energy. Energy in Agriculture 1:251-265. 
Conrad R, Phelps TJ, Zeikus JG. 1985. Gas metabolism evidence in support of the juxtaposition of hydrogen-producing and methanogenic bacteria in sewage sludge and lake sediments. Applied and Environmental Microbiology 50:595-601.

Coppinger E, Brantigan J, Lenart J, Baylon D. 1979. Report on the Disign and Operation of a Full-Scale Anaerobic Dairy Manure Digester. Golden, CO: Solar Energy Research Institute. Report nr SERI/TR-312-471.

Corso PS, Kramer MH, Blair KA, Addiss DG, Davis JP, Haddix AC. 2003. Cost of illness in the 1993 Waterborn Cryptosporidium Outbreak, Milwaukee, Wisconsin. Emerging Infectious Diseases 9(4).

Dague RR, McKinney RE, Pfeffer JT. 1970. Solids retention in anaerobic waste treatment systems. Journal of the Water Pollution Control Federation 42(2 Part 2):R29-R46.

de los Reyes FL, de los Ritter W, Raskin L. 1998. Group-specific small subunit rRNA hybridization probes to characterize filamentous foaming in activated sludge systems. Applied and Environmental Microbiology 63:1107-1117.

Diaz L, Kurz F, Trezek G. 1974. Methane gas production as part of a refuse recycling system. Compost Science:7-13.

Diaz L, Trezek G. 1977. Biogasification of a selected fraction of municipal solid wastes. Compost Science:8-13.

Dolfing J. 1992. The energetic consequences of hydrogen gradients in methanogenic ecosystems. FEMS Microbiology Ecology 101:183-87.

EPA. Animal Waste Management. EPA.

EPA. Global Warming -- Emissions. EPA.

EPA. 2003. Concentrated Animal Feeding Operations (CAFO) - Final Rule. EPA.

EPA U. 1979. Process Design Manual for Sludge Treatment and Disposal. EPA 625/1-79-011.

Fannin KF. 1987. Ch 10: Start-Up, Operation, Stability, and Control. In: Ronisaacson DPCa, editor. Anaerobic Digestion of Biomass. New York: EIsevier Applied Science.

Finny CD, Evans RSI. 1975. Anaerobic Digestion: The Rate-Limiting Process and the Nature of Inhibition. Science 4219:1088.

Fontenot JP, Ross IJ. Animal waste utilization. Livestock wastes: A renewable resourse; 1980; St. Joseph, MI. ASCE.

Franklin G, Koeroets WAA, van Gils WMA, van der Pas A. 1992. Application of the Biobed upflow fluidized-bed process for anaerobic waste water treatement. Water Science and Technology 25:373-82.

Ghaly AE, Ben-Hasson RM. 1989. Continuous production of biogas from dairy manure using an innovative no-mix reactor. Applied Biochemistry and Biotechnology 20/21:541-559.

Griffin ME, McMahon KD, Mackie RI, Raskin L. 1998. Methanogenic population dynamics during start-up of anaerobic digesters treating 
municipal solid waste and biosolids. Biotechnology and Bioengineering 57:342-55.

Haghighi-Podeh MR, Bhattacharya SK, Mingbo Q. 1995. Effect of nitrophenols on acetate utilizing methanogenic systems. Water Research 29:391-397.

Harikishan S, Sung S. 2003. Cattle waste treatment and Class A biosolid production using temperature-phased anaerobic digester. Advances in Environmental Research 7(3):701-706.

Hashimoto AG. 1982. Effect of mixing duration and vacuum on methane production rate from beef cattle waste. Biotechnology and Bioengineering 24:9-23.

Hawkes FR, Donnelly T, Anderson GK. 1995. Comparative perfomance of anaerobic digesters operating on ice-cream wastewater. Water Science and Technology 29(2):525-33.

Heijnen JJ, Mulder A, Enger W, Hoeks F. 1989. Review on the application of anaerobic fluidized bed reactors in wastewater treatement. Chemical Engineering Journal 41:B37-B50.

Ho CC, Tan YK. 1985. Anaerobic treatment of palm oil mill effluent by tank digesters. Journal of Chemical Technology and Biotechnology 35b(2):155164.

James S, Wiles C, Swartzbaugh J, Smith R. 1980. Mixing in large-scale municipal solid waste-sewage sludge anaerobic digesters. Biotechnology and Bioengineering Symposium 10:259-72.

Karim K, Gupta SK. 2001. Biotransformation of nitrophenols in upflow anaerobic sludge blanket reactors. Bioresource Technology 80(3):179-186.

Karim K, Hoffmann R, Klasson K, Al-Dahhan MH. Anaerobic digestion of animal waste: Effect of mode of mixing; 2003 May 14-16; Halkidiki, Greece. p 175-185.

Karim K, Hoffmann R, Klasson K, Al-Dahhan MH. 2005a. Anaerobic digestion of animal waste: waste strength vs. impact of mixing. Bioresource Technology In Press.

Karim K, Klasson K, Hoffmann R, Drescher S, DePaoli D, Al-Dahhan MH. 2005b. Anaerobic digestion of animal waste: effect of mixing. Bioresource Technology In Press.

Karim K, Varma R, Veskivar M, Al-Dahhan MH. 2004. Flow pattern visualization of a simulated digester. Water Research 38:3659-3670.

Kaye J. 2004. Pigs and Politices. News Hour: PBS.

Konstandt HG, Roediger AG. 1977. Engineering operation and ecomomics of methane gas production. In: Shlegel HG, Barnea J, editors. Microbial Energy Conversion. New York: Pergammon Press. p 379.

Lanting J. Optimization of biological activity for anaerobic sludge digestion; Annual Conference of the Water Environment Federation October 12, 2003; Los Angeles, CA. 
Lanting J, Murphy JLI; (Biothane Corporation, USA). assignee. 2002. Anaerobic digestion apparatus, methods for anaerobic digestion and for minimizing the use of inhibitory polymers in digestion. Wo patent 2002100784.

Lapp HM, Schulte DD, Sparling AB, Buchanan LC. 1975. Methane production from animal wastes. 1. Fundamental considerations. Canadian Agricultural Engineering 17(2):97-102.

Lema JM, Mendez R, Iza J, Garcia P, Fernandez-Polonco F. 1991. Chemical reactor engineering concepts in design and operation of anaerobic treatment processes. Water Science and Technology 24(8):79-86.

Lettinga G. Anaerobic digestion for energy saving and production. In: Chartier WPaP, editor; 1981. Applied Science Publishers Ltd.

Lettinga G, van Velsen AFM, Homba SW, de Zeeuw W, Klapeijik A. 1980. Use of the upflow sludge blanked reactor concept for biological wastewater treatment especially for anaerobic treatment. Biotechnology and Bioengineering 22:699-734.

Lin KC, Pearce MEJ. 1991. Effects of mixing on anaerobic treatment of potatoprocessing wastewater. Canadian Journal of Civil Engineering 18:504514.

Linke B. 1997. A model for anaerobic digestion of animal waste slurries. Environmental Technology 18(8):849-854.

Lusk P. 1998. Methane Recovery from Animal Manuers -- The Current Opportunities Casebook. Golden Colorado: National Renewable Energy Laboratory. Report nr NREL/SR-580-25145.

Lusk P. 1999. Latest Progress in Anaerobic Digestion. BioCyle 40(7).

MacKenzie WR, Hoxie NJ, Proctor M, Gradus MS, Blair KA, Peterson DE. 1994. A massive outbreak in Milwaukee of Cryptosporidium infection transmitted through the public water supply. New England Journal of Medicine 331(3):161-7.

Madigan MT, Martinko JM, Parker J. 2003. Brock Biology of Microorganisms. 10, editor: Prentice Hall.

McCarty PL, Mosey FE. 1991. Modelling of anaerobic digestion processes (A discussion of concepts). Water Science and Technology 24:17-33.

McMahon KD, Stroot PG, Mackie RI, Raskin L. 2001. Anaerobic codigestion of municipal solid waste and biosolids under various mixing conditions-II:Microbial Population Dynamics. Water Research 35(7):1817-1827.

Mills PJ. 1979. Minimization of energy input requirements of an anaerobic digester. Agricultureal Wastes 1:57-59.

Miner JR, Humenik FJ, Overcash MR. 2000. Manaving Livestock Wastes to Preserve Environmental Quality. Ames, Iowa: Iowa State University Press.

Misi SN, Forster CF. 2001. Batch co-digestion of multi-component agro-wastes. Bioresource Technology 80(1):19-28. 
Moller HB, Sommer SG, Ahring BK. 2004. Methane productivity of manure, straw and solid fractions of manure. Biomass and Bioenergy 26:485-495.

Monteith H, Stephenson JP. 1981. Mixing efficiencies in full-scale anaerobic digesters by tracer methods. Journal of the water pollution control federation 53:78.

Nicolella C, Loosdrecht MCMv, Heijnen JJ. 2000. Wastwater treatment with particulate biofilm reactors. Jounal of Biotechnology 80(1):1-33.

Novaes RFV. 1986. Microbiology of anaerobic digesters. Water Science and Technology 18:1-14.

Parkin GF, Owen WF. 1986. Fundementals of anaerobic digestion of wastewater sludges. Journal of Environmental Engineering 112:869-920.

Pillars R. Farm-Based Anaerobic Digesters. Michigan State University Extension.

Rivard CJ, Kay BD, Kerbaugh DH, Nagle NJ, Himmel ME. 1995. Horsepower requirements for high-solids anaerobic digestion. Applied Biochemistry and Biotechnology 51/52:155-62.

Salminen EA, Rintala JA. 2002. Semi-continuous anaerobic digestoin of organic solid poultry slaughterhouse waste -- a review. Water Research 36(1):1326.

Schink B. 1992. Syntrophism among prokaryotes. In: A. Balows HGT, M. Dworkin, W. Harder and K.H. Schleifer, editor. The Prokaryotes: A Handbook on the Biology of Bacteria:Ecophysiology, Isolation, Identification, Applications. New York: Springer-Verlag. p 276-299.

Schink B. 1997. Energetics of syntrophic cooperation in methanogenic degradation. Microbiology and Molecular Microbiology Reviews 61:262280.

Sen BP, Baskaran TR. 1962. Anaerobic digestion of liquid molasses distillery wastes. Inc. Water Pollut. Control Fed. 34(10):1014.

Smith RJ, Hein MJ, Greinier TH. 1979. Experimental methane production from animal excreta in pilot-scale and farm-size units. Journal of Animal Science 48:202-217.

Speece RE. 1996. Anaerobic biotechnology for industrial wastewaters. Nashville, TN: Archae Press.

Stafford DA. 1981. The effects of mixing and volatile fatty acid concentrations on anaerobic digester performance. Tribune du CEBEDEAU 34(456):493500.

Stafford DA, Hawkes DL, Horton R. 1980. Methane Production from Waste Organic Matter. Boca Raton, FL: CRC Press.

Stahl DA, Amann RI. 1991. In: Stackebrandt E, Goodfellow, M.,, editor. Nucleic Acid Techniques in Bacterial Systematics. New York: John Wiley \& Sons. p 205-248. 
Strenstrom M, Ng A, Bhunia P, Abramson S. 1983. Anaerobic digestion of municipal solid waste. Journal of Environmental Engineering 109:11481158.

Stroot PG, McMahon KD, Mackie RI, Raskin L. 2001. Anaerobic codigestion of municipal solid waste and biosolids under various mixing conditions--I. Digester Performance. Water Research 35(7):1804-1816.

Sung S, Dague RR. 1995. Laboratory studies on the anaerobic sequencing batch reactor. Water Environ. Res 67(3):294-301.

Sung S, Liu T. 2003. Ammonia inhibition on thermophilic anaerobic digestion. Chemosphere 53(1):43-52.

Torpey WN. 1955. Loading to failure of a pilot high-rate digester. Sewage and Industrial wastes 27:121.

USDA. 2002/2003. Statistical Highlights of United States Agriculture 2002/2003. Washington, D.C: National Agricultural Statistics Service.

van Brakel J. 1980. Biogas before 1970: a review. Tropical Science 22(2):105-48. Van den Berg L. 1986. High rate reactors for methane production. Biotechnology and renewable energy:268-75.

Whitmore TN, Lloyd D, Jones G, Williams TN. 1987. Hydrogen-dependent control of the continuous anaerobic digestion process. Applied Microbiology and Biotechnology 26:383-388.

Young JC, and P.L. McCarty. The anaerobic filter for waste treatment; 1967. p 559-574.

Zhang RH, Yin Y, Sung S, Dague RR. 1997. Anaerobic treatment of swine waste $b$ the anaerobic sequencing batch reactor. Transactions of the ASAE 40:761-767.

Zheng D, Alm EW, Stahl DS, Raskin L. 1996. Characterization of Universal Small-Subunit rRNA Hybridization Probes for Quantitative Molecular Microbial Ecology Studies. Applied and Environmental Microbiology 62(12):4504-4513.

Zheng D, Raskin L. 2000. Quantification of Methanosaeta species in anaerobic bioreactors using genus- and species-specific hybridization probes. Microbial ecology 39:246-262.

Zinder SH. 1984. Microbiology of anaerobic conversion of organic wastes to methane: recent developements. American Society of Microbiology 50:294-298.

Zinder SH. Conversions of acetic acid to methane by thermophiles; 1988 May 2226; Bologna, Italy. 


\section{VITA \\ Rebecca Hoffmann}

\section{EDUCATION}

Washington University, St. Louis, MO

B.S. in Chemical Engineering

Graduation: May 2003
Webster University, St. Louis, MO

B.A. in Mathematics

Graduation: May 2003

\section{HONORS}

2003 AIChE Student Design Competition, Safety and Health Division Award for Safety - "Recycling Nitric Acid from a Radioactive Liquid Waste Stream".

Best Presentation Award, $9^{\text {th }}$ Annual Mid-America Environmental Engineering Conference, Sept. 18, 2004, SIU-Edwardsville. "Effect of Shear on Performance and Microbial Ecology in Anaerobic Digesters Treating Cow Manure

$2^{\text {nd }}$ place in Environmental Division 2002 AIChE National Student Poster Competition, 2002 AIChE Annual Meeting, Nov. 3-8, Indianapolis, IN - "Mixing in Anaerobic Digesters Treating Animal Waste".

Departmental Honors - Mathematics, Webster University

\section{PUBLICATIONS AND PRESENTATIONS}

Presenter, $9^{\text {th }}$ Annual Mid-America Environmental Engineering Conference, Sept. 18, 2004, SIU-Edwardsville. "Effect of Shear on Performance and Microbial Ecology in Anaerobic Digesters Treating Cow Manure".

Presenter, AIChE 2003 National Conference, Nov. 16-21, San Francisco, CA, Session 412 - Advances in Environmental Biotechnology II: Green Processing - "Anaerobic Digestion of Animal Waste: Effect of Mode of Mixing".

AIChE 2002 National Student Poster Competition, Indianapolis, IN - "Mixing in Anaerobic Digesters Treating Animal Waste".

Karim, K., Klasson, K.T., Hoffmann, R., Dresher, S., DePaoli, D., and Al-Dahhan, M.H. (2004) "Anaerobic digestion of animal waste: Effect of Mixing", Bioresource Technology, Accepted.

Karim, K., Hoffmann, R., Klasson, K.T., and Al-Dahhan, M.H. (2004) "Anaerobic digestion of animal waste: Effect of mode of mixing", Bioresource Technology, Communicated.

Hoffmann, R., Karim, K., Veskivar, M., Al-Dahhan, M.H., and Angenent, L. (2005)

"Effect of Shear on Performance, Microbial Community, and Hydrodynamics in Anaerobic Digesters Treating Cow Manure”, Bioresource and Bioengineering, Intended. 


\section{Appendix 2}

Thesis: Vesvikar M. (2006) Understanding the hydrodynamics of anaerobic digester for bioenergy production.[D.Sc. Thesis], St. Louis -USA, Washington University. 
WASHINGTON UNIVERSITY

THE HENRY EDWIN SEVER GRADUATE SCHOOL DEPARTMENT OF COMPUTER SCIENCE AND ENGINEERING

UNDERSTANDING THE HYDRODYNAMICS OF ANAEROBIC DIGESTERS FOR BIOENERGY PRODUCTION

by

Mehul S. Vesvikar

Prepared under the direction of Professor Muthanna H. Al-Dahhan

A dissertation presented to the Henry Edwin Server Graduate School of Washington University in partial fulfillment of the requirements for the degree of DOCTOR OF SCIENCE

December 2006

Saint Louis, Missouri 
WASHINGTON UNIVERSITY

THE HENRY EDWIN SEVER GRADUATE SCHOOL DEPARTMENT OF COMPUTER SCIENCE AND ENGINEERING

UNDERSTANDING THE HYDRODYNAMICS OF ANAEROBIC DIGESTERS FOR BIOENERGY PRODUCTION

by

Mehul S. Vesvikar

Prepared under the direction of Professor Muthanna H. Al-Dahhan

A dissertation presented to the Henry Edwin Server Graduate School of Washington University in partial fulfillment of the requirements for the degree of DOCTOR OF SCIENCE

December 2006

Saint Louis, Missouri 
WASHINGTON UNIVERSITY

THE HENRY EDWIN SERVER GRADUATE SCHOOL DEPARTMENT OF CHEMICAL ENGINEERING

\begin{tabular}{c}
\hline ABSTRACT \\
\hline UNDERSTANDING THE HYDRODYNAMCS OF ANAEROBIC DIGESTERS \\
FOR BIOENERGY PRODUCTION \\
by \\
Mehul S. Vesvikar
\end{tabular}

ADVISOR: Professor Muthanna H. Al-Dahhan

December 2006

St. Louis, Missouri

Anaerobic digestion is an efficient way of treating animal wastes and biomass byproducts to reduce its pollution threat and obtain renewable bioenergy in the form of methane (biogas). The high failure rate of anaerobic digesters coupled with the lack of fundamental research prohibits the widespread use of anaerobic digestion in USA. Assessing the mixing and hydrodynamics of gaslift anaerobic digesters and their influence on digester design, scale and operation via experimental studies is the focus of this work. A new and unique Multiple Particle Tracking (MP-CARPT) technique to track up to eight particles simultaneously was successfully developed and validated by tracking single and dual particles of same and different densities. For the first time, Computer automated radioactive particle tracking (CARPT) and Computational Fluid Dynamics (CFD) are used to study the effects of operating and design variables, internals, and scales on the mixing pattern and the detailed hydrodynamics of the anaerobic digesters. The CARPT experimental results show that gaslift digester with draft tube diameter half of the reactor diameter and multiple point sparger provides better mixing than other digester configurations. CARPT data concluded that the geometric similarity and equal power input per unit volume is not sufficient to obtain the same digester performance at two different scales. Further, successful development and implementation of the multiple-particle tracking technique (MP-CARPT) in this work will overcome the limitations of the single-particle CARPT in future research on dense multiphase systems including anaerobic digesters. Performance studies in laboratory and pilot-scale digesters treating cow manure show that large-scale experimentation is required to obtain reliable information for design and scale-up of digesters. The knowledge gained from this dissertation will be useful for further investigations that can lead to better understanding and design of anaerobic digesters. 
Appendix-2: Vesvikar(2006), D.Sc. Thesis

\section{Dedicated to}

my dear Aaee (Mom) and Baba (Dad) 


\section{Contents}

List of Tables .......................................................................................................

List of Figures.....................................................................................................vii

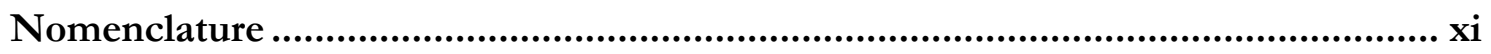

Acknowledgements.......................................................................................................

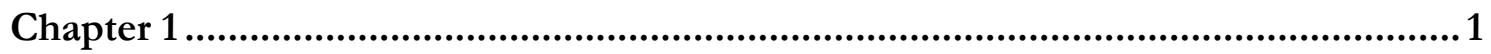

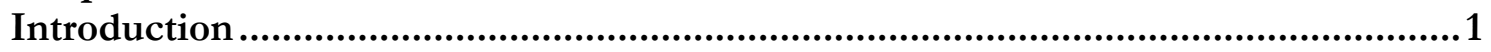

1.1 Introduction and Motivation........................................................................ 1

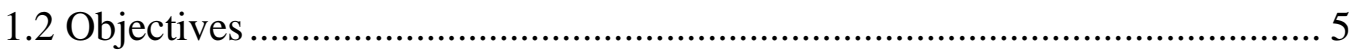

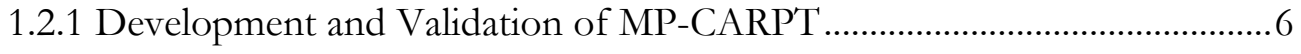

1.2.2 Single Particle CARPT and CFD Studies ..................................................

1.3 Thesis Organization...................................................................................... 7

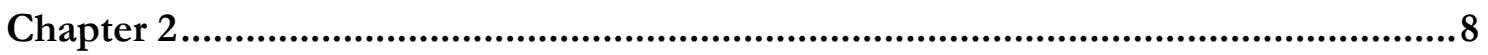

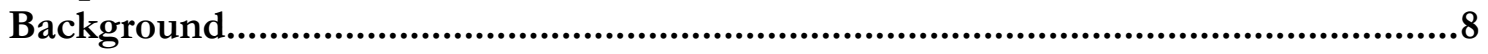

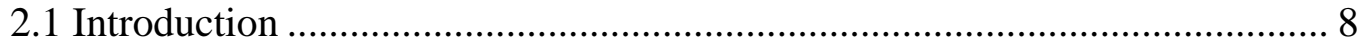

2.2 Anaerobic Digestion............................................................................. 8

2.2.1 Pollution, Renewable Energy and Anaerobic Digestion................................. 8

2.2.2 Anaerobic Digestion Mechanism ....................................................................10

2.2.3 Anaerobic Digesters (ADrs) ..........................................................................13

2.2.4 Importance of Mixing in Anaerobic Digestion...............................................16

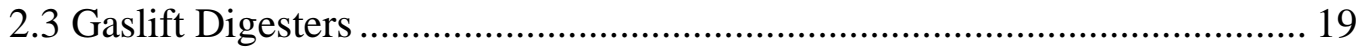

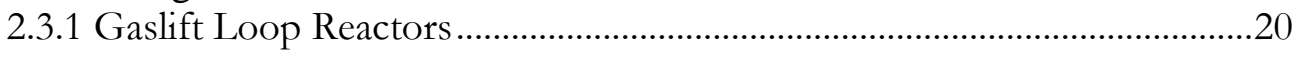

2.3.2 Hydrodynamics in Gas-Solid-Liquid IGLRs ..................................................2

2.4 Scale-up of IGLRs ................................................................................. 26

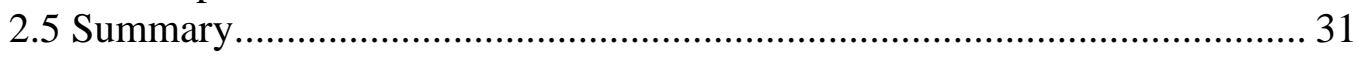

Chapter 3 ...................................................................................................................3

Multiple-Particle Tracking Technique: Development, Validation and

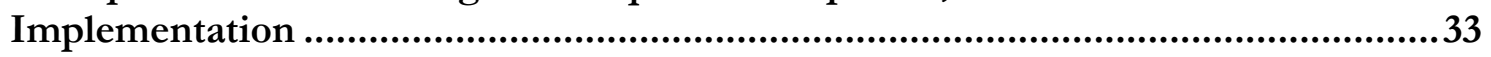

3.1 Introduction and Motivation........................................................................... 33

3.2 Selection of Radioactive Sources ................................................................ 36

3.3 MP-CARPT Electronics ........................................................................... 39

3.4 MP-CARPT Validation .......................................................................... 41

3.4.1 Tracking Stationary Particles............................................................................4

3.4.2 Tracking Particles in Motion.........................................................................57 
3.4.3 Tracking Two Moving Particles with Different Densities ..........................62

3.4.4 Tracking More than Two Particles............................................................6

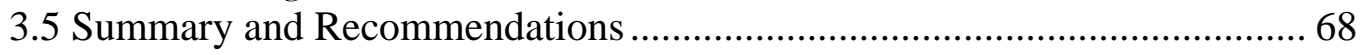

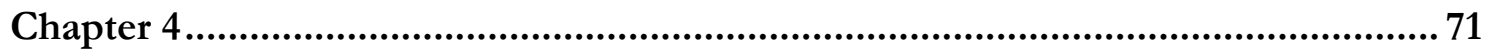

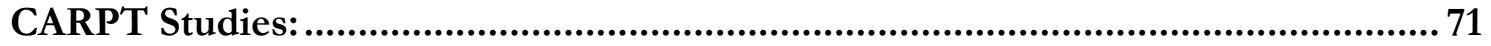

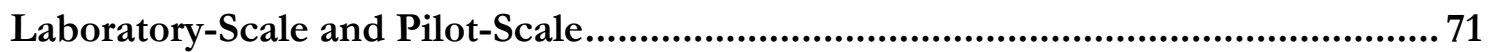

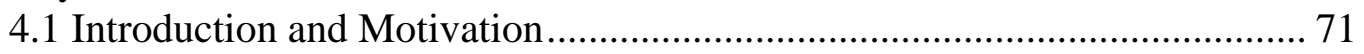

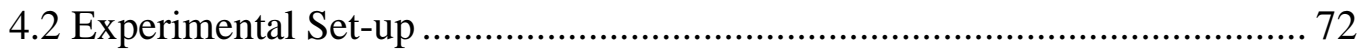

4.2.1 Laboratory-scale Digester............................................................................. 72

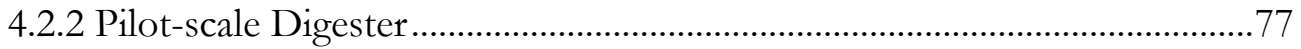

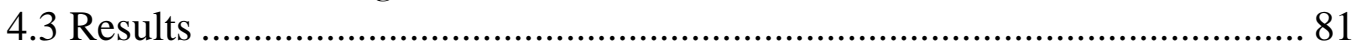

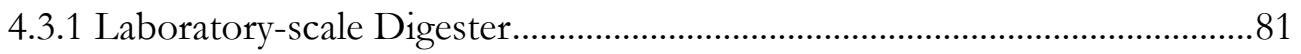

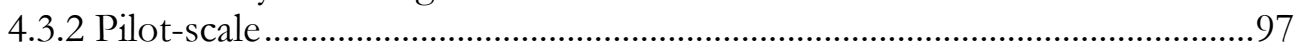

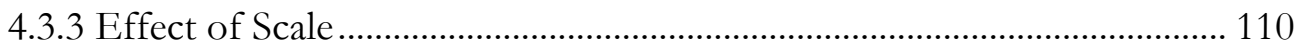

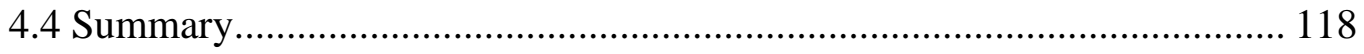

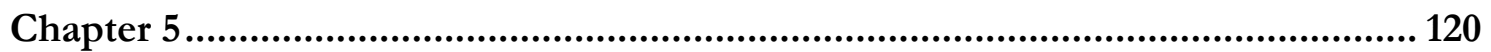

Computational Fluid Dynamics Studies: ....................................................... 120

Laboratory-scale and Pilot-scale ................................................................... 120

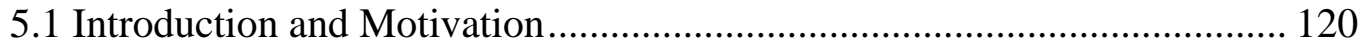

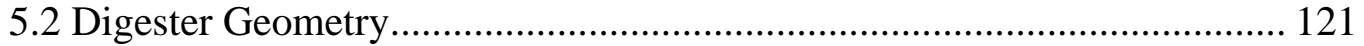

5.2.1 Laboratory-scale (6-inch Diameter) Digester ......................................... 121

5.2.2 Pilot-scale (18-inch Diameter) Digester ..................................................... 122

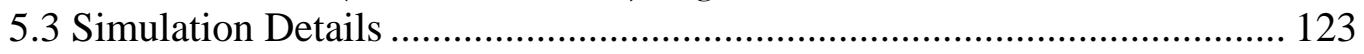

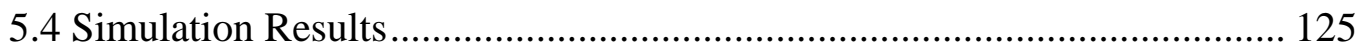

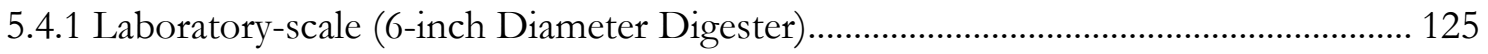

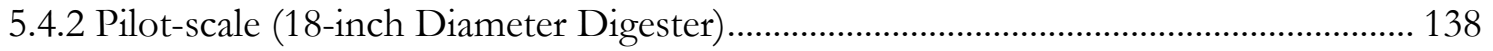

5.4.3 Effect of Scale (6-inch v/s 18-inch Diameter Digester) .............................................. 144

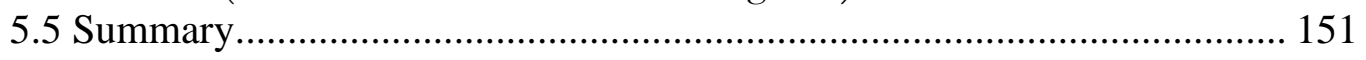

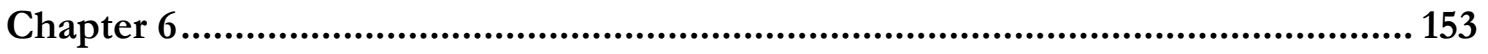

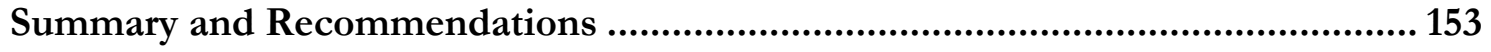

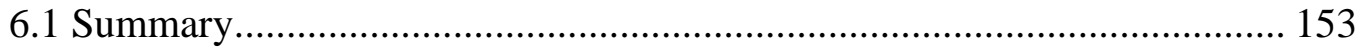

6.1.1 Development of Multiple-Particle Tracking.............................................. 154

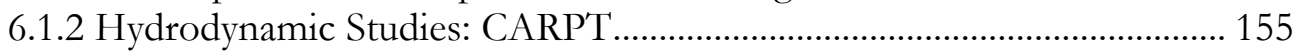

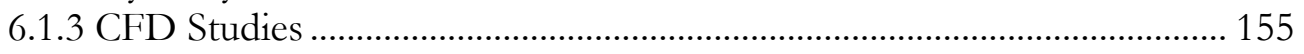

6.2 Recommendations for Future Work ................................................... 156

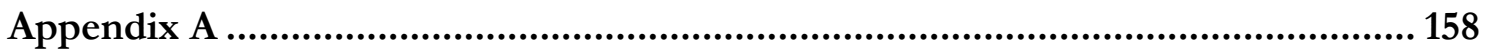

Performance Studies: Laboratory-scale and pilot-scale ..................................... 158

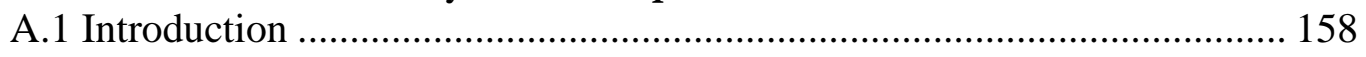

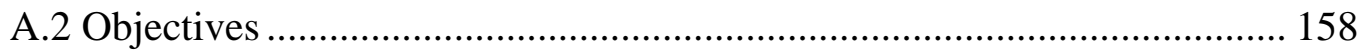

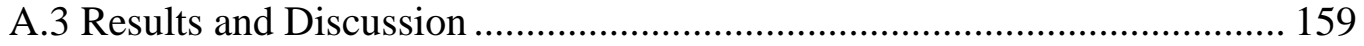




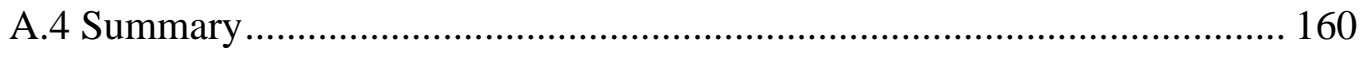

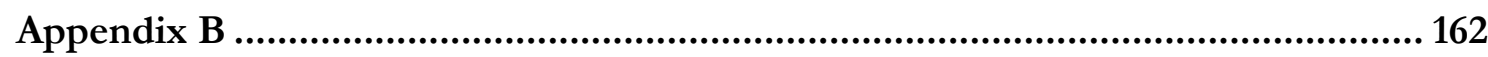

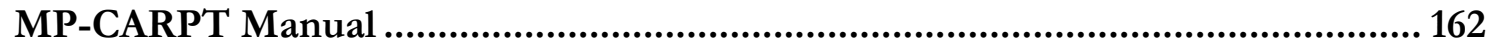

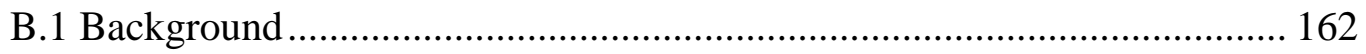

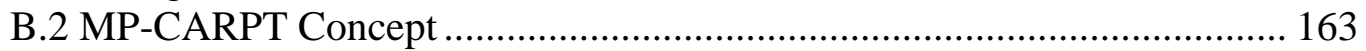

B.3 Proof of Concept Experiments.............................................................. 164

B.4 NaI Detector Readout Concept ........................................................... 167

B.5 Readout Electronics and Data Acquisition ......................................... 168

B.5.1 Timing Filter Amplifier ........................................................................... 169

B.5.2 Pulse Processor Module/NaI readout Module .......................................... 173

B.5.3 MP-CARPT Electronics Arrangement..................................................... 180

B.6 Module Programming and Data Access .............................................. 181

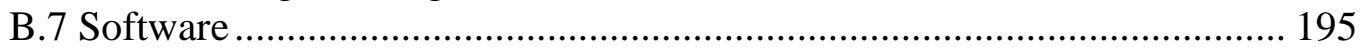

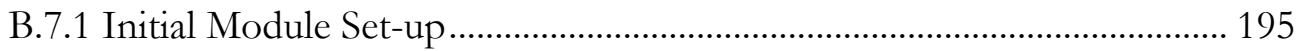

B.7.2 Module Configuration ............................................................................... 195

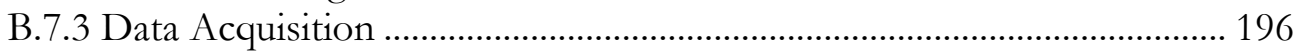

B.7.4 Summary of the Programming Procedure ................................................ 199

B.7.5 Test Mode Operation........................................................................................ 199

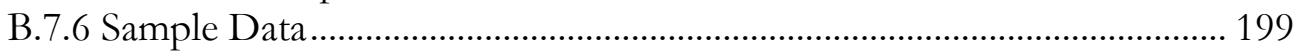

B.8 Problems encountered with MP-CARPT ............................................... 201

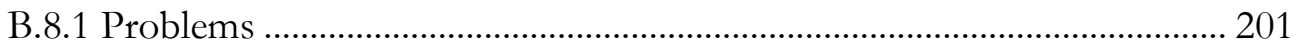

B.8.2 Rectification of Problems ...................................................................... 203

Appendix B.1 .......................................................................................... 205

Example Configuration File. ..................................................................... 205

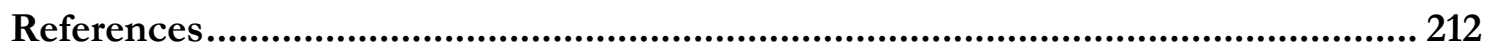

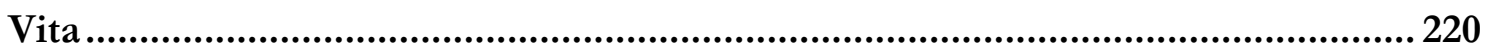

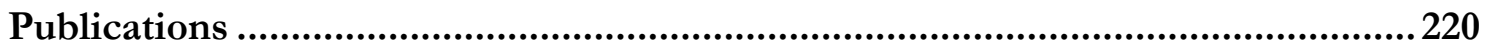

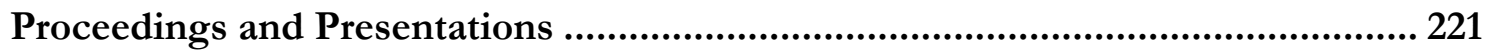




\section{List of Tables}

Table 3.1 List of possible radioactive candidates to be used for MP-CARPT .......................38

Table 3.2 Cost comparison of old and new unit for 16 detectors ...........................................42

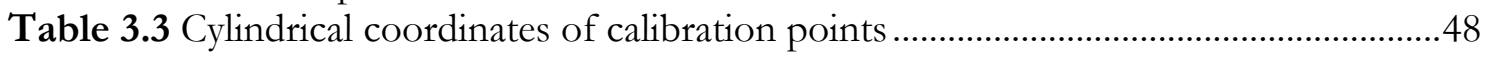

Table 4.1 Details of CARPT experiments for laboratory-scale digester ................................77

Table 4.2 Details of CARPT experiments for pilot-scale digester...........................................81

Table 4.3 Dead or stagnant volumes for laboratory-scale digester configurations ..............93

Table 4.4 Dead or stagnant volumes for pilot-scale digester configurations ..................... 106

Table 4.5 Effect of scale on dead volumes for different digester configurations............... 111

Table 5.1 Simulation details for laboratory-scale (LS) digesters ........................................... 122

Table 5.2 Simulation details for pilot-scale (PS) digesters................................................... 122

Table 5.3 Comparison of dead zone volumes predicted by CFD with experimental data for 6-inch laboratory-scale digester

Table 5.4 Comparison of dead zone volumes predicted by CFD with experimental data for pilot-scale digester

Table A.1 Effect of mixing on performance of laboratory-scale and pilot-scale anaerobic

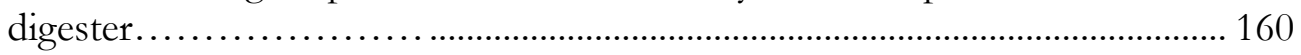

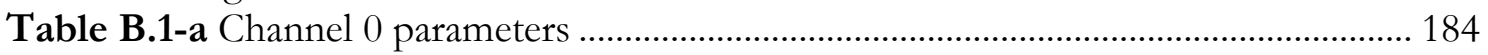

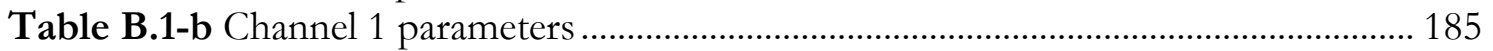

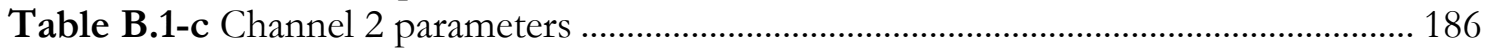

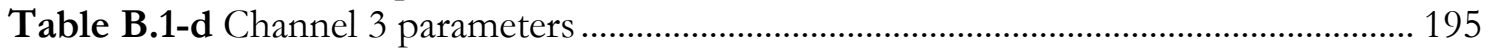

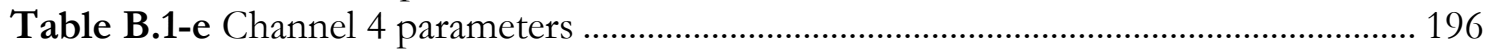

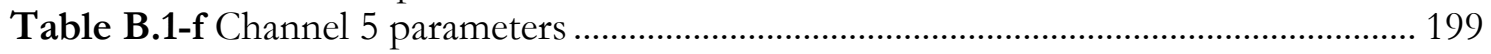

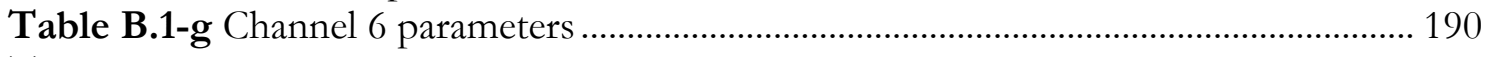

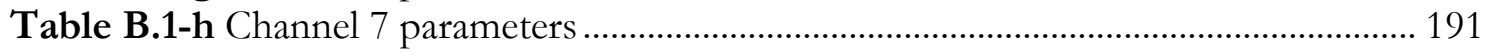

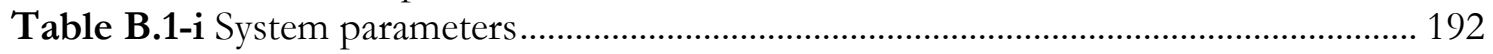

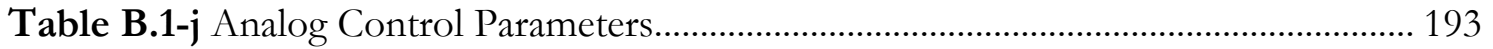

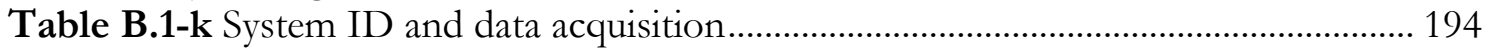

Table B.2 Parameter default values (in hexadecimal) ......................................................... 195

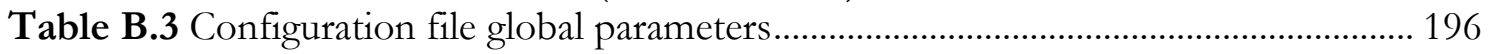

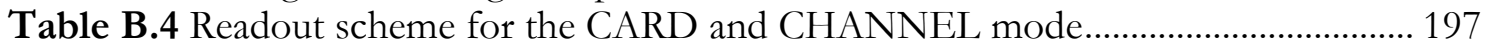

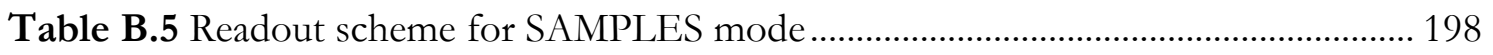

Table B.6 Suggested Parameter values (in hexadecimal)..................................................... 198 


\section{List of Figures}

Figure 1.1 Integration of the objectives and tasks that are considered in this study .............6

Figure 2.1 Three-stage anaerobic digestion mechanism.......................................................11

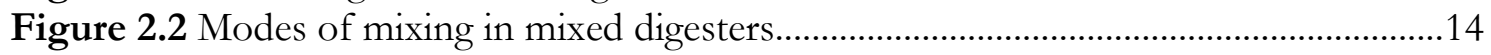

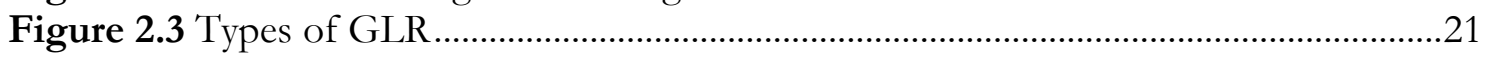

Figure 2.4 Relationship between independent and dependent variables in the

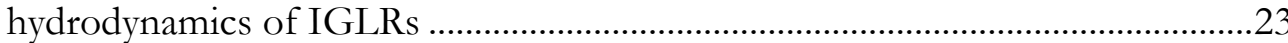

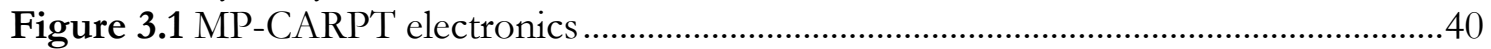

Figure 3.2 MP-CARPT electronics components and connections........................................41

Figure 3.3a Schematic of the arrangement of detectors on detector stand...........................43

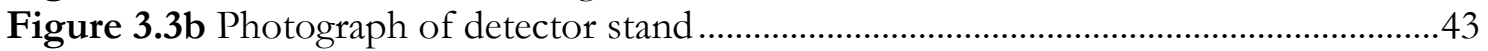

Figure 3.4 Gamma peaks of Sc-46 and Co-60 individually, together and summation of

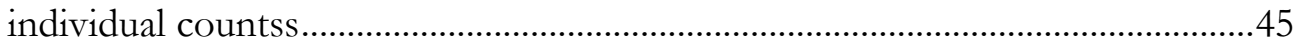

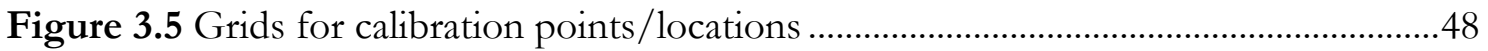

Figure 3.6 (a) calibration plot for Co-60 (b) calibration plot for Sc-46 ................................49

Figure 3.7 Reconstruction algorithm for dual-particle tracking s ..........................................50

Figure 3.8a Reconstructed positions of Co-60 and comparison with original experimental CARPT positions

Figure 3.8b Reconstructed positions of Sc-46 and comparison with original experimental

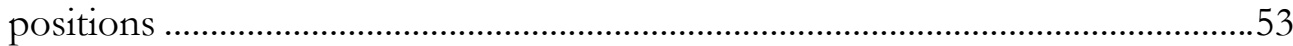

Figure 3.9 Modified reconstruction algorithm for dual-particle tracking ...............................54

Figure 3.10 Calibration plane for detector 1 for low energy window total counts of Co-60 and Sc-46.....

Figure 3.11 Comparison of original Sc-46 locations with reconstructed positions using new

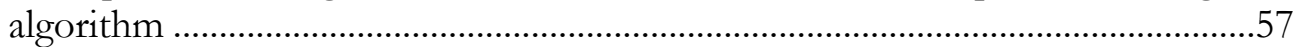

Figure 3.12 Experimental set-up for dual-particle tracking ...................................................58

Figure 3.13a\&b Flow pattern obtained from single particle CARPT unit for Co-60 and Sc46 , respectively.

Figure 3.13c\&d Flow pattern obtained from MP-CARPT unit for Co-60 and Sc-46, tracked separately, respectively.

Figure 3.13e\&f Flow pattern obtained from MP-CARPT unit for Co-60 and Sc-46, tracked together, respectively

Figure 3.14 Comparison of time averaged azimuthally averaged axial velocity at the center of the tank

Figure 3.15 Experimental set-up for tracking two particles with different densities ...........63

Figure 3.16 (a) Co-60, single-particle tracking (b) Co-60, dual-particle tracking (c) Sc-46, single-particle tracking (d) Sc-46, dual-particle tracking..

Figure 3.17 Comparison of axial velocity profiles obtained from single-particle and dualparticle tracking for Co-60 and Sc-46 with different densities .

Figure 3.18 Gamma energy peaks for 3 hypothetical particles $\mathrm{Aa}, \mathrm{Bb}$ and $\mathrm{Cc}$.....................68

Figure 4.1a Digester geometrys........................................................................................73

Figure 4.1b Cross sparger (top view, $\mathrm{D}$ is the diameter of the draft tube)............................73 
Figure 4.2 Photograph of laboratory-scale digester experimental set-up .............................76

Figure 4.3a\&b Digester geometry and cross sparger geometry...............................................

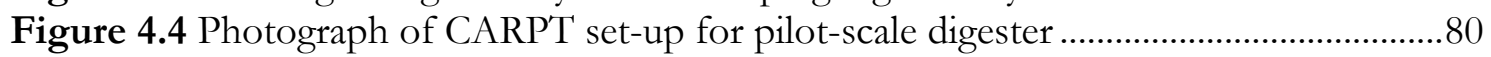

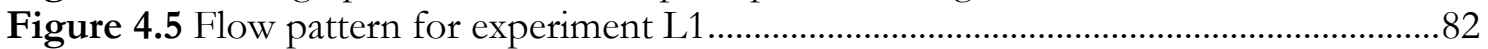

Figure 4.6 Circumferentially averaged axial liquid velocity profile for experiment L1 ........83

Figure 4.7 Effect of gas flow rate on axial liquid velocity (L1 and L2) at the center of the $\operatorname{tank}(\mathrm{z}=11 \mathrm{~cm})$

Figure 4.8 Effect of draft tube diameter on the flow pattern .............................................86

Figure 4.9a Effect of draft tube diameter on the axial liquid velocity at the bottom of the draft tube

Figure 4.9b Effect of draft tube diameter on the axial liquid velocity at the center of the

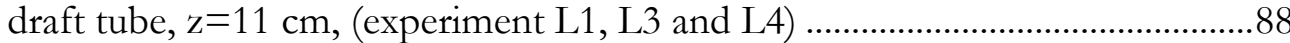

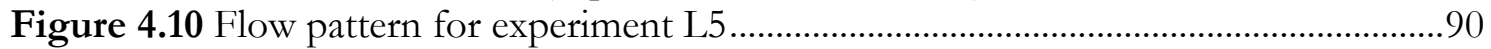

Figure 4.11 Expected effect of sparger geometry and $D / T$ ratio on gas distribution..........91

Figure 4.12 Effect of sparger geometry on axial liquid velocity at center of the tank, $z=11$

$\mathrm{cm}$, (experiment L1 and L5)

Figure 4.13 Dead zone map for Experiment L1 (dark spaces are the dead zones)..........94

Figure 4.14 Axial and radial eddy diffusivities at the center of the tank for experiment ....96

Figure 4.15 Axial diffusivity map for experiment L1 .............................................................96

Figure 4.16 Comparison of axial eddy diffusivities for different digester configurations ...97

Figure 4.17 Flow pattern for experiment $\mathrm{P} 1$ ( $D / T=0.25,4.5 \mathrm{lpm}$, cross sparger) $\ldots \ldots \ldots . .98$

Figure 4.18 Circumferentially averaged axial velocity radial profile for experiment P1 at different axial locations

Figure 4.19 Effect of gas flow rate on axial liquid velocity (experiment P1, P2 and P3) at

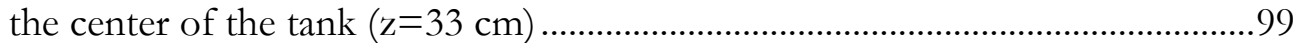

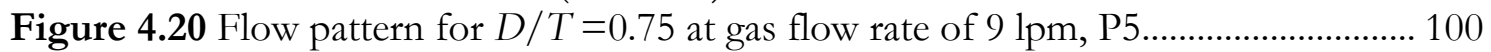
Figure 4.21 Circumferentially averaged axial velocity radial profile for experiment P5...101 Figure 4.22 Effect of draft tube diameter on the axial liquid velocity at the center of the draft tube at different gas flow rates

Figure 4.23 Flow pattern for a single point sparger and $D / T=0.25$ at $9 \mathrm{lpm}(\mathrm{P} 8) \ldots \ldots \ldots .103$

Figure 4.24 Circumferentially averaged axial liquid velocity radial profile for expt P8 .... 103

Figure 4.25 Effect of sparger on the axial liquid velocity at the center of the draft tube at different gas flow rates......

Figure 4.26 Effect of solids concentration on the axial liquid velocity at the center of the

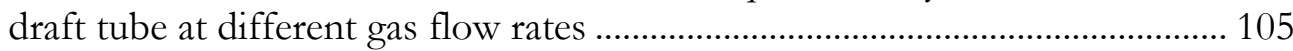

Figure 4.27 Dead zone map for Experiment P2 .

Figure 4.28 Axial and radial eddy diffusivities at the center of the tank for experiment P1, $\mathrm{P} 2$ and $\mathrm{P} 3$

Figure 4.29 Axial diffusivity map for experiment P2 108

Figure 4.30a Effect of gas flow rate on turbulent axial turbulent diffusivities for configurations with $D / T$ of 0.75 and cross sparger.

Figure 4.30b Effect of gas flow rate on turbulent axial turbulent diffusivities for configurations with $D / T$ of 0.25 and single point sparger

Figure 4.31 Comparison of axial eddy diffusivities for different digester configurations at gas flow rate of $9 \mathrm{lpm}$. 
Figure 4.32 Comparison of axial liquid velocity radial profile for laboratory scale and pilot scale digesters.....

Figure 4.33 Comparison of axial liquid velocity radial profile for laboratory scale and pilot scale digesters

Figure 4.34 Comparison of axial liquid velocity radial profile for laboratory scale and pilot scale digesters

Figure 4.35a Comparison of magnitudes of axial liquid velocity for laboratory scale and pilot scale digesters

Figure 4.35b Comparison of magnitudes of axial liquid velocity for laboratory scale and pilot scale digesters

Figure 5.1 Comparison of (a) simulated flow pattern (LS1) and (b) flow pattern obtained from CARPT (L1) for 6-inch digester

Figure 5.2 Comparison of simulated axial liquid velocity profile for Simulation LS1 with experimental CARPT data (L1) ..................................... 195

Figure 5.3 Gas hold up distribution predicted by CFD for Simulation LS1 ...................... 128

Figure 5.4 Effect of gas flow rate on axial liquid velocity at the middle height of tank for $D / T=0.25$, cross sparger.

Figure 5.5 Effect of gas flow rate on axial liquid velocity at the middle height of tank for $D / T=0.25$, single point sparger

Figure 5.6 Effect of $D / T$ ratio on flow pattern with cross sparger at $1 \mathrm{lpm}$.. 132

Figure 5.7 Effect of $D / T$ ratio on gas holdup distribution with cross sparger at $1 \mathrm{lpm} . .133$

Figure 5.8 Effect of $D / T$ ratio on flow pattern with single point sparger at $1 \mathrm{lpm} . . . \ldots \ldots . . . .134$

Figure 5.9 Effect of $D / T$ ratio on gas holdup distribution with cross sparger at $1 \mathrm{lpm} . .134$

Figure 5.10a Effect of $D / T$ ratio on liquid velocity with cross sparger and $1 \mathrm{lpm}$ gas flow

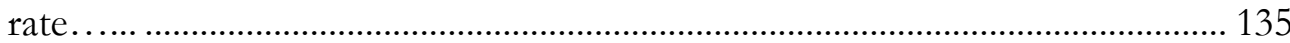

Figure 5.10b Effect of $D / T$ ratio on liquid velocity with single point sparger $1 \mathrm{lpm}$ gas

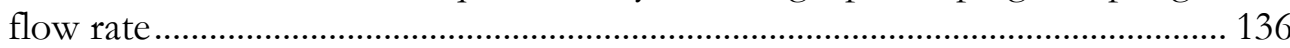

Figure 5.11 Effect of sparger geometry for different $D / T$ ratios on liquid velocity at gas flow rate of $1 \mathrm{lpm}$

Figure 5.12 Comparison of (a) simulated flow pattern (PS2) and (b) flow pattern obtained from CARPT (P2) ................................................. 140

Figure 5.13 Pilot-scale, comparison of simulated axial liquid velocity profile for simulation PS2 with experimental CARPT data ................................................................... 140

Figure 5.14 Gas hold up distribution predicted by CFD for Simulation PS2 .................... 142

Figure 5.15a Effect of gas flow on axial liquid velocity at the middle height of tank for $D / T=0.25$, cross sparger.

Figure 5.15b Effect of gas flow on axial liquid velocity at the center of tank for $D / T=0.25$ single point sparger

Figure 5.16 Effect of $D / T$ ratio on liquid velocity at $4.5 \mathrm{lpm}$ gas flow rate ..................... 143

Figure 5.17 Effect of sparger geometry for $D / T$ ratio of 0.25 on liquid velocity at different gas flow rates.

Figure 5.18a Effect of scale on dimensionless axial liquid velocity profile.............. 146

Figure 5.18b Effect of scale on dimensionless axial liquid velocity profile......................... 199

Figure 5.18c Effect of scale on dimensionless axial liquid velocity profile ....................... 199

Figure 5.18d Effect of scale on dimensionless axial liquid velocity profile........................ 147 
Figure 5.19 Effect of scale on axial liquid velocity profile

Figure A.1 Comparison of cumulative methane production rates for laboratory-scale and pilot-scale digesters

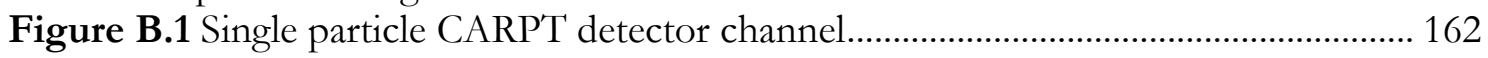

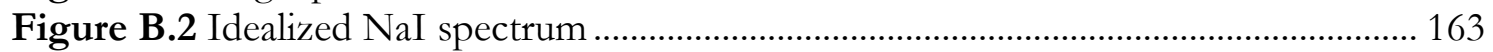

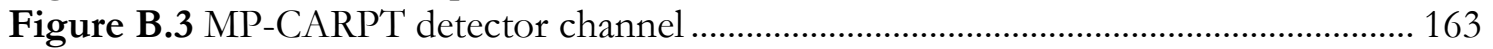

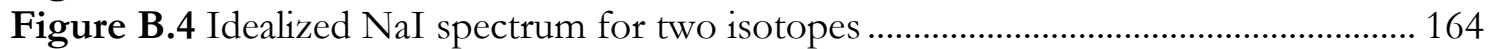

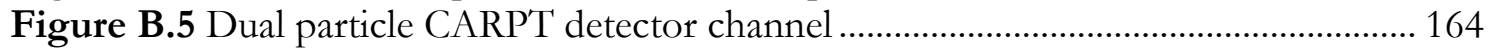

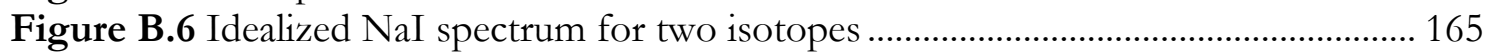

Figure B.7 Dual-particle CARPT electronics showing NIM and CAMAC elements ...... 166

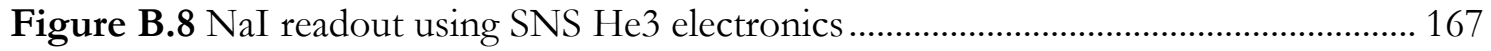

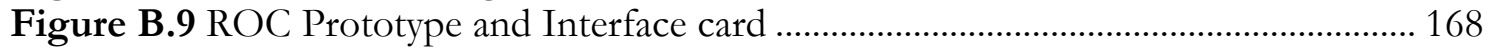

Figure B.10 Spectrum of Potassium-40 obtained using prototype electronics................... 168

Figure B.11 MP-CARPT arrangement using NIM and CPCI format NaI readout

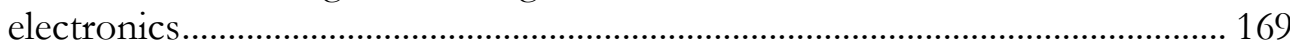

Figure B.12 Block diagram of shaping amplifier channel ................................................. 170

Figure B.13 Circuit diagram of one shaping amplifier channel.......................................... 170

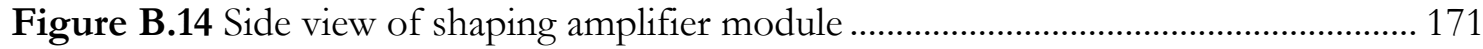

Figure B.15 Shaping amplifier module front and read panels........................................... 172

Figure B.16 Close-up view of circuit board showing adjustment locations ........................ 172

Figure B.17 Block diagram of the pulse processor module .............................................. 174

Figure B.18 Analog electronics for one channel .............................................................. 175

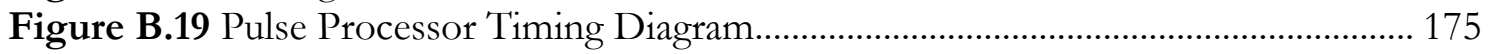

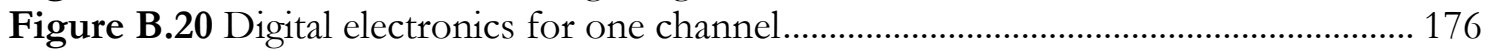

Figure B.21 Timing diagram for pulse acquisition in a channel......................................... 177

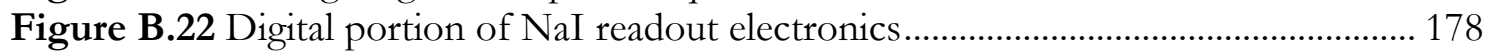

Figure B.23 CPCI crate and single board computer........................................................... 179

Figure B.24 Module Controller FPGA block diagram...................................................... 179

Figure B.25 MP-CARPT electronics showing NIM and CPCI elements. ......................... 182

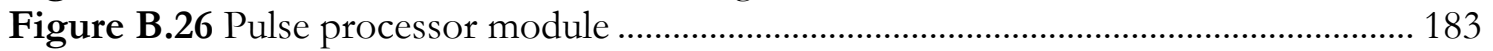

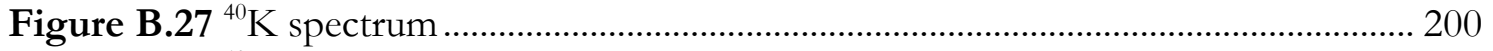

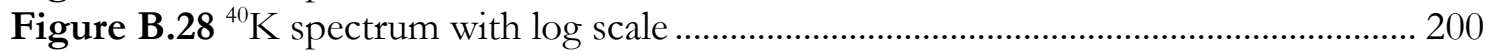

Figure B.29 Shaper amplifier noise .................................................................................... 199

Figure B.30 Shift in gamma ray energy spectrum of the ${ }^{60} \mathrm{Co}$ and ${ }^{46} \mathrm{Sc}$ at two different

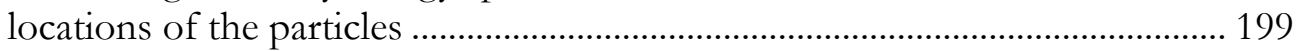

Figure B.31 Photo peaks of ${ }^{46} \mathrm{Sc}$ and ${ }^{60} \mathrm{Co}$ taken with individual source both separately and

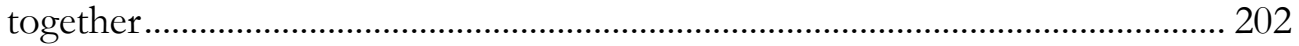

Figure B.32 Individual Gamma peaks of Sc-46 and Co-60 placed at different locations.199 Figure B.33 Gamma peaks of Sc-46 and Co-60 individually and together, and summation of individual counts 199 


\section{Nomenclature}

\begin{tabular}{|c|c|c|}
\hline a & Constant & - \\
\hline A & Cross sectional area & $m^{2}$ \\
\hline $\mathrm{D}$ & Draft tube diameter & $m$ \\
\hline $\mathrm{d}$ & Distance & $m$ \\
\hline d & Diameter of bubble & $m$ \\
\hline $\mathrm{f}$ & Function & - \\
\hline$g$ & Acceleration due to gravity & $m / s^{2}$ \\
\hline$g$ & Gravity vector & $\mathrm{m} / \mathrm{s}^{2}$ \\
\hline $\mathrm{H}$ & Liquid level in the reactor & $m$ \\
\hline h & Draft tube height & $m$ \\
\hline h & Specific static enthalpy & $J / k g$ \\
\hline K & Constant & - \\
\hline $\mathrm{k}$ & Kinetic energy & dynes $/ \mathrm{cm}^{2}$ \\
\hline $\mathrm{k}$ & Turbulent kinetic energy & $\mathrm{m}^{2} / \mathrm{s}^{2}$ \\
\hline M & Interfacial force term & $N / m^{3}$ \\
\hline $\mathrm{N}$ & Number & - \\
\hline $\mathrm{N}_{\mathrm{P}}$ & Number of phases & \\
\hline $\mathrm{P}$ & Pressure & $N / m^{2}$ \\
\hline $\mathrm{p}$ & Static pressure & $N / m^{2}$ \\
\hline $\mathrm{p}_{\text {ref }}$ & Reference pressure & $N / m^{2}$ \\
\hline $\mathrm{p}_{\text {tot }}$ & Total Pressure & $N / m^{2}$ \\
\hline Q & Volumetric flow rate & $m^{3} / s$ \\
\hline$r_{\alpha}$ & Volume fraction of phase $\alpha$ & \\
\hline $\mathrm{R}_{0}$ & Universal gas constant & l.atm $/ \mathrm{kmol} .{ }^{\circ} \mathrm{K}$ \\
\hline $\mathrm{S}_{\mathrm{M}}$ & Momentum source & $N / m^{3}$ \\
\hline $\mathrm{S}_{\mathrm{MS}}$ & Mass source & $\mathrm{kg} / \mathrm{m}^{3} \mathrm{~s}$ \\
\hline $\mathrm{T}$ & Tank/ reactor diameter & $m$ \\
\hline $\mathrm{t}$ & Time & Sec \\
\hline $\mathrm{t}$ & Time & $S$ \\
\hline $\mathrm{T}$ & Temperature & ${ }^{\circ} K$ \\
\hline $\mathrm{U}$ & Superficial velocity & $m / s$ \\
\hline $\mathrm{U}$ & Velocity & $\mathrm{m} / \mathrm{s}$ \\
\hline $\mathrm{u}$ & Fluctuating velocity component in turbulent flow & $\mathrm{m} / \mathrm{s}$ \\
\hline u' & Fluctuating velocity & $m / s$ \\
\hline $\mathrm{V}$ & Volume & $m^{3}$ \\
\hline $\mathrm{w}$ & Molecular weight & $\mathrm{kg} / \mathrm{kmol}$ \\
\hline
\end{tabular}




\section{Greek Symbols}

$\begin{array}{lll}\rho & \text { Density } & \mathrm{Kg} / \mathrm{m}^{3} \\ \varepsilon & \text { Holdup } & -\end{array}$

\section{Subscripts / Superscripts}

$\theta$

avg

b

c

ct

d

g

G

int

L

$\max$

$\min$

$\mathrm{r}$

S

Z
Azimuthal

Average

Bottom

Circulation

Circulation time

Downcomer

Gas

Gas

Interval

Liquid

Maximum

Minimum

Riser/radial

Solid

Axial

\section{Acronyms}

$\mathrm{AD}$

ADrs

ALR

BOD

CARPT

CFD

Co-60

COD

CT

CTD

CTDF

EALR

GC
Anaerobic digestion

Anaerobic digesters

Airlift loop reactor

Biological oxygen demand

Computer automated radioactive particle tracking

Computational fluid dynamics

Cobalt with mass number 60 (radioactive)

Chemical oxygen demand

Computed tomography

Circulation time distribution

Circulation time distribution function

External airlift loop reactor

Gas chromatography 
HRT Hydraulic retention time

IALR Internal airlift loop reactor

LS Laboratory-scale

MP-CARPT Multiple particle CARPT

NIM Nuclear instrument modules

ORNL Oak Ridge National Laboratory

PCI Peripheral component interconnect

PMT Photo multiplier tube

PS Pilot-scale

SBCR Slurry bubble column reactor

Sc-46 Scandium with mass number 46 (radioactive)

TFA

TS

Timing filter amplifier

TVS

Total solids

Total volatile solids

VFA Volatile fatty acids 


\section{Acknowledgements}

Five years of graduate life felt like five decades at one point, but now that it's over, they seem shorter than a moment. Long or short, stressful or joyous, tedious or enlightening, depressing or motivating, these years will always be remembered. I am thankful to everyone and everything that accompanied me in my journey towards the doctorate.

My advisor, Prof. Muthanna Al-Dahhan, deserves first acknowledgement for the support, advice, help, and enthusiasm that he showered on me. He introduced me to the world of multi-tasking and made me an inhabitant there. I thank him with a deep sense of gratitude for showing me the path towards my destination.

I also want to take this opportunity to thank Prof. Milorad Dudukovic and Prof. P. A. Ramachandran for their guidance and help throughout my studies and also for being on my defense committee. I also want to thank my other committee members, Dr. Largus Angenent, Dr. Daniel Giammar, and Dr. David Depaoli for investing their precious time to read my thesis and offering their thoughtful comments and suggestions.

I wish to acknowledge the DOE for its financial support and ANSYS for providing the CFX software. I also wish to thank the whole Oak Ridge National Laboratory group, including Dr. Alan Wintenberg, Dr. Abhijeet Borole, Dr. Chuck Alexander, Dr. Thomas Klasson, Dr. David Depaoli, and others for their contribution towards the development of the new MP-CARPT and for conducting digester experimental runs. I am thankful to Dr. Khursheed Karim, who provided lot of help and guidance during the initial and intermediate stages of this project. I wish to thank Ryan Mackin and Rebecca Hoffmann for helping me with the experimental work.

This journey would not have been the same without my fellow passengers, the CREL students and scholars. Although it is not possible to mention every name, a few need 
Appendix-2: Vesvikar(2006), D.Sc. Thesis

special recognition. Debangshu has always been there to brainstorm with me and offer very much needed criticism, as well as meaningless but stress-relieving chit-chat. Rajneesh, Satish, Huping, and Ashfaq always offered their help in spite of their busy schedules. My other friends Saurabh, Mao, RC, Vidya, Rahul, Radmila, Lu Han and many others have made a great contribution by their encouraging presence and delightful company.

I cannot thank enough the department secretaries, Rose, Mindy, Jean, Ruth and Angela, for their timely help, always offered with a big smile and warm greetings. I wish to thank Steve Picker, Jim Linders, and Pat Harkins for their help with the fabrication, design and the setup of the experiments. I also wish to thank Prof. Jim Ballard from the Technical Writing Center for providing me with help and guidance on writing. I cannot forget my TA experience and all my students, who always made me feel like a student myself, and helped me enjoy the teaching process while I was learning with them. Three of them, Amy, Kristin and Rebecca will always be the "Charmed" (or the charming) ones.

This acknowledgement would be incomplete without the mention of the friends who were there for me at every turn to guide me and to hold me at every ups and downs of this journey. Without their support, encouragement, love or sheer presence, this would have never been possible. My roommates and dearest friends, Swapnil and Mukund; my loving friends, Shilpi, Sukanya and Tanika; my buddies Samir, Anand and Abhishek; my American friends, Angie and Ed; the "old is gold" ones, Sanket, Nandu, Medha, Subodh, Sagar, Vivek, and Sreepriya; the best one, Rahul Mahajan; my soulmate, Sarita; my mentor, Prof. J.B. Joshi; the only one, Sharada Vahini; all my neighbors and friends from India, my grandma, aunties and uncles, cousins, nephews and nieces, and innumerable others share a special place in my heart, my life and my accomplishments.

There are no words to express my feeling towards my family - Deepa, Dada, Tai, Aaee and Baba. Their selfless love and unshaken trust in me has brought me this and every other success in my life. My dear sister has been a constant source of inspiration to face obstacles bravely, with a smile. My father sacrificed everything he had to provide me with anything I 
wanted with the only expectation of seeing me successful. Every time I see my mom, my faith becomes stronger in the saying "God could not be everywhere, so he created mother." I owe everything I am and I will be to my mother. Thanking my family would be disrespectful, so I ask for their blessings.

Finally, I bow down in front of Him and pray that He gives the peace, love, knowledge, joy and fulfillment to everyone that he has bestowed upon me.

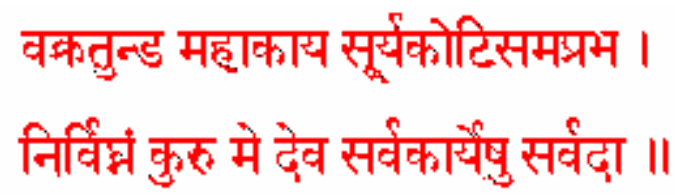

Vakratunda Mahakaaya Suryakoti Samaprabha |

Nirvighnam Kuru Mey Deva Sarva Kaaryeshu Sarvada ||

Meaning: The Lord Ganesh, with the curved trunk and a mighty body, who has the luster of a million suns. I pray to thee Oh Lord, to remove the obstacles from all the actions I intend to perform.

Mehul S. Vesvikar

Washington University in St. Louis

December 2006 


\section{Chapter 1}

\section{Introduction}

\subsection{Introduction and Motivation}

The growth and concentration of the livestock industries provide a large source of affordable and renewable energy with a need to safe disposal of the large quantities of animal waste (manure) generated at dairy, swine, and poultry farms. According to 1997 census, in the United States itself over 900 million tons of such waste is produced every year (USDA, 2003). The energy equivalent of this waste is approximately 100 megatons of coal per year. Unsafe and improper disposal of decomposable animal waste causes major environmental pollution problems, including surface and groundwater contamination, odors, dust, and ammonia leaching. There is also threat from methane emissions, which contribute to the greenhouse effect. The increasing growth of animal industries has resulted in the formulation of new laws and regulations governing safe handling and disposal of animal waste. A survey of dairy and swine farms in the country reaffirmed that Anaerobic Digestion (AD) is a technology with considerable potential. Ignoring caged layer poultry, about 426 metric tons of methane is potentially recoverable from 3,000 dairy and swine farms in 19 states of the United States (Lusk, 1998).

Over the past 25 years, AD processes have been developed and applied to a wide array of industrial and agricultural wastes to reduce pollution and recover methane (Speece, 1996; Ghosh, 1997). AD is a process of conversion of biomass to biogas, a mixture of 
methane and carbon dioxide, involving several biological steps occurring slowly (Parkin and Owen, 1986), more details are given in Chapter 2. Byproduct of AD is a semisolid residue which can be used as a fertilizer.

$\mathrm{AD}$ has been implemented on only a small percentage of farms in the United States. One of the important reasons for this is the high rate of failure of farm based digesters, (Lusk, 1998). These high failure rates can be attributed mostly to poor design (Lusk, 1998) and absence of a well acclimated microbial community (Angenent et al., 2002).

The performance of ADrs is affected primarily by the retention (residence) time of digestible slurry (substrate) in the digester and the degree of contact between incoming substrate and a viable bacterial population. These parameters are functions of the hydraulic regime (mixing) in the reactors. Mixing in the digester is required to distribute organisms, substrate, and nutrients uniformly, to transfer heat, and to maintain uniform $\mathrm{pH}$ and temperature. Thus, mixing is regarded as essential in ADrs (Meynell, 1976; Sawyer and Grumbling, 1960). Furthermore, mixing aids in particle size reduction as digestion progresses and in the removal of gas from the mixture. Mixing is also required to prevent stratification and scum formation. In short, adequate mixing provides a uniform environment, one of the keys to good digestion (Parkin and Owen, 1986).

The effects of mixing on the $\mathrm{AD}$ process are not well understood and the information available is contradictory. This discrepancy and contradiction regarding the role of mixing in ADr performance needs to be resolved through carefully planned experimentation. Karim et al. (2005a and 2005b) concluded from a series of their extensive laboratory-scale performance experiments that pilot-scale experiments are required to reach unambiguous conclusions. Mixing can be accomplished by a variety of mechanical mixers, by recirculation of the digester contents, or by recirculation of the produced biogas using recirculation pumps. Gas-mixed digesters are easy to operate and require comparatively less energy for mixing (Casey, 1986; Kondandt and Roediger, 1977; Lee et al., 1995; Morgan and Neuspiel, 1958). Therefore, by comparing the 
performance of identically operated gas-mixed ADrs in both mixed and unmixed modes, the true effect of mixing was evaluated (Appendix A).

The performance of an ADr is affected by mixing at larger scales of operation, thus the knowledge of the hydrodynamics is important in the design and scale-up of such ADrs. The hydrodynamics are in turn affected by the geometry and the physical properties of the system, along with operating conditions. The digesters mixed by recirculation of biogas are commonly referred as gaslift internal loop reactors in the literature. In these processes, the gaslift loop reactors are extensively used in industrial chemical and biochemical applications. These reactors are equipped with a concentric draft tube and gas sparger to create liquid movement. These reactors are usually tall, with slurry level to reactor diameter $(L / D)$ ratio normally greater than two. Thus vast information of hydrodynamics of these reactors is available in literature. However, the anaerobic digesters have low $L / D$ ratio, approximately equal to one to two. The hydrodynamic information of low $L / D$ ratio gaslift loop reactors is not available in literature, although the effect of $L / D$ on the hydrodynamics is very important. Therefore studying the hydrodynamics of low $L / D$ ratio gaslift internal loop reactors is necessary. Some of the other important parameters which can affect the mixing pattern inside such type of digester includes, biogas recycling rate, bottom clearance of the draft tube, slope of the hopper bottom, draft tube to tank diameter ratio, position and type of gas sparger and solids loading rate.

ADrs are highly opaque systems due to the presence of the solids and the dense color of the biomass, which creates problems in using common experimental techniques to reveal hydrodynamic information. Hence, advanced non-invasive techniques such as Computer Automated Radioactive Particle Tracking (CARPT) and Computed Tomography (CT) have to be used to "see" inside the digester, (Karim et al., 2004).

A wide variety of solids is encountered in ADrs treating animal waste, such as husk, straw, and fibers coming from the feed, and sand particles, saw dust, wood 
shavings/chips, rice hulls, etc. from bedding material. Due to these solids, regions of very slow flow or even solids settling in the digester are highly possible. If CARPT is applied in such situations, the single radioactive particle can represent only one type of solids, and slow flows will reduce the data collection rate. In addition, solids settling can bring data collection to a halt. The data collection rate, representation of more than one solids type, and ability to deal with settling can be greatly improved by the introduction of multiple tracers that can be tracked simultaneously. Furthermore, Multiple-particle tracking (MP-CARPT) can offer other important advantages, such as the capability to simultaneously track the motion of particles of different sizes, shapes, densities. It can also determine segregation of particles and probe particle interactions. The techniques can be of valuable use in other process applications as well, such as slurry bubble columns, gas-solid and gas-liquid-solid fluidized beds, solid-liquid and gas-liquid-solid stirred tanks, etc.

Although advanced non-invasive techniques are very useful in understanding the hydrodynamics of digesters, these techniques are time consuming, expensive, and are not always available. Thus, it is hard to use these experiments to characterize all digester configurations and operating conditions. As an alternative, Computational Fluid Dynamics (CFD) code, once it is validated, proves to be a valuable and efficient tool to understand and evaluate the hydrodynamics of digesters. For multiphase systems, like ADrs, CFD can be used with confidence for design and scale-up only after validation with the experimental data, which can be obtained by CARPT and CT.

Advanced non-invasive experimental techniques and computational modeling together can provide valuable information about the digester hydrodynamics. Further, if this information is intended to be used with confidence for the design of ADrs, then scaleup studies are necessary. A general rule in industry is that the scale of operation has a significant impact on the performance of reactor and process equipment, as the mixing scales are affected by scale of the operation. However, the effects of digester scale on digester mixing pattern/intensity have not been studied and quantified. Thus, 
experimentation on the pilot scale is required to test the applicability of the laboratory scale results at large scales, in order to use this data efficiently for design of commercial/farm scale ADrs.

To provide information regarding the effective design and scale-up of ADrs and to evaluate the feasibility of different design and operating conditions, further research related to the mixing and hydrodynamics of ADrs is necessary. Advanced non-invasive experimental techniques computational modeling approaches performed on laboratory and pilot scale digesters can fill the missing gaps in the literature regarding the hydrodynamics of anaerobic digesters.

\subsection{Objectives}

The primary objectives of this study are to advance the understanding of Anaerobic Digester (ADr) hydrodynamics, to evaluate the effects of various operating conditions, design and scale up parameters on their hydrodynamics and to develop a new technique based on MP-CARPT. Figure 1.1 shows the integration of the overall objectives and tasks that are considered in this work. Regarding the performance study, the details of results and findings are reported in Appendix A. It has been shown that the performance of lab-scale digesters is not affected by the mixing provided by gas recirculation, while the performance of pilot-scale digesters is significantly affected by the mixing provided by gas recirculation. The lab-scale digesters showed better performance than the pilot plant scale digesters in terms of methane production. The findings of this study suggest that only large scale digesters should be considered for reliable evaluation of the effect of design and scale-up parameters on the performance of the digesters while small scale digesters could be useful for bioreaction rate related studies. Since, this work does not represent the main theme of this study yet it is related to it, it has been reported as Appendix A. Therefore, the other components of Figure 1.1 are reported as part of the thesis body which represents the overall objectives of this work. 


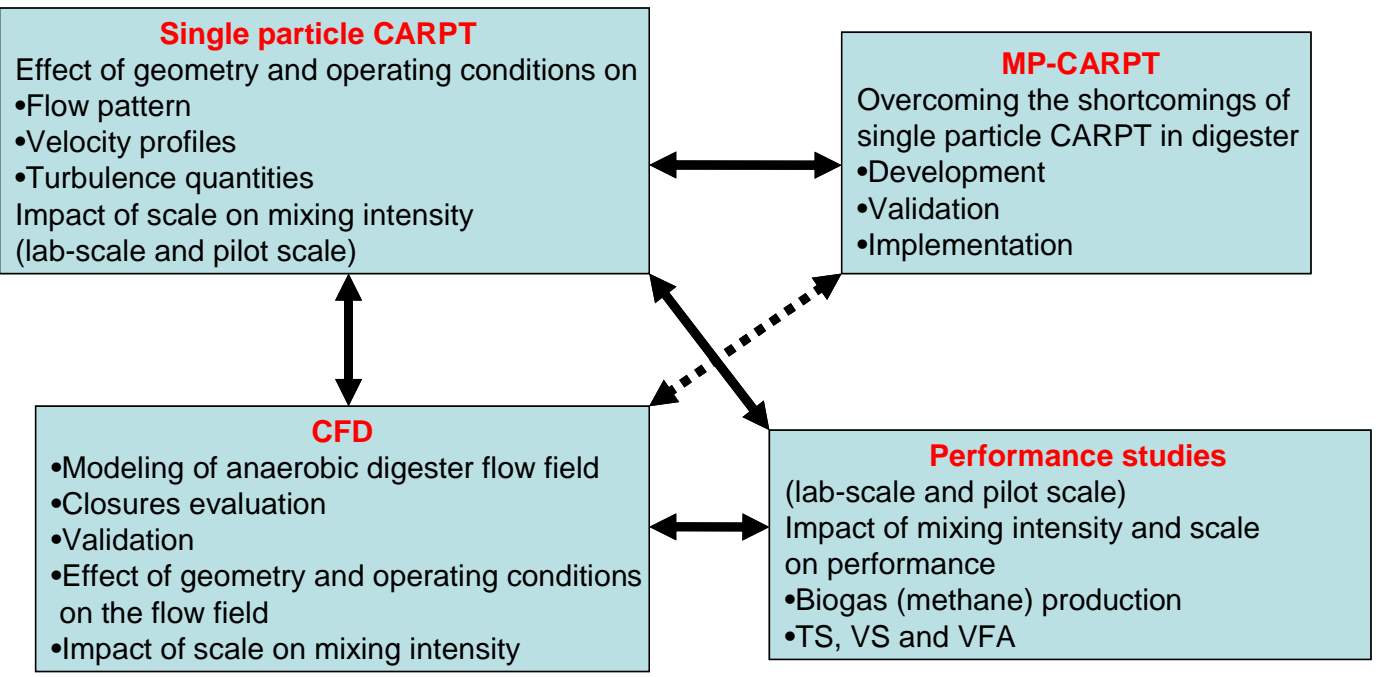

Figure 1.2 Integration of the objectives and tasks that are considered in this study

The specific techniques and procedures of these studies are described in the following sections.

\subsubsection{Development and Validation of MP-CARPT}

With the help of Oak Ridge National Laboratory (ORNL), single particle tracking technique currently used in the Chemical Reaction Engineering Laboratory (CREL) was advanced to:

- Develop a new and unique multiple-particle tracking technique (MP-CARPT), where up to eight radioactive tracer particles can be tracked simultaneously.

- Evaluate the current particle reconstruction methodology and develop new one for MP-CARPT technique.

- Modify and develop new electronics to reduce the overall cost of the equipment and electronics and to simplify the operation as compared to the original single particle CARPT.

- Validate and implement this technique. 


\subsubsection{Single Particle CARPT and CFD Studies}

The objectives of single particle CARPT and CFD are as follows:

- $\quad$ Study the flow pattern and detailed fundamental hydrodynamics of ADrs at laboratory and pilot scales using real cow manure. The investigated digesters are equipped with a draft tube and a sparger to allow mixing by gas recirculation. The CARPT data will be also used as a benchmark for evaluation and validation of CFD models.

- Investigate the effect of gas flow rate, shape of tank bottom, draft tube diameter to tank diameter ratio, type of sparger, solids content of the slurry, and scale on the mixing pattern and hydrodynamics of the digester.

- $\quad$ Evaluate CFD models and closures using CFX Code (Ansys Inc.) for simulating the flow pattern and hydrodynamics of the studied anaerobic digesters.

\subsection{Thesis Organization}

Chapter 2 provides a literature survey of anaerobic digestion and the performance and design of anaerobic digesters. It also reviews the hydrodynamics of internal gaslift loop reactors. Chapter 3 introduces the new MP-CARPT technique, and describes its validation and implementation. Chapter 4 outlines CARPT investigations for two scales of digester and also discusses the findings of the experiments related to hydrodynamics and scale-up. Chapter 5 outlines the CFD efforts to simulate the flow in ADrs. Chapter 6 summarizes the research findings and makes recommendations for future work. The appendices consist of the following, results of the performance study of lab-scale and pilot-scale digesters and the MP-CARPT manual. 


\section{Chapter 2}

\section{Background}

\subsection{Introduction}

The importance of anaerobic digestion and the need for a better understanding of the design and scale-up of anaerobic digesters have been discussed in Chapter 1. The role of mixing and hydrodynamics in the performance of anaerobic digesters has also been introduced. The aim of this chapter is to briefly document the literature related to the anaerobic digestion process for bioenergy production, the design of anaerobic digesters, and their hydrodynamics.

\subsection{Anaerobic Digestion}

\subsubsection{Pollution, Renewable Energy and Anaerobic Digestion}

Growth and concentration of the livestock industry in the US create opportunities for the proper disposal of the large quantities of manures generated at dairy, swine, and poultry farms. The potential pollutants from decomposing livestock manures are Biological Oxygen Demand (BOD), pathogens, nutrients, methane, and ammonia emissions. The major pollution problems associated with these wastes are surface and groundwater contaminations and surface air pollution caused by odors, dust and 
ammonia. There is also concern about the contribution of methane emissions to global climate change.

The methane contained in biogas is a potent greenhouse gas, 21 times more harmful than carbon-dioxide in causing the greenhouse effect. The Environmental Protection Agency (EPA) has estimated that the atmospheric concentration of methane, has more than doubled in the past two centuries, and is now is increasing 1\% per year (Lusk, 1998).

Consequently, manure management systems that enable pollution prevention are necessary. Moreover, the EPA passed the Concentrated Animal Feeding Operations (CAFOs) Rule in December 2003, requiring the Nation's largest CAFOs to acquire Clean Water Act permits. This forces CAFO's to develop manure management plans that ensure proper management and land application of manure (Hoffmann, 2005; Moser and Roos, 1997).

Several animal waste treatments are practiced, including chemical treatment, incineration, aerobic biological fermentation and Anaerobic Digestion (AD). AD offers several advantages over other waste treatment processes (Chynoweth et al., 1993). AD converts odor-causing materials (food source for methane-producing bacteria) in organic matter to methane as a bioenergy source and carbon-dioxide, which are odorless. Odor reduction using Anaerobic Digesters (ADrs) can be a cost-effective alternative compared to aeration, chemicals. or enzyme treatments. In fact, the biogas produced can be a by-product of a system designed for odor control. Odor control is the main reason livestock farmers have installed ADrs in the US.

With better management practices, methane from manure can be a clean, renewable source of energy. Depending on the digestion process, the methane content of biogas is generally between $55 \%-80 \%$. The remaining composition is primarily carbon dioxide, 
with trace quantities (0-15,000 ppm) of corrosive hydrogen sulfide and water (Lusk, 1998).

The animal manure is rich in nitrogen, but the nitrogen is bound up in proteins and is not readily available to plants without undergoing biological conversion. During the digestion process, much of the organic bound nitrogen is released as ammonia. The digestant left after the digestion process has combined nitrogen, phosphorus, and potassium percentages ranging from $3-4.5 \%$ on a dry matter basis and can be spread directly onto farmland for its nutrient value. Due to the lower viscosity of digestant as compared to the raw manure, it penetrates faster into the soil. Soil ammonium adsorption is also high, hence, washout is low. Thus, the by-product of the AD process proves to be a very good soil fertilizer.

Biomass is a form of renewable energy, unlike fossil fuels such as oil, natural gas and coal. Vanishing reserves of fossil fuels and increasing demand for energy make it critical to consider the use of renewable energy. Based on life-cycle cost analysis of proven anaerobic digesters (ADrs) producing biogas (bioenergy) and nutrient rich by-product slurry, an avoidable livestock production liability can become a profit-making asset.

\subsubsection{Anaerobic Digestion Mechanism}

The primary objective of anaerobic digestion is the stabilization of organic matter, with a concurrent reduction in odors, pathogen concentration, and mass of solid organic material. This is accomplished through biological conversion of organics to methane and carbon dioxide in an oxygen-free environment. Conversion of organics to methane involves five groups of bacteria carrying out rather specific reactions. Conceptually, anaerobic digestion can be represented in a three step process for simplicity (Hill, 1982; Parkin and Owen, 1986). The three-stage process (shown in Figure 2.1) involves: (1) hydrolysis, liquefaction and fermentation; (2) hydrogen and acetic acid formation; (3) methane formation. 


\section{Hydrolysis, Liquefaction and Fermentation}

Hydrolysis and liquefaction of complex and/or insoluble organics are necessary to convert these materials to a size and form that can pass through bacterial cell walls for use as energy or nutrient sources.

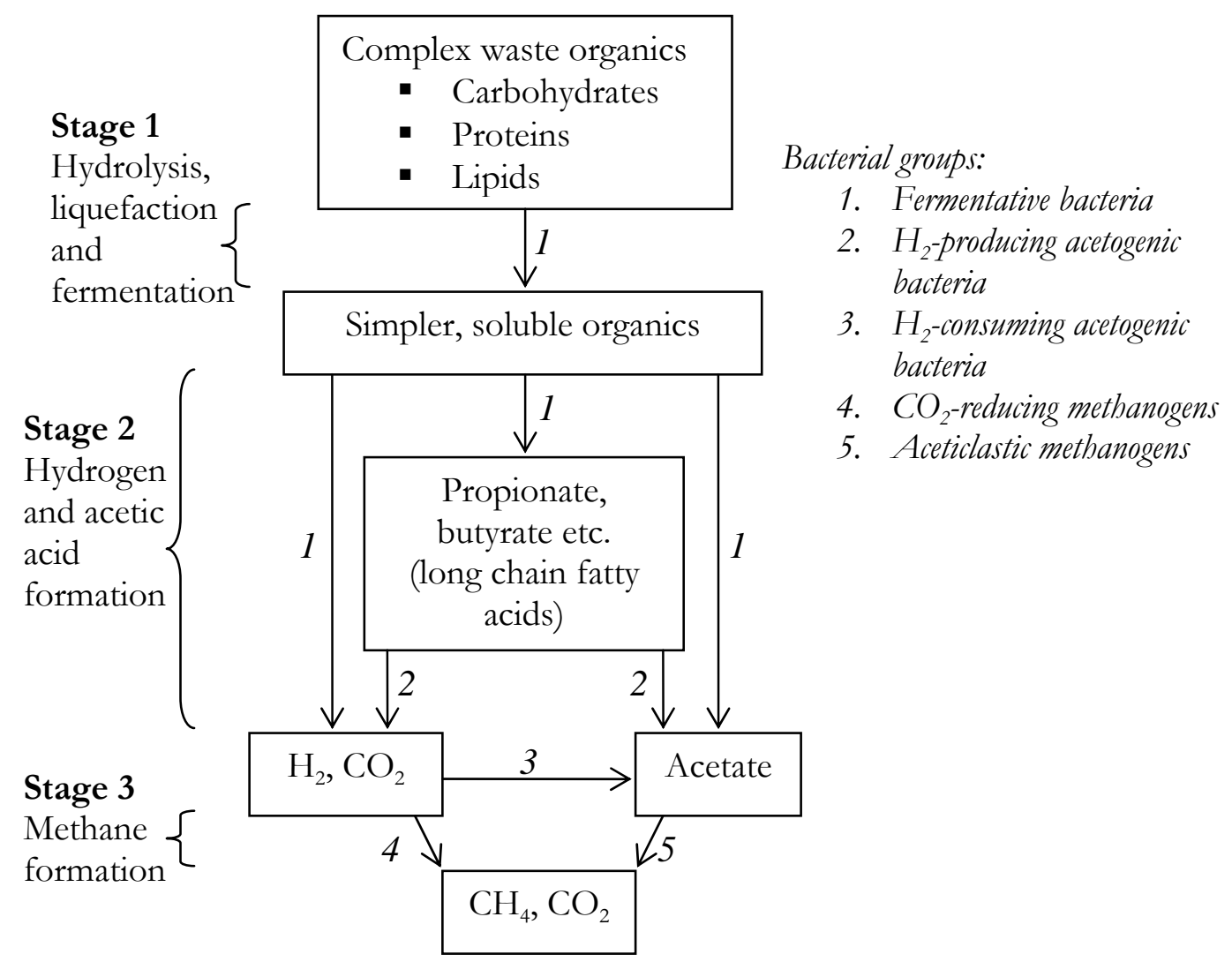

Figure 2.1 Three-stage anaerobic digestion mechanism

Hydrolysis and liquefaction are accomplished by extracellular, hydrolytic enzymes produced and excreted by the bacterial population for this specific purpose. It is important to recognize that stabilization of complex organics cannot occur unless this initial hydrolysis step is functioning properly. Therefore the overall rate of stabilization and methane fermentation can be limited by the hydrolysis rate of complex organics.

Once complex organics are hydrolyzed, they are fermented to long chain organic acids, sugars, amino acids, and eventually to smaller organic acids such as propionic, butyric, 
and valeric acid. This phase is commonly called the 'acid-forming' or fermentation phase, and also results in essentially no stabilization. The population of bacteria responsible for acid production, called acetogenic bacteria, may be facultative anaerobes (viable in the presence of oxygen), strict or obligate anaerobes (to which oxygen is toxic), or a combination of both. Acetic acid, hydrogen, and carbon dioxide are also formed during the production of organic acids.

Hydrogen is inhibitory to many of the acid-forming bacteria and must be removed from the system if acid production is to continue. Fortunately, hydrogen is an energy source for some methanogenic bacteria and is rapidly consumed in the reduction of carbon dioxide to methane. The optimum $\mathrm{pH}$ of acidogenic bacteria is 5.2 to 6.5, and its specific growth rate is around 2 days (Demirer and Chen, 2004).

\section{Hydrogen and Acetic Acid Formation}

Hydrogen is produced by fermentative bacteria and consumed by acetogenic bacteria. Acetate is also produced by these groups, as well as by acetogenic bacteria. Hydrogen plays a key role in regulating organic acid production and consumption. If the partial pressure of hydrogen exceeds $10^{-4}$ atm, methane production is inhibited and the concentration of organic acids (e.g., propionic and butyric) will increase. Thus, to maintain efficient anaerobic digestion of sludges to methane, hydrogen levels must be maintained below this level. A syntrophic association with a large, stable population of $\mathrm{CO}_{2}$-reducing methanogens will ensure maintenance of low hydrogen concentrations. Because of its key regulatory role, hydrogen offers promise as a process performance indicator. Acetogenic bacteria grow very slowly, with a minimum doubling time of 3.6 days (Demirer and Chen, 2004).

\section{Methane Formation}

Waste stabilization occurs during the methanogenic phase by conversion of the acetic acid into methane, which is essentially insoluble in water and readily separates from the sludge as a gas which leaves the system. Carbon dioxide is also produced, and either 
escapes as gas or is converted to bicarbonate alkalinity. Methanogenic bacteria are strict anaerobes to which oxygen is inhibitory. One of the most important characteristics of the methanogenic phase is that very few substrates can act as energy sources for the various methanogens. Of these, acetic acid and hydrogen serve as the major substrates. Methanogenic bacteria, which are very sensitive to environmental stresses, grow more slowly than acidogenic bacteria, and at a rate similar to acetogens (Demirer and Chen, 2004).

Methane formed in anaerobic digestion comes from acetate cleavage (Equation 2.1) and from reduction of carbon dioxide by $\mathrm{CO}_{2}$-reducing methanogens using hydrogen as their energy source (Equation 2.2).

$\mathrm{CH}_{3} \mathrm{COOH} \rightarrow \mathrm{CH}_{4}+\mathrm{CO}_{2}$

$\mathrm{CO}_{2}+\mathrm{H}_{2} \rightarrow \mathrm{CH}_{4}+\mathrm{H}_{2} \mathrm{O}$

\subsubsection{Anaerobic Digesters (ADrs)}

In practice, ADrs are operated in both mixed and unmixed modes. The choice of operation also depends on the type of waste. Dairy and swine manure management systems are often liquid or slurry based, which simplifies the necessary manure movement.

ADr designs can be classified as two main types, unmixed and mixed digesters. (Complete-mix digester is a generally used misnomer since it indicates only the provision of an additional form of mixing but does not imply complete mixing of the digester contents). ADrs used on farms are also commonly classified as continuous fed or batch fed (Gunaseelan, 1997; Parkin and Owen, 1986). As the name suggests, no mixing is provided in unmixed ADrs. Unmixed ADrs are operated in two main designs, plug-flow and anaerobic lagoon digesters. The choice between the two is mostly governed by the solids content of the waste. There are also other modifications of 
designs and operation of ADrs, such as a fixed film digester or an anaerobic filter, and temperature-phased digesters (Gunaseelan, 1997).

\section{Mixed Digesters}

Mixed digester vessels are insulated and maintained at a constant elevated temperature, in the mesophilic $\left(77^{\circ} \mathrm{F}\right.$ to $\left.104^{\circ} \mathrm{F}\right)$ or thermophilic $\left(122^{\circ} \mathrm{F}\right.$ to $\left.149^{\circ} \mathrm{F}\right)$ range. The digester vessel is usually a round insulated tank, above or below ground, and made from reinforced concrete, steel or fiberglass. Heating coils with circulating hot water can be placed inside the digester or, depending on the consistency of the feedstock, the contents can be circulated through an external heat exchanger to maintain the desired temperatures. They can be mixed by a motor driven mixer, a liquid recirculation pump, or by biogas recirculation (see Figure 2.2). A gas tight cover (floating or fixed) traps the biogas. The mixed digester is best suited to process manure with $3-10 \%$ total solids. Retention time is usually 10 to 20 days. The biogas created by the digester can be used to heat the digester to the desired temperature (McNeil, 2005).

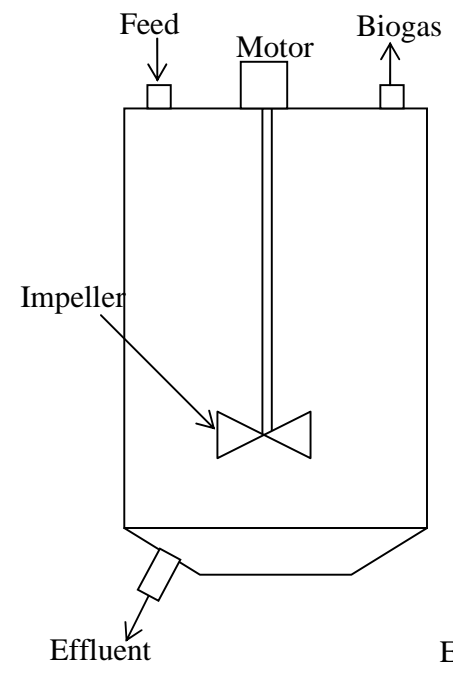

(a) Impeller-mix

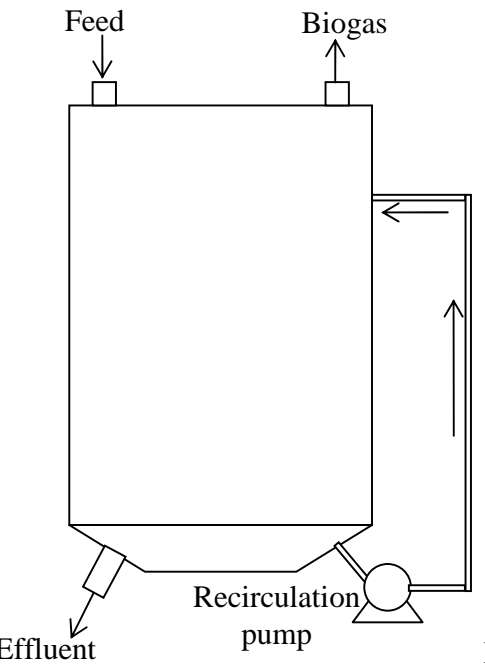

(b) Liquid recirculation

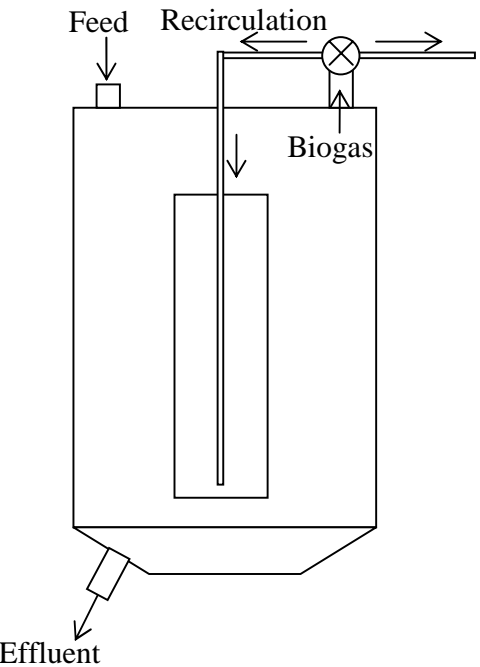

(c) Gas recirculation

Figure 2.2 Modes of mixing in mixed digesters 
Gaslift digesters are popular in AD applications because they offer several advantages over other designs of mixed digesters (Carroll and Ross, 1984). Gas recirculation digesters do not have any moving parts, which makes cleaning and maintenance easy. Sealing the digester to avoid leakage of biogas or contamination by air is very difficult in case of impeller mixed digesters. Power consumption is a very important factor in selection of digester design. The power required to drive the impeller motors is significantly higher than the power required for pumping the gas. The energy required to run the digester should be kept to minimum to maximize the energy gain from the biogas produced by the digester. Since the slurry used in mixed digesters has solids, slurry recirculation needs special pumps that can handle slurry and also needs higher power.

Non-uniform dispersion of mixing energy causes problems such as solids settling to the bottom and formation of a floating layer at the top. Thus, the distribution of mixing energy throughout the digester volume is key in the selection of a mixing system (Casey, 1986). Spreading a relatively low energy input throughout the highly viscous manure slurry is not an easy task. This is especially true for concentrated power inputs such as impellers and slurry recirculation systems. These systems show high shear rate and hence high power dissipation rate near the input location, and shear rate tapers off with distance from the power input location. In the case of slurry recirculation systems, the mixing input usually leads to a mass circulation of the digester contents, with consequent uneven shear stress and relatively poor performance in preventing float layer formation. High shear stress is also detrimental to the microorganisms. Gas recirculation systems provide low shear distributed throughout the digester volume due to distribution of the dissipated power input.

Another important factor is the interaction between the separate mixing effects of the evolved biogas and that of the imposed mixing. Ideally, their mixing effects should be complementary. This will not be the case with a slurry recirculation system or an impeller mixed system. By contrast, in a gaslift digester the pumped biogas rises 
vertically upwards inside the draft tube, carrying slurry upwards with it, thus facilitating evolution of biogas bubbles. (The liquid circulation outside the draft tube is in downward direction opposite to the direction of the gas bubbles, but the liquid velocities are very low in this region to affect the motion of gas bubbles.) Hence, digester mixed by gas recirculation seems to be the option within mixed digesters and therefore it has been selected to be investigated in this work according to the objectives outlined earlier.

\subsubsection{Importance of Mixing in Anaerobic Digestion}

Number of factors affects the performance and efficiency of AD, such as temperature, $\mathrm{pH}$, feed characteristics, feed rate and feeding mode, toxicity, and mixing in the digester. Hoffmann (2005) and Parkin and Owen (1986) have explained this in detail. Parkin and Owen (1986) provided a check list of key factors that govern bacterial growth and thus, the AD's performance. Favorable conditions for the following factors will maximize chances for achieving optimum design and efficient operation: 1) optimum retention time, 2) adequate mixing (bacteria-substrate contact), 3) proper $\mathrm{pH}$, 4) proper temperature control, 5) adequate concentration of proper nutrients, 6) absence (or assimilation) of toxic materials, and 7) proper feed characteristics. Factors one to five are directly related to mixing in the digester. Thus, we will review the effect of mixing on $\mathrm{AD}$ performance in this discussion.

Despite the slow bioreaction rate, the reasons for providing mixing in digesters are to provide efficient utilization of the entire digester volume, to prevent stratification and temperature gradients, to maintain uniform $\mathrm{pH}$, to disperse metabolic end products and any toxic materials contained in the influent sludge, and to maintain intimate contact between the bacteria, bacterial enzymes, and their substrates (Bello-Mendoza and Sharratt, 1998; Casey 1986; Meynell, 1976; Parkin and Owen, 1986; Sawyer and Grumbling, 1960; Smith et al., 1996). Additional concerns associated with inefficient mixing are foaming and scum formation, and excessive solids deposition. Mixing also 
helps in evolution of biogas bubbles. In short, adequate mixing provides a uniform environment, one of the keys to good digestion.

Inefficient mixing decreases effective system volume, which reduces the sludge retention time (SRT) and pushes the system towards failure. Studies with full-scale digesters have shown that inefficient mixing may reduce the effective volume of the digester by as much as 70\%, leaving an actual volume utilization of only $30 \%$ (Monteith and Stephenson, 1981). Parkin and Owen (1986) illustrated the effect of the SRT on digester performance and proved that inefficient mixing causes digester failure. From a digester study at a larger scale, James et al. (1980) also suggest that mixing is required for efficient operation of the digester to avoid settling and flocculation.

The optimum $\mathrm{pH}$ of acidogenic bacteria is 5.2 to 6.5 , and the specific growth rate is around 2 days. Acetogenic bacterias grow very slowly, with a minimum doubling time of 3.6 days. Methanogenic bacteria, the group of anaerobes most sensitive to environmental stresses, grow more slowly than acidogenic bacteria, at a rate similar to acetogens (Demirer and Chen, 2004). The optimum pH environment for methanogens is 7.5-8.5. ADrs are generally operated in fed-batch or batch mode. If the added feed concentration is not kept uniform throughout the digester volume, then fast growing acidogenic bacteria will produce acids at a higher rate than the rate at which acids can be consumed by acetogenic or methanogenic bacteria. Increasing acids concentration lowers the $\mathrm{pH}$, killing the methanogenic activity and pushing the digester towards failure. Thus fast and uniform distribution of feed is required through proper mixing (Merchuk and Gluz, 1999).

In spite of the crucial role played by mixing in the operation of ADrs, contradictory findings in small scale digesters are reported in the literature about the necessity of mixing and the mixing intensity required to enhance the digester performance. Chen et al. (1990) found that a non-mixed digester exhibited a higher methane yield than a continuously mixed digester. Ho and Tan (1985) reported greater gas production for a 
continuously mixed digester than for an unmixed digester for palm oil mill effluents. Dague (1970) observed that shifting from continuous mixing to intermittent mixing resulted in significantly higher gas production during the anaerobic treatment of a liquid municipal waste stream. Ben-Hasson et al. (1985) observed a 75\% lower methane production rate from a continuously mixed reactor than from an unmixed reactor when treating dairy cattle manure anaerobically. Pierkiel and Lanting (2004) observed in a pilot-scale digester that higher volumetric power input induces stronger mixing, reducing the hydraulic retention time and raising biological activity.

While discussing the importance of mixing in digesters, it has to be remembered that some degree of internal mixing is always present in all ADrs. The evolution of biogas bubbles creates some amount of mixing. Under favorable conditions - at high gas evolution rates and in the absence of readily floatable solids or low solids content evolved biogas may provide sufficient process mixing on own, thus eliminating the need for an external mixing input. However, in the case of slurries, self-mixing cannot be relied upon to prevent the development of bottom deposits or a floating scum layer. Some mixing of digester contents also takes place during the addition of feed and removal of effluent; however this mixing is intermittent in nature, unlike the continuous mixing provided by evolving biogas.

Karim et al. (2005a, 2005b) and Hoffmann (2005) conducted a range of systematic experiments on 6-inch diameter laboratory-scale units to assess the effect of mixing on the digesters' performance. Karim (2005a) operated several digesters with conical bottoms; fed with slurry containing $5 \%$ or $10 \%$ (i.e., 50 or $100 \mathrm{gm} / \mathrm{L}$ ) total solids with different mode of mixing and with different geometries. The mode of mixing or the digester geometry showed no significant effect on the performance of the digesters. They concluded that the true effect of mixing cannot be observed at laboratory scales, and more performance experiments need to be performed at larger scales to arrive at confirmatory conclusions. 
The impact of mixing intensity in small and large digesters on the performance of ADrs mixed by gas recirculation was studied (Appendix A). The findings of this study showed that the performance of laboratory-scale digesters is not affected by mixing, but the performance of pilot-scale digesters is significantly affected by mixing. The laboratoryscale digesters showed better performance than the pilot-scale digesters in terms of methane production. And thus it was conclude that only large-scale digesters should be used to study the performance and to obtain reliable data that can be used for the design for the design of digesters.

Reactor configuration itself does not play an important role in causing changes in the microbial community (Morgan et al., 1991). The effect of reactor configuration on the performance of ADrs is due to changes in the hydrodynamics or mixing performance. For example, higher shear produced by impeller mixed digesters is harmful to the microorganisms, or a reactor configuration causing higher dead zones can reduce the effective reactor volume, thus reducing the effective sludge retention time (SRT) and causing digester failure. Tilche and Vieira (1991) observed a change in process performance upon scale-up and related it to a change in mixing patterns. Smith et al. (1996) also observed a change in digester performance upon scale-up. They conducted tracer studies and measured dead zone volumes and concluded that the change in hydrodynamics of the digester upon scale-up results in a change in their performance.

Thus, the hydrodynamics or mixing performance of a digester is important in understanding, designing and scale-up of ADrs. The next section discusses the hydrodynamics of gaslift digesters.

\subsection{Gaslift Digesters}

Gaslift loop reactors are used for anaerobic digestion process to provide mixing by recirculation of gas. For digester applications the reactor height (slurry level) is maintained approximately equal to one to two times the reactor diameter. For gaslift 
loop reactors, conventionally used in chemical or biochemical industrial applications, the reactor height to diameter ratio $(H / T)$ is normally greater than two. (In this discussion, low $H / T$ (approximately equal to one) gaslift loop reactors will be referred to as gaslift digesters). Because of the prominent use of high $H / T$ ratio gaslift loop reactors in industry, much information on gaslift loop reactors is available. In contrast, there is very little information on gaslift digesters $(H / T \cong 1)$. Thus, it is difficult to evaluate the effect of $H / T$ ratio on the mixing and hydrodynamics of gaslift loop reactors. The liquid or slurry level in the gaslift loop reactors is one of the important parameters affecting the hydrodynamics (Merchuk et al., 1996).

Since the gaslift loop reactors and the gaslift digesters are basically the same type of reactors operating with the same working principle, their global hydrodynamic behavior should not be considerably different. The global hydrodynamic characteristics such as the nature of the flow pattern, flow regimes, the nature of correlations and equations to determine hydrodynamic parameters (holdup, circulation velocities, circulation time, turbulence parameters, transport coefficients), would not be expected to be considerably affected by the change in $H / T$ ratio. However the effect of operating conditions and design and scale-up variables on the flow pattern, flow regime transition

criteria, constants and exponents in correlations, and hydrodynamic parameters will be different for considerably different $H / T$ ratios.

Knowledge of gaslift loop reactors will be helpful in understanding the hydrodynamics of gaslift digesters. Thus the hydrodynamics of gaslift loop reactors is discussed in the following sections.

\subsubsection{Gaslift Loop Reactors}

Gaslift Loop Reactors (GLR) can be divided into two main types on the basis of their structure (Figure 2.3), external gaslift loop reactors (EGLR) and internal gaslift loop reactors (IGLR) 


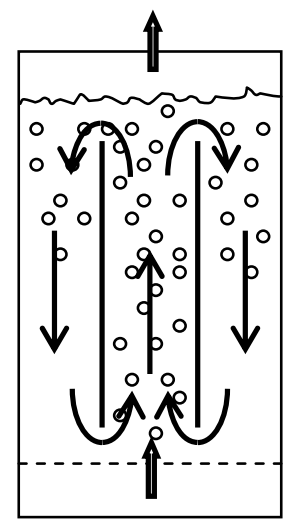

(a)

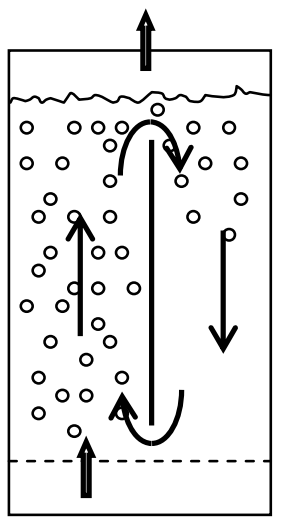

(b)

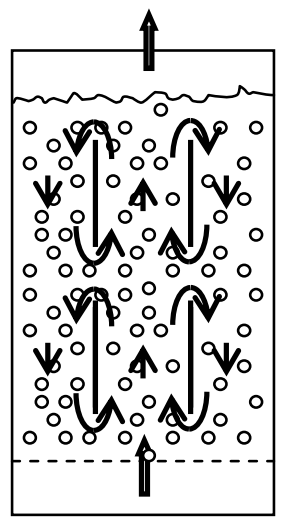

(c)

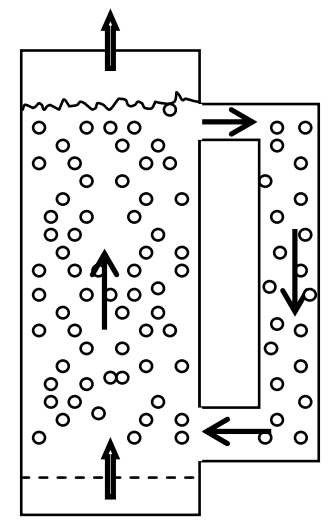

(d)

$\longrightarrow$ Slurry flow direction

Gas flow direction

OGas bubbles

Figure 2.3 Types of GLR (a) Concentric tube IGLR, (b) Split IGLR

(c) Stages IGLR (d) External GLR

In EGLRs circulation takes place through separate and distinct conduits, whereas in IGLRs a baffle or concentric tube is placed strategically in a single vessel to create the channels required for the circulation. The designs of both types of reactors can be modified further, leading to variation in hydrodynamics, in the extent of gas disengagement from the fluid, and in the flow rates of various phases. Some of the variations of IGLR are rectangular and square cross-sectioned gaslift, split-cylinder gaslift, concentric-tube gaslift, and multiple concentric tubes. (see Figure 2.3).

\section{Advantages of GLRs}

Advantages offered by GLRs are discussed below:

- Low shear stress: The uniformly distributed, low shear stress present in GLRs is one of their most important advantages, and makes them popular for biological applications. In GLRs the gas is injected at a single point, but the direct contribution of gas injection to the hydrodynamics of the system is small. Circulation of liquid and gas is created by the difference in the gas holdup between the riser and the downcomer, which creates a pressure difference at the bottom of the equipment. 
$\Delta P=\rho_{l} g\left(\varepsilon_{r}-\varepsilon_{d}\right)$

Where $\Delta P$ is the pressure difference, $\rho_{l}$ is the liquid density, $g$ is the gravitational constant, $\varepsilon_{r}$ is the gas holdup in the riser and $\varepsilon_{d}$ is the gas holdup in the downcomer.

The pressure difference forces the fluid from the bottom of the downcomer towards the riser, generating circulation. Since the average gas holdup along the length of the riser and downcomer contributes to the pressure difference, there are no focal points of energy dissipation, and thus shear distribution is homogeneous throughout the GLR. In contrast, in bubble columns and stirred tanks, the energy source inducing fluid motion is focal. The shear forces in bubble columns are highest adjacent to the gas sparger and dissipate with distance from the sparger. In stirred tanks, a region of very high shear exists near the impeller, which decreases with increasing distance from the impeller. Thus GLRs are used in biological systems where microorganisms are very sensitive to shear.

- Simple design: GLRs are mechanically simple in design, without any rotating internal parts. The absence of a shaft and the associated sealing, which is always a weak element from the point of view of sterility, confers on the GLR an obvious advantage over stirred tanks. The vertical orientation of these reactors, as well as lack of internals, facilitates easier cleaning and sterilization.

- Low energy consumption: The energy consumption per unit volume to create circulation and mixing is significantly lower in GLRs than in stirred tanks and bubble columns. In bubble columns the difference in gas holdup creates liquid circulation. Thus, in GLRs even very low gas velocities can initiate liquid circulation in the whole reactor. The injected gas serves the dual functions of aeration and agitation, which promotes efficiency in the overall energy balance and eliminates the need for a separate expenditure of energy for agitation.

- GLRs show good mass transfer and heat transfer characteristics and are easily adaptable to three-phase systems. 


\subsubsection{Hydrodynamics in Gas-Solid-Liquid IGLRs}

The interconnections between the design variables, the operating variables, and the observable hydrodynamic variables in an IGLR are shown schematically in Figure 2.4 (adapted from Merchuk et al., 1996). The design variables are the reactor height, the $D / T$ (draft tube diameter to tank diameter) ratio, the geometrical design of the gasliquid separator, and the bottom clearance of the draft tube (it is proportional to the free area for flow in the bottom and represents the resistance to flow in this part of the reactor). The main variables are primarily the gas input rate and, to a lesser extent the top clearance of the draft tube from the liquid surface. These two independent variables set the conditions that determine the liquid velocity in the IGLR via the mutual influence of pressure drop and holdup. Viscosity is not shown as independent variable because in the case of gas-liquid mixtures, it is a function of the gas holdup (and of liquid velocity in the case of non-Newtonian liquids), and because in a real process it will change with time due to changes in compression of liquid.

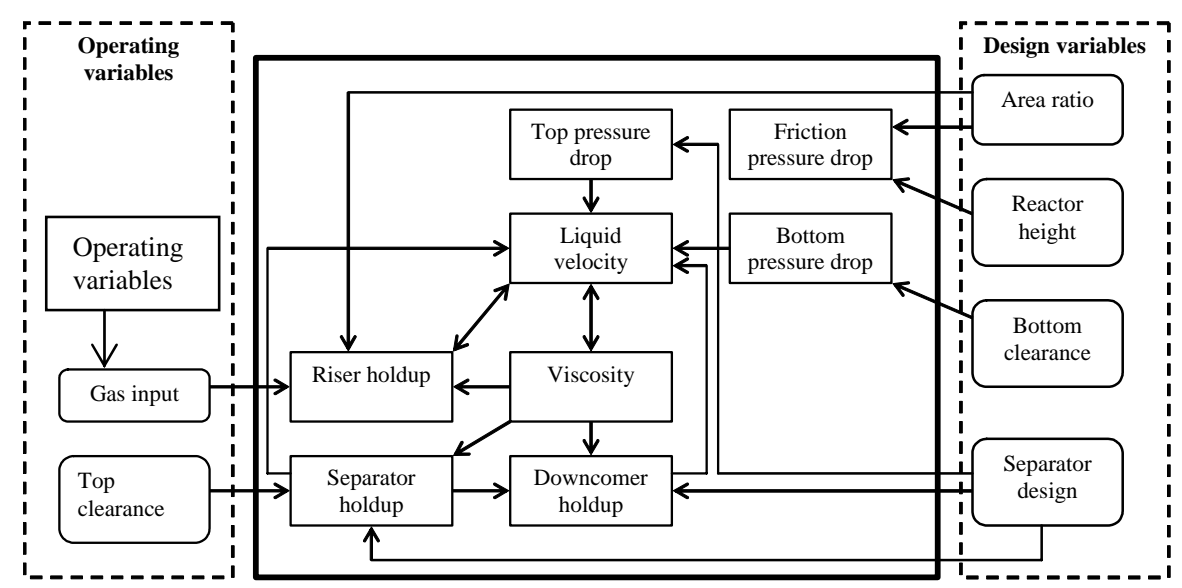

Figure 2.4 Relationship between independent and dependent variables in the hydrodynamics of IGLRs (Merchuk et al., 1996)

Because of the advantages of IGLRs, they are becoming increasingly popular in three phase gas-liquid-solid applications. Recent literature has focused on the hydrodynamics 
and modeling of three phase IGLRs (Feitkenhauer et al., 2003; Freitas et al., 1999; Heijnen et al., 1997; Kennard and Janekeh, 1991; Klein et al., 2003a and 2003b; Lu et al., 1995; Luo, 2005; Merchuk et al. 2003; Merchuk and Shechter, 2003; Petersen and Margaritis, 2001; Siegel and Robinson et al., 1992; Sun et al., 2005; Trilleros et al., 2005). This literature discusses the effect of solids of varying densities ranging from lighter than water to heavier than glass $(0.8$ to $4.5 \mathrm{~g} / \mathrm{cc})$, on the hydrodynamics of IGLR. The consensus exists between all the researchers that, if the density of the solids is higher than that of the liquid, liquid rising velocity will be smaller and the holdup of solids in the riser will be larger than in the downcomer, and vice-versa. The presence of solids however, always diminishes the driving force for circulation, independently of their density.

The importance of the gas holdup in gaslift reactors is twofold: 1 . The value of the gas holdup gives an indication of the potential for mass transfer; and 2. The difference in the gas holdup between the riser and the downcomer generates the driving force for liquid circulation. It should be stressed, however, that when referring to gas holdup as the driving force for liquid circulation, only the total volume of gas is relevant. This is not the case for mass transfer phenomena, in which case the interfacial area is of paramount importance, and therefore some information on bubble size distribution is required for complete understanding of the process. Because gas holdup values vary within a reactor, average values, referring to whole volume of the reactor, are usually reported. Values referring to a particular section, such as the riser or the downcomer, are much more valuable, since they provide a basis for determining liquid velocity and mixing. The geometric design of the IGLR has a significant influence on the gas holdup. Changes in $D / T$ ratio will change the liquid and gas residence time in each part of the reactor and hence their contributions to the overall holdup. Gas holdup increases with decreasing $D / T$.

The liquid velocity is one of the most important parameters in the design of IGLRs. It affects the gas holdup in the riser and downcomer, the mixing time, the mean residence 
time of the gas phase, the interfacial area, and the mass and heat transfer coefficients. Circulation in IGLRs is induced by the difference in hydrostatic pressure between the riser and the downcomer as a consequence of a difference in gas holdup. Unlike gas holdup, liquid velocity is not an independent variable, because the gas flow rate is the only variable that can be manipulated. The geometry of the reactor also influences the liquid velocity, but this remains constant during operation. Experiments have been carried out in devices specially designed to artificially change the resistance to flow, with the aim of studying the effect of the velocity at a fixed rate of aeration (Merchuk and Stein, 1981). The information emerging from these experiments indicates that an increase in the liquid velocity leads to a decrease in the mean residence time of bubbles in the riser. In practice, when the gas flow is increased, the higher liquid velocity increases the carryover of the bubbles from the gas separator into the downcomer. The carryover dampens the liquid flow by reducing the hydrostatic driving force. As a result, the overall change in liquid velocity is tempered.

One of the major factors in the design of IGLRs is the effect of the geometry of the system on the various characteristics of the flow phases. Reactor geometry affects overall performance, and is also influenced by the operating variables and fluid dynamic properties (explained in Figure 2.4). Geometric variables that affect the hydrodynamic performance of IGLR are draft tube diameter to reactor diameter ratio $(D / T)$, liquid height to reactor diameter ratio $(H / T)$, draft tube top and bottom clearance, draft tube height $(b)$, shape of the bottom, location and geometry of sparger, etc.

Trilleros et al. (2005) proposed several correlations to predict the effect of $D / T$ and $\mathrm{b} / \mathrm{H}$ on the liquid velocity and gas holdup. Comparing the exponents of each term in the correlations, he concluded that the effect of physical properties of the GLS system on the hydrodynamics is more important than the effect of geometry. The crosssectional area of the draft tube determines the superficial fluid velocity in the reactor. In three-phase systems it also plays an important role in determining the minimum velocity necessary to fluidize the solid particles. It has been shown that to achieve optimum gas 
holdup, D/T should be greater than 0.75 (Kennard and Janekeh, 1991; Weiland, 1984). Rousseau and Bu'Lock (1980) have shown that minimal mixing time is achieved when $D / T$ is between 0.6 and 1; this has been confirmed by Lin et al. (1976). Weiland (1984) states that $D / T$ of less than 0.6 should only be used if high liquid velocities in the draft tube are required to avoid sedimentation of large microbial aggregates. Kojima et al. (1999) observed an increase in liquid velocity with an increase in $D / T$.

To study the effect of top and bottom clearance, $D / T$, and $\mathrm{h} / \mathrm{D}$, Gavrilescu and Tudose (1998b) performed experiments on three scales of IGLRs, with volumes ranging from 0.07 to $5.2 \mathrm{~m}^{3}$. They found that the draft tube clearance and $D / T$ have major influences on liquid superficial velocity, circulation time, friction coefficient, and the radial profiles of liquid velocity and gas holdup. Interestingly, Kojima et al. (1999) found no effect of draft tube clearance on the liquid circulation velocity, whereas Luo (2005) found from CARPT and CT experiments that both the top and bottom clearance has significant impact on the liquid circulation and gas holdup in IGLRs. Lu et al. (1995) found that liquid velocity increased with increase in draft tube height, whereas the effect of static liquid height on liquid velocity was negligible. Kojima et al. (1999) also confirmed that liquid velocity increases with an increase in draft tube height, but no explanation was provided for this behavior.

\subsection{Scale-up of IGLRs}

A thorough knowledge of mixing behavior is of particular importance during the process of scale-up from laboratory-scale to industrial-scale IGLRs. In small scale reactors, due to ease of mixing, it is easier to maintain the optimal conditions of $\mathrm{pH}$, temperature, and substrate concentration required for maximum productivity. However, because of the compromises made during scale-up, it is difficult to keep the same hydrodynamic conditions established in the laboratory-scale. Thus, full-scale mixing may not be as good as mixing on a laboratory-scale. 
Merchuk and Gluz (1999) pointed out two main groups of problems encountered in scale-up of bioreactors. First, economic and mechanical limitations make it difficult to maintain the same high power input per unit volume in large scale reactors that is used in laboratory-scale units. This problem is not encountered in ADrs, because power input is kept to minimum for economical operation. Second, the lack of knowledge of hydrodynamics of large-scale reactors prevents design of bioreactors from first principles. Thus, simplistic hydrodynamic models and empirical correlations are used for scale-up.

Despite of many successful full-scale applications of IGLRs (the Pachuca tank used in metallurgy; the waste water treatment at Gist Brocades, The Netherlands; the production of single cell proteins by Pruteen process, Klein et al., 2001), the use of GLRs is limited. One of the most important reasons is lack of reliable scale-up models or scale-up methods to predict key operational parameters in the range of different geometries and operational conditions. Blazej et al. (2004); Gavrilescu and Tudose (1998); Heijnen et al. (1997); Merchuk et al. (1996) and Merchuk and Gluz (1999) are among the few who have addressed the scale-up issues of IGLR.

Heijnen et al. (1997) reported that the flow regimes occurring in IGLR are the same for all scales of reactors (ranging from a liter to $100 \mathrm{~m}^{3}$ ), but flow regime transition conditions are not the same for all scales. Blazej et al. (2004) performed experiments on three different scales of IGLR, ranging from 10.5 liters to 200 liters, and concluded that larger reactor volumes operating in the bubble recirculation regime provide higher liquid circulation velocities and higher, more uniformly distributed gas holdup than smaller reactors. Better performance of large scale reactors was attributed to lower values of friction factors from the walls and internals. Heijnen et al. (1997) made similar observations with a pilot-scale (400 liters) and full-scale $\left(284 \mathrm{~m}^{3}\right)$ IGLR. Merchuk et al. (1996) observed higher gas holdup and lower liquid circulation velocities in a larger reactor $\left(300 \mathrm{~m}^{3}\right)$ than a smaller scale one $\left(30 \mathrm{~m}^{3}\right)$. 
Gavrilescu and Tudose (1998) encountered the change in hydrodynamics of the system when passing from laboratory to larger scales. As the reactor scale increased from 70 liters to $2.5 \mathrm{~m}^{3}$, the overall gas holdup decreased, whereas from 2.5 to $5.2 \mathrm{~m}^{3}$ no effect of scale on the gas holdup was seen. They also observed that the influence of the geometry of the system on the flow of different phases is important in design and scaleup of IGLRs (see Figure 2.4).

Merchuk et al. (1996) presented an extensive list of design, operational, and hydrodynamic variables and interconnections between them; the effect of these variables on each other is important in scale-up of IGLRs.

Although the above knowledge of IGLRs cannot be directly extrapolated to gaslift digesters, it can help in understanding the challenges involved in their design and scaleup. In a specific well-mixed laboratory-scale digester, the optimum growth rate of microorganisms or the optimum production rate of a specific product usually relates to well-defined environmental conditions, such as $\mathrm{pH}$ range, temperature, substrate level and limiting factors. Laboratory-scale digesters are very attractive for experimentation because of their convenient small size, ease of operation, and low cost. They are also efficiently mixed and thus contain a uniform environment. These characteristics make them valuable in estimating kinetic parameters and nutrient and alkalinity requirements, and in discovering potential problems like toxicity. Small-scale experiments performed to reveal the hydrodynamics provide insight into flow patterns and the shape of velocity and holdup profiles. On the other hand, experimentation on a large scale digester is necessary to elucidate the operational problems and difficulties, such as the effects of improper mixing (Ben-Hasson and Ghaly, 1989, Karim et al., 2005a \& 2005b).

The literature on IGLRs is focused on phenomenological hydrodynamic modeling and validation of these models through experiments (Freitas et al., 1999; Garcia et al., 1996; Garcia et al., 1999; Heijnen et al., 1997; Klein et al., 2003; Sun et al., 2005 and many others). Hydrodynamic models are used to predict the two most important design 
parameters, liquid circulation velocity and gas holdup. Another prevalent approach in designing IGLRs is formulating correlations, to evaluate desired quantities such as liquid velocity, gas holdup and mass transfer coefficients, by correlating the experimental data (Choi et al., 1996; Feitkenhauer et al., 2003; Kojima et al., 1999; Miron et al., 2004; Trilleros et al., 2005; Gavrilescu and Tudose, 1998a, 1998b; Wei et al., 2000; Wen et al., 2005).

If a hydrodynamic model is formulated from first principles, it can offer many advantages such as ease and reliability of reactor design and scale-up and the ability to predict the effect of operating conditions. However, such formulations are made difficult by the inherent geometric complexity of the system and by the fact that these processes typically involve turbulent flow (Saez et al., 1998). As a result, these models rely on one or more input parameters that are fitted from the experimental data or obtained from empirical correlations. Therefore, hydrodynamic models, just like empirical correlations, cannot be used for or extrapolated to different geometries, scales, and operating conditions (Cockx et al., 1997; van Baten et al., 2003).

Considering the shortcomings of conventional experiments, phenomenological models, and advanced non-invasive experimental techniques like Computer Automated Radioactive Particle Tracking (CARPT) and Computed Tomography (CT) are required to understand the hydrodynamics of IGLRs in detail. CARPT provides time-averaged knowledge of flow patterns, velocity profiles and turbulence parameters, while CT provides local or averaged phase holdup. Karim et al. (2004) and Luo (2005) applied CARPT and CT for visualizing flow patterns and phase holdup profiles in an IGLR type anaerobic digester. Laser Doppler Velocimetry (LDV) and Ultrasound Doppler Velocimetry (UDV) can also be used under limited conditions for obtaining flow patterns and velocity and holdup profiles (Vial et al., 2003).

Advanced non-invasive experimental techniques like CARPT and CT help to understand the hydrodynamics in more detail, but their application is limited by time 
and resource constraints. Thus these techniques cannot be used to evaluate the effect of every parameter on the hydrodynamics. This is where Computational Fluid Dynamics (CFD) can be utilized, once they are validated.

For single-phase systems, CFD models and closures are well established and validated with benchmark experimental data, so that CFD can be used with a high level of confidence for simulating single-phase systems. However, this is not the case with multiphase systems. The complex flow structure and interactions within different phases, in addition to the turbulence, make it very difficult to develop models for multiphase systems that can mimic reality. The closures used for these equations are modeled hypothetically or correlated from experimental data at different conditions and thus cannot be universally applied to all cases. Multiphase CFD simulations need to be developed for individual situations and validated against experimental data. Once the CFD results are validated for a particular system, CFD can be used to optimize the system by varying parameters and operating conditions to achieve proper design and scale-up.

Only a few CFD modeling attempts are described in the literature on IGLRs (Bagatin et al., 1999; Blazej et al., 2004a; Cockx et al., 1999; Glover et al., 2003; Jakobsen et al., 1993; Luo (2005); Mudde and Van Den Akker, 2001; Oey et al., 2001 and 2003b; Svendsen et al., 1992; van Baten et al., 2003a and 2003b). Some of the researchers (Mudde and Van Den Akker, 2001; Oey et al., 2001) compared CFD predictions with the results of a 1D mechanical energy balance model. This cannot be a conclusive way to evaluate the predictions of the CFD model, because of the over-simplifying assumptions and empiricism involved with 1D hydrodynamics models.

Few other researchers have compared CFD results with experimental data. The comparison has been done mostly for the overall liquid circulation velocity and/or overall gas holdup in the riser and/or downcomer section (Bagatin et al., 1999; Blazej et al., 2004a; Glover et al., 2003; van Baten et al., 2003a and 2003b). The predictions of average quantities match very well with the experimental data in most cases. But CFD is 
put to real test to make conclusive evaluations, when the predicted local quantities, like liquid velocity profiles or gas holdup profiles, are compared with experimental values. The comparison in this case is often satisfactory qualitatively and only reasonable quantitatively. Svendsen et al. (1992) compared liquid velocity profiles and reported unsatisfactory predictions of CFD simulations in the case of IGLRs.

Blazej et al. (2004a) simulated 2D flow in IGLR using the algebraic slip model and compared the simulated predictions with the experimental data. The average liquid velocities were obtained by magnetic tracer particle method, and the average gashold up was measured by an inverted U-tube manometer. Computational predictions for liquid velocity and gas holdup in the riser matched reasonably with the experimental data, but the computations always overpredicted the liquid velocities and gas holdup at higher gas superficial velocities. This was attributed to lack of proper modeling of gas entrainment in the downcomer region at high gas flow rates. To resolve this issue, Glover et al. (2003) performed 3D simulations in a similar system and found that it increased the accuracy of predictions in downcomer region but the predictions in the riser section were less accurate than the predictions of $2 \mathrm{D}$ simulations.

van Baten et al. (2003a) performed both 2D and 3D simulations for different configurations of IGLR and observed that the geometry effects were properly accounted for by the CFD model. van Baten et al. (2003b) and Bagatin et al. (1999) found that the scale effects were accounted for by CFD, in addition to the geometry effect. This feature of CFD is very helpful in design and scale-up of IGLRs and needs to be evaluated further.

\subsection{Summary}

The literature review of the anaerobic digestion process has helped to highlight the importance of mixing in the process Considering the advantages and disadvantages offered by various designs of ADrs, a digester mixed by gas recirculation was selected 
for experimental studies. Gaslift digesters are geometrically similar to the IGLR, with the exception of the $H / T$ ratio. For gaslift digesters, the $H / T$ ratio is normally close to one, whereas for IGLRs it is greater than two. Because of the considerable literature available related to IGLRs, their hydrodynamics related to flow regimes, gas hold holdup, liquid velocity, liquid mixing, etc. was discussed. The G-L mass transfer characteristics were not discussed because in the case of ADrs, the gas is recirculated only to facilitate liquid mixing. Mass transfer of gas in the liquid phase is not important. Due to lack of information about low $L / D$ ratio IGLRs, the hydrodynamics of gaslift digesters is not known in detail, making their design and scale-up difficult. This is the main motivation behind studying the hydrodynamics of gaslift digesters (low $L / D$ ratio IGLRs) using experimental and computational techniques.

To understand the impact of mixing, hydrodynamic experiments need to be conducted on the selected digester configuration. CARPT and CT were identified as suitable techniques for revealing hydrodynamics of ADrs. In addition, CFD can also be used to study the hydrodynamics of ADrs, but the CFD models need to be evaluated against the experimental data obtained from CARPT and CT.The hydrodynamics of a reactor are significantly affected by its scale of operation; thus, to get a true feel for the magnitudes of hydrodynamic variables like phase velocity and holdup and turbulence parameters in full scale reactors, experimentation/modeling on larger scale is necessary. If these kinds of experiments/modeling are carried out on both small and large scale, the comparison of these results can help in design and scale-up of ADrs. 


\section{Chapter 3}

\section{Multiple-Particle Tracking Technique: Development, Validation and Implementation}

\subsection{Introduction and Motivation}

The CARPT technique has been utilized for a number of years at the CREL to map flow fields and mixing in various opaque single phase and multiphase systems using a single radioactive tracer particle. Useful hydrodynamic information can be obtained from CARPT studies. CARPT is one of the technologies for application to opaque systems like anaerobic digesters as well. However, the nature of the slurry and the flow in the digester presents some technical challenges that were not encountered in the previous applications of CARPT, including:

1. With gaslift digester designs studied and discussed in chapter 4, we observed very slow flows in some portions of the digesters. These slow flows may have caused possible solids settling. This caused two difficulties: (a) data collection was very slow in certain portions of the reactor and/or under certain operating conditions, and (b) the tracer particle would settle in more than one instances and thus will cause a halt to data collection. These problems are enhanced with increase in the scale of operation. 
2. The slurry in the anaerobic digester consists of particles having different properties (size, shape, and density), while the current CARPT technique used only single-particle tracking.

Thus the data collection process was slow and all the required information such as the hydrodynamics behavior of the solids of different physical properties, and the segregation and interaction of the solid particles could not be obtained by tracking a single radioactive particle.

The current CARPT data-acquisition assembly has many components, which not only makes it bulky, but also expensive. The assembly and the synchronization of the components of single particle CARPT unit is very time consuming and laborious. Since the CARPT was introduced at the CREL in 1990 by Yubo Yang, Moslemian and Devenathan, very few things have been changed with the CARPT hardware. Thus, a development of a new system was required, which will not only extend the capabilities and overcome few of the limitations of the current CARPT system, but will also improve the current CARPT assembly, in terms of accuracy and cost.

The data collection rate and the capability to deal with the settling of the tracer can be greatly improved by the introduction of multiple tracers that can be tracked simultaneously. Multiple-particle tracking can be pursued by introduction of particles containing different isotopes emitting gamma radiation of different energies that can be discriminated. In addition to speeding up the data collection rate for slow flows and reducing the impact of the particle settling, multiple-particle tracking will offer other important advantages, such as the capability to simultaneously track the motion of particles of different size, shape, and density, determining segregation of particles, and probing particle interactions. Use of the advanced technology in designing a new system will also provide an opportunity to make the new assembly compact, cheaper, faster, and easy to operate and understand. 
The multiple-particle tracking technique will be a valuable tool for characterization of number of multiphase processes/reactor systems of industrial interests, which use a range of particles with different properties. For example, gas-solid fluidized beds are widely used in process industries for large-scale applications like coal gasification to small scale, polymer and pharmaceutical, production (Lee et al., 2005). These reactors contain a large amount of solids with a wide range of sizes and some times different densities; characterization of flow of these solids of different physical properties can provide valuable information for designing and understanding these systems. Similarly MP-CARPT can be very useful in the evaluation of multiphase processes in gas-liquidsolid (GLS) and liquid-solid (LS) fluidized beds, stirred tanks, slurry bubble columns, etc.

To accomplish the above objectives, a new data acquisition system for tracking multiple radioactive particles was designed and manufactured. Because of its ability to track more than one radioactive particle, it was named as Multiple-Particle Tracking Technique and abbreviated as MP-CARPT after CARPT. The system was developed with the help of the team from the Oak Ridge National Laboratory (ORNL) consisting of electronic engineers, software engineers and nuclear engineers. Dr. Alan Wintenberg designed the hardware and electronics, Dr. Lloyd Clonts helped with the design of the acquisition software, and Dr. Chuck Alexander provided the input on the radiation and radioactive particles. Dr. David Depaoli oversaw the activities at the ORNL as a Co-PI with Dr. Muthanna Al-Dahhan as project PI. The hardware was assembled at the CREL and the necessary modifications to hardware and software were also made at the CREL.

This chapter covers the validation and implementation of the MP-CARPT. Various issues related to the design and selection of the MP-CARPT system and its components are discussed in this chapter. The details of the hardware and software are provided in the Appendix B. The procedure and the guidelines to operate the MP-CARPT unit is also explained in the Appendix B. The principle of the MP-CARPT, results of the validation and implementation are presented in the following discussion. 


\subsection{Selection of Radioactive Sources}

A number of characteristics should be considered carefully for the selection of the radioactive sources to be used in the MP-CARPT experiments. Following are the main considerations:

1. Gamma energy peak: The MP-CARPT works on the principle of discrimination between different sources based on the gamma energy peak (explained in detail in the next section). This requires that the gamma peaks of different particles should be well separated from each other. At least one peak of any one particle should be completely separated from all other peaks of other particles. In addition to that, for MP-CARPT to work, no more than two gamma peaks of two different particles should be overlapped. This criterion narrows the radioactive sources as possible candidates for the MPCARPT.

2. Half-life period: The activity of a radioactive source is reduced by $50 \%$ in time equal to its half-life. Half-life of a radioactive particle can be in the range of few seconds to many years. Since each experiment takes at least a period of 48 hours, neglecting the technical difficulties, the half-life of possible radioactive candidate should be preferably more than 48 hours. But considering the time required for the shipping of activated source, legal formalities, particle preparation, etc., only sources with the half-life of over a month are suitable for the experimentation. Relatively longer half-life guarantees multiple use of particle and reduces the costs of frequent activation. On the contrary, very long half-life means a longer liability on the part of the user for its protection, handling and maintenance.

3. Physical state: The radioactive source to be used as a tracer must be easy to handle and be able to mimic the phase to be tracked. It cannot be miscible with the system. Thus radioactive sources existing in gas or liquid phase are not suitable for the MPCARPT. Radioactive sources available in solid phase, such that the density of source can be adjusted (explained in next section), are suitable candidates. In addition, a 
radioactive source should also be recoverable after the experimentation, thus it has to be in a solid state or in a solid composite particle. Liquid tracers can be used if enclosed properly in a leak proof casing, but until the safe procedures to do so are identified and benchmarked, and approval is obtained from the Radiation Safety Department, the radioactive sources available in solid state are the only viable choices at this time.

4. Density: The density of the radioactive tracer should match the phase being tracked. For this reason the density of the source is manipulated in different ways to make it either lighter or heavier to match the density of the phase to be tracked. A radioactive source (in solid state) can be coated with suitable material or it can be enclosed in a tiny plastic ball to adjust its density. But if the density of the radioactive source is very high, it would not be easy to adjust its density to the required value. Density is certainly a factor important in the selection of the radioactive source, but it is dependent on the requirements of system to be studied.

5. Personnel safety: The safety of the personnel handling and using the radioactive material is of prime importance. Excessive exposure to radiation causes serious health problems. Thus, the selected radioactive source should possess minimum health risks. It should be easy to handle and easy to clean up in case of contamination.

There are many other considerations in the selection of radioactive source such as physical and chemical properties of the source, cost, ease of availability, ease of activation and legal formalities. The Radiation Safety Department at Washington University controls the possession and use of any radioactive material. The radioactive source should pass the approval of the Radiation Safety Department before being used.

Table 3.1 gives a condensed list of radioactive sources that may or may not be suitable for the MP-CARPT. Only the elements occurring in a solid form with a half life greater than 30 days and less than 5 years are listed in the Table 3.1. The sources which do not produce gamma or with very low percentage of gamma production are also not listed in Table 3.1. 
Table 3.1 List of possible radioactive candidates to be used for MP-CARPT (obtained from Wang, 1969)

\begin{tabular}{|c|c|c|c|c|}
\hline $\begin{array}{l}\text { Element (mass } \\
\text { number) }\end{array}$ & $\begin{array}{l}\text { Half } \\
\text { life }\end{array}$ & $\begin{array}{c}\text { Gamma energy } \\
\mathrm{MeV}(\%)\end{array}$ & $\begin{array}{c}\text { Density } \\
(\mathrm{g} / \mathrm{cc})\end{array}$ & Comments \\
\hline Beryllium (7) & $53 \mathrm{~d}$ & $0.48(10)$ & 1.8 & Poisonous \\
\hline Sodium (22) & $2.58 y$ & $0.511(180), 1.27(100)$ & 0.97 & Reactive with water \\
\hline Scandium (46) & $84 d$ & $0.89(100), 1.12(100)$ & 2.9 & Can be used \\
\hline Manganese (54) & $303 d$ & $0.83(100)$ & 7.3 & Can be used \\
\hline Cobalt (56) & $77.3 \mathrm{~d}$ & $0.85(100)-3.3(13)$ & 8.7 & $\begin{array}{c}\text { Many gamma energies, not } \\
\text { suitable }\end{array}$ \\
\hline Cobalt (57) & $267 d$ & $0.12(87), 0.14(11)$ & 8.7 & Very low gamma energies \\
\hline Cobalt (58) & $71 d$ & $0.81(99), 1.7(0.6)$ & 8.7 & Can be used \\
\hline Cobalt (60) & $5.26 y$ & $1.17(100), 1.33(100)$ & 8.7 & suitable \\
\hline Zinc (65) & $245 \mathrm{~d}$ & $1.12(49)$ & 7.1 & Low gamma percentage \\
\hline Selenium (75) & $120 \mathrm{~d}$ & $0.14(57), 0.27(60)$ & 4.8 & low gamma energies \\
\hline Rubidium (83) & $83 \mathrm{~d}$ & $0.53(93), 0.79(1)$ & 1.5 & \multirow{2}{*}{$\begin{array}{l}\text { Spontaneously flammable } \\
\text { in air, explosive in water }\end{array}$} \\
\hline Rubidium (84) & $33 d$ & $0.9(74), 0.5(42)$ & 1.5 & \\
\hline Strontium (85) & $64 d$ & $0.51(100)$ & 2.6 & Reactive with water \\
\hline Yttrium (88) & $108 \mathrm{~d}$ & $0.9(91), 1.84(100)$ & 4.5 & Suitable \\
\hline Zirconium (95) & $65 \mathrm{~d}$ & $0.72(49), 0.76(49)$ & 6.4 & Can be used \\
\hline Niobium (95) & $35 \mathrm{~d}$ & $0.77(100)$ & 8.6 & Can be used \\
\hline Ruthenium(103) & $40 \mathrm{~d}$ & $0.5(88), 0.61(6)$ & 12.2 & Very high density \\
\hline Antimony (124) & $60 d$ & $0.6(97), 1.7(50), 8$ to 2.1 & 6.68 & Many gamma energies \\
\hline Cesium (134) & $2.1 \mathrm{y}$ & $0.6(98), 0.8(98)$ & 1.87 & $\begin{array}{l}\text { Explosive in water, reacts } \\
\text { with air }\end{array}$ \\
\hline Cerium (139) & $140 \mathrm{~d}$ & $0.165(80)$ & 6.9 & Very low gamma energy \\
\hline Hafnium (175) & $70 \mathrm{~d}$ & $0.34(85)$ & 11.4 & $\begin{array}{c}\text { Low gamma energy, high } \\
\text { density }\end{array}$ \\
\hline Osmium (185) & $94 d$ & $0.65(80), 0.88(14)$ & 22.48 & $\begin{array}{l}\text { Heaviest element, oxide is } \\
\text { poisonous }\end{array}$ \\
\hline Iridium (192) & $74 d$ & $0.32(80), 0.47(49)$ & 22.4 & Extremely high density \\
\hline
\end{tabular}

Upon careful consideration of all the above criteria, Co-60 and Sc-46 were selected for dual-particle tracking to evaluate the developed technique. High-energy gamma peak of Co-60 is completely distinguished from other gamma peaks of Sc-46, which satisfies the most crucial criterion. The half life of Sc-46 is only 84 days, which is suitable. Co-60 has 
a very long half life of 5.27 years is not desirable from safety consideration, but is suitable for frequent use at no additional cost of activation. Co-60 and Sc-46 are both available in solid state with densities of 8.9 and $2.98 \mathrm{~g} / \mathrm{cm}^{3}$, respectively. Co-60 is heavier, due to which smaller size particles are required. Smaller the particle, longer is the activation time and harder it is to handle. Thus, Co-60 and Sc-46 may not be the ideal candidates, but they are the best possible alternatives that meet most of the requirements mentioned above at this time for the development, validation and implementation of the MP-CARPT.

\subsection{MP-CARP'T Electronics}

Figure 3.1 below shows the schematic of the new MP-CARPT electronics. The connections of the electronics components are shown in Figure 3.2. The MP-CARPT unit essentially consists of detectors, formed by a photomultiplier tube (PMT) connected to the base amplifier. This base amplifier is powered by a power supply unit and the output signal from the base amplifier goes to timing filter amplifier (TFA) input for amplification. Both power supply unit and timing amplifier sit in a NIM bin. Each timing amplifier has 8 channels (one for each detector). The timing amplifier is connected to the pulse processor card (one card is required for one timing amplifier, thus 8 detectors need only one card). The pulse processor card functions as a discriminator, scaler and an interface to the PC. This pulse processer card sits in a compact PCI box and it is connected to the back plane of compact PCI which also holds a PC on a card. 


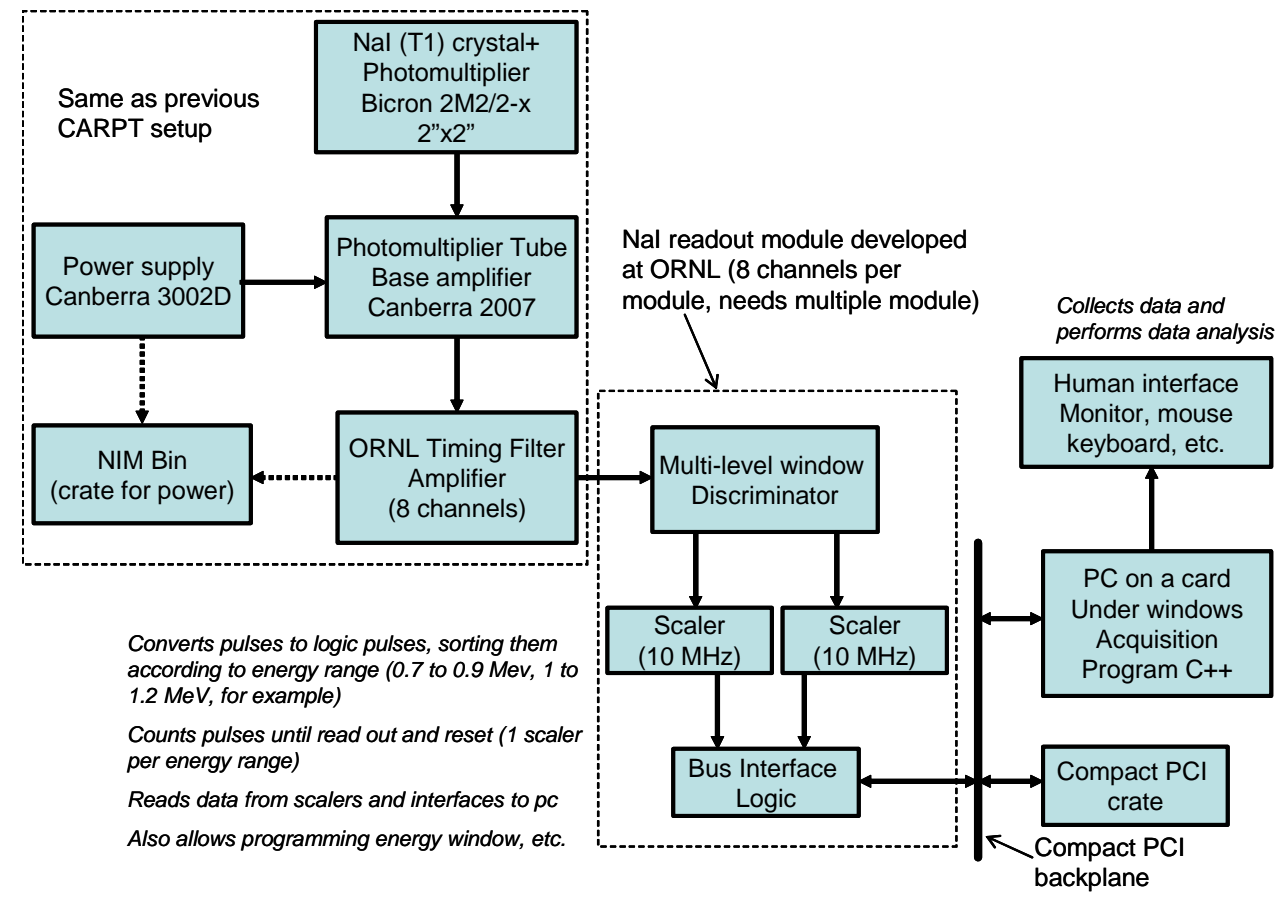

Figure 3.1 MP-CARPT electronics

A single $\mathrm{C}++$ program compiled and run by the user performs the data acquisition according to the needs of the user. Each component of the MP-CARPT unit, its operation and its functions are explained in Appendix B.

Even though the list of electronics is long, it is all contained in only tow boxes/crates. This reduces the wiring connections and avoids the lengthy set-up procedures. Less number of components also cuts the costs significantly. Cost estimation of MP-CARPT electronics and its comparison with the cost of the single particle CARPT unit shown in Table 3.2 shows the cost savings of $\$ 25,000$ obtained with new unit for set-up of 16 detectors. Moreover, the new electronics is advanced, thus it is more efficient and faster in data acquisition. 


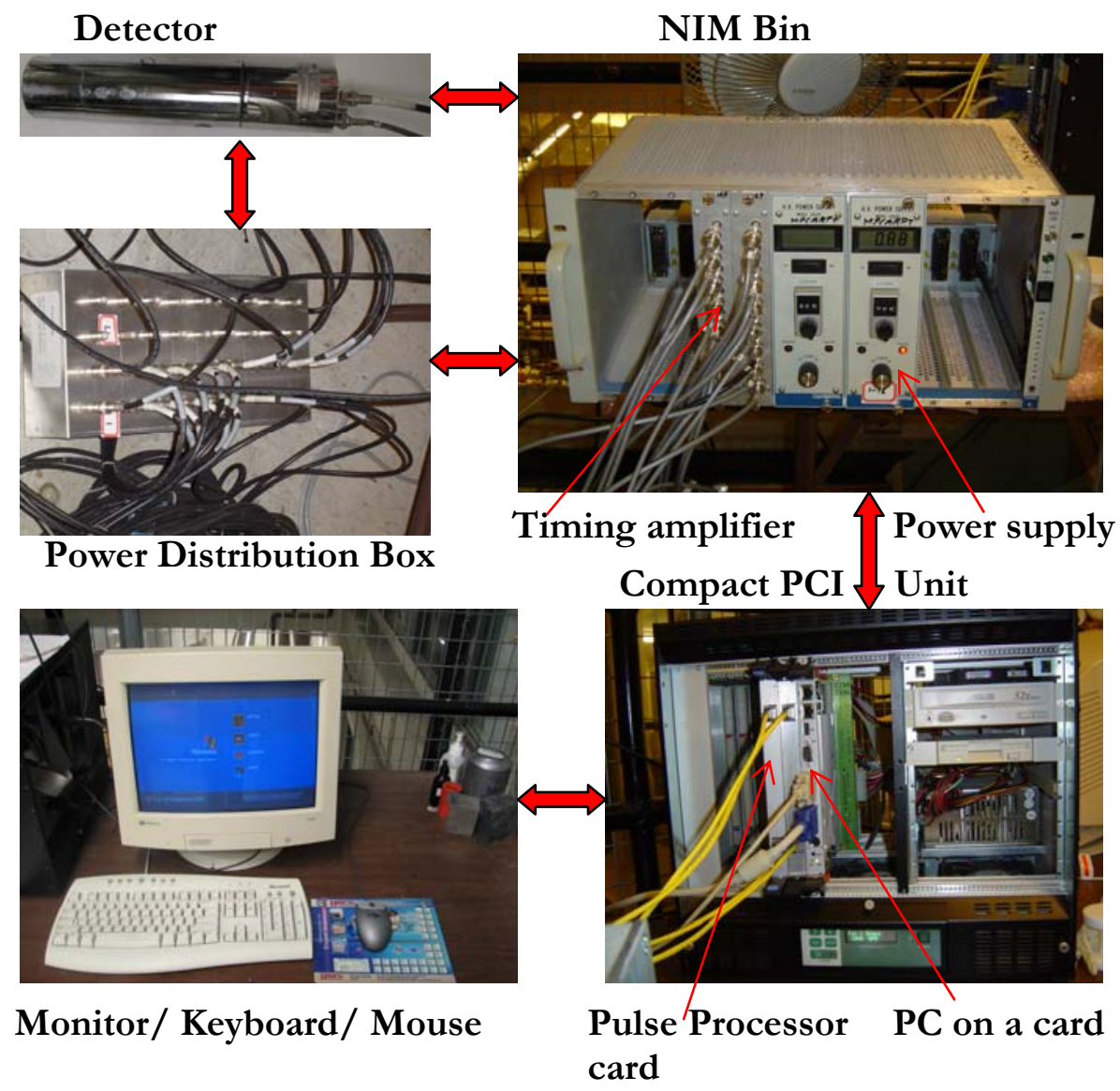

Figure 3.2 MP-CARPT electronics components and connections

\subsection{MP-CARPT Validation}

\subsubsection{Tracking Stationary Particles}

The MP-CARPT technique, its principles, operation, and data-processing will be discussed here in reference to the tracking of stationary Co-60 and Sc-46 particles, both for the single-particle tracking and dual-particle tracking. By tracking stationary particles at known locations, the error in the reconstruction can be evaluated and the MPCARPT electronics and reconstruction algorithm can be validated. 
Table 3.2 Cost comparison of old and new unit for 16 detectors

\begin{tabular}{|c|c|c|c|c|}
\hline \multirow[t]{2}{*}{ Component } & \multicolumn{2}{|c|}{$\begin{array}{c}\text { Old single particle CARPT } \\
\text { unit }\end{array}$} & \multicolumn{2}{|c|}{ New MP-CARPT unit } \\
\hline & quantity & cost (USD) & quantity & cost (USD) \\
\hline $\begin{array}{l}\text { PMT and its } \\
\text { base }\end{array}$ & 16 & 16,080 & 16 & 16,080 \\
\hline TFA & $\begin{array}{c}16 \text { (at } \$ 1,100 \\
\text { each) }\end{array}$ & 17,600 & $\begin{array}{l}2 \text { (at } \$ 1,200 \\
\text { each) }\end{array}$ & 2,400 \\
\hline Power supply & 1 & 1,540 & 1 & 1,540 \\
\hline NIM Bin & 2 & 4,550 & 1 & 2,275 \\
\hline Computer & 1 & 500 & $\begin{array}{l}1 \text { (PC on a } \\
\text { card })\end{array}$ & 5,500 \\
\hline Power Cables & 20 & 200 & 20 & 200 \\
\hline Signal Cables & 16 & 1,704 & 16 & 1,704 \\
\hline Other Cables & $\begin{array}{c}16 \text { (at \$55 } \\
\text { each) }\end{array}$ & 880 & 8 (at $\$ 4$ each) & 32 \\
\hline $\begin{array}{l}\text { Pulse Processor } \\
\text { Module }\end{array}$ & 1 & 15,450 & $\begin{array}{l}2 \text { (at } \$ 2,000 \\
\text { each) }\end{array}$ & 4,000 \\
\hline Total & & 58,504 & & 33,731 \\
\hline Savings & & $\$ 24$ & 75 & \\
\hline
\end{tabular}

\section{Experimental Set-up}

16 number of $\mathrm{NaI}$ detectors were mounted circumferentially on a stand in 8 columns. Each column had two detectors mounted one over other and separated by 3.9 inches. Two consecutive columns were $45^{\circ}$ apart, thus covering whole $360^{\circ}$ by 8 columns. The arrangement of detectors is shown schematically in Figure 3.3a and a photograph is shown in Figure 3.3b. 


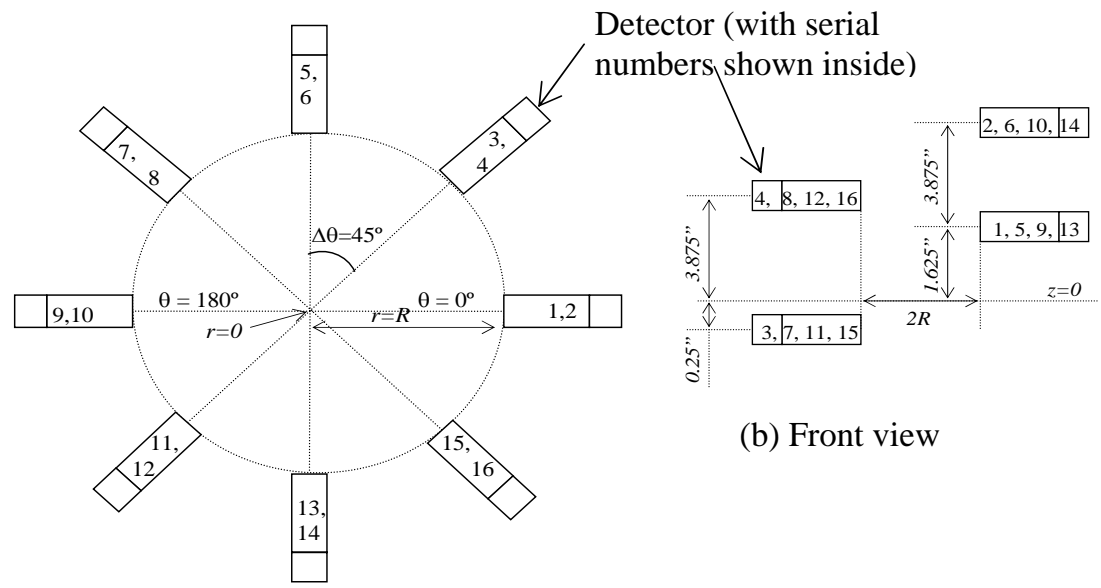

(a) Top view

Figure 3.3a Schematic of the arrangement of detectors on detector stand

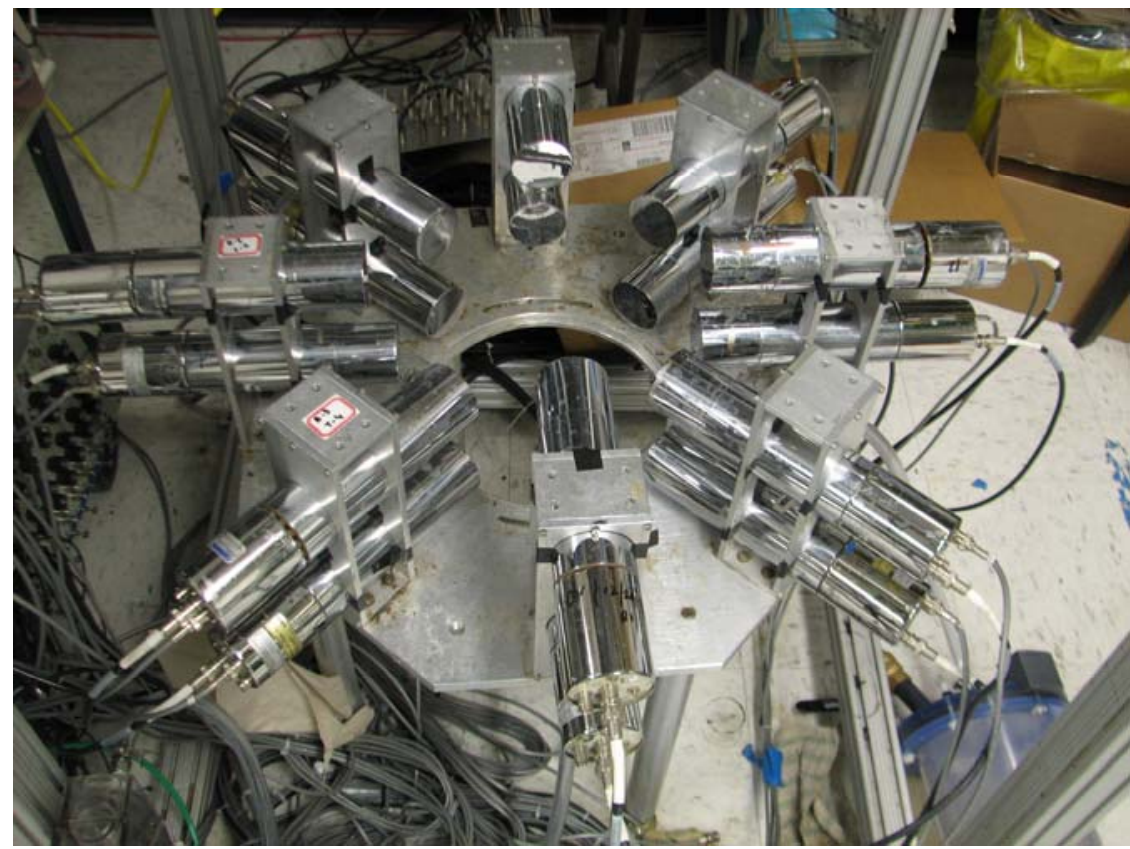

Figure 3.3b Photograph of detector stand

An automated calibration device was used for carrying out the calibration. The device is equipped with a rod to hold the radioactive source at one end. This rod is connected to three separate motors for independent movement of rod in axial, radial and azimuthal direction. The design and details of calibration device are given in detail by Luo (2005). 
Co-60 and Sc-46 were used as the radioactive sources. $100 \mu \mathrm{m}$ Co-60 particle with approximate activity of $100 \mu \mathrm{Ci}$ was enclosed in a $1 \mathrm{~mm}$ polypropylene ball to match the density of the water which represented liquid phase in the experiments. The Sc-46 particle was $150 \mu \mathrm{m}$ in diameter with approximate activity of $150 \mu \mathrm{Ci}$ was also enclosed in a $1 \mathrm{~mm}$ polypropylene ball. Enclosing the particles in plastic balls makes it convenient to handle and see the particles and also ensures safe handling.

\section{Principle and Methodology}

Gamma peaks obtained by recording the photon counts of Sc-46 and Co-60 in fine mode (fine mode records the counts of all energies as opposed to coarse mode, where only the counts in a selected energy window are recorded) are shown in Figure 3.4. The procedure to obtain scans and operate the MP-CARPT unit is explained in Appendix B. To obtain the counts for generating gamma peaks, the radioactive sources can be placed anywhere within the vicinity of all the detectors, but not too close to the detectors.

The counts obtained form radioactive particles are additive. The total counts of Sc-46 and Co-60 obtained individually are equal to the counts obtained from both sources together, illustrated and proved by Figure 3.4. Figure 3.4 reveals one more important point that forms the principle for discriminating between different radioactive sources. The high energy peak of Co-60 (1.332 MeV) is completely distinguished from other peaks of Sc-46. Thus, if counts of Sc-46 and Co-60 are obtained in such a way that the high energy counts of Co-60 are recorded separately, then reconstruction of Co-60 is a trivial problem similar to reconstruction of single particle in CARPT (see CARPT manual, 2005 for details of reconstruction of single particle tracking). The additive property of counts can be used for reconstruction of Sc-46 particle. 


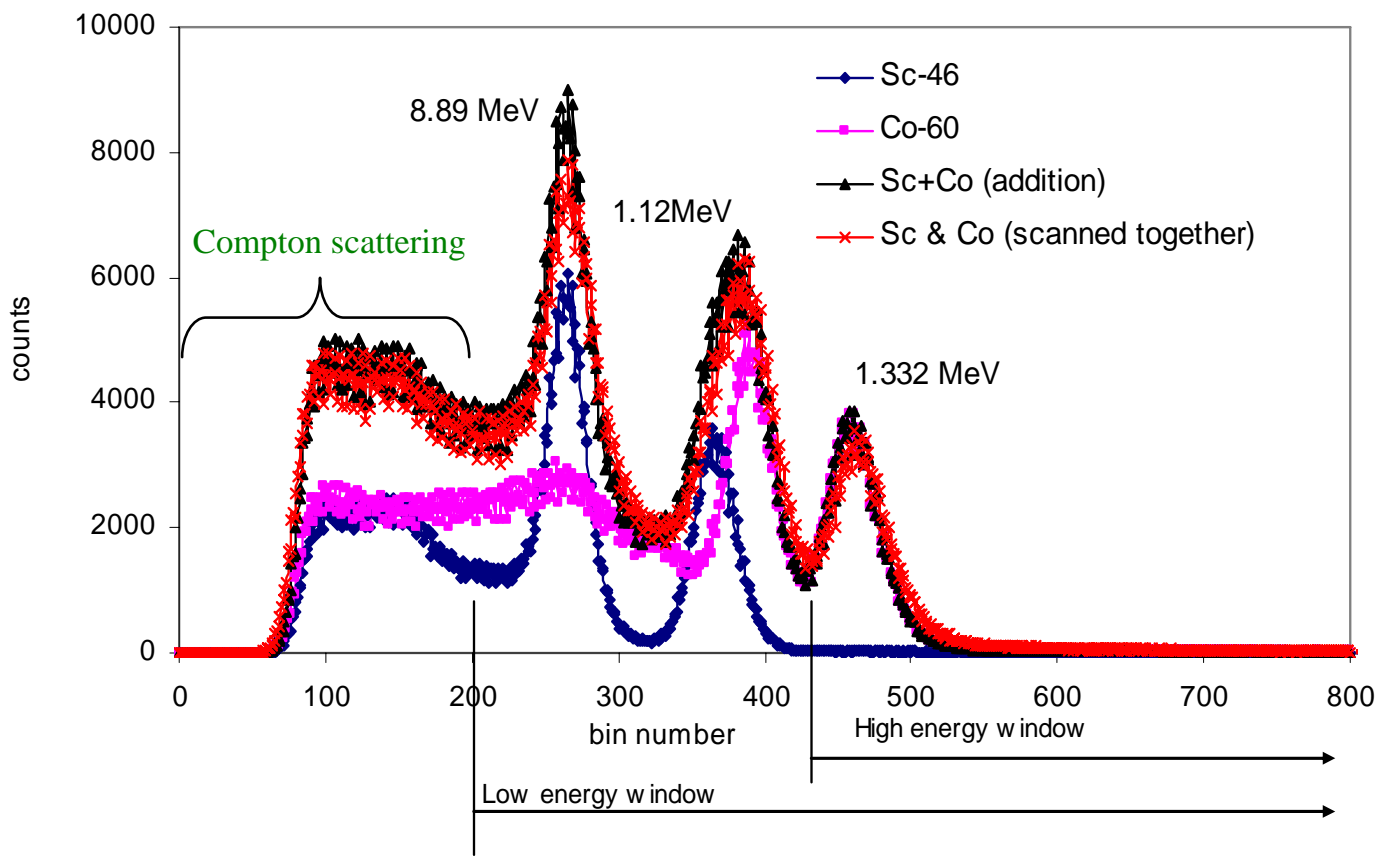

Figure 3.4 Gamma peaks of Sc-46 and Co-60 individually, together and summation of individual counts

\section{Selection of Energy Windows}

The counts of high energy and low energy peaks are separated by setting up the energy windows for discriminator. The new MP-CARPT unit is capable of recording counts in eight separate energy windows. The lower and upper limit of each window can be specified by the user; the windows can also be overlapped if necessary. This gives us the ability of tracking eight different radioactive sources simultaneously. However, tracking and reconstruction of only two radioactive sources is discussed here. Once dual particle tracking is tested and validated then this technique can be extended easily to track more than two radioactive sources.

The first step in the MP-CARPT is to obtain position of energy peaks of Sc-46 and Co60 for each detector, as shown in Figure 3.4. The limits of energy window for calibration and tracking experiment are obtained from Figure 3.4. The complete energy spectrum is spread from 0 to 1023 bins by discriminator. Lower and higher limit of high 
energy window can be $425^{\text {th }}$ and $600^{\text {th }}$ bin, respectively. Thus all the counts corresponding to the energy level from $425^{\text {th }}$ to $600^{\text {th }}$ bin (both inclusive) will be recorded in high energy window. The higher limit can be extended till $1023^{\text {rd }}$ bin, this will necessarily make no difference because the counts of both the sources are zero from bin number 525 . But the lower limit has to be specified higher than $425^{\text {th }}$ bin, as only this way the counts of Co-60 can be recorded distinctly without any overlapping from Sc-46. The lower and higher limit for low energy window can be $200^{\text {th }}$ and $425^{\text {th }}$ bin, respectively. Again the lower limit can be as low as bin number zero. But the Compton scatter present in lower bin numbers below 200 introduces error during reconstruction and has to be avoided (see CARPT manual, 2005 for more discussion on Compton scatter). The lower limit can be set as $315^{\text {th }}$ bin to exclude Compton scattering by Sc-46 as well; but it has to remembered that low span of energy window reduces the number of counts in the window. Lower counts also introduce error in the reconstruction. If the activity of the sources used is high, then the low span of energy window is acceptable. Very high activity of sources however, will cause the problem of peak shift (discussed in Appendix B).

All these points should be considered carefully to select the limits of energy windows. Every detector can have different specifications of limits of energy windows based on the detector settings. Thus, synchronization of detectors is not required when using MP-CARPT unit. Synchronization of detectors means matching the position of gamma peaks for all the detectors. Synchronization of detectors (traditionally referred to as MCA in the CREL) is a major time consuming step with old single particle CARPT unit. A set of 16 detectors needed about 24 to 48 hours for synchronization, more number of detectors required more time. With the new unit, the energy peaks as shown in Figure 3.4 can be obtained even in a fraction of second, but to obtain enough number of counts, the counts should be obtained for at least 15 seconds. 60 seconds of data acquisition is more than sufficient for obtaining Figure 3.4 in all cases; this time is independent of number of detectors. Thus, MP-CARPT unit offers a huge time-saving advantage over the old single particle CARPT unit. 


\section{Calibration}

Two sets of calibration are required for (stationary or moving) dual particle tracking; one for each source, Sc-46 and Co-60, separately. Same limits of energy windows and data acquisition frequency should be used for both calibration and tracking. The suitable value of data acquisition frequency is selected, $50 \mathrm{~Hz}$ (50 samples per second) in this case. The data acquisition frequency can be changed by adjusting the acquisition time for each sample in the acquisition program (data acquisition time of 0.02 seconds corresponds to sampling frequency of $50 \mathrm{~Hz}$ ). Data acquisition frequency cannot be too high or too low for tracking moving particles. Very high values, normally above $200 \mathrm{~Hz}$, introduce noise in the acquired data. The lower limit of allowable acquisition frequency depends on the maximum velocity of moving particle in the system. Low frequencies

can cause error in reconstruction, referred to as dynamic bias (Rammohan et al. 2001, Rammohan, 2003).

For calibration, each particle is placed individually (in absence of other source) at several known locations and tracked until desired number of samples are obtained. The data is acquired in coarse mode for the calibration and experiment (details given in Appendix B). The average of all the samples for each calibration location is used for reconstruction. Thus, maximum possible number of samples should be obtained during calibration for better accuracy. 512 number of samples were generally obtained for each calibration location at acquisition frequency of $50 \mathrm{~Hz}$.

The number of calibration points depends on the geometry of the system. Maximum possible number of calibration points should be used. Generally, the geometry of system is divided into number of cells in radial, azimuthal and axial direction as shown in Figure 3.5, the calibration points can be located at either at the centers of the cells or at the nodes of the cells for convenience. The closer the calibration points, more the number of calibration points, thus lesser is the error in reconstruction of particle positions. 250 calibration points were used for tracking stationary particle, in this case. The cylindrical coordinates of calibration points are listed in Table 3.3. 


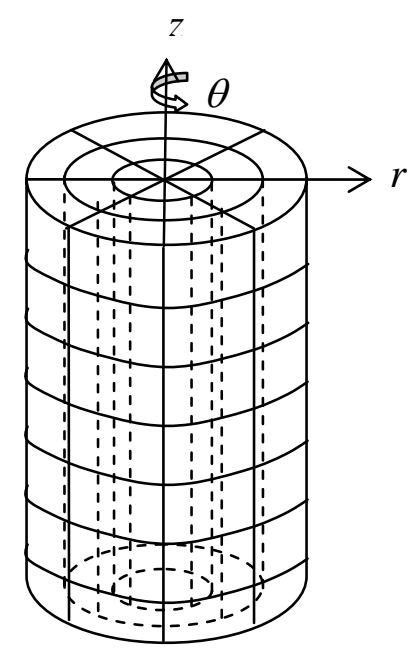

Figure 3.5 Grids for calibration points/locations

Table 3.3 Cylindrical coordinates of calibration points

\begin{tabular}{|l|l|l|l|}
\hline $\begin{array}{l}\mathrm{r} \\
\text { (inch) }\end{array}$ & $\theta$ (degrees) & $Z$ (inch) & $\begin{array}{l}\# \text { of calibration } \\
\text { points }\end{array}$ \\
\hline 0 & 0 & 0 to 4.5 (with $\Delta \mathrm{z}$ of 0.5$)$ & $1 \mathrm{x} 1 \mathrm{x} 10=10$ \\
\hline 1 & 0 to 330 (with $\Delta \theta$ of 30$)$ & 0 to 4.5 (with $\Delta \mathrm{z}$ of 0.5$)$ & $1 \mathrm{x} 12 \mathrm{x} 10=120$ \\
\hline 2 & 0 to 330 (with $\Delta \theta$ of 30$)$ & 0 to 4.5 (with $\Delta \mathrm{z}$ of 0.5$)$ & $1 \mathrm{x} 12 \mathrm{x} 10=120$ \\
\hline \multicolumn{2}{|l|}{ Total number of calibration points } & 250 \\
\hline
\end{tabular}

The calibration process is fully automated and performed with the help of calibration device (Luo, 2005). Calibration device is equipped with rod, which can be moved in radial, azimuthal and axial direction with the help of three separate motors. Radioactive particle is placed in a small plastic vial and the vial is attached to the end of the rod during calibration. The movement of motor is computerized and the motor movement program is integrated with data acquisition program. Thus, the calibration location ( $\mathrm{r}$, $\theta$ and $z$ ) is recorded automatically along with data acquisition.

This way the calibration for each particle is carried out separately. The calibration locations for both the particles should preferably be the same, but it is not required to be the same. If counts of Sc-46 and Co-60 together at 'position $x$ ' are required, they can 
be readily evaluated by summing up the individual counts of Sc-46 and Co-60, each recorded at the same 'position $x$ '.

The calibration data is obtained in two separate energy windows as explained earlier. Figure 3.6a and 3.6b shows the calibration plot for Sc-46 and Co-60, respectively, for both energy windows for a given detector. The ordinate in Figure 3.6a and 3.6b is the averaged value of counts obtained for 512 samples. The abscissa is the distance of a source from a given detector. The number of counts varies inversely with the distance from the detector; the counts recorded are higher when the source is nearer the detector and vice-a-versa. In Figure 3.6b, for Sc-46, the counts in high energy window are very low; they should be ideally zero, as the Sc-46 peaks do not fall in the high energy window (see Figure 3.4). Non-zero counts are recorded due to the background radiation or the random nature of radioactivity and introduce error in the reconstruction.

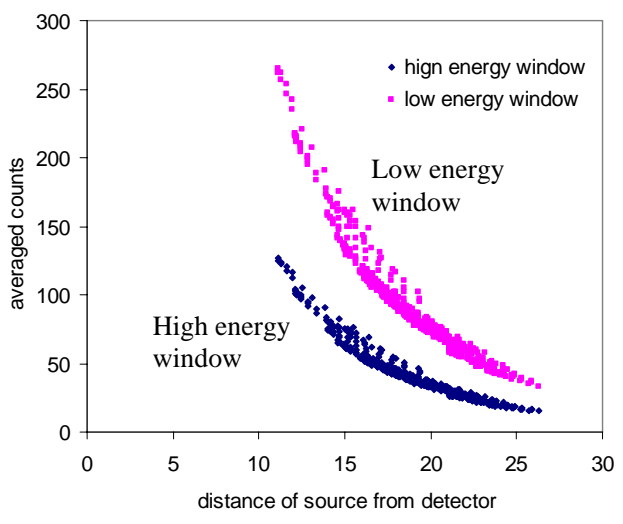

(a)

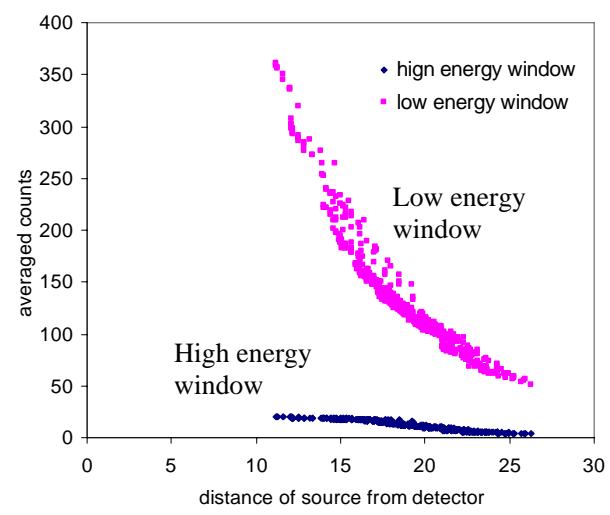

(b)

Figure 3.6 (a) calibration plot for Co-60 (b) calibration plot for Sc-46

\section{Stationary Tracking Experiment}

During the actual tracking experiment, Co-60 and Sc-46 particles were placed together at 48 known locations and counts data was obtained in coarse mode. The limits of low and high energy windows and the data acquisition frequency were equal to what was used during calibration. 64 samples of data at frequency of $50 \mathrm{~Hz}$ were obtained for 
each of 48 known locations. 24 of these locations were same as calibration points. Reconstruction of points which do not overlap the calibration points helps to test the accuracy of the reconstruction algorithm. The particles were placed at $r=1 \mathrm{inch}, \theta=0^{\circ}$ to $345^{\circ}$ with $\Delta \theta=15^{\circ}$, and $z=2$ and 3 inches, thus total $1 \times 24 \times 2=48$ locations.

Traditionally for tracking a moving particle in any reactor system, the particle is released into the system and it is tracked for at least a period of 24 hours at a suitable data acquisition frequency. The particles were kept at known stationary locations in this experiment for validation of the technique and to evaluate the error in the reconstruction.

\section{Reconstruction}

Obtaining the location of the radioactive particles from the acquired count data is called particle position reconstruction. The reconstruction algorithm is shown in Figure 3.7. Since the limits of high energy window are selected such that only counts of Co-60 are recorded in that window, the reconstruction procedure of Co-60 is exactly similar to that of single particle CARPT. Reconstruction procedure of single particle tracking is explained in short here, see CARPT manual (2005) for more details.

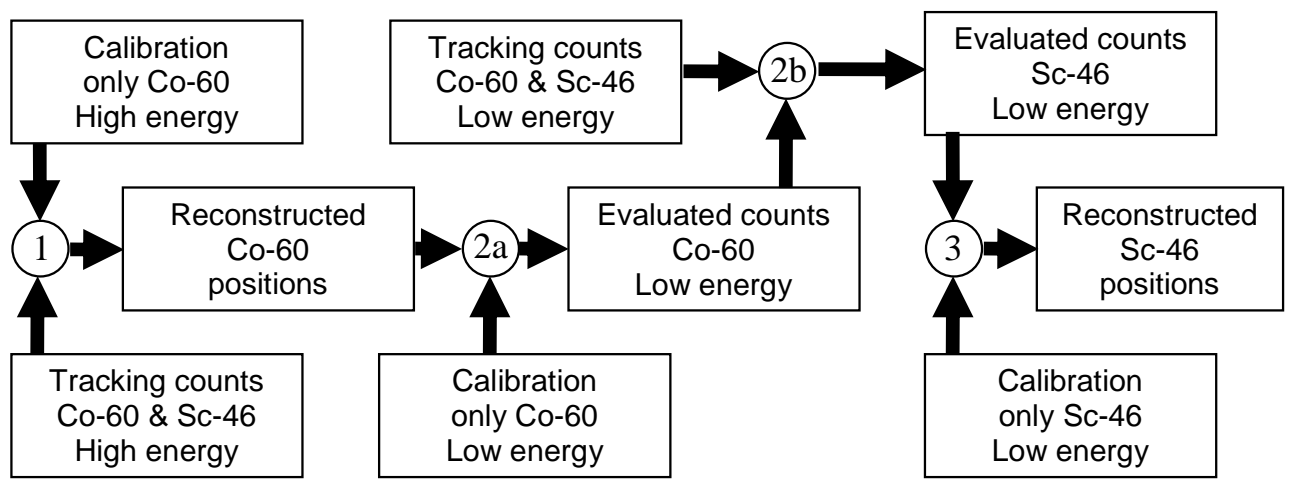

Data processing (step number showed inside the circle)

Figure 3.7 Reconstruction algorithm for dual-particle tracking 
The first step is to reconstruct the Co-60 positions. The calibration curve of Co-60 (for high energy window) is fitted using spline fitting and spline coefficients are obtained for each detector (Rados, 2003). Using these coefficients, if the counts of Co-60 in high energy window for a particular detector are known, the distance of particle from a given detector can be evaluated. Then the counts from the experiment are used to calculate the distance of the particle from each detector using spline fit coefficients. Now we have $N$ number (equal to number of detectors) of known distances and three unknown coordinates $(x, y$ and $z)$ to evaluate (see equation 5.1).

$$
d_{i}=\sqrt{\left(x-x_{i}\right)^{2}+\left(y-y_{i}\right)^{2}+\left(z-z_{i}\right)^{2}} \quad \text { for } i=1 \text { to } N
$$

where, $d_{i}$ is the distance of particle from $i^{\text {th }}$ detector

$$
\left(x_{i}, y_{i}, z_{i}\right) \text { are the coordinates of } i^{\text {th }} \text { detector }
$$

$N$ is the number of detectors

Thus, it becomes a problem of solving a system of $N$ nonlinear equations using a least square approximation method to evaluate three unknowns (where $N>3$ ). The least square approximation function is given in equation 5.2.

$$
f(x, y, z)=\sum_{i=1}^{N}\left\{\left[\left(x-x_{i}\right)^{2}+\left(y-y_{i}\right)^{2}+\left(z-z_{i}\right)^{2}\right]-d_{i}{ }^{2}\right\}
$$

The reconstructed positions evaluated in this manner are then filtered to remove any noise in the processed data, encountered due to the random nature of radioactivity. More details of reconstruction and filtering are given in CARPT Manual (2005), Rados (2003), Degaleesan (1997) and Bhusarapu (2005). An alternate, more accurate method of reconstruction was formulated by Bhusarapu (2005). But this method is computationally very time-consuming. 
Next step is to obtain the counts of Sc-46 only from the low energy window (Figure 3.7). The counts in low energy window are contributed both by Co-60 and Sc-46. The counts of Co-60 in the low energy window are evaluated using the reconstructed Co-60 locations, spline fit coefficients and low energy window calibration of Co- 60 . Then for each experimental data, the Co-60 counts in low energy window are subtracted from the total counts in low energy window to obtain the counts of Sc-46 in this window. Step three, is to reconstruct the positions of Sc-46. It is exactly similar to step one, since the counts are known, and calibration curve (for Sc-46 low energy window) is available.

The reconstructed positions of $\mathrm{Co}-60$ and Sc-46 (tracked together) using above reconstruction method are shown in Figures 3.8a and 3.8b, respectively. The error in reconstruction of Co-60 is less than $5 \%$ for $\mathrm{x}$ and $\mathrm{y}$ coordinates whereas $15 \%$ for $\mathrm{z}$ coordinates because the calibration grid in $\mathrm{z}$ direction was coarser than in $\mathrm{x}$ and $\mathrm{y}$ direction. The error in reconstruction of Co-60 is less than Sc-46 because the Sc-46 counts do not interfere with Co-60 in high energy window. But the error in reconstruction of Sc-46 is less than 5\% for x-coordinates, about $25 \%$ for $y$-coordinates and $20 \%$ for z-coordinates. This error is very large and unacceptable. There are two main reasons for this large error in reconstruction of Sc-46.

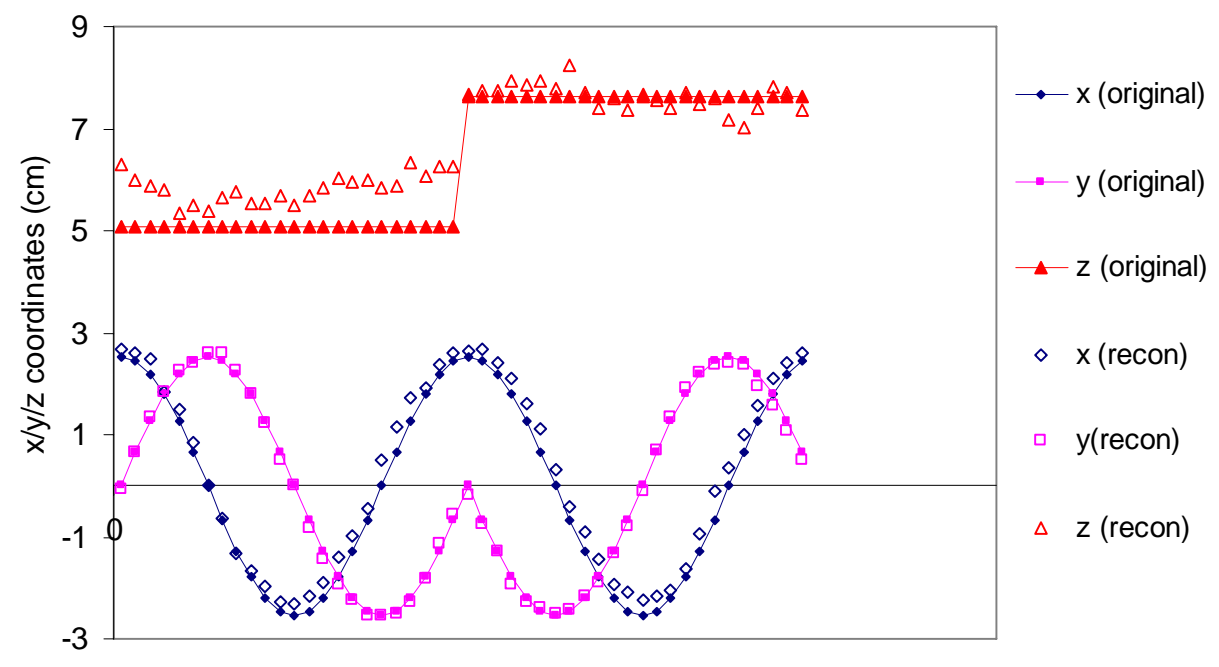

Figure 3.8a Reconstructed positions of Co-60 and comparison with original experimental positions 


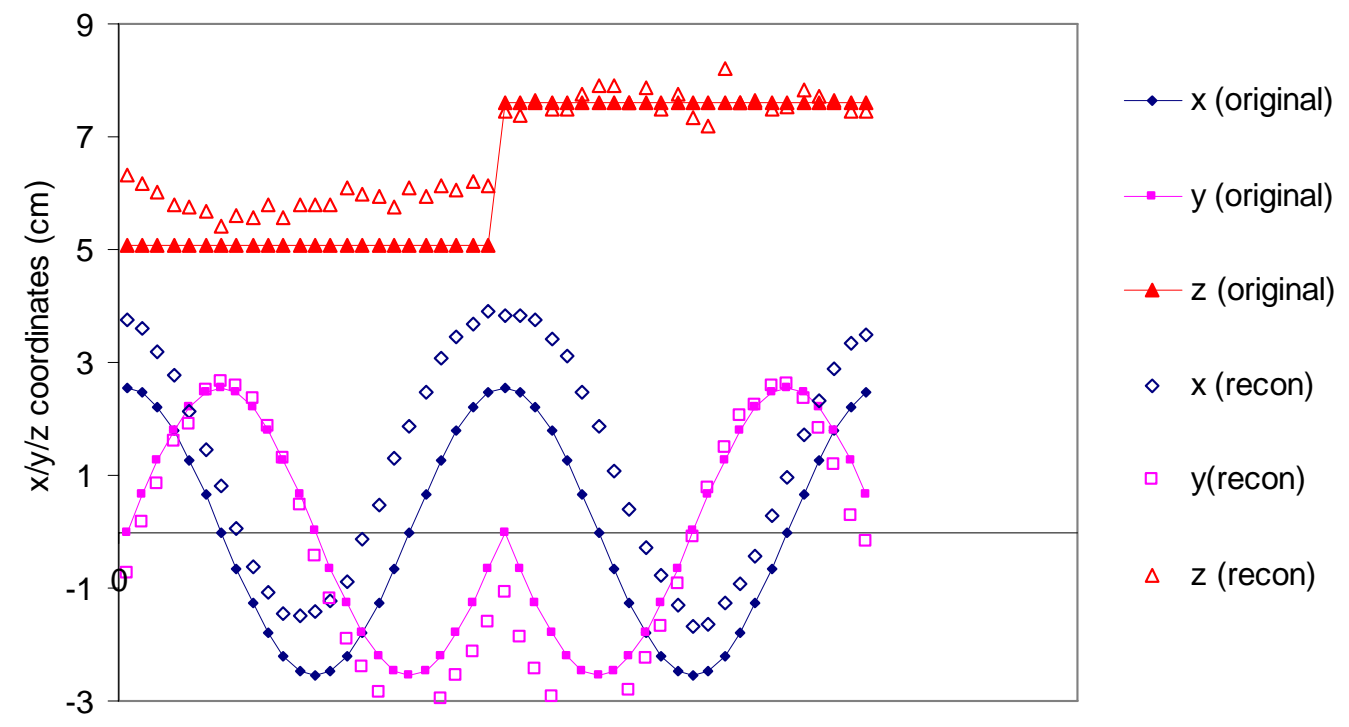

Figure 3.8b Reconstructed positions of Sc-46 and comparison with original experimental positions

First, the reconstructed Co-60 positions are used in the reconstruction of Sc-46. The small error in Co-60 reconstructed positions contributes and amplifies the error caused due to numerical approximations during reconstruction and due to the random nature of radioactivity. The second reason for large error is due to the subtraction of counts carried out in step two of reconstruction (see step $2 \mathrm{~b}$ in Figure 3.7). The result of subtraction is sometimes a negative number, which is treated as zero counts in reconstruction program and introduces error. Recall that the calibration counts are average of large number of samples where as the experimental counts are very random in nature, which is the main reason of error in reconstruction. Therefore, a new methodology for particle reconstruction is needed.

\section{Development of a New Reconstruction Methodology}

To avoid the large error in the reconstruction of Sc-46, the subtraction of counts of Co60 from total low energy window counts has to be avoided. Thus, a new reconstruction scheme has been developed. Since the Co-60 reconstruction involves negligible error, same procedure as described before (step 1) can be used for Co-60 reconstruction. A 
new reconstruction algorithm for Sc-46 positions is described below and shown in Figure 3.9.

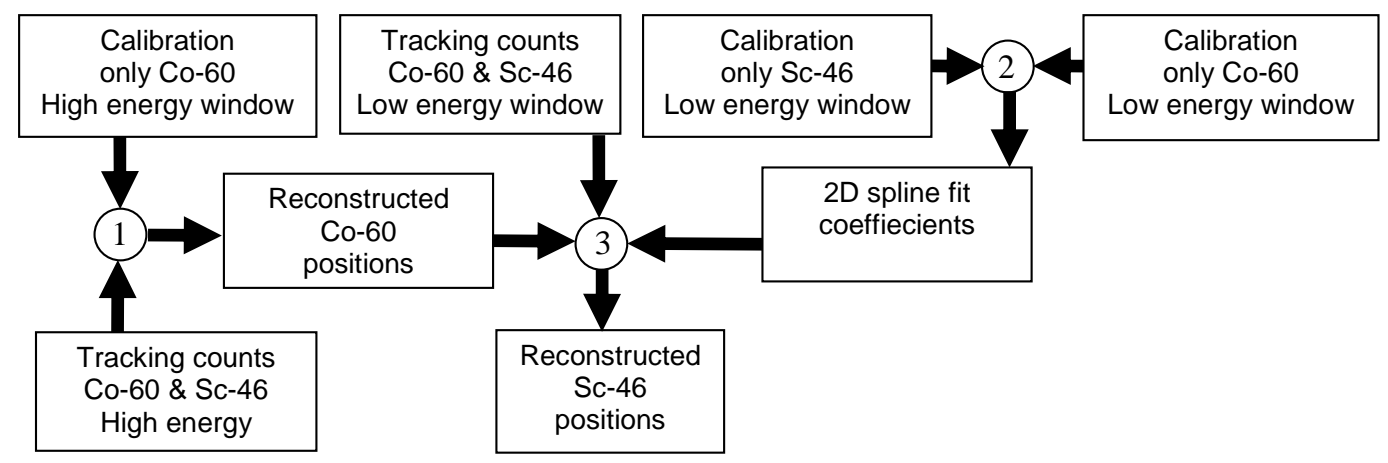

Data processing (step number showed inside the circle)

Figure 3.9 Modified reconstruction algorithm for dual-particle tracking

2D (two-dimensional) spline fitting is done using three variables, the total counts of Co60 and Sc-46, distance of Co-60, and distance of Sc-46 from a particular detector. By knowing two of these variables, third unknown can be evaluated by the spline coefficients obtained through $2 \mathrm{D}$ spline fitting. Distance of Co-60 from any detector is known, because Co-60 positions are reconstructed. Total counts of Co-60 and Sc-46 are available in low energy window from tracking experiment. Thus, the third unknown distance of Sc-46 from every detector can be evaluated using 2D spline fit coefficients. Important point to note her is, distances of Sc-46 are directly obtained from spline fitting. The step to obtain counts by subtraction is eliminated. Thus the error in reconstruction due to subtraction as well as obtaining distances from Sc-46 counts is eliminated.

The first task would be to generate a $2 \mathrm{D}$ spline fit plane. Calibration counts of only Co60 and only Sc-46 from low energy window can be added to obtain total counts of Co60 and Sc-46 in low energy window, as shown below.

Calibration counts of Co-60 for $i^{\text {th }}$ detector, $C_{i}=\left[\begin{array}{lllll}c_{1} & c_{2} & C_{3} \ldots \ldots . c_{j} \ldots . & c_{n}\end{array}\right]_{i}$ 
Calibration counts of Sc-46 for $i^{\text {th }}$ detector, $S_{i}=\left[\begin{array}{lllll}s_{1} & s_{2} & S_{3} \ldots \ldots . . . s_{j} \ldots . & s_{n}\end{array}\right]_{i}$ Total counts of Co-60 and Sc-46 for $i^{\text {th }}$ detector,

$$
T_{i}=\left[\begin{array}{ccccc}
t_{1,1} & t_{1,2} & \ldots t_{1, k} \ldots & \ldots & t_{1, n} \\
t_{2,1} & t_{2,2} & \ldots & \ldots & \ldots \\
\ldots t_{j, 1} \ldots & \ldots & t_{j, k} & \ldots & t_{j, n} \\
\ldots & \ldots & \ldots & \ldots & \ldots \\
t_{n, 1} & t_{n, 2} & \ldots & \ldots & t_{n, n}
\end{array}\right]_{i} \text { where, } t_{j, k}=c_{j}+s_{k}
$$

Where $c_{j}$ are the counts of Co- 60 at the calibration location $j$

$\mathrm{s}_{k}$ are the counts of Sc-46 at the calibration location $k$

$t_{j, k}$ are the total counts of Co-60 and Sc-46 with Co-60 at the calibration location $j$ and Sc-46 at the calibration location $k$ $n$ is the total number of calibration points

Every count $c_{j}$ in matrix $C_{i}$ is associated with distance $d^{c}{ }_{j, i}$ i.e. distance of Co-60 at $j^{\text {th }}$ location from $i^{\text {th }}$ detector. Similarly, every count $s_{j}$ in matrix $S_{i}$ is associated with distance $d_{j, j}^{s}$ i.e. distance of Sc- 46 at $j^{\text {th }}$ location from $i^{\text {th }}$ detector, such that;

Distance of Co-60 calibration locations from $i^{\text {th }}$ detector, $D_{i}^{c}=\left[\begin{array}{lllll}d_{1, i}^{c} & d_{2, i}^{c} & \ldots d_{j, i}^{c} \ldots & d_{n, i}^{c}\end{array}\right]$

Distance of Sc-46 calibration locations from $I^{\text {th }}$ detector, $D_{i}^{s}=\left[\begin{array}{lllll}d_{1, i}^{s} & d_{2, i}^{s} & \ldots & d_{j, i}^{s} \ldots & d_{n, i}^{s}\end{array}\right]$ Using matrix $D_{i}^{c}, D_{i}^{s}$ and $T_{i}, 2 \mathrm{D}$ spline fit plane can be generated and spline fit coefficients can be obtained. Unlike 1D spline fitting, we have generated a calibration plane, as shown in Figure 3.10, instead of a calibration curve. 


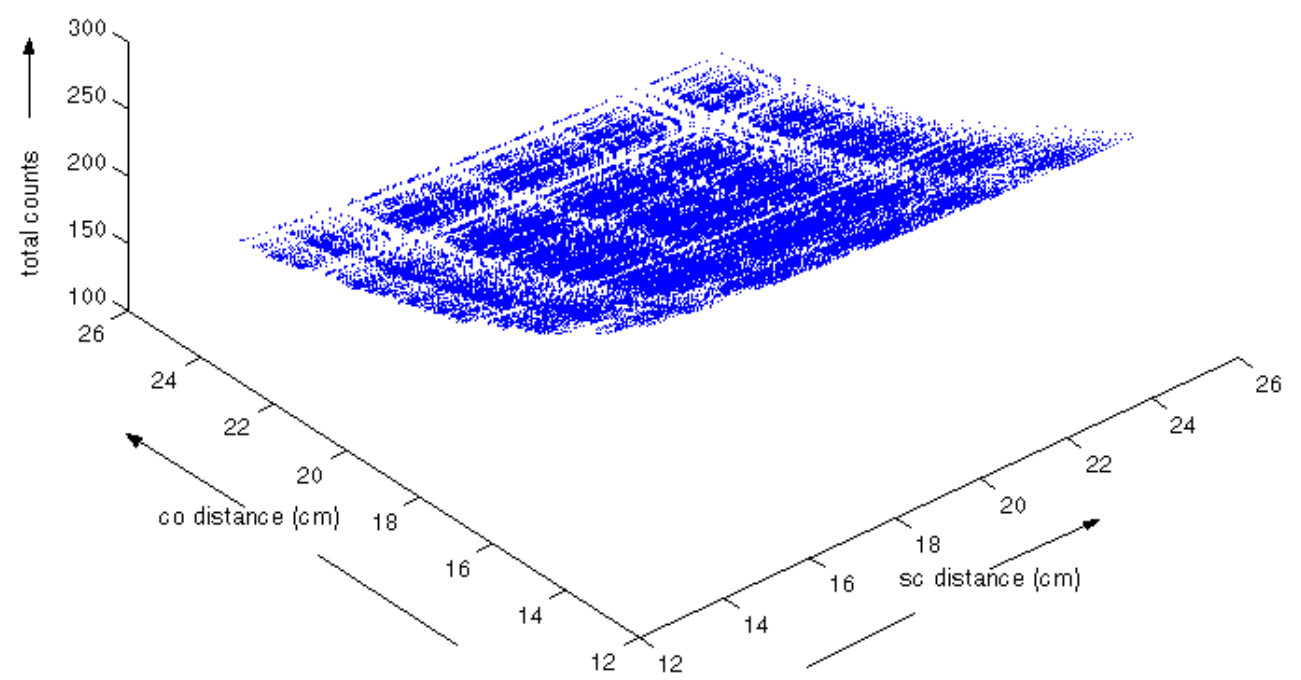

Figure 3.10 Calibration plane for detector 1 for low energy window total counts of Co60 and Sc-46.

Next, using the total counts of Co-60 and Sc-46 in low energy window from tracking experiment and corresponding reconstructed distance of Co-60 for each count data, distance of Sc-46 from each detector can be evaluated. Again, as described above, by least square approximation of these distances, coordinates of Sc-46 can be evaluated.

The reconstruction of Sc-46 locations using this new algorithm is shown in Figure 3.11. The error in reconstruction of $\mathrm{x}$ and $\mathrm{y}$ coordinates is very small (less than $5 \%$ ) as compared to error in z co-ordinate (about 12\%). There is significant improvement in the reconstructed positions with new algorithm as compared to one with old algorithm (Figure 3.8, error in z co-ordinate was $25 \%$ ). The large error in z co-ordinate is due to the larger calibration grid size (1 inch) in z direction (see Table 3.3). This error can be reduced by using a more number of calibration points and high-activity radioactive particles. 


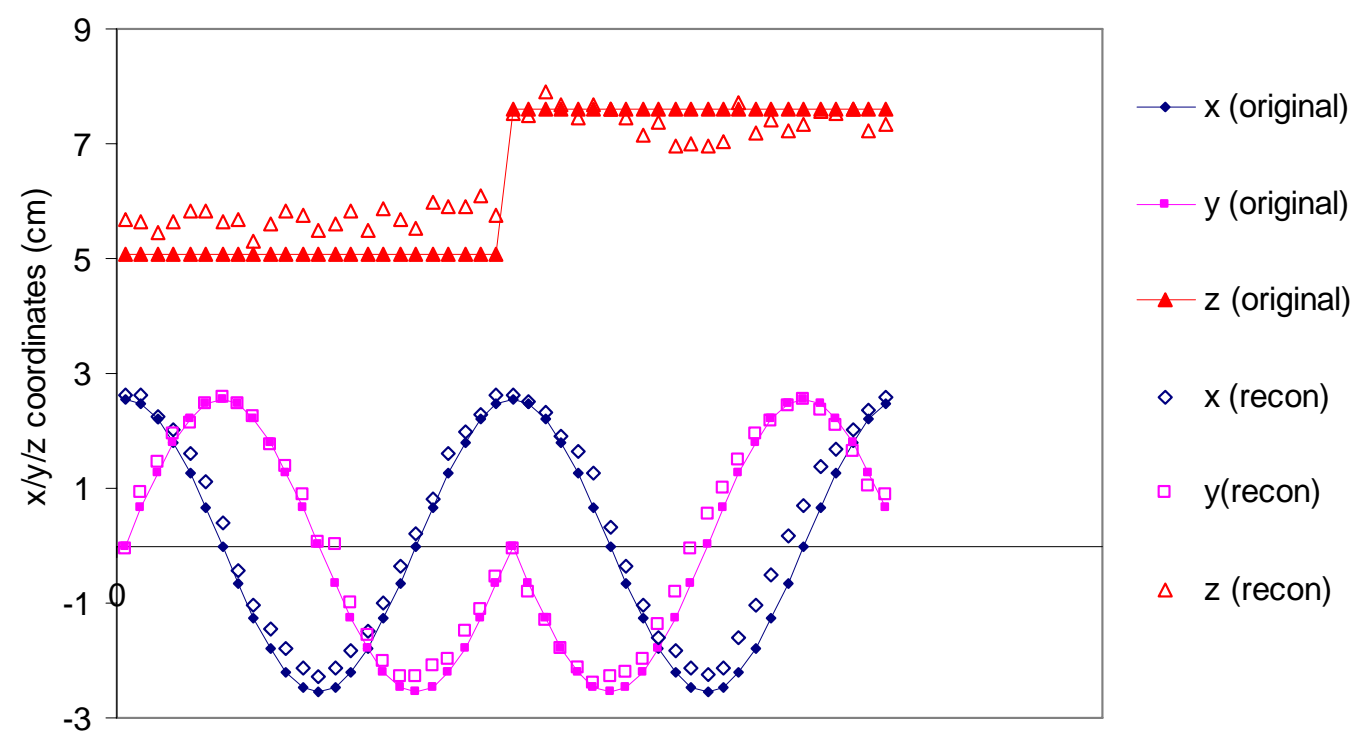

Figure 3.11 Comparison of original Sc-46 locations with reconstructed positions using new algorithm

The results of reconstruction of Co-60 and Sc-46 scanned together with the new MPCARPT unit shows that the MP-CARPT unit can be satisfactorily used to track two particles simultaneously. Thus the new technique MP-CARPT is validated for tracking two stationary radioactive sources successfully. However, the same development of particle reconstruction methodology can be extended to track more than two particles (up to eight particles as the limit of the current MP-CARPT hardware) simultaneously as discussed in section 3.4.4.

In the next section MP-CARPT reconstruction methodology will be implemented to track Co-60 and Sc-46 together moving independently in a cold reactor system.

\subsubsection{Tracking Particles in Motion}

Both old single particle CARPT and MP-CARPT units were used for this validation experiment. The objective was to evaluate the results of new unit with the benchmarked 
data obtained by old CARPT unit and ensure that new unit is providing correct results for tracking moving particles.

\section{Experimental Set-up}

An acrylic tank of $15.2 \mathrm{~cm}$ diameter and $34 \mathrm{~cm}$ in height, as shown in Figure 3.12, was used for this experiment. Tank was equipped with a sparger to circulate air and draft tube with $7.6 \mathrm{~cm}$ diameter and $14 \mathrm{~cm}$ height. Tank was filled with water upto a level of 22 inches. Air was sparged at a rate of $5 \mathrm{lpm}$. The tank was placed on a detector stand in the center surrounded by $16 \mathrm{NaI}$ detectors arranged circumferentially.

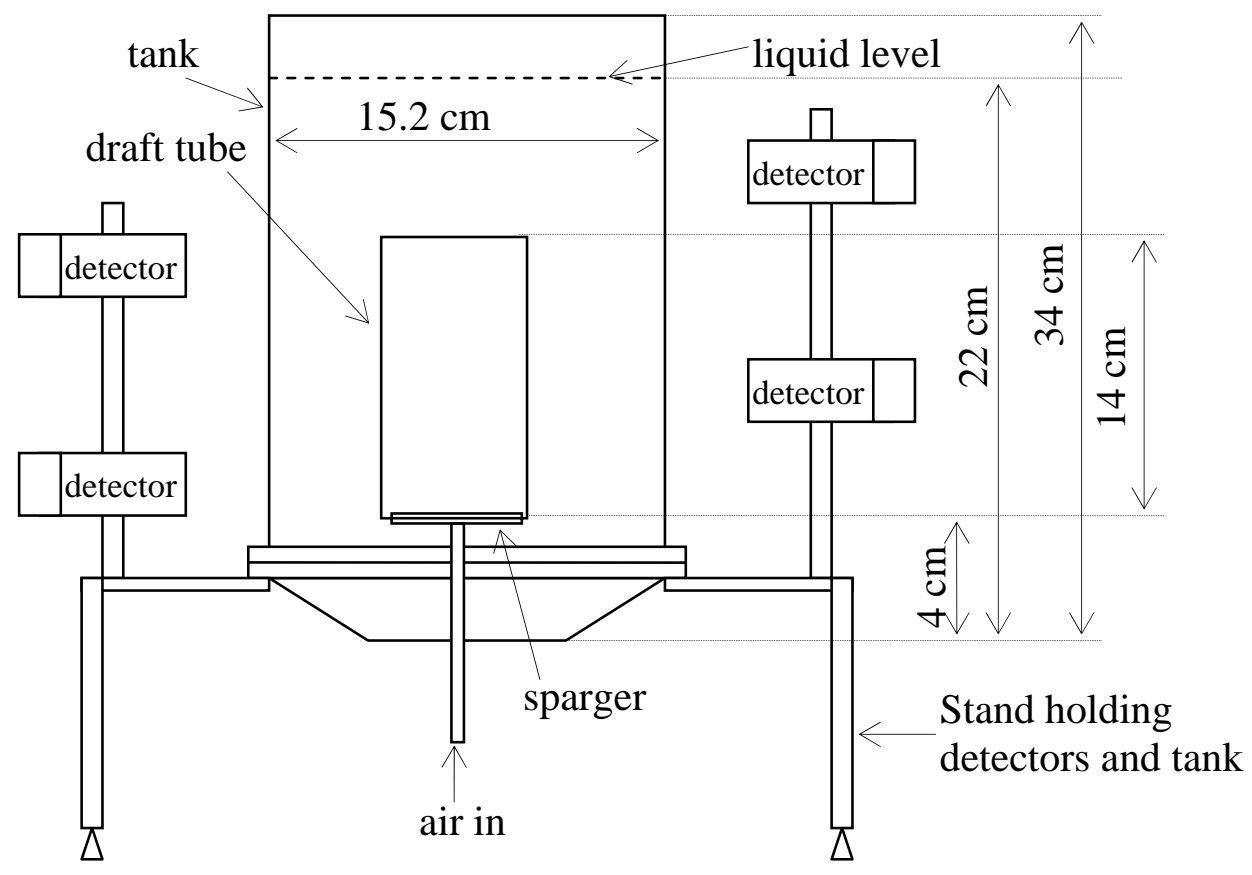

Figure 3.12 Experimental set-up for dual particle tracking

Co-60 and Sc-46 were used as the radioactive sources. $100 \mu \mathrm{m}$ Co-60 particle with approximate activity of $100 \mu \mathrm{Ci}$ was enclosed in a $1 \mathrm{~mm}$ polypropylene ball to adjust its density equal to that of water. The Sc-46 particle was $150 \mu \mathrm{m}$ in diameter with approximate activity of $150 \mu \mathrm{Ci}$ was also enclosed in a $1 \mathrm{~mm}$ polypropylene ball. The density of both particles was adjusted equal to that of water to mimic the water phase. 
Both old single particle CARPT unit and new MP-CARPT unit was used to track the Co-60 and Sc-46 particles individually. MP-CARPT unit was used to track Co-60 and Sc-46 together. For MP-CARPT unit, the limits of low energy window were set from bin number 200 to 425, whereas the limits of high energy window were from bin number 425 to 600. Calibration was done separately for old and new unit at 500 different known locations for individual Co-60 and Sc-46 particle using both units. The data acquisition frequency was $50 \mathrm{~Hz}$.

Co-60 and Sc-46 were tracked individually with the old and new unit and together with new unit. For the tracking experiment radioactive particles were introduced in the system and data for every condition with both units was acquired for a period of 24 hours at a frequency of $50 \mathrm{~Hz}$.

\section{Reconstruction Results}

The modified algorithm (Figure 3.9) was used for reconstruction of Sc-46 positions from the dual particle tracking data. For all other tracking experiments, the reconstruction was treated as in single particle tracking. The reconstructed position data is actually the instantaneous position data for the particle. Since the acquisition frequency is known $(50 \mathrm{~Hz})$, the time lap between 2 consecutive positions is also known (0.02 seconds). The instantaneous position data can be processed to obtain instantaneous velocities. Time averaged, azimuthally averaged axial and radial velocities can be obtained from instantaneous velocity data and this can used to obtain time averaged flow pattern of moving particles and turbulence quantities. Post-processing of reconstructed data is explained in detail in the CARPT manual (2005).

The flow patterns obtained from each of the tracking experiment viz. for Co-60 and Sc46 with old CARPT unit, Co-60 and Sc-46 tracked separately with new MP-CARPT unit and Co-60 and Sc-46 tracked together with new MP-CARPT unit are shown in Figure 3.13a to 3.13f, respectively. All the flow patterns look more or less the same and actual difference between the data is not clearly noticeable. 


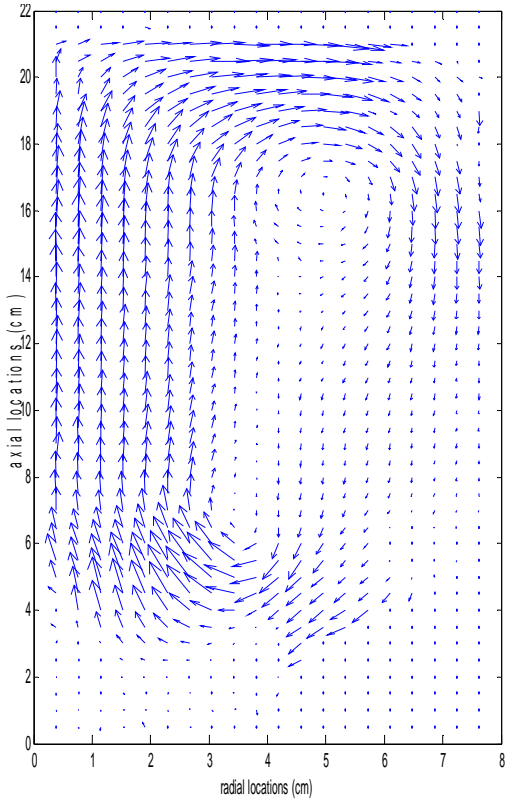

(a) $\mathrm{Co}-60$

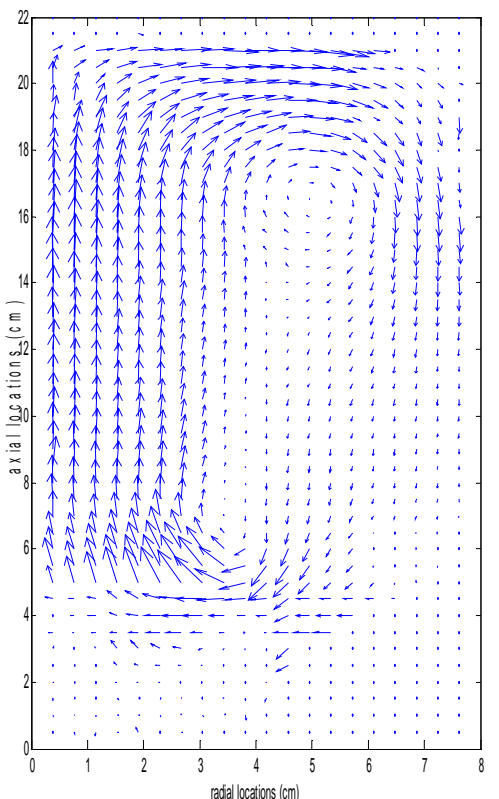

(b) Sc-46

Figure 3.13a \& 3.13b Flow pattern obtained from single particle CARPT unit for (a) Co-60 and (b) Sc-46, respectively

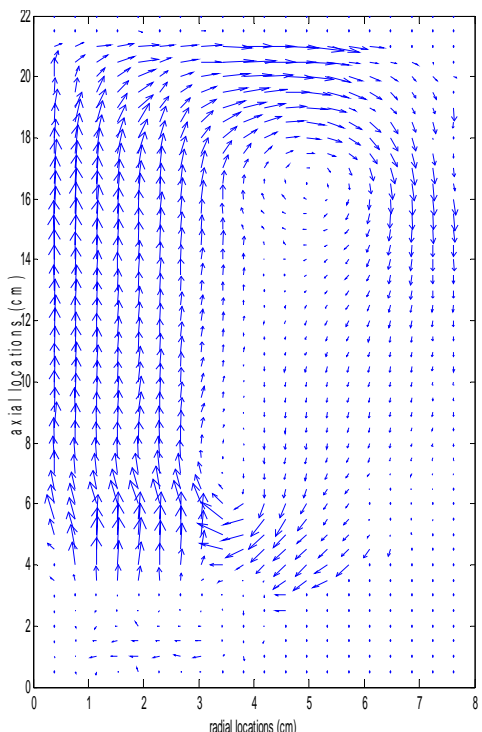

(c) $\mathrm{Co}-60$

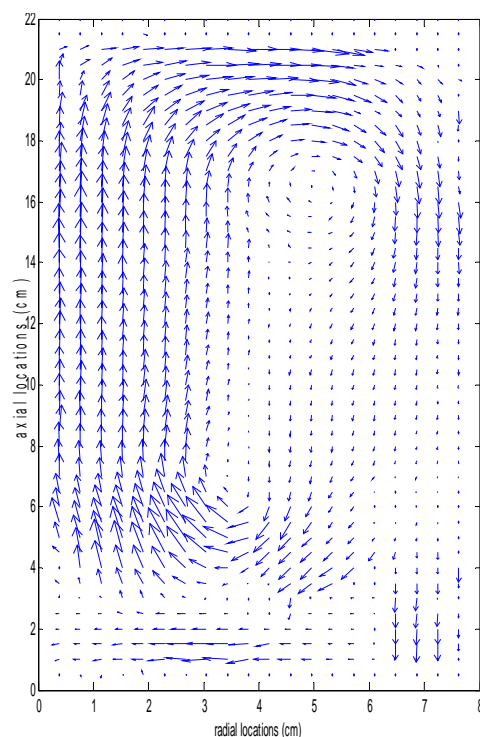

(d) Sc-46

Figure 3.13c \& 3.13d Flow pattern obtained from MP-CARPT unit for (c) Co-60 and (d) Sc-46, tracked separately, respectively 


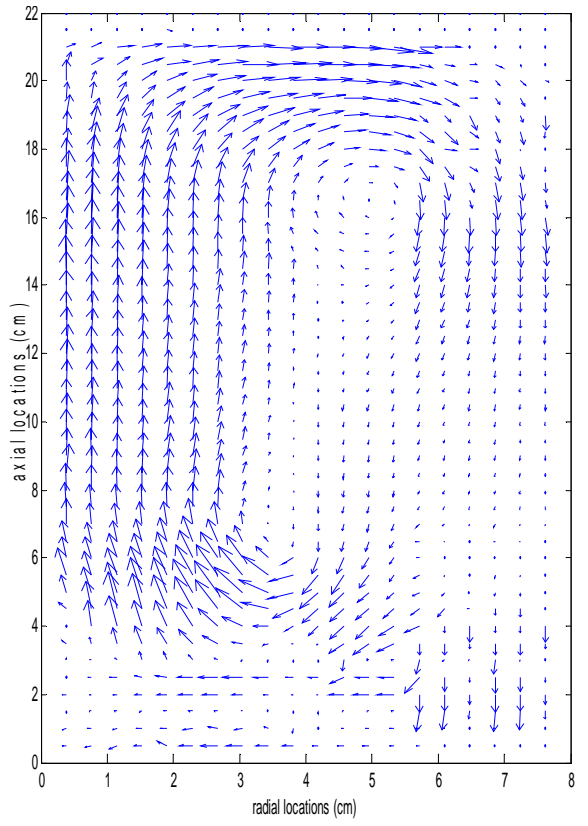

(e) $\mathrm{Co}-60$

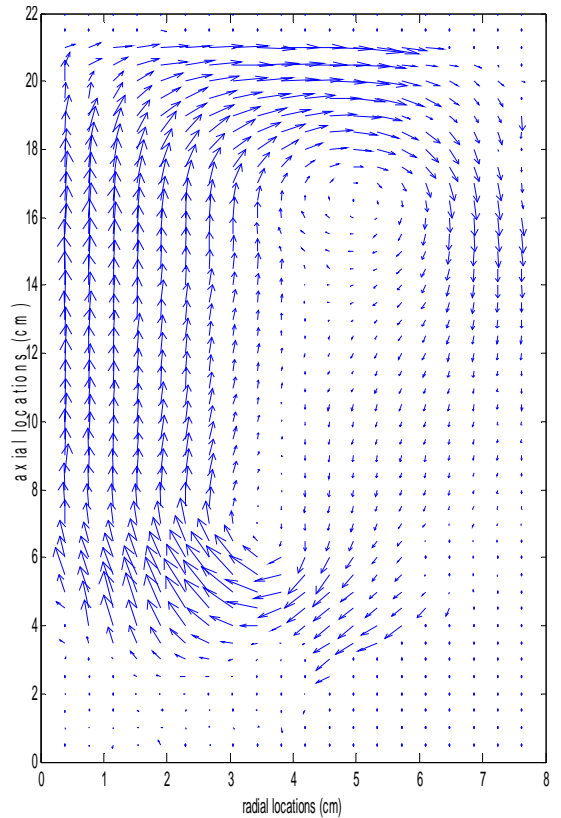

(f) Sc-46

Figure 3.13e \& 3.13f Flow pattern obtained from MP-CARPT unit for (e) Co-60 and (f) Sc-46, tracked together, respectively.

Radial profiles of average axial velocity can be compared to evaluate the quantitative differences between the data obtained from old unit and new unit, and between the single particle tracking and dual particle tracking. Figure 3.14 shows the comparison of time averaged azimuthally averaged axial velocities at the middle height of the tank for Co-60 and Sc-46 particles for different set of experiments. The magnitude of axial velocity is slightly different for every case. If the velocities at a given location obtained from single particle tracking of Co-60 and Sc-46 are averaged and used as a basis to evaluate the error, then the reconstruction error is less than $10 \%$. This $( \pm 10 \%)$ error is acceptable and is within the range associated with CARPT itself (Degaleesan, 1997; Rados, 2003, Bhusarapu, 2005).

It can also be noted in Figure 3.14 that the error is higher at the center and negligible near the wall. Because, higher counts are obtained when particle is near the detectors (near the wall) as compared to when particle is away from the detector (at the center). 


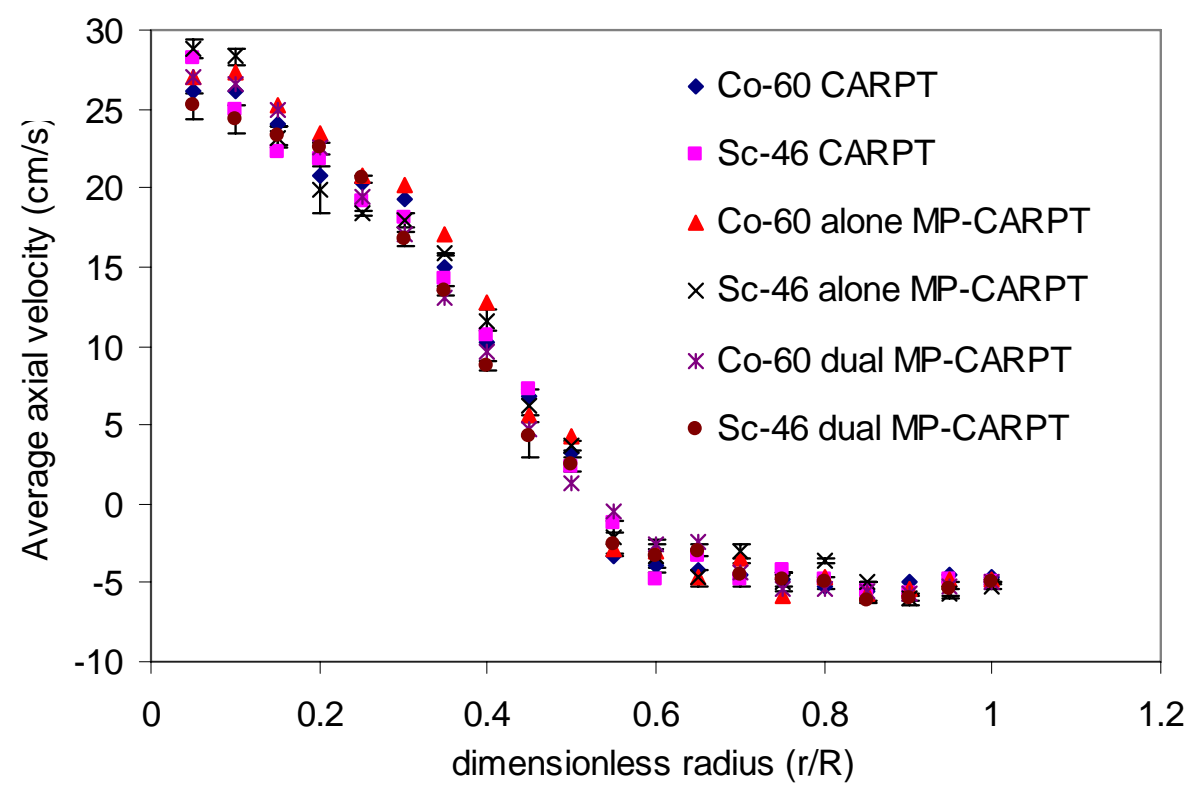

Figure 3.14 Comparison of time averaged azimuthally averaged axial velocity at the middle height of the tank

\subsubsection{Tracking Two Moving Particles with Different Densities}

Majority of the processes of industrial interest are multiphase in nature and normally consists of solid particles suspended in liquid or gas phase. In such processes it is of particular interest to evaluate the effect of presence of one phase on the hydrodynamics of the other phase. This can be done using the single particle CARPT by repeating the tracking for each phase separately as only one phase can be tracked at a time. Using the MP-CARPT both phases can be tracked together at the same time, thus the time required for such experiments is considerably reduced. However there are certain limitations in performing such experiments, especially to track liquid phase in a LS or GLS system. These limitations will be discussed in the following sections. 
To demonstrate the use of MP-CARPT to track two tracers representing different phases a low $H / T$ slurry bubble column reactor (SBCR) with low solids loadings was used. SBCR consists of solids moving in a liquid phase due to the sparging of a gas.

\section{Experimental Set-up}

A six-inch diameter acrylic cylindrical vessel shown in Figure 3.15 was used for this study. The system was operated as a slurry bubble column reactor (SBCR). The distributor plate had 139 holes of $1.32 \mathrm{~mm}$ diameter each, arranged in a triangular pitch of $1 \mathrm{~cm}$. The distributor plate had open area of 1.04\%. The tank was filled with 4 liters of water. $40 \mathrm{gms}$ of 300 micron glass spheres $(2.5 \mathrm{gm} / \mathrm{cc}$ density) were added to the water, such that slurry had $1 \%$ (by weight) solids. Air was sparged at the rate of 50 SCFH, such that superficial gas velocity in the tank was $2.154 \mathrm{~cm} / \mathrm{sec}$. The average gassed liquid height was $22 \mathrm{~cm}$.

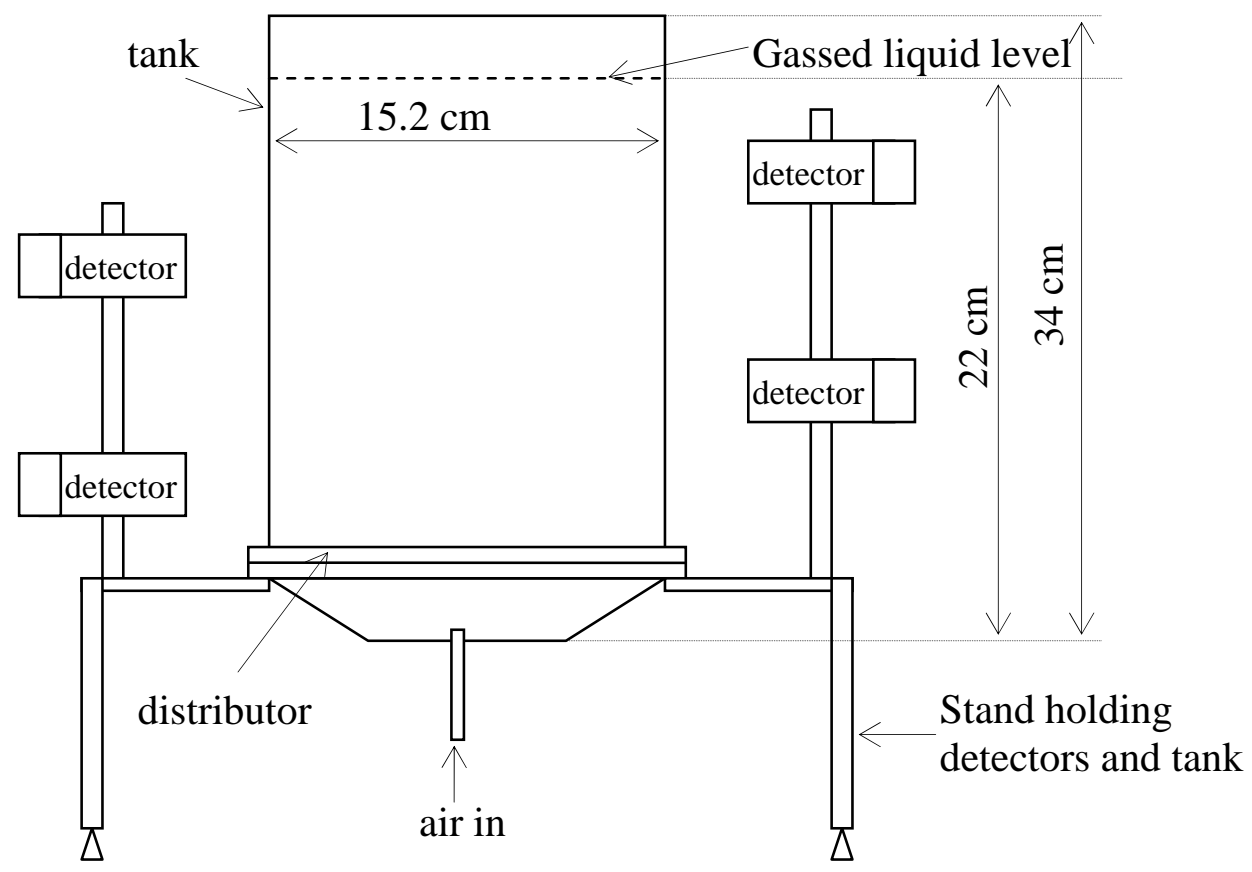

Figure 3.15 Experimental set-up for tracking two particles with different densities 
A $300 \mu \mathrm{m} \mathrm{Sc}-46$ particle with approximate activity of 100 microCi was used to mimic the solid phase. The Sc-46 particle was actually $276 \mu \mathrm{m}$ in diameter and then coated with polypropylene up to $300 \mu \mathrm{m}$ to adjust its density to $2500 \mathrm{Kg} / \mathrm{m}^{3}$. The Co-60 particle was $100 \mu \mathrm{m}$ in diameter and $100 \mu \mathrm{Ci}$ in strength. Co-60 particle was enclosed in a $1 \mathrm{~mm}$ polypropylene ball and its density was adjusted to $1000 \mathrm{Kg} / \mathrm{m}^{3}$ using glue to fill the air gap. The Co-60 particle was used to mimic water used as the liquid phase.

Similar 16-detector set-up (shown in Figure 3.3) was used in this study as used in the previous experiments. Three sets of experiments were performed, all of them using MPCARPT unit. Two experiments were conducted where Co-60 and Sc-46 particles were tracked separately as liquid phase and solids phase, respectively. Then both the particles were released in the system and were tracked together in the third experiment. This allowed the validation of results of dual-particle tracking of different densities against the single-particle tracking results.

527 calibration points were obtained for each particle and 512 samples were collected for each calibration point at data acquisition frequency of $50 \mathrm{~Hz}$. The limits of low energy window were set from bin number 250 to 475, whereas the limits of high energy window were from bin number 475 to 640 .

In each of the three experiments particles were tracked for total of 20 hours at frequency of $50 \mathrm{~Hz}$.

\section{Results}

Single-particle tracking reconstruction algorithms were used for single-particle tracking and for Co-60 reconstruction in dual-particle tracking, whereas the newly developed algorithm was used for reconstruction of Sc-46 in dual-particle tracking. The flow patterns obtained for Sc-46 and Co-60 are shown in Figure 3.16a to 3.16d. The flow patterns for Co-60 from single-particle and dual-particle tracking look the same. This is also the case for Sc-46 particles. It is very interesting to note that the flow patterns for a 
low L/D SBCR are significantly different than the flow patterns of solids or liquid phase in a high L/D SBCR. The flow patterns in a high L/D SBCR were obtained by Rados (2003). Figure 3.17 shows the radial profile of azimuthally averaged axial velocity at the middle height of the column. The error bars are also shown in Figure 3.17. The dual particle tracking experiment was repeated two times to obtain the error. It can be seen that the difference between velocities obtained from the single-particle and dualparticle tracking is not significant (less than 5\%). The error associated with CARPT itself is $\pm 10 \%$ (Degaleesan, 1997; Rados, 2003; Bhusarapu, 2005).

The error associated with Sc-46 reconstruction is more than the error associated with Co-60. The reasons for this were explained before.

These results show the ability of the new MP-CARPT unit to track two radioactive particles of different densities. However, it has to be remembered that the solids fraction in the system was kept low to $1 \%$, so that the collisions between the Sc-46 particle tracking liquid phase and the solids in the system can be kept to minimum. If the solids hold up is too high then the true hydrodynamics of liquid phase cannot be obtained due to the interference created by solids in the system to the tracer mimicking liquid phase. 


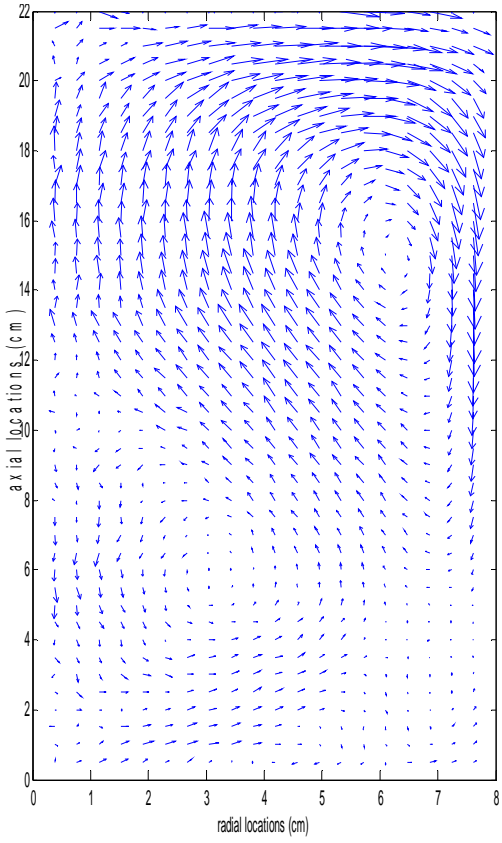

(a)

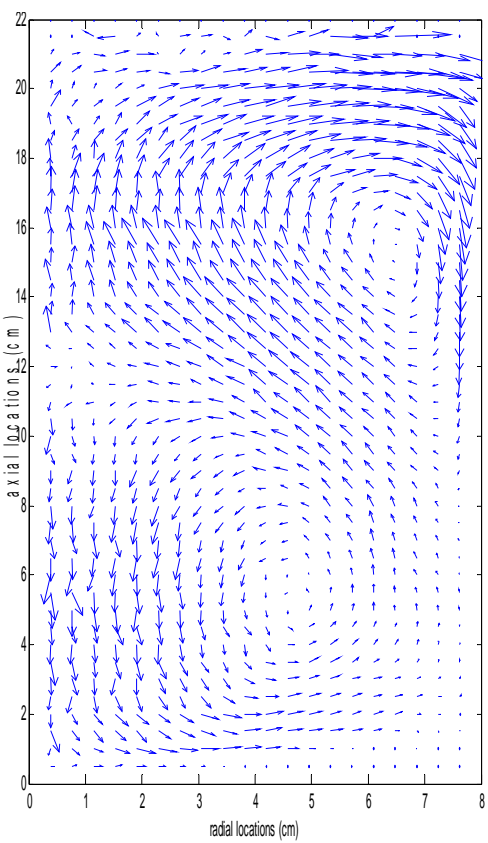

(c)

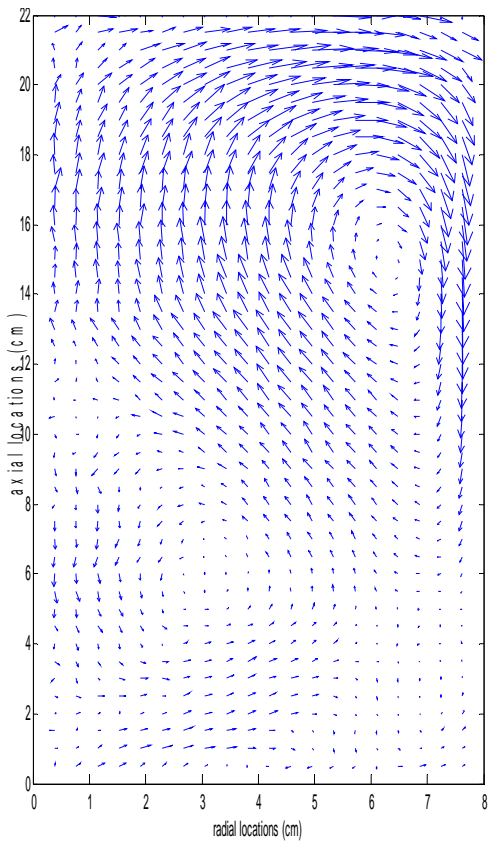

(b)

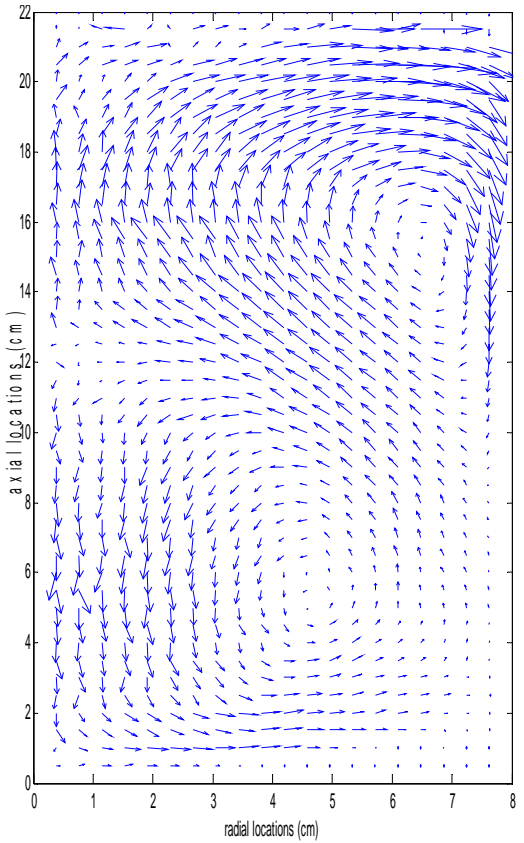

(d)

Figure 3.16 (a) Co-60, single-particle tracking (b) Co-60, dual-particle tracking (c) Sc-46, single-particle tracking (d) Sc-46, dual-particle tracking 


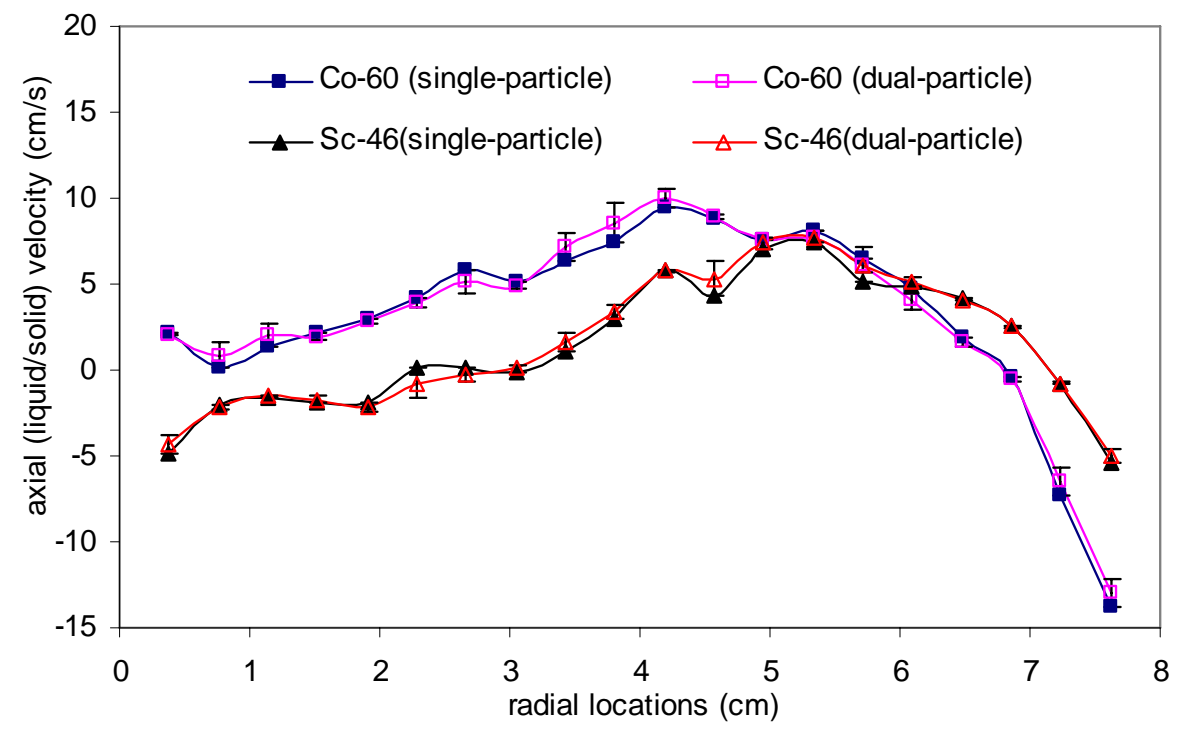

Figure 3.17 Comparison of axial velocity profiles obtained from single-particle and dual-particle tracking for Co-60 and Sc-46 with different densities.

\subsubsection{Tracking More than Two Particles}

Previous section discussed the methodology of MP-CARPT for tracking two particles. This can be easily extended to track more than two particles. Lets consider 3 radioactive particles for example with the gamma peaks as shown in Figure 3.18. As mentioned earlier for MP-CARPT to work, at least one gamma peak of highest energy particle should be completely separated from rest of the gamma peaks and the not more than 2 gamma peaks of any particle can be overlapped by each other. This is shown schematically in Figure 3.18 for three hypothetical particles $\mathrm{Aa}, \mathrm{Bb}$ and Cc.

Given the gamma peaks as shown in Figure 3.18, the energy window ranges can be chosen such that not more than two peaks of any particle appear in one energy window. Since only counts of particle Aa appear in Window I, Aa can be reconstructed easily as a single particle. In Window II, only counts of $\mathrm{Aa}$ and $\mathrm{Bb}$ appear, thus $\mathrm{Bb}$ can be reconstructed as dual particle tracking as explained in section 3.3. In Window III, only 
counts of $\mathrm{Bb}$ and $\mathrm{Cc}$ appear, thus $\mathrm{Cc}$ can also be reconstructed similar to $\mathrm{Bb}$ as dual particle tracking.

Using this sequential procedure a number of radioactive particles can be tracked simultaneously. The current hardware limits tracking only up to eight particles.

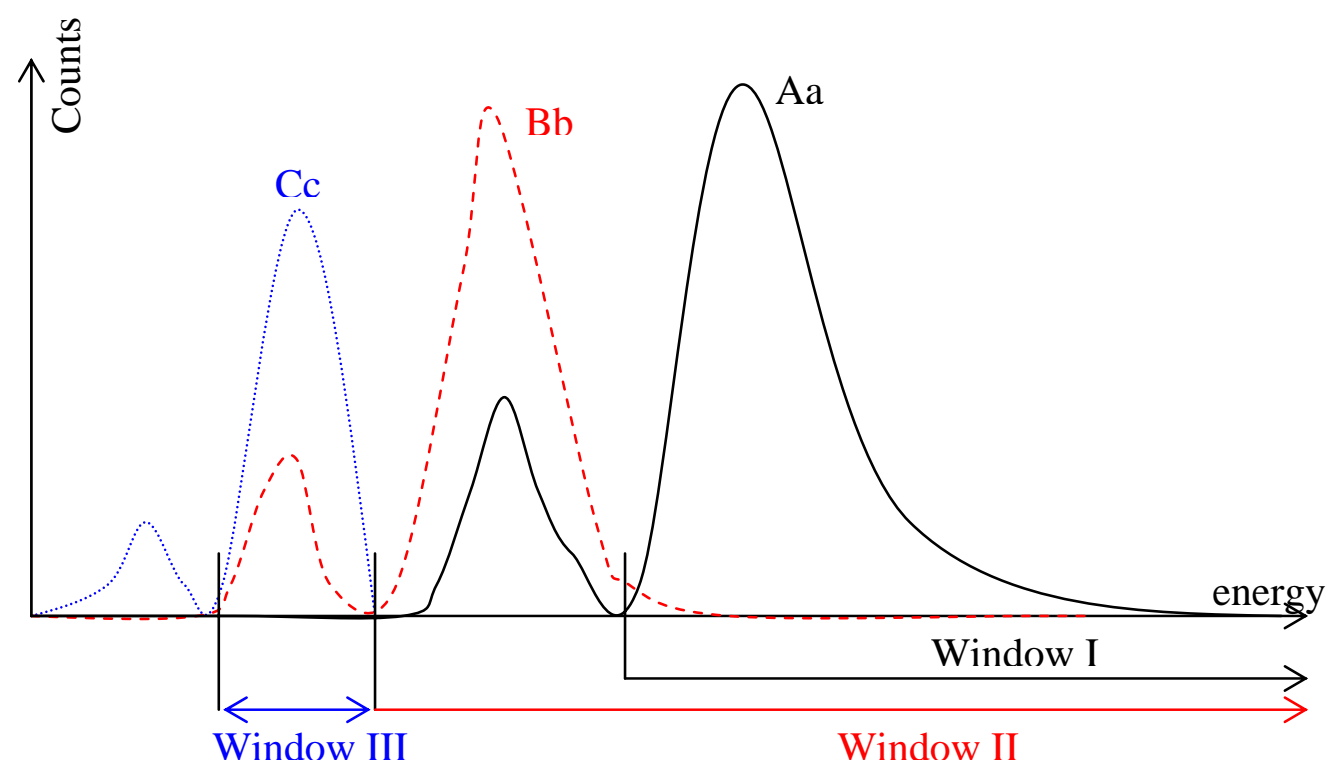

Figure 3.18 Gamma energy peaks for 3 hypothetical particles $\mathrm{Aa}, \mathrm{Bb}$ and $\mathrm{Cc}$

\subsection{Summary and Recommendations}

New MP-CARPT unit offers number of advantages over the old single particle CARPT unit. The new unit is compact, cheaper, faster, and easy to use and operate. It provides ability to track eight different radioactive sources simultaneously.

The MP-CARPT electronics and technique was validated to track two stationary particles simultaneously. A new reconstruction algorithm was developed which shown small error (less than 10\%) in reconstruction of Co-60 and Sc-46 particles. The validation was taken further to next step to track two moving particles representing the same liquid phase. The MP-CARPT was successful in tracking two particles in motion 
as well. Next, two radioactive particles of different densities, one mimicking liquid phase and other solid phase, were tracked in SBCR. The particles representing different phases could also be tracked simultaneously using MP-CARPT unit.

The solids fraction is SBCR was kept low to $1 \%$ to obtain true hydrodynamic information of liquid phase. When tracking two different phases, ex. solid and liquid, care should be taken to design the experiment in such a way that the tracer follows the represented phase as closely as possible. Collisions of tracer representing liquid phase with the solid particles in the system can be minimized by using very low solids fraction.

MP-CARPT can be used conveniently to track two or more solids phases in a system with different properties (for example size, shape or density). However, how much difference in size or density of tracers is required so that the tracers can provide true hydrodynamics of phase being tracked needs to be evaluated. This issue can be addressed by tracking tracers of same size and different densities or same density and different sizes and observing the difference in hydrodynamics.

Having validated the dual particle tracking, this technique can be easily extended to track more than two radioactive sources simultaneously. The current unit is capable of tracking maximum of eight sources at a time, but it is limited due to availability and suitability of radioactive sources for this technique.

The error in the reconstruction of the MP-CARPT can be further reduced by some modifications of the experimental set-up, procedures, and the reconstruction algorithms. If the number of detectors for tracking are increased, such that the detector are packed closely together, then the error in the reconstruction will be reduced due to increased spatial resolution (CARPT manual, 2005). The current MP-CARPT reconstruction algorithm is based on the principle of addition of the calibration counts of Co-60 and Sc-46 obtained separately to represent the counts obtained together. Instead if the calibration is performed with the Co-60 and Sc-46 particles present 
together, keeping one particle fixed at one location and placing other particle at all the calibration locations one by one and thus covering all the possible permutations, then more accurate calibration region can be obtained. This calibration technique will take into consideration the effect of presence of two particles together on their total counts. In addition, if the number of calibration points is increased, it will also help to increase the accuracy of reconstruction. The reconstruction method developed by Bhusarapu (2005) also can be evaluated for the increased accuracy.

Since the technique is validated and the protocols for operation of MP-CARPT unit are understood, a manual for MP-CARPT is prepared (Appendix B). This manual will help future novice users to understand and operate the MP-CARPT electronics and also and provide guidelines to process the raw data obtained from tracking experiments using the new electronics. 


\section{Chapter 4}

\section{CARPT Studies:}

\section{Laboratory-Scale and Pilot-Scale}

\subsection{Introduction and Motivation}

The results of the performance studies (Appendix A) showed that the scale of operation has a significant effect on the performance of digesters. Mixing affects the performance of large-scale digesters but not of laboratory-scale digesters. Performance of digesters is partly governed by the mixing characteristics/hydrodynamics inside the digester, which in turn is affected by the scale of operation. To evaluate the effect of scale on the hydrodynamics information of hydrodynamics in the digester is required. As mentioned in chapter 2 , the hydrodynamics information about the low $L / D$ ratio gaslift digesters is lacking. Thus, there is a need to investigate the hydrodynamics of these gaslift digesters in detail. Due to opaque nature of the slurry in the digester, advanced non-invasive techniques like Computer Automated Radioactive Particle Tracking (CARPT) and Computed Tomography (CT) are needed to discern the hydrodynamics of digester. CARPT provides 3D flow pattern, velocity profiles and turbulence parameters, while CT provides time averaged cross sectional phase holdup distribution. This chapter is focused on the digester hydrodynamic investigation using CARPT measurements performed on laboratory-scale and pilot-scale digesters, which are geometrically similar 
to the digesters used in the performance studies. However, the phase holdup distribution study using CT at same conditions of CARPT is part of other doctoral thesis (by Rajneesh Varma at CREL). The geometric and operating conditions are varied to evaluate their effect. Flow patterns, liquid velocity profiles, turbulence parameters such as shear stress, turbulent kinetic energy, and eddy diffusivities were evaluated to understand the nature of the flow in the digesters at two scales and differentiate between them. Mixing intensity is quantified in terms of dead space volume and turbulent diffusivities to understand the effect of scale.

\subsection{Experimental Set-up}

\subsubsection{Laboratory-scale Digester}

A six inch $(15.24 \mathrm{~cm})$ diameter acrylic tank equipped with a draft tube and a conical bottom with a slope of $25^{\circ}$, as shown in Figure $4.1 \mathrm{a}$ was used as a digester. The geometry of the digester and the operating conditions were maintained similar to the performance experiments described in Appendix A. The slurry level was $22 \mathrm{~cm}$ and working liquid volume was 3.78 liters. Gas was introduced at the bottom of the tank using a sparger. Two different types of spargers were used; viz., a single point sparger and a cross sparger. Single point sparger was a pipe with a single opening of $5 \mathrm{~mm}$ diameter, while the cross sparger had 4 holes (facing towards the bottom of the tank) of $1.7 \mathrm{~mm}$ each. Schematic of the cross sparger is shown in Figure 4.1b. Four arms of cross sparger extended inside the draft tube and covered $50 \%$ of the draft tube cross sectional area. The spacing of sparger hole from the center of the tank was $\sqrt{2} D / 2$; where $\mathrm{D}$ is the diameter of the draft tube. Draft tube diameter was changed from 3.8 $\mathrm{cm}$ to $7.6 \mathrm{~cm}$ and $11.4 \mathrm{~cm}$, such draft tube diameter to tank diameter ratio $(D / T)$ is 0.25 , 0.5 and 0.75 , respectively. The length of the arms of cross sparger was also changed with respect to the draft tube diameter (see Figure 4.1). 


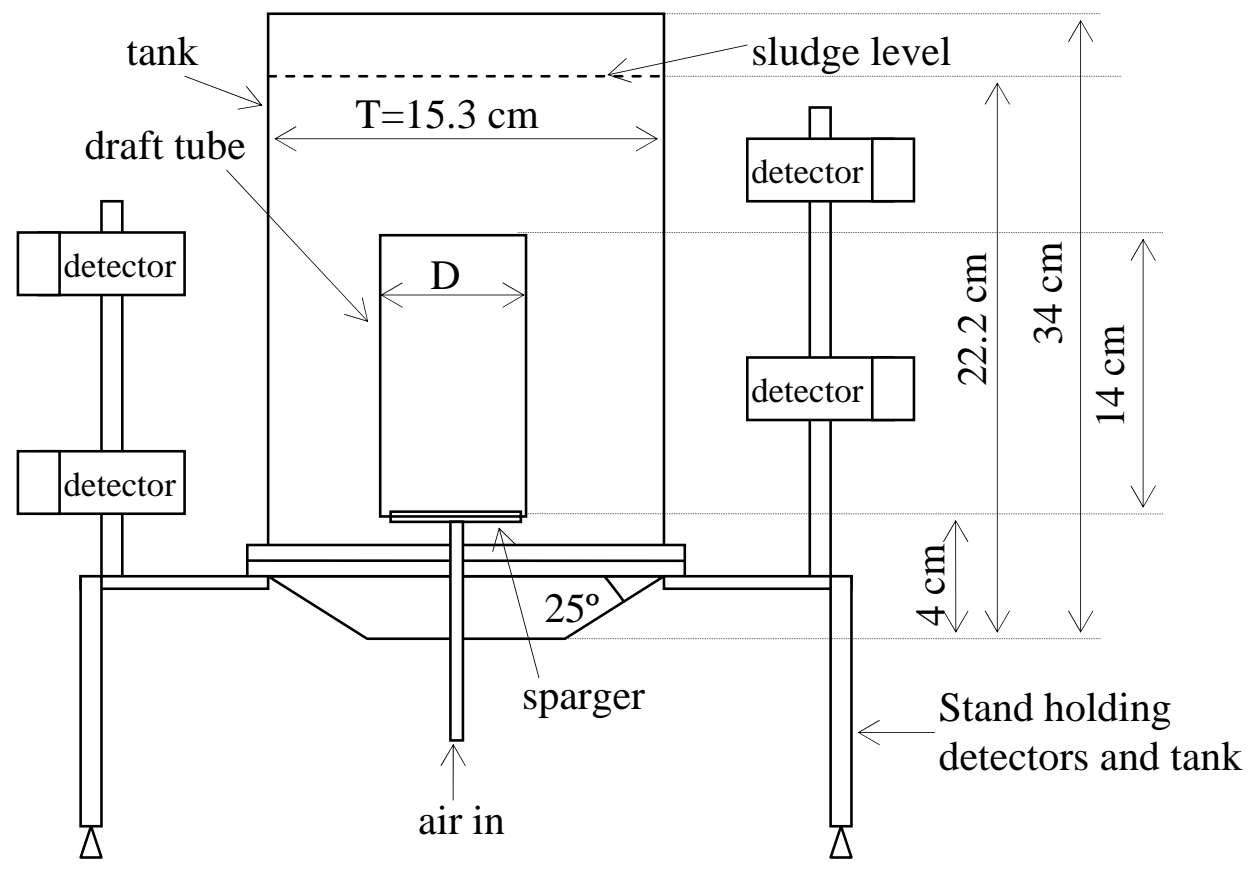

Figure 4.1a Digester geometry

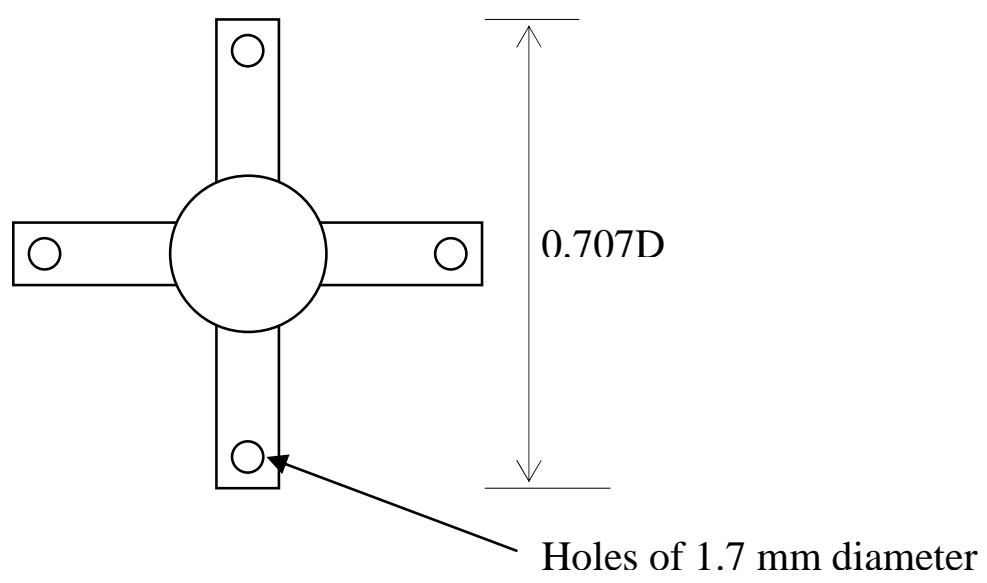

Figure 4.1b Cross sparger (top view, D is the diameter of the draft tube)

The experiments were conducted with slurry obtained form dairy waste. The slurry was screened to eliminate larger solids and then diluted to adjust the total solids concentration to $100 \mathrm{~g} / 1$ (or 10\% solids). To account for mixing created by the gas sparging only, anaerobic biogas production was hindered using sodium azide (2g/l). 
Air was used as gas phase; air can be used to mimic biogas in digester. Biogas is a mixture of methane and carbon-dioxide, thus there is no significant difference in density of air and biogas. (Density of air at atmospheric pressure and room temperature is 1.18 $\mathrm{Kg} / \mathrm{m}^{3}$, whereas biogas with $60 \%$ methane and $40 \% \mathrm{CO}_{2}$ has density of $1.11 \mathrm{Kg} / \mathrm{m}^{3}$ at standard conditions). The air flow rate was varied from $1 \mathrm{lpm}$ to $3 \mathrm{lpm}$. These flow rates resulted in superficial gas velocity (based on tank diameter) of 0.91 and $2.74 \mathrm{~mm} / \mathrm{sec}$, respectively. The gas flow rate of $1 \mathrm{lpm}$ corresponds to energy input density of $8 \mathrm{~W} / \mathrm{m}^{3}$ (minimum suggested by US, EPA 1979 for proper digester mixing). Equation 4.1 was used to calculate the power input density as suggested by Karim (2005a).

$$
\frac{P}{V}=\frac{G_{r} P_{2}}{(\lambda-1)}\left[\left(\frac{P_{1}}{P_{2}}\right)^{(\lambda-1) / \lambda}-1\right]
$$

where $\mathrm{P}$ is the power and $\mathrm{V}$ the volume of the slurry mixed, $\mathrm{Gr}$ the specific biogas recirculation rate $(\mathrm{m} 3 / \mathrm{dm} 3), \quad \mathrm{P} 2$ the head space pressure $=101,416.83 \mathrm{~N} / \mathrm{m} 2$ (atmospheric $)=101,325 \mathrm{~Pa}, \mathrm{P} 1$ the pressure at the injection point $=(\mathrm{P} 2+$ static head of slurry), and $1=1.03$ (Casey, 1986)

At this low gas superficial velocity the IGLR operates in regime one called as bubbly flow regime or no gas entrainment regime (Heijnen et al., 1997; Pironti et al., 1995; Siegel, 1992; van Benthum et al., 1999).

CARPT experiments were performed in accordance to experiments carried out by Karim et al. (2004). $150 \mu \mathrm{m}$ diameter Sc-46 particle with approximate activity of 200 $\mu \mathrm{Ci}$, enclosed in $1 \mathrm{~mm}$ diameter polypropylene ball was used as a tracer. The density of particle was adjusted close to that of water by using epoxy-resin to fill the air gap inside the ball. The density of the sealed tracer particle was checked by determining its terminal settling velocity in water. The tracer particle represented both the liquid in the slurry (water) and the microorganisms; microorganisms have density close to that of water. The solid particles and microorganisms in the slurry are small enough to behave similar to liquid flow elements, thus the two-phase solid-liquid slurry behaves like a 
single phase in which the fluid phase and the solid phase are in thermal equilibrium state and flow with the same velocity rather than a conventional solid-liquid mixture (Wen et al., 2005 and Klein et al., 2003).

Sixteen numbers of $\mathrm{NaI}$ detectors were arranged surrounding the six inch digester as shown in Figure 4.1a. A picture of experimental set-up is shown in Figure 4.2. First of all calibration was performed in situ by positioning the tracer particle at 400 known positions and spline fit curves were generated. An automated calibration device was used for this purpose (the details of calibration device are given by Luo, 2005). After that the tracer particle was released into the digester and the track data were collected at a frequency of $50 \mathrm{~Hz}$ for 24 hours followed by data processing and reconstruction of the tracer particle trajectories. More details of CARPT and reconstruction algorithms are discussed by Karim et al. (2004) and Luo (2005) and detailed information is available in CARPT manual (2005). 


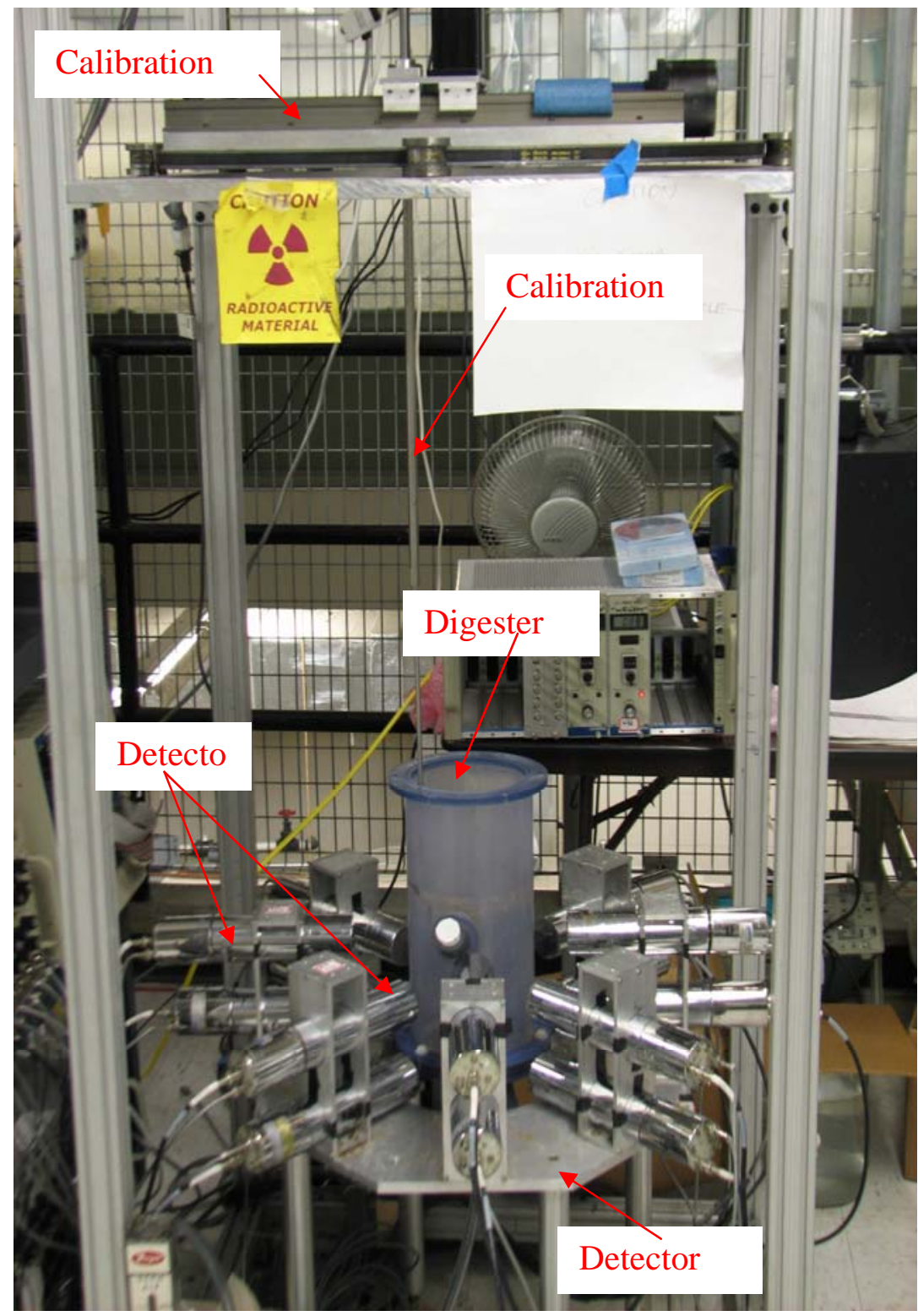

Figure 4.2 Photograph of laboratory-scale digester experimental set-up

Total five CARPT runs were performed; the operational details are given in Table 4.1. The operating conditions were varied in order to study the effect of gas recirculation rate, draft tube diameter and type of sparger. Vesvikar and Al-Dahhan (2005) carried out CFD studies on similar digester configuration and studied the effect of gas recirculation rate and draft tube diameter. They concluded that the gas recirculation rate did not show any appreciable effect on the liquid velocity may be because of the non- 
uniform local gas distribution. Thus both non-uniform single-point sparger and a uniform multi-point cross sparger were used in this study. Effect of gas flow rate was studied for cross sparger and the effect of sparger type on the hydrodynamics was studied at the lowest gas flow rate.

Table 4.1 Details of CARPT experiments for laboratory-scale digester

\begin{tabular}{|c|c|c|c|c|}
\hline $\begin{array}{c}\text { Experiment } \\
\text { no. }\end{array}$ & $\begin{array}{c}\text { Gas flow } \\
\text { rate (lpm) }\end{array}$ & $\begin{array}{c}\text { Total solids in } \\
\text { the slurry }(\mathrm{g} / \mathrm{l})\end{array}$ & $\begin{array}{c}D / T \\
\text { ratio }\end{array}$ & $\begin{array}{c}\text { Sparger } \\
\text { geometry }\end{array}$ \\
\hline L1 & 1 & 100 & 0.25 & Cross \\
\hline L2 & 3 & 100 & 0.25 & Cross \\
\hline L3 & 1 & 100 & 0.5 & Cross \\
\hline L4 & 1 & 100 & 0.75 & Cross \\
\hline L5 & 1 & 100 & 0.25 & Single point \\
\hline
\end{tabular}

\subsubsection{Pilot-scale Digester}

An 18-inch $(45.72 \mathrm{~cm})$ diameter acrylic tank was used as a pilot-scale digester; it was geometrically similar to the laboratory-scale digester. The working volume of pilot-scale digester was 97 liters; whereas it was 3.78 liters for laboratory-scale digester (volumetric scale-up ratio of approximately 25 was employed). Digester was equipped with a draft tube and a conical bottom with a slope of $25^{\circ}$, as shown in Figure 4.3a. Air was recirculated at the bottom of the tank using a sparger. Two different types of spargers were used; viz., a single point sparger and a cross sparger. Single point sparger was a pipe with a single opening of $3 / 4$ inch diameter, while the cross sparger had 4 holes of $2.2 \mathrm{~mm}$ each (facing downwards towards the bottom of the tank). Schematic of the cross sparger is shown in Figure 4.3b. Four arms of cross sparger extended inside the draft tube and covered $50 \%$ of the draft tube cross sectional area. The spacing of sparger hole from the center of the tank was $\sqrt{2} \mathrm{D} / 2$; where $\mathrm{D}$ is the diameter of the draft tube. Draft tube diameter was changed from $11.5 \mathrm{~cm}$ to $34.3 \mathrm{~cm}$, such that draft 
tube diameter to tank diameter ratio $(D / T)$ is 0.25 and 0.75 , respectively. The overall diameter of sparger was also changed in accordance with the draft tube diameter.

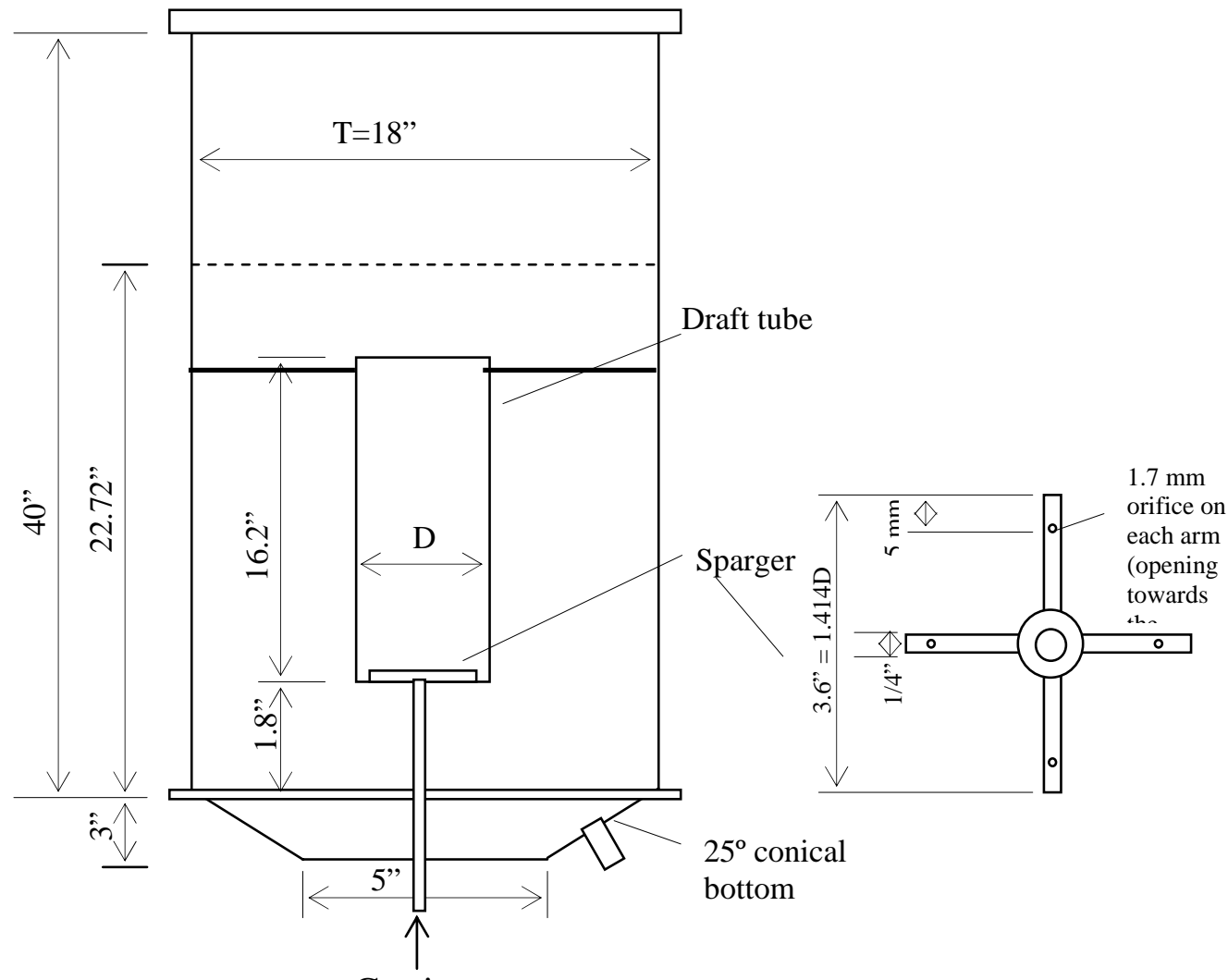

Gas in

(a) Pilot-scale digester

(b) Cross-sparger

Figure 4.3a \& 4.3b Digester geometry and cross sparger geometry

The experiments were conducted with similar slurry obtained from dairy waste, which was used for laboratory-scale experiment. The slurry was screened to eliminate larger solids and then diluted to adjust the total solids to desired concentration, $50 \mathrm{gm} / 1$ (5\% solids) or $100 \mathrm{~g} / 1$ (10\% solids). To account for mixing created by the gas sparging only, anaerobic biogas production was hindered using sodium azide $(2 \mathrm{~g} / \mathrm{l})$.

Air was used as the gas phase to mimic the biogas; air flow rate was varied from $4.5 \mathrm{lpm}$ to $9 \mathrm{lpm}$ and $18 \mathrm{lpm}$. These flow rates resulted in superficial gas velocity (based on tank 
diameter) of $0.45 \mathrm{~mm} / \mathrm{sec}$ to $0.91 \mathrm{~mm} / \mathrm{sec}$ and $1.82 \mathrm{~mm} / \mathrm{sec}$, respectively. Gas flow rate of $9 \mathrm{lpm}$ corresponds to power input density of $8 \mathrm{~W} / \mathrm{m}^{3}$ (minimum suggested by US, EPA 1979 for proper digester mixing) and superficial gas velocity of $0.91 \mathrm{~mm} / \mathrm{sec}$, similar to laboratory-scale digester for gas flow rate of $1 \mathrm{lpm}$. At this low gas superficial velocity the IGLR operates in regime one also known as bubbly flow regime or no gas entrainment regime (Heijnen et al., 1997; Siegel and Robinson, 1992; van Benthum et al., 1999).

CARPT experiments were performed in accordance to experiments carried out in laboratory-scale digester. $150 \mu \mathrm{m}$ diameter Sc-46 particle with approximate activity of $250 \mu \mathrm{Ci}$, enclosed in $1 \mathrm{~mm}$ diameter polypropylene ball was used as tracer. The density of particle was adjusted close to that of water by using epoxy-resin to fill the air gap inside the ball. The tracer particle represented both the liquid in the slurry (water) and the microorganisms, which has density close to that of water.

24 numbers of $\mathrm{NaI}$ detectors were arranged surrounding the pilot-scale digester as shown in Figure 4.4. First of all calibration was performed in situ by positioning the tracer particle at 500 known positions, using an automated calibration device, and spline fit curves were generated. After that the tracer particle was released into the digester and the track data were collected at a frequency of $50 \mathrm{~Hz}$ for 24 hours followed by data processing and reconstruction of the tracer particle trajectories (see CARPT Manual, 2005). 


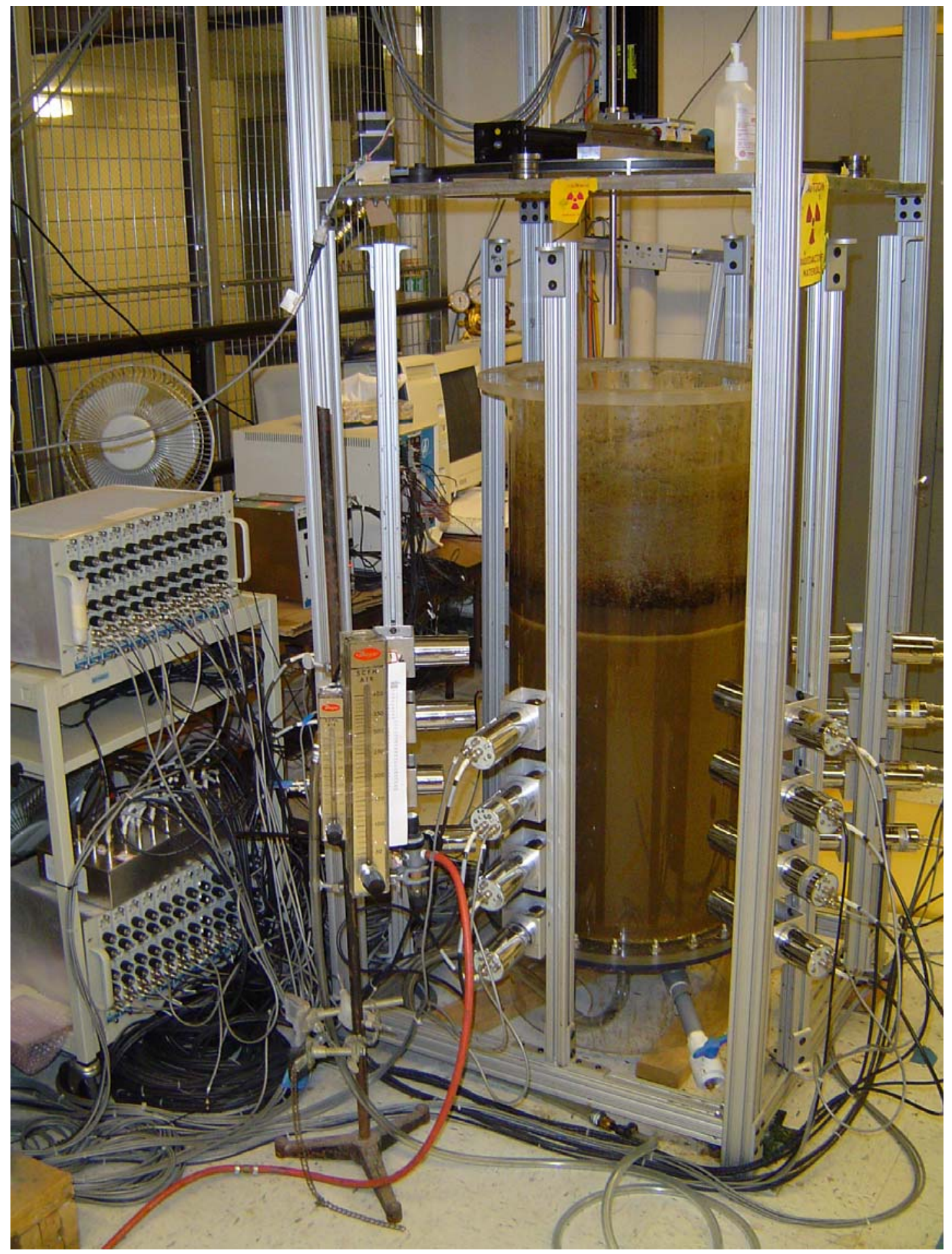

Figure 4.4 Photograph of CARPT set-up for pilot-scale digester 
Total ten CARPT runs were performed; the operational details are given in Table 4.2. The operating conditions were varied in order to study the effect of gas recirculation rate, draft tube diameter, and type of sparger. CARPT was performed on laboratoryscale digester configuration to study the effect of sparger type, gas recirculation rate and draft tube diameter. Similar studies were carried out at pilot-scale for comparison with laboratory-scale hydrodynamic performance. In addition, effect of solids content in the slurry on the hydrodynamics was also investigated.

Table 4.2 Details of CARPT experiments for pilot-scale digester

\begin{tabular}{|c|c|c|c|c|}
\hline $\begin{array}{l}\text { Experime } \\
\text { nt no. }\end{array}$ & $\begin{array}{l}\text { Gas flow rate } \\
(\mathrm{lpm})\end{array}$ & $\begin{array}{l}\text { Total solids in } \\
\text { the slurry }(\mathrm{g} / \mathrm{l})\end{array}$ & $D / T$ ratio & $\begin{array}{l}\text { Sparger } \\
\text { geometry }\end{array}$ \\
\hline P1 & 4.5 & 100 & 0.25 & Cross \\
\hline P2 & 9 & 100 & 0.25 & Cross \\
\hline P3 & 18 & 100 & 0.25 & Cross \\
\hline P4 & 4.5 & 100 & 0.75 & Cross \\
\hline P5 & 9 & 100 & 0.75 & Cross \\
\hline P6 & 18 & 100 & 0.75 & Cross \\
\hline P7 & 4.5 & 100 & 0.25 & Single point \\
\hline P8 & 9 & 100 & 0.25 & Single point \\
\hline P9 & 18 & 100 & 0.25 & Single point \\
\hline P10 & 9 & 50 & 0.25 & Cross \\
\hline
\end{tabular}

\subsection{Results}

\subsubsection{Laboratory-scale Digester}

\section{Flow Pattern and Liquid Velocity Profile}

Figure 4.5 shows the flow pattern inside the digester for experiment L1 (Table 4.1); digester with $D / T$ ratio of 0.25 , cross sparger, and gas flow rate of $1 \mathrm{lpm}$. The overall flow pattern consists of two circulation loops; the bigger circulation loop extends from the top of the digester to the bottom of draft tube, and it is directed upwards inside the draft tube. Another smaller circulation loop exists at the top of the draft tube close to 
the surface. The liquid moves upward in the center and downwards towards the wall. There are big dead zones outside the draft tube and close to the digester wall having very low or zero velocities (represented with smaller arrows or dots in Figure 4.5). This flow pattern has been reported and discussed in detail by Karim et al. (2004) and Vial et al. (2002). Similar flow patterns were observed for experiment L2 to L5, and will be discussed in the following sections.

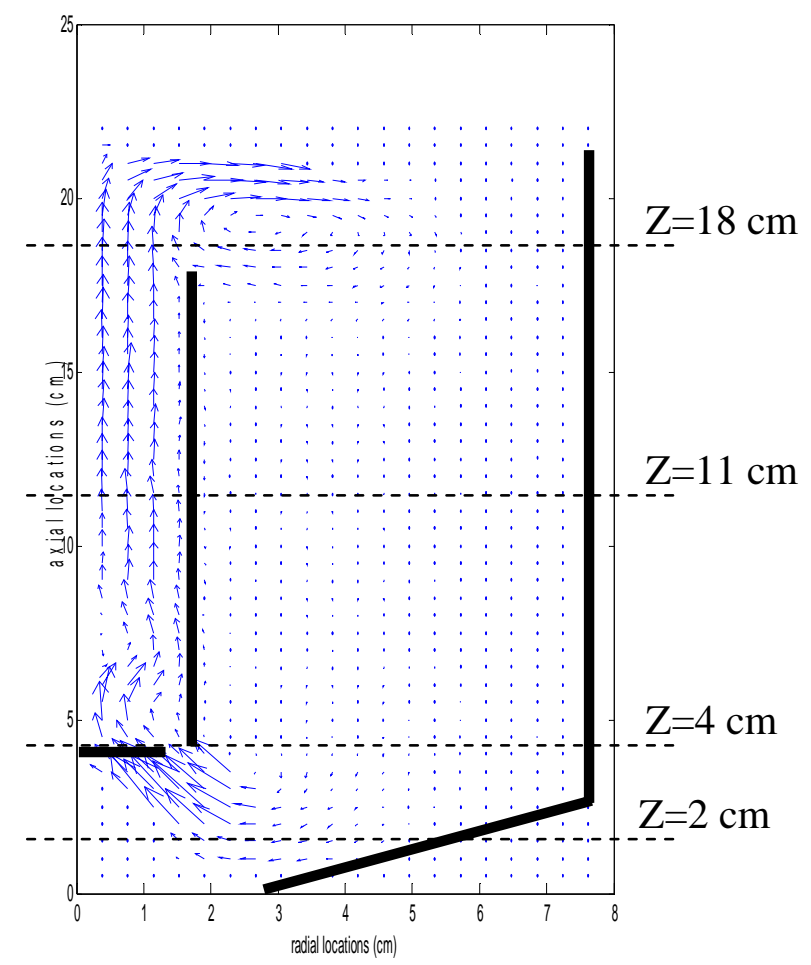

Figure 4.5 Flow pattern for experiment L1

$(D / T=0.25,1 \mathrm{lpm}$ gas flow rate, cross sparger $)$

Radial profile of time averaged azimuthally averaged axial liquid velocity at different axial locations is shown in Figure 4.6. The level of $\mathrm{z}=2 \mathrm{~cm}$ represents the horizontal level inside the conical bottom region at $2 \mathrm{~cm}$ form the bottom of the digester, similarly $z=4 \mathrm{~cm}$ is just below the draft tube, $z=11 \mathrm{~cm}$ is at the centre of the draft tube or tank, and $\mathrm{z}=18 \mathrm{~cm}$ is just above the draft tube. The velocities are positive inside the draft tube and negative outside the draft tube, indicating the upward and downward motion 
of the liquid, respectively. The velocities are zero near the wall region and near the bottom of the digester which shows the location of the dead zones. Higher velocities inside the draft tube are due the turbulence created by the gas bubbles rising inside with high velocities and the maximum axial liquid velocities existed near the sparger hole openings. Similar liquid velocity profiles were obtained by Karim et al. (2004) for 8 inch diameter gas recirculation digester.

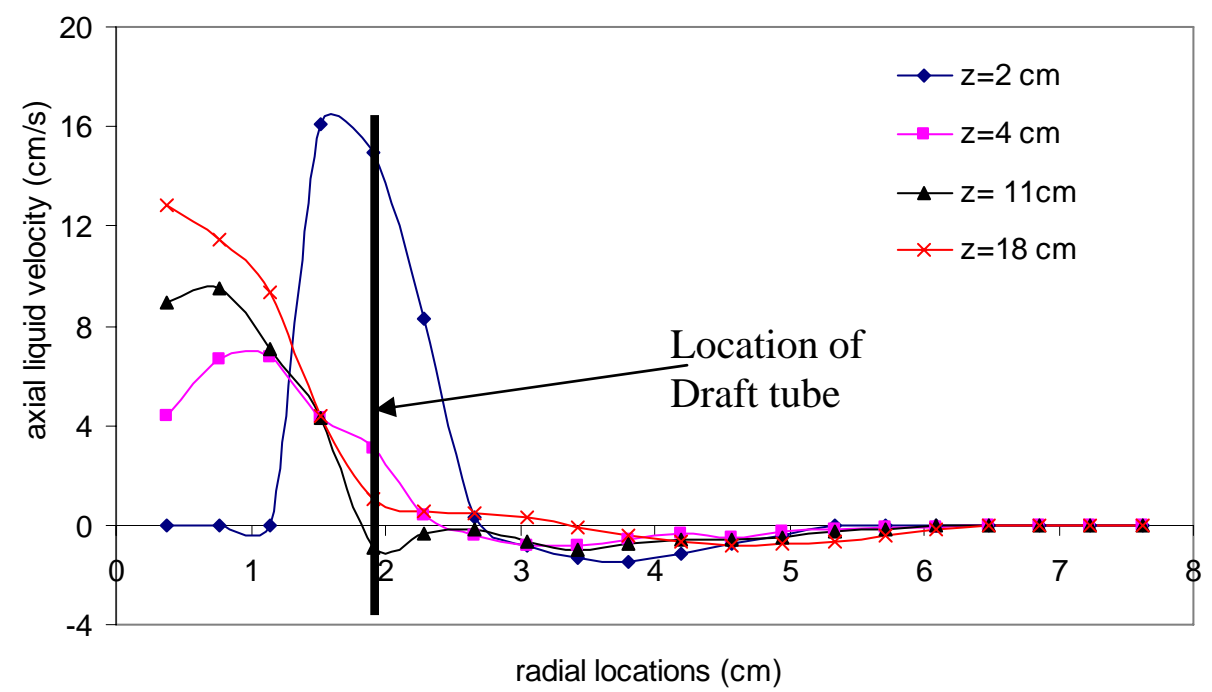

Figure 4.6 Circumferentially averaged axial liquid velocity radial profile for experiment

L1

\section{Effect of Gas Flow Rate}

The effect of gas flow rate on the liquid velocity is shown in Figure 4.7. It is clear from Figure 4.7 that the time averaged axial liquid velocity in the riser increases significantly with the increase in the gas flow rate. However, increase in liquid velocity in the downcomer was negligible. As mentioned before, due to very low superficial gas velocities, the digester was operating in bubbly flow regime. In this regime, as the gas superficial velocity is increased, increasing gas hold up in the riser increases the driving force for the liquid flow, and thus increasing the liquid velocity (Heijnen et al., 1997). At higher gas flow rates the gas bubbles coalesce and also occupy some part of downcomer, without any substantial increase in liquid velocity. The liquid velocity in the downcomer should also increase with increasing the gas flow rate in bubbly flow 
regime, but the magnitude of increase depends on the flow area in downcomer. With $D / T$ of 0.25 , because of large flow area of downcomer, the increase in liquid velocity with increase in gas flow rate is not appreciable. This effect of gas superficial velocity on average liquid circulation velocity was observed by many researchers (Freitas et al., 1999; Klein et al., 2003; Lu et al., 1995; Sun et al., 2005; Wen et al., 2005).

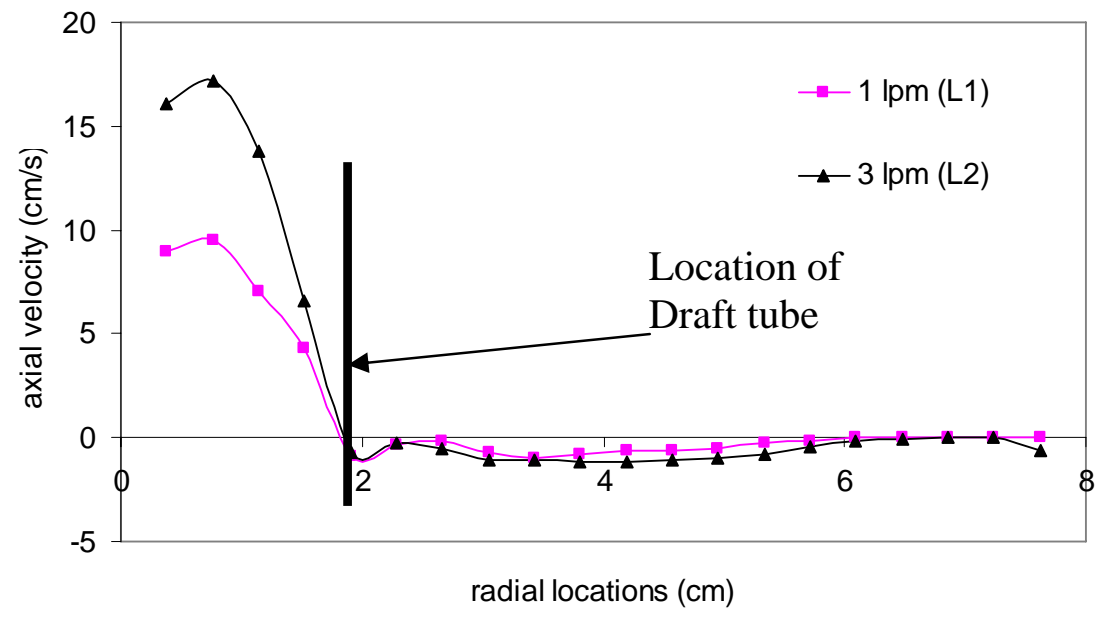

Figure 4.7 Effect of gas flow rate on axial liquid velocity (L1 and L2) at the middle height of the tank $(\mathrm{z}=11 \mathrm{~cm})$

The increase in the liquid velocity with gas flow rate depends on the distribution of gas in the draft tube. If the gas holdup is uniform inside the draft tube, higher liquid velocities can be obtained in the downcomer region due to better gas-liquid dispersion and higher density difference between the draft tube and downcomer regions This information about gas hold-up profile can be obtained from CT, which is a part of the other doctoral thesis by Rajneesh Varma.

\section{Effect of Draft Tube Diameter}

The cross sectional area of draft tube determines the superficial velocity of gas in the riser. Therefore, its size becomes a key parameter, which influences the hydrodynamics of IGLRs. In three-phase systems like digesters, it also plays an important role in 
determining the minimum velocity necessary to fluidize the solid particles. Kennard and Janekeh (1991) reported that $D / T>0.75$ is required to achieve optimum gas hold up. This value is best for aerobic fermenters, since the fluid has a minimum residence time in the downcomer where bubble concentration is low. Minimal mixing time is achieved, when $0.6<D / T<1$. $D / T<0.6$ should be used if high liquid velocities in the riser are required in order to avoid sedimentation of heavy solid particles or large microbial aggregates. Trilleros et al. (2005) correlated his experimental gas holdup data with the geometric variables like draft tube height and diameter in pilot-scale IGLR and found that the effect of $D / T$ on liquid velocity is more important than the effect of draft tube height or solids or liquid holdup in the riser. Thus, the effect of $D / T$ on liquid velocity is investigated here.

Figure 4.8 shows the flow pattern obtained for $D / T$ ratios of 0.5 and 0.75 (experiment L3 and L4, Table 4.1), respectively. The overall flow pattern in digester with larger draft tube diameter is different as compared to the one with smaller draft tube. There is one circulation loop inside the draft tube (figure 4.8a and 4.8b), which is absent in digester with smaller draft tube diameter $(D / T=0.25)$, Figure 4.5. There are two other circulation loops present, one stronger loop at the top of the digester and other weaker loop outside the draft tube. The circulation is very good inside the draft tube, but poor outside the draft tube region; for $D / T$ of 0.75 (Figure $4.10 \mathrm{~b}$ ) almost no liquid movement is observed outside the draft tube. 


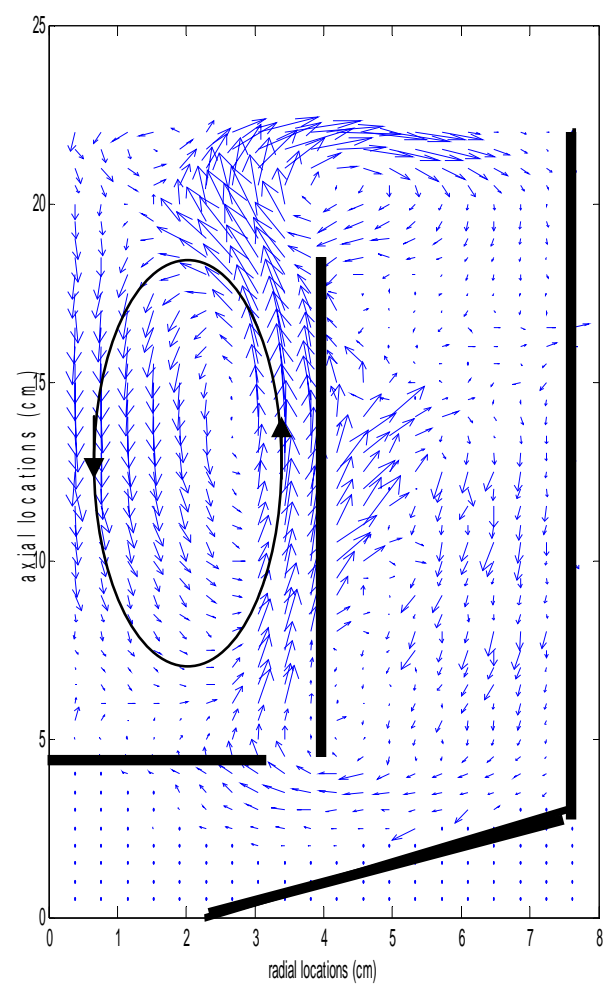

(a)

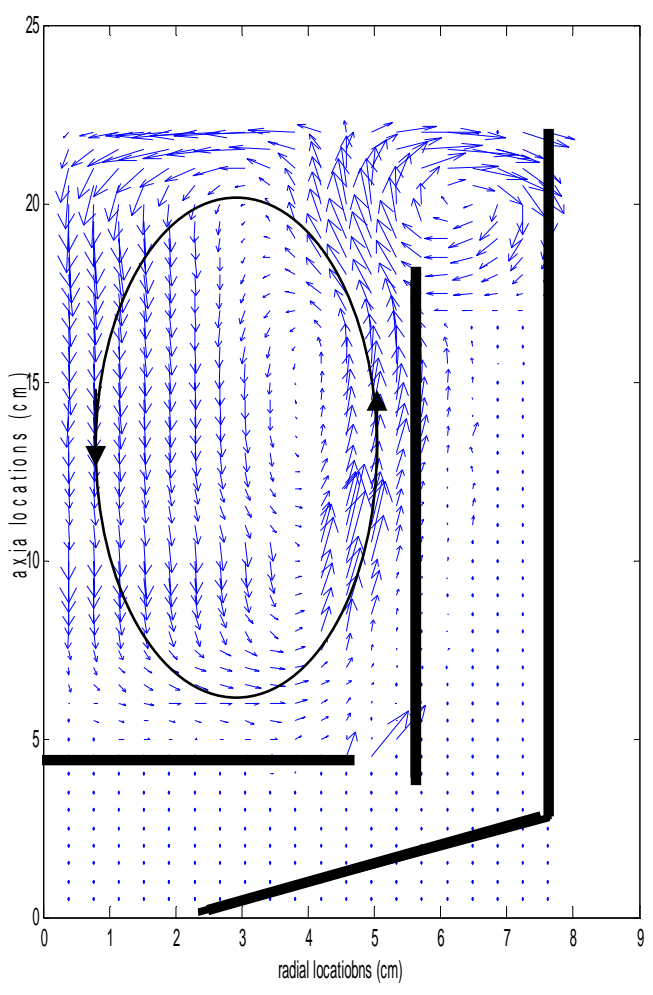

(b)

Figure 4.8 Effect of draft tube diameter on the flow pattern

(a) $D / T=0.5, \mathrm{~L} 3$ (b) $D / T=0.75, \mathrm{~L} 4$

The presence of the circulation loop inside the draft tube would be due to the cross sparger design and large diameter of the riser. The sparger holes are spaced at $5.4 \mathrm{~cm}$ and $8.1 \mathrm{~cm}$ away from the center of the draft tube for $D / T$ of 0.5 and 0.75 , respectively. That means the gas is sparged away from the center and close to sparger walls. The rising gas bubbles close to riser walls, carries liquid upwards with it. The dispersed sparging created by the cross sparger, creates an additional loop inside the draft tube. Thus, the liquid flow is directed downwards in the center and upwards near the wall. This behavior is not seen in the Figure 4.5 for $D / T$ of 0.25 . For $D / T$ of 0.25 , the arms of sparger extend only for a very short length $(2.75 \mathrm{~cm}$ from the center $)$ and the cross sparger behaves more like a single point sparger (concentrated sparging at the center) 
due to smaller diameter. This will be explained further in next section under effect of the sparger design.

Effect of $D / T$ ratio on the time averaged liquid axial velocity radial profiles at the bottom of the draft tube and at the middle height of the tank is shown in Figure 4.9a and $4.9 \mathrm{~b}$, respectively. These figures show the magnitudes of axial velocity inside the digester, the axial liquid velocities are higher at the center of draft tube. The velocities at the bottom of the tank are low for $D / T$ of 0.5 but for $D / T$ of 0.75 these velocities are practically zero, indicating the dead zones. The negative velocities for $D / T$ of 0.5 outside the draft tube region, indicates the downward velocities and existence of circulation loop.

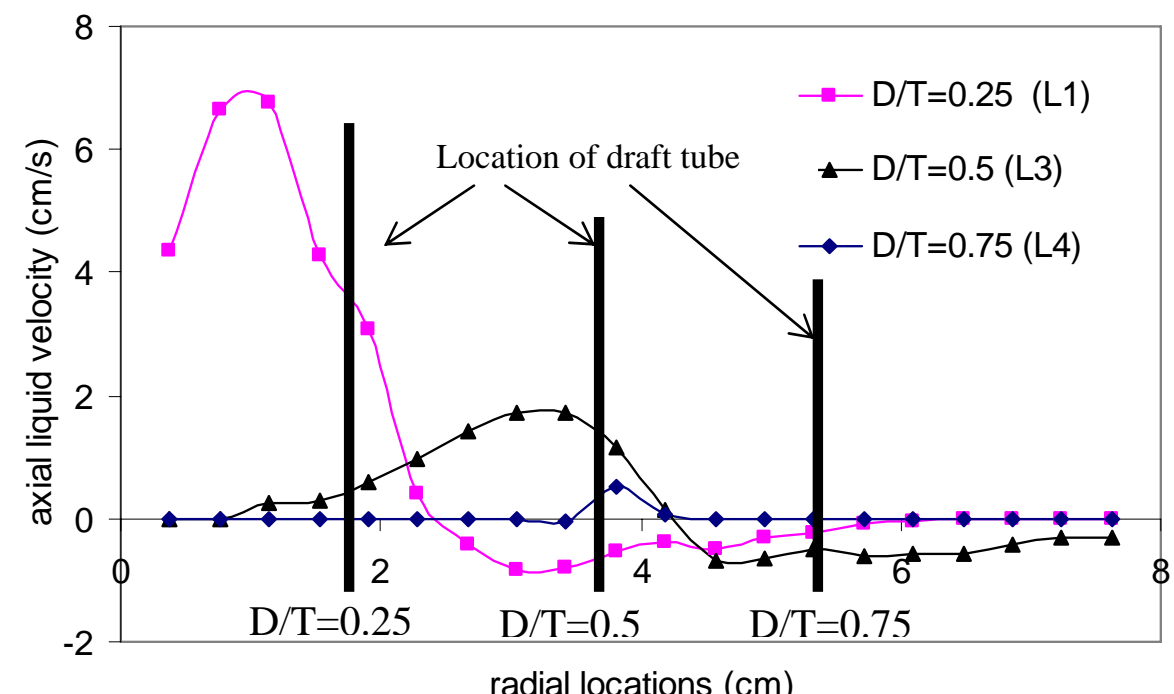

Figure 4.9a Effect of draft tube diameter on the axial liquid velocity at the bottom of the draft tube, $z=4 \mathrm{~cm}$, (experiment no L1, L3 and L4) 


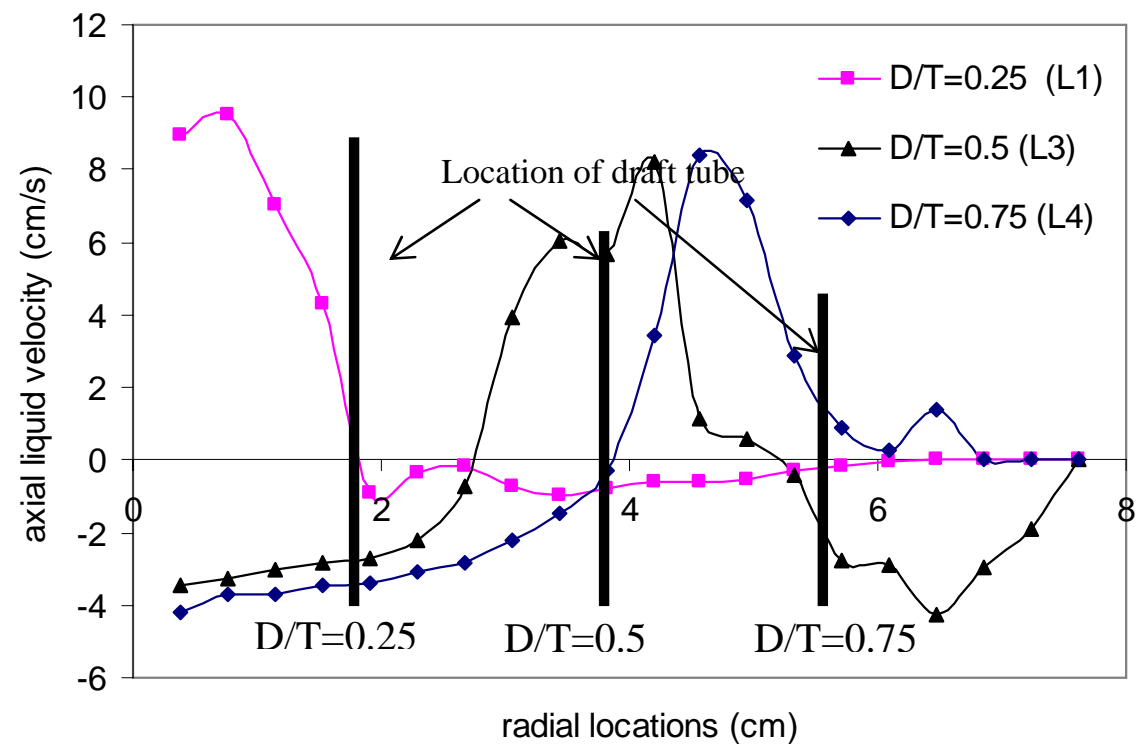

Figure 4.9b Effect of draft tube diameter on the axial liquid velocity at the middle height of the draft tube, $z=11 \mathrm{~cm}$, (experiment L1, L3 and L4)

As the $D / T$ ratio increases, the magnitude of axial velocity inside the draft tube decreases. This was expected, with same gas flow rate as the area for flow increases with the increasing draft tube diameter the velocity decreases, according to Bernoulli's principle. This was also observed by Kojima et al. (1999), Kennard and Janekeh (1991), and Merchuk and Gluz (1999).

One would expect that as $D / T$ increases, the liquid velocity in the downcomer should increase due to reduction in flow area. But higher $D / T$ also means larger riser cross section, lower riser gas holdup at the same superficial gas velocity (i.e. lower gas velocity based on the riser diameter) and hence lower driving force for the liquid circulation. That is why, an optimum $D / T$ exists for achieving higher liquid velocities in the downcomer. 
Feitkenhauer et al. (2003) suggested that the riser and downcomer should have same equivalent diameter to minimize the energy loss by friction, this also prevents higher liquid velocities in one part of reactor and hence higher energy dissipation.

\section{Effect of Sparger Geometry}

Since the overall flow pattern and hydrodynamics of gaslift digester depends on the gas hold up and gas hold up is partly decided by the design of sparger, the effect of sparger at the digester conditions design on hydrodynamics of gaslift digester is very important. In spite of this, effect of sparger design in IGLR is not often discussed in literature. Becker et al. (1994) investigated the effect of sparger geometry on the hydrodynamics of IGLR using CFD. He found that a multipoint sparger creates higher gas holdup as compared to a single point sparger. Whereas, Merchuk (1986) only varied the hole diameters of multipoint sparger and found no effect on gas holdup or liquid velocity in 2D split GLR.

The flow pattern for digester with pipe sparger and $D / T$ of 0.25 (experiment L5) shown in Figure 4.10 is similar to flow pattern obtained for experiment L1 and L2, Figure 4.5. No effect of sparger on the flow pattern was observed for $D / T$ ratio of 0.25 . In experiments L1 and L2 the effect of sparger is not very important due to smaller draft tube diameter $(D / T$ ratio of 0.25$)$. As explained in the previous section, the arms of cross sparger extend only for a very short length $(2.75 \mathrm{~cm}$ from the center) and cover $50 \%$ of the area inside the draft tube with $D / T$ of 0.25 . The gas distribution is concentrated at the middle height of the tank over a small region, see Figure 4.1. This may be the reason for absence of the internal circulation loop in the draft tube with $D / T$ of 0.25 . As the draft tube diameter increases, the length of arms of sparger extend away from center and each other and create more dispersed sparging, see Figure 4.11. This gives rise to the internal circulation loop inside the draft tube, which becomes more prominent with increasing draft tube diameter. 


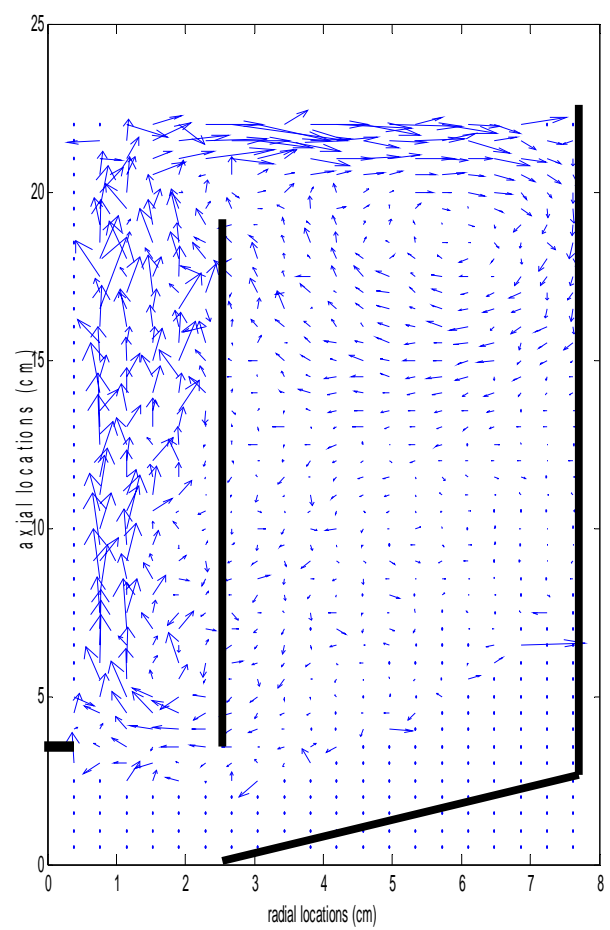

Figure 4.10 Flow pattern for experiment L5

$(D / T=0.25,1 \mathrm{lpm}$ gas flow rate, single point sparger $)$

Figure 4.11 shows the possible pattern of gas dispersion in the digester, without considering bubble breakup and coalescence. Definite conclusions about the effect of sparger geometry can be made only when the gas hold-up profile inside the riser is known. CT measurements need to be performed for this purpose. 


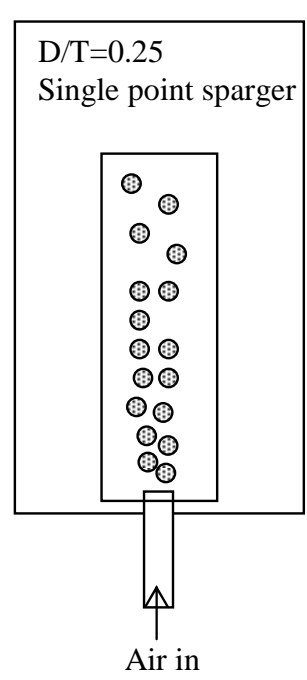

(a)

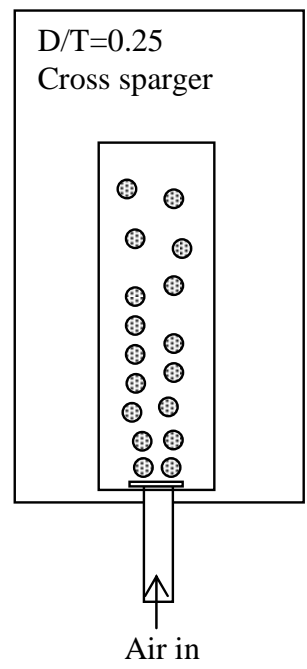

(b)

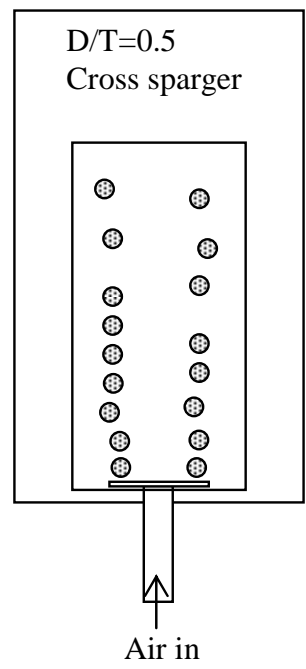

(c)

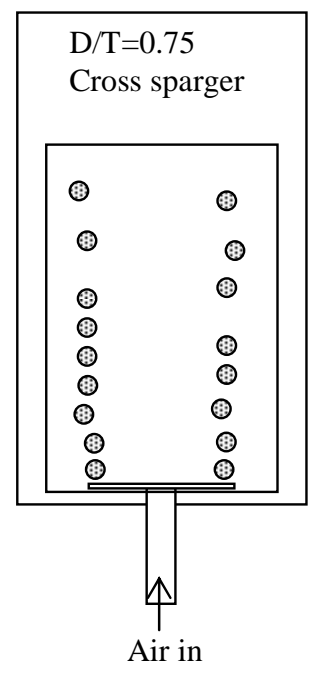

(d)

Figure 4.11 Expected effect of sparger geometry and $D / T$ ratio on gas distribution

The effect of sparger on the axial liquid velocity profile is shown in Figure 4.12. The axial liquid velocities are higher for pipe sparger as compared to that for the cross sparger inside the draft tube, whereas the liquid velocities are the same for both the spargers outside the draft tube. The higher velocities can be a result of different gas holdup distribution or change in bubble dynamics. Further confirmation is required from CT measurements to explain effect of sparger design.

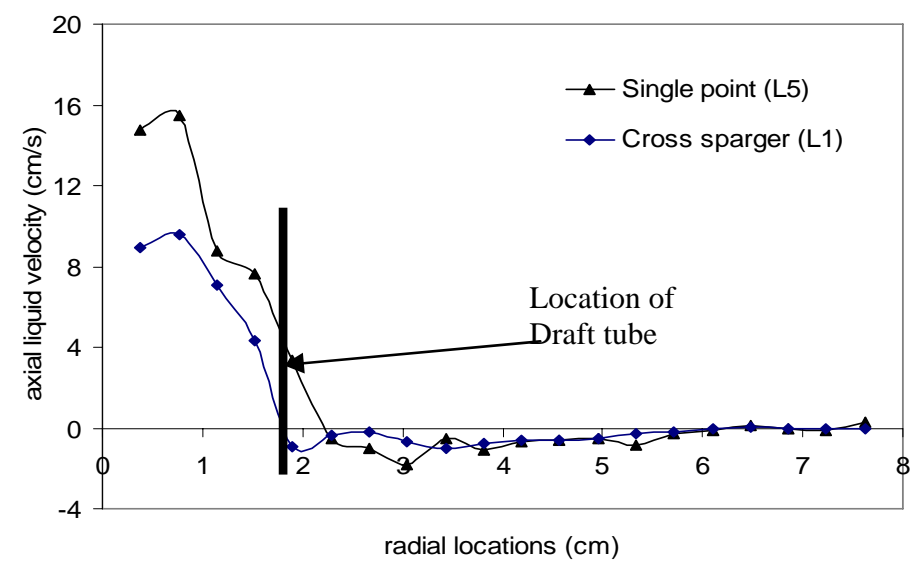

Figure 4.12 Effect of sparger geometry on axial liquid velocity at middle height of the tank, $z=11 \mathrm{~cm}$, (experiment L1 and L5). 


\section{Volume of Dead Zones or Stagnant Regions}

The different configurations of digester can be compared conveniently in terms of dead volume. The dead zones or stagnant zones are the part of the reactor with no flow or very low velocities due to which particle settling occures. Dead zones are undesirable as the dead regions remain secluded from rest of the reactor volume with no mixing thus reducing the effective reactor volume. These are the regions which cause building of $\mathrm{pH}$ and temperature, thus degrading the digester performance. Dead zones should also be avoided to prevent solids from settling due to low liquid velocities (Feitkenhauer et al., 2003). Bello-Mendoza and Sharratt (1998) also used volume of dead zones as the mixing parameter for the study of anaerobic digesters.

It is important to select a correct criterion to evaluate the total volume of dead zones. If the settling velocity of the solid particles is known then it can be used as a limiting velocity to define a dead region. Calculation of settling velocity using Stokes law requires particle size, particle density and fluid viscosity information. The digester slurry contains varying concentration of a variety of solids from different sources such as husk, straw, and fibers coming from the feed, and sand particles, saw dust, wood, shavings, chips, rice hulls from bedding material. The solids are also of different sizes. As the digestion progresses the size of the solids reduces due to the digestion. The microbes form agglomerates and grow on size. Due to different sources of solids

present in the digester and agglomerates of microbes acting as solids, specifying a representative solid density is difficult. Digester sludge generally have non-Newtonian properties (Seyssiecq et al., 2003), thus shear stress and shear rate are needed to calculate the sludge viscosity. All these factors along with lack of related literature make it difficult to calculate solids settling velocity in the digesters treating cow manure. Karim et al. (2006) used settling velocity to evaluate the dead zones in the simulated digester. He used $0.32 \mathrm{~cm} / \mathrm{s}$ as the representative solids settling velocity for the sludge. The settling velocity was calculated using Stokes law using the density and viscosity of water and randomly selecting the particle size based on the particle size distribution data reported by Li and Yuan (2002) for the sludge medium 
In the present study the dead zone volume was qualitatively evaluated by locating the cells with very low velocities (some arbitrary low value, $1 \mathrm{~cm} / \mathrm{sec}$ was used here), and summing up the volumes of these cells. For the sake of comparison, different values of low velocities were used to evaluate the dead zones. $5 \%$ of maximum liquid velocity in the digester was used as the low limit to identify the dead zones, which gives values ranged from 0.5 to $2.5 \mathrm{~cm} / \mathrm{s}$. It is noteworthy that even with this range of velocity, same comparisons and conclusions were obtained as discussed in this section.

Hence, Table 4.3 shows the qualitatively dead zone volumes for different digester configurations based on limited velocity of $1 \mathrm{~cm} / \mathrm{s}$. The values reported in the Table 4.3 are qualitative in nature as opposed to being strictly quantitative. It should be noted that for the reasons discussed earlier the dead zone volume is used in this thesis only for comparison between different configurations and not for digester design. Thus the limiting value of liquid velocity used for calculating dead zone volume is not critical as long as same value is used for all configurations to be compared. For the digester design and sizing purposes detailed characterization of the rheology of the anaerobic digesters is needed to properly quantify the solids settling velocities and their distribution to properly quantify the dead zones. Such proper quantification is needed for the development of a detailed reactor model with the dead zones.

Table 4.3 Dead or stagnant volumes for laboratory-scale digester configurations

\begin{tabular}{|c|c|c|c|c|}
\hline $\begin{array}{c}\text { Expt } \\
\text { no. }\end{array}$ & $\begin{array}{c}\text { Gas flow } \\
\text { rate }(\mathrm{lpm})\end{array}$ & $\begin{array}{c}D / T \\
\text { ratio }\end{array}$ & Sparger & $\begin{array}{c}\% \text { Dead } \\
\text { volume }\end{array}$ \\
\hline L1 & 1 & 0.25 & cross & 50 \\
\hline L2 & 3 & 0.25 & cross & 42 \\
\hline L3 & 1 & 0.5 & cross & 30 \\
\hline L4 & 1 & 0.75 & cross & 60 \\
\hline L5 & 1 & 0.25 & $\begin{array}{c}\text { single } \\
\text { point }\end{array}$ & 55 \\
\hline
\end{tabular}

Table 4.3 shows that the dead zone volume decreases by increasing the gas flow rate, but it is clear from flow pattern in Figure 4.5 that high liquid velocities are present only 
inside the draft tube region for $D / T$ of 0.25 , thus decrease in dead zone volume does not indicate more homogeneity in this case. Only 30\% of the digester volume is inactive/dead in case of $D / T$ of 0.5 , whereas this number is highest for $D / T$ of 0.75 . Flow patterns shown in Figure 4.10 indicate that the digester with $D / T$ of 0.5 is mixed more homogenously thorough out the volume than the digester with $D / T$ of 0.75 .

It is also very interesting to see the location of dead zones in addition to their total volume. Figure 4.13 shows the map of dead zones in digester with $\mathrm{D} / \mathrm{T}$ ratio of 0.25 and 0.5. The dead zones exist near the bottom and towards the wall of the digester for $\mathrm{D} / \mathrm{T}$ ratio of 0.25 . For $\mathrm{D} / \mathrm{T}$ ratio of 0.5 , the dead zones occupy only the bottom portion of the digester. This can also be clearly seen in the flow pattern of digester, thus flow pattern in digester gives a clear indication of dead zones location.

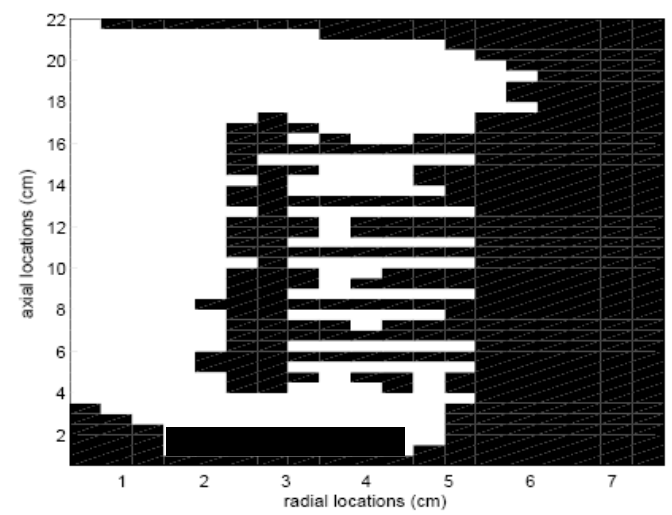

(a)

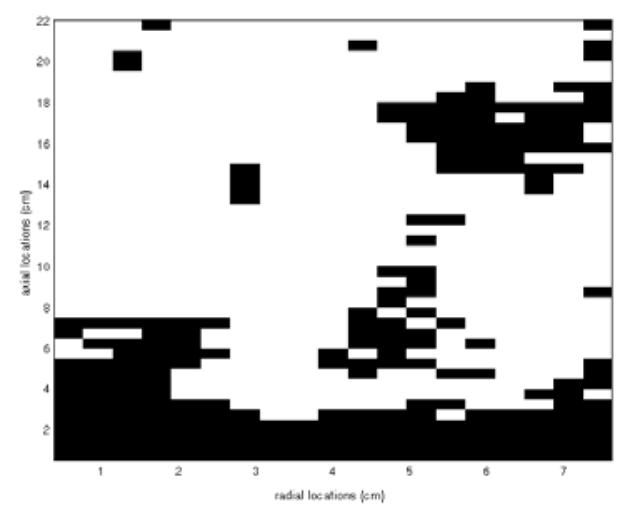

(b)

Figure 4.13 Dead zone map for Experiment (a) L1 and (b) L3 (dark spaces are the dead zones)

In addition to dead zone volume turbulent diffusivities can also be used to evaluate the mixing performance of digesters, as described in next section.

\section{Eddy Diffusivity}

The key property of turbulent motion is its ability to transport or mix momentum, energy, scalar quantities, etc. The rates of transfer and mixing in the presence of 
turbulence are orders of magnitude larger than the rates due to molecular transport. The values of diffusivity at the location near and inside the dead zones will help to understand the diffusion time scales for transfer of material within and through the dead zones. If the material entering the dead zone stays inside the dead zone for a period longer than the time scale of limiting digestion reaction due to low diffusion rate, then the performance of digester will be degrade due to two reasons. First, the effective volume of the digester will be reduced causing the reduction in the effective sludge retention time. Second, if the fatty acids formed in the dead zones are not diffused uniformly as they are formed, they will kill the methanogens. Uniformity of $\mathrm{pH}$ and temperature within the reactor is also important.

In the present section, the turbulent eddy diffusivities are defined as measured in a Lagrangian framework. The details of calculation of eddy diffusivities are given by Degaleesan (1997). The radial and axial eddy diffusivities calculated for experiment L1 at the middle height of the tank are shown in Figure 4.14. The radial diffusivities are very small as compared to axial diffusivities and can be neglected. The distribution if axial diffusivities over the digester volume for experiment L1 and L3 is shown in Figure 4.15. The axial diffusivities are very low almost zero inside and near the dead zones. This signifies that the exchange of material through and within the dead zones is very slow.

The axial diffusivities for different configurations are shown in Figure 4.16. The diffusivity increases with increasing gas flow rate. The diffusivity is highest for configuration with $D / T$ ratio of 0.5 , indicating better mixing behavior. Sparger design has no effect on the diffusivity. These results are consistent with the qualitative findings based on the comparison of dead zones, where digester with $D / T$ ratio of 0.5 shows lowest value of dead zones. 


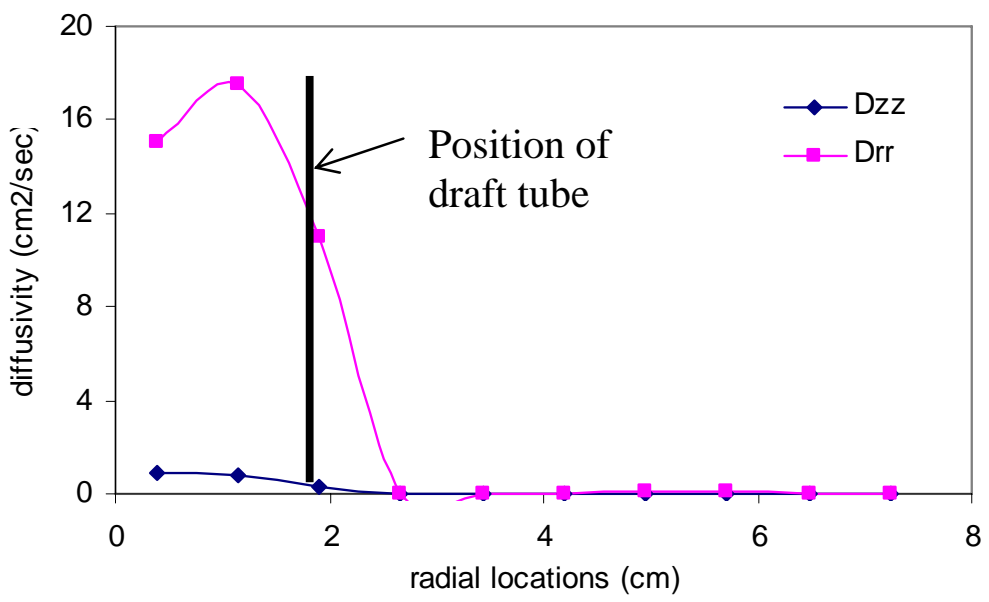

Figure 4.14 Axial and radial eddy diffusivities at the middle height of the tank for experiment

L1

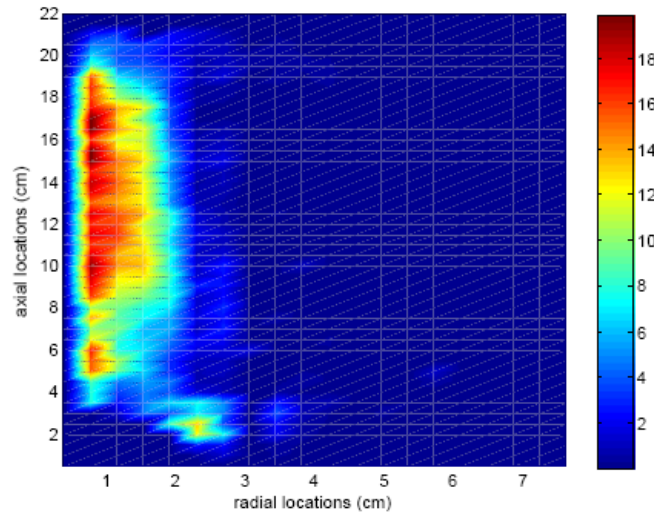

(a)

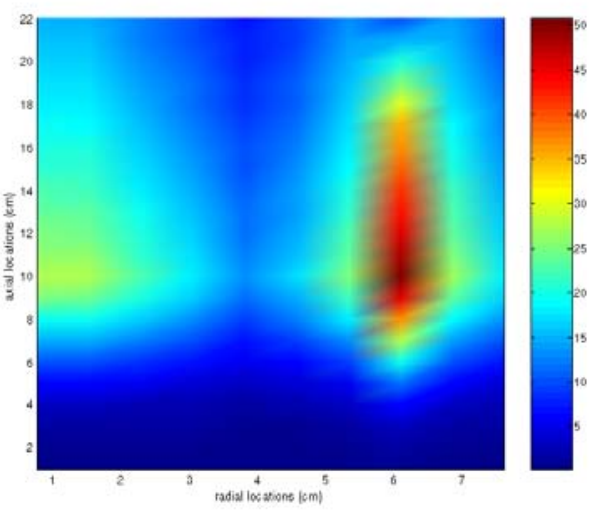

(b)

Figure 4.15 Axial diffusivity map for experiment (a) L1 and (b) L3 


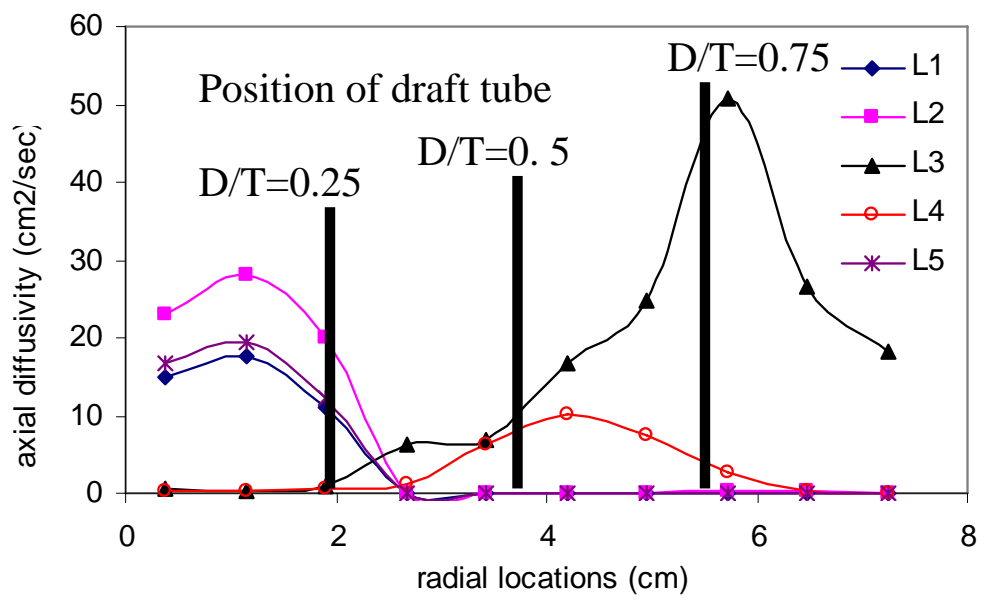

Figure 4.16 Comparison of axial eddy diffusivities for different digester configurations

\subsubsection{Pilot-scale}

\section{Flow Pattern and Liquid Velocity Profile}

Figure 4.17 shows the flow pattern inside the digester for experiment P1 (Table 4.2); digester with $D / T$ ratio of 0.25 , cross sparger, and gas flow rate of $4.5 \mathrm{lpm}$. The overall flow pattern consists of two circulation loops; the bigger circulation loop extends from the top of the digester to the bottom of draft tube, and it is directed upwards inside the draft tube. Another smaller circulation loop exists at the top of the draft tube close to the surface. The liquid moves upward in the riser and downwards in the downcomer. Dead zones exist in the downcomer and close to the digester wall having very low or zero velocities (represented with smaller arrows or dots in Figure 4.17). Similar flow pattern in a small-scale digester has been reported and discussed in detail by Karim et al. (2004). Similar flow patterns were observed for experiment P2 and P3 (Table 4.2), and will be discussed in following sections. 


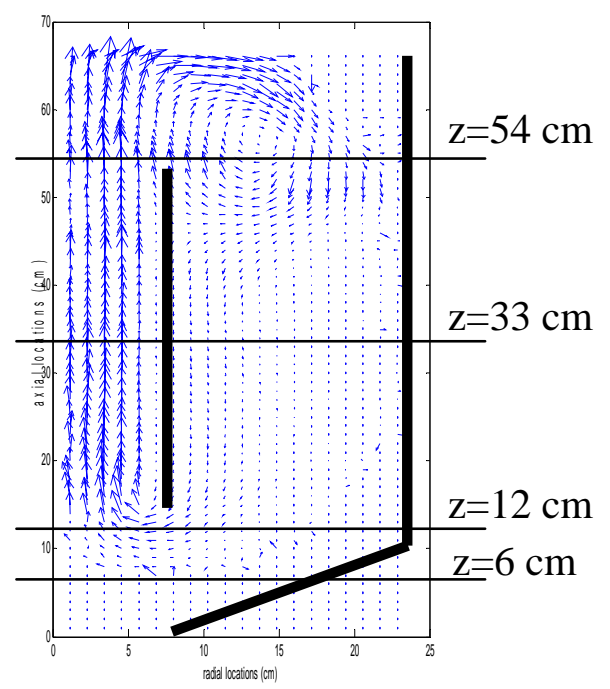

Figure 4.17 Flow pattern for experiment $\mathrm{P} 1(D / T=0.25,4.5 \mathrm{lpm}$, cross sparger $)$

Radial profile of time and azimuthally averaged axial liquid velocity at different axial locations is shown in Figure 4.18. The level of $z=6 \mathrm{~cm}$ represents the horizontal level inside the conical bottom region at $6 \mathrm{~cm}$ form the bottom of the digester, similarly $\mathrm{z}=12$ $\mathrm{cm}$ is just below the draft tube, $z=33 \mathrm{~cm}$ is at the middle height of the draft tube or tank, and $z=54 \mathrm{~cm}$ is just above the draft tube. The velocities are high and positive inside the draft tube, where as low and negative outside the draft tube, indicating the upward and downward motion of the liquid respectively. The velocities are zero near the wall region and near the bottom of the digester which shows the location of the dead zones. Higher velocities inside the draft tube are due the turbulence created by the air bubbles rising inside with high velocities.

\section{Effect of Gas Flow Rate}

The effect of gas flow rate on the liquid velocity at the middle height of the tank $(z=33$ $\mathrm{cm}$, all liquid velocity profiles are reported at the middle height of the tank unless or otherwise mentioned) is shown in Figure 4.19. It is clear from Figure 4.19 that the time averaged axial liquid velocity inside the draft tube increases significantly with the increase in the gas flow rate on liquid velocity. However, there is almost no effect of gas 
flow rate outside the draft tube. The reasons for this behavior are already discussed in laboratory-scale section of this chapter.

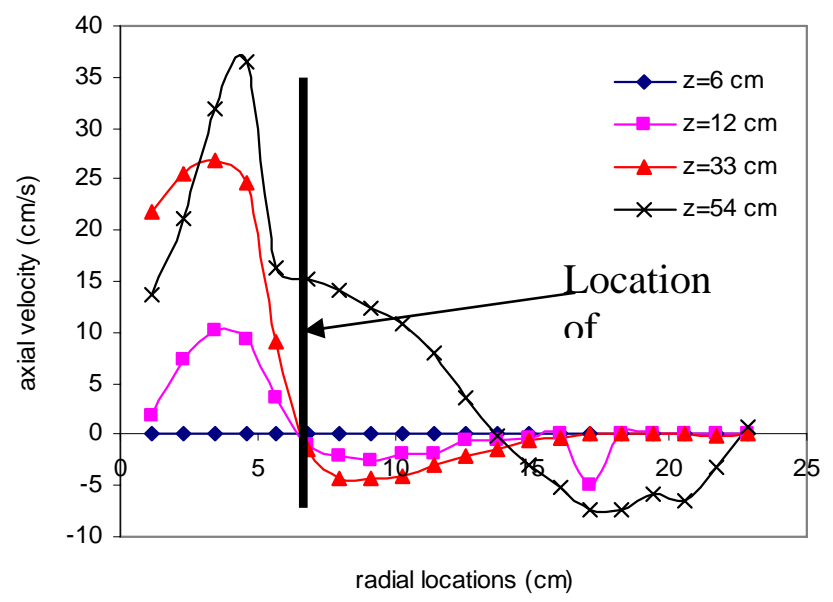

Figure 4.18 Circumferentially averaged axial velocity radial profile for experiment P1 at different axial locations

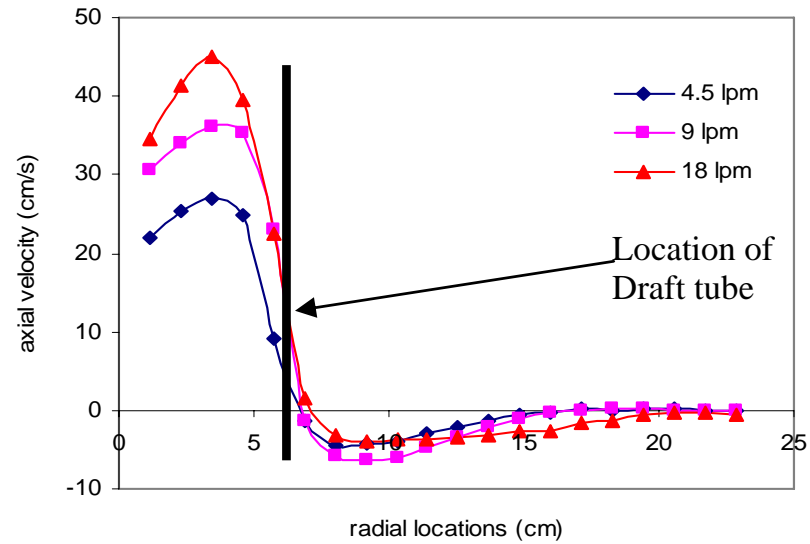

Figure 4.19 Effect of gas flow rate on axial liquid velocity (experiment P1, P2 and P3) at the middle height of the $\operatorname{tank}(\mathrm{z}=33 \mathrm{~cm})$.

\section{Effect of Draft Tube (Riser) Diameter}

The draft tube diameter to tank diameter ratio $(D / T)$ was varied to 0.75 to see the effect of draft tube diameter. The flow pattern obtained for $D / T$ of 0.75 with cross sparger is shown in Figure 4.20 for gas flow rate of $9 \mathrm{lpm}$ (experiment P5), respectively. The flow patterns are different than the one obtained with $D / T$ ratio of 0.25 (Figure 4.12). Two 
major circulation loops are seen in Figure 4.20. The liquid flow is directed upwards inside the draft tube by the sparger and then the liquid is distributed into 2 loops. One loop is directed upwards inside the draft tube near the draft tube wall and flows downward in the downcomer region (that is the region between the draft tube and the tank walls). Another loop exists in the riser at the top, which flows downward near the middle height of the tank and upwards at the region halfway between the draft tube wall and middle height of the tank. There is significant liquid flow in the downcomer region, which was absent for smaller draft tube diameter as shown in Figure 4.16.

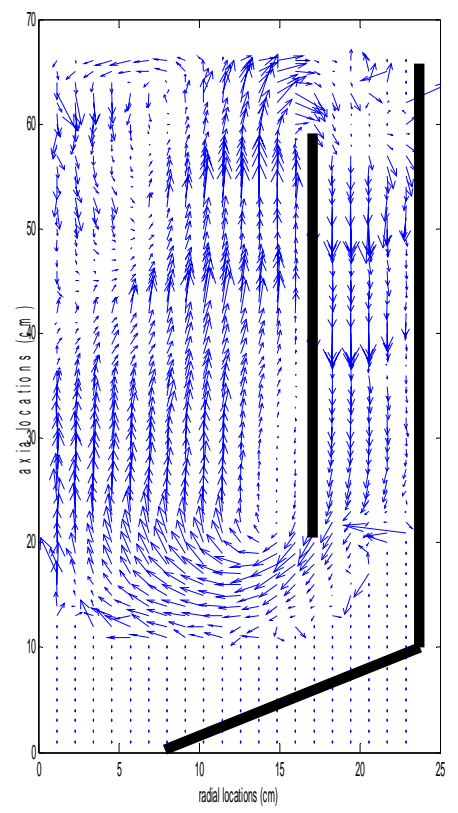

Figure 4.20 Flow pattern for $D / T=0.75$ at gas flow rate of 9 lpm, P5

The circulation loop inside the riser exists because of the combined effect of large riser cross sectional area and cross sparger design. Four holes of sparger are placed at $24 \mathrm{~cm}$ from middle height of the tank for $D / T$ of 0.75 . Thus, cross sparger is expected to create dispersed sparging as shown in Figure 4.11d, due to large spacing between the holes. The dispersed sparging created by the cross sparger, creates an additional loop inside the draft tube. This behavior is not seen in the Figure 4.13 for $D / T$ of 0.25 . For $D / T$ of 0.25 , due to smaller riser diameter and closer spacing of holes (only $7.5 \mathrm{~cm}$ from 
the center, see Figure 4.11b), the gas is expected to be dispersed more uniformly over the smaller cross section of riser as compared to riser of $D / T=0.75$.

Figure 4.21 shows the radial profile of azimuthally averaged axial liquid velocity at different axial levels for gas flow rate of $9 \mathrm{lpm}$ (experiment P5), respectively. These plots show that the liquid velocity is zero inside the conical bottom region. The positive and the negative values of velocity inside the riser, show that the liquid flows upwards near the center and downwards near the wall of the draft tube.

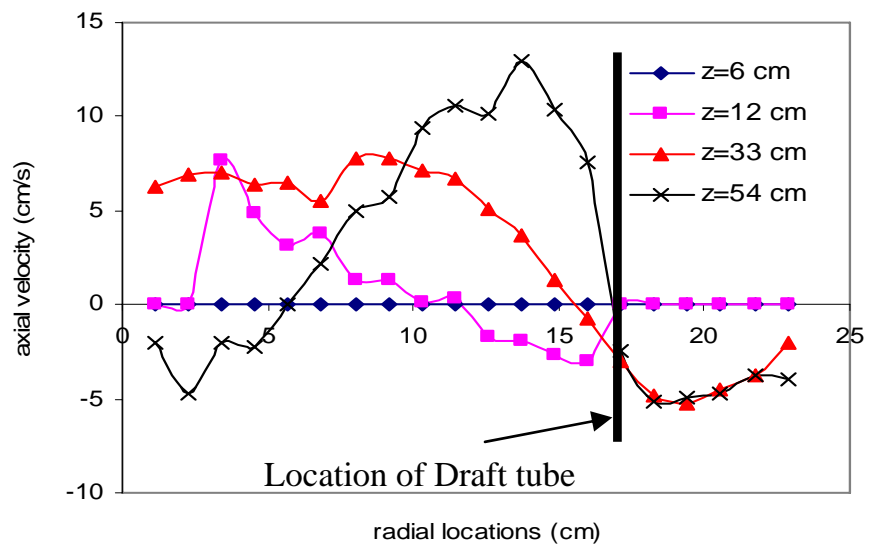

Figure 4.21 Circumferentially averaged axial velocity radial profile for experiment P5

The axial liquid velocity profiles for $D / T$ of 0.25 and 0.75 are compared in Figure 4.22 for different gas flow rates. As the $D / T$ ratio increases, the magnitude of axial velocity inside the riser decreases but it increases in the downcomer region. This is was expected, with same gas flow rate as the area for flow increases with the increasing draft tube diameter the velocity decreases, according to Bernoulli's principle. The higher liquid velocity in downcomer region is an advantage offered by increasing the draft tube diameter. This was also observed by Kojima et al. (1999).

Figure 4.22 also explains the effect of gas flow rate on the axial velocity, increase in the gas flow rate increases the liquid velocity inside the draft tube and hence the circulation 
in the digester. Similar observations were made by Freitas et al. (1999), Klein et al. (2003a), Lu et al. (1995), Sun et al. (2005), and Wen et al. (2005).

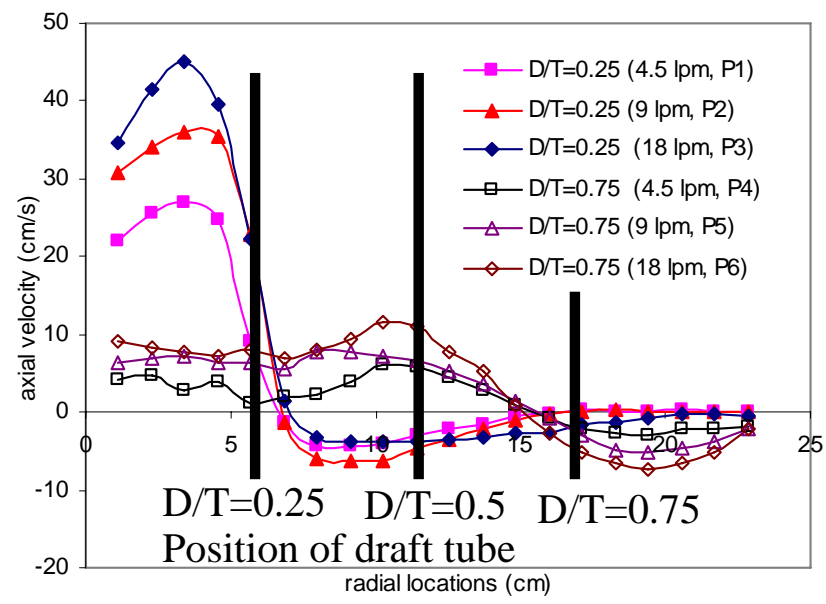

Figure 4.22 Effect of draft tube diameter on the axial liquid velocity at the middle height of the draft tube at different gas flow rates, (experiments P1 to P6)

\section{Effect of Sparger Geometry}

Cross sparger was replaced with single point sparger to study the effect of sparger on the flow pattern of a digester. Figures 4.23 show the flow patterns obtained for digester with single point sparger and $D / T$ of 0.25 for gas flow rate of $9 \mathrm{lpm}$ (experiment P8), respectively. The flow patterns look similar to one with the cross sparger for experiment P1, P2 and P3 as shown in Figure 4.17. The sparger does not affect the flow pattern significantly for $D / T$ ratio of 0.25 in qualitative manner. 


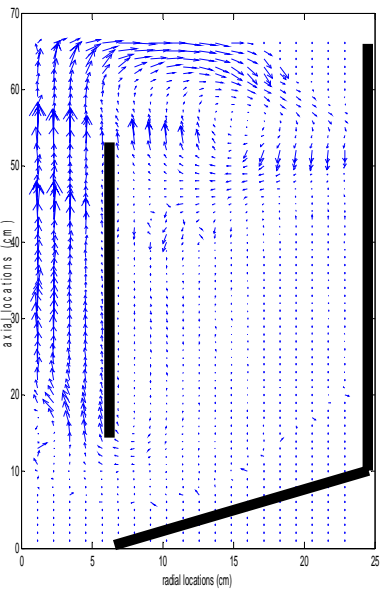

Figure 4.23 Flow pattern for a single point sparger and $D / T=0.25$ at $9 \mathrm{lpm}$ (P8)

Figure 4.24 shows the liquid velocity profile at different axial locations for configurations with single point sparger at gas flow rate of $9 \mathrm{lpm}$. Figure 4.25 shows the effect of sparger type on the axial liquid velocity for different gas flow rates. For cross sparger the maximum liquid velocity exists away from the centre of the tank, whereas for single point sparger the location of maximum liquid velocity is at the middle height of the tank. The liquid velocities are higher for single point sparger than cross sparger only in small region at the center of the riser. But for cross sparger, the liquid velocities are higher than single point sparger for majority of annular cross-section of the riser.

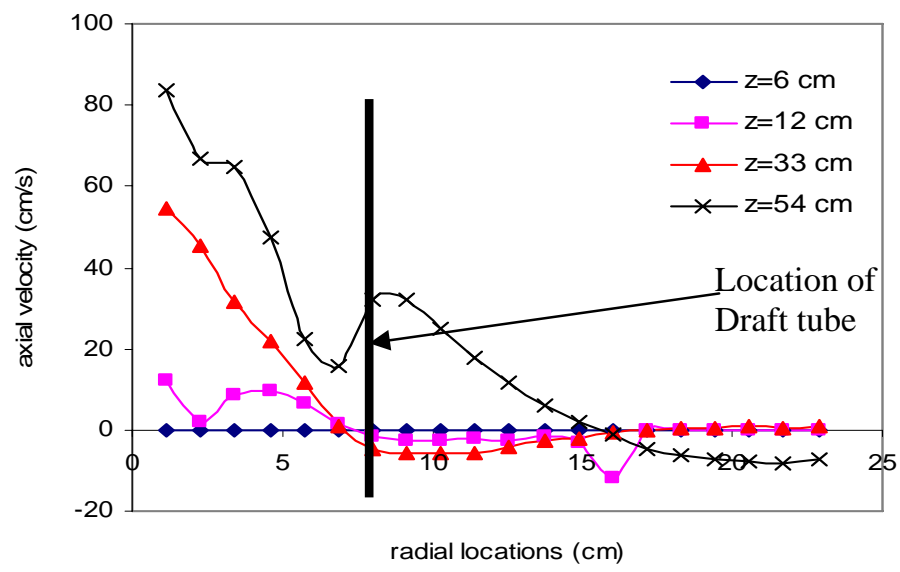

Figure 4.24 Circumferentially averaged axial liquid velocity radial profile for expt P8 


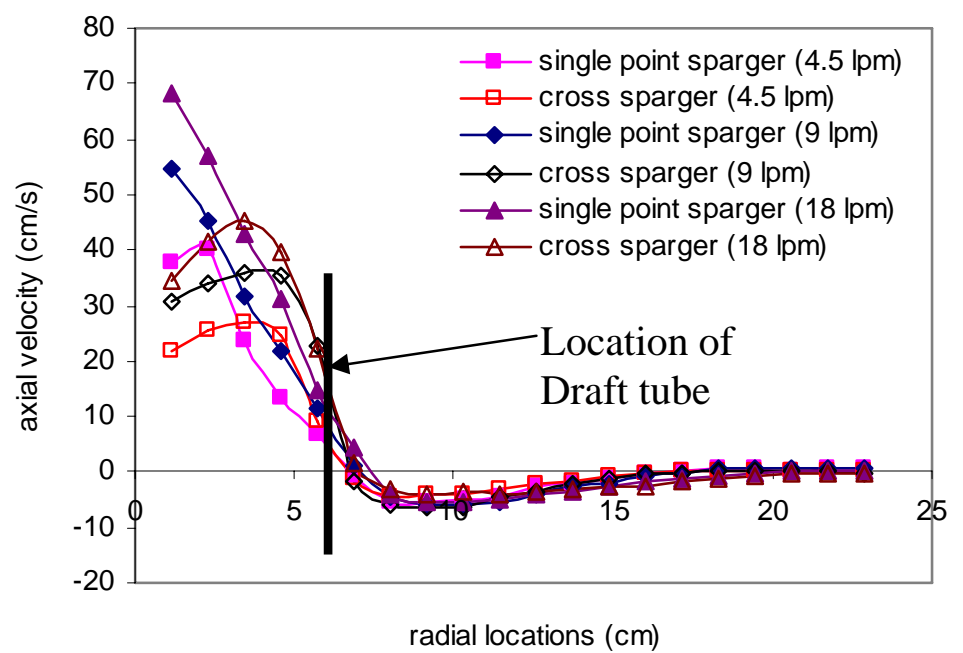

Figure 4.25 Effect of sparger on the axial liquid velocity at the middle height of draft tube at different gas flow rates (experiments P1 to P3 and P7 to P9)

The reason for this behavior is obvious, the holes on the cross sparger are $7.5 \mathrm{~cm}$ away from the middle height of the tank and near the draft tube walls on the arms of the cross sparger for $D / T$ of 0.25 , whereas the sparging is done at one localized point at the center of the tank in case of single point sparger. The rising gas bubble from sparging hole accelerates the liquid and creates high liquid velocity. Whereas, cross sparger creates gas dispersion at four separate uniformly spaced point inside the riser. Thus better gas dispersion is expected in case of cross sparger, which creates better liquid circulation and higher average liquid circulation velocities as compared to single-point sparger. The difference in gas holdup distribution can be obtained from CT experiments and this issue can be addressed in more detail.

\section{Effect of Solids Concentration in the Slurry}

In three-phase gaslift digesters with very small solid particles, as in present case of dairy manure slurry, a defined flow pattern is obtained along with complete suspension of solids at low gas superficial velocities (Wen et al., 2005). For low density particles (between 1000 to $1600 \mathrm{Kg} / \mathrm{m}^{3}$ ), the liquid and solid phase are often assumed to be one pseudo-homogeneous phase. 
Solids concentration in the slurry was changed from $100 \mathrm{gm} / \mathrm{lit}(10 \%)$ to $50 \mathrm{gm} / \mathrm{lit}(5 \%)$ for a digester with cross sparger and draft tube with $D / T$ ratio of 0.25 at gas flow rate of $9 \mathrm{lpm}$ (experiment P10) to see the effect of solids concentration on the flow pattern of the digester. The flow pattern was not considerably affected by solids concentration, except with a slight increase in the liquid axial velocity for 5\% slurry, shown in Figure 4.26 (comparison of axial liquid velocity profile at the middle height of the tank for different solids concentration, experiment P2 and P10). Higher solids concentration causes the reduction in flow area for the solid and liquid phase thereby increasing the frictional loss and decreasing the velocity. Higher solids concentration also causes the increase in the viscosity of pseudo-homogeneous liquid-solid phase. As discussed earlier, increase in liquid velocity with decreasing solids fraction was also observed by Merchuk (2003) and Sun et al. (2005).

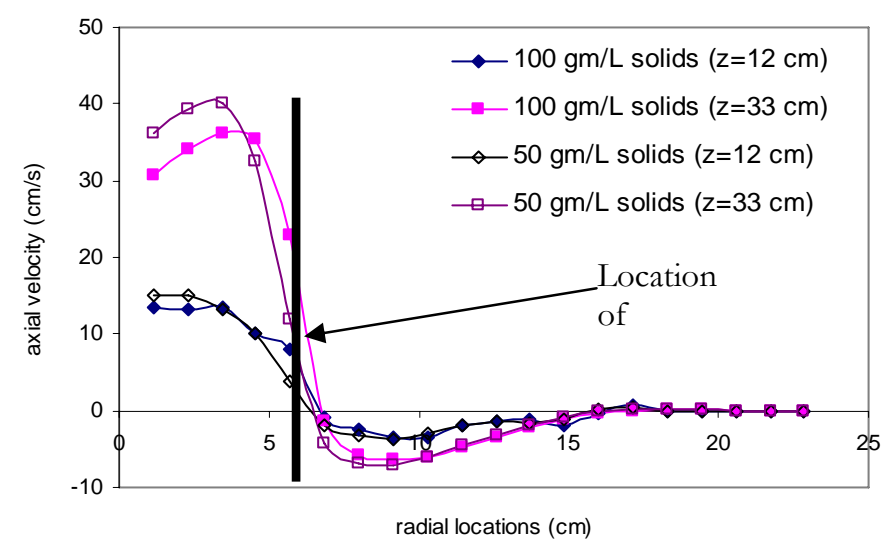

Figure 4.26 Effect of solids concentration on the axial liquid velocity at the middle height of draft tube at different gas flow rates (experiment P1 and P10)

\section{Volume of Dead Zones or Stagnant Regions}

The different configurations of digester can be compared qualitatively, for the reasons mentioned earlier in section 4.3.1, in terms of dead volume. Just like laboratory-scale, for pilot-scale also the dead zone volume was evaluated by locating the cells with very low velocities (some arbitrary low value, $1 \mathrm{~cm} / \mathrm{sec}$ was used here), and summing up the 
volumes of those cells. Table 4.4 shows the dead zone volumes for different digester configurations. Table 4.4 shows that the dead zone volume decreases upon increasing the gas flow rate for all the configurations. For $D / T$ of 0.25 the higher liquid velocities exist only inside the riser, since decrease in dead zone volume with increasing gas flow rate does not indicate more homogeneity in this case, increased flow rate is not advantageous. The dead zone volume increases with increase in the $D / T$ ratio. The values in the Table 4.4 show that the sparger type and slurry solids concentration does not have an appreciable effect on the dead zone volume, for $D / T$ ratio of 0.25

Table 4.4 Dead or stagnant volumes for pilot-scale digester configurations

\begin{tabular}{|c|c|c|c|c|c|}
\hline $\begin{array}{c}\text { Expt } \\
\text { no. }\end{array}$ & $\begin{array}{c}\text { Gas flow } \\
\text { rate } \\
(\mathrm{lpm})\end{array}$ & $\begin{array}{c}\text { Total solids } \\
\text { in the slurry } \\
(\mathrm{g} / \mathrm{l})\end{array}$ & $\begin{array}{c}D / T \\
\text { ratio }\end{array}$ & $\begin{array}{c}\text { Sparger } \\
\text { geometry }\end{array}$ & $\begin{array}{c}\% \text { Dead } \\
\text { volume }\end{array}$ \\
\hline P1 & 4.5 & 100 & 0.25 & cross & 61 \\
\hline P2 & 9 & 100 & 0.25 & cross & 55 \\
\hline P3 & 18 & 100 & 0.25 & cross & 50 \\
\hline P4 & 4.5 & 100 & 0.75 & cross & 65 \\
\hline P5 & 9 & 100 & 0.75 & cross & 60 \\
\hline P6 & 18 & 100 & 0.75 & cross & 54 \\
\hline P7 & 4.5 & 100 & 0.25 & Single point & 63 \\
\hline P8 & 9 & 100 & 0.25 & Single point & 58 \\
\hline P9 & 18 & 100 & 0.25 & Single point & 53 \\
\hline P10 & 9 & 50 & 0.25 & cross & 58 \\
\hline
\end{tabular}

Figure 4.27 shows the map of dead zones in pilot scale configuration P2 and P5. Again the dead zones are located neat the bottom and towards the wall of the digester and can be easily located from the flow pattern of digester. 


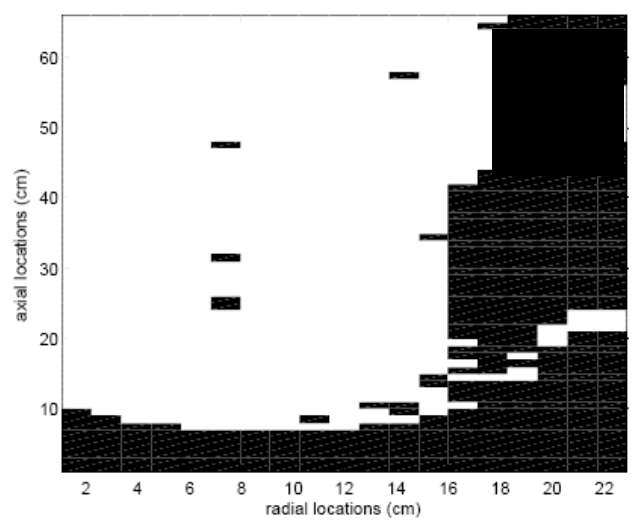

(a)

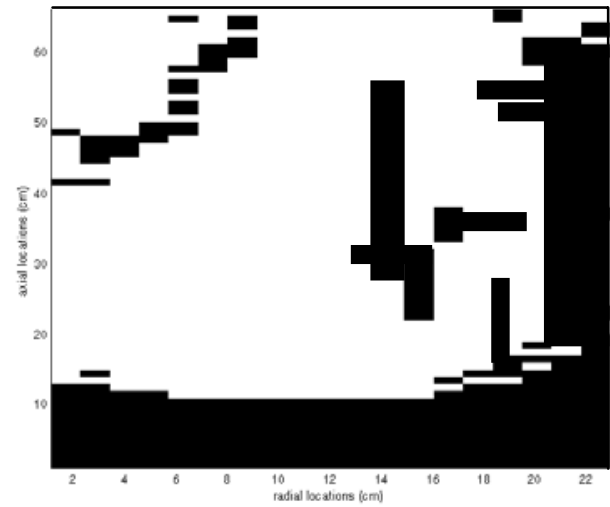

(b)

Figure 4.27 Dead zone map for Experiment (a) P2 and (b) P5 (dark spaces are the dead zones)

\section{Eddy Diffusivity}

The importance and role of eddy diffusivities and the calculations are discussed in the laboratory-scale section 4.3.1. The radial and axial eddy diffusivities calculated for experiment P1, P2 and P3 at the middle height of the tank are shown in Figure 4.28. The radial diffusivities are very small as compared to axial diffusivities for all gas flow rates and can be neglected. The distribution of axial diffusivity for experiment P2 and P5 is shown in Figure 4.29. The diffusivities are very low inside and near the dead zones, as observed for laboratory-scale configuration. The effect of gas flow rate on axial diffusivities for different configurations is show in Figure 4.30a and 4.30b. The effect of gas flow rate on axial diffusivities is not very clear. The axial diffusivities for different configurations at gas flow rate of $9 \mathrm{lpm}$ are shown in Figure 4.31. The diffusivities are higher for $D / T$ ratio of 0.75 in major portion of the digester as compared to $D / T$ of 0.25 . The axial diffusivities are the lowest for the single point sparger. Solids concentration in the slurry has no effect on the axial diffusivities. 


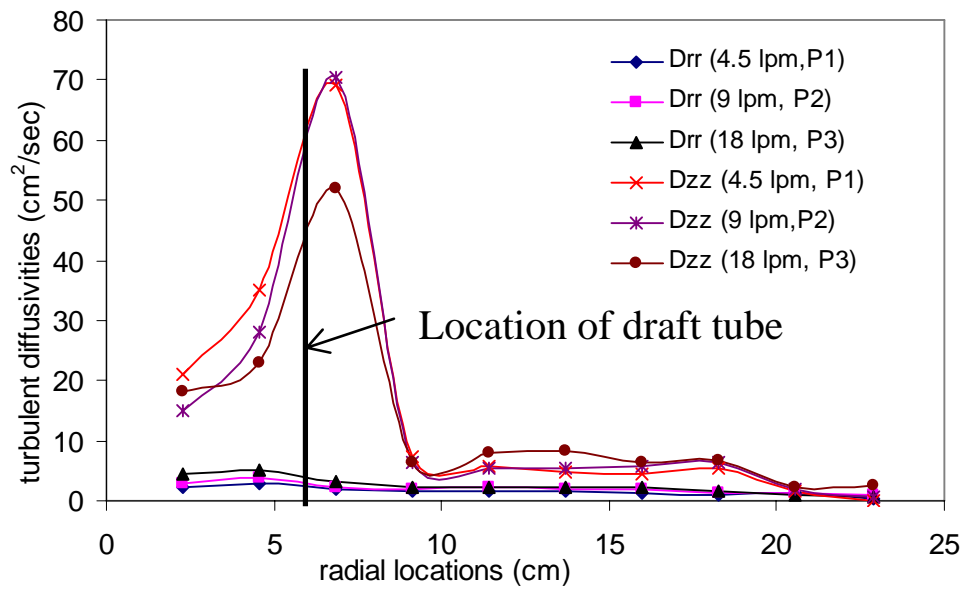

Figure 4.28 Axial and radial eddy diffusivities at the middle height of the tank for experiment P1, P2 and P3

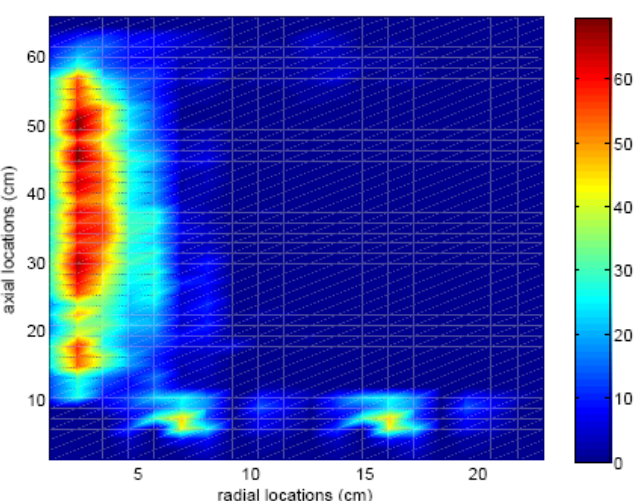

(a)

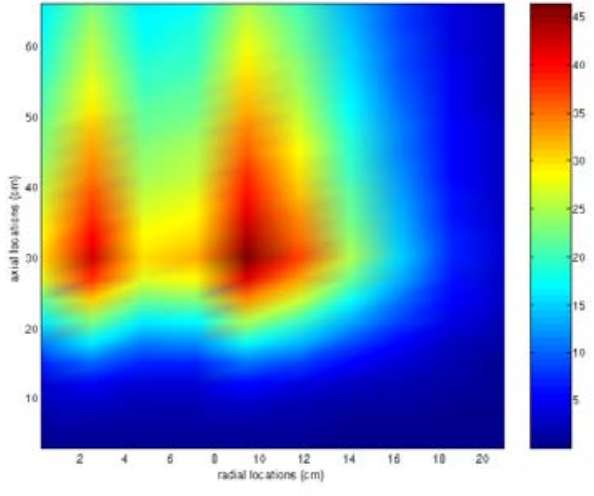

(b)

Figure 4.29 Axial diffusivity map for experiment (a) P2 and (b) P5 


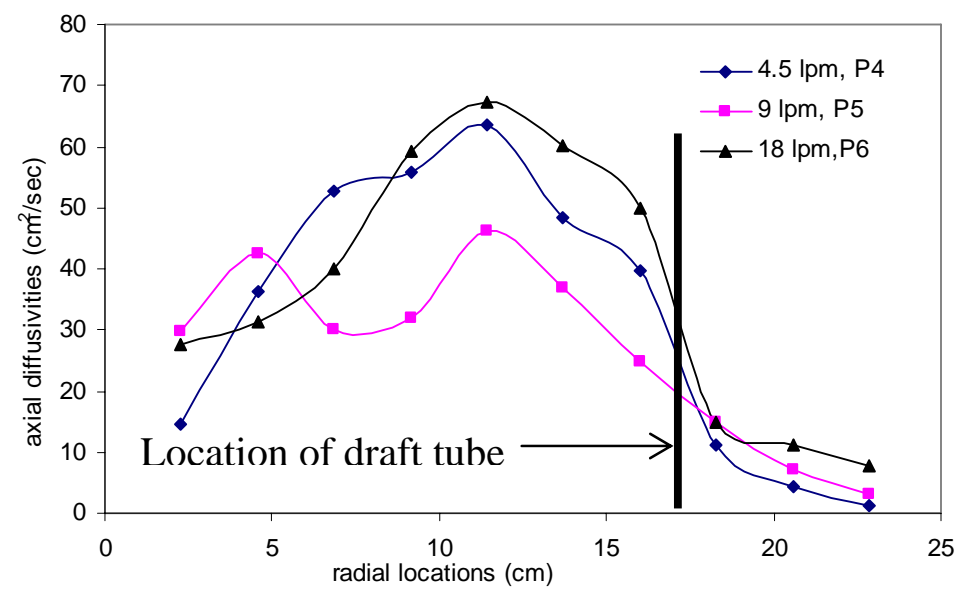

Figure 4.30a Effect of gas flow rate on turbulent axial turbulent diffusivities for configurations with $D / T$ of 0.75 and cross sparger

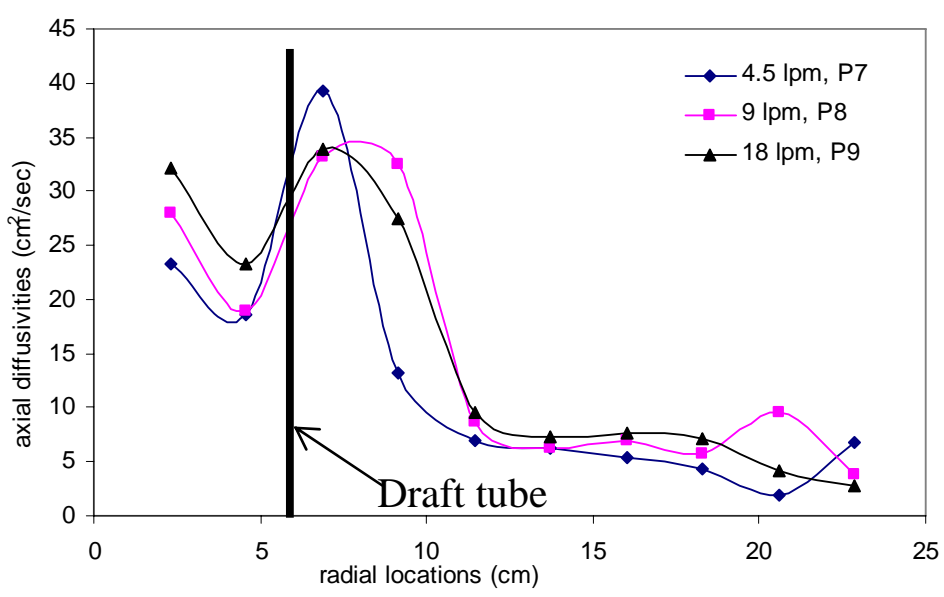

Figure 4.30b Effect of gas flow rate on axial turbulent eddy diffusivities for configurations with $D / T$ of 0.25 and single point sparger 


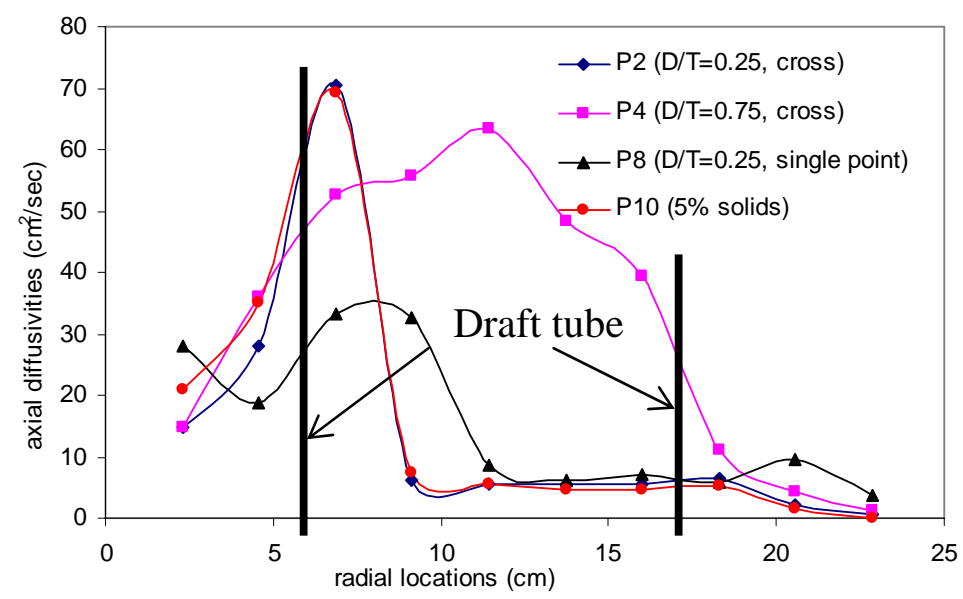

Figure 4.31 Comparison of axial turbulent eddy diffusivities for different digester configurations at gas flow rate of $9 \mathrm{lpm}$

\subsubsection{Effect of Scale}

The results of laboratory scale and pilot scale CARPT studies were compared to investigate the effect of scale. The working volume of laboratory scale unit was $3.78 \mathrm{~L}$ whereas for pilot scale it was $97 \mathrm{~L}$, thus a volumetric scale up factor of approximately 25 was employed. Both the units were geometrically similar; the diameter of pilot unit was three times the diameter of laboratory unit. The gas flow rate of $1 \mathrm{lpm}$ in small scale and $9 \mathrm{lpm}$ in pilot scale corresponds to same superficial gas velocity of $0.91 \mathrm{~mm} / \mathrm{sec}$ based on tank diameter $(14.6 \mathrm{~mm} / \mathrm{sec}$ based on draft tube diameter for $D / T$ ratio of 0.25 and $1.6 \mathrm{~mm} / \mathrm{sec}$ for $D / T$ of 0.75$)$.

Thus the laboratory-scale and pilot-scale configurations with cross sparger for $D / T$ of 0.25 and 0.75 with $10 \%$ waste and operating with superficial gas velocity if $0.91 \mathrm{~mm} / \mathrm{sec}$ can be compared to evaluate the effect of scale. Configurations with single point sparger for $D / T$ of 0.25 containing slurry with $10 \%$ solids can also be compared; see Table 4.5. While the percentage dead volumes are similar for two scales, the actual volume dead zones in pilot-scale is 25 times larger than in the laboratory-scale. 
Table 4.5 Effect of scale on dead or stagnant volumes for different digester configurations

\begin{tabular}{|c|c|c|c|}
\hline \multirow[b]{2}{*}{ sparger } & \multirow[b]{2}{*}{$D / T$ ratio } & \multicolumn{2}{|c|}{$\%$ Dead volume } \\
\hline & & $\begin{array}{l}\text { Laboratory- } \\
\text { scale } \\
1 \mathrm{lpm}\end{array}$ & $\begin{array}{l}\text { Pilot-scale } \\
9 \mathrm{lpm}\end{array}$ \\
\hline Cross sparger & 0.25 & 50 (L1) & $55(\mathrm{P} 2)$ \\
\hline Cross sparger & 0.75 & $60(\mathrm{~L} 4)$ & 65 (P5) \\
\hline Single point & 0.25 & 55 (L5) & 58 (P8) \\
\hline
\end{tabular}

Comparison of flow patterns of configurations with cross sparger with $D / T$ of 0.25 and superficial gas velocity of $0.91 \mathrm{~mm} / \mathrm{sec}$ shows that the flow patterns are qualitatively similar. Liquid axial velocity profiles can be compared for quantitative analysis. To facilitate the comparison, the axial liquid velocity is made dimensionless. Axial velocities of laboratory-scale at middle height of the $\operatorname{tank}(\mathrm{z}=11 \mathrm{~cm})$ are divided by the maximum liquid velocity for laboratory-scale at the middle height of the tank to obtain dimensionless velocity. Similarly, axial velocities for pilot-scale at middle height of the $\operatorname{tank}(z=33 \mathrm{~cm})$ are divided by the maximum liquid velocity for pilot-scale at the middle height of the tank to obtain dimensionless velocity. Figure 4.32 shows the comparison of dimensionless axial liquid velocity profile.

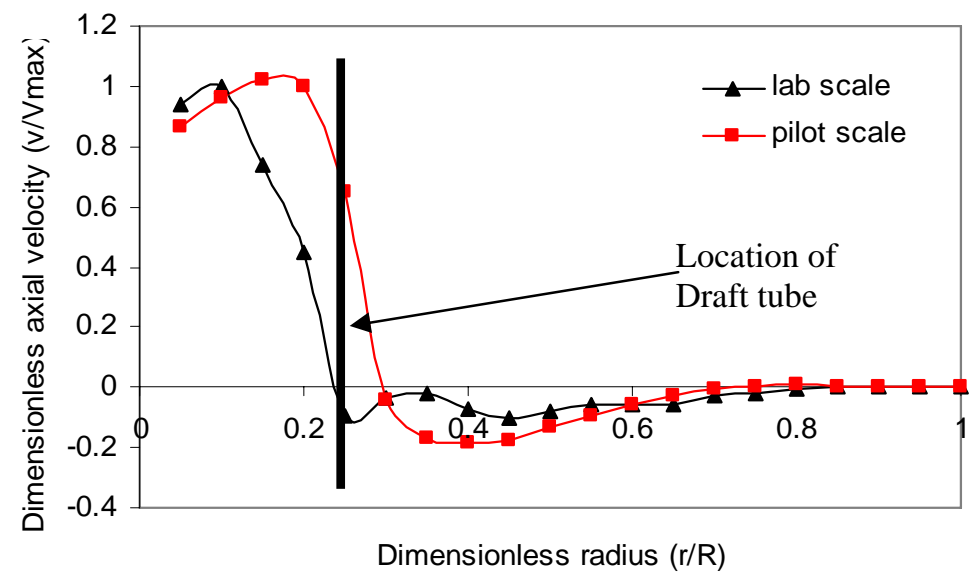

Figure 4.32 Comparison of axial liquid velocity radial profile for laboratory scale and pilot scale digesters (experiment L1 and P2), $D / T=0.25$, cross sparger, $10 \%$ slurry and superficial gas velocity of $0.91 \mathrm{~mm} / \mathrm{sec}$. 
The velocity profile has the same shape and the dimensionless velocities are also comparable. Not only the velocity profiles and holdup profiles of two scales should overlap each other for the two scales to be hydrodynamically similar, but their magnitudes of velocities should also match (elaborated further in the following discussion). Gas holdup profiles will be obtained as a part of another doctoral dissertation by Rajneesh Varma. The velocity profile for pilot scale is shifted to the right (the velocity should be zero at the wall of draft tube, $r / R=0.25$ ), this would be attributed to the error in the CARPT measurement. The error for laboratory-scale velocity profile is negligible but not for pilot-scale. Larger scale of pilot unit causes more error in reconstruction because of coarser calibration grid and more attenuation of the radiation signal in the system. Thus, if there was no error, then the shape of velocity profiles of both the scales would be postulated to match with each other for $D / T$ of 0.25. This needs to be further evaluated for further investigations.

Comparison of flow patterns of configurations with single point sparger with $D / T$ of 0.25 and superficial gas velocity of $0.91 \mathrm{~mm} / \mathrm{sec}$ also shows that the flow patterns are qualitatively similar. The dimensionless velocity profile of both scales matches well, Figure 4.33. The geometry of the laboratory-scale digester with single point sparger did not allow acquiring enough calibration points inside and near the draft tube. Therefore, the error in the reconstruction in this configuration would be more than the other laboratory-scale configurations. Hence, the zero velocity at the draft tube wall is observed at $r / R$ of 0.3 instead of 0.25 . 


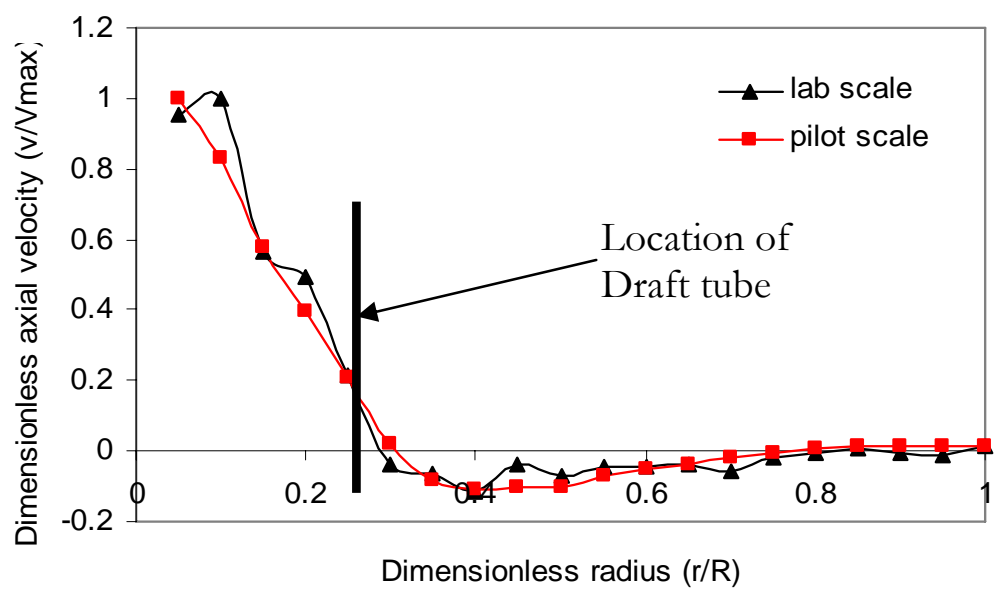

Figure 4.33 Comparison of axial liquid velocity radial profile for laboratory scale and pilot scale digesters (experiment L5 and P8), $D / T=0.25$, single point sparger, $10 \%$ slurry and superficial gas velocity of $0.91 \mathrm{~mm} / \mathrm{sec}$.

Unlike for $D / T$ of 0.25 , the flow patterns are quite different for $D / T$ of 0.75 . The laboratory-scale digester has only one circulation loop inside the draft tube, with liquid moving down in the center and up towards the wall. For pilot-scale, the circulation pattern is more complex. The liquid moves downwards in the center in upper part of the riser in a smaller independent circulation loop, while the liquid is directed upwards in the center of the riser at the bottom. The dimensionless liquid velocity profiles are compared in Figure 4.34; the profiles are completely different for two scales. This is in accordance with the observations of Blazej et al. (2004), Gavrilescu and Tudose (1998) and Heijnen et al. (1997). 


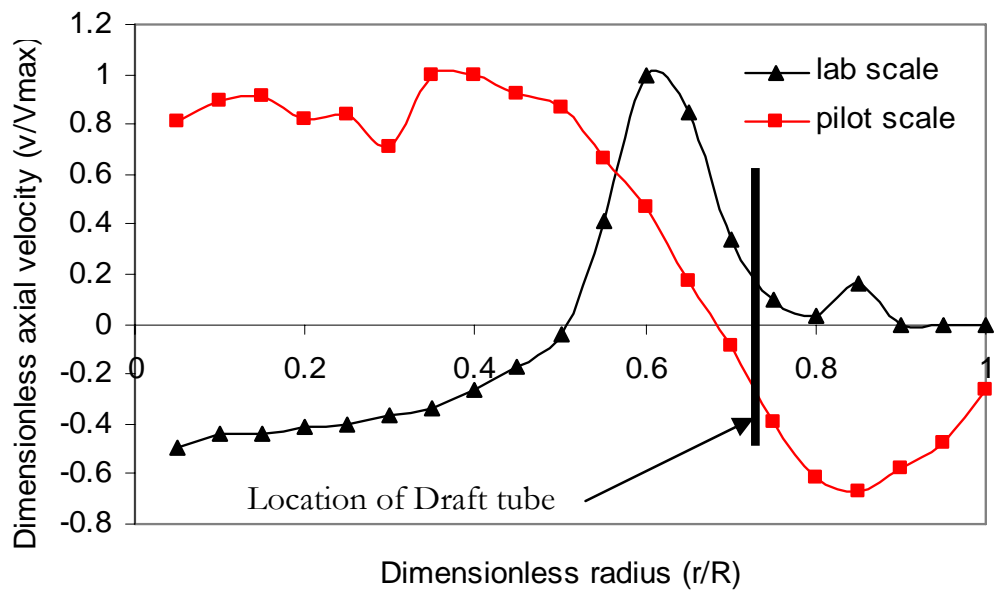

Figure 4.34 Comparison of axial liquid velocity radial profile for laboratory scale and pilot scale digesters, $D / T=0.75$, cross sparger, $10 \%$ slurry and superficial gas velocity of $0.91 \mathrm{~mm} / \mathrm{sec}$, (experiment L4 and P5).

Figure $4.35 \mathrm{a}$ and $4.35 \mathrm{~b}$ explain the true effect of scale on the axial liquid velocity for experiment L1/P2 and L5/P8, respectively. Since the flow pattern of experiment L4 and P5 are completely different, their axial velocity magnitudes are not compared. In Figure $4.35 \mathrm{a}$ and $4.35 \mathrm{~b}$, the magnitude of axial velocity is compared rather than dimensionless velocities. The liquid velocities in pilot-scale are about 3 to 4 times higher as compared to laboratory-scale in the riser. The increase liquid velocity is not so significant in downcomer. This has been observed experimentally by Blazej et al. (2004) and Gavrilescu and Tudose (1998). 


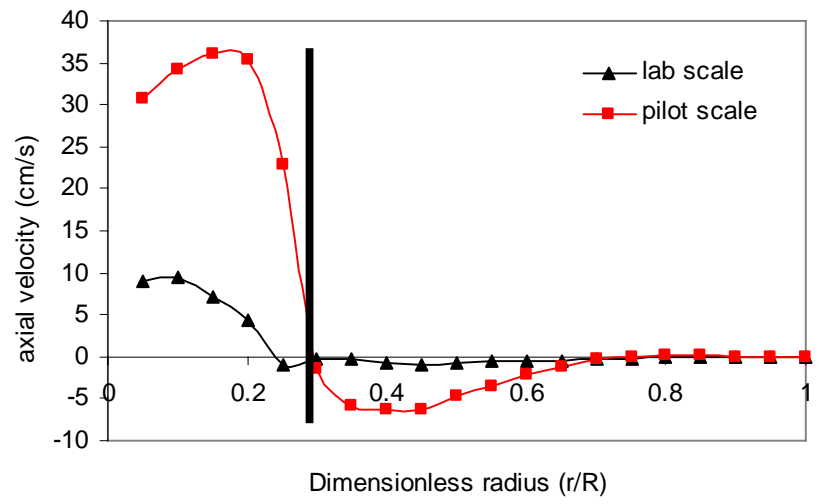

Figure 4.35a Comparison of magnitudes of axial liquid velocity for laboratory scale and pilot scale digesters (experiment L1 and P2), $D / T=0.25$, cross sparger, $10 \%$ slurry and superficial gas velocity of $0.91 \mathrm{~mm} / \mathrm{sec}$.

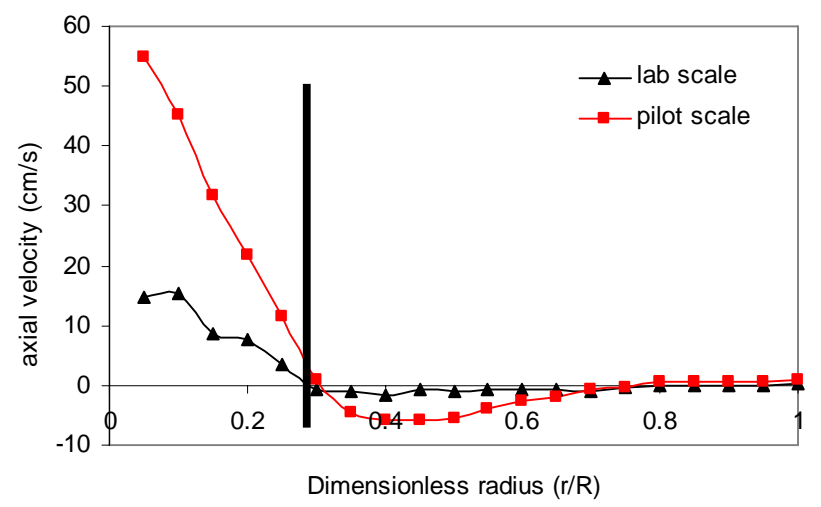

Figure 4.35b Comparison of magnitudes of axial liquid velocity for laboratory scale and pilot scale digesters (experiment L5 and P8), $D / T=0.25$, single point sparger, $10 \%$ slurry and superficial gas velocity of $0.91 \mathrm{~mm} / \mathrm{sec}$.

Blazej et al. (2004) and Gavrilescu and Tudose (1998) observed increase in liquid circulation velocity and decrease in gas hold up with increasing scale. The frictional losses of the liquid phase encountered in pilot-scale are much lower as compared to small-scale. A significant amount of frictional loss is due to the wall friction, tank walls and the draft tube walls. The surface area of a cylinder per unit volume is inversely proportional to its diameter. Thus the frictional losses per unit volume of liquid in larger 
reactors are much less as compared to smaller reactors. These reduced frictional losses in pilot-scale result in increased liquid circulation as compared to laboratory-scale digesters. Blazej et al. (2004) evaluated the frictional loss constants in IGLR using empirical correlations and hydrodynamic model of Heijnen et al. (1997) and proved that the frictional loss constant (proportional to frictional loss) decreases with increasing scale of reactor (from 10.5 liters to 200 liters).

Mixing and hydrodynamics can be characterized by dead zone volume and turbulent eddy diffusivity together. Table 4.5 shows the effect of scale on dead zone volume. For all three cases, even though the liquid velocities in pilot scale are significantly higher than in laboratory-scale, pilot-scale configuration had significantly higher dead zone volume (25 times larger) and larger size of dead zones than the laboratory-scale configurations. The axial diffusivities in pilot-scale are slightly higher than the laboratory-scale. But the diffusivities in the dead zones are very low for both digester scales. This means that the diffusion or dispersion of material (substrate or intermediates of digestion reaction such as fatty acids) is much slower and poor in pilotscale digester. Due to smaller size of laboratory-scale digester (and hence smaller size of their dead zones) diffusion to/from the center of dead zone in smaller reactor may be sufficient to maintain significant activity, while that in the larger reactor may not be sufficient. Higher dead zone volume and low axial diffusivities in the pilot-scale digester can explain its poor performance than the identically operated laboratory-scale digester (Appendix A)

The increase in liquid velocities with scale is significant in riser but insignificant in downcomer. As pointed out before dead zones are observed in downcomer section due to low liquid velocities. Higher liquid velocities only in the riser does not help to reduce to dead zone volume in pilot-scale. Probably for a scale-up ratio of 25, only 3-4 times increase in liquid velocities in a small section of pilot-scale reactor is not enough to achieve the same mixing intensity that was obtained in small-scale reactors. 
Small-scale configurations show better mixing characteristics as compared to large-scale units. Small-scale units are well mixed due to their small size and can be considered to be perfectly or ideally mixed (Bello-Mendoza and Sharratt, 1998; Nielsen and Villadsen, 1992). Schlattmann et al. (2004) studied four different scales of digesters ranging from 2 $\mathrm{L}$ to $900 \mathrm{~m}^{3}$ and concluded that the process efficiency of small scale reactors is higher than that for large scale reactors. Residence time distribution studies conducted by Monteith and Stephenson (1981) found only 23\% of the reactor volume actively mixed in a large scale digester, indicating inefficient mixing and larger mixing time constants. Performance studies carried out in laboratory-scale and pilot-scale anaerobic digesters (Appendix A) showed that laboratory-scale digester produced more amount of better quality biogas as compared to pilot-scale digester. This may be due to the better mixing performance of laboratory-scale digester as compared to pilot-scale digester, as observed here.

Next question arises, what scale-up criteria should be used to obtain similar performance at different scales of reactor? Geometric similarity is essential but that alone does not guarantee the same hydrodynamic performance. Same superficial gas velocity (power dissipation per unit volume) was used as scale-up criteria in this study. Obviously, same superficial gas velocity did not provide same flow patterns or liquid velocity profiles in geometric similar configurations of experiment L4 and P5. Moreover, sparger geometry did not affect the hydrodynamics with $D / T$ of 0.25 significantly. But sparger geometry had an appreciable effect on the liquid velocities, dead zone volumes, and mean circulation times for pilot-scale configuration with $D / T$ of 0.25 . These observations suggest that the superficial gas velocity (energy input per unit volume) may not be the correct scale-up criteria to obtain similar flow patterns in gas recirculation type reactors. Hence, further investigations are needed to possibly answer above questions to identify the reliable criteria for hydrodynamics and performance similarity in different scales under same conditions of anaerobic digesters. 


\subsection{Summary}

The flow pattern and liquid velocity profiles were obtained in a laboratory-scale and pilot-scale gaslift digester operating in bubbly flow regime. Effect of geometry and operating variables was studied on the liquid velocity, dead zone volume, mean circulation time, and turbulence parameters.

Increased gas flow rate increases the liquid velocity; decreases mean circulation time for all configurations at both the scales, but does not offer any advantage of lowering the dead zone volume significantly. The larger draft tube diameters (with $D / T$ of 0.5 or 0.75) reduce the dead zones and produce relatively homogenous mixing throughout the digester volume. Digester with $D / T$ of 0.5 has minimum percentage of dead volume for laboratory-scale digester. For pilot-scale digester lowest percentage of dead volume was obtained for $D / T$ ratio of 0.75 at highest gas flow rate. Mean circulation time increases with increase in $D / T$ ratio. Decreasing solids content in the slurry decreases the mean circulation time.

If different laboratory-scale configurations of digester are compared on the basis of flow pattern, liquid velocities, dead zone volume, then the configuration with $D / T$ ratio of 0.5 with low gas flow rate seems to be best configuration for anaerobic digester operation. Because, it provides good liquid circulation throughout the volume of the digester, lowest volume of dead zones and low mean circulation time. Higher gas flow rates can provide more circulation but they are not desirable from the energy consumption consideration.

Flow pattern, liquid velocity profile, dead zone volume and diffusivities were used to evaluate the effect of scale on the hydrodynamics. Geometric similarity and same gas superficial velocity were used as scale-up criteria in this study. Scale of operation affected the flow pattern and liquid velocity profile significantly for $D / T$ of 0.75 . The 
liquid velocities were significantly higher in pilot-scale in the riser but change was less significant in the downcomer section. Comparing the values of dead zone volume and axial diffusivities to quantify mixing, proved that the mixing performance is better in small-scale digesters as compared to large-scale units in spite of liquid velocities being higher for pilot-scale units. Sparger geometry affected the hydrodynamic performance significantly in pilot-scale but not in laboratory-scale digesters. Thus it can be concluded that only geometric similarity and same gas superficial velocity (energy input per unit volume) does not guarantee similar mixing intensity or hydrodynamic performance at different scales of operation.

Only two $D / T$ ratios were tested in pilot-scale experiments. Thus, the optimum $D / T$ ratio for pilot-scale configuration to provide improved circulation and improved mixing performance was not examined. CFD studies will be carried out for this purpose. The CFD predictions will be first evaluated with CARPT results and then validated CFD code will be used to understand the hydrodynamics of gaslift digesters in detail and to compare additional laboratory-scale and pilot-scale configurations. The CFD studies are presented in Chapter 5. 


\section{Chapter 5}

\section{Computational Fluid Dynamics Studies: Laboratory-scale and Pilot-scale}

\subsection{Introduction and Motivation}

Advanced non-invasive experimental techniques like CARPT and CT help to understand the hydrodynamics in detail but their application is limited by the time and resource constraints. Thus these techniques cannot be used to evaluate the effect of every parameter on the hydrodynamics. This is where Computational Fluid Dynamics (CFD) can help in such evaluation and for design and scale-up once it is validated.

CFD proves to be a valuable and efficient tool to understand and evaluate hydrodynamics of a flow system. For single-phase systems, CFD models and closures are well established and validated with benchmark experimental data, such that CFD can be used with high level of confidence for simulating single-phase systems. However, this is not the case with multiphase systems. The complex flow structure and interactions within different phases in addition to the turbulence makes it very difficult to develop models for multiphase systems that can mimic reality. The closures used for these equations are modeled hypothetically or correlated from experimental data at different conditions thus cannot be universally applied to all cases. Therefore multiphase CFD simulations need to be developed for individual situations and validated against experimental data. Once the CFD results are validated for a particular 
system, CFD can be used to optimize the system by varying parameters and operating conditions to achieve proper design and scale-up.

Considering the need of evaluating the CFD model with proper experimental data, so that CFD can be used in design and scale-up of gaslift digesters, a 3D two-phase CFD model provided by CFX is used and the simulation predictions are compared with the CARPT data. Ability of CFD to account for the effect of geometry, operating conditions and scale is revisited here.

Karim et al. (2004) performed CARPT studies on 8-inch diameter gaslift digester. Thus, preliminary CFD studies were performed by Vesvikar and Al-Dahhan (2005) to simulate the digester used by Karim et al. (2004) in order to evaluate the predictability of CFD simulations. The experimental data for CFD calculation is obtained from CARPT; CARPT studies on two scales of digesters are presented in Chapter 4. This work is an attempt to understand the hydrodynamics of IGLRs with the help of CFD for the configurations and operating conditions of IGLR type anaerobic digester that were not covered by the experiments in Chapter 4 .

\subsection{Digester Geometry}

\subsubsection{Laboratory-scale (6-inch Diameter) Digester}

The geometry of 6-inch laboratory-scale digesters used in the performance studies (Chapter 3) and CARPT studies (Chapter 4) was used in these simulations. The details of the digester geometry are given in Figure 4.1. All the laboratory-scale configurations listed in Table 4.1 were simulated along with additional simulations with different geometrical and operating variables. The 6-inch laboratory-scale simulation details are given in Table 5.1 (Acronym LS in simulation number stands for Laboratory-scale Simulation). The additional simulations were performed after validating the CFD code 
with the CARPT data reported in Chapter 4. See section 4.2.1 of Chapter 4 for details of the experimental conditions and results.

Table 5.1 Simulation details for laboratory-scale (LS) digesters

\begin{tabular}{|c|c|c|c|c|}
\hline Simulation no. & $\begin{array}{c}D / T \\
\text { ratio }\end{array}$ & $\begin{array}{c}\text { Gas flow } \\
\text { rate }(\mathrm{lpm})\end{array}$ & $\begin{array}{c}\text { Gas superficial } \\
\text { velocity }(\mathrm{mm} / \mathrm{sec})\end{array}$ & $\begin{array}{c}\text { Sparger } \\
\text { geometry }\end{array}$ \\
\hline LS1, LS2, LS3 & 0.25 & $1,2,3$ & $0.91,1.82,2.84$ & Cross sparger \\
\hline LS4, LS5, LS6 & 0.5 & $1,2,3$ & $0.91,1.82,2.84$ & Cross sparger \\
\hline LS7, LS8, LS9 & 0.75 & $1,2,3$ & $0.91,1.82,2.84$ & Cross sparger \\
\hline LS10, LS11, LS12 & 0.25 & $1,2,3$ & $0.91,1.82,2.84$ & Single point \\
\hline LS13, LS14, LS15 & 0.5 & $1,2,3$ & $0.91,1.82,2.84$ & Single point \\
\hline LS16, LS17, LS18 & 0.75 & $1,2,3$ & $0.91,1.82,2.84$ & Single point \\
\hline
\end{tabular}

\subsubsection{Pilot-scale (18-inch Diameter) Digester}

The geometry of the 18-inch pilot-scale digester used in the performance studies (Chapter 3) and CARPT studies (Chapter 4) was used in these simulations. The details of the digester geometry are given in Figure 4.3. All the pilot-scale configurations listed in Table 4.2 were simulated along with additional simulations with different geometrical and operating variables. The pilot-scale simulations details are given in Table 5.2 (Acronym PS in simulation number stands for Pilot-scale Simulation). The additional simulations were performed after validating the CFD code with the CARPT data reported in Chapter 4. See section 4.2.2 of Chapter 4 for details of experimental conditions and results.

Table 5.2 Simulation details for pilot-scale (PS) digesters

\begin{tabular}{|c|c|c|c|c|}
\hline Simulation no. & $\begin{array}{c}D / T \\
\text { ratio }\end{array}$ & $\begin{array}{c}\text { Gas flow } \\
\text { rate }(\mathrm{lpm})\end{array}$ & $\begin{array}{c}\text { Gas superficial } \\
\text { velocity }(\mathrm{mm} / \mathrm{sec})\end{array}$ & $\begin{array}{c}\text { Sparger } \\
\text { geometry }\end{array}$ \\
\hline PS1, PS2, PS3 & 0.25 & $4.5,9,18$ & $0.45,0.91,1.82$ & Cross sparger \\
\hline PS4, PS5, PS6 & 0.5 & $4.5,9,18$ & $0.45,0.91,1.82$ & Cross sparger \\
\hline PS7, PS8, PS9 & 0.75 & $4.5,9,18$ & $0.45,0.91,1.82$ & Cross sparger \\
\hline PS10, PS11, PS12 & 0.25 & $4.5,9,18$ & $0.45,0.91,1.82$ & Single point \\
\hline
\end{tabular}




\subsection{Simulation Details}

Density of the $(5 \%$ or $10 \%)$ slurry used in the CARPT experiments was approximately equal to that of the water (see section 4.2.1). The slurry contained microorganism clusters and very small sized solid particles uniformly dispersed in the liquid phase, such that the two-phase solid-liquid slurry can be treated as a single pseudo-homogeneous phase (Klein et al., 2003 and Wen et al., 2003). Oey et al. (2001) also followed the same approach for simulating flow in three-phase IGLR. After obtaining the flow field of pseudo-homogenous liquid phase, Oey et al. (2001) used this flow field to compute the solids distribution inside the mixture by solving a transport equation for the solid volume fraction. This pseudo-two-phase approach is computationally much cheaper than treating the gas, liquid, and solid phases with separate mass and momentum balances and it also circumvents the modeling of even more complicated closure laws. Using this approach, they found that the solids fraction vary locally (in each computational cell) only from $0.81 \%$ to $0.99 \%$. Thus, the pseudo-liquid phase was simulated with physical properties of water. The gas phase was simulated with physical properties of air.

The validated CFD code and closures were then used to simulate different geometries of the digester by varying the draft tube diameter, type of sparger and gas flow rate. Table 5.1 and 5.2 lists the CFD simulations performed with different geometries and operating conditions at different scales.

Three dimensional (3D) steady-state simulations were carried out using CFD software version 5.7 for 6-inch and 18-inch digester. van Baten et al. (2003a) compared 2D simulation results with $3 \mathrm{D}$ simulation results and found that the assumption of $2 \mathrm{D}$ axis symmetry leads to radial profile that have a more parabolic character than that for fully 3D simulations. Mudde and Van Den Akker (2001) also found differences of about $30 \%$ in the liquid circulation velocities calculated by $2 \mathrm{D}$ and $3 \mathrm{D}$ simulations. Sokolichin 
et al. (2004) observed that 3D models are able to capture important flow and mixing characteristics. They also observed that 3D dynamic simulations do not require an adjustment through additional lift forces, generally needed in 2D simulations to match the experimental results. Mudde and Van Den Akker (2001) suggested this difference in $2 \mathrm{D}$ and $3 \mathrm{D}$ simulations results is due to the lower friction in $2 \mathrm{D}$ simulations because of absence of front and back wall of the reactor.

The governing equations used in the simulations are shown by Vesvikar and Al-Dahhan (2005). Only drag force term was considered to account for interphase forces, as drag force is dominant as compared to other interphase forces (Kuipers and Swaaij, 1998; Oey et al. 2003; Rafique, et al. 2003; Ranade, 2002). Drag force was modeled with Grace drag model. Preliminary simulations were carried out by Vesvikar and Al-Dahhan using different bubble diameters ranging from 2 to $12 \mathrm{~mm}$, but the change in diameter did not affect their results significantly. The same observation was made by van Baten, et al. (2003) and Sokolichin, et al. (2004). The explanation for the lack of dependence on bubble diameter is that the bubble rise velocity is practically independent of bubble diameter in the range of 3-10 $\mathrm{mm}$. Thus bubble diameter of $10 \mathrm{~mm}$ was chosen for the results reported here.

There is a possibility of coalescence of the bubbles generated by the pipe sparger. But the high liquid circulation velocity and low gas fraction in the draft tube maintains high bubble-bubble distance and reduces the bubble coalescence. This was also visually during the experiments.

An Eulerian-Eulerian approach was used to simulate the flow in a three-phase IGLR type anaerobic digesters at two scales. Eulerian method is less computationally intensive as compared to Langrangian-Langrangian approach, especially when the void fraction of dispersed phase is high (Oey et al., 2001 and Sokolichin and Eigenberger, 1994). Bagatin et al. (1999), Mudde and Van Den Akker (2001), Oey et al. (2001), van Baten et al. (2003a), and many others used this approach for simulation of three-phase IGLR. 
Psuedo-liquid phase was modeled as continuous phase using k- $\varepsilon$ (k-epsilon) model, where as gas phase was modeled as dispersed phase using zero equation model (see Vesvikar and Al-Dahhan, 2005). The inlet boundary condition for air was provided by specifying the inlet air velocity at the sparger hole. The outlet boundary condition was the degassing condition for air phase at the surface of water. A no-slip boundary condition was used for air at all the wall boundaries and free-slip boundary condition was applied for air-phase at the draft tube wall.

The mesh was prepared in two stages: a surface mesh of triangular elements is generated and then the volume mesh of tetrahedral elements is generated from the surface mesh. The mesh generated was non uniform. The mesh in the center (i.e. in the region of draft tube) was finer as compared to the region outside the draft tube. Mesh refinement was carried out until a mesh independent solution was obtained as discussed later.

The Finite volume method was used as the numerical technique. The momentum and continuity equations were discretized using finite differences. A first order upwind scheme was used for convective terms. The simulation results are discussed below.

\subsection{Simulation Results}

\subsubsection{Laboratory-scale (6-inch Diameter Digester)}

\section{Simulation Results and Comparison with CARPT Data}

Figure 5.1a shows the flow pattern obtained from 3D CFD simulation for 6-inch laboratory-scale (simulation LS1). Flow pattern is obtained from velocity vector plot of azimuthally averaged liquid velocities. The main features of the flow pattern shown in Figure 5.1a are exactly similar to flow pattern of 8-inch digester given by Vesvikar and Al-Dahhan (2005). 


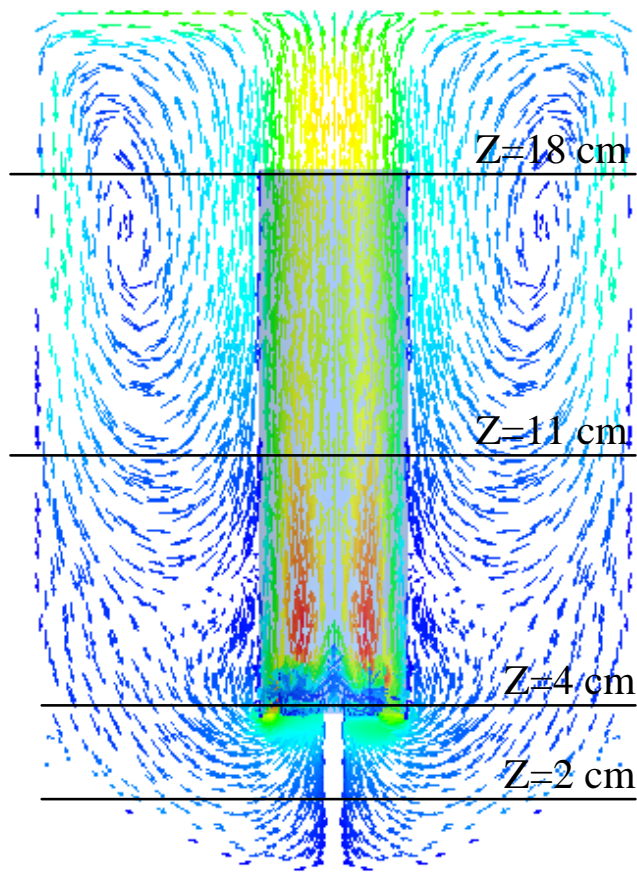

(a)

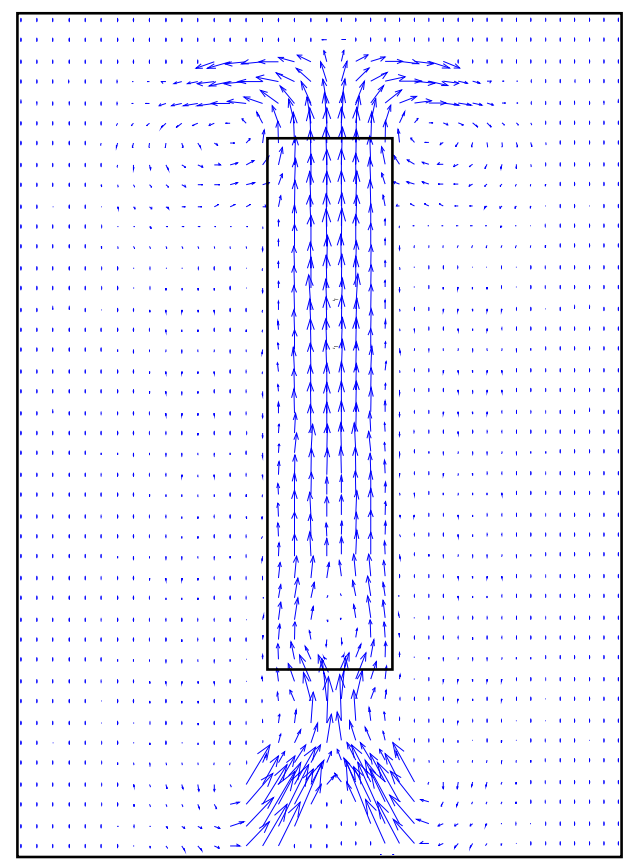

(b)

Figure 5.1 Comparison of (a) simulated flow pattern (LS1) and (b) flow pattern obtained from CARPT (L1) for 6-inch digester

Similar flow pattern was obtained from CARPT results. Simulated flow pattern, Figure 5.1a is compared with experimentally obtained flow pattern, Figure 5.1b. Figure 5.1a and 5.1b, shows good qualitative agreement between the simulation and the experimental results for flow pattern, location of stagnant zones, and circulation loops. The flow pattern obtained agrees with simulation results of Mudde and Van Den Akker (2001), Oey et al. (2001), Oey et al. (2003a), and Svendsen et al. (1992).

Figure 5.2 shows radial profile of azimuthally averaged axial liquid velocities at different axial locations obtained from CFD simulation LS1. The level of $z=2 \mathrm{~cm}$ represents the horizontal level inside the conical bottom region at $2 \mathrm{~cm}$ from the bottom of the digester, similarly $z=4 \mathrm{~cm}$ is just below the draft tube, $z=11 \mathrm{~cm}$ is at the middle height of the draft tube or tank, and $z=18 \mathrm{~cm}$ is just above the draft tube. Figure 5.2 also presents the quantitative comparison of CFD predictions of liquid velocity with the 
CARPT results. The CFD predictions match reasonably with the CARPT experimental data. The trend of velocity profile matches very well with the experimental data, but the values of liquid velocity are over predicted by CFD. The simulated liquid velocities match experimental data better in downcomer section as compared in the riser. This was also reported by Glover et al. (2003) from his 3D simulations. There is still a lot of room for improvement in the CFD predictions. Use of different closures and models for interphase forces should be evaluated for further improvement (Luo, 2005). The contribution of different interphase forces should also be considered to improve the predictability of CFD models. This will be discussed later.

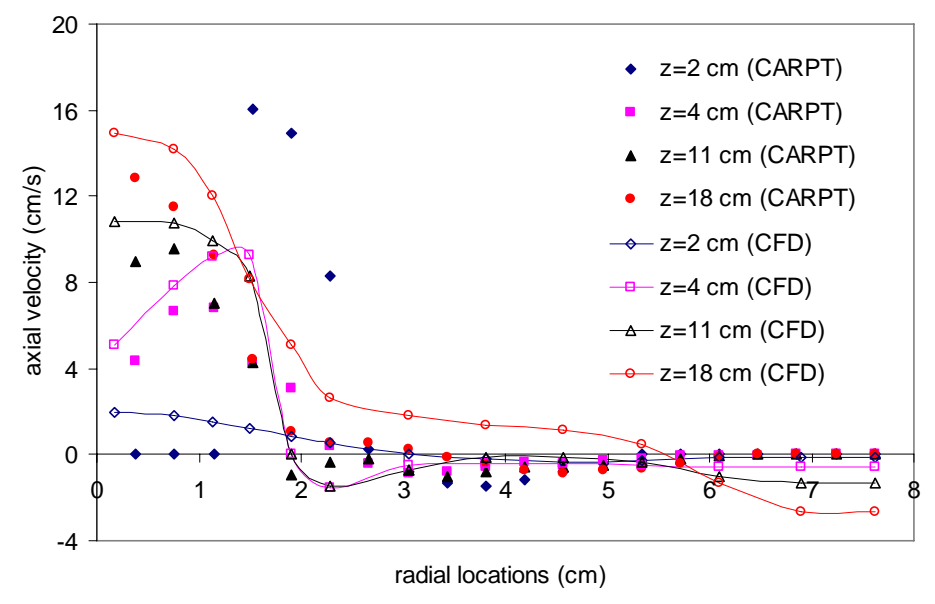

Figure 5.2 Comparison of simulated axial liquid velocity profile for Simulation LS1 with experimental CARPT data (L1)

This level of (dis)agreement between the experimental and CFD simulation results is also reported by Bagatin et al. (1999), Blazej et al. (2004a), Jakobsen et al. (1993), Glover et al. (2003), and Svendsen et al. (1992).

The local gas holdup distribution predicted by CFD for Simulation LS1 is shown in Figure 5.3 (the maximum value of holdup scale is manipulated to improve the readability of plot). The gas is present only in the riser and there is no gas entrainment in the downcomer due to low superficial gas velocity. The gas hold up is maximum at the sparger holes and then gets uniformly distributed in the upper half portion of the 
riser. The experimental data for comparison of predicted gas holdup is not available at this time but will be obtained by CT as a part of other work (by Rajneesh Varma). The overall gas hold up is only $0.06 \%$.
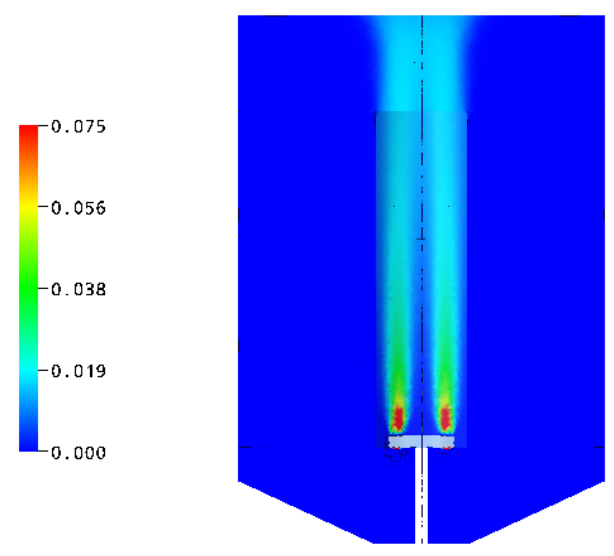

Figure 5.3 Gas hold up distribution predicted by CFD for Simulation LS1

Laboratory-scale Simulations LS3, LS4, LS7, and LS10 were also performed to check the predictability of the CFD simulations. For all these conditions, CFD predictions agree only reasonably with the experimental data on quantitative basis but predict qualitatively the trends of liquid velocity profile very well. The comparison of experimental data and predictions of these simulations is presented in the further discussion.

The dead zone volumes (explained in detail in later section) obtained from CFD simulation results for selected configurations are compared with experimental values in Table 5.3. The agreement between the simulated and experimental values is reasonably accurate. 
Table 5.3 Comparison of dead zone volumes predicted by CFD with experimental data for 6-inch laboratory-scale digester

\begin{tabular}{|c|c|c|c|c|}
\hline \multirow{2}{*}{$\begin{array}{l}\text { Sparger } \\
\text { geometry }\end{array}$} & \multirow{2}{*}{$D / T$} & \multirow{2}{*}{$\begin{array}{c}\text { Gas } \\
\text { superficial } \\
\text { velocity } \\
(\mathrm{mm} / \mathrm{sec})\end{array}$} & \multicolumn{2}{|c|}{$\%$ Dead volume } \\
\hline & & & CFD & CARPT \\
\hline \multirow{9}{*}{$\begin{array}{l}\text { Cross } \\
\text { sparger }\end{array}$} & \multirow{3}{*}{0.25} & 0.91 & 46 (LS1) & 50 (L1) \\
\hline & & 1.82 & 42 (LS2) & - \\
\hline & & 2.74 & 39 (LS3) & 42 (L2) \\
\hline & \multirow{3}{*}{0.5} & 0.91 & 36(LS4) & 30 (L3) \\
\hline & & 1.82 & 30 (LS5) & - \\
\hline & & 2.74 & 25 (LS6) & - \\
\hline & \multirow{3}{*}{0.75} & 0.91 & 52 (LS7) & 60 (L4) \\
\hline & & 1.82 & 48 (LS8) & - \\
\hline & & 2.74 & 42 (LS9) & - \\
\hline \multirow{9}{*}{$\begin{array}{l}\text { Single } \\
\text { point } \\
\text { sparger }\end{array}$} & \multirow{3}{*}{0.25} & 0.91 & $52(\mathrm{LS} 10)$ & 55 (L5) \\
\hline & & 1.82 & 44 (LS11) & - \\
\hline & & 2.74 & 40 (LS12) & - \\
\hline & \multirow{3}{*}{0.5} & 0.91 & 39 (LS13) & - \\
\hline & & 1.82 & 36 (LS14) & - \\
\hline & & 2.74 & 30 (LS15) & - \\
\hline & \multirow{3}{*}{0.75} & 0.91 & 55 (LS16) & - \\
\hline & & 1.82 & 51 (LS17) & - \\
\hline & & 2.74 & 49 (LS18) & - \\
\hline
\end{tabular}

Since the applicability of the CFD simulations to predict the flow pattern and liquid velocities at this scale have been established to match the trend of liquid velocity profiles, these CFD models can be now used to simulate flow in gaslift digesters at other operating conditions listed in Table 5.2 and to evaluate the effect of different operating and geometric variables and the effect of scale.

\section{Effect of Gas (Air) Flow Rate}

Effect of air flow rate for a given $D / T$ ratio and a particular type of sparger can be evaluated using the simulations listed in Table 5.2. The flow pattern remains unaffected by the air flow rate (for all $D / T$ ratios and both sparger geometries), only the magnitude of the liquid velocity changes as discussed below. This was also observed for two different gas flow rates from CARPT experiments in Chapter 4. 
Figure 5.4 shows the effect of air flow rate on the radial profile of circumferentially averaged axial liquid velocity at the middle height of the tank $(z=11 \mathrm{~cm}$, all liquid velocity profiles are reported at the middle height of the tank unless or otherwise mentioned) for configurations equipped with cross sparger and $D / T$ ratio of 0.5 (Simulations LS1/LS2/LS3). The liquid velocity inside the draft tube (riser) increases with the increasing air flow rate but remains unaffected in the downcomer region. The downcomer region is the region with the low velocities and dead zones, whereas there is good circulation inside the riser even at low liquid velocities. Thus increase in air flow rate offers no advantage in increasing the circulation in the downcomer region. Also for $D / T$ ratio of 0.5 and 0.75 , the liquid velocities increased both in riser and downcomer with increasing gas flow rate but the change was marginal as compared to $D / T$ of 0.25 . Configurations with single point sparger also showed same effect of gas flow rate; see Figure 5.5 .

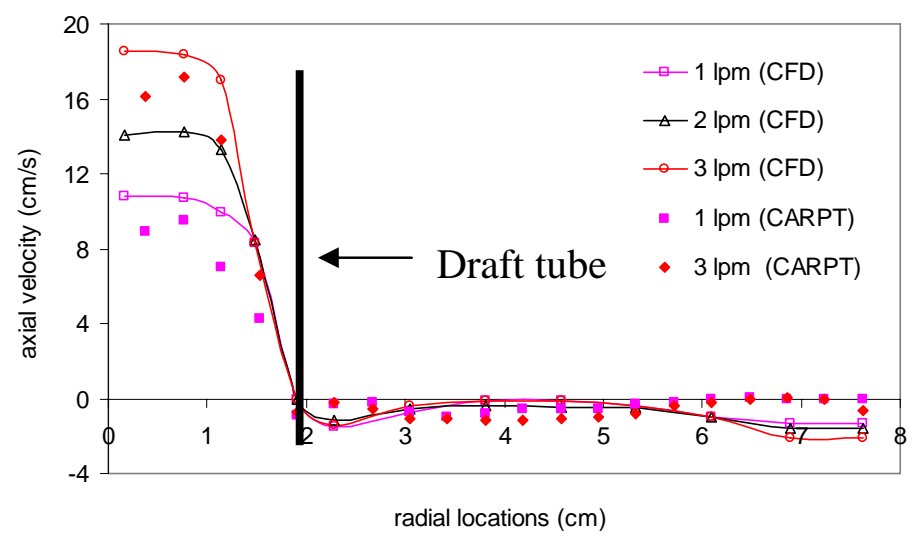

Figure 5.4 Effect of gas flow rate on axial liquid velocity at the middle height of tank for $D / T=0.25$, cross sparger (LS1, LS2 and LS3) 


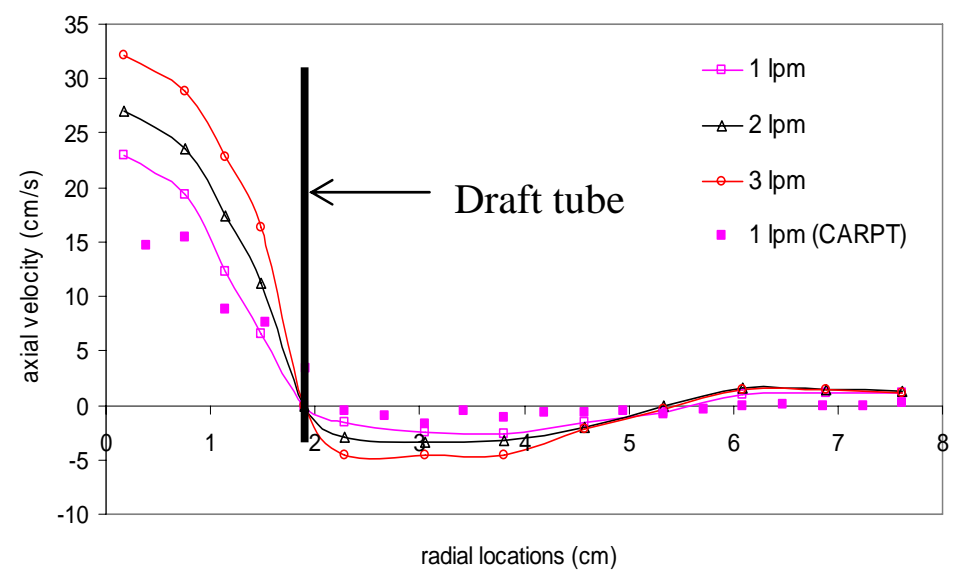

Figure 5.5 Effect of gas flow rate on axial liquid velocity at the middle height of tank for $D / T=0.25$, single point sparger (LS10, LS11 and LS12)

All three gas flow rates correspond to bubbly flow regime or regime one (no gas entrainment in downcomer). Thus the liquid velocity increases with increasing gas flow rate, this has been proven experimentally also by Freitas et al. (1999), Klein et al. (2003), Lu et al. (1995), Sun et al. (2005) and Wen et al. (2005).

The nature of gas distribution remains the same, only the value of gas holdup increases with increasing gas flow rate. The overall gas holdup increases from $0.1 \%$ to $0.16 \%$ as gas flow rate increases from $2 \mathrm{lpm}$ to $3 \mathrm{lpm}$. This increase in gas holdup increases the driving force for liquid circulation. Even at highest gas flow rate of $3 \mathrm{lpm}$ there is no gas entrainment in the downcomer, corresponding to regime one.

\section{Effect of Draft Tube (Riser) Diameter}

Effect of draft tube diameter on the flow can be evaluated by changing the draft tube diameter to tank diameter ratio $(D / T)$ at a given gas flow rate and for a particular type of sparger. Flow pattern at air flow rate of $1 \mathrm{lpm}$, for $D / T$ of 0.25 with cross sparger (simulation LS1) is shown in Figure 5.1a and was discussed earlier. The flow pattern at 1 lpm for $D / T$ ratio of 0.5 and 0.75 (simulation LS4 and LS7) with cross sparger is shown in Figure 5.6a and Figure 5.6b, respectively. Since the flow pattern remains unaffected 
by air flow rate, the flow patterns for higher gas flow rates of 2 and $3 \mathrm{lpm}$ are not shown here.

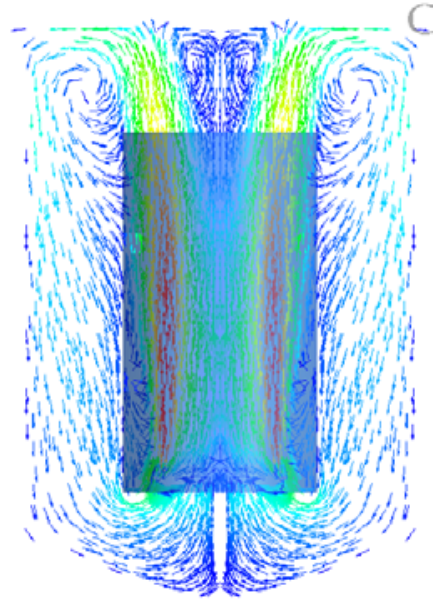

(a)

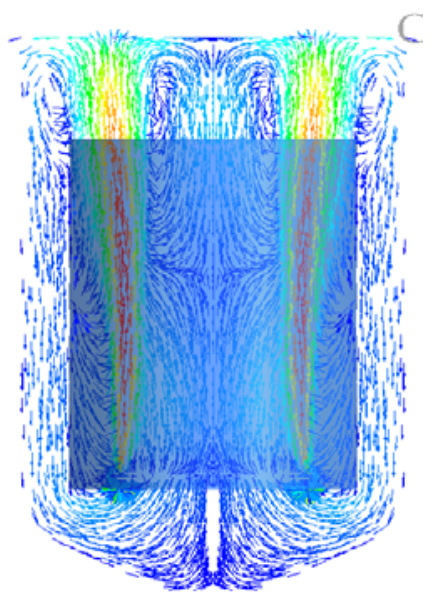

(b)

Figure 5.6 Effect of $D / T$ ratio on flow pattern with cross sparger at $1 \mathrm{lpm}$; (a) simulation LS4, $D / T=0.5$ (b) simulation $\mathrm{LS} 7, D / T=0.75$

The overall flow pattern in digester with larger draft tube diameter is different as compared to the one with smaller draft tube. There is one circulation loop inside the riser in Figure 5.6a and 5.6b, which is absent in digester with smaller draft tube diameter $(D / T=0.25)$, Figure 5.1a. This internal circulation loop inside the riser was also observed by Blazej et al. (2004a). There are two other circulation loops present in Figures 5.6a and 5.6b, one stronger loop at the top of the digester and other weaker loop in the downcomer. For more explanation, see Section 4.3.1 of Chapter 4.

The existence of circulation loops inside the riser can explained by the nature of gas distribution inside the riser, see Figure $5.7 \mathrm{a}$ and $5.7 \mathrm{~b}$. As $D / T$ increases, the distance between the sparger holes also increases. Thus, gas is sparged towards the riser wall, leaving an unsparged region in the center. This nature of gas distribution initiates the circulation loops inside riser. More the distance between the sparged regions, stronger is the circulation loop. 


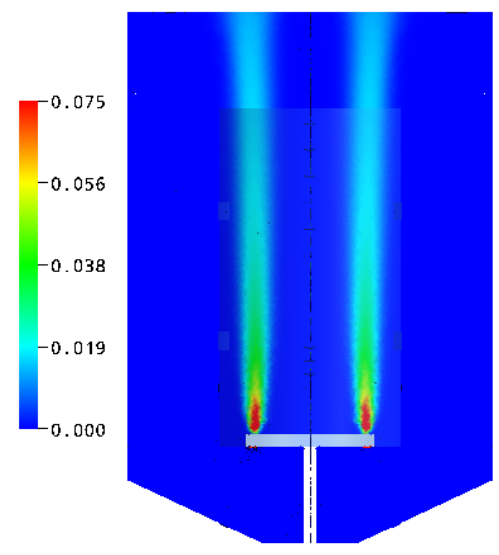

(a)

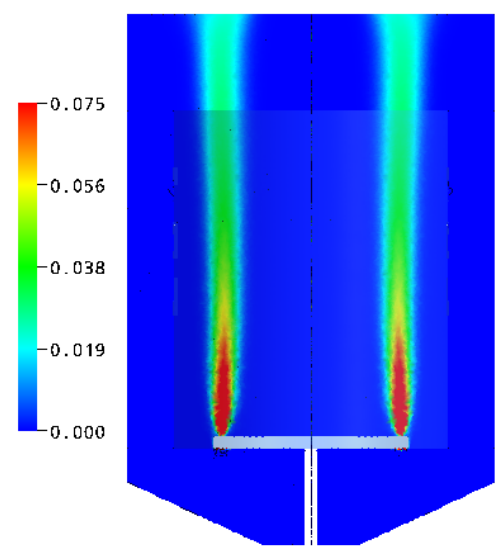

(b)

Figure 5.7 Effect of $D / T$ ratio on gas holdup distribution with cross sparger at $1 \mathrm{lpm}$; (a) simulation $\mathrm{LS} 4, D / T=0.5$ (b) simulation $\mathrm{LS} 7, D / T=0.75$

As the $D / T$ ratio increases, the magnitude of axial velocity inside the riser decreases. This was expected, with same gas flow rate as the area for flow increases with the increasing draft tube diameter the velocity decreases, according to Bernoulli's principle.

Figure $5.8 \mathrm{a}$ to $5.8 \mathrm{c}$ shows the flow pattern at $1 \mathrm{lpm}$ for configuration with single point sparger and $D / T$ ratio of $0.25,0.5$, and 0.75 (simulation LS10, LS13, and LS16), respectively. Changing draft tube diameter does not change the flow pattern appreciably for configurations with single point sparger. 


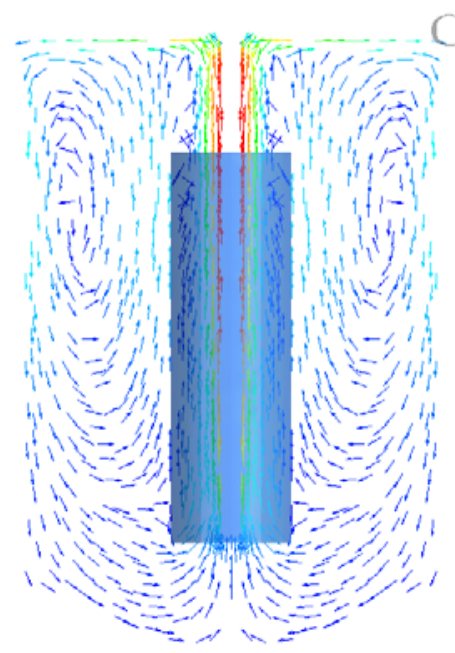

(a)

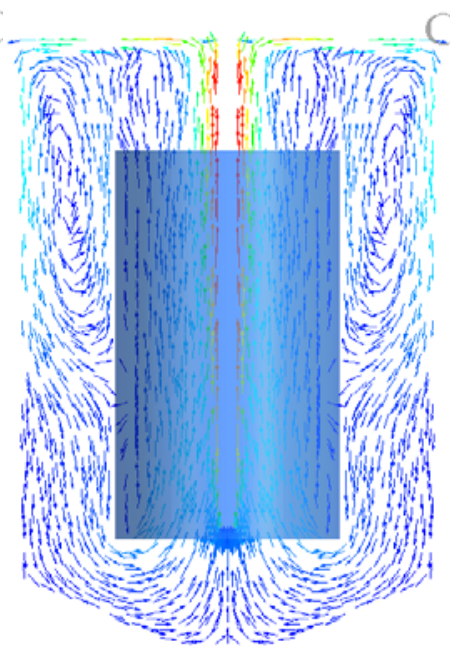

(b)

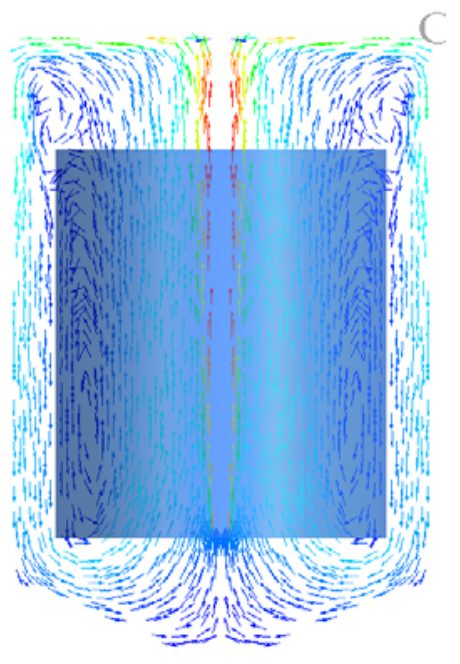

(c)

Figure 5.8 Effect of $D / T$ ratio on flow pattern with single point sparger at 1lpm;
(a) LS10, $D / T=0.25$; (b)
(b) LS13 $D / T=0.5$;
(c) $\mathrm{LS} 16, \mathrm{D} / \mathrm{T}=0.75$

The difference between the flow patterns is mainly due to the different gas distribution. Since, for single point sparger the gas distribution is not affected by the $D / T$ ratio, (see Figure $5.9 \mathrm{a}, 5.9 \mathrm{~b}$ and $5.9 \mathrm{c}$ ) the flow pattern is also not significantly affected by the $D / T$ ratio. Although, as the $D / T$ ratio increases, the unsparged portion of the riser increases and initiates some liquid circulation in the riser due to difference in gas holdup inside the riser at different sections.

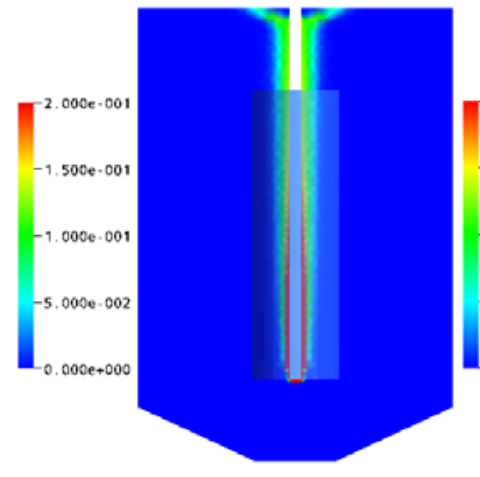

(a)

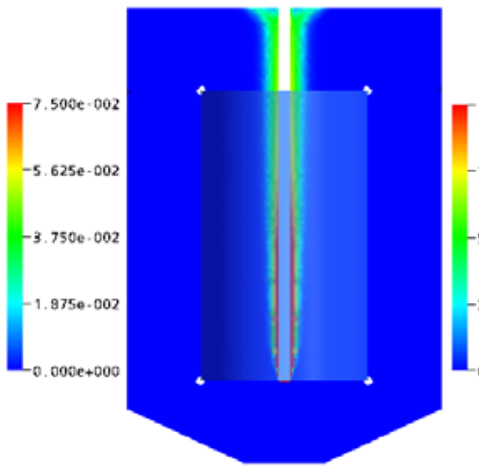

(b)

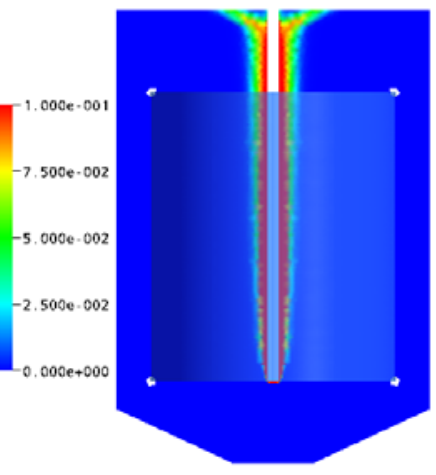

(c)

Figure 5.9 Effect of $D / T$ ratio on gas holdup distribution with single point sparger at 1lpm; (a) LS10, $D / T=0.25$; (b) $\operatorname{LS13} D / T=0.5$; (c) $\operatorname{LS} 16, D / T=0.75$ 
Effect of $D / T$ ratio on the time averaged liquid axial velocity radial profiles at $1 \mathrm{lpm}$ at the middle height of the tank is shown in Figure 5.10a and 5.10b for cross sparger and single point sparger, respectively. In Figure 5.10a for configurations with cross sparger the trend of velocity profiles, as well as the magnitude of liquid velocities are different for different $D / T$ ratio. Whereas, in Figure $5.10 \mathrm{~b}$ for single point sparger, the trend of velocity profiles is same for all $D / T$ ratios. The liquid axial velocities are higher for lower $D / T$ ratios. The negative velocities for $D / T$ of 0.5 and 0.75 in the downcomer, indicates the downward velocities and existence of circulation loop. Draft tube diameter affects the velocity profile for configurations with cross sparger but not with single point sparger. It is due to the nature of gas distribution, which is affected by sparger design.

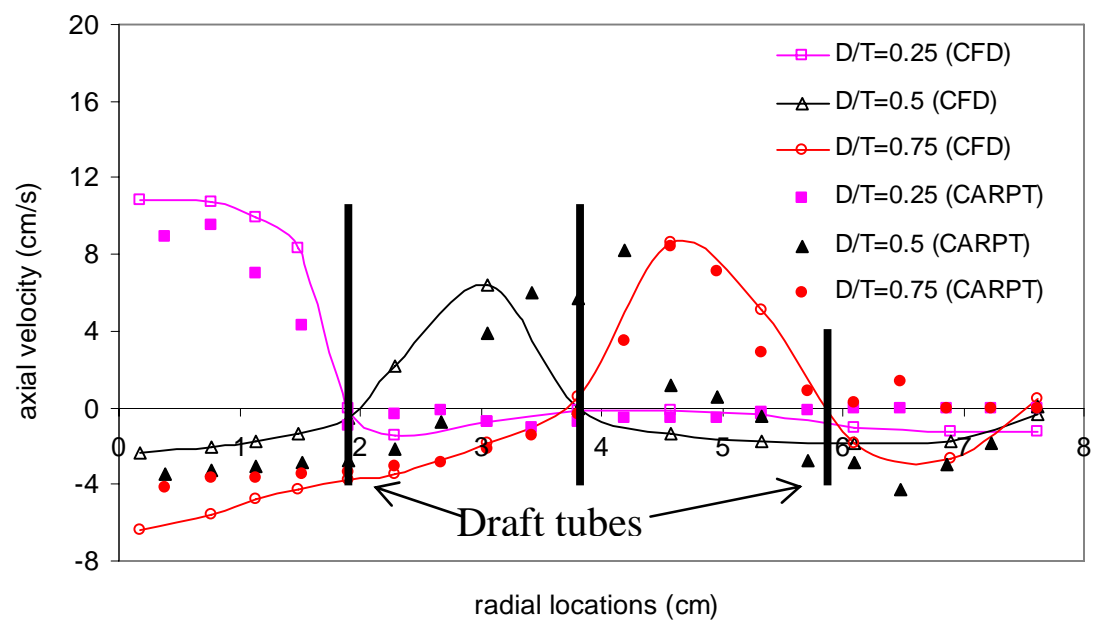

Figure 5.10a Effect of $D / T$ ratio on liquid velocity with cross sparger and $1 \mathrm{lpm}$ gas flow rate (simulation LS1, LS4, and LS7) 


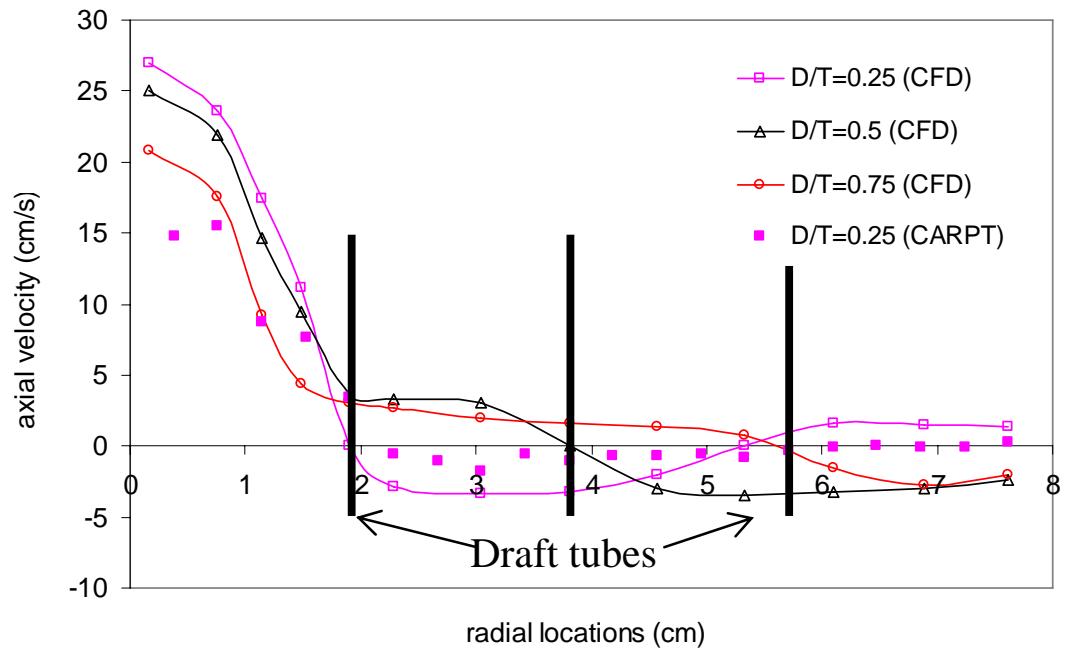

Figure 5.10b Effect of $D / T$ ratio on liquid velocity with single point sparger $1 \mathrm{lpm}$ gas flow rate (simulation LS10, LS13, and LS16)

\section{Effect of Sparger Geometry}

The flow patterns are similar for both sparger designs at $D / T$ ratio of 0.25 . For $D / T$ ratio of 0.5 , (Figure 5.6a and Figure 5.8b), the flow patterns are completely different. This is also true for $D / T$ ratio of 0.75 (Figure 5.6b and Figure 5.8c). Sparger does not affect the flow pattern for smaller $D / T$ ratio of 0.25 but does affect appreciably for higher $D / T$ ratios of 0.5 and 0.75 .

In simulation LS1 to LS3 and LS10 to LS12, the effect of sparger is not very important due to smaller draft tube diameter $(D / T$ ratio of 0.25$)$. Same observations were made by Mudde and Van Den Akker (2001) in their simulation results, and reasons for this behavior are discussed in Chapter 4.

The effect of sparger on the axial liquid velocity profile is shown in Figure 5.11 for different $D / T$ at $1 \mathrm{lpm}$ gas flow rate for laboratory-scale. It can be observed from Figure 5.11, that the high liquid velocities in case of single point sparger are present only in small central core in the riser. In the annular region near the riser wall and in the downcomer, liquid velocities are higher for cross sparger as compared to single point 
sparger. The higher velocities are the result of higher gas flow rate dispersed at one single location by the pipe sparger as opposed to the cross sparger, where only onefourth of given flow rate is dispersed at each of the four sparger holes. Cross sparger creates fairly dispersed gas distribution that also results in higher liquid velocities away from the center of the tank as compared to that with the single point sparger. Becker et al. (1994) observed from his experiments and simulations that multipoint sparger creates higher liquid velocities away from the center of the tank due to homogeneous gas dispersion as compared to pipe sparger.

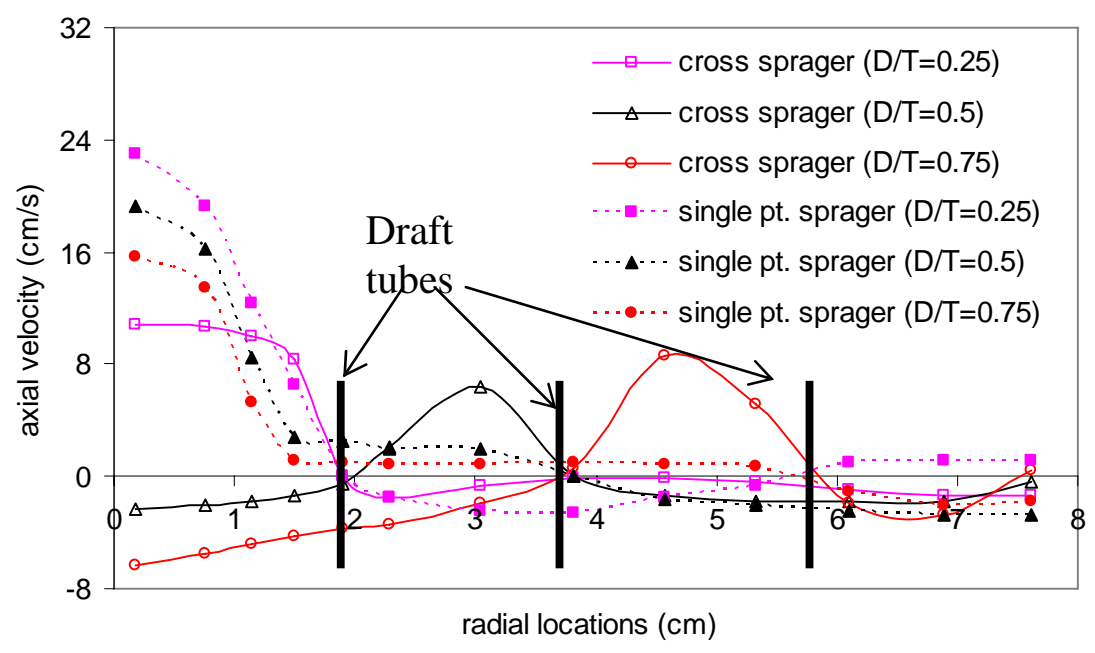

Figure 5.11 Effect of sparger geometry for different $D / T$ ratios on liquid velocity at gas flow rate of 1 lpm (Simulation LS1/LS4/LS7 versus LS10/LS13/LS16)

\section{Volume of Dead Zones or Stagnant Regions}

In the present study the dead zones volume was evaluated by locating the cells with low velocities $(1 \mathrm{~cm} / \mathrm{sec}$ was used here, as this value was used in Chapter 4 using CARPT data), and summing up the volumes of these cells. Table 5.3 shows the qualitative dead zone volumes (refer to section 4.3.1 for more explanation about the dead zone volumes) for different digester configurations (the number in the cells represents the percentage dead volume and simulation numbers are given in the bracket). 
The dead zone volume decreases by increasing the gas flow rate, but it is clear from the flow patterns that circulation is localized only inside the riser for $D / T$ of 0.25 , thus decrease in dead zone volume does not indicate more homogeneity in this case. For higher $D / T$ ratios $(0.5$ and 0.75$)$, the effect of gas flow rate on dead volume is not very appreciable.

$D / T$ ratio of 0.75 has highest dead volume, whereas it is lowest for $D / T$ of 0.5 . Only $30 \%$ of the digester volume is inactive/dead in case of $D / T$ of 0.5 , whereas this number is higher for $D / T$ of 0.75 . Flow patterns in Figure 5.1 and 5.6 indicate that the digester with $D / T$ of 0.5 is mixed more homogenously through out the volume than the digester with $D / T$ of 0.75 .

For $D / T$ ratio of 0.25 , the sparger design has no effect on the dead zone volumes. For higher $D / T$ ratios of 0.5 and 0.75 , configurations with cross sparger has lower dead zone volume as compared to configurations with single point sparger.

\subsubsection{Pilot-scale (18-inch Diameter Digester)}

\section{Simulation Results and Comparison with CARPT Data}

Figure 5.12a shows the flow pattern obtained from 3D CFD simulation for pilot-scale digester (simulation PS2). Similar flow patterns were obtained from CARPT results. Simulated flow patterns, Figure 5.12a is compared with experimentally obtained flow patterns, Figure 5.12b. Figure 5.12a and 5.12b, shows good qualitative agreement between the simulation and the experimental results for flow pattern, location of stagnant zones, and circulation loops. 


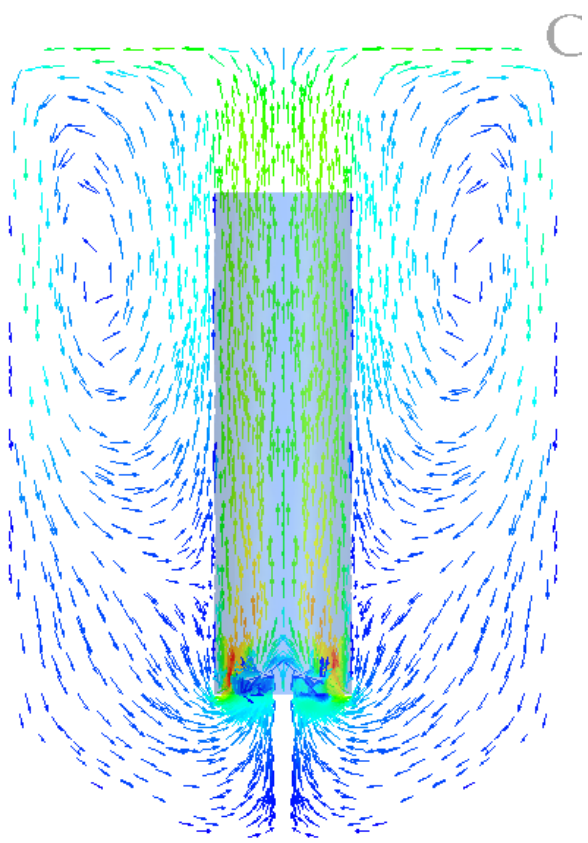

(a)

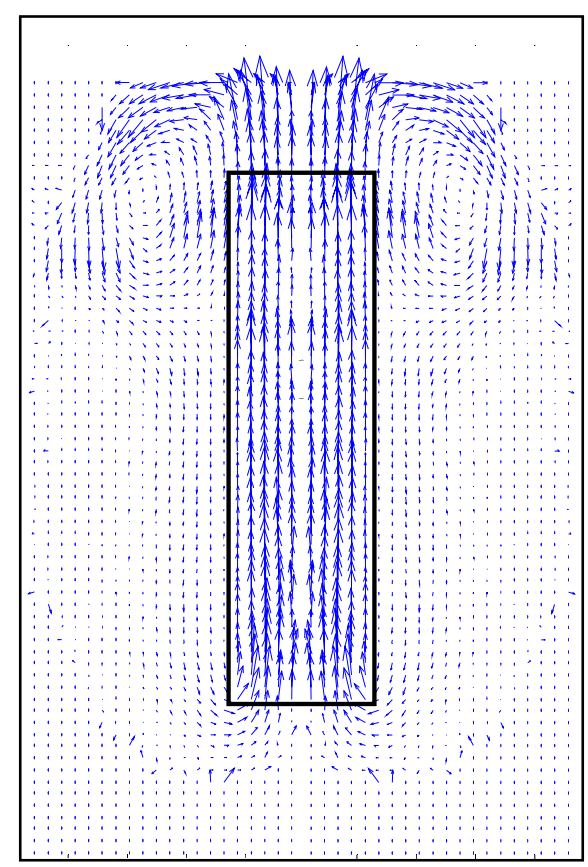

(b)

Figure 5.12 Comparison of (a) simulated flow pattern (PS2) and (b) flow pattern obtained from CARPT (P2)

The flow pattern obtained agrees with simulation predictions for 8-inch and 6-inch diameter laboratory-scale digesters.

Figure 5.13 shows radial profile of azimuthally averaged axial liquid velocities at different axial locations obtained from CFD simulation PS2. The level of $z=6 \mathrm{~cm}$ represents the horizontal level inside the conical bottom region at $6 \mathrm{~cm}$ form the bottom of the digester, similarly $z=12 \mathrm{~cm}$ is just below the draft tube, $z=33 \mathrm{~cm}$ is at the middle height of the draft tube or tank, and $z=54 \mathrm{~cm}$ is just above the draft tube. Figure 5.13 also presents the quantitative comparison of CFD predictions of liquid velocity with the CARPT results. The CFD predictions match reasonably with the CARPT experimental data. The trend of velocity profile matches very well with the experimental data, but the values of liquid velocity are over predicted by CFD. The simulated liquid 
velocities match experimental data better in downcomer section as compared in the riser. Just like laboratory-scale simulation predictions.

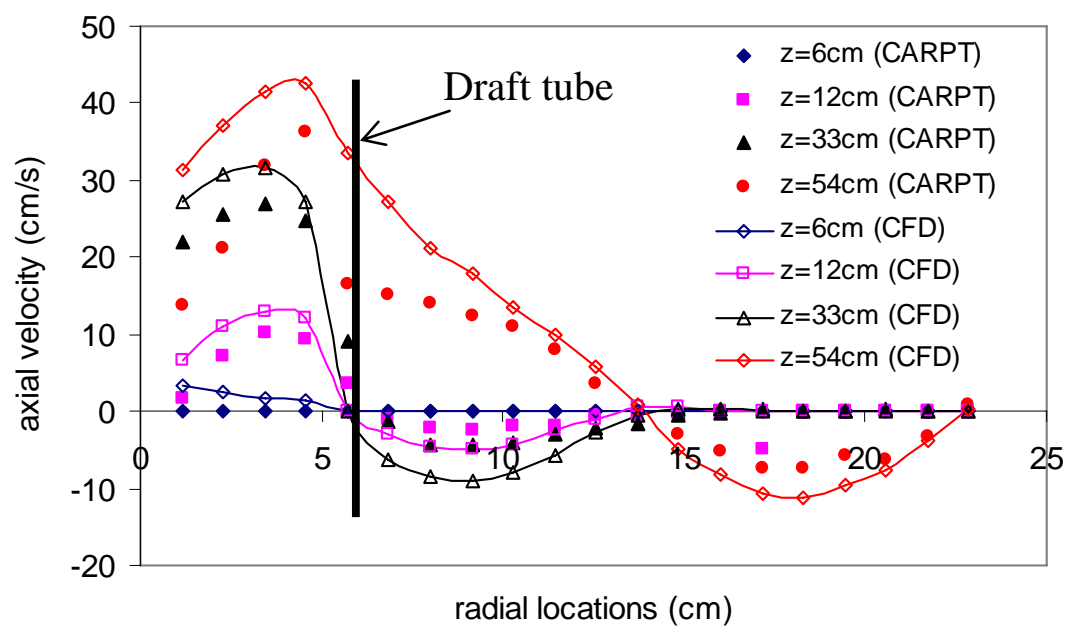

Figure 5.13 Pilot-scale, comparison of simulated axial liquid velocity profile for simulation PS2 with experimental CARPT data.

The gas prediction of Simulation PS2 is shown in Figure 5.14. The same pattern was obtained in similar laboratory-scale configuration (see Figure 5.3). The overall gas holdup is even smaller than the laboratory-scale, only 0.005 .

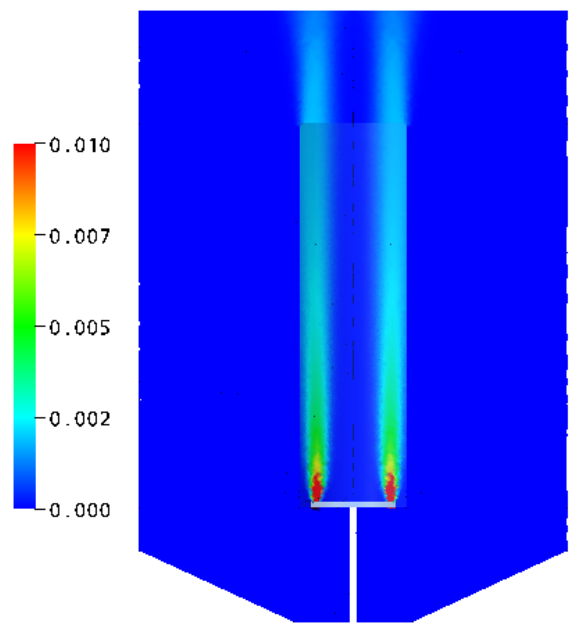

Figure 5.14 Gas hold up distribution predicted by CFD for Simulation PS2 
The scale does not affect the accuracy of the predictions of CFD simulations because the simulation grid was made sufficiently small in both scales.

Pilot-scale simulations PS1 to PS3 and PS7 to PS12 were also performed to check the predictability of the CFD simulations. For all these conditions, CFD predictions agree only reasonably with the experimental data on quantitative basis but predict qualitatively the trends of liquid velocity profile very well.

The dead zone volumes (explained in detail in the earlier section) obtained from CFD simulation results for selected configurations are compared with experimental values in Table 5.4. The agreement between the simulated and experimental values is reasonably well.

Table 5.4 Comparison of dead zone volumes predicted by CFD with experimental data for pilot-scale digester

\begin{tabular}{|c|c|c|c|c|}
\hline \multirow{2}{*}{$\begin{array}{l}\text { Sparger } \\
\text { geometry }\end{array}$} & \multirow{2}{*}{$D / T$} & \multirow{2}{*}{$\begin{array}{c}\text { Gas } \\
\text { superficial } \\
\text { velocity } \\
(\mathrm{mm} / \mathrm{sec})\end{array}$} & \multicolumn{2}{|c|}{$\%$ Dead volume } \\
\hline & & & CFD & CARPT \\
\hline \multirow{9}{*}{$\begin{array}{l}\text { Cross } \\
\text { sparger }\end{array}$} & \multirow{3}{*}{0.25} & 0.45 & 62 (PS1) & 61(P1) \\
\hline & & 0.91 & 58 (PS2) & $55(\mathrm{P} 2)$ \\
\hline & & 1.82 & 51 (PS3) & $50(\mathrm{P} 3)$ \\
\hline & \multirow{3}{*}{0.5} & 0.45 & $50(\mathrm{PS})$ & - \\
\hline & & 0.91 & 44 (PS5) & - \\
\hline & & 1.82 & 36 (PS6) & - \\
\hline & \multirow{3}{*}{0.75} & 0.45 & 68 (PS7) & $65(\mathrm{P} 4)$ \\
\hline & & 0.91 & 62 (PS8) & 60 (P5) \\
\hline & & 1.82 & 59 (PS9) & $54(\mathrm{P} 6)$ \\
\hline \multirow{3}{*}{$\begin{array}{l}\text { Single } \\
\text { point } \\
\text { sparger }\end{array}$} & \multirow{3}{*}{0.25} & 0.45 & $60($ PS10) & $63(\mathrm{P} 7)$ \\
\hline & & 0.91 & 57 (PS11) & $58(\mathrm{P} 8)$ \\
\hline & & 1.82 & 53 (PS12) & $53(\mathrm{P} 9)$ \\
\hline
\end{tabular}

Since the applicability of the CFD simulations to predict the flow pattern and liquid velocities at both the scales have been established to match the trend of liquid velocity profiles, these CFD models can be now used to simulate flow in gaslift digesters at 
other operating conditions listed in Table 5.2 and to evaluate the effect of different operating and geometric variables.

\section{Effect of Air Flow Rate}

Effect of air flow rate for a given $D / T$ ratio and a particular type of sparger can be evaluated using the simulations listed in Table 5.2. The flow pattern remains unaffected by the air flow rate (not shown here), only the magnitude of the liquid velocity changes as discussed below. Similar observations were made for the effect of gas flow rate on liquid velocity for laboratory-scale simulations. The results are shown in Figure 5.15a and 5.15b.

All three gas flow rates correspond to bubbly flow regime or regime one (no gas recirculation in downcomer) for pilot-scale digester also. Thus the liquid velocity increases with increasing gas flow rate.

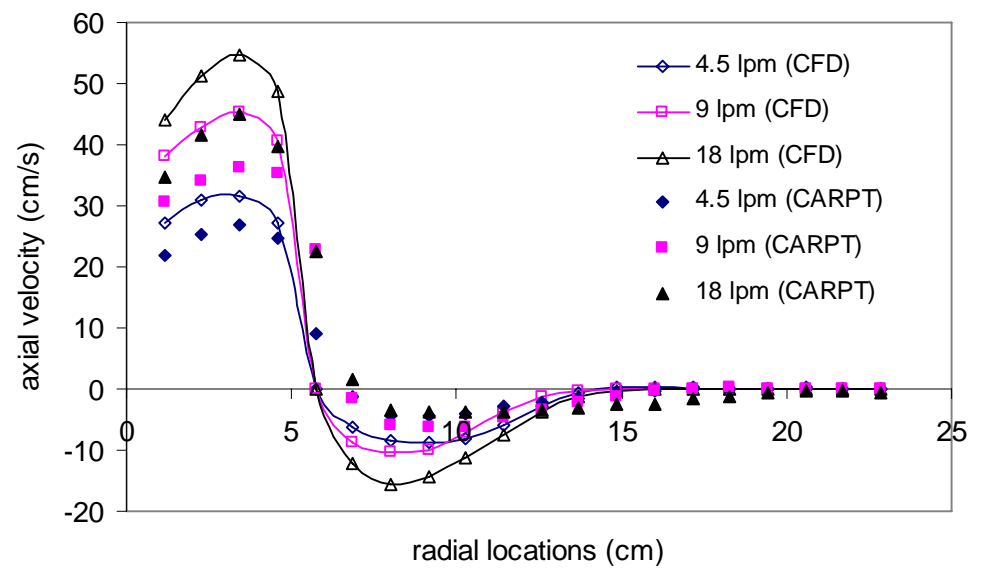

Figure 5.15a Effect of gas flow on axial liquid velocity at the middle height of tank for $D / T=0.25$, cross sparger (PS1, PS2 and PS3) 


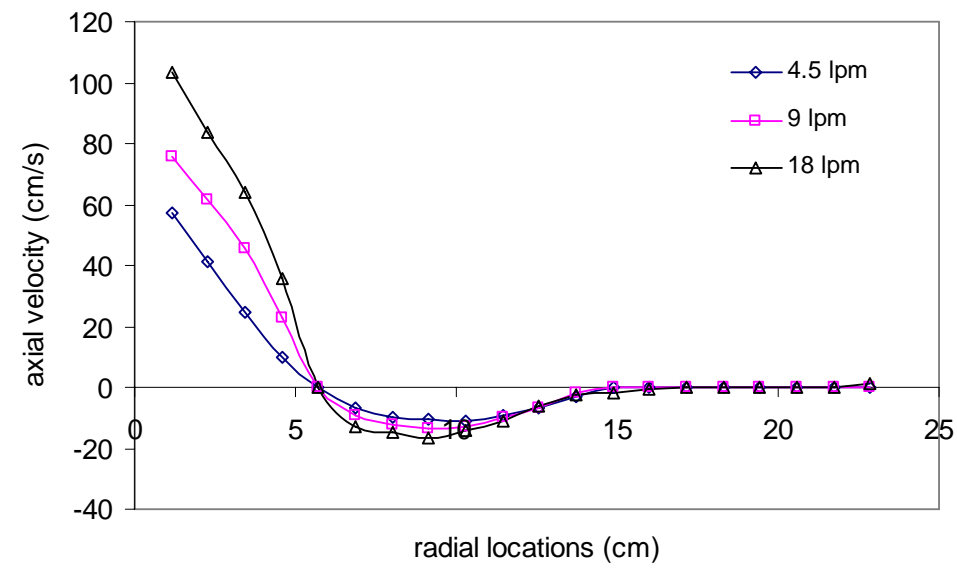

Figure 5.15b Effect of gas flow on axial liquid velocity at the middle height of tank for $D / T=0.25$, single point sparger (PS10, PS11 and PS12)

\section{Effect of Draft Tube (Riser) Diameter}

Effect of $D / T$ ratio of axial liquid velocity profile for cross sparger in pilot-scale is shown in Figure 5.16. The discussion for effect of $D / T$ ratio for laboratory-scale simulations also holds for pilot-scale.

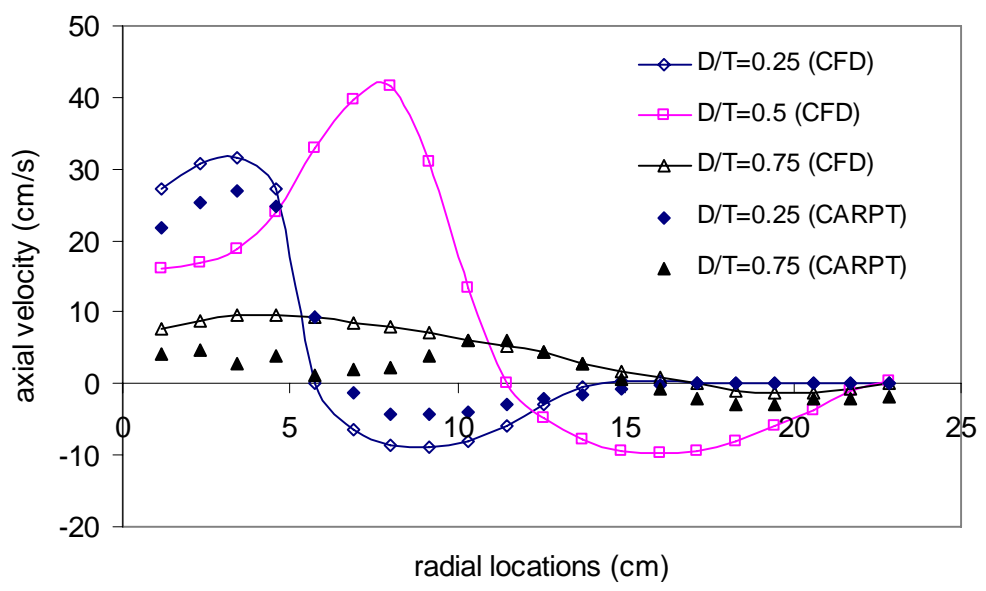

Figure 5.16 Effect of $D / T$ ratio on liquid velocity with cross sparger $4.5 \mathrm{lpm}$ gas flow rate (simulation PS1, PS4, and PS7) 


\section{Effect of Sparger Geometry}

Effect of sparger geometry for pilot-scale simulations at $D / T$ ratio of 0.25 is same as discussed for laboratory-scale simulations; see Figure 5.17.

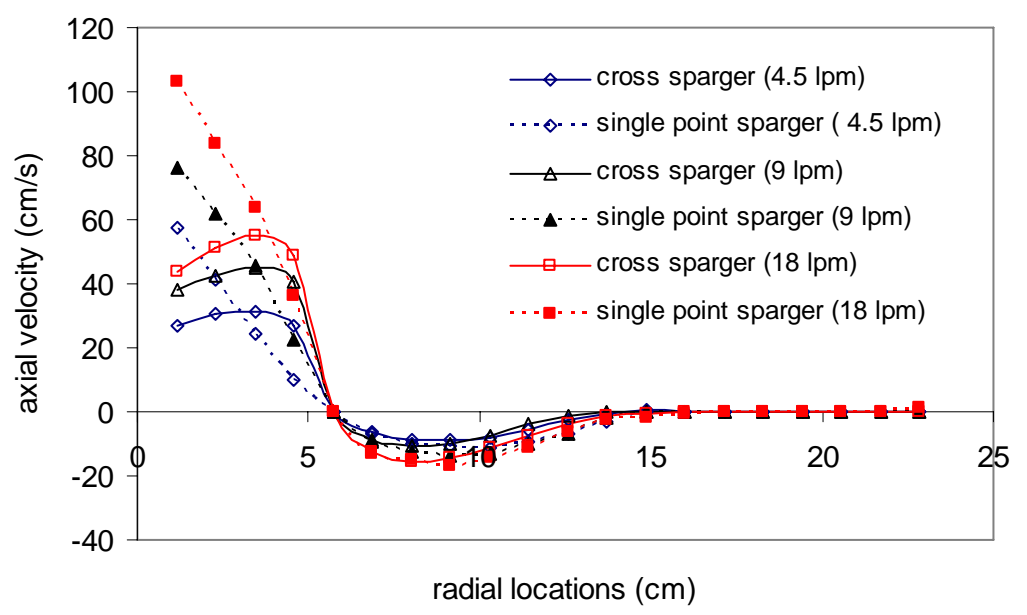

Figure 5.17 Effect of sparger geometry for $D / T$ ratio of 0.25 on liquid velocity at different gas flow rates (simulation PS1, PS2, and PS3 versus PS10, PS11 and PS12)

\section{Volume of Dead Zones or Stagnant Regions}

The above discussion is applicable to both laboratory-scale and pilot-scale simulations. The discussion for laboratory-scale in Section 5.5.2 is applicable here for pilot-scale also. See Table 5.4 for the values of volume of dead zones.

\subsubsection{Effect of Scale (6-inch v/s 18-inch Diameter Digester)}

The results of laboratory-scale and pilot-scale CFD simulations can be compared to investigate the effect of scale. The liquid volume in laboratory-scale was $3.78 \mathrm{~L}$ whereas for pilot-scale it was $97 \mathrm{~L}$, thus a volumetric scale up factor of approximately 25 was employed. Geometry of simulated digester at both the scales was similar; the diameter of pilot scale digester was three times the diameter of laboratory-scale. The gas flow rate of $1 \mathrm{lpm}$ and $2 \mathrm{lpm}$ in small scale and gas flow rate of $9 \mathrm{lpm}$ and $18 \mathrm{lpm}$ in pilot scale 
corresponds to same superficial gas velocity of 0.91 and $1.82 \mathrm{~mm} / \mathrm{sec}$, respectively, based on tank diameter (14.6 and $29.2 \mathrm{~mm} / \mathrm{sec}$ based on draft tube diameter for $D / T$ ratio of 0.25$)$.

Thus the laboratory-scale and pilot-scale configurations with cross sparger for $D / T$ of $0.25,0.5$ and 0.75 operating with superficial gas velocity of 0.91 and $1.82 \mathrm{~mm} / \mathrm{sec}$ are compared to evaluate the effect of scale. Configurations with single point sparger for $D / T$ of 0.25 is also compared.

Comparison of flow patterns of configurations with cross sparger with $D / T$ of 0.25 and superficial gas velocity of $0.91 \mathrm{~mm} / \mathrm{sec}$ (simulation LS1 and PS2) shows that the flow patterns are qualitatively similar; Figure 5.1a and 5.12a. Liquid axial velocity profiles can be compared for qualitative analysis. To facilitate the comparison, the axial liquid velocity at all the radial locations is made dimensionless. Axial velocities of laboratoryscale at middle height of the tank are divided by the maximum liquid velocity for laboratory-scale at the $\mathrm{c}$ middle height of the tank to obtain dimensionless velocity. Similarly, axial velocities for pilot-scale at middle height of the tank are divided by the maximum liquid velocity for pilot-scale at the middle height of the tank to obtain dimensionless velocity. Figure 5.17a shows the comparison of dimensionless axial liquid velocity profile (for simulation LS1 and LS2 versus PS2 and PS3).

The velocity profile has the same shape. Not only the velocity profiles of two scales should overlap each other for the two scales to be hydrodynamically similar, but their magnitudes of velocities should also match (elaborated further in the following discussion). The slight difference between the dimensionless profiles shows the effect of scale on the flow pattern and trend of liquid velocity.

Comparison of flow patterns of configurations with single point sparger with $D / T$ of 0.25 and superficial gas velocity of $0.91 \mathrm{~mm} / \mathrm{sec}$ (simulation LS10 and PS11) shows that 
the flow patterns are qualitatively similar. The dimensionless velocity profile of both scales matches well, Figure 5.17b (simulation LS10 and LS11 versus PS11 and PS12).

The laboratory-scale and pilot-scale flow patterns are quite different for higher $D / T$ ratios of 0.5 and 0.75 , which is not the case for $D / T$ of 0.25 . The laboratory-scale digester has only one circulation loop inside the draft tube, with liquid moving down in the center and up towards the wall. Pilot-scale has more complex circulation pattern with liquid moving downwards in the center in upper part of draft in a smaller independent circulation loop, while the liquid is directed upwards in the center of draft tube at the bottom. Figure $5.17 \mathrm{c}$ and $6.17 \mathrm{~d}$ clearly shows the difference in axial velocity profile due to different flow patterns.

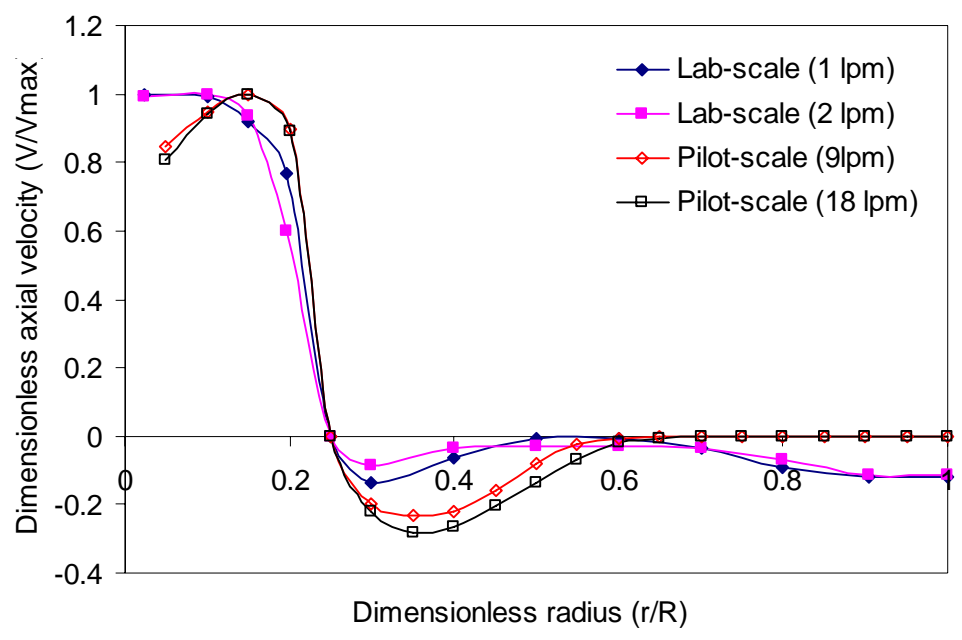

Figure 5.18a Effect of scale on dimensionless axial liquid velocity profile (simulation LS1 and LS2 versus PS2 and PS3, with corresponding maximum liquid velocities of $10.8,14.2,45.2$ and 54.7, respectively) 


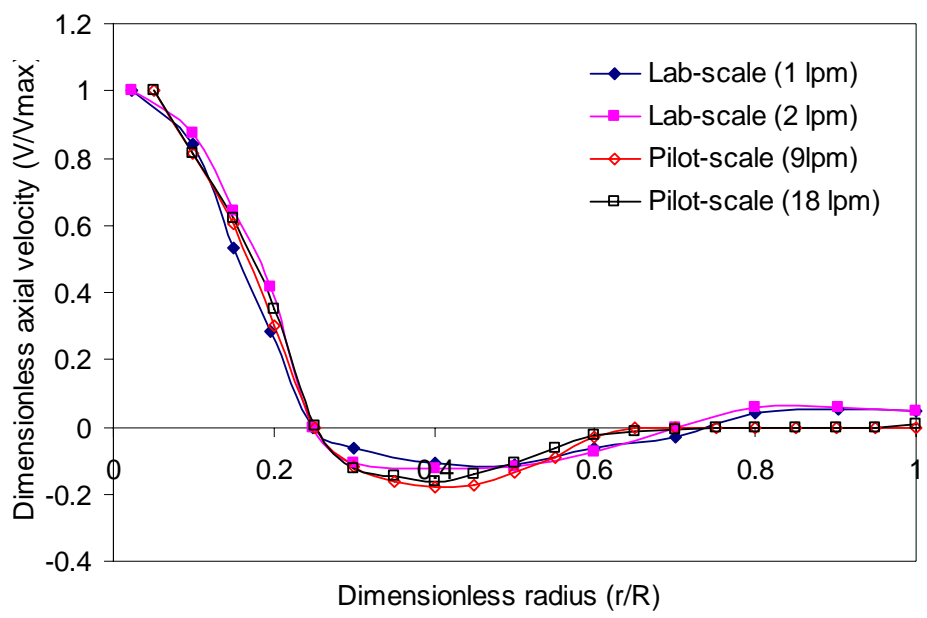

Figure 5.18b Effect of scale on dimensionless axial liquid velocity profile (simulation LS11 and LS12 versus PS12 and PS13, with corresponding maximum liquid velocities of 23, 27, 76 and 103.4, respectively)

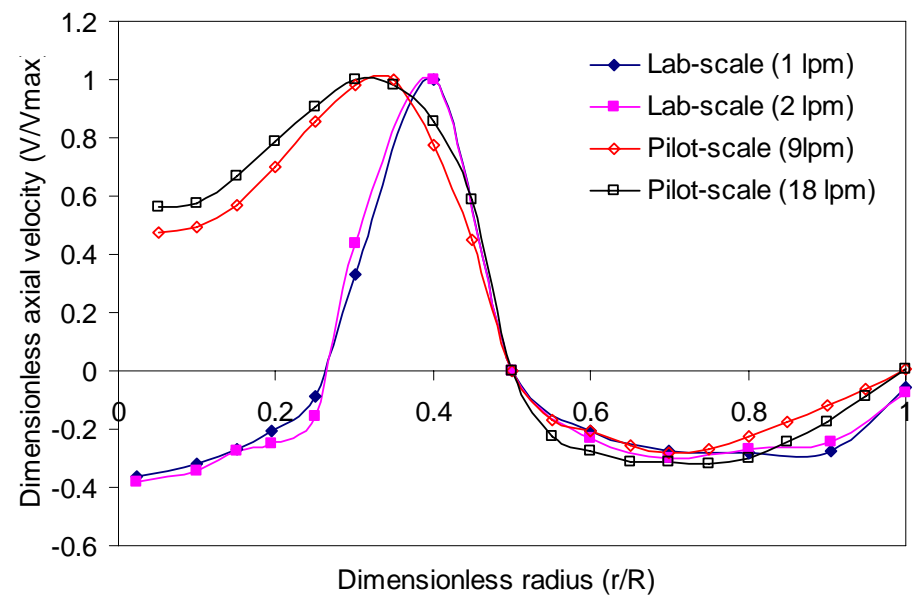

Figure 5.18c Effect of scale on dimensionless axial liquid velocity profile (simulation LS4 and LS5 versus PS5 and PS6, with corresponding maximum liquid velocities of 6.4, 7.6, 50 and 57.2, respectively) 


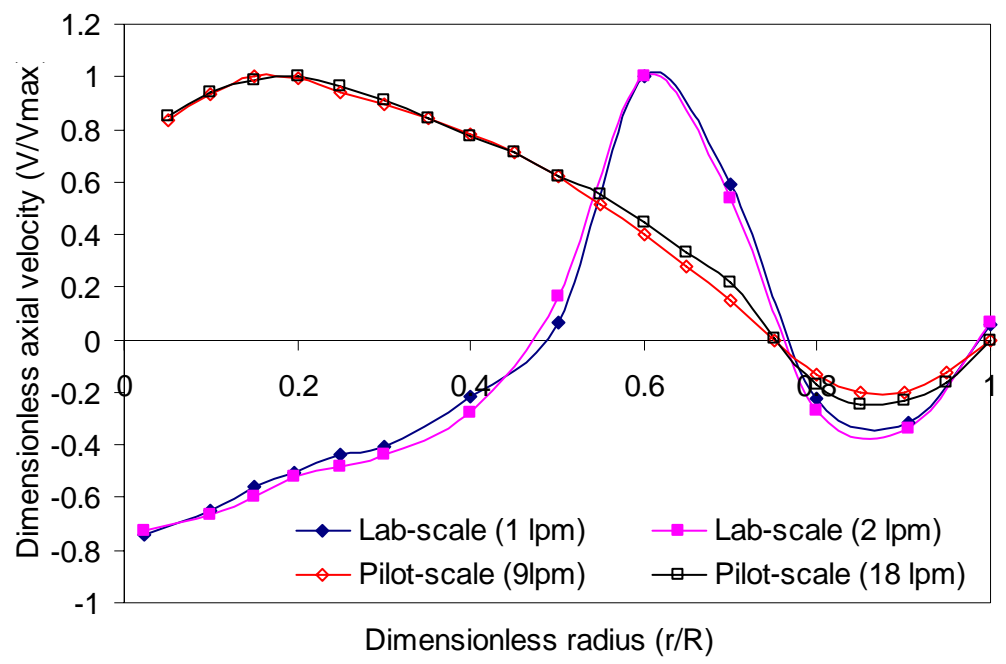

Figure 5.18d Effect of scale on dimensionless axial liquid velocity profile (simulation LS7 and LS8 versus PS8 and PS9, with corresponding maximum liquid velocities of 8.6, $10.1,12.1$ and 14.9 , respectively)

Figure 5.18 explains the true effect of scale on the axial liquid velocity for simulation LS1, LS2, PS2 and PS3. In Figure 5.18 the magnitude of axial velocity is compared rather than dimensionless velocities. The liquid velocities in pilot-scale are about 2 to 3 times higher as compared to laboratory-scale. This has been observed experimentally and also by CFD simulations. (For other configurations the difference in the magnitude of liquid velocities can be compared by multiplying the dimensionless velocity with respective maximum axial liquid velocity; the values of corresponding maximum velocities are provided with the Figures $6.17 \mathrm{a}$ to $6.17 \mathrm{~d}$.) 


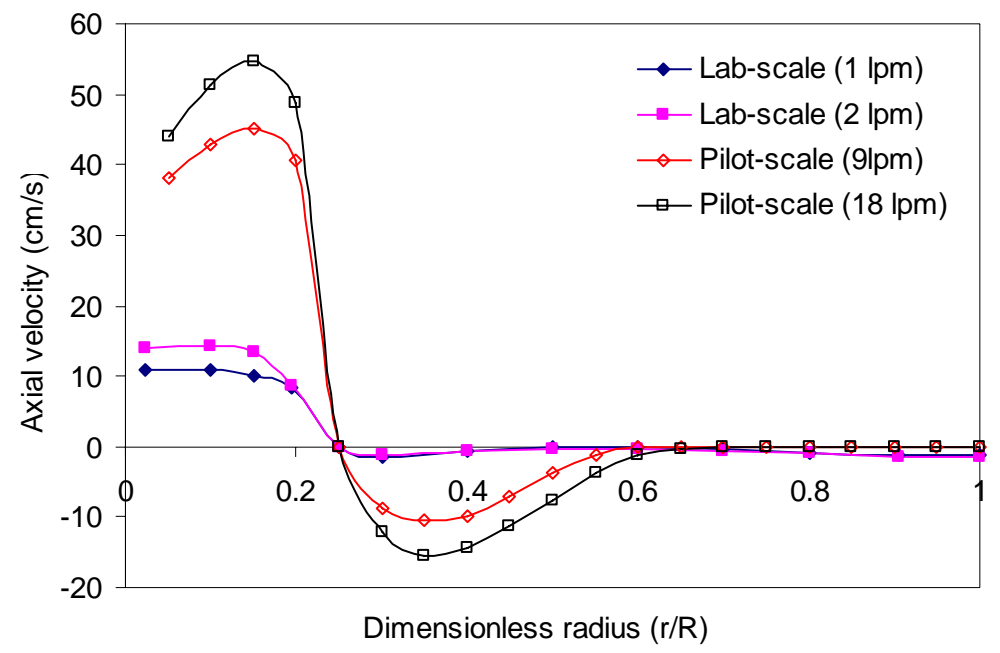

Figure 5.19 Effect of scale on axial liquid velocity profile (simulation LS1 and LS2 versus PS2 and PS3)

Blazej et al. (2004b) and Gavrilescu and Tudose (1998) observed increase in liquid circulation velocity and decrease in gas hold up with increasing scale. van Baten et al. (2003b) found that the radial profiles of gas holdup and liquid velocity simulated by CFD in pilot-scale are much more parabolic in shape as compared to laboratory-scale. The frictional losses of the liquid phase encountered in pilot-scale are much reduced, and this causes much higher liquid circulation and a significantly smaller gas holdup. Blazej et al. (2004b) evaluated the friction factor coefficients in IGLR using empirical correlations and hydrodynamic model of Heijnen et al. (1997) and proved that the friction factor coefficients decreases with increasing scale of reactor (from 10.5 liters to 200 liters). This is explained in detail in Section 4.3.3 of Chapter 4.

The dead zone volumes listed in Table 5.3 and 5.4 can help to understand the effect of scale on dead zones. For pilot-scale the dead zone volumes are higher as compared to laboratory-scale for same superficial gas velocity. Even though the liquid velocities in pilot-scale are higher, it does not help in lowering the dead zones significantly. This is also true for average circulation time (evaluated from CARPT data in Chapter 4 for laboratory-scale and pilot-scale). In most of the biological applications of IGLR, gas 
holdup or mass transfer coefficients are chosen to evaluate the mixing performance. In case of anaerobic digester, gas is sparged only to mix the reactor contents and not for reaction. The mass transfer coefficient is not appropriate criteria to evaluate its mixing performance.

Higher liquid velocities in pilot-scale do not imply that the pilot-scale reactors are better mixed than the laboratory-scale for same energy input per unit volume. (Superficial gas velocity of $0.91 \mathrm{~cm} / \mathrm{sec}$ corresponds to same power input per unit volume of $8 \mathrm{~W} / \mathrm{m}^{3}$ in both laboratory-scale and pilot-scale.) The scale-up ratio is 25 , thus, just $2-3$ times increase may not be sufficient to achieve same mixing intensity or same mixing performance at pilot-scale.

Thus the questions one needs to ask in relation to scale-up of IGLRs are: What should be the appropriate scale-up criterion? How can mixing be quantified correctly for a given application? Although this study cannot answer these questions satisfactorily, few points are clear from experimental and CFD results. Such as, same superficial gas velocity or same energy input per unit volume cannot be used as a scale-up criterion in this case. Next issue is about the applicability of CFD for design and scale-up of IGLRs.

CFD simulations were able to account reasonably for the effect of scale for a scale-up factor of 25. The comparison of experimental data and simulation predictions was reasonable for both scales and the predictability of simulations was same at both scales. This may not happen at scales larger than this. Bagatin et al. (1999) observed that the CFD simulations showed high degree of reliability at laboratory and pilot-scale IGLRs, but when reactors of even larger (full-scale) dimension were considered, the agreement was poor. According to them it was due to the poor description of the bubble size distribution using a single-size model. 


\subsection{Summary}

3D CFD simulations were used to predict the flow in gaslift digesters. The CFD predictions showed good qualitative comparison with the experimental data but gave only reasonable quantitative agreement. Different closures and addition of interphase force terms in the CFD model did not improve the CFD predictions.

CFD was able to account for the effect of geometry and operating variables at both laboratory-scale and pilot-scale. The geometrical variations and operating conditions that were not used in experiments to evaluate their effect on hydrodynamics of IGLRs were evaluated with CFD. The increase in the gas flow rate for all configurations of gaslift digesters increased the axial liquid velocities in the riser but showed no added advantage in increasing the dead zone volumes effectively in the downcomer. The flow pattern was affected appreciably by changing the draft tube diameter. The configuration with $D / T$ ratio of 0.5 showed lowest volume of dead zones. From the conclusions of experiments from Chapter 4 and the results of CFD simulations, it becomes clear that the $D / T$ ratio of 0.5 offers better liquid circulation as compared other $D / T$ of 0.25 and 0.75 .

Sparger design affects flow pattern for higher $D / T$ ratios of 0.5 and 0.75 , but showed no effect for smaller $D / T$ ratio of 0.25 . Better gas dispersion created by cross sparger is advantageous in increasing the circulation and decreasing the dead zone volume over the local dispersion created by the single point sparger. This effect is duet o the increased difference in density of the medium between the draft tube and downcomer zone which represent the driving force for circulation.

CFD simulations were able to account for the effects of scale. The scale affects the flow pattern and as a result, liquid velocities are also affected. At same superficial gas velocity, the liquid velocities are about 2-3 times higher in pilot-scale as compared to 
laboratory-scale. Does it imply that the large-scale reactors are better mixed than the small reactors at same energy input? Is 2-3 times increase in liquid velocity for a scale-up ratio of 25 enough to achieve same intensity of mixing at two scales? If the dead zone volumes and liquid circulation are treated as parameters that quantify mixing, then the answer to above questions is 'No'. Same gas superficial velocity or same energy input per unit volume did not provide same mixing intensities in the two scales of reactor. Thus superficial gas cannot be used a scale-up criterion. 


\section{Chapter 6}

\section{Summary and Recommendations}

\subsection{Summary}

Anaerobic digestion is a preferable method for animal waste treatment because it reduces the environmental pollution and also provides energy in the form of methane. To reduce the anaerobic digester failure rate and increase its use on the farms, proper understanding of their performance and effect of variables that affect the performance is necessary. Mixing is one of the important variables affecting digester performance. Thus, main objective of this study was to advance the knowledge and understanding of the role of hydrodynamics in the anaerobic digester performance.

In this work the literature was reviewed and the key parameters contributing towards better design of anaerobic digesters were identified. Role of mixing in performance of anaerobic digesters needed more investigation, thus performance experiments to evaluate the contribution of mixing in digesters performance were designed, as explained in Appendix A. From the results of preliminary lab-studies and considering the advantages of low energy consumption and easy operation offered by gaslift internal loop reactor, it was chosen for the performance studies. The results of these performance experiments suggested the need of detailed investigation of hydrodynamics of the digesters, which was accomplished through experimental and computational studies. 
The important findings of these experimental and computational studies are summarized in this chapter. Detailed summaries of each of these studies are also provided at the end of each chapter from Chapter 3 to Chapter 5.

\subsubsection{Development of Multiple-Particle Tracking}

A new unique technique of MP-CARPT, which is the first of its kind in the world was developed to overcome the limitations of the old single particle CARPT. Newly developed MP-CARPT was successfully validated and tested for tracking of single or two, stationary and moving tracer particles. This technique is now available for implementation on multiphase systems. The main highlights of MP-CARPT are as follows:

- MP-CARPT provides ability to track eight different radioactive tracer particles simultaneously. The radioactive tracers are distinguished on the basis of their gamma energy peaks.

- MP-CARPT is designed such that the data acquisition is faster and free of any interruptions due to nature of flow in the reactor with dead spaces.

- MP-CARPT enables to track more than two solid particles in the system simultaneously, thus the particle-particle interactions can be measured.

- MP-CARPT system is faster, cheaper, compact and advanced as compared to old single particle CARPT system.

MP-CARPT was implemented on a low $H / T$ (height to diameter ratio) slurry bubble column reactor to test its ability to track tracers representing different phases (solid and liquid in this case). MP-CARPT successfully provided the flow patterns and velocities of solid and liquid phase in SBCR (slurry bubble column reactor). 


\subsubsection{Hydrodynamic Studies: CARPT}

Hydrodynamic studies using CARPT were also performed at both laboratory-scale and pilot-scale digester configurations to understand the difference in hydrodynamics at different scales, which can be related to the performance of digesters. CARPT also provided the information about the effect of geometrical and operating variables on the hydrodynamic and turbulent parameters. The main findings were as follows:

- Diameter of draft tube and the geometry of sparger were two important variables that had significant effect on the flow and hydrodynamics in gaslift digester at both the scales.

- Dead zone volume and eddy diffusivity were used to quantify mixing. On basis of these parameters it was found that configuration with draft tube diameter half of the reactor diameter provides good mixing performance. A sparger with uniform distribution of gas over the cross section of riser provides better mixing than the gas dispersion concentrated at a single point.

- Same energy input per unit volume in geometrically similar gas recirculation type digesters was used as a scale-up criterion. The liquid circulation velocities were higher in pilot-scale than the laboratory-scale configurations. But the dead space volume and circulation mixing time were also higher in pilot-scale, suggesting that the laboratory-scale digester shows better mixing performance than the pilot-scale. These findings explain the need of external mixing in case of pilot-scale anaerobic digesters in performance studies.

- Thus, maintaining same energy input and geometric similarity is not sufficient to obtain to the same mixing performance at two scales of operation of gaslift digester.

\subsubsection{CFD Studies}

CFD studies were performed because of the time and resources constraints associated with CARPT. Before CFD could be used to simulate various geometries of digesters, 
operating at different operating conditions, the CFD models need to be evaluated against the experimental data. The main findings from the CFD studies were as follows:

- The CFD predictions showed good agreement with the experimental data only qualitatively, whereas the quantitative agreement was only satisfactory or reasonable.

- The CFD predictions also clarified that the draft tube diameter and the sparger geometry are the two more important parameters affecting the hydrodynamics of gaslift digesters.

- CFD simulations were able to account for the effect of scale on the geometry and proved that the power input per unit volume is not the correct scale-up criteria.

To summarize, the mixing or hydrodynamics have a significant impact on the performance of large-scale anaerobic digesters. Thus, the effect of mixing should be accounted during the design of anaerobic digesters. The variables that affect the hydrodynamics, such as geometry and type of digester, operating conditions, energy input should also be considered in addition to the factors that affect the anaerobic digestion reaction, such as feed characteristics, $\mathrm{pH}$, temperature, level of toxins and nutrients, etc. The coupling of performance knowledge with the hydrodynamics, as followed in this work, will lead to successful design and scale-up of anaerobic digesters.

\subsection{Recommendations for Future Work}

Research does not have an end! Solution of one problem raises more questions, provides new goals for investigation of another aspect of same problem or in some cases creates identifies more problems. As any other research project, the work accomplished in this thesis was also constrained by the time and resources. For complete understanding of anaerobic digesters, more research needs to done. Some of the points that need further investigation are listed in the following paragraphs and are identified as future work. 
There is still room for improvement of CFD predictions. New closure laws or modified interphase terms should be identified to improve the CFD predictions (Luo, 2005).

The MP-CARPT system has a great potential to provide important hydrodynamic information of industrially important processes that use multiphase reactors particularly in the field related to renewable energy and chemicals. This potential should be exploited to take full advantage of capability of MP-CARPT. For example, in case of gas-solid fluidized beds, the solids occur in different sizes, shapes and properties. Using MP-CARPT hydrodynamics and interaction of these solids of different properties can be evaluated; this information is very valuable for design of such reactors and also for validation of CFD simulations.

The reconstruction of MP-CARPT can be further improved to get more accurate results. Use of new "matrix-calibration" technique can significantly reduce the reconstruction errors. This technique will require modifications to current calibration system. Modification of reconstruction algorithm provided by Bhusarapu (2005) can also increase the reconstruction accuracy. The current reconstruction program is only applicable for tracking two radioactive tracers simultaneously. Slight modifications are needed to extend this program for reconstruction of 2 or more tracers tracked simultaneously. 


\section{Appendix A}

\section{Performance Studies: Laboratory-scale and Pilot-scale}

\section{A.1 Introduction}

Mixing in anaerobic digester is required for number of important reasons viz. to provide efficient utilization of entire digester volume, to prevent stratification and temperature gradients, to disperse metabolic end products and any toxics contained in the feed, to maintain intimate contact between the bacteria and the substrate, to prevent foaming and scum formation and to avoid solids settling. In short, adequate mixing provides a uniform environment, one of the keys to good digestion.

In spite of the crucial role of mixing in digester operation, contradictory findings are reported in the literature about the necessity of mixing and the required mixing intensity to enhance the digester performance. There are many reasons for these controversies and uncertainties. One of them is, mixing is not adequately quantified and characterized in these systems. Another important reason is, most of these digester performance studies are performed in small laboratory-scale reactors and/or using low solids concentration. These approaches do not contribute greatly in understanding influence of mixing on digester performance or in providing criteria for full scale digester design.

Laboratory-scale reactors are valuable in estimating kinetic parameters, in estimation of nutrient and alkalinity requirements and discovering potential problems like toxicity, because they are easy to control, efficient mixing and uniform environment can be guaranteed. On the other hand, experimentation on a large scale digester is necessary to elucidate the operational problems and difficulties like effects of improper mixing, clogging of feed and outlet ports, solids accumulation, foaming and so on.

\section{A.2 Objectives}

1. To study the effect of mixing on the performance of anaerobic digester.

2. To demonstrate the effect of digester size on the role of mixing by comparing the lab-scale and pilot-scale digester performance. 


\section{A.3 Results and Discussion}

Two identical laboratory-scale digesters with working volume of 3.87 liters (6 inches in diameter) were used. One was mixed by gas recirculation at a rate of $11 / \mathrm{min}$; digester was equipped with draft tube with diameter one fourth of digester diameter and a multipoint sparger to facilitate mixing. Another digester was unmixed; unmixed condition implies that no mixing is provided by external means, but digester is naturally mixed due to the evolution of biogas bubbles and addition of feed and effluent removal. Pilot scale digester had working volume of 97 liters (18 inches in diameter) and was geometrically similar to the laboratory-scale digester. The pilot-scale digester operation was started with biogas recirculation. After 70 days of operation of the pilot-scale digester in mixed condition, biogas recirculation was stopped and it was operated in unmixed condition for more than 70 days. Again the biogas recirculation was started and the digester was operated in mixed condition for more than 12 days, this was done to check the reproducibility of the results obtained. The biogas recirculation rate in pilotscale digester was $9.07 \mathrm{l} / \mathrm{min}$, resulting in an input power density of $8 \mathrm{~W} / \mathrm{m}^{3}$, which corresponds to $1 \mathrm{l} / \mathrm{min}$ biogas recirculation rate in the 6-inch laboratory scale unit at same energy input rate.

Both the digesters were operated in same manner using same cow manure collected from a local dairy farm in the Oak Ridge, TN area. The raw sludge was processed and diluted with water to obtain $6.6 \%$ total volatile solids (total solids of about 12-13\%) concentration. This feeding rate was maintained corresponding to a hydraulic retention time of 16 days. Gas samples were analyzed for methane and carbon dioxide content. Slurry samples were analyzed for total solids (TS), total volatile solids (TVS), Volatile Fatty acids (VFA), and total alkalinity (TA).

Table A.1 shows the results of the performance results of two scales of digesters, whereas Figure A.1 compares their cumulative methane production rates. Laboratoryscale digester produced more biogas with higher methane content than the pilot-scale digester. The TS, TVS and VFA content in the effluent of laboratory-scale was also lower than the pilot-scale digester. The laboratory-scale digester in mixed and unmixed condition showed same performance in terms of methane production. Pilot-scale digester in mixed condition performed significantly better than in unmixed condition with approximately 100\% higher methane production. Increase in VFA in the effluent reaching the values of feed VFA indicated that unmixed pilot-scale digester was failing.

Since the rate of bioreaction is low, anaerobic digesters are kinetically controlled. But, still sufficient amount of mixing is required to maintain a uniform environment inside the digester to guarantee efficient distribution of substrate, $\mathrm{pH}$ and temperature. Even the small amount of mixing produced by the motion of evolving gas bubbles and the addition of feed in the unmixed digester is sufficient for efficient operation of the laboratory scale digester. Since the reaction is kinetically controlled, any additional amount of mixing does not further improve the performance of the mixed laboratory- 
scale digester over an unmixed digester. As the size of the reactor increases, difficulty in achieving complete mixing increases, and additional mixing is required. Since, no additional mixing was provided in pilot-scale unmixed reactor, it showed poorer performance than the pilot-scale mixed reactor.

Table A.1 Effect of mixing on performance of laboratory-scale and pilot-scale anaerobic digester

\begin{tabular}{|c|c|c|c|c|}
\hline Scale & \multicolumn{2}{|c|}{$\begin{array}{c}\text { Laboratory-scale } \\
(6 \text {-inch, 3.78 L) }\end{array}$} & \multicolumn{2}{c|}{$\begin{array}{c}\text { Pilot-scale } \\
(18 \text {-inch, 97 L) }\end{array}$} \\
\hline Condition & Mixed & Unmixed & Mixed & Unmixed \\
\hline Gas recirculation rate $(\mathrm{L} / \mathrm{min})$ & 1 & - & 9 & - \\
\hline Feed/effluent rate $(\mathrm{L} / 2$ days $)$ & 0.470 & 0.470 & 12 & 12 \\
\hline Biogas production rate $(\mathrm{L} / \mathrm{L} /$ day $)$ & 1.2 & 1.1 & 0.55 & 0.3 \\
\hline Methane content (\%) & 76 & 73 & 65 & 52 \\
\hline $\begin{array}{c}\text { Cumulative methane production } \\
\text { rate }(\mathrm{L} / \text { day })\end{array}$ & 3.3 & 3.1 & 40 & 20 \\
\hline $\begin{array}{c}\text { Cumulative methane production } \\
\text { rate per unit volume }(\mathrm{L} / \mathrm{L} / \text { day })\end{array}$ & 0.87 & 0.82 & 0.41 & 0.2 \\
\hline
\end{tabular}

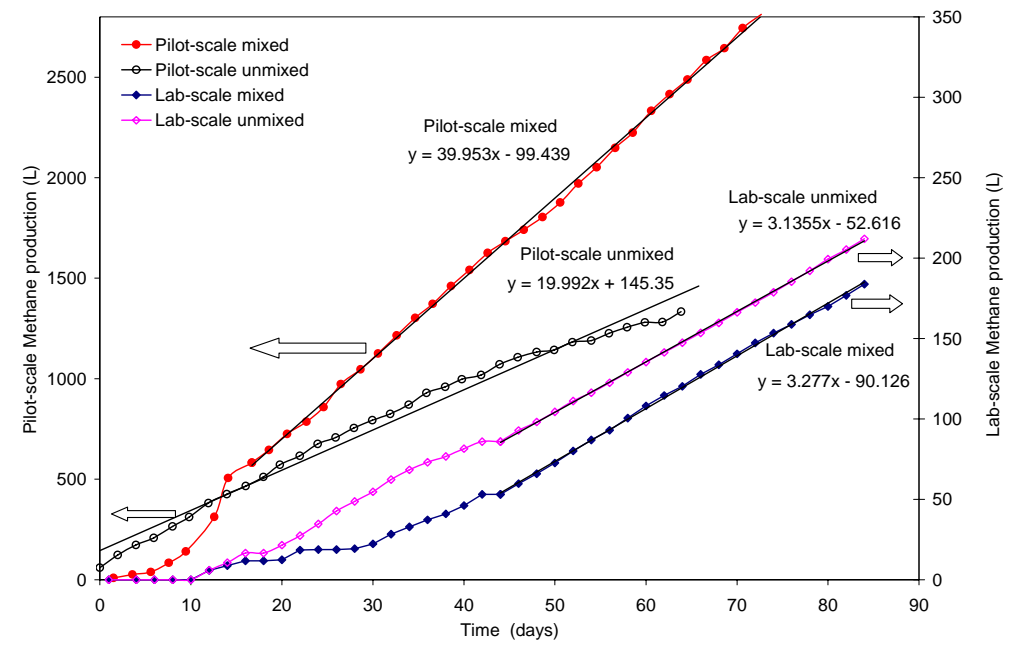

Figure A.1 Comparison of cumulative methane production rates for laboratory-scale and pilot-scale digesters

\section{A.4 Summary}

Significant differences between the results obtained for mixed and unmixed condition in the pilot-scale digester were observed. Mixing provided in the digester results in its efficient operation and avoids its failure. Mixing played no significant role in the 
performance if laboratory-scale digesters. At the smaller scale the mixing created by the evolution of gas bubbles is sufficient for proper operation of the unit. Any additional amount of mixing does not benefit the digesters to create more gas, necessarily because the digestion process is kinetically controlled. Excessive amount of mixing is also not recommended as mixing needs energy and spending more energy will not be profitable. This concludes that large scale operation of digester is necessary to obtain meaningful results and findings that can be used for proper design of commercial scale units.

The following essential question arises: what is the best or optimum mixing intensity to ensure efficient or less energy input to maximize the energy output obtained from the biogas. This question is yet to be answered and it needs further investigation using large scale digester. The findings in the pilot scale digester and their comparison with those obtained with 6-inch digester suggest that laboratory scale digesters are of no use to determine the optimum mixing intensity needed for efficient digester performance. 


\section{Appendix B}

\section{MP-CARPT Manual}

\section{B.1 Background}

Concept for MP-CARPT is based on energy discrimination. By using a different isotope (with different gamma peaks) for each particle, it should be possible to discriminate which particle a detected gamma came from. Before examining the method for doing this, it is useful to review a few aspects of single-particle CARPT. Figure B.1 shows a detector channel used for single-particle CARPT, while Figure B.2 shows an idealized spectrum for a hypothetical isotope. The detector channel registers one count for each detected gamma exceeding the discriminator threshold. This arrangement rejects lower energy gammas that are due to Compton scattering or background sources (depending on how close to the peak the discriminator threshold is set.)

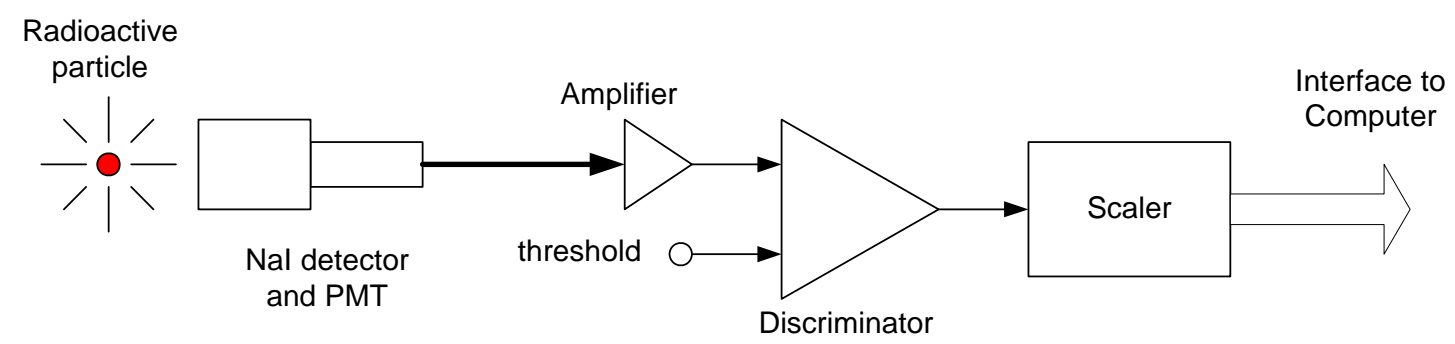

Figure B.1 Single particle CARPT detector channel.

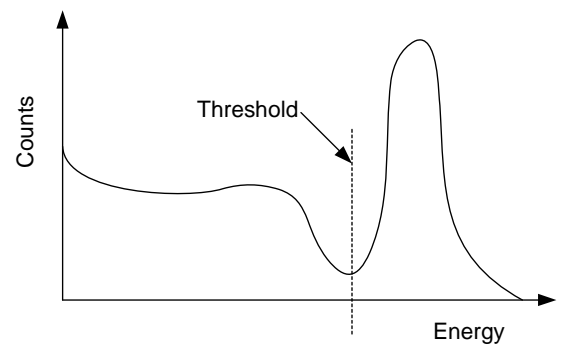

Figure B.2 Idealized NaI spectrum. 


\section{B.2 MP-CARPT Concept}

Figure B.3 shows the concept for the MP-CARPT (MP-CARPT) detector channel. The detected and amplified signal is introduced to an energy analyzer that can sort the events according to non-overlapping energy windows. For example, gammas with energies between 1 and $1.2 \mathrm{MeV}$ would be events counted by scaler 1 while gammas with energies between 0.7 and $0.9 \mathrm{MeV}$ would be events counted by scaler 2. Figure B.4 shows the $\mathrm{NaI}$ spectrum for two hypothetical isotopes individually and combined. It also shows the range of the two energy windows. This illustrates only two particles and two isotopes, but the concept could be extended to greater numbers. For those familiar with conventional NIM electronics, the energy analyzer could be implemented as multiple single-channel analyzers (SCA) - one per each isotope or energy window. This would be a very costly approach for CARPT where multiple detectors (16 or more) are used and 2 or more isotopes would be used. An alternative approach would be to use multi-channel analyzers (MCA). A standard multi-channel analyzer determines the energy of each detected gamma and sorts them into multiple energy windows (called channels). Generally these windows are evenly spaced, and there are usually 2000 or more of them. This type of instrument is generally used for spectroscopy, but the spectral data could be processed in software to reduce the many windows to the few needed for MP-CARPT. This is still a complex and costly approach as commerciallyavailable MCAs generally have far more capabilities than needed (and therefore more cost), and they are generally meant to be used singly or in very small numbers - it would be difficult to interface to 16 or more of them.

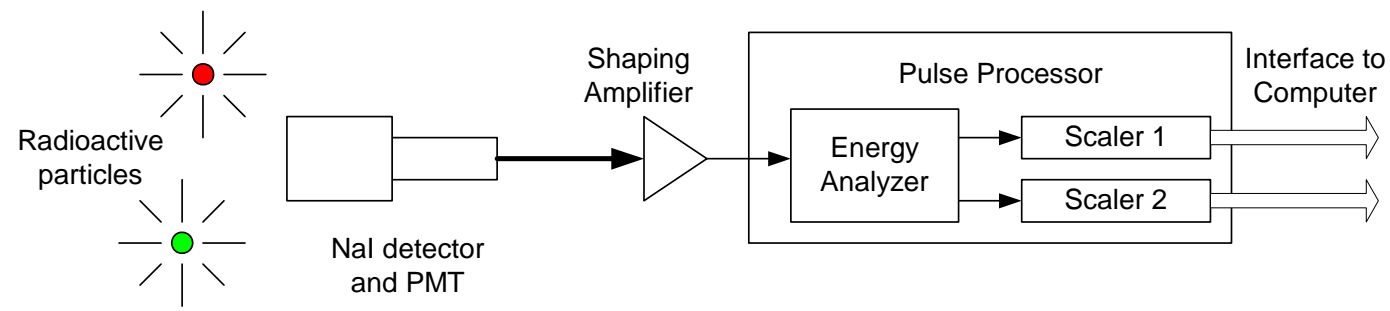

Figure B.3 MP-CARPT detector channel. 


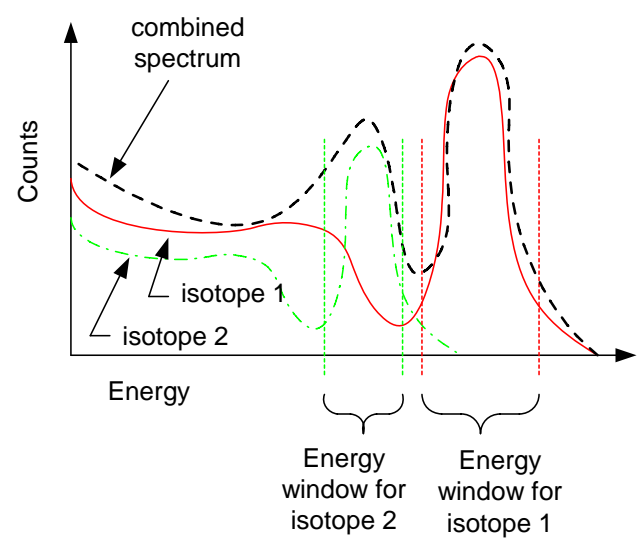

Figure B.4 Idealized NaI spectrum for two isotopes.

\section{B.3 Proof of Concept Experiments}

As a proof of the energy discrimination concept, an experiment was set up based on the old CAMAC (Computer Automated Measurement and Control) and NIM (Nuclear Instrumentation Module) electronics. This experiment allowed CARPT to be extended to two particles with a minimum of additional hardware. Figure B.5 shows a detector channel modified for dual-particle CARPT, while Figure B.6 shows an idealized spectrum for two different hypothetical isotopes. Gammas resulting from one of the particles or background are detected by the $\mathrm{NaI}$ detector and photomultiplier, and are then amplified by a timing-filter amplifier (TFA). The signal from the TFA is split into two equal parts and these are input to two discriminators. As shown in Figure B.6, the two thresholds are different. The threshold for discriminator 1 (threshold 1) is set just below the photopeak for isotope 1 . This insures that the counts recorded by scaler 1 will be mainly due to isotope (and particle) 1. The threshold for discriminator 2 (threshold 2) is set just below the photopeak for isotope 2. This means that the counts recorded by scaler 2 will be due to isotope (and particle) 2 plus those of particle 1. By subtracting the counts of scaler 1 from those of scaler 2, we get the counts that are due to particle 2 alone.

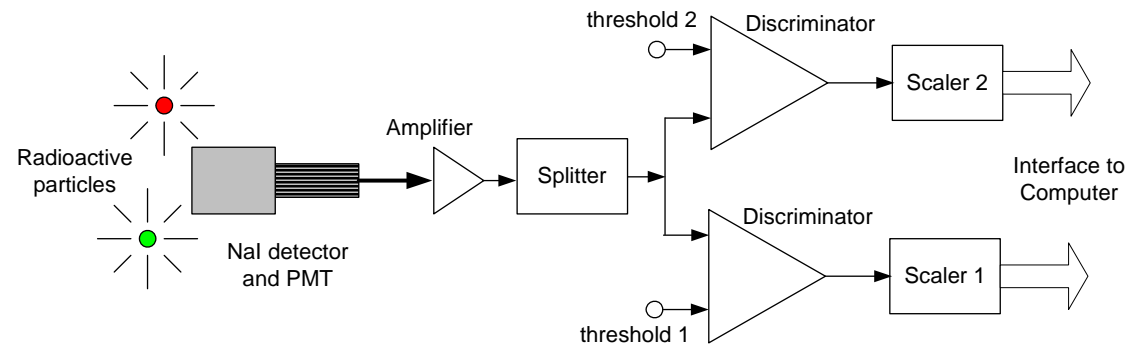

Figure B.5 Dual particle CARPT detector channel. 


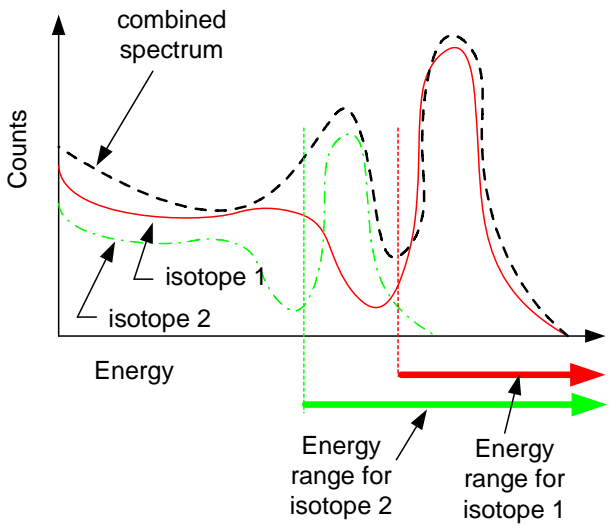

Figure B.6 Idealized NaI spectrum for two isotopes.

Figure B.7 shows the modules and their connections for the dual particle experiment. Up to 16 detectors may be used. The existing single particle CARPT data acquisition program was modified slightly to allow setting the two different thresholds.

We were able to use one detector in the dual-particle setup to distinguish the two peaks of Scandium-46. The Sc-46 spectrum resembles Figure B.2, and by setting the two discriminators to values corresponding to the valley below each peak we were able to get about twice as many counts in one scaler than the other. (The two peaks are equally intense.) This would indicate that the scaler corresponding to the higher threshold counted only the upper peak, while the scaler with the lower threshold counted both peaks. 


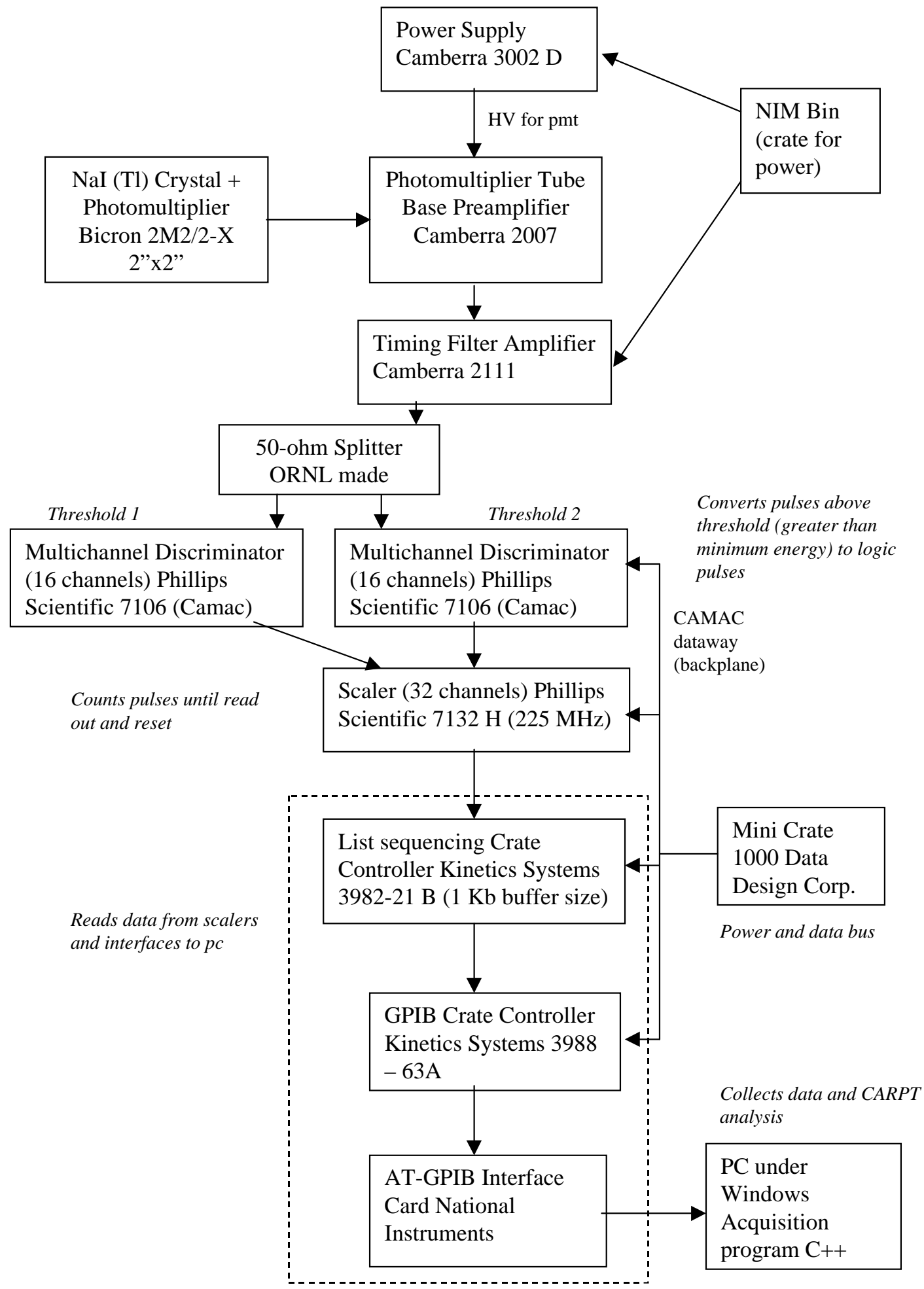

Figure B.7 Dual-particle CARPT electronics showing NIM and CAMAC elements 


\section{B.4 NaI Detector Readout Concept}

Many physics experiments require a large number of detector channels (hundreds, thousands or even tens of thousands.), and due to the large number of channels, each must be relatively inexpensive and have a good computer interface. For the Spallation Neutron Source, it is expected that there will be several thousand He3 detectors to read out. This readout was being developed at ORNL. Fortunately, the signal from the preamp used with those detectors resembles the signal from the $\mathrm{NaI}$ detectors and photomultipliers used for CARPT. Figure B. 8 shows an experimental setup using a NaI detector and part of the SNS He3 readout electronics.

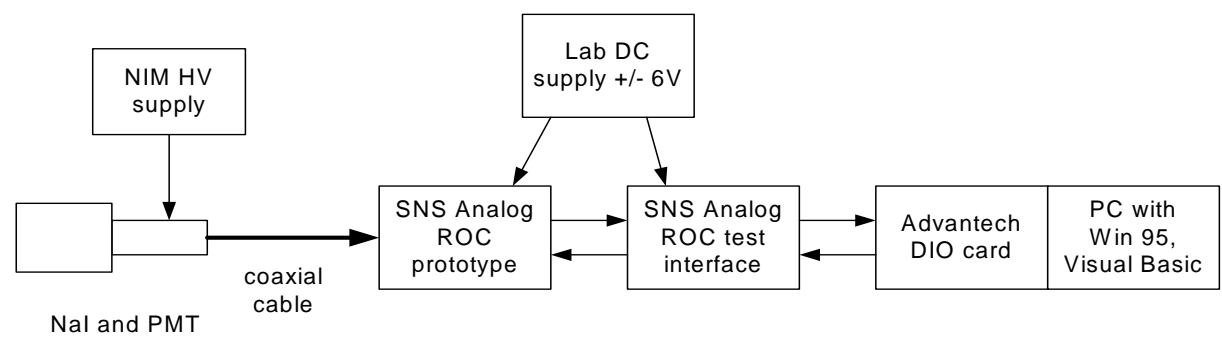

Figure B.8 NaI readout using SNS He3 electronics

Initial tests of this setup use it as an MCA to view energy spectra. It operates in the following way. The PC arms ROC card, making it ready to process pulses from the photomultiplier (PMT). Pulses from the PMT are detected by a discriminator in the Analog Readout Card (ROC). The discriminator threshold is programmable and is set just above the system noise level. When a pulse is detected (this corresponds to a gamma), the pulse is integrated and the integrated value is digitized. This digital value is proportional to the energy of the gamma. The digital value is sent to the PC via the digital input/output (DIO) card. This sequence terminates by disarming the ROC. The software in the pc controls how many pulses are processed. The software also histograms the events and writes the results to a file. The software could be easily rewritten to categorize the values according to two or more energy windows.

Figure B.9 shows the Analog ROC prototype card and the ROC interface. These were used along with a 3-inch $\mathrm{NaI}$ detector to obtain the spectrum shown in Figure B.10. The peak near channel 260 is due to Potassium-40. 

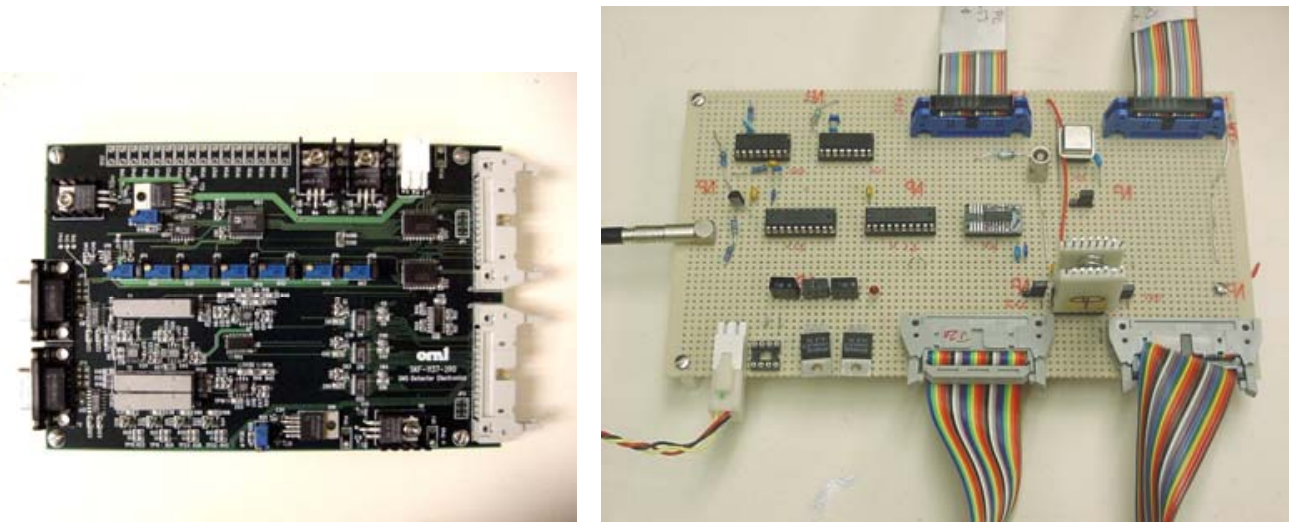

(a) ROC prototype card.

(b) ROC interface card

Figure B.9

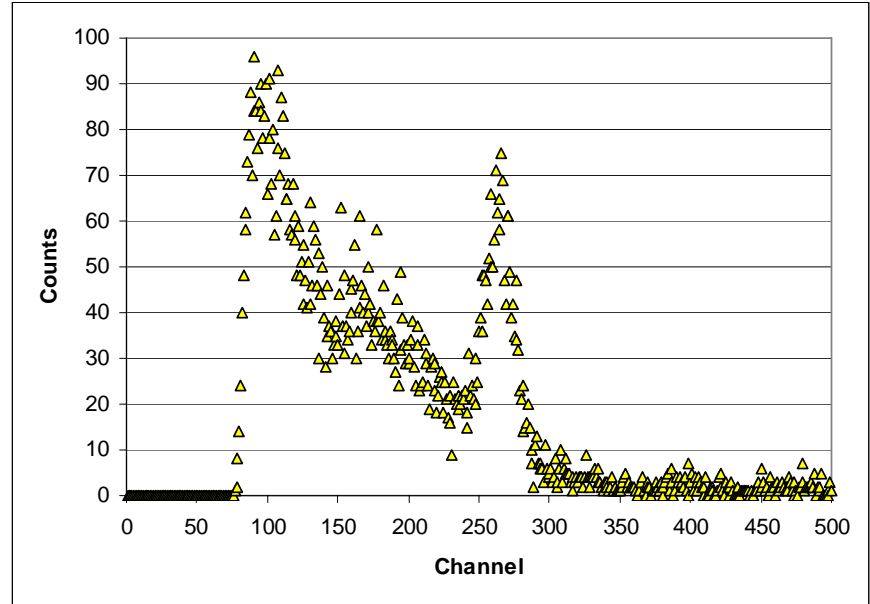

Figure B.10 Spectrum of Potassium-40 obtained using prototype electronics

\section{B.5 Readout Electronics and Data Acquisition}

The readout electronics and data acquisition for MP-CARPT consists of timing filter/shaping amplifiers, pulse processor or $\mathrm{NaI}$ readout modules, a crate and a PC as shown in Figure B.11. The shaping amplifiers are used to increase the signal levels before pulse processing occurs. The readout modules perform the pulse processing and energy discrimination and are comprised of analog, digital and interface sections. Several readout modules are plugged into a CPCI (Compact Peripheral Component Interconnect) crate. The crate contains single board PC that controls the readout modules and communicates to a network via Ethernet. These modules and other elements are discussed in more detail in the following subsections. 


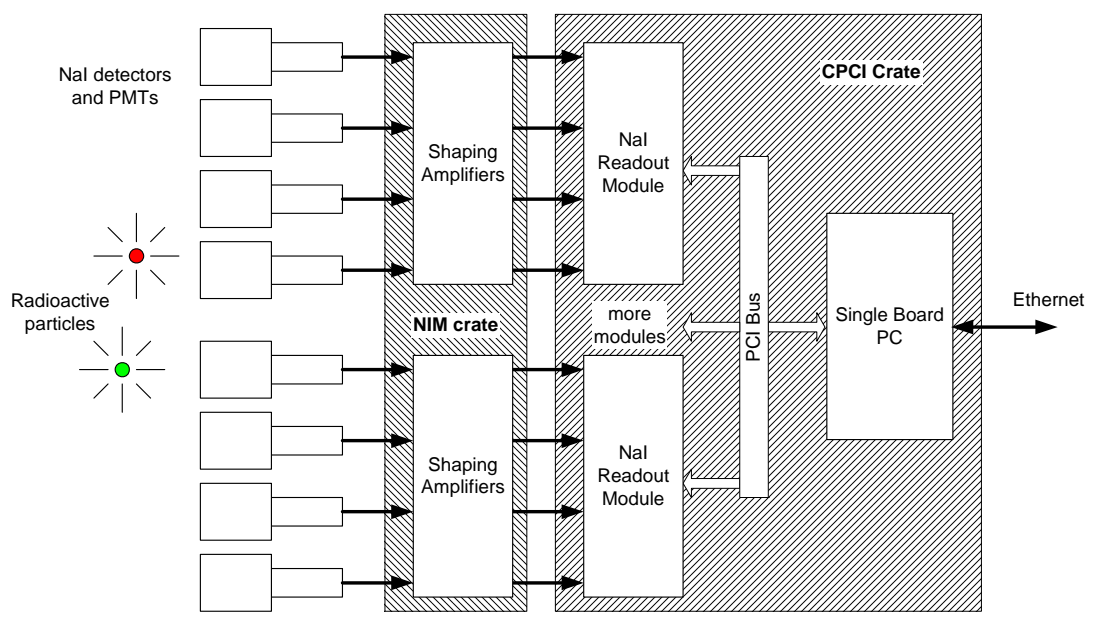

Figure B.11 MP-CARPT arrangement using NIM and CPCI format NaI readout electronics

\section{B.5.1 Timing Filter Amplifier}

\section{Introduction}

In single particle CARPT experiments, a commercial timing filter amplifier (TFA) is used. This amplifier is normally adjusted to provide an adjustable gain on the order of 100 and to provide minimal filtering to the signal from the $\mathrm{NaI}$ detector. (Nominal settings are 50-ns integration and no differentiation.) The TFA is capable of a wide variety of time-constants for filtering, but that ability is not required for this application, and a much simpler amplifier would serve as well.

A prototype timing filter or shaping amplifier was developed as a possible replacement for the NIM timing filter amplifiers. (The large number of single-channel TFAs needed for a CARPT experiment is a considerable expense.) The prototype shaping amp has four channels, a maximum gain of 100 and filtering that is equivalent to $50 \mathrm{~ns}$ integration and no differentiation. The gain of each channel is continuously adjustable from 100 to $<1$. The prototype operates from a $+/-6 \mathrm{Vdc}$ power supply. It was evaluated during the dual particle CARPT experiments and found to be the functional equivalent of the TFAs.

Currently, an 8-channel version of the shaping amplifier is developed. This amplifier is housed in a NIM module and is designed for use with the NaI pulse processor module. Following sections describe it and explain its use.

\section{Design Overview}

Figure B.12 shows a block diagram of a single channel of the shaping amplifier. It consists of a differential receiver amplifier, an adjustable attenuator, and a differential line driver plus input and output connectors. The differential receiver has two 
functions: it provides gain and allows the polarity of the input signal to be reversed. The adjustable attenuator allows the signal level to be reduced as needed to set the overall gain. The gain stage also has two functions: it provides gain and serves as a lowpass filter. (Low-pass filtering is the pulse shaping function.) The differential line driver converts the signal from the gain stage to a differential signal that can be transmitted through a twisted pair cable.

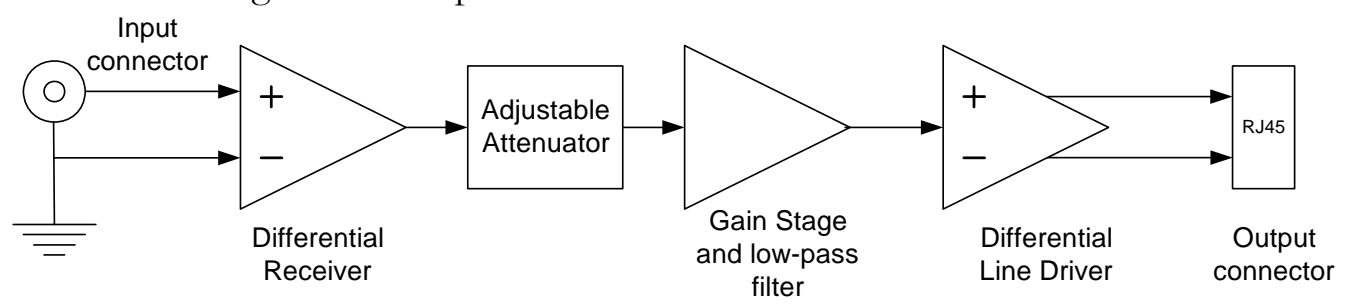

Figure B.12 Block diagram of shaping amplifier channel.

Figure B.13 is a circuit diagram for one channel of the shaping amplifier. Integrated circuits U1 is the differential receiver, RP1 is the adjustable Attenuator, U2 is the core of the gain stage and $\mathrm{U} 3$ is the differential line driver. These circuits will be described in more detail in the remainder of this section.

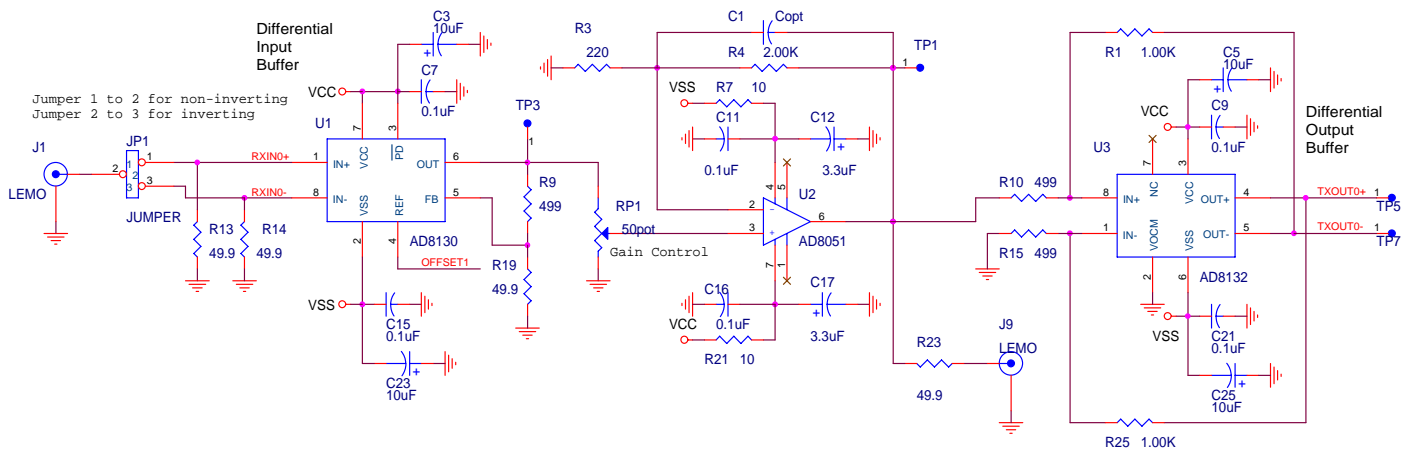

Figure B.13 Circuit diagram of one shaping amplifier channel.

Differential receiver U1 uses an Analog Devices AD8130 differential amplifier to provide a gain of 11 . The AD8130 has a bandwidth of approximately $10 \mathrm{MHz}$ when connected for a gain of 10, so that the bandwidth of the receiver amplifier should be just slightly less than $10 \mathrm{MHz}$. The signal polarity can be reversed by using jumper JP1 to connect the input signal to the inverting input of the amplifier (pin 8) instead of the non-inverting input (pin 1). The receiver circuit also includes 50-ohm input resistors that properly terminate the coaxial cable used to bring the signal from the detector to the shaping amplifier. The signal from the differential receiver passes through potentiometer RP1 to the input of the gain stage. Depending upon the position of the wiper, the signal may not be attenuated or it may be attenuated by as much as a factor of 100 .

The gain stage uses an AD8051 opamp connected for a non-inverting gain of 10 . According to the data sheet, the AD8051 has a bandwidth of approximately $7 \mathrm{MHz}$ for that gain. Combined with the bandwidth of the receiver amplifier, this gives an overall 
bandwidth of about $6 \mathrm{MHz}$ and that corresponds to a shaping time of approximately 25 ns. The line driver is implemented using an AD8132 differential amplifier that is connected to provide a gain of two. The AD8132 has a gain-bandwidth of approximately $300 \mathrm{MHz}$, so that the bandwidth of the line driver amplifier is approximately $150 \mathrm{MHz}$, which is large enough to have a negligible effect on the overall shaping amplifier bandwidth.

\section{Module Implementation and Use}

The shaping amplifier is using a 4-layer printed circuit board (SKF-1166-4R0) and is housed in a single-wide NIM module. Figure B.14 shows the module with the side panel removed. The eight channels are arranged linearly with channel 1 at the top and channel 8 at the bottom. The Lemo input connectors are visible at the right and extend through the front panel. The potentiometers (blue rectangles) used for gain adjustment are located just below each input connector. The RJ-45 output connectors are visible at the left, above the NIM power connector. Channels 1 through 4 use the upper RJ-45 connector, while channels 5 through 8 use the lower one. Figure B.15 shows the front and rear panels of the module.

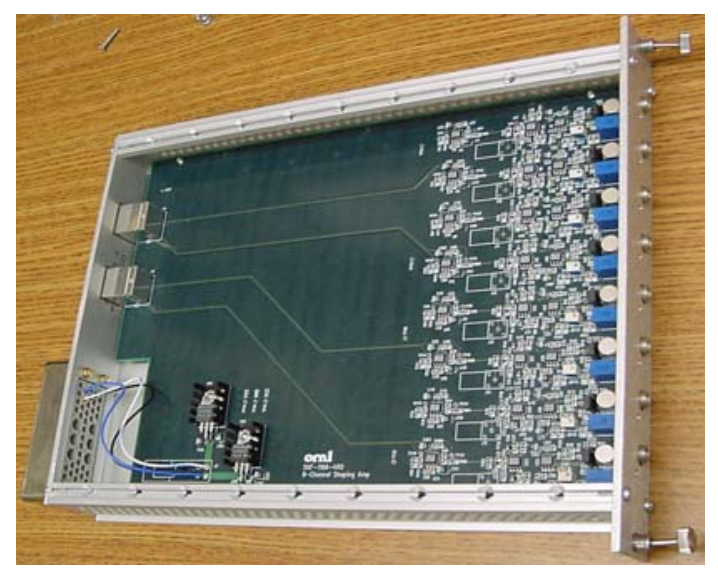

Figure B.14 Side view of shaping amplifier module 
Figure B.15 Shaping amplifier module front and read panels

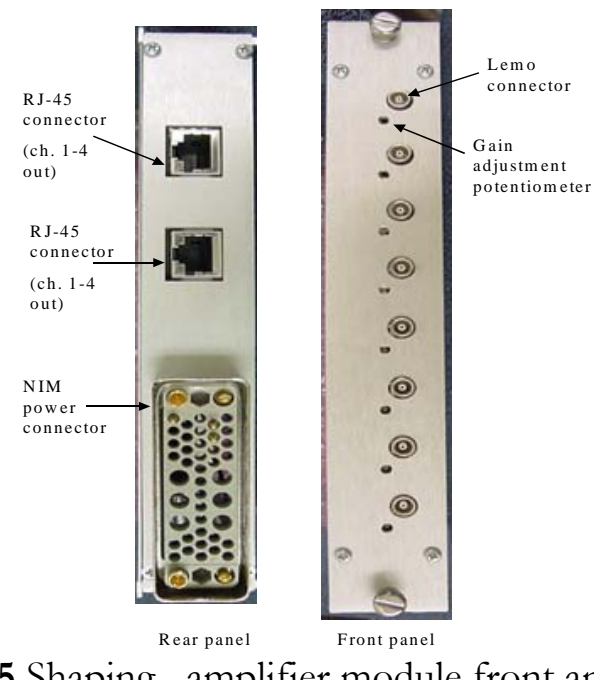

Gain adjustments to the channels are made using the variable attenuators accessible through the front panel. The full range of adjustment is 20 turns, and a clockwise rotation increases the gain. The potentiometers idle if turned pass the upper or lower limit, so there is no damage to the module from turning the control too far.

Each channel has a DC offset adjustment that allows adjusting the dc output voltage to zero. This affects the baseline of an amplified pulse and should be done prior to setting discriminator levels. To use this control, the side cover of the NIM module must be removed to gain access. Figure B.16 shows the location of the DC offset adjustment potentiometer for a typical channel. It is immediately to the left of the blue gain-adjust potentiometer for the corresponding channel.

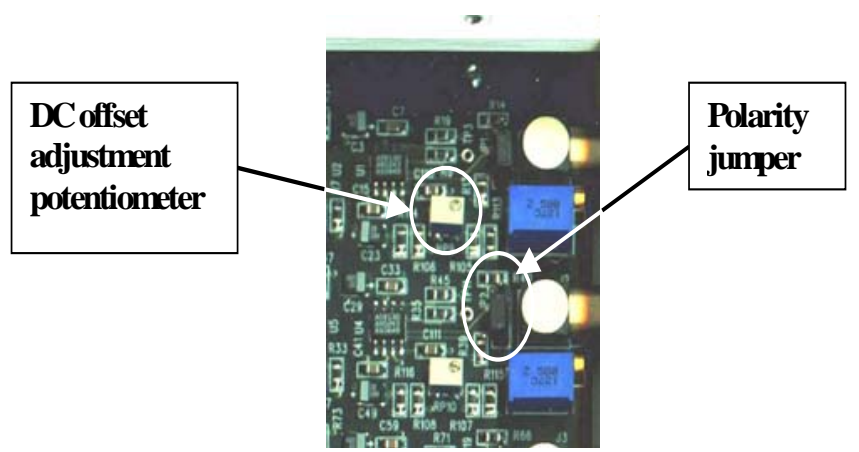

Figure B.16 Close-up view of circuit board showing adjustment locations.

The shaper amplifier module is initially set to provide non-inverting gain. If the polarity of the pulse needs to be inverted (to match the detector to the discriminator or other circuits following the shaper), it can be accomplished by changing a jumper setting. The polarity jumper for each channel is located just behind the input Lemo connector for that channel. For non-inverting gain, the jumper should be set on the lower two pins of the 3-pin header (lower meaning towards the bottom of the NIM module). For 
inverting gain, the jumper should be set on the upper two pins of the 3-pin header (upper meaning towards the top of the NIM module).

\section{Specifications}

DC power supply

Input impedance

Output capability

Gain

Shaping time constant

DC offset adjustment

$+/-6 \mathrm{~V} @ 230 \mathrm{~mA}$
50 ohms nominal
up to $+/-4 \mathrm{~V}$ into 100 ohms differential load
2 to 200 , adjustable via front panel potentiometers
25 ns (integration)
via internal potentiometers

+/-6V@230mA

up to $+/-4 \mathrm{~V}$ into 100 ohms differential load

2 to 200 , adjustable via front panel potentiometers

25 ns (integration)

Module/NaI readout Module

\section{Introduction}

The pulse processor module for MP-CARPT separates pulses through energy discrimination. It integrates detected and amplified pulses in order to produce a voltage that is proportional to the detected energy. This voltage is digitized and introduced to an energy analyzer that can sort the events according to non-overlapping energy windows. The pulse processor module keeps a count of how many pulses fall into each energy window.

A simplified diagram of this equipment is shown in Figure B.3. As an example of its use for MP-CARPT, consider the NaI spectrum for two hypothetical isotopes individually and combined that is shown in Figure B.4. Isotope 1 has a peak around 1.1 $\mathrm{MeV}$ while isotope 2 has a peak around $0.8 \mathrm{MeV}$. If two energy windows like those shown in Figure B.4 are set in the pulse processor, then gammas with energies between 1 and $1.2 \mathrm{MeV}$ will be routed to scaler 1, and gammas with energies between 0.7 and 0.9 $\mathrm{MeV}$ would be events counted by scaler 2 . Thus scaler 1 will count primarily events due to isotope 1 and scaler 2 will count mainly events due to isotope 2 . This illustrates the concept of counting energy-discriminated events using only two particles and two isotopes, but the concept can be extended to greater numbers.

For particle tracking, the pulse processor module must acquire energy discriminated data multiple time intervals and for multiple detectors. For example, counts might be accumulated for $50 \mathrm{~ms}$ before being read out and the counter reset. This process would be repeated over and over again for the duration of the experiment, which might last minutes or even hours. Determination of the particle's position requires using multiple, spatially separate detectors, typically 8 to 32 .

\section{Design Overview}

Figure B.17 is a block diagram of the pulse processor module. It has 8 channels with each consisting of an analog section, an ADC (Advanced Data Controller) and a digital section implemented using a Field-Programmable Gate Array (FPGA). One channel is 
used with each detector. Each channel is connected to another FPGA that controls the operation of the module. This FPGA controls the counting intervals, downloads setup parameters to the channel FPGAs and collects data from them. In turn, the control FPGA is connected to an IC (PLX9030) that interfaces to the PCI bus. The PLX9030 handles the PCI bus protocol allowing data transfers to and from the PC that is the bus master. These circuits are discussed in greater detail in the following subsections.

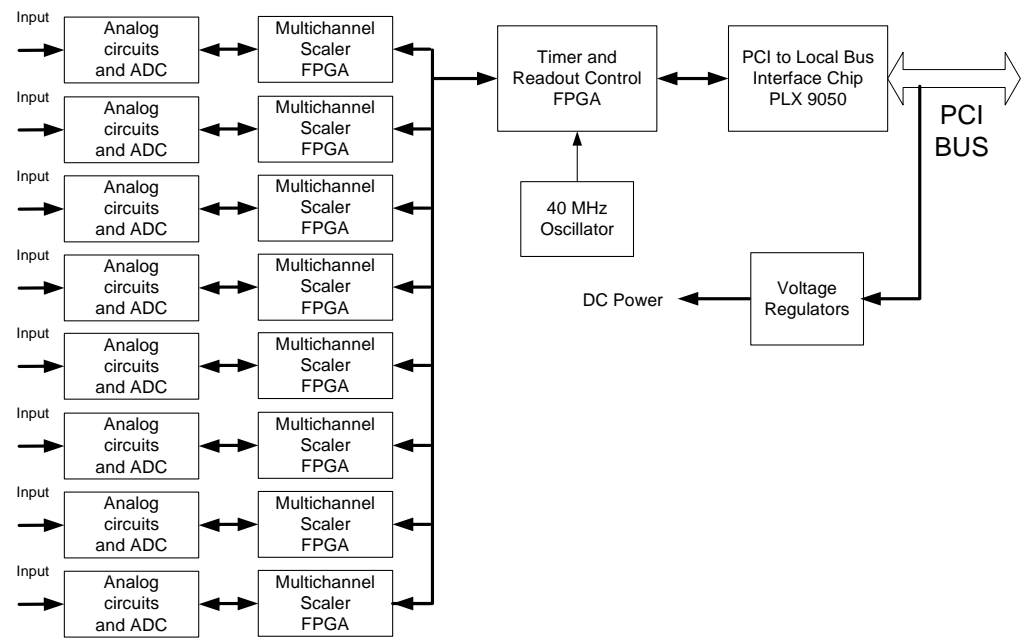

Figure B.17 Block diagram of the pulse processor module.

\section{Pulse Processor Module Analog Circuits}

Figure B.18 is a block diagram of one channel of the analog section showing connections to the shaping amplifier and to the digital section. Each channel has a differential receiver followed by both a gain stage and a delay line. The output of the gain stage is compared the initial limit threshold by a discriminator. If a pulse exceeds this threshold, it is assumed that it might be due to a suitable pulse (and not just noise), and the pulse processing process is started. An example of this type of event is shown as a timing diagram in Figure B.19. The gain stage output is low-pass filtered and discriminated again (this is the minimum discriminator.) If minimum discriminator fires within approximately 200 ns of the initial discriminator firing, it is assumed that the pulse was due to a real pulse and processing of the event should continue. If the second discriminator does not fire, then it is assumed that first discriminator fired on noise and the event should be rejected and not processed. 


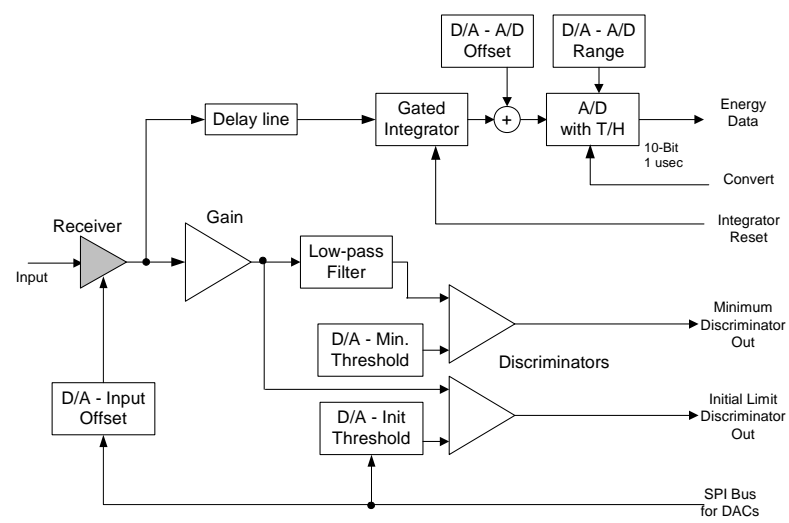

Figure B.18 Analog electronics for one channel.

The differential receiver also drives a delay line of approximately $400 \mathrm{~ns}$ in length. The delay line is then followed by a gated integrator. The use of delay lines allows the pulse height discriminators to gate the integrators on without losing any signal due to delays in gating. As seen in the timing diagram, the digital logic times from the initial pulse discriminator and opens the integrator reset switch after approximately $300 \mathrm{~ns}$. (Several of these parameters are programmable and are discussed further in the section on the pulse processor digital circuits.) This delay plus the discriminator delay should be less than the length of the delay line so the pulse is completely integrated. If the Minimum discriminator output does not go true during the $300 \mathrm{~ns}$ delay, the integrators are reset and the system waits for another pulse. If it does go true, the integrators are not reset until after they are sampled by their ADCs.

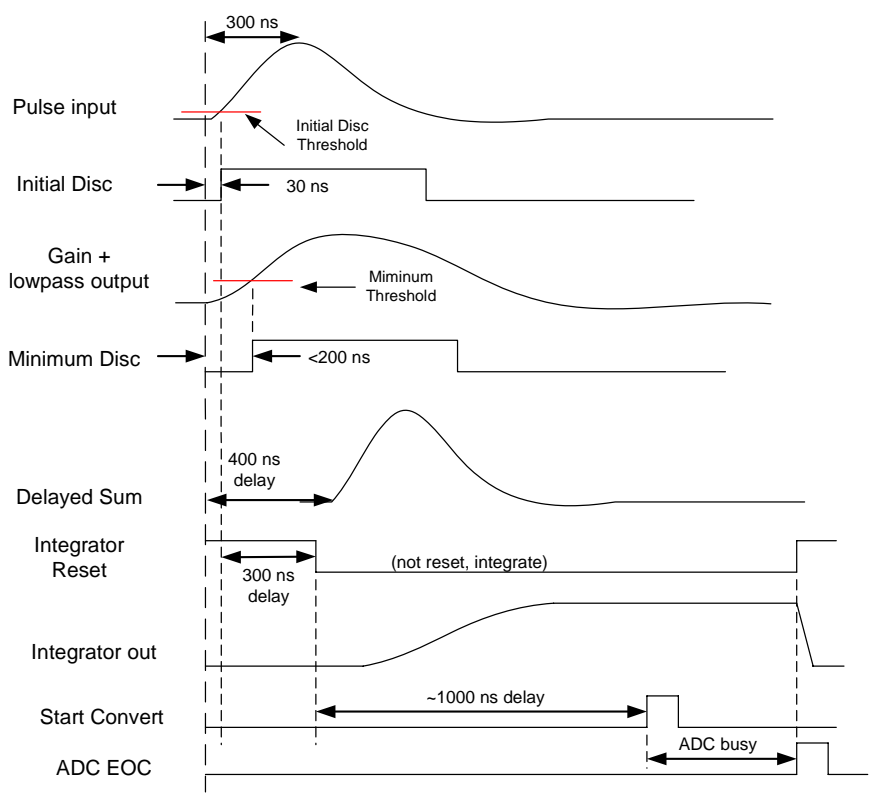

Figure B.19 Pulse Processor Timing Diagram. 
After sufficient integration time (this is programmable), the integrator output is sampled by the 10-bit ADC. This is shown in the timing diagram by the Start Convert signal for the case of a 1-microsecond integration time. The conversion takes approximately half a microsecond, and when the converter is finished, it gives an End of Conversion (EOC) signal. This signal causes the logic to reset the integrators, and the pulse processor channel is ready to process another pulse. The time taken between pulse detection and EOC is approximately 2 microseconds for this arrangement.

\section{Pulse Processor Module Digital Channel Circuits}

A digital portion implemented using a single FPGA follows the analog portion of the channel circuits. Figure B.20 shows the block diagram of the channel FPGA. The timing control block determines when a pulse is detected and controls the integration and digitization process. The energy window logic accepts digitized pulses and determines which scaler(s) should be incremented. The interface to the module controller transfers data to from the channel to the controller and control parameters from the module controller to the channels.

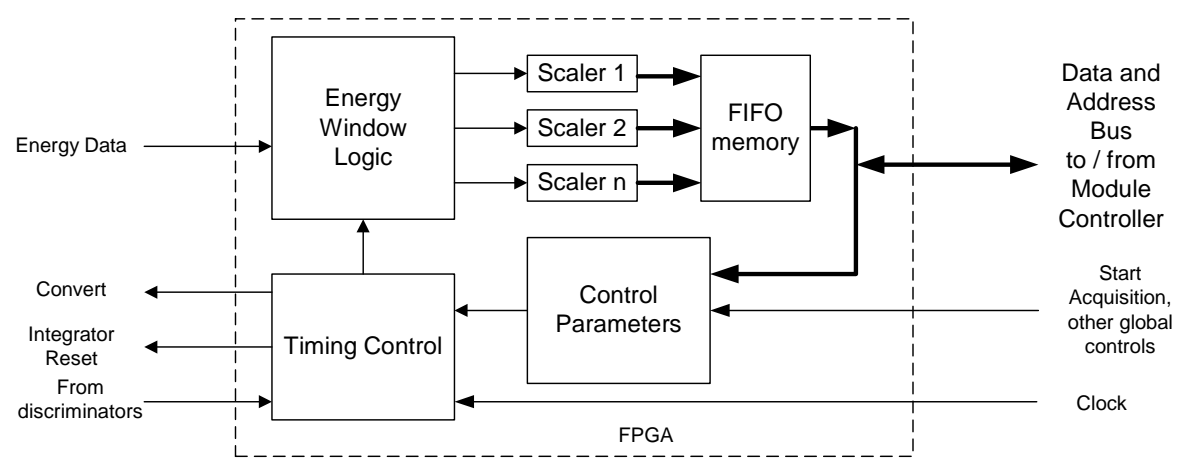

Figure B.20 Digital electronics for one channel.

The timing control logic uses the output of the initial discriminator to start a sequence of events. The pulse from this discriminator indicates that an input pulse has been detected. (See Figure B.19.) Shortly after this, the timing control releases the integrator reset (allow it to start integration) and then waits for the minimum discriminator to fire. If it fires within the prescribed amount of time, the process continues, if not, the process is aborted. After the integration is complete, the timing control logic starts the analog-to-digital converter and resets the integrator.

The logic that implements this process uses a $20-\mathrm{MHz}$ clock (the acquisition clock). Figure B.21 shows the timing diagram for the pulse acquisition process. The acquisition process starts with rising edge of the initial discriminator being synchronized to the acquisition clock. Because of the synchronization, starting the acquisition time counter (at $20 \mathrm{MHz}$ ) is delayed by 1 to 2 clock cycles (one clock cycle is $50 \mathrm{~ns}$ ). All acquisition time parameters (Pulse Timeout, Integrator release time, and Sampling time) are based on the acquisition time counter. This counter is zeroed before the pulse acquisition process begins and counts increments of $50 \mathrm{~ns}$. The Pulse Timeout parameter defines 
how long the process is to wait for the minimum discriminator signal (MIN_DISC) to occur. If the MIN_DISC signal does not occur, then the input pulse is defined as invalid and the process is reset. Otherwise, the integrated value of the input pulse will be converted and sent to the energy window logic. The integrator release time is the acquisition count value $(+1)$ where the integrator is released (changed from reset to not reset) to start the integration process. The sampling time $(+1)$ is the acquisition count value where the integrator analog input is sampled. After the analog input has been sampled, the integrator is reset. The ADC conversion process takes 14 acquisition clock cycles, and the energy windowing (binning) process takes either 1 clock cycle in coarse mode or 6 clock cycles in fine mode to finish the process. The total time for the acquisition process is approximately the Sampling Time +22 acquisition clock cycles. Any input pulses occurring more closely spaced than this value will result in distorted data.

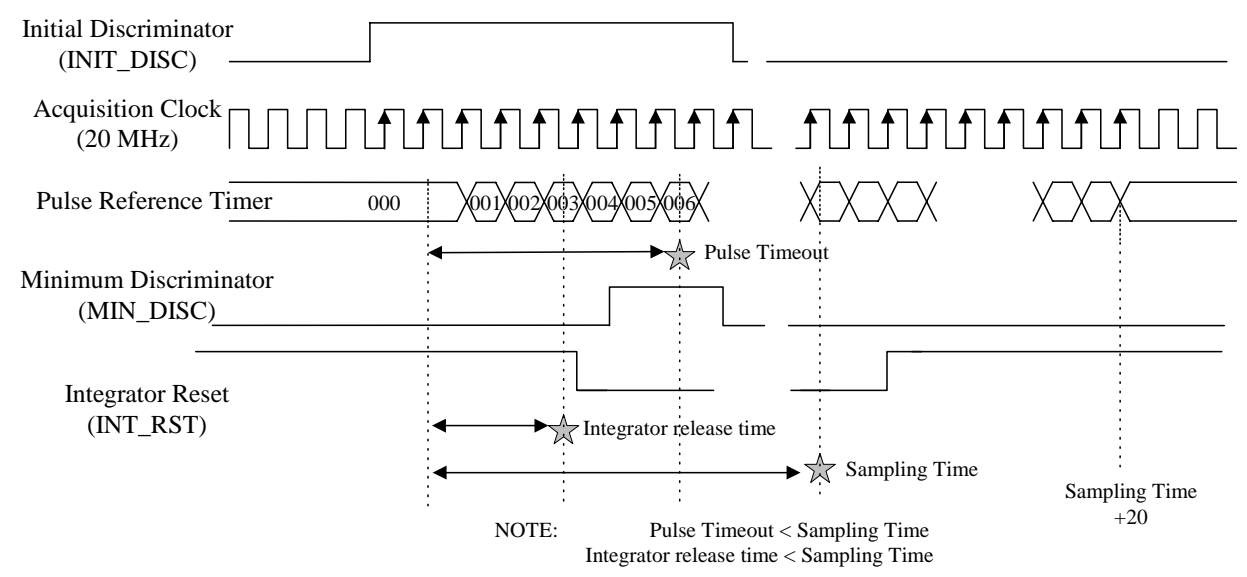

Figure B.21 Timing diagram for pulse acquisition in a channel.

Upon completion of the conversion, the digitized energy data goes to the energy window logic. This logic sorts the energy into windows. The pulse processor has two modes of sorting and counting pulses - coarse and fine windows. The coarse window mode takes data in the format needed for CARPT, while the fine window mode allows the processor to function as a multi-channel analyzer (MCA). The coarse mode will be described first.

In the coarse mode, for example, one window might correspond to the range of 1 to 1.2 $\mathrm{MeV}$ while another could be the range of 0.7 to $0.9 \mathrm{MeV}$. Each time a pulse is processed and if its energy falls within a window, the corresponding scaler is incremented by one count. The coarse mode has eight windows with independently adjustable upper and lower limits. Window limits are given in terms of ADC values (0 to 1023). If desired, the windows may overlap. Pulses are processed and counts accumulated as long as the module controller dictates. Each scaler can accumulate up to 65,535 counts $\left(2^{16}-1\right)$ before overflowing or being reset. An overflow condition is indicated by a count of 65,535 - the counters do not rollover. 
In the coarse mode, data is taken over multiple time intervals (sampling sessions) before being read out by the module controller. At the end of each time interval, the scaler values are transferred to a FIFO (first-in, first-out) memory that is part of the FPGA. The scalers are then reset and a new counting interval starts with minimal dead time. The size of the FIFO (4 Kbytes) limits the number of intervals to a maximum of 256 between readouts.

In the fine mode, the channel logic is used in a slightly different way to collect MCAtype data and to transfer it to the computer. Pulses are acquired and digitized in exactly the same way as in the coarse mode, but the energy window logic uses the FIFO to simply record how many times each possible ADC value (bin) occurred during the experiment. Each bin (memory location) can accumulate up to 65,535 counts before overflowing. The fine mode is a very useful feature as it allows collecting an energy spectrum that can be used to set up the energy windows for CARPT.

In fine mode, data is taken over one time interval (sampling session) before being read out by the module controller. This is due to the size of the FIFO memory holding the data. This memory is arranged as 1024 two-byte words.

\section{Bus Interface}

Figure B.22 shows a computer bus interface that is needed to transfer data for analysis and for adjustment of instrument parameters. Several interface and packaging formats were considered for the $\mathrm{NaI}$ readout electronics. It seemed most useful if the circuit cards were made as modules and plugged into a crate. One possibility was CAMAC. That had the advantage that the existing CARPT equipment uses CAMAC and the bus interface is relatively simple to design. However, there are a number of disadvantages. The CAMAC bus is relatively slow and interfacing the CAMAC crate to a pc or workstation generally requires conversion to another interface such as GPIB and then another conversion to the native bus for the pc or workstation used for data analysis. Another disadvantage is that the CAMAC format is not up to date and is increasing less commonly used.

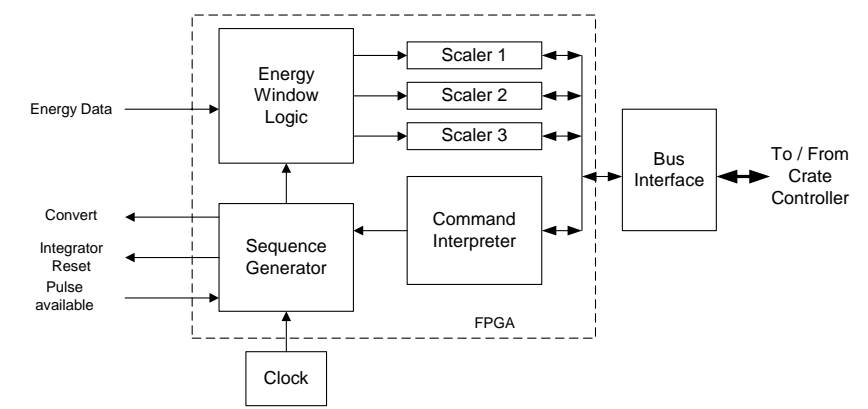

Figure B.22 Digital portion of $\mathrm{NaI}$ readout electronics.

Ultimately, it was decided to build the $\mathrm{NaI}$ readout electronics using a compact PCI (CPCI) format. This is an up-to-date standard that is more suited to the modern pc world and allows fast data transfers. This implementation uses commercially available 
crates and processor cards (Figure B.23). A big advantage of this arrangement is that a single board computer (SBC) can serve both as crate controller and as a data analysis engine.

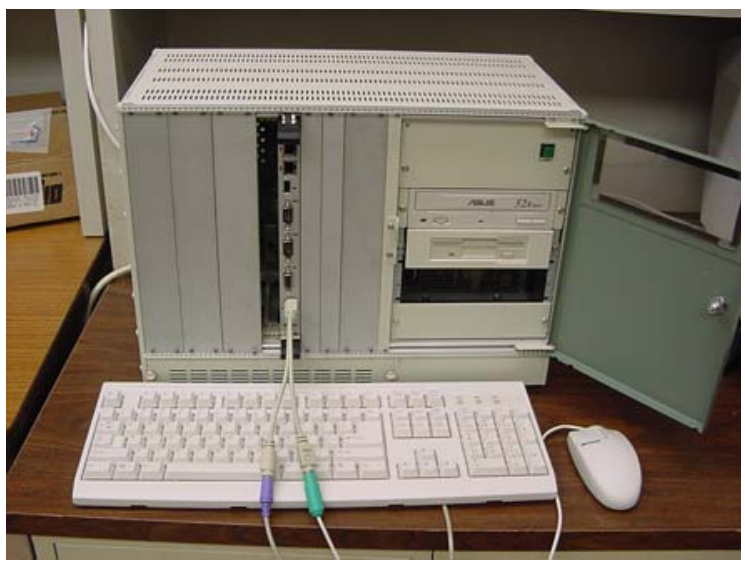

Figure B.23 CPCI crate and single board computer.

\section{Module Control and Bus Interface}

The module controller FPGA (Figure B.24) handles the interface to the PLX chip and the FPGA channels. For both interfaces, a 32-bit bus is used with a variety of address and enabling signals. The controller also configures all Digital to Analog Controllers (DACs) in the system via an SPI bus interface. These DACs set analog values such as thresholds and offsets. The controller's other function is to define general acquisition parameters and modes for the eight FPGA channels (global control signals). For example, the signal that enables counting is broadcast from the module controller FPGA to the channel FPGAs. The module controller FPGA also contains FIFO memory that is used to buffer data on readout of the channel FPGAs.

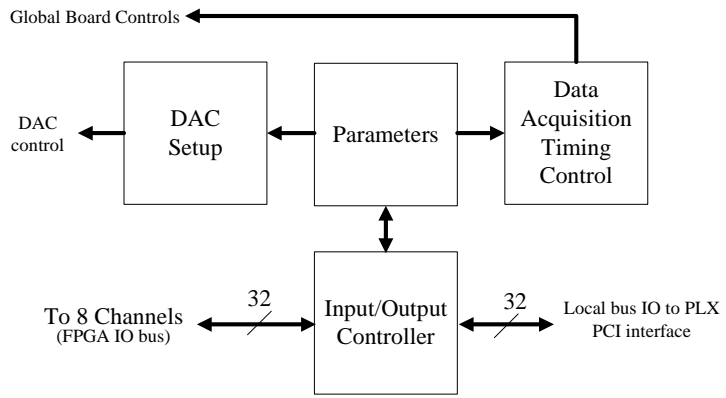

Figure B.24 Module Controller FPGA block diagram.

When the module controller enables the channel FPGAs to start counting pulses, it starts its own counter that counts cycles of a clock with a 6.4 microsecond period. The time interval (sampling session) duration is 6.4 microseconds times the value programmed into the sequence acquisition time register. This value may range from 1 to $2.15 * 10^{9}\left(2^{31}-1\right)$ that corresponds to a time interval of 6.4 microseconds to 3.8 hours. Smaller values (on the order of milliseconds) are appropriate for CARPT while larger 
values (minutes or hours) are appropriate for acquiring energy spectra. The contents of the various control registers are given fully in the section on module programming.

The number of time intervals for which counts are to be acquired is given by the number of sequences parameter in the Modes and Sequence Definition Register. The parameter may range from 0 to 255 if the coarse mode is selected. The actual number of intervals is one plus the value in the register. When the programmed number of intervals is reached, the data must be read out before any more can be taken. This results in a small amount of dead time.

The module controller can also operate in a "fake trigger mode" which causes data to be taken, analyzed, counted and read out with no input pulses. This is useful for adjusting offsets, measuring system noise and debugging the module. In this mode, the module functions normally, except that the module controller periodically provides a "trigger" that takes the place of the signals from the initial and minimum discriminators. The integrator and ADC are operated normally. The rate of fake triggers can be programmed via the Trigger Firing Count register.

\section{PCI Bus Interface}

The interface to the PCI bus is through a PLX PCI9030. This IC has a non-multiplexed local bus (separate address and data lines) connecting it to the module controller FPGA. It also provides a 32-bit, 33-MHz connection to the PCI bus and handles the PCI bus protocol. Commands and set-up parameters pass through the PCI bus to the module while data is transferred from the module through the PCI bus to the host pc.

\section{B.5.3 MP-CARPT Electronics Arrangement}

A somewhat more detailed picture of the MP-CARPT electronics is shown in Figure B.25. This drawing indicates the parts of the arrangement that are the same as in singleparticle CARPT and those that are different. It also indicates the main functions of the different elements.

\section{Module Implementation and Use}

Figure B.26 is a photograph of the pulse processor module. It is a $6 \mathrm{U}$ Compact PCI module. The connections to the shaping amplifier are made using the two RJ-45 connectors on the front panel while the connections to the PCI bus are via the P1 connector at the rear of the module. The module is not hot-swap compliant, and the CPCI crate should be powered down to remove or insert a pulse processor module. The pulse processor is designed to be used as part of a radiation detection system. A detailed diagram of electronics for a MP-CARPT experiment is shown in Figure B.25. This drawing indicates the parts of the arrangement that are the same as in singleparticle CARPT and those that are different. It also indicates the main functions of the different elements. 


\section{B.6 Module Programming and Data Access}

Operation of the module is memory mapped. Set-up and the initiation of data acquisition are accomplished by PCI-bus write operations, while data are acquired from the module via PCI-bus read operations. All data transfers use 32-bit (4 byte) words and are done in PCI non-bursting mode.

Normal data taking involves writing the various set-up parameters to the module and initiating data collection with write to the appropriate address. The software can monitor a status bit indicating when data collection has finished or may simply wait long enough before starting to read out data. The data may be read out by multiple reads to the appropriate address. (One read per data word.) Data collection may then be started again.

Reading address 110 or any address $800000 \mathrm{H}$ through FFFFFFH represents a data acquisition read once the module is collecting data. Each data acquisition read operation reads one data word. The next read operation reads the next word, etc. Data acquisition read operations are always sequential regardless of the address specified. For example, reading address $2000000 \mathrm{H}$ instead of $3000000 \mathrm{H}$ will not change the contents of the read. The address space was partitioned in this manner so bursting operations would possible if required. When the acquired data from the pulse processor module is being readout, the configuration parameters should NOT be read. 


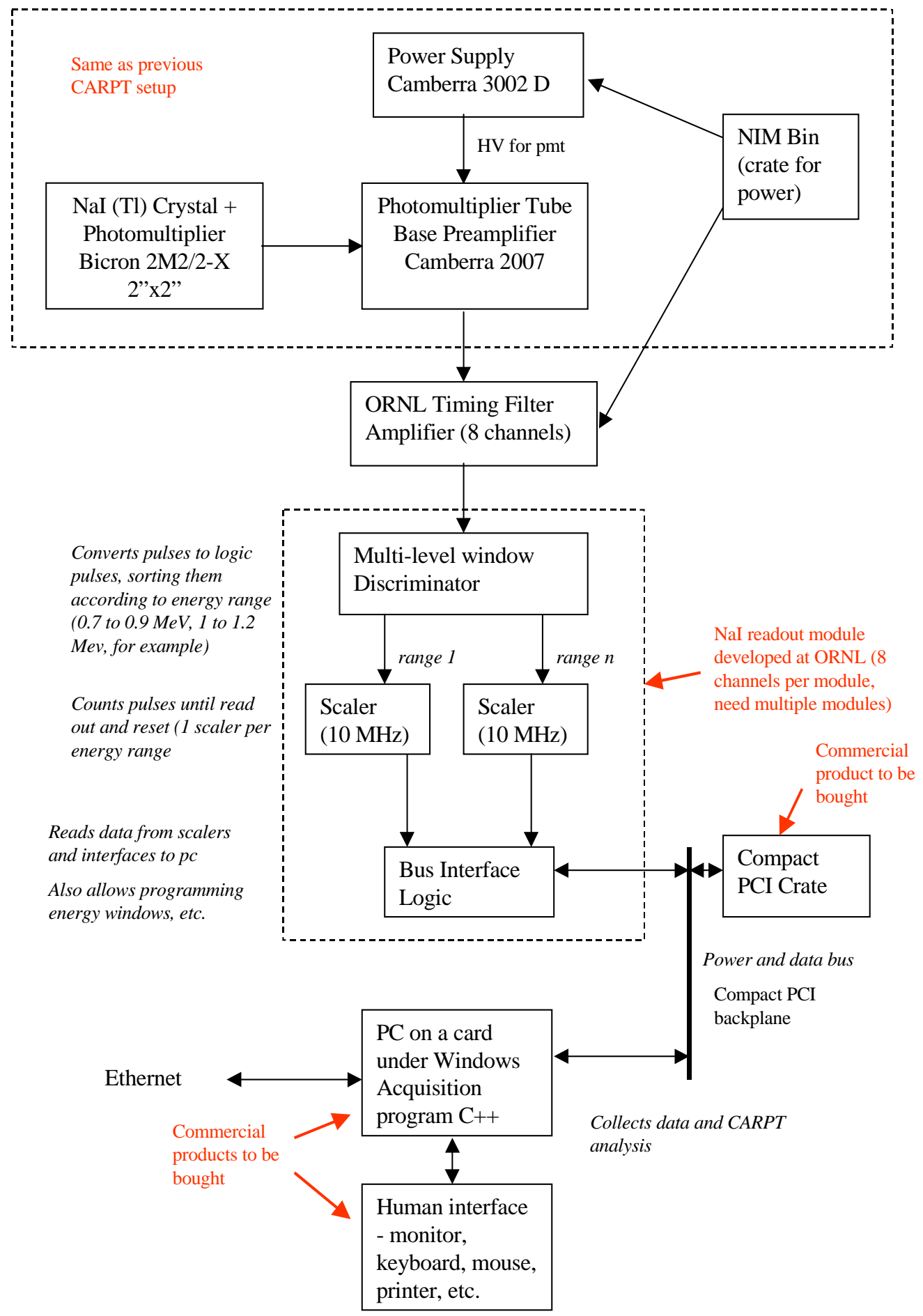

Figure B.25 MP-CARPT electronics showing NIM and CPCI elements. 
The register addresses to access a particular parameter or to start an operation are shown in Table B.1. These are the local-bus addresses for 4-byte words - each one corresponds to four PCI (1 byte) addresses. Some of register addresses store two or more parameters due to the parameters requiring less than 32 bits.

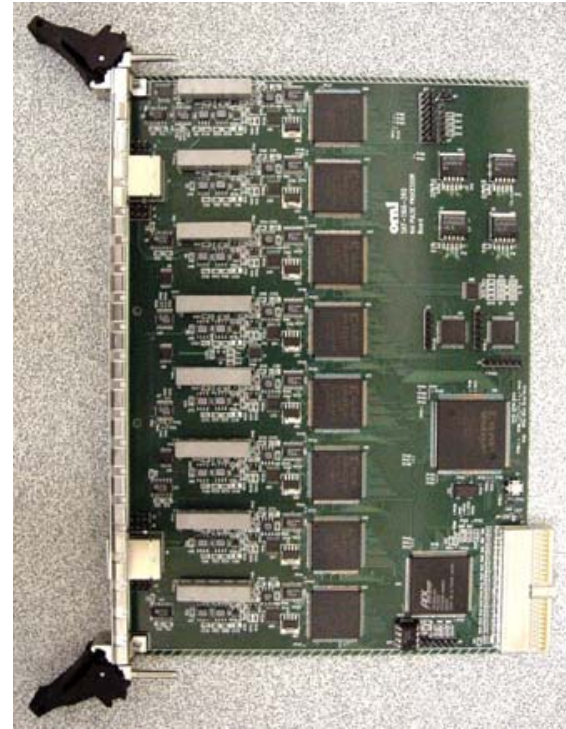

Figure B.26 Pulse processor module. 
Table B.1-a Channel 0 parameters

\begin{tabular}{|c|c|}
\hline ADDR (Hex) & Description \\
\hline 000 & $\begin{array}{c}\text { Channel 0 } \\
\text { Coarse BIN0 Threshold HIGH (31:16) } \\
\text { Coarse BIN0 Threshold LOW (15:0) }\end{array}$ \\
\hline 001 & $\begin{array}{c}\text { Channel 0 } \\
\text { Coarse BIN1 Threshold HIGH (31:16) } \\
\text { Coarse BIN1 Threshold LOW (15:0) }\end{array}$ \\
\hline 002 & $\begin{array}{c}\text { Channel 0 } \\
\text { Coarse BIN2 Threshold HIGH (31:16) } \\
\text { Coarse BIN2 Threshold LOW (15:0) }\end{array}$ \\
\hline 003 & $\begin{array}{c}\text { Channel 0 } \\
\text { Coarse BIN3 Threshold HIGH (31:16) } \\
\text { Coarse BIN3 Threshold LOW (15:0) }\end{array}$ \\
\hline 004 & $\begin{array}{c}\text { Channel 0 } \\
\text { Coarse BIN4 Threshold HIGH (31:16) } \\
\text { Coarse BIN4 Threshold LOW (15:0) }\end{array}$ \\
\hline 005 & $\begin{array}{c}\text { Channel 0 } \\
\text { Coarse BIN5 Threshold HIGH (31:16) } \\
\text { Coarse BIN5 Threshold LOW (15:0) }\end{array}$ \\
\hline 006 & $\begin{array}{c}\text { Channel 0 } \\
\text { Coarse BIN6 Threshold HIGH }(31: 16) \\
\text { Coarse BIN6 Threshold LOW }(15: 0)\end{array}$ \\
\hline 007 & $\begin{array}{c}\text { Channel 0 } \\
\text { Coarse BIN7 Threshold HIGH (31:16) } \\
\text { Coarse BIN7 Threshold LOW (15:0) }\end{array}$ \\
\hline 008 & $\begin{array}{l}\text { Channel } 0 \\
\text { Valid pulse timeout. This parameter is represents maximum time after } \\
\text { INIT_DISC for MIN_DISC to occur so the pulse is deemed valid. Each bit } \\
\text { corresponds to 50ns. (15:0) }\end{array}$ \\
\hline 009 & $\begin{array}{l}\text { Channel } 0 \\
\text { Sample time. Define the sample time for the ADC with respect to INIT_DISC. } \\
\text { Each bit corresponds to 50ns. }(15: 0)\end{array}$ \\
\hline $00 \mathrm{a}$ & $\begin{array}{c}\text { Channel } 0 \\
\text { Integrator release time. Define the time with respect to INIT_DISC where the } \\
\text { integrator is enabled. Each bit corresponds to 50ns. }(15: 0)\end{array}$ \\
\hline
\end{tabular}


Table B.1-b Channel 1 parameters

\begin{tabular}{|c|c|}
\hline ADDR (Hex) & Description \\
\hline 020 & $\begin{array}{c}\text { Channel 1 } \\
\text { Coarse BIN0 Threshold HIGH (31:16) } \\
\text { Coarse BIN0 Threshold LOW (15:0) }\end{array}$ \\
\hline 021 & $\begin{array}{c}\text { Channel 1 } \\
\text { Coarse BIN1 Threshold HIGH (31:16) } \\
\text { Coarse BIN1 Threshold LOW (15:0) }\end{array}$ \\
\hline 022 & $\begin{array}{c}\text { Channel 1 } \\
\text { Coarse BIN2 Threshold HIGH (31:16) } \\
\text { Coarse BIN2 Threshold LOW (15:0) }\end{array}$ \\
\hline 023 & $\begin{array}{c}\text { Channel 1 } \\
\text { Coarse BIN3 Threshold HIGH (31:16) } \\
\text { Coarse BIN3 Threshold LOW (15:0) }\end{array}$ \\
\hline 024 & $\begin{array}{c}\text { Channel 1 } \\
\text { Coarse BIN4 Threshold HIGH (31:16) } \\
\text { Coarse BIN4 Threshold LOW (15:0) }\end{array}$ \\
\hline 025 & $\begin{array}{c}\text { Channel 1 } \\
\text { Coarse BIN5 Threshold HIGH (31:16) } \\
\text { Coarse BIN5 Threshold LOW (15:0) }\end{array}$ \\
\hline 026 & $\begin{array}{c}\text { Channel 1 } \\
\text { Coarse BIN6 Threshold HIGH (31:16) } \\
\text { Coarse BIN6 Threshold LOW (15:0) }\end{array}$ \\
\hline 007 & $\begin{array}{c}\text { Channel 1 } \\
\text { Coarse BIN7 Threshold HIGH (31:16) } \\
\text { Coarse BIN7 Threshold LOW (15:0) }\end{array}$ \\
\hline 028 & $\begin{array}{l}\text { Channel } 1 \\
\text { Valid pulse timeout. This parameter is represents maximum time after } \\
\text { INIT_DISC for MIN_DISC to occur so the pulse is deemed valid. Each bit } \\
\text { corresponds to 50ns. (15:0) }\end{array}$ \\
\hline 029 & $\begin{array}{l}\text { Channel } 1 \\
\text { Sample time. Define the sample time for the ADC with respect to } \\
\text { INIT_DISC. Each bit corresponds to 50ns. (15:0) }\end{array}$ \\
\hline $02 \mathrm{a}$ & $\begin{array}{l}\text { Channel 1 } \\
\text { Integrator release time. Define the time with respect to INIT_DISC where the } \\
\text { integrator is enabled. Each bit corresponds to 50ns. (15:0) }\end{array}$ \\
\hline
\end{tabular}


Table B.1-c Channel 2 parameters

\begin{tabular}{|c|c|}
\hline ADDR (Hex) & Description \\
\hline 040 & $\begin{array}{c}\text { Channel } 2 \\
\text { Coarse BIN0 Threshold HIGH (31:16) } \\
\text { Coarse BIN0 Threshold LOW (15:0) }\end{array}$ \\
\hline 041 & $\begin{array}{c}\text { Channel } 2 \\
\text { Coarse BIN1 Threshold HIGH (31:16) } \\
\text { Coarse BIN1 Threshold LOW (15:0) }\end{array}$ \\
\hline 042 & $\begin{array}{c}\text { Channel 2 } \\
\text { Coarse BIN2 Threshold HIGH (31:16) } \\
\text { Coarse BIN2 Threshold LOW (15:0) }\end{array}$ \\
\hline 043 & $\begin{array}{c}\text { Channel } 2 \\
\text { Coarse BIN3 Threshold HIGH (31:16) } \\
\text { Coarse BIN3 Threshold LOW (15:0) }\end{array}$ \\
\hline 044 & $\begin{array}{c}\text { Channel 2 } \\
\text { Coarse BIN4 Threshold HIGH (31:16) } \\
\text { Coarse BIN4 Threshold LOW (15:0) }\end{array}$ \\
\hline 045 & $\begin{array}{c}\text { Channel } 2 \\
\text { Coarse BIN5 Threshold HIGH (31:16) } \\
\text { Coarse BIN5 Threshold LOW (15:0) }\end{array}$ \\
\hline 046 & $\begin{array}{c}\text { Channel } 2 \\
\text { Coarse BIN6 Threshold HIGH }(31: 16) \\
\text { Coarse BIN6 Threshold LOW }(15: 0)\end{array}$ \\
\hline 047 & $\begin{array}{c}\text { Channel } 2 \\
\text { Coarse BIN7 Threshold HIGH (31:16) } \\
\text { Coarse BIN7 Threshold LOW (15:0) }\end{array}$ \\
\hline 048 & $\begin{array}{l}\text { Channel } 2 \\
\text { Valid pulse timeout. This parameter is represents maximum time after } \\
\text { INIT_DISC for MIN_DISC to occur so the pulse is deemed valid. Each bit } \\
\text { corresponds to 50ns. (15:0) }\end{array}$ \\
\hline 049 & $\begin{array}{l}\text { Channel } 2 \\
\text { Sample time. Define the sample time for the ADC with respect to INIT_DISC. } \\
\text { Each bit corresponds to 50ns. }(15: 0)\end{array}$ \\
\hline $04 a$ & $\begin{array}{l}\text { Channel } 2 \\
\text { Integrator release time. Define the time with respect to INIT_DISC where the } \\
\text { integrator is enabled. Each bit corresponds to 50ns. }(15: 0)\end{array}$ \\
\hline
\end{tabular}


Table B.1-d Channel 3 parameters

\begin{tabular}{|c|c|}
\hline ADDR (Hex) & Description \\
\hline 060 & $\begin{array}{c}\text { Channel 3 } \\
\text { Coarse BIN0 Threshold HIGH (31:16) } \\
\text { Coarse BIN0 Threshold LOW (15:0) }\end{array}$ \\
\hline 061 & $\begin{array}{c}\text { Channel 3 } \\
\text { Coarse BIN1 Threshold HIGH (31:16) } \\
\text { Coarse BIN1 Threshold LOW (15:0) }\end{array}$ \\
\hline 062 & $\begin{array}{c}\text { Channel 3 } \\
\text { Coarse BIN2 Threshold HIGH (31:16) } \\
\text { Coarse BIN2 Threshold LOW (15:0) }\end{array}$ \\
\hline 063 & $\begin{array}{c}\text { Channel 3 } \\
\text { Coarse BIN3 Threshold HIGH (31:16) } \\
\text { Coarse BIN3 Threshold LOW (15:0) }\end{array}$ \\
\hline 064 & $\begin{array}{c}\text { Channel } 3 \\
\text { Coarse BIN4 Threshold HIGH (31:16) } \\
\text { Coarse BIN4 Threshold LOW (15:0) }\end{array}$ \\
\hline 065 & $\begin{array}{c}\text { Channel } 3 \\
\text { Coarse BIN5 Threshold HIGH (31:16) } \\
\text { Coarse BIN5 Threshold LOW (15:0) }\end{array}$ \\
\hline 066 & $\begin{array}{c}\text { Channel 3 } \\
\text { Coarse BIN6 Threshold HIGH (31:16) } \\
\text { Coarse BIN6 Threshold LOW (15:0) }\end{array}$ \\
\hline 067 & $\begin{array}{c}\text { Channel 3 } \\
\text { Coarse BIN7 Threshold HIGH (31:16) } \\
\text { Coarse BIN7 Threshold LOW (15:0) }\end{array}$ \\
\hline 068 & $\begin{array}{l}\text { Channel } 3 \\
\text { Valid pulse timeout. This parameter is represents maximum time after } \\
\text { INIT_DISC for MIN_DISC to occur so the pulse is deemed valid. Each bit } \\
\text { corresponds to 50ns. }(15: 0)\end{array}$ \\
\hline 069 & $\begin{array}{l}\text { Channel } 3 \\
\text { Sample time. Define the sample time for the ADC with respect to } \\
\text { INIT_DISC. Each bit corresponds to 50ns. (15:0) }\end{array}$ \\
\hline $06 a$ & $\begin{array}{l}\text { Channel } 3 \\
\text { Integrator release time. Define the time with respect to INIT_DISC where } \\
\text { the integrator is enabled. Each bit corresponds to 50ns. (15:0) }\end{array}$ \\
\hline
\end{tabular}


Table B.1-e Channel 4 parameters

\begin{tabular}{|c|c|}
\hline ADDR (Hex) & Description \\
\hline 080 & $\begin{array}{c}\text { Channel } 4 \\
\text { Coarse BIN0 Threshold HIGH (31:16) } \\
\text { Coarse BIN0 Threshold LOW (15:0) }\end{array}$ \\
\hline 081 & $\begin{array}{c}\text { Channel } 4 \\
\text { Coarse BIN1 Threshold HIGH (31:16) } \\
\text { Coarse BIN1 Threshold LOW (15:0) }\end{array}$ \\
\hline 082 & $\begin{array}{c}\text { Channel } 4 \\
\text { Coarse BIN2 Threshold HIGH (31:16) } \\
\text { Coarse BIN2 Threshold LOW (15:0) }\end{array}$ \\
\hline 083 & $\begin{array}{c}\text { Channel } 4 \\
\text { Coarse BIN3 Threshold HIGH (31:16) } \\
\text { Coarse BIN3 Threshold LOW (15:0) }\end{array}$ \\
\hline 084 & $\begin{array}{c}\text { Channel } 4 \\
\text { Coarse BIN4 Threshold HIGH (31:16) } \\
\text { Coarse BIN4 Threshold LOW (15:0) }\end{array}$ \\
\hline 085 & $\begin{array}{c}\text { Channel } 4 \\
\text { Coarse BIN5 Threshold HIGH (31:16) } \\
\text { Coarse BIN5 Threshold LOW (15:0) }\end{array}$ \\
\hline 086 & $\begin{array}{c}\text { Channel } 4 \\
\text { Coarse BIN6 Threshold HIGH (31:16) } \\
\text { Coarse BIN6 Threshold LOW (15:0) }\end{array}$ \\
\hline 087 & $\begin{array}{c}\text { Channel } 4 \\
\text { Coarse BIN7 Threshold HIGH (31:16) } \\
\text { Coarse BIN7 Threshold LOW (15:0) }\end{array}$ \\
\hline 088 & $\begin{array}{l}\text { Channel } 4 \\
\text { Valid pulse timeout. This parameter is represents maximum time after } \\
\text { INIT_DISC for MIN_DISC to occur so the pulse is deemed valid. Each } \\
\text { bit corresponds to 50ns. }(15: 0)\end{array}$ \\
\hline 089 & $\begin{array}{l}\text { Channel } 4 \\
\text { Sample time. Define the sample time for the ADC with respect to } \\
\text { INIT_DISC. Each bit corresponds to 50ns. (15:0) }\end{array}$ \\
\hline $08 \mathrm{a}$ & $\begin{array}{l}\text { Channel } 4 \\
\text { Integrator release time. Define the time with respect to INIT_DISC } \\
\text { where the integrator is enabled. Each bit corresponds to 50ns. (15:0) }\end{array}$ \\
\hline
\end{tabular}


Table B.1-f Channel 5 parameters

\begin{tabular}{|c|c|}
\hline ADDR (Hex) & Description \\
\hline $\mathrm{OaO}$ & $\begin{array}{c}\text { Channel } 5 \\
\text { Coarse BIN0 Threshold HIGH (31:16) } \\
\text { Coarse BIN0 Threshold LOW (15:0) }\end{array}$ \\
\hline 0a1 & $\begin{array}{c}\text { Channel } 5 \\
\text { Coarse BIN1 Threshold HIGH (31:16) } \\
\text { Coarse BIN1 Threshold LOW (15:0) }\end{array}$ \\
\hline $0 \mathrm{a} 2$ & $\begin{array}{c}\text { Channel } 5 \\
\text { Coarse BIN2 Threshold HIGH (31:16) } \\
\text { Coarse BIN2 Threshold LOW (15:0) }\end{array}$ \\
\hline $0 \mathrm{a3}$ & $\begin{array}{c}\text { Channel } 5 \\
\text { Coarse BIN3 Threshold HIGH (31:16) } \\
\text { Coarse BIN3 Threshold LOW (15:0) }\end{array}$ \\
\hline $0 \mathrm{a} 4$ & $\begin{array}{c}\text { Channel } 5 \\
\text { Coarse BIN4 Threshold HIGH (31:16) } \\
\text { Coarse BIN4 Threshold LOW (15:0) }\end{array}$ \\
\hline $0 a 5$ & $\begin{array}{c}\text { Channel } 5 \\
\text { Coarse BIN5 Threshold HIGH (31:16) } \\
\text { Coarse BIN5 Threshold LOW (15:0) }\end{array}$ \\
\hline $0 \mathrm{a} 6$ & $\begin{array}{c}\text { Channel } 5 \\
\text { Coarse BIN6 Threshold HIGH (31:16) } \\
\text { Coarse BIN6 Threshold LOW (15:0) }\end{array}$ \\
\hline $0 \mathrm{a} 7$ & $\begin{array}{c}\text { Channel } 5 \\
\text { Coarse BIN7 Threshold HIGH (31:16) } \\
\text { Coarse BIN7 Threshold LOW (15:0) }\end{array}$ \\
\hline $0 \mathrm{a} 8$ & $\begin{array}{l}\text { Channel } 5 \\
\text { Valid pulse timeout. This parameter is represents maximum time after } \\
\text { INIT_DISC for MIN_DISC to occur so the pulse is deemed valid. Each bit } \\
\text { corresponds to 50ns. (15:0) }\end{array}$ \\
\hline 0a9 & $\begin{array}{l}\text { Channel } 5 \\
\text { Sample time. Define the sample time for the ADC with respect to INIT_DISC. } \\
\text { Each bit corresponds to 50ns. (15:0) }\end{array}$ \\
\hline Oaa & $\begin{array}{l}\text { Channel } 5 \\
\text { Integrator release time. Define the time with respect to INIT_DISC where the } \\
\text { integrator is enabled. Each bit corresponds to 50ns. }(15: 0)\end{array}$ \\
\hline
\end{tabular}


Table B.1-g Channel 6 parameters

\begin{tabular}{|c|c|}
\hline ADDR (Hex) & Description \\
\hline $0 \mathrm{c} 0$ & $\begin{array}{c}\text { Channel } 6 \\
\text { Coarse BIN0 Threshold HIGH (31:16) } \\
\text { Coarse BIN0 Threshold LOW (15:0) }\end{array}$ \\
\hline $0 \mathrm{c} 1$ & $\begin{array}{c}\text { Channel 6 } \\
\text { Coarse BIN1 Threshold HIGH (31:16) } \\
\text { Coarse BIN1 Threshold LOW (15:0) }\end{array}$ \\
\hline $0 \mathrm{c} 2$ & $\begin{array}{c}\text { Channel } 6 \\
\text { Coarse BIN2 Threshold HIGH (31:16) } \\
\text { Coarse BIN2 Threshold LOW (15:0) }\end{array}$ \\
\hline $0 \mathrm{c3}$ & $\begin{array}{c}\text { Channel } 6 \\
\text { Coarse BIN3 Threshold HIGH (31:16) } \\
\text { Coarse BIN3 Threshold LOW (15:0) }\end{array}$ \\
\hline $0 \mathrm{c} 4$ & $\begin{array}{c}\text { Channel } 6 \\
\text { Coarse BIN4 Threshold HIGH (31:16) } \\
\text { Coarse BIN4 Threshold LOW (15:0) }\end{array}$ \\
\hline $0 \mathrm{c5}$ & $\begin{array}{c}\text { Channel } 6 \\
\text { Coarse BIN5 Threshold HIGH (31:16) } \\
\text { Coarse BIN5 Threshold LOW (15:0) }\end{array}$ \\
\hline $0 \mathrm{c} 6$ & $\begin{array}{c}\text { Channel 6 } \\
\text { Coarse BIN6 Threshold HIGH (31:16) } \\
\text { Coarse BIN6 Threshold LOW (15:0) }\end{array}$ \\
\hline $0 \mathrm{c} 7$ & $\begin{array}{c}\text { Channel 6 } \\
\text { Coarse BIN7 Threshold HIGH (31:16) } \\
\text { Coarse BIN7 Threshold LOW (15:0) }\end{array}$ \\
\hline $0 \mathrm{c} 8$ & $\begin{array}{l}\text { Channel } 6 \\
\text { Valid pulse timeout. This parameter is represents maximum time after } \\
\text { INIT_DISC for MIN_DISC to occur so the pulse is deemed valid. Each bit } \\
\text { corresponds to 50ns. }(15: 0)\end{array}$ \\
\hline 0c9 & $\begin{array}{l}\text { Channel } 6 \\
\text { Sample time. Define the sample time for the ADC with respect to } \\
\text { INIT_DISC. Each bit corresponds to 50ns. (15:0) }\end{array}$ \\
\hline $0 \mathrm{ca}$ & $\begin{array}{l}\text { Channel } 6 \\
\text { Integrator release time. Define the time with respect to INIT_DISC where the } \\
\text { integrator is enabled. Each bit corresponds to 50ns. }(15: 0)\end{array}$ \\
\hline
\end{tabular}


Table B.1-h Channel 7 parameters

\begin{tabular}{|c|c|}
\hline ADDR (Hex) & Description \\
\hline $0 \mathrm{e} 0$ & $\begin{array}{c}\text { Channel } 7 \\
\text { Coarse BIN0 Threshold HIGH (31:16) } \\
\text { Coarse BIN0 Threshold LOW (15:0) }\end{array}$ \\
\hline $0 \mathrm{e} 1$ & $\begin{array}{c}\text { Channel } 7 \\
\text { Coarse BIN1 Threshold HIGH (31:16) } \\
\text { Coarse BIN1 Threshold LOW (15:0) }\end{array}$ \\
\hline $0 \mathrm{e} 2$ & $\begin{array}{c}\text { Channel } 7 \\
\text { Coarse BIN2 Threshold HIGH (31:16) } \\
\text { Coarse BIN2 Threshold LOW (15:0) }\end{array}$ \\
\hline $0 \mathrm{e} 3$ & $\begin{array}{c}\text { Channel } 7 \\
\text { Coarse BIN3 Threshold HIGH (31:16) } \\
\text { Coarse BIN3 Threshold LOW (15:0) }\end{array}$ \\
\hline 0e4 & $\begin{array}{c}\text { Channel } 7 \\
\text { Coarse BIN4 Threshold HIGH (31:16) } \\
\text { Coarse BIN4 Threshold LOW (15:0) }\end{array}$ \\
\hline $0 \mathrm{e} 5$ & $\begin{array}{c}\text { Channel } 7 \\
\text { Coarse BIN5 Threshold HIGH (31:16) } \\
\text { Coarse BIN5 Threshold LOW (15:0) }\end{array}$ \\
\hline $0 \mathrm{e} 6$ & $\begin{array}{c}\text { Channel } 7 \\
\text { Coarse BIN6 Threshold HIGH (31:16) } \\
\text { Coarse BIN6 Threshold LOW (15:0) }\end{array}$ \\
\hline $0 \mathrm{e} 7$ & $\begin{array}{c}\text { Channel } 7 \\
\text { Coarse BIN7 Threshold HIGH (31:16) } \\
\text { Coarse BIN7 Threshold LOW (15:0) }\end{array}$ \\
\hline $0 \mathrm{e} 8$ & $\begin{array}{l}\text { Channel } 7 \\
\text { Valid pulse timeout. This parameter is represents maximum time after } \\
\text { INIT_DISC for MIN_DISC to occur so the pulse is deemed valid. Each bit } \\
\text { corresponds to 50ns. }(15: 0)\end{array}$ \\
\hline $0 \mathrm{e} 9$ & $\begin{array}{l}\text { Channel } 7 \\
\text { Sample time. Define the sample time for the ADC with respect to } \\
\text { INIT_DISC. Each bit corresponds to 50ns. (15:0) }\end{array}$ \\
\hline Oea & $\begin{array}{l}\text { Channel } 7 \\
\begin{array}{l}\text { Integrator release time. Define the time with respect to INIT_DISC where the } \\
\text { integrator is enabled. Each bit corresponds to 50ns. (15:0) }\end{array}\end{array}$ \\
\hline
\end{tabular}


Table B.1-i System parameters

\begin{tabular}{|c|c|c|}
\hline ADDR (Hex) & & Description \\
\hline 100 & \multicolumn{2}{|r|}{ Sequence acquisition time in $6.4 \mu$ s increments. $(31: 0)$} \\
\hline \multirow[t]{4}{*}{101} & \multicolumn{2}{|r|}{ Modes and Sequence Definition } \\
\hline & $7: 0$ & $\begin{array}{l}\text { Number of sequences. If NS is the number of sequences } \\
\text { defined, then a total of NS }+1 \text { sequences will be run. }\end{array}$ \\
\hline & 8 & $\begin{array}{c}\text { Acquisition type } \\
0=\text { Coarse, } 1=\text { Fine }\end{array}$ \\
\hline & $10: 9$ & $\begin{array}{c}\text { Acquisition mode } \\
00=\text { Normal } \\
01=\text { Fixed coarse pattern. (for module testing only) } \\
10=\text { Fake trigger mode. } \\
11=\text { Normal }\end{array}$ \\
\hline \multirow[t]{8}{*}{102} & \multicolumn{2}{|r|}{ Acquisition Status } \\
\hline & 18:0 & $\begin{array}{l}\text { Word count (number of } 32 \text { bit words) read by the host } \\
\text { computer. }\end{array}$ \\
\hline & 19 & Data Ready for transfer. 1=Ready \\
\hline & 20 & BIOMASS card FIFO empty. \\
\hline & 21 & Acquisition enabled. \\
\hline & 22 & $\begin{array}{l}\text { FIFO hold-off. BIOMASS FIFO has occurred sufficient data } \\
\text { to be read. }\end{array}$ \\
\hline & 23 & FIFO Full Error. The BIOMASS card had a FIFO go full. \\
\hline & $31: 24$ & Channel error indicators. \\
\hline 103 & \multicolumn{2}{|c|}{$\begin{array}{l}\text { Start Acquisition. } \\
\begin{array}{c}\text { Writing any data to this address starts the acquisition. Reading this address } \\
\text { returns the last word written to the card. }\end{array}\end{array}$} \\
\hline 104 & \multicolumn{2}{|r|}{ Stop Acquisition. (abort current operation) } \\
\hline 105 & \multicolumn{2}{|c|}{$\begin{array}{l}\text { Write DAC. } \\
\text { The parameters stored in the control FPGA (Addresses } 180 \mathrm{H} \text { to } 1 \mathrm{~A} 7 \mathrm{H}) \text { are } \\
\text { written to the DACs via the board SPI bus. Changing the DAC values } \\
\text { requires first writing new values to the appropriate addresses }(180 \mathrm{H} \text {, etc.) }\end{array}$} \\
\hline 106 & \multicolumn{2}{|c|}{$\begin{array}{c}\text { Trigger Firing Count. } \\
\text { In Fake trigger mode, this value defines how often a channel fakes valid pulse } \\
\text { and begins the integration process. Specifically, this variable is used in } \\
\text { calibration and debugging modes. }\end{array}$} \\
\hline 108 & \multicolumn{2}{|c|}{ Word Count value at end of Channel 0 (bits 18:0) DEBUGGING } \\
\hline 109 & \multicolumn{2}{|c|}{ Word Count value at end of Channel 1 (bits 18:0) DEBUGGING } \\
\hline $10 \mathrm{a}$ & \multicolumn{2}{|c|}{ Word Count value at end of Channel 2 (bits 18:0) DEBUGGING } \\
\hline $10 \mathrm{~b}$ & \multicolumn{2}{|c|}{ Word Count value at end of Channel 3 (bits 18:0) DEBUGGING } \\
\hline $10 \mathrm{c}$ & \multicolumn{2}{|c|}{ Word Count value at end of Channel 4 (bits 18:0) DEBUGGING } \\
\hline $10 \mathrm{~d}$ & \multicolumn{2}{|c|}{ Word Count value at end of Channel 5 (bits 18:0) DEBUGGING } \\
\hline $10 \mathrm{e}$ & \multicolumn{2}{|c|}{ Word Count value at end of Channel 6 (bits 18:0) DEBUGGING } \\
\hline
\end{tabular}


Appendix-2: Vesvikar(2006), D.Sc. Thesis

Table B.1-j Analog Control Parameters

\begin{tabular}{|c|c|}
\hline $\begin{array}{c}\text { Address } \\
\text { (Hex) }\end{array}$ & Description \\
\hline 180 & DAC Parameter DACSEL $=1, A D D R=0$ : Input offset Channel 0 \\
\hline 181 & DAC Parameter DACSEL=1, ADDR=1: Input offset Channel 1 \\
\hline 182 & DAC Parameter DACSEL $=2$, ADDR $=0$ : Input offset Channel 2 \\
\hline 183 & DAC Parameter DACSEL=2, ADDR=1: Input offset Channel 3 \\
\hline 184 & DAC Parameter DACSEL $=3$, ADDR $=0$ : Input offset Channel 4 \\
\hline 185 & DAC Parameter DACSEL=3, ADDR=1: Input offset Channel 5 \\
\hline 186 & DAC Parameter DACSEL=4, ADDR=0: Input offset Channel 6 \\
\hline 187 & DAC Parameter DACSEL=4, ADDR=1: Input offset Channel 7 \\
\hline 188 & DAC Parameter DACSEL=5, ADDR=0: Minimum Threshold Channel 0 \\
\hline 189 & DAC Parameter DACSEL=5, ADDR=1: Minimum Threshold Channel 1 \\
\hline $18 \mathrm{a}$ & DAC Parameter DACSEL=5, ADDR=2: Minimum Threshold Channel 2 \\
\hline $18 \mathrm{~b}$ & DAC Parameter DACSEL=5, ADDR=3: Minimum Threshold Channel 3 \\
\hline $18 \mathrm{c}$ & DAC Parameter DACSEL=5, ADDR=4: Minimum Threshold Channel 4 \\
\hline $18 \mathrm{~d}$ & DAC Parameter DACSEL=5, ADDR=5: Minimum Threshold Channel 5 \\
\hline $18 \mathrm{e}$ & DAC Parameter DACSEL=5, ADDR=6: Minimum Threshold Channel 6 \\
\hline $18 \mathrm{f}$ & DAC Parameter DACSEL=5, ADDR=7: Minimum Threshold Channel 7 \\
\hline 190 & DAC Parameter DACSEL $=6$, ADDR $=0$ : Initial Threshold Channel 0 \\
\hline 191 & DAC Parameter DACSEL=6, ADDR=1: Initial Threshold Channel 1 \\
\hline 192 & DAC Parameter DACSEL=6, ADDR=2: Initial Threshold Channel 2 \\
\hline 193 & DAC Parameter DACSEL=6, ADDR=3: Initial Threshold Channel 3 \\
\hline 194 & DAC Parameter DACSEL=6, ADDR=4: Initial Threshold Channel 4 \\
\hline 195 & DAC Parameter DACSEL=6, ADDR=5: Initial Threshold Channel 5 \\
\hline 196 & DAC Parameter DACSEL=6, ADDR=6: Initial Threshold Channel 6 \\
\hline 197 & DAC Parameter DACSEL=6, ADDR=7: Initial Threshold Channel 7 \\
\hline 198 & DAC Parameter DACSEL=5, ADDR=8: Full scale reference Channel 0 \\
\hline 199 & DAC Parameter DACSEL $=5$, ADDR=9: Full scale reference Channel 1 \\
\hline $19 \mathrm{a}$ & DAC Parameter DACSEL=5, ADDR=10: Full scale reference Channel 2 \\
\hline $19 \mathrm{~b}$ & DAC Parameter DACSEL=5, ADDR=11: Full scale reference Channel 3 \\
\hline $19 \mathrm{c}$ & DAC Parameter DACSEL=6, ADDR=8: Full scale reference Channel 4 \\
\hline $19 \mathrm{~d}$ & DAC Parameter DACSEL $=6$, ADDR=9: Full scale reference Channel 5 \\
\hline $19 \mathrm{e}$ & DAC Parameter DACSEL $=6$, ADDR=10: Full scale reference Channel 6 \\
\hline $19 \mathrm{f}$ & DAC Parameter DACSEL=6, ADDR=11: Full scale reference Channel 7 \\
\hline $1 \mathrm{~A} 0$ & DAC Parameter DACSEL=7, ADDR=0: ADC offset Channel 0 \\
\hline 1A1 & DAC Parameter DACSEL=7, ADDR=1: ADC offset Channel 1 \\
\hline $1 \mathrm{~A} 2$ & DAC Parameter DACSEL=7, ADDR=2: ADC offset Channel 2 \\
\hline $1 \mathrm{~A} 3$ & DAC Parameter DACSEL=7, ADDR=3: ADC offset Channel 3 \\
\hline $1 \mathrm{~A} 4$ & DAC Parameter DACSEL=7, ADDR=4: ADC offset Channel 4 \\
\hline 1A5 & DAC Parameter DACSEL=7, ADDR=5: ADC offset Channel 5 \\
\hline $1 \mathrm{~A} 6$ & DAC Parameter DACSEL=7, ADDR=6: ADC offset Channel 6 \\
\hline $1 \mathrm{~A} 7$ & DAC Parameter DACSEL=7, ADDR=7: ADC offset Channel 7 \\
\hline
\end{tabular}


Table B.1-k System ID and data acquisition

\begin{tabular}{|c|c|}
\hline ADDR (Hex) & Description \\
\hline 110 & Read-only. The acquisition information. \\
\hline 111 & Read-only. BIOMASS fixed ID-1 (0x89ABCDEF) \\
\hline 112 & Read-only. BIOMASS fixed ID-2 (0x12345678) \\
\hline 113 & Read-only. BIOMASS fixed ID-3 (0xB77BEFDF) \\
\hline 0800000 to & Read-only. The acquisition information. \\
0FFFFFF & \\
\hline
\end{tabular}

The default values for the different thresholds and other module parameters are defined in Table B.2. Data in this system is always read and written as a 32-bit word - these values are padded with zeroes to fill out the word. The default values are loaded into the module control FPGA on power up. As shown in Table 1, many of these parameters, such as valid pulse timeout or Integrator release time, can be set independently on a per channel basis, but the same default values are used for all eight channels.

Table B.2 Parameter default values (in hexadecimal).

\begin{tabular}{|l|c|}
\hline Parameter & Default value in Hex \\
\hline BIN0 Threshold LOW (15:0) & 000 \\
\hline BIN0 Threshold HIGH (31:16) & $07 \mathrm{~F}$ \\
\hline BIN1 Threshold LOW (15:0) & 080 \\
\hline BIN1 Threshold HIGH (31:16) & $0 \mathrm{FF}$ \\
\hline BIN2 Threshold LOW (15:0) & 100 \\
\hline BIN2 Threshold HIGH (31:16) & $17 \mathrm{~F}$ \\
\hline BIN3 Threshold LOW (15:0) & 180 \\
\hline BIN3 Threshold HIGH (31:16) & $1 \mathrm{FF}$ \\
\hline BIN4 Threshold LOW (15:0) & 200 \\
\hline BIN4 Threshold HIGH (31:16) & $27 \mathrm{~F}$ \\
\hline BIN5 Threshold LOW (15:0) & 280 \\
\hline BIN5 Threshold HIGH (31:16) & $2 \mathrm{FF}$ \\
\hline BIN6 Threshold LOW (15:0) & 300 \\
\hline BIN6 Threshold HIGH (31:16) & $37 \mathrm{~F}$ \\
\hline BIN7 Threshold LOW (15:0) & 380 \\
\hline BIN7 Threshold HIGH (31:16) & $3 \mathrm{FF}$ \\
\hline Valid pulse timeout & 6 \\
\hline Sample time & 28 \\
\hline Integrator release time & 1 \\
\hline Sequence acquisition time & 3000 \\
\hline Acquisition Mode \& number of sequences & 0 \\
\hline Input Offset Channel & 80 \\
\hline Minimum Threshold & 80 \\
\hline Initial Threshold & 80 \\
\hline Full Scale Reference & 80 \\
\hline ADC offset & 80 \\
\hline
\end{tabular}




\section{B.7 Software}

The "BIOMASS" software for the pulse processor module is written in C code. This code provides a simple interface to configure parameters on each pulse processor module and acquire information from all pulse processor module (s). These software routines handle all communications with the Window's PLX API via the PLX DLL. A different subroutine is used at each step of the procedure, so integration with higherlevel code is possible. At present, all input and output functions of the software are file based, i.e., setup parameters are taken from a file and downloaded to the module, and data from the module is written to a file without graphical or other display.

\section{B.7.1 Initial Module Set-up}

To set-up pulse processor modules for the first time, you insert the modules in the crate (power must be off) and make certain they are seated properly and latched. You then turn on the power. Windows should start and a message box should say that new hardware (PCI bridge device) has been found. Another message box will indicate that it is "OEM custom PCI9030". Click "ok" as needed.

Next the PLXMON program is used to set-up the API for PLX cards. When this starts, a window pops up and should say that a driver is detected. This window closes by itself, and the next window says that PCI devices don't have listings. This window lists the number of cards and some other information. Click "ok" and this window goes away. Next, the properties window appears. Once again, click "ok" to exit this window. You can now exit the program - the card set-up is complete.

\section{B.7.2 Module Configuration}

A configuration file is used to set-up the pulse processor module for data taking. This section describes the generation, modification and use of configuration files.

The pulse processor modules in the CPCI crate are located and configured by using the BIOMASS program with the configuration option. The command to create a configuration file is "BIOMASS -C CONFIG_FILE" where CONFIG_FILE is the name of the output file where the configuration data is stored for future use. This command also runs a test procedure that adjusts the input and ADC offsets in the module using the corresponding DACs. For this procedure, the shaping amplifiers should be powered on and connected to the pulse processor module(s). If detectors are connected to the shaping amplifiers, they should not be powered up so as to prevent any pulses from detectors disturbing the adjustment procedure. 
The configuration procedure generates a configuration file (CONFIG_FILE.ACQ) that contains energy window limits, integration time, thresholds, ADC full-scale and offsets for each channel of each card. The file also contains the parameters acquisition time, acquisition mode, acquisition type and acquisition number of samples. These global parameters apply to all pulse processor modules in a crate. Table B.3 describes the global parameters.

Table B.3 Configuration file global parameters.

\begin{tabular}{|l|l|l|l|}
\hline Parameter & Description & $\begin{array}{l}\text { Values } \\
\text { Hex or (decimal) }\end{array}$ & $\begin{array}{l}\text { module address } \\
\text { (Hex) }\end{array}$ \\
\hline Acquisition time & $\begin{array}{l}\text { time interval for data taking - } \\
\text { time is this integer value } \\
\text { multiplied by } 6.4 \mu \mathrm{s}\end{array}$ & $\begin{array}{l}1 \text { to 3FFFFFF } \\
(1 \text { to 2147483647) }\end{array}$ & 100 \\
\hline Acquisition mode & Determines type of data taking & $\begin{array}{l}0=\text { normal } \\
1=\text { fixed pattern } \\
2=\text { fake trigger }\end{array}$ & 101, bits 9 and 10 \\
\hline Acquisition type & $\begin{array}{l}\text { Determines if coarse or fine bins } \\
\text { are used }\end{array}$ & $\begin{array}{l}0=\text { coarse } \\
1=\text { fine }\end{array}$ & 101 , bit 8 \\
\hline $\begin{array}{l}\text { Acquisition } \\
\text { number of }\end{array}$ & $\begin{array}{l}\text { Number of time intervals during } \\
\text { shich data is to be acquired } \\
\text { samples }\end{array}$ & $\begin{array}{l}0 \text { to } 7 \mathrm{~F} \\
(0 \text { to } 127)\end{array}$ & 101 , bits 0 to 7 \\
\hline
\end{tabular}

The configuration file is an ordinary text file and may be manually edited to set-up the parameters for a given experiment. Parameters such as energy window limits and the global parameters would generally be modified for a particular experiment. Parameters such as integration time, discriminator thresholds, and ADC full-scale might need to be adjusted to allow for detector variations or for other optimization needs. Generally, the automatically determined offset values should be sufficient and not need to be adjusted manually. Appendix B.1 is a sample configuration file.

\section{B.7.3 Data Acquisition}

Once the modules are initialized and a configuration file is created and modified as needed, data acquisition is simple. An experiment can be run by using the command "BIOMASS -R-I CONFIG_FILE -O OUT_FILE". This command runs the program in the normal run mode (-R option) with input file (-I option) "CONFIG_FILE" and output file (-O option) "OUT_FILE".". Please note that a .LOG extension is added to the name of the output file.

Presently, there are two possible output file formats for the pulse processor card available in the software. One mode has CARD and CHANNEL information in the columns with BIN and SAMPLE information in block rows as shown in Table 4. This format should be useful for a fine-mode (MCA type) experiment. For example, if two cards used in the fine mode with one sampling interval, the output file will have 16 columns of data with 1024 rows. The other mode has SAMPLE information in the 
columns with the CARD, CHANNEL, and BIN information in the rows in Table B.5. The name of the mode reflects what values are stored in the columns.

The output file format can be set with the file format option (-f value). The format shown in Table B.4 (Samples in columns, Cards and Channels in rows) is the default format ( $f$ 0). To use the format shown in Table B.5 (Samples in rows, Cards and Channels in columns), you specify $-\mathrm{f} 1$ when running the program. Running the BIOMASS program with the - h option will give "help" information on various options. To do this, type "BIOMASS -H". Table B.6 gives a list of suggested configuration values for MP-CARPT.

Table B.4 Readout scheme for the CARD and CHANNEL mode

\begin{tabular}{|c|c|c|c|c|c|c|c|c|c|c|c|c|c|c|c|c|c|}
\hline \multicolumn{18}{|c|}{ Time interval \#1 } \\
\hline & \multicolumn{8}{|c|}{ Card \#0 } & & \multicolumn{8}{|c|}{ Card \#N } \\
\hline & 0 & 1 & 2 & 3 & 4 & 5 & 6 & 7 & & 0 & 1 & 2 & 3 & 4 & 5 & 6 & 7 \\
\hline \multicolumn{17}{|l|}{ BIN 0 (Sample 1) } & \\
\hline \multicolumn{17}{|l|}{ BIN 1 (Sample 1) } & \\
\hline \multicolumn{17}{|l|}{ ETC } & \\
\hline \multicolumn{17}{|l|}{ BIN X (Sample 1) } & \\
\hline \multicolumn{18}{|c|}{ Time interval \#2 } \\
\hline \multirow[b]{2}{*}{ Channels } & \multicolumn{8}{|c|}{ Card \#0 } & & \multicolumn{8}{|c|}{ Card \#N } \\
\hline & 0 & 1 & 2 & 3 & 4 & 5 & 6 & 7 & & 0 & 1 & 2 & 3 & 4 & 5 & 6 & 7 \\
\hline \multicolumn{17}{|l|}{ BIN 0 (Sample 1) } & \\
\hline \multicolumn{17}{|l|}{ BIN 1 (Sample 1) } & \\
\hline \multicolumn{17}{|l|}{ ETC } & \\
\hline \multicolumn{17}{|l|}{ BIN X (Sample 1) } & \\
\hline \multicolumn{18}{|c|}{ (data from additional time intervals) } \\
\hline \multicolumn{18}{|c|}{ Time interval \#S } \\
\hline & \multicolumn{8}{|c|}{ Card \#0 } & \multirow{6}{*}{ ETC } & \multicolumn{8}{|c|}{ Card \#N } \\
\hline Channels & 0 & 1 & 2 & 3 & 4 & 5 & 6 & 7 & & 0 & 1 & 2 & 3 & 4 & 5 & 6 & 7 \\
\hline BIN 0 (Sample S) & & & & & & & & & & & & & & & & & \\
\hline BIN 1 (Sample S) & & & & & & & & & & & & & & & & & \\
\hline ETC & & & & & & & & & & & & & & & & & \\
\hline BIN X (Sample S) & & & & & & & & & & & & & & & & & \\
\hline
\end{tabular}


Table B.5 Readout scheme for SAMPLES mode

\begin{tabular}{|c|c|c|c|c|}
\hline \multicolumn{5}{|c|}{ Card \#0 } \\
\hline & Sample 1 & Sample 2 & \multirow{14}{*}{ ETC } & Sample S \\
\hline Channel 0 BIN 0 & & & & \\
\hline Channel 0 BIN 1 & & & & \\
\hline ETC & & & & \\
\hline Channel 0 BIN X & & & & \\
\hline Channel 1 BIN 0 & & & & \\
\hline Channel 1 BIN 1 & & & & \\
\hline ETC & & & & \\
\hline Channel 1 BIN X & & & & \\
\hline \multicolumn{3}{|c|}{ * ETC* } & & \\
\hline Channel 7 BIN 0 & & & & \\
\hline Channel 7 BIN 1 & & & & \\
\hline ETC & & & & \\
\hline Channel 7 BIN X & & & & \\
\hline \multicolumn{5}{|c|}{ Card \#1 } \\
\hline Channel 0 BIN 0 & & & \multirow{3}{*}{ ETC } & \\
\hline & & & & \\
\hline Channel 7 BIN X & & & & \\
\hline \multicolumn{5}{|c|}{ ** ETC ** } \\
\hline \multicolumn{5}{|c|}{ Card \#N } \\
\hline Channel 0 BIN 0 & & & \multirow{3}{*}{ ETC } & \\
\hline & & & & \\
\hline Channel 7 BIN X & & & & \\
\hline
\end{tabular}

Table B.6 Suggested Parameter values (in hexadecimal).

\begin{tabular}{|c|c|c|}
\hline Parameter & $\begin{array}{l}\text { Suggested value } \\
\text { in Hex }\end{array}$ & Comment \\
\hline $\begin{array}{l}\text { BIN Thresholds LOW and } \\
\text { HIGH }\end{array}$ & $?$ & Set as needed \\
\hline Valid pulse timeout & 6 & default \\
\hline Sample time & 28 & Default, s 2 \\
\hline Integrator release time & 1 & Default \\
\hline Sequence acquisition time & $30 \mathrm{D}$ & $\sim 5 \mathrm{~ms}$ per interval \\
\hline Acquisition mode & 0 & Normal data \\
\hline Acquisition type & 0 & Coarse mode \\
\hline $\begin{array}{l}\text { Acquisition Mode \& number } \\
\text { of sequences }\end{array}$ & FF & 256 intervals \\
\hline Input Offset Channel & A0 & $\begin{array}{c}\text { mid scale, program will } \\
\text { autoset }\end{array}$ \\
\hline Minimum Threshold & 8 & Lower values are possible \\
\hline Initial Threshold & 8 & Lower values are possible \\
\hline Full Scale Reference & FF & Lower value to increase gain \\
\hline ADC offset & 80 & $\begin{array}{c}\text { mid scale, program will } \\
\text { autoset }\end{array}$ \\
\hline
\end{tabular}




\section{B.7.4 Summary of the Programming Procedure}

The procedure for setting up and acquiring data is described in the following sequence: 1) Use the PLXMON program from PLX to setup the API for the PLX cards. You will need to use this program each time a new pulse processor card is added to the system. 2) Initialize the pulse processor cards in the system and create a configuration file. The command is "BIOMASS -C CONFIG_FILE" where CONFIG_FILE is the name of the output file.

3) Modify the control parameters for each card in the configuration file. Be sure and set the mode to fine.

4) Connect the detectors to the amplifiers and/or turn on detectors.

5) Take data using each radioactive source one at a time. Plot energy spectra and determine desired energy windows.

6) Modify the control parameters for each BIOMASS card in the configuration file for normal (CARPT-type) acquisition. Remember to change the ACQ_MODE back to 0! It may be useful to save different versions of the configuration file for similar, but slightly different experiments (like one for MCA mode and another for CARPT).

7) Run the experiment. The command is "BIOMASS -R-I CONFIG_FILE -O LOG_FILE". Please note that a .LOG extension is added to the name of the output $\log$ file.

\section{B.7.5 Test Mode Operation}

There are a couple of test modes that are useful. The "fake trigger" mode is useful to determine the system noise and mean value for a zero signal. This can be accomplished by setting the module for fine mode and "fake trigger" mode and running a short experiment. In the "fake trigger" mode, the module controller causes the channels to fake the detection of a valid input pulse, so the integration and digitization process is started and the resulting data stored. This process is repeated according to the TRIGGER FIRING COUNT parameter. The time between "fake triggers" is $25 \mathrm{~ns}$ times the TRIGGER FIRING COUNT. The default value is $32 \mu \mathrm{s}$, so sufficient data can be taken in a few seconds.

If this experiment is run without a shaping amplifier connected to pulse processor module (the module may need to be configured without the shaping amplifier), essentially all the counts should be in one or two bins. If the shaping amplifier is connected, the noise level will be greater and the counts will be spread over perhaps 20 bins.

\section{B.7.6 Sample Data}

Figure B.27 shows spectra resulting from using the shaping amplifier and pulse processor module with two 2-inch NaI detectors and naturally-occurring Potassium-40 
(in salt substitute). The peak for this isotope is $1.46 \mathrm{MeV}$, and it occurs at channel 950 for one detector and channel 400 for the other due to the detector gains not being balanced. (Both detectors used the same high voltage and same shaping amplifier gain.) The same source was counted for approximately 16 hours and the data shown in Figure B.28 was obtained. Other well-known background peaks are labeled.

The "fake trigger" mode was used to obtain the plot shown in Figure B.29. The distributions show the level of amplifier noise. (Ideally all counts would occur in a single bin as the input is a constant - zero.) The root-mean-square (rms) variation for the typical channel is about 6 bins. This variation is significantly less than the variation due to the $\mathrm{NaI}$ detectors, so it is sufficiently good to not effect peaks like those shown in Figure B.27. Two channels show significantly lower rms variations. These channels have modified shaping amplifiers. These modifications may be included in future shaping amplifier modules.

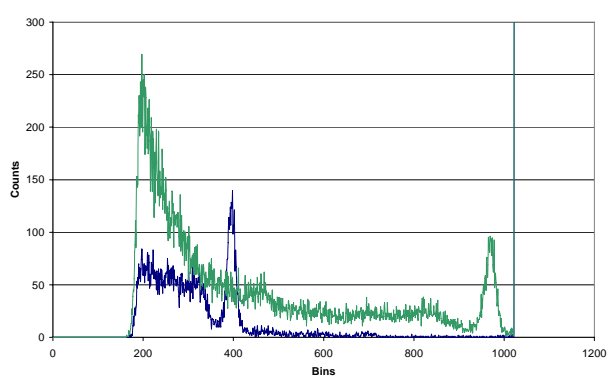

Figure B.27 ${ }^{40} \mathrm{~K}$ spectrum

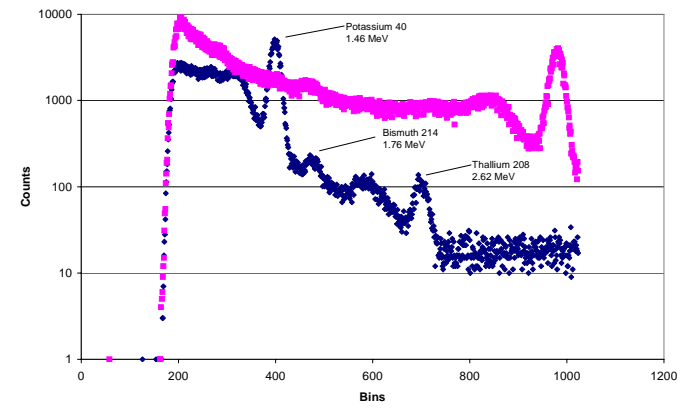

Figure B.28 ${ }^{40} \mathrm{~K}$ spectrum with log scale.

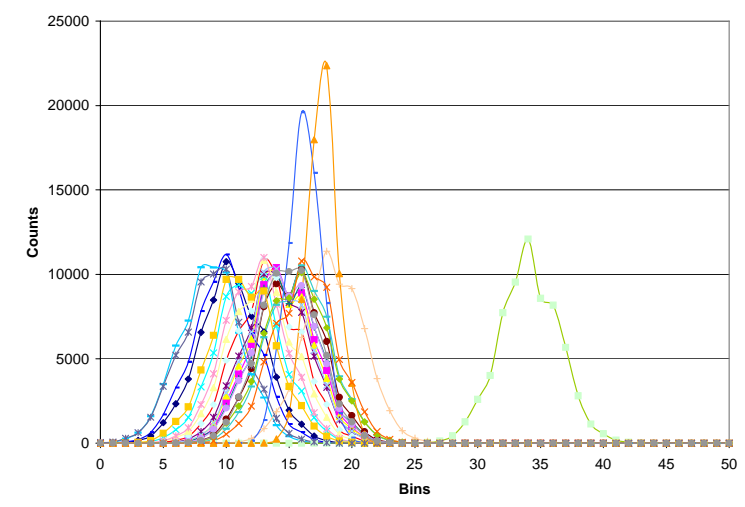

Figure B.29 Shaper amplifier noise. 


\section{B.8 Problems encountered with MP-CARPT}

\section{B.8.1 Problems}

The principle of MP-CARPT is based on the energy discrimination. Two or more radioactive particles can be distinguished from each other based on their gamma energy peaks. Co-60 has its energy peaks at 1.17 and $1.33 \mathrm{MeV}$ and Sc-46 has its peaks at 0.889 and $1.12 \mathrm{MeV}$. With the adjustment in gain of the timing amplifiers, the peaks of Co-60 and Sc-46 can be shifted together horizontally but not independently. During the course of normal operation this peak should remain at the same position, given that all other settings (like gain etc.) in data acquisition electronics are kept constant, irrespective of the position of the radioactive particle and the intensity of the gamma ray counts received. This property is essential for the success for the MP-CAPRT.

The limits of energy windows during the experiment phase are set such that only the high energy Co-60 peak is captured in one window and in the other window both Co-60 and Sc-46 peaks are captured. This approach helps in distinguishing between the counts obtained from different sources. For this to succeed, the position of the energy peaks must remain the same for different locations of radioactive source. Hence the variation in the intensity of the gamma ray counts (due to changing distance) shouldn't affect the location of the peaks on the energy (bins) scale.

However, during experimentation it was discovered that this was not the case with the electronics developed at the ORNL which is currently in use for MP-CARPT. The Figure B.30 below shows the shift in energy peaks of Co-60 and Sc-46 for two different source locations (hence gamma ray counts intensity). For a location of the particles closer to the detector the peaks shift towards a higher energy (towards the right on the scale). The number of gamma ray photon counts obtained by the detectors is dependent on the distance of the source from the detector. Therefore, more counts are obtained for source near the detector and less photon counts for longer distances. In this data acquisition system the change in counts brings about a shift in the location of the peak. Hence the photo peak shifts out of the energy window when the particle location is closer to the detector. This causes a drop in the counts detected for particle locations close to the detector. This is counter intuitive to the basic principles. 


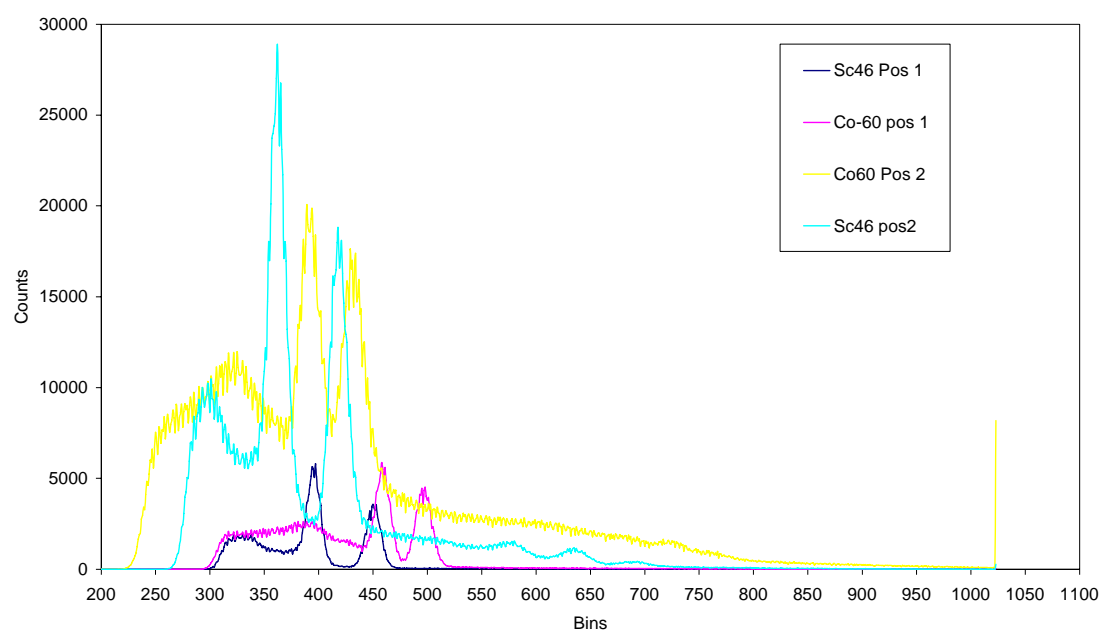

Figure B.30 Shift in gamma ray energy spectrum of the ${ }^{60} \mathrm{Co}$ and ${ }^{46} \mathrm{Sc}$ at two different locations of the particles.

Another problem encountered has been explained with the aid of Figure B.31. The counts obtained from two separate single sources independently should add up to the total gamma counts obtained with 2 sources together (provided their locations remain unchanged). When this experiment was carried out, it was found that they don't add up with this set up. Figure B.31 shows the gamma ray photon peaks for Co-60 and Sc-46, taken simultaneously and separately with the current MP-CARPT data acquisition system. It can be seen that the peaks of the individual source are not superimposed when the scans are obtained simultaneously. This shift creates problems during the data processing, as the counts of Sc-46 are obtained by subtracting the counts of co-60 alone from the total counts of Sc-46 and Co-60 together.

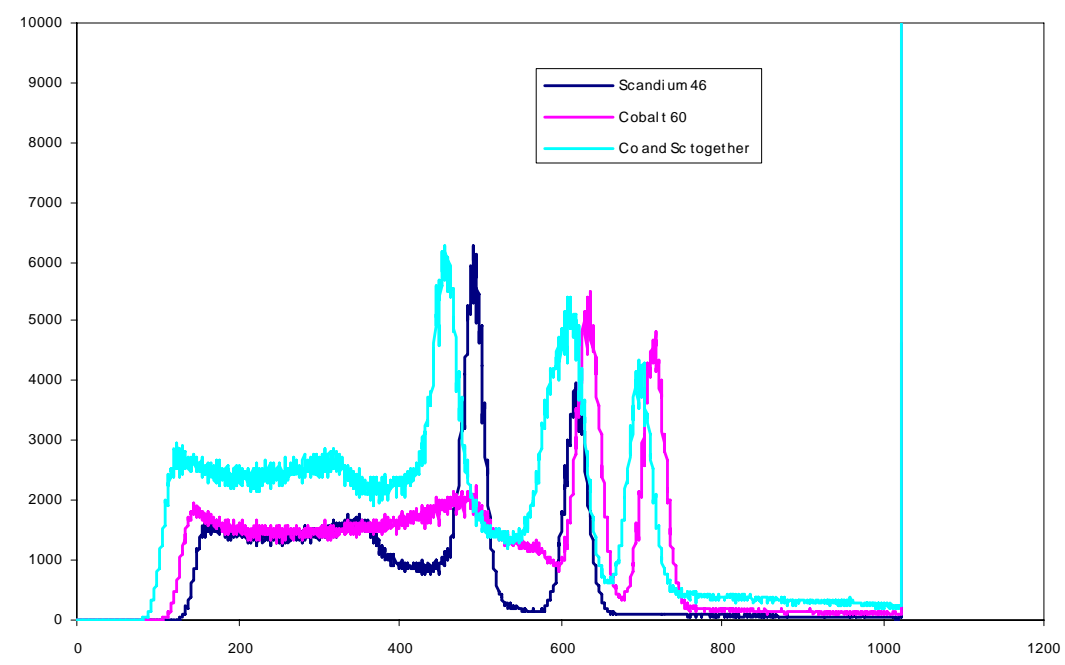

Figure B.31 Photo peaks of ${ }^{46} \mathrm{Sc}$ and ${ }^{60} \mathrm{Co}$ taken with individual source both separately and together. Notice a shift in the spectrum of the combined sources. 


\section{B.8.2 Rectification of Problems}

Above problems can be solved by changing the parameters in the configuration file, which provides input to the data acquisition program.

Figure B.31 shows the problem of shifting of gamma peak with changing distance of Sc46 and Co-60 sources from the detector. The value of parameter "sample_time" was changed from 40 to 16 , this value was decided after a trial and error procedure to eliminate the shifting of gamma peaks. Figure B.33 shows the gamma peaks of Sc-46 and Co-60 obtained separately for different positions, position 1 is closer to the detector whereas position 2 is farther. The shifting of the peak is almost eliminated by changing the value of sample_time.

Figure B.32 shows the shift in the gamma spectrum when Sc-60 and Co-60 are scanned together with respect to their individual spectrum. This suggests that the counts are not additive. With the change of sample_time, the Sc-46 and Co-60 counts were found to be additive as shown in Figure B.33. Counts were obtained for Sc-46 and Co-60 separately and together. The counts of Sc-46 and Co-60 were added to examine the overlap with counts that were obtained by placing Sc-46 and Co-60 together at the same location. The summed counts match very well with the counts of Sc-46 and Co-60 scanned together, indicating that the superposition rule of gamma peaks for different sources is satisfied.

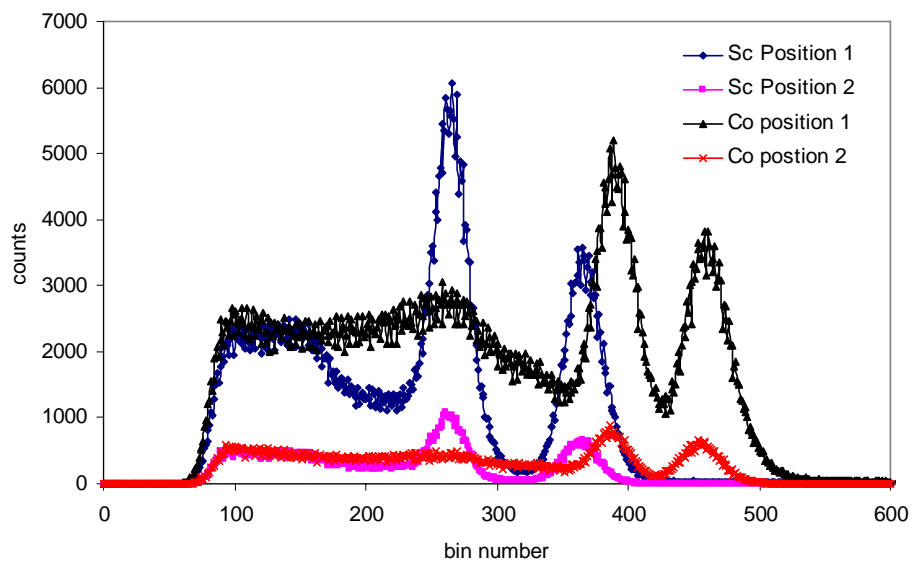

Figure B.32 Individual Gamma peaks of Sc-46 and Co-60 placed at different locations 


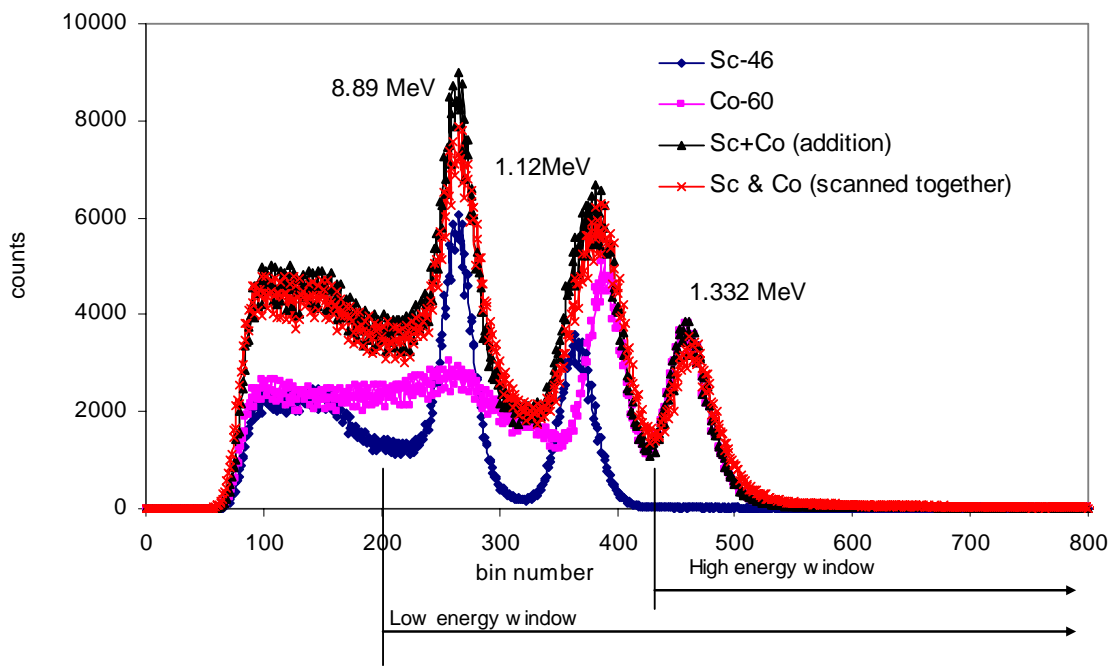

Figure B.33 Gamma peaks of Sc-46 and Co-60 individually and together, and summation of individual counts 


\section{Appendix B.1 \\ Example Configuration File.}

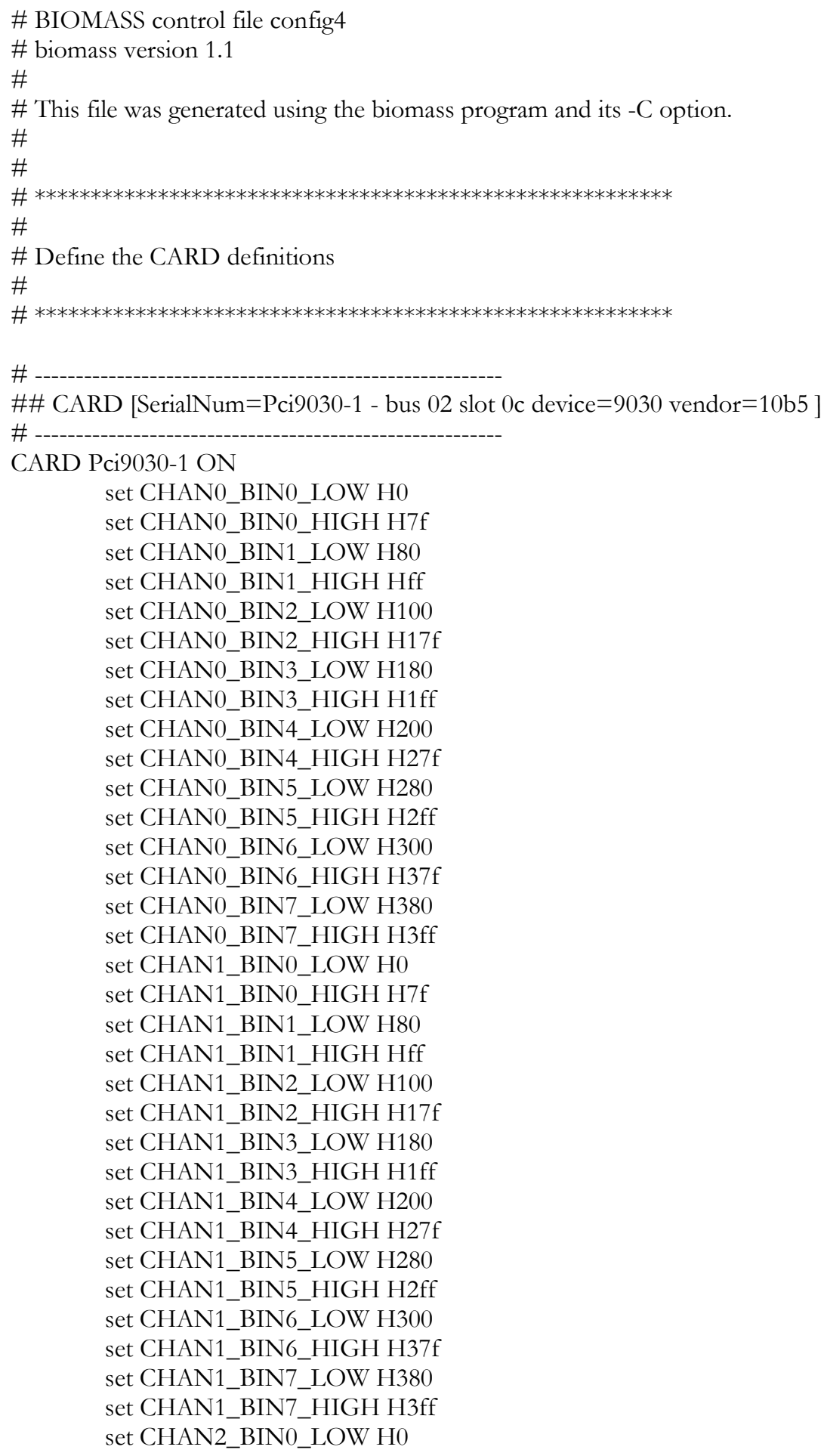


Appendix-2: Vesvikar(2006), D.Sc. Thesis

set CHAN2_BIN0_HIGH H7f set CHAN2_BIN1_LOW H80 set CHAN2_BIN1_HIGH Hff set CHAN2_BIN2_LOW H100 set CHAN2_BIN2_HIGH H17f set CHAN2_BIN3_LOW H180 set CHAN2_BIN3_HIGH H1ff set CHAN2_BIN4_LOW H200 set CHAN2_BIN4_HIGH H27f set CHAN2_BIN5_LOW H280 set CHAN2_BIN5_HIGH H2ff set CHAN2_BIN6_LOW H300 set CHAN2_BIN6_HIGH H37f set CHAN2_BIN7_LOW H380 set CHAN2_BIN7_HIGH H3ff set CHAN3_BIN0_LOW H0 set CHAN3_BIN0_HIGH H7f set CHAN3_BIN1_LOW H80 set CHAN3_BIN1_HIGH Hff set CHAN3_BIN2_LOW H100 set CHAN3_BIN2_HIGH H17f set CHAN3_BIN3_LOW H180 set CHAN3_BIN3_HIGH H1ff set CHAN3_BIN4_LOW H200 set CHAN3_BIN4_HIGH H27f set CHAN3_BIN5_LOW H280 set CHAN3_BIN5_HIGH H2ff set CHAN3_BIN6_LOW H300 set CHAN3_BIN6_HIGH H37f set CHAN3_BIN7_LOW H380 set CHAN3_BIN7_HIGH H3ff set CHAN4_BIN0_LOW H0 set CHAN4_BIN0_HIGH H7f set CHAN4_BIN1_LOW H80 set CHAN4_BIN1_HIGH Hff set CHAN4_BIN2_LOW H100 set CHAN4_BIN2_HIGH H17f set CHAN4_BIN3_LOW H180 set CHAN4_BIN3_HIGH H1ff set CHAN4_BIN4_LOW H200 set CHAN4_BIN4_HIGH H27f set CHAN4_BIN5_LOW H280 set CHAN4_BIN5_HIGH H2ff set CHAN4_BIN6_LOW H300 set CHAN4_BIN6_HIGH H37f set CHAN4_BIN7_LOW H380 set CHAN4_BIN7_HIGH H3ff set CHAN5_BIN0_LOW H0 set CHAN5_BIN0_HIGH H7f set CHAN5_BIN1_LOW H80 set CHAN5_BIN1_HIGH Hff set CHAN5_BIN2_LOW H100 set CHAN5_BIN2_HIGH H17f set CHAN5_BIN3_LOW H180 
Appendix-2: Vesvikar(2006), D.Sc. Thesis

set CHAN5_BIN3_HIGH H1ff set CHAN5_BIN4_LOW H200 set CHAN5_BIN4_HIGH H27f set CHAN5_BIN5_LOW H280 set CHAN5_BIN5_HIGH H2ff set CHAN5_BIN6_LOW H300 set CHAN5_BIN6_HIGH H37f set CHAN5_BIN7_LOW H380 set CHAN5_BIN7_HIGH H3ff set CHAN6_BIN0_LOW H0 set CHAN6_BIN0_HIGH H7f set CHAN6_BIN1_LOW H80 set CHAN6_BIN1_HIGH Hff set CHAN6_BIN2_LOW H100 set CHAN6_BIN2_HIGH H17f set CHAN6_BIN3_LOW H180 set CHAN6_BIN3_HIGH H1ff set CHAN6_BIN4_LOW H200 set CHAN6_BIN4_HIGH H27f set CHAN6_BIN5_LOW H280 set CHAN6_BIN5_HIGH H2ff set CHAN6_BIN6_LOW H300 set CHAN6_BIN6_HIGH H37f set CHAN6_BIN7_LOW H380 set CHAN6_BIN7_HIGH H3ff set CHAN7_BIN0_LOW H0 set CHAN7_BIN0_HIGH H7f set CHAN7_BIN1_LOW H80 set CHAN7_BIN1_HIGH Hff set CHAN7_BIN2_LOW H100 set CHAN7_BIN2_HIGH H17f set CHAN7_BIN3_LOW H180 set CHAN7_BIN3_HIGH H1ff set CHAN7_BIN4_LOW H200 set CHAN7_BIN4_HIGH H27f set CHAN7_BIN5_LOW H280 set CHAN7_BIN5_HIGH H2ff set CHAN7_BIN6_LOW H300 set CHAN7_BIN6_HIGH H37f set CHAN7_BIN7_LOW H380 set CHAN7_BIN7_HIGH H3ff set ACQ_TRIGGER_CNT H80 set CHANO_INPUT_OFFSET Ha0 set CHAN1_INPUT_OFFSET H9e set CHAN2_INPUT_OFFSET H95 set CHAN3_INPUT_OFFSET H99 set CHAN4_INPUT_OFFSET H9b set CHAN5_INPUT_OFFSET H92 set CHAN6_INPUT_OFFSET H9e set CHAN7_INPUT_OFFSET H99 set CHAN0_INIT_THRESHOLD H08 set CHAN1_INIT_THRESHOLD H40 set CHAN2_INIT_THRESHOLD H40 set CHAN3_INIT_THRESHOLD H40 
Appendix-2: Vesvikar(2006), D.Sc. Thesis

set CHAN4_INIT_THRESHOLD H40

set CHAN5_INIT_THRESHOLD H40

set CHAN6_INIT_THRESHOLD H40

set CHAN7_INIT_THRESHOLD H40

set CHANO_MIN_THRESHOLD H08

set CHAN1_MIN_THRESHOLD H20

set CHAN2_MIN_THRESHOLD H20

set CHAN3_MIN_THRESHOLD H20

set CHAN4_MIN_THRESHOLD H20

set CHAN5_MIN_THRESHOLD H20

set CHAN6_MIN_THRESHOLD H20

set CHAN7_MIN_THRESHOLD H20

set CHANO_ADC_OFFSET H94

set CHAN1_ADC_OFFSET Hbc

set CHAN2_ADC_OFFSET He3

set CHAN3_ADC_OFFSET Hd6

set CHAN4_ADC_OFFSET Ha2

set CHAN5_ADC_OFFSET He2

set CHAN6_ADC_OFFSET Ha5

set CHAN7_ADC_OFFSET Hcf

set CHANO_FULL_SCALE Hff

set CHAN1_FULL_SCALE Hff

set CHAN2_FULL_SCALE Hff

set CHAN3_FULL_SCALE Hff

set CHAN4_FULL_SCALE Hff

set CHAN5_FULL_SCALE Hff

set CHAN6_FULL_SCALE Hff

set CHAN7_FULL_SCALE Hff

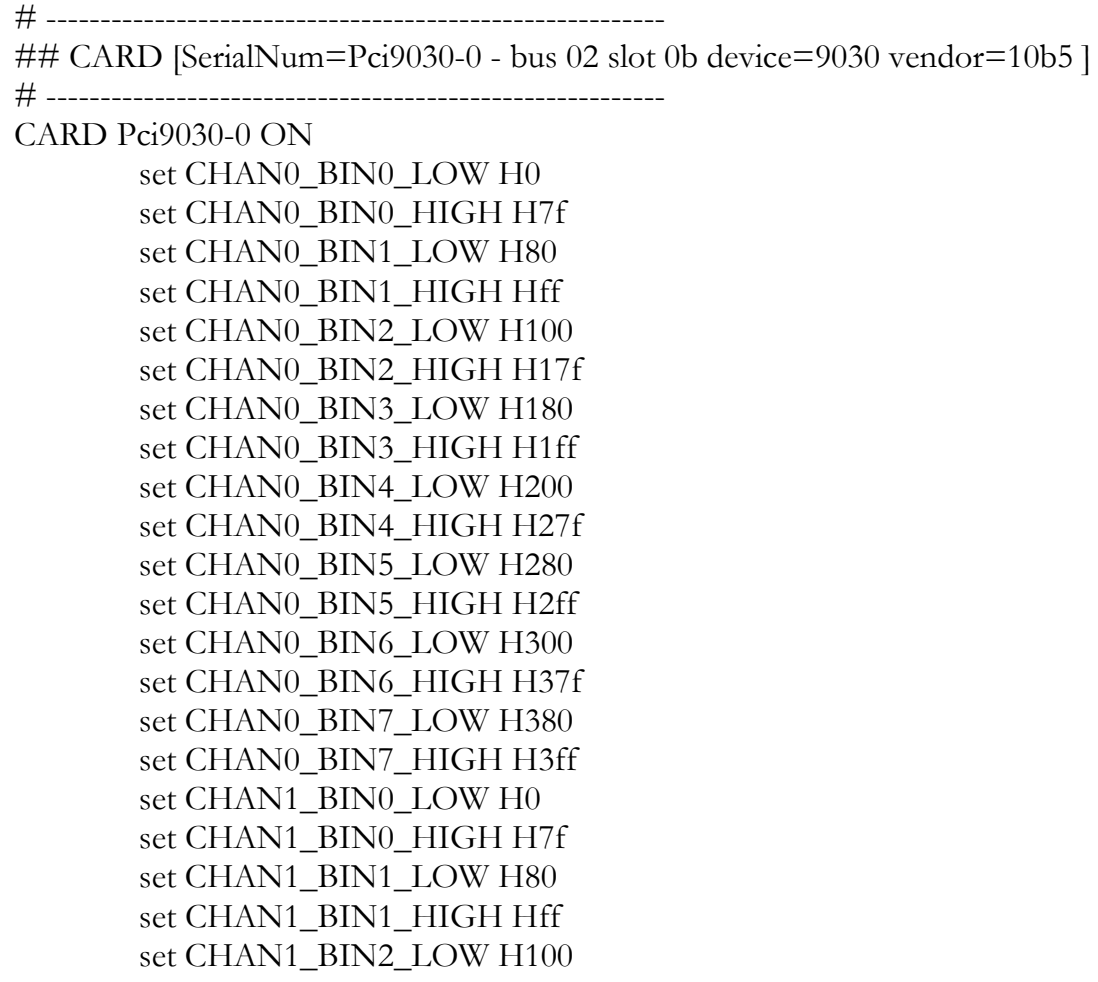


Appendix-2: Vesvikar(2006), D.Sc. Thesis

set CHAN1_BIN2_HIGH H17f set CHAN1_BIN3_LOW H180 set CHAN1_BIN3_HIGH H1ff set CHAN1_BIN4_LOW H200 set CHAN1_BIN4_HIGH H27f set CHAN1_BIN5_LOW H280 set CHAN1_BIN5_HIGH H2ff set CHAN1_BIN6_LOW H300 set CHAN1_BIN6_HIGH H37f set CHAN1_BIN7_LOW H380 set CHAN1_BIN7_HIGH H3ff set CHAN2_BIN0_LOW H0 set CHAN2_BIN0_HIGH H7f set CHAN2_BIN1_LOW H80 set CHAN2_BIN1_HIGH Hff set CHAN2_BIN2_LOW H100 set CHAN2_BIN2_HIGH H17f set CHAN2_BIN3_LOW H180 set CHAN2_BIN3_HIGH H1ff set CHAN2_BIN4_LOW H200 set CHAN2_BIN4_HIGH H27f set CHAN2_BIN5_LOW H280 set CHAN2_BIN5_HIGH H2ff set CHAN2_BIN6_LOW H300 set CHAN2_BIN6_HIGH H37f set CHAN2_BIN7_LOW H380 set CHAN2_BIN7_HIGH H3ff set CHAN3_BIN0_LOW H0 set CHAN3_BIN0_HIGH H7f set CHAN3_BIN1_LOW H80 set CHAN3_BIN1_HIGH Hff set CHAN3_BIN2_LOW H100 set CHAN3_BIN2_HIGH H17f set CHAN3_BIN3_LOW H180 set CHAN3_BIN3_HIGH H1ff set CHAN3_BIN4_LOW H200 set CHAN3_BIN4_HIGH H27f set CHAN3_BIN5_LOW H280 set CHAN3_BIN5_HIGH H2ff set CHAN3_BIN6_LOW H300 set CHAN3_BIN6_HIGH H37f set CHAN3_BIN7_LOW H380 set CHAN3_BIN7_HIGH H3ff set CHAN4_BIN0_LOW H0 set CHAN4_BIN0_HIGH H7f set CHAN4_BIN1_LOW H80 set CHAN4_BIN1_HIGH Hff set CHAN4_BIN2_LOW H100 set CHAN4_BIN2_HIGH H17f set CHAN4_BIN3_LOW H180 set CHAN4_BIN3_HIGH H1ff set CHAN4_BIN4_LOW H200 set CHAN4_BIN4_HIGH H27f set CHAN4_BIN5_LOW H280 
Appendix-2: Vesvikar(2006), D.Sc. Thesis

set CHAN4_BIN5_HIGH H2ff set CHAN4_BIN6_LOW H300 set CHAN4_BIN6_HIGH H37f set CHAN4_BIN7_LOW H380 set CHAN4_BIN7_HIGH H3ff set CHAN5_BIN0_LOW H0 set CHAN5_BIN0_HIGH H7f set CHAN5_BIN1_LOW H80 set CHAN5_BIN1_HIGH Hff set CHAN5_BIN2_LOW H100 set CHAN5_BIN2_HIGH H17f set CHAN5_BIN3_LOW H180 set CHAN5_BIN3_HIGH H1ff set CHAN5_BIN4_LOW H200 set CHAN5_BIN4_HIGH H27f set CHAN5_BIN5_LOW H280 set CHAN5_BIN5_HIGH H2ff set CHAN5_BIN6_LOW H300 set CHAN5_BIN6_HIGH H37f set CHAN5_BIN7_LOW H380 set CHAN5_BIN7_HIGH H3ff set CHAN6_BIN0_LOW H0 set CHAN6_BIN0_HIGH H7f set CHAN6_BIN1_LOW H80 set CHAN6_BIN1_HIGH Hff set CHAN6_BIN2_LOW H100 set CHAN6_BIN2_HIGH H17f set CHAN6_BIN3_LOW H180 set CHAN6_BIN3_HIGH H1ff set CHAN6_BIN4_LOW H200 set CHAN6_BIN4_HIGH H27f set CHAN6_BIN5_LOW H280 set CHAN6_BIN5_HIGH H2ff set CHAN6_BIN6_LOW H300 set CHAN6_BIN6_HIGH H37f set CHAN6_BIN7_LOW H380 set CHAN6_BIN7_HIGH H3ff set CHAN7_BIN0_LOW H0 set CHAN7_BIN0_HIGH H7f set CHAN7_BIN1_LOW H80 set CHAN7_BIN1_HIGH Hff set CHAN7_BIN2_LOW H100 set CHAN7_BIN2_HIGH H17f set CHAN7_BIN3_LOW H180 set CHAN7_BIN3_HIGH H1ff set CHAN7_BIN4_LOW H200 set CHAN7_BIN4_HIGH H27f set CHAN7_BIN5_LOW H280 set CHAN7_BIN5_HIGH H2ff set CHAN7_BIN6_LOW H300 set CHAN7_BIN6_HIGH H37f set CHAN7_BIN7_LOW H380 set CHAN7_BIN7_HIGH H3ff set ACQ_TRIGGER_CNT H80 


\section{Appendix 3}

Investigation of the phases' distribution using gamma ray computed tomography (CT) techniques 


\section{Appendix 3.1}

\subsubsection{Gas distribution in a 6 inch anaerobic digester using a single point sparger and a single source $\mathrm{CT}$.}

The objective of this work was to visualize the gas phase holdup distribution in the digesters on which performance studies were carried out and reported in the quarterly reports and published papers (chapter 2). The 6 inch $(153 \mathrm{~mm})$ anaerobic digester mixed by gas recirculation at rates of $1 \mathrm{liter} / \mathrm{min}$ and $3 \mathrm{liter} / \mathrm{min}$ were selected. These biogas recirculation rates represent the lowest and the highest flow rates that were used for the performance studies. The data has been collected for the above conditions at 5 levels (Figure 3.1). The gas phase is introduce at the bottom of the draft tube through a $1 / 4$ inch tubing inserted from the top of the digester with a single hole of $5 \mathrm{~mm}$ diameter. Biogas (methane) was used along with animal waste slurry of $5 \% \mathrm{w} / \mathrm{v}$ concentration. Due to low solids concentration used, the slurry was assumed as a homogeneous phase comparable to water's properties. Figure 3.1 shows a schematic (not to scale) of the biodigester used and the levels at which CT scans were taken. Five levels along the vertical axes of the digester were chosen for the tomographic scans. Levels 1 and 2 were chosen to visualize the gas phase distribution just above and below the upper section of the draft tube. Similarly levels 4 and 5 were chosen to visualize the gas phase distribution at the lower section of the draft tube. Level 3 was chosen to visualize the mid section of the daft tube. The gas is released in the center at the bottom of the draft tube.

The gamma ray attenuation for each pixel for any given system is computed based on the EM (Estimation Maximization) algorithm developed at CREL (Kumar, 1994). Further details of the CT technique and its hardware, software, reconstruction and its applications can be found in:

Kumar S. 1994. Computed Tomographic Measurements of Void Fraction and Modelinof the Flow in Bubble Columns [Ph.D Thesis performed in St. Louis]: Florida Atlantic University

Roy S. 2006. Phase distribution and performance studies of gas-liquid monolith reactor. [D.Sc. Thesis]: Washington University - St. Louis. 203 p. 


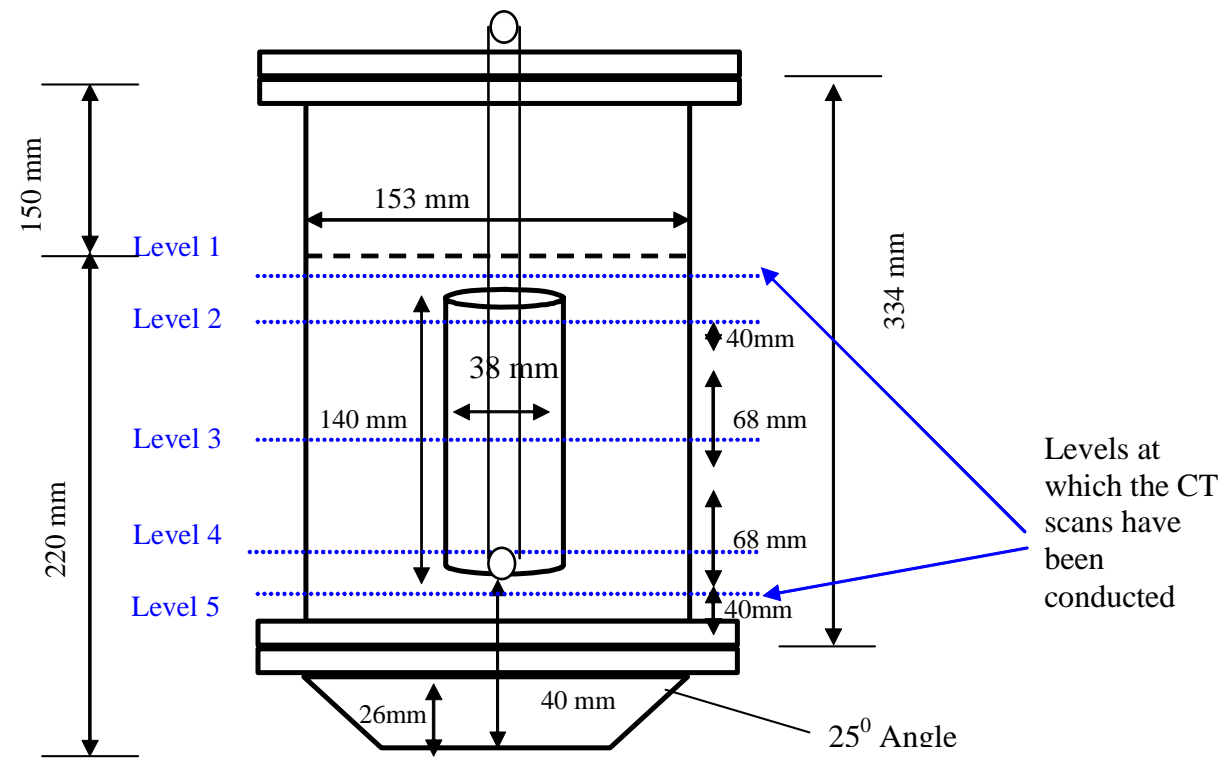

Figure 3.1. Schematic (not to scale) of the biodigester used with indications of the positions of the CT scans carried out.

The cross-sectional time averaged gas holdup distribution obtained from the CT scans are shown in Figures 3.2 to 3.6 representing the levels 1-5.

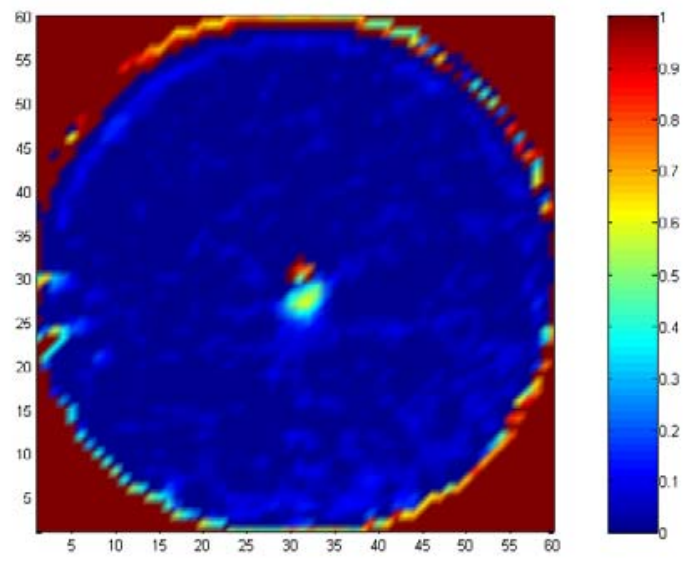

Figure 3.2: Image of the cross-sectional time averaged gas phase distribution at level 1 with Biogas recirculation rate of 1 lit/min (Pixel size $153 \mathrm{~mm}$ x $153 \mathrm{~mm}$ ). Colorbar indicates gas holdup. 


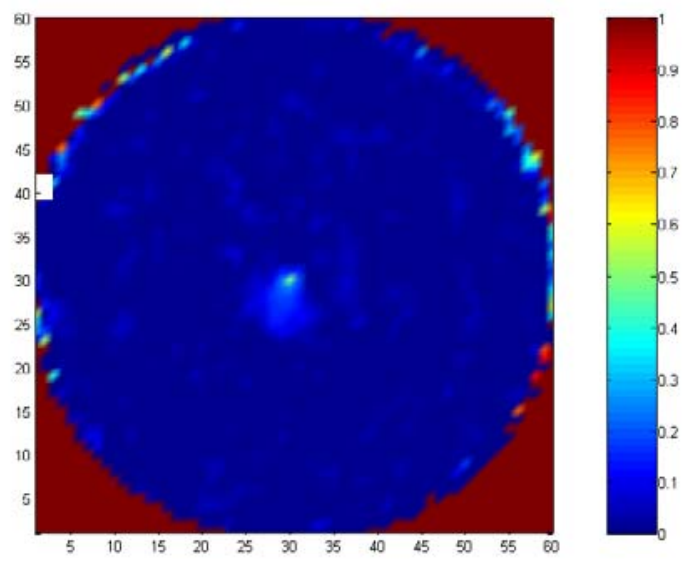

Figure 3.3: Image of the cross-sectional time averaged gas phase distribution at level 2 with Biogas recirculation rate of 1 lit/min (Pixel size 153 mm x 153 mm)

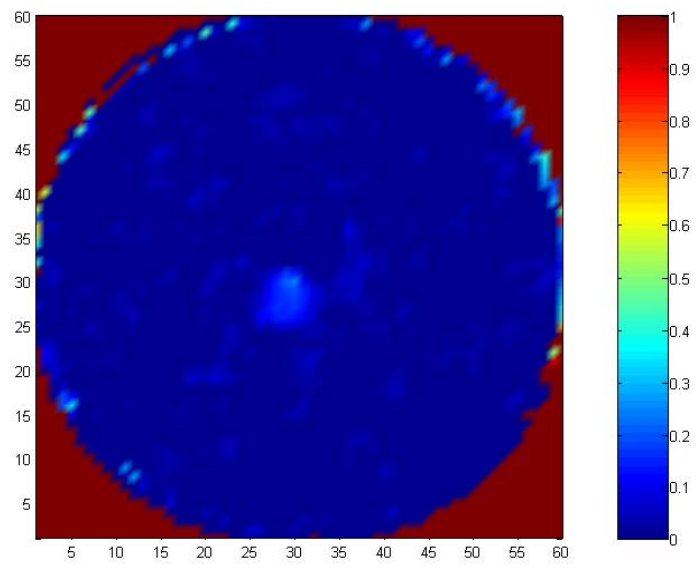

Figure 3.4: Image of the cross-sectional time averaged gas phase distribution at level 3 with Biogas recirculation rate of 1 lit/min (Pixel size 153 mm x $153 \mathrm{~mm}$ )

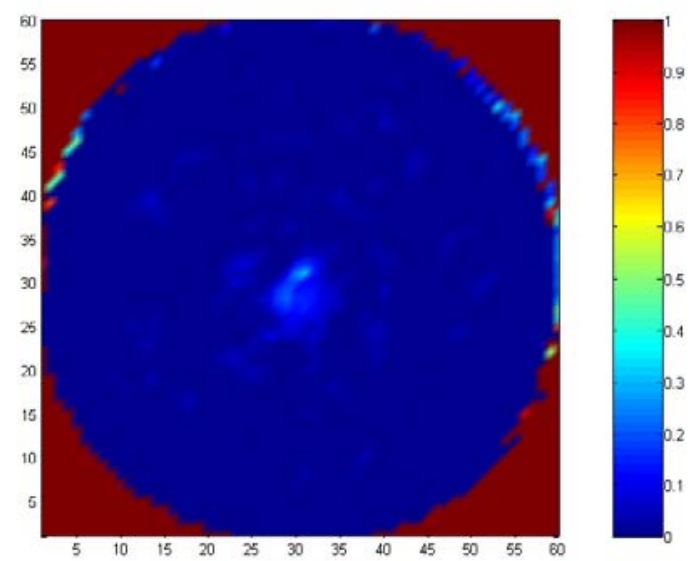

Figure 3.5: Image of the cross-sectional time averaged gas phase distribution at level 4 with Biogas recirculation rate of 1 lit/min (Pixel size $153 \mathrm{~mm}$ x $153 \mathrm{~mm}$ ) 


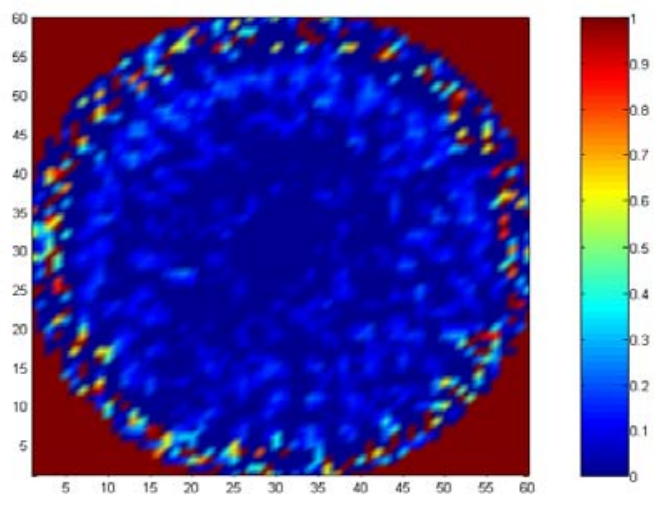

Color bar indicating the magnitude of gas holdup

Figure 3.6: $\quad$ The image obtained at Level 5 using the two phase flow system assumption for data processing (Pixel size $153 \mathrm{~mm}$ x $153 \mathrm{~mm}$ )

It is clearly evident that the gas is confined to a very small region in the draft tube. At Level 1 the gas hold up appears to be the highest as there is bubble break up. Also the gas injection port passing through this region has the highest gas holdup that appears as a red region with a gas hold up of unity. The gas phase seems to form a channel around the gas injection tube in the draft tube and escape through it as it can be seen at all the levels 1-4. This is due the type of sparger used, which has a single opening of $5 \mathrm{~mm}$ inner diameter. These findings indicate that careful attention needs to be given to the sparger design to ensure uniform distribution of the gas phase at the base of the draft tube.

The image of the scan at level 5 shows that there is no gas below the draft tube hence the circulation patterns that are created due to the buoyancy gradient between the region enclosed by the draft tube and the surrounding region is not sufficient to entrain the gas in this region. Thus this would indicate a stagnant zone. It is also confirmed by the fact that the region around the draft tube doesn't have gas. However, slurry recirculation does exist, between the draft tube and part of the surrounding area due to the density gradient. The intensity of such recirculation and the size and location of the zones involved in such recirculation can be identified and quantified with the aid of CARPT. Moreover, the advances made on CFD will help further quantify and fundamentally analyze the flow pattern obtained in these conditions.

In addition, the image of gas hold up at Level 5 also has a lot of aberrations in it which could be miss understood as gas. This aberration however has been brought about by the presence of solids (organic and sand) due the stagnancy in that region. The animal wastes slurry that had been obtained from the field had a lot of sand in it that invariably escaped the prescreening process that was carried out on the animal waste before it was fed to the reactors.

The presence of sand (silica) makes the system a true three phase system and hence the two phase assumption that was used may not be valid in this region. For a system/situation like this invariably a Dual Source CT (DSCT) unit has to be used; the development of which has been achieved. 


\section{Appendix 3.2 \\ 3.2 Gas distribution with a multipoint gas sparger in a 6 inch anaerobic digester}

The objective of this study was to asses how the gas phase distribution inside the draft tube can be enhanced by using a ring sparger with multiple holes. To achieve this, a ring sparger was designed as shown in Figure 3.7. The details of the ring sparger are shown in Figure 3.8. It was expected that the improvement of the gas phase distribution and its holdup in the draft tube region or the riser region of the reactor will reduce the apparent density of the mixture. This would create a larger density gradient that shall cause enhanced circulation of the liquid/slurry, and hence, improved mixing and reduced dead zones (in active volumes). For this study a 6 inch $(153 \mathrm{~mm})$ diameter digester equipped with a $38 \mathrm{~mm}$ diameter draft tube, same as the one used for performance studies. Gas was recirculated from the bottom using a ring sparger having 25 orifices of $1 \mathrm{~mm}$ diameter. Total open area of the holes was kept same as it was earlier in the case of a single point gas injection. It was planned to conduct CT experiments using an air-water system first and to repeat the CT scans at selected conditions in an animal slurry biogas system. This will allow us to understand the difference being caused in the gas holdups by the presence of solids (biomass, sand, etc.). The orifices were made to face downwards to reduce the probability of getting clogged, as the same sparger will be used in the slurry biogas system. Tomographic scans were taken at three levels (just above sparger, close to mid height of the draft tube and just above the draft tube) at air recirculation rates of 1,3 and 5 liter/min. These air recirculation rates include the lowest and the highest biogas flow rates that were used for the performance studies. The collected CT scans data were processed using same reconstruction algorithm as discussed in Kumar (1994).

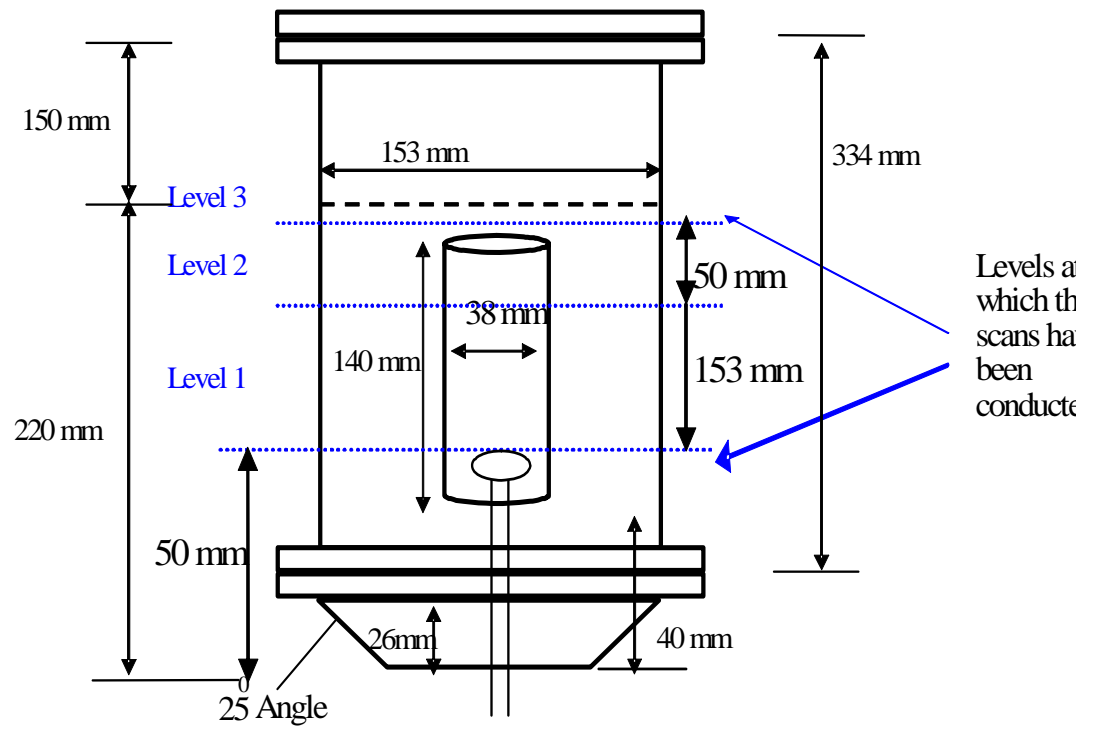

Figure 3.7: Schematic diagrams of 6 inch digester with ring sparger . 


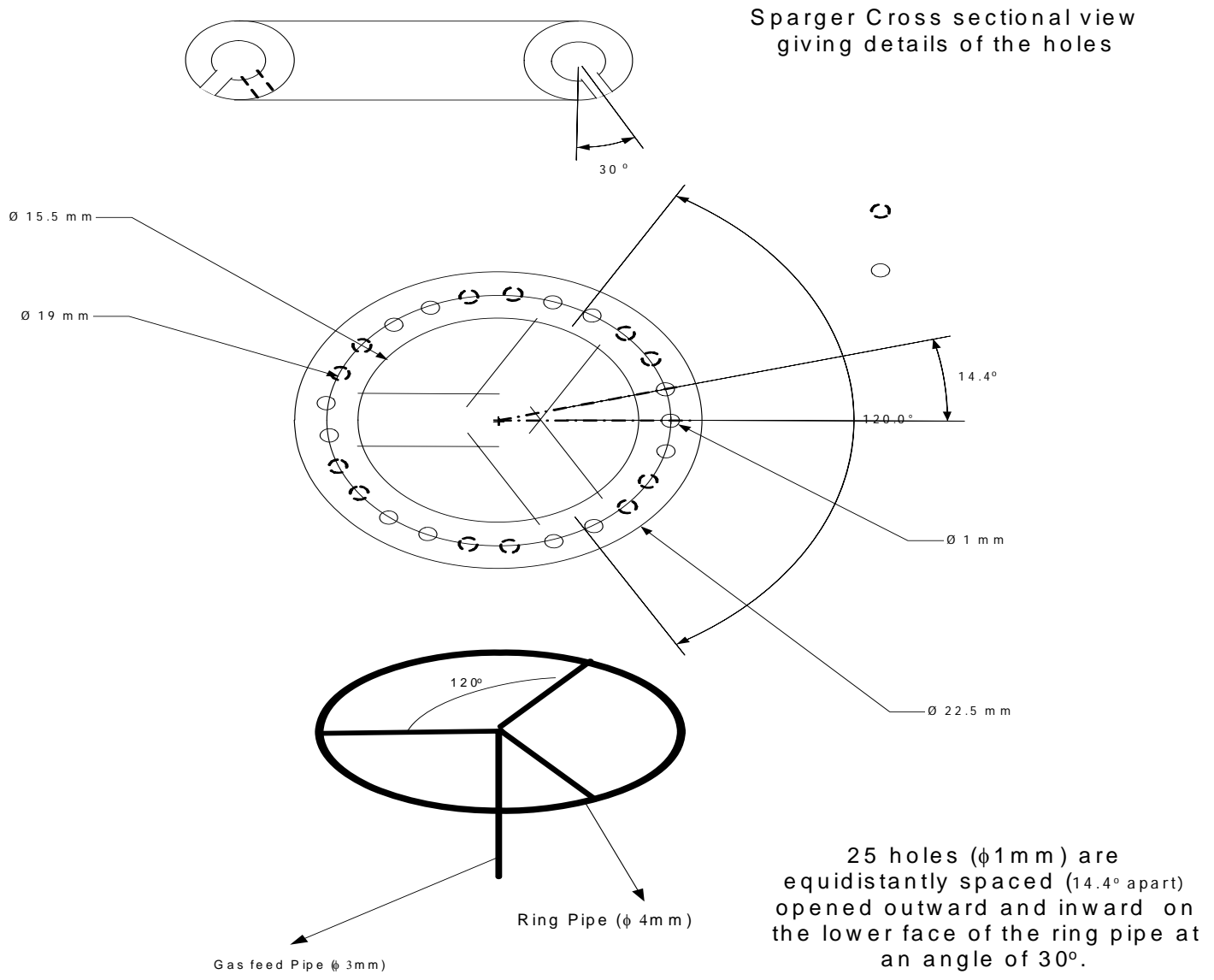

Figure 3.8: Schematic diagram of the sparger with the same open area as the single point injection system studied earlier

The cross-sectional time averaged gas holdup distributions obtained from the CT experiments at three different gas flow rates of 1,3 and $5 \mathrm{l} / \mathrm{min}$ are shown in Figures 3.9 to 3.11. The images in the figures are arranged at levels in descending order, as shown in Figure 3.7. From the tomography scans it is clearly visible that the sparger system has improved the gas hold up distribution. Figures 3.9 to 3.11 show improvement in the gas distribution and increase in the gas holdup inside the draft tube. However, it can also be observed that at a gas flow rate of $1 \mathrm{l} / \mathrm{min}$ (Figure 3.9 Level 1) the holes of the ring sparger were not all open, where maldistribution occurred and hence, the sparger was under utilized. However, at higher gas flow rates ( 3 and $5 \mathrm{l} / \mathrm{min}$ ) all the holes were open and a uniform gas distribution was achieved as shown in Figures 3.10 and 3.11. Figure 3.12 shows the photograph of the air-water system with ring sparger at $5 \mathrm{l} / \mathrm{min}$ air recirculation rate. These results show the advantage of using a multiple holes sparger in place of a single point sparger. However, to show the improvement in the mixing pattern inside the digester due to the density gradient between the region of the draft tube and the region outside the draft tube as a result of the used multiple holes ring sparger could only be seen via computer automated radioactive particle tracking (CARPT) experiments. Therefore, a set of CARPT experiments were conducted and have been reported in Appendix 3.3. 


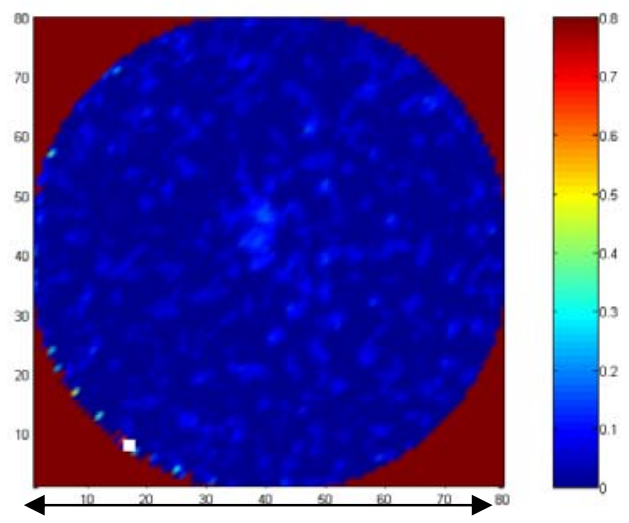

Level 3

153 mm diameter

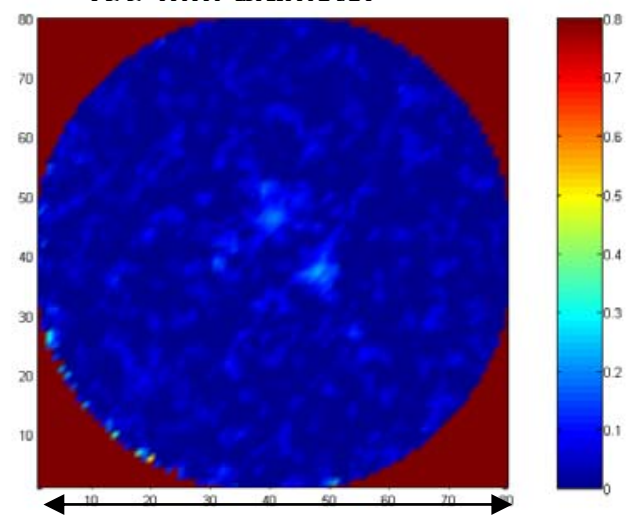

Level 2

$153 \mathrm{~mm}$ diameter

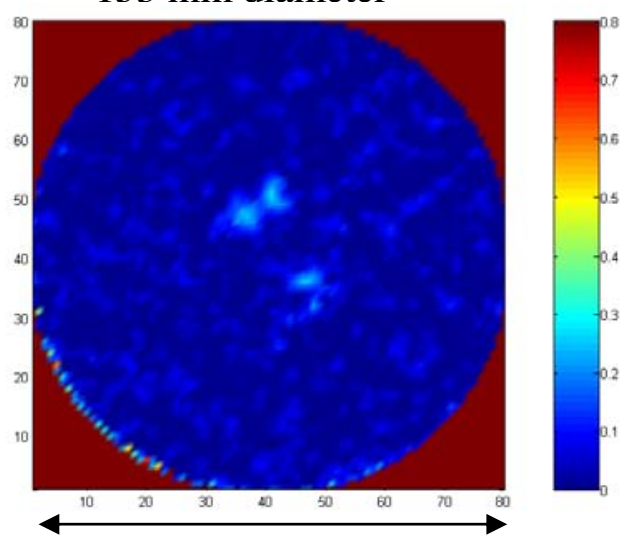

Level 1

$153 \mathrm{~mm}$ diameter

Figure 3.9. Images of the cross-sectional time averaged gas phase distribution in the biogas-slurry system with gas recirculation rate of 1 l/min (80x80 pixels) 


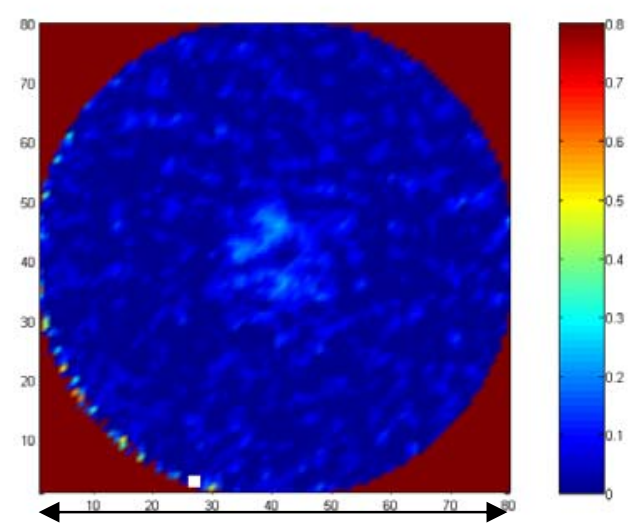

Level 3

153 mm diameter

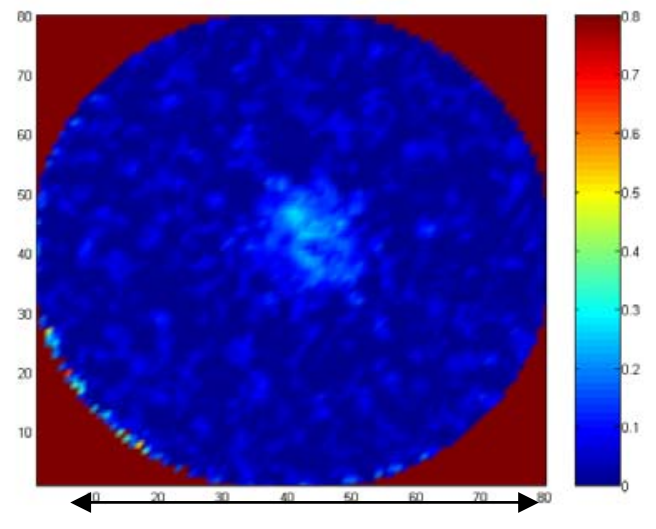

Level 2

153 mm diameter

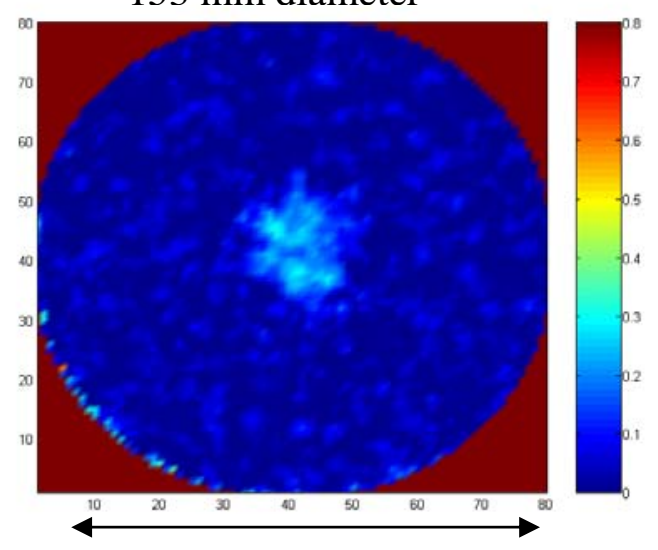

$153 \mathrm{~mm}$ diameter

Level 1

Figure 3.10: Images of the cross-sectional time averaged gas phase distribution in the biogas-slurry system with gas recirculation rate of $3 \mathrm{l} / \mathrm{min}$ (80x80 pixels) 


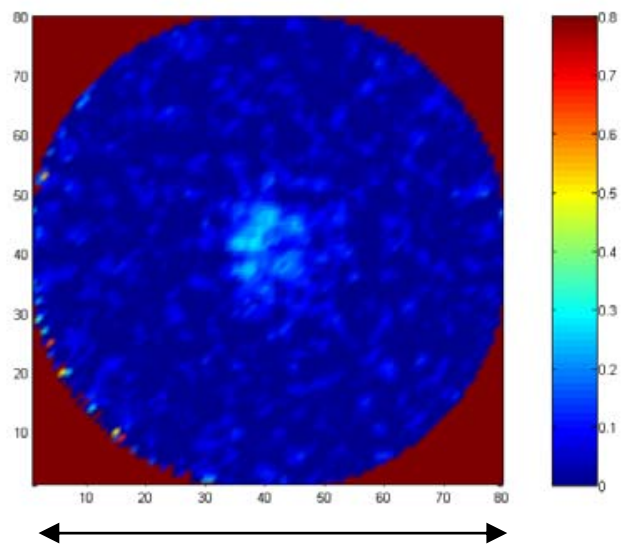

153 mm diameter

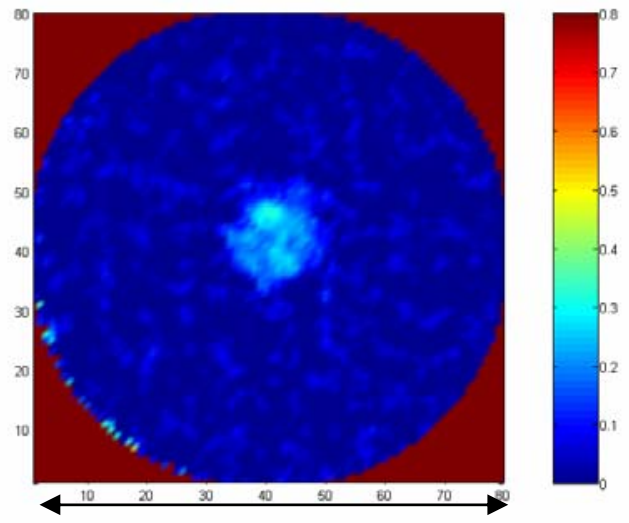

$153 \mathrm{~mm}$ diameter

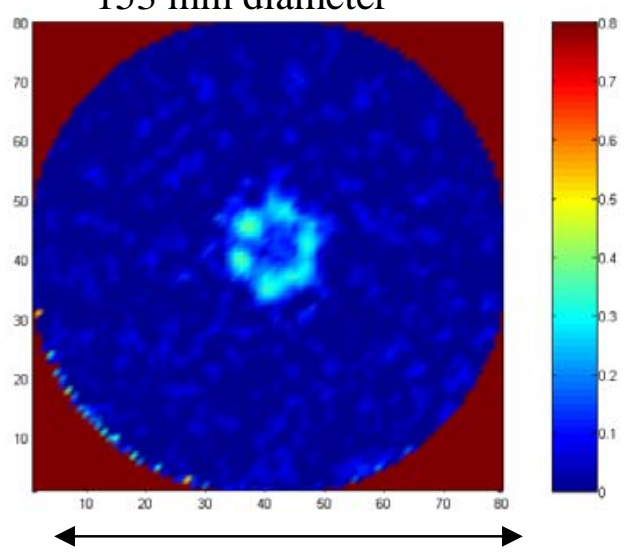

153 mm diameter
Level 3

Level 2

Level 1

Figure 3.11: Images of the cross-sectional time averaged gas phase distribution in the biogas-slurry system with gas recirculation rate of 5 l/min (80x80 pixels) 


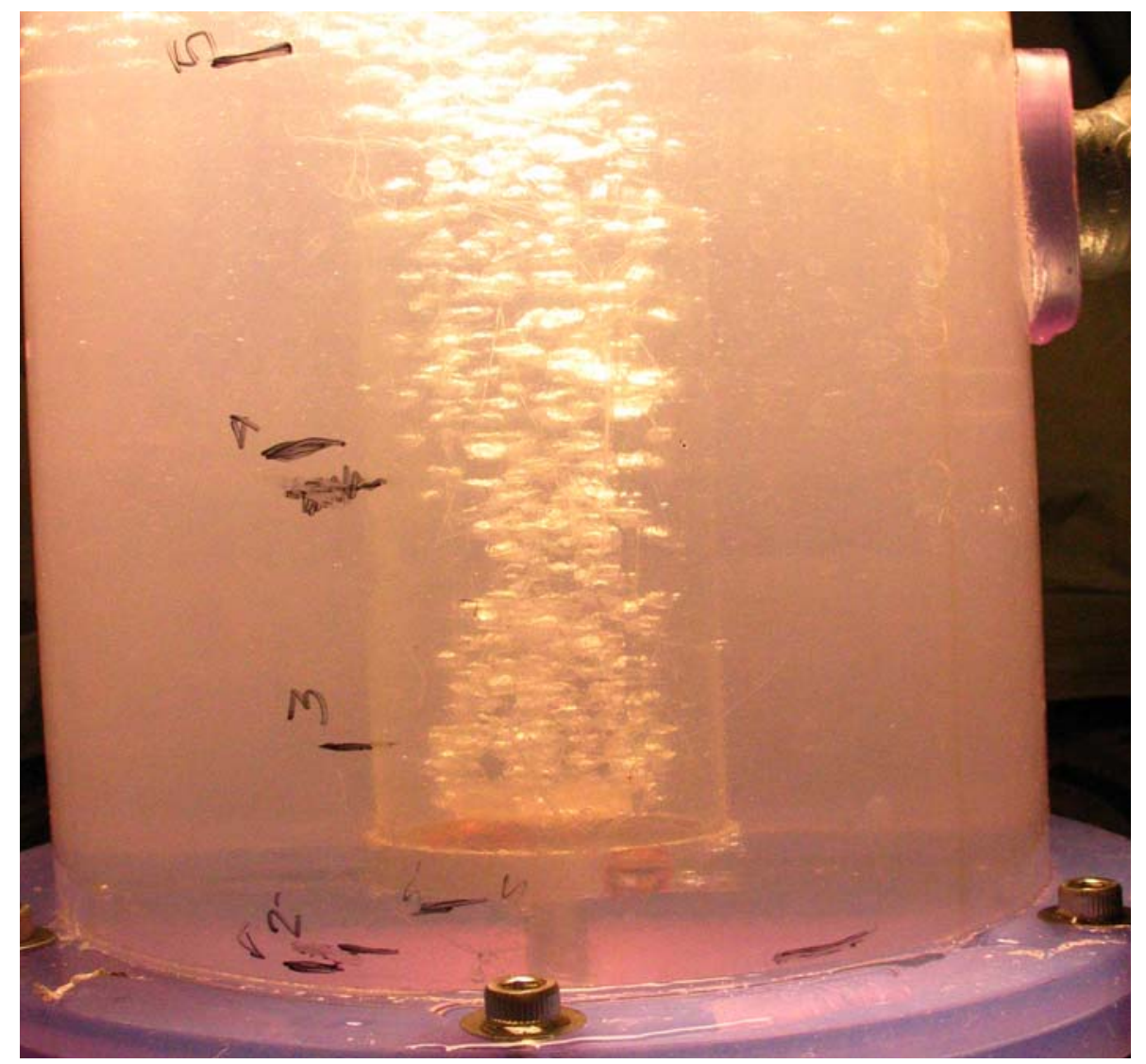

Figure 3.12: Photograph of the gas bubbles in the air-water system at an air recirculation rate of $5 \mathrm{lit} / \mathrm{min}$. 


\section{Appendix 3.3}

Manuscript: - -Varma R, Al-Dahhan MH. (2007). Effect of sparger design on hydrodynamics of a gas recirculation anaerobic bioreactor. In review Biotechnology Bioengineering. 


\title{
Effect Of Sparger Design On Hydrodynamics Of A Gas Recirculation Anaerobic Bioreactor
}

\author{
Rajneesh Varma, Muthanna Al-Dahhan* \\ Bioreactors and Bioprocess Engineering Laboratory (BBEL) \\ Chemical Reaction Engineering Laboratory (CREL) \\ Department Of Energy Environmental and Chemical Engineering \\ Urbauer Hall \#208 Campus Box 1198 \\ One Brookings Dr. \\ Washington University, St. Louis, MO 63130 \\ USA
}

*Corresponding Author

Tel: +1 (314) 935-7187; Fax: +1 (314) 935-4832; e-mail: muthanna@seas.wustl.edu

Contract grant sponsor: US Department of Energy (DOE)

Contract grant number: DE-FC36-01GO11054 


\section{Abstract:}

The effects of sparger design and gas flow rate on, gas holdup distribution and liquid (slurry) recirculation velocity have been studied in a surrogate anaerobic bioreactor used for treating bovine waste with a conical bottom mixed by gas recirculation. A single orifice sparger (SOS) and a multi-orifice ring sparger (MORS) with the same orifice open area and gas flow rates (hence the same process power input) are compared in this study. The advanced non-invasive techniques of Computer Automated Tomography (CT) and Computer Automated Radioactive Particle Tracking (CARPT) were employed to determine gas holdup, liquid recirculation velocity and the poorly mixed zones. Gas flows $\left(Q_{g}\right)$ ranging of $0.017 \times 10^{-3} \mathrm{~m}^{3} / \mathrm{s}$ to $0.083 \times 10^{-3} \mathrm{~m}^{3} / \mathrm{s}$ were used which correspond to draft tube superficial gas velocities ranging from $1.46 \mathrm{x}$ $10^{-2} \mathrm{~m} / \mathrm{s}$ to $7.35 \times 10^{-2} \mathrm{~m} / \mathrm{s}$ (based on draft tube diameter). Air was used for the gas, as the molecular weights of air and biogas (consisting mainly of $\mathrm{CH}_{4}$ and $\mathrm{CO}_{2}$ ) are in the same range (biogas: 28.32-26.08 $\mathrm{kg} / \mathrm{kmol}$ and air: $28.58 \mathrm{~kg} / \mathrm{kmol}$ ). For a given gas flow rate, the MORS gave better gas holdup distribution in the draft tube and hence, enhanced liquid (slurry) recirculation and reduced the fraction of the poorly mixed zones compared to the SOS. The improved gas holdup distribution in the draft tube was found have increased the overall liquid velocity. Hence, for the same process power input the MORS system performed better by enhancing the liquid recirculation and reducing the poorly mixed zones.

Keywords: CARPT; CT; Bioreactor; Sparger design; Power input 


\section{Introduction}

Animal waste from agricultural sources is a source of largely unexplored renewable energy. It is estimated that about 230 million tons of animal waste (dry weight basis) are generated in the United States annually (Sheffield 2002). Most of it is discharged untreated, a potential source of pollution. Methane, a gas with higher green house potential than carbon dioxide, is generated from the waste and could be utilized as an energy source and thereby reduce its potential green house effect. Anaerobic bioreactors readily generate methane for energy utilization from such waste. Moreover, the liquid/semisolid byproduct is an excellent soil conditioner.

Over the last couple of decades the anaerobic digestion process has been applied to different kinds of agricultural, animal, and industrial wastes (Ghosh, 1997; Speece, 1996). Many configurations have been tested and employed in lab scale and large scale systems. However, in a Department of Energy sponsored study by Lusk (1998), which covered about a hundred field anaerobic bioreactors, it was reported that $60 \%$ of them have failed. Mechanical problems associated with mixing was identified as one of the major reasons.

As in all heterogeneous reaction systems, mixing plays an important role in the performance of anaerobic bioreactors. The process of degradation is facilitated by anaerobic microorganisms that are in nature. Mixing facilitates contact between the microbial flocs and the solid suspended matter, as well as the dissolved matter that constitutes the substrate. Mixing also prevents scum formation, settling of the solids and reduction in poorly mixed zones, thereby enabling smooth operation. Hence, a better understanding of the mixing process is required to improve the performance of such bioreactors.

Since the objective of commercially viable anaerobic degradation is to generate net energy while disposing of waste, the amount of process energy that can be invested is limited by the quantity of energy 
generated from the methane produced. Although the other byproducts such as the solid sludge, has great potential as soil conditioner and fertilizer, its commercial value is limited. Typically, the modes of mixing in anaerobic bioreactors can be broadly classified as impeller based agitation, liquid jets or slurry recirculation, and gas recirculation. High mixing rates may yield better performance in biogas generation; however when the process energy requirement is weighed against the energy from the biogas generated, a process with high mixing rates becomes economically unviable. Also, very high mixing rates may cause destruction of the microbial population (Whitmore et al., 1989; Stroot et al., 2001), which would lead to the failure of the bioreactor. It is for this reason that impeller based reactors and circulating liquid jets are not popular.

Gas recirculation bioreactors are a more appealing option as they have no moving parts and their energy requirements are minimal. In these reactors the biogas generated is recirculated with the aid of blowers. It must be noted that the gas in anaerobic processes is used purely to induce mixing and does not consists of any species that participates in reaction or in the cell growth process. In contrast, gas (air) used in aerobic process has a species (oxygen) that participates in the cell growth processes. Therefore requirements for high gas holdup, or a high gas-liquid interfacial area, to facilitate mass transfer of any species from the gas phase to the liquid phase do not exist in the anaerobic bioreactors. This basic reality makes a significant impact on the operating conditions and design of gas mixed bioreactors for anaerobic digestion applications. According to an estimate by Chisti (1998), aerobic reactors that are gas (air) agitated require $3000-2000 \mathrm{~W} / \mathrm{m}^{3}$ to meet the dissolved oxygen and mixing requirements for systems that produce primary metabolites from microorganisms or that treat effluent. Systems that involve animal cell cultures normally require power input in the range $100 \mathrm{~W} / \mathrm{m}^{3}$ (Chisti, 1998). For gas mixed anaerobic systems the EPA (US EPA, 1979) recommends a power input range of $5-8 \mathrm{~W} / \mathrm{m}^{3}$. This shows that the difference of magnitude between the power requirements of gas mixed aerobic and anaerobic bioreactors is of few orders.

In this work the effect of a single orifice sparger (SOS) system, also called an ejector, on mixing and hydrodynamics has been compared with that of a multi orifice ring sparger (MORS). The gas phase 
distribution, the liquid velocity profile, and the liquid flow pattern visualization studies have been conducted for the same superficial gas velocities in both the systems. Such comparison will indicate the impact of the degree of uniformity of gas holdup in the draft tube on the formation of the poorly mixed zones in the system. These studies have been done with the synergistic use of single source gamma ray Computer Tomography (CT) and Computer Automated Radioactive Particle Tracking (CARPT). CARPT and CT are not hindered by typical opacity of reactor walls and its contents. CARPT enables mapping the flow field of a particular phase (solid or liquid) in a given system. CT employs the penetrable property of gamma-ray photons to image the holdup, or spatial distribution, of a particular phase at a given cross sectional level in any system.

\section{Materials and methods}

\subsubsection{Details of the surrogate anaerobic bioreactor}

The experiments were carried out in a 6 in. $\left(15.24 \times 10^{-2} \mathrm{~m}\right)$ diameter bioreactor with a conical bottom and a draft tube (Fig.1and Fig.2). The draft tube diameter was selected so that the ratio of the draft tube's internal diameter $\left(D_{d i}\right)$ to that of the internal diameter of the reactor $\left(D_{T}\right)$ was 0.25 . Various researchers in the past have worked with air lift reactors with various draft tube diameter to reactor diameter ratios (Pironti et al.,1995; kojima et al., 1999). Their observation was that lower draft tube diameters to reactor diameter ratios tend to give a higher liquid velocity in the draft tube. In a study by Karim et al. (2004) in an 8 in. $\left(20.32 \times 10^{-2} \mathrm{~m}\right)$ diameter gas recirculation bioreactor, the draft tube diameter was maintained at 0.2. Walker Process Inc., (Aurora, IL 60506-285), one of a commercial manufacture of biogas recirculation anaerobic bioreactors for municipal wastes, makes bioreactors with ejector tubes (similar to the SOS system) with a draft tube diameter to reactor diameter ratio of 0.07 to 0.08 . Since our aim was to study the effect of sparger design, the draft tube to reactor diameter ratio was chosen in the range of that studied by previous researchers. The draft tube was spaced equidistant from the top and the bottom of the active region of the bioreactor, along its vertical axis at the center. The effect of the conical 
bottom has been studied in a flow pattern visualization study, using computational fluid dynamics, by Vesvikar and Al-Dahhan (2005) for surrogate gas recirculation anaerobic bioreactors. One of the conclusions reached was that a $25^{\circ}$ sloping angle from the horizontal gave the better performance for a given gas flow rate. Hence a conical bottom with a sloping angle of $25^{\circ}$ from the horizontal plane was used for this study.

\subsubsection{Single orifice sparger (SOS)}

The single orifice sparger (SOS), shown in Fig.1, consists of a long $0.5 \times 10^{-2} \mathrm{~m}$ i.d. steel tube which opens into the reactor at the bottom of the draft tube. The steel tube is fixed to the upper lid of the reactor. There are four flat tie rods at the upper end and the lower end of the draft tube. The rods are welded on one end to the steel tube and to the draft tube at the other. This arrangement to supports the draft tube and keeps it in the desired position during operation. The gas is introduced through this tube from the top of the reactor, and it enters the reactor at the lower end of the draft tube.

\subsubsection{Multi - orifice ring sparger (MORS)}

The multi-orifice ring sparger (MORS), as shown in Fig.2(a), consists of a tubular ring supplied with gas by three central tubular arms. The diameter of the orifices on the ring is $0.1021 \times 10^{-2} \mathrm{~m}$. Twentyfour equally spaced orifices are drilled in the ring (Fig.2 (b)). This way the open area (all orifices included) of the MORS is same as that of the SOS. The gas is introduced from the bottom of the bioreactor through a steel tube into the center of the MORS where the three cross arms meet. The MORS is set at $0.048 \mathrm{~m}$ from the bottom of the bioreactor. The orifices face downwards at an angle of $30^{\circ}$ from the vertical (Fig. 2(b)), and face alternately inwards and outwards in pairs. The orifices open downwards to minimize the possibility of being choked or clogged with solid debris that circulates in the bioreactor slurry. Since the anaerobic bioreactor involves a gas-liquid-solid system like slurry bubble columns with fine catalyst 
particles, the precedent of downward facing pores in the sparger set by George et. al. (2001) and Ong (2003) was followed in this case. The orifices of the MORS open into the system at a height of $0.042 \mathrm{~m}$ from the bottom of the reactor. Hence, the gas in the MORS system also enters at the same axial position as in the SOS system.

The draft tube support system consists of four flat tie rods that connected to a flat circular strip which has an internal diameter that matches the draft tube's outer diameter. This strip is attached with fasteners (not shown in Fig. 2(a)) to the bottom of the draft tube. The four tie rods converge at the center, where they are welded to the wall of a steel tube. This steel tube is attached to the bottom of the reactor (at the center of the conical section) with fittings. The steel tube attached to the center of the MORS is of smaller diameter than the steel tube connected to the tie rods of the draft tube support system and is able to fit into it. This assembly is clamped to the outer tube. The gas line is attached to the tube that is connected to the MORS.

\subsection{Experimental conditions}

This study used bovine manure from the University of Tennessee (UT) dairy farm at Oak Ridge, TN. The waste was pre-treated before use by wet screening through a $2 \times 10^{-3} \mathrm{~m}$ sieve, followed by dilution. For this slurry the total solids (TS) level was set to $50 \mathrm{~kg} / \mathrm{m}^{3}$, and the volatile suspended solids (VSS) concentration was found to be $3.45 \mathrm{~kg} / \mathrm{m}^{3}$. A total volume of $4.2 \times 10^{-3} \mathrm{~m}^{3}$ for each reactor was used in the study. The gas flow rates ranged from $0.017 \times 10^{-3} \mathrm{~m}^{3} / \mathrm{s}$ to $0.083 \times 10^{-3} \mathrm{~m}^{3} / \mathrm{s}$. Details of flow rates and the superficial gas velocity based on the draft tube diameter are given in Table I. The gad flow rate was regulated with the air of a rota-meter attached to the air line.

Air was used in this study to substitute the biogas (a mixture of $\mathrm{CH}_{4}$ and $\mathrm{CO}_{2}$ ). Karim et al. (2005) conducted performance studies with four anaerobic bioreactors mixed by different modes processing bovine manure from the same dairy farm. One of these included a configuration similar to the SOS system. The authors reported a composition ratio of $\mathrm{CH}_{4}: \mathrm{CO}_{2}$ ranging from 56-64\%: 44-36\% in the biogas 
produced. In another performance study by Borole et. al. (2006) involving a gas mixed reactor with bovine manure, the ratio of $\mathrm{CH}_{4}: \mathrm{CO}_{2}$ was found to be $60: 40$. The molecular weight of biogas with these compositions is in the range $28.32-26.08 \mathrm{~kg} / \mathrm{kmol}$. Based on these findings air was used to substitute the biogas. Another reason for using air is that the CAPRT procedure used for liquid velocity measurement (described later) involves a calibration step that involves the insertion of a rod into the system. A hermetic system would not allow access to this calibration rod and hence would complicate the CARPT calibration process. The study by Karim et al. (2005) also indicates that the biogas generated in 24 hrs (once the reactor operation has reached steady state) is only about 3\% of the total volume of the gas recirculated through the sparger at a rate of $0.017 \times 10^{-3} \mathrm{~m}^{3} / \mathrm{s}$. Hence it can be concluded that the hydrodynamic effects due to the gas in the system are mainly caused by the recirculated biogas, and the contribution of the biogas generated by waste degradation is minimal.

The process power input into the reactor, via gas, was calculated using the expression (Eq. 1) developed by Casey (1986)

$$
\frac{P}{V}=\frac{G_{r} P_{2}}{((\lambda-1) / \lambda)}\left[\left(\frac{P_{1}}{P_{2}}\right)^{((\lambda-1) / \lambda)}-1\right] .
$$

$P$ is power, $V$ is the active volume of the reactor, $G_{r}$ is the biogas recirculation rate, $P_{2}$ is the pressure in the head space, $\mathrm{P}_{1}$ is the pressure at the point where gas in introduced $\left(\mathrm{P}_{2}\right.$ static ahead of slurry) and $\lambda=1.03$ (Casey, 1986). The range of power input (based on gas flow rate) used in this study is within the range of 5-8 W/m³ (summarized in Table 1) suggested by the EPA (US EPA, 1979) for such systems. Therefore it can be safely said that the surrogate anaerobic bioreactor system used in this study very closely represents a lab scale anaerobic bioreactor. Since this expression (equation. 1) doesn't have terms that are dependant on the open area or other design parameters of the sparger, it is assumed that for a given gas flow rate the same amount of energy is introduced into both the systems. Hence the conclusions drawn in this study represent an actual system with a great degree of accuracy. 


\subsection{Overview of computer automated radioactive particle tracking (CARPT) technique and experimental procedure}

CARPT is a powerful method employed for measuring the flow field, instantaneous time averaged velocities and turbulent parameters, dead zones, residence time distribution, and other parameters of a particular phase in a given system. It tracks the motion of a radioactive particle that represents an element, or small packet, of a phase in a system. It is usually used to track liquid or solid phases (Devanathan, 1991). For tracking liquids, the density of the radioactive particle (usually a few hundred microns in size) is made to match the density of the liquid by encapsulation in a polypropylene (PP) ball. Such a particle is tracked with the aid of an array of $\mathrm{NaI}$ scintillation detectors placed at strategic locations around the system. A typical CARPT study involves the following sequence of steps: preparation of radioactive particle, in situ calibration (at experimental conditions), experimental runs, and processing of the data generated from the preceding two steps (Roy, 2000). These aspects have been discussed in brief here to maintain continuity, for further details the reader is advised to refer to the works of Karim et. al. (2004), Devanathan (1991), and Degaleesan (1997).

\subsubsection{Particle Preparation}

In this study the liquid phase was tracked. A ${ }^{46} \mathrm{Sc}$ radioactive particle of $300 \mu \mathrm{Ci}$ strength and 150 micron diameter was encapsulated in a PP ball of $0.001 \mathrm{~m}$ diameter with a calculated air gap. The density of the composite particle was made to match that of water. The density of the composite particle was checked based the terminal settling velocity and Stoke's law (Equation 2)

$U_{t}=\frac{g D_{p}\left(\rho_{p}-\rho_{\mathrm{w}}\right)}{18 \mu_{\mathrm{w}}}$.

Eq. 2

To determine the settling velocity the particle was released in a $1 \mathrm{~m}$ long acrylic tube filled with water. The terminal settling velocity was calculated based on the time taken to settle in the last $0.5 \mathrm{~m}$ of the tube. If 
the particle floated, it was coated with commercial spray paint to make it heavy. If it was heavy, it was made lighter by coating it with a commercial epoxy glue spray which has a density less than that of water. The final composite particle that was used for the experiments had a density of $1.0016 \mathrm{~kg} / \mathrm{m}^{3}$ (average absolute error $=0.0016$, standard deviation $=0.0012$ : based on five terminal settling velocity measurements).

\subsubsection{Calibration, particle tracking and data processing}

The gamma ray photon counts received by the $\mathrm{NaI}$ scintillation detectors are a function of the distance of the radioactive particle from the detector, and of the attenuation of the photons due to the material between the detector and particle. Hence the calibration process is carried out in situ, i.e. the reactor was operating at the given conditions and configuration for which the experiment was to be carried. Thus, the number of counts received during calibration matches the counts received if the free particle were to be at the same location in the tracking experiment (described later). For a given signal strength (or the number of counts) detected for a particular detector, the particle could be anywhere along the surface of a hypothetical sphere with a radius represented by the distance the corresponding counts or signal strength refer to. Hence a minimum of three detectors are required to pinpoint the location of a

particle. A total of sixteen detectors were used in this system. In this study an automated calibration device developed by Lou (2005) was used.

To test for error in the calibration, the counts data for the known positions of the particle was split into two groups, which constitute $80 \%$ and $20 \%$ of the complete data set. The $80 \%$ data set was used to calibrate the system by developing a distance-counts map. The $20 \%$ data set was traced based on this distance count map. The traced positions were compared to the positions (distance) at which these data points (the $20 \%$ set) were taken. Error was determined by taking the mean of the differences in the position. The results summarized in columns 5 and 6 in Table I show that the average error was $0.003 \mathrm{~m}$. 
Once the calibration procedure was completed the particle was removed from the calibration rod and introduced into the system. The particle was allowed to move freely within the system, unhindered by external influence. While the particle was moving in the reactor operating at the set conditions, the counts received by all the detectors were recorded at the same sampling rate as it was calibrated (in this case, $50 \mathrm{~Hz}$ ). This tracking process was carried out for 18 hours for all the conditions described in Table I, which provides enough data to reach asymptotic values of time averaged velocity for each location in the system.

To reconstruct the position of particle based on the tracking data, the distance-count map generated by the calibration process was used. Each location of the particle was at a time interval of 0.02 seconds; hence the instantaneous position (i.e., Lagrangian trajectory) of the particle was obtained. The process involves the application of a weighted least-squares algorithm; a wavelet based position filtering, and by a particle position reconstruction algorithm described elsewhere (Devanathan, 1991; Degaleesan, 1997; Rados, 2003). Time differencing between two positions yielded instantaneous velocities, which were averaged at each spatial location over the whole time span of the experiment to yield the ensemble average velocity flow map of the system. To get Eulerian information, the volume of the reactor was divided into 28160 cells ( 20 divisions in $r, 32$ in $\theta$, and 44 in the $z$ direction, respectively). This enables better resolution in visualizing the velocity profile. The number of cells used is determined on the basis of work done by Degaleesan (1997). The estimated instantaneous velocity information was then assigned to the cell falling at the midpoint of two successive particle positions. The average liquid velocity data thus generated was used for comparison in this study.

\subsection{Overview of computer tomography (CT) technique and experimental procedure}

\subsubsection{Data acquisition process}

The details of the of the mechanical hardware and the data acquisition system for the single source $\gamma$-ray CT unit used in this study has been described at length by Karim et. al.(2004) and Roy (2006). The E- 
M algorithm proposed by Lange \& Carson, (1984) and implemented by Kumar (1994) was used for image reconstruction.

\subsubsection{CT Data processing}

Single source CT can be used to compute the individual phase holdup of only a two phase system, and can be extended to three phases where one of the phases is stationary, like a solid phase in structured packing (Roy 2004). In this study the liquid and solids were considered as a single slurry phase, as the solids concentration was only $5 \%$ (w/v basis), and the solids contribution to attenuation was minimal. Also, the pretreatment avoided large debris and sand particles, etc., in the system that would cause high attenuation. Per Beer-Lambert's law, the attenuation by any substance is a linear sum of the attenuation of the individual pure components. Therefore, the attenuation value computed for a given pixel in the image domain via the process of tomography by the use of the E-M algorithm is a liner sum of the attenuation of the pure components in the pixel (Equation 3)

$\mu_{E M, l-g}\langle x\rangle=\mu_{l} \varepsilon_{l}\langle x\rangle+\mu_{g} \varepsilon_{g}\langle x\rangle$.

Additionally, because the fact that the system consists of only two phases, gas and slurry, the sum of the volumetric fractions of these two phases is unity (Equation 4) for any given pixel. The pure component values are usually available from literature. However in this case, for accuracy the reactor was scanned with the individual phases, i.e. empty (with air) and with the slurry. The scans for such systems are represented by Equations 5 and 6, where the values of the holdups are unity.

$$
\begin{aligned}
& \varepsilon_{l}\langle x\rangle+\varepsilon_{g}\langle x\rangle=1 \\
& \mu_{E M, g}\langle x\rangle=\mu_{l} \varepsilon^{\circ}{ }\langle x\rangle \\
& \mu_{E M, g}\langle x\rangle=\mu_{g} \varepsilon^{o}{ }_{g}\langle x\rangle \\
& \varepsilon^{o}{ }_{l}\langle x\rangle=\varepsilon_{g}^{o}\langle x\rangle=1
\end{aligned}
$$


Hence, the holdup of the gas phase can be calculated based on Equation 8, which is derived from Equations $3,4,5,6$ and 7

$\varepsilon_{g}\langle x\rangle=1-\left(\frac{\mu_{E M, I-g}\langle x\rangle-\mu_{E M, g}\langle x\rangle}{\mu_{E M, l}\langle x\rangle-\mu_{E M, g}\langle x\rangle}\right)$.

Eq. 8

Scans of the systems with SOS and MORS were carried out at level 1 (5 x10-2 $\mathrm{m}$ from the base of the reactor) and at level $2\left(15 \times 10^{-2} \mathrm{~m}\right.$ from the base of the reactor) as indicated in Fig. 1 and Fig.2 respectively.

\section{Results and discussion}

\subsection{Computed tomography}

3.1.1 Effect of gas flow rate and sparger configuration on gas holdup distribution.

Fig. 3 shows a three dimensional perspective of the tomograms showing the time averaged gas holdup distribution of the system with SOS and the MORS, at levels 1 and 2 in the draft tube region with a gas flow rate of $0.05 \times 10^{-3} \mathrm{~m}^{3} / \mathrm{s}$. These images have been reconstructed on the basis of Equation 8 . The draft tube region alone has been shown here as the tomograms generated for the entire cross section (not sown here) that gas holdup in the region outside the draft tube in the reactor is negligible. This can be seen in Fig.5 and Fig.6 which shows the radial gas holdup profile (discussed later). The gas spreads within the draft tube as it is released and rises in the draft tube for both the systems. The MORS system has more gas distributed in the draft tube than does the SOS. In Fig.3(a) there is a local spot of high gas holdup visible at the center within the SOS system at level $2\left(15 \times 10^{-2} \mathrm{~m}\right.$ from the base of the reactor). This represents the gas inside SOS injector tube that extends from the top of the reactor to the bottom region of the draft tube (Fig.1). There is also a concentration of the gas phase near the center of the draft tube surrounding the SOS injection tube. This shows that there is certain degree of channeling of the gas within the central region of the draft tube in the reactor with the SOS system, as it rises within the draft tube after it is introduced at the bottom of the draft tube. 
The gas holdup distribution in the draft tube of the MORS system is quite different. It can be seen as circles over the ring sparger at level 1 (0.05 $\mathrm{m}$ from the base), as level 1 is located just above the MORS. The gas distributes itself over the region of the draft tube for the system with MORS as it rises, as seen in at level 2 in Fig.3(b). For both SOS and MORS, the gas flow rates were maintained at $0.05 \times 10^{-3} \mathrm{~m}^{3} / \mathrm{s}$ in Fig.3, hence a better gas holdup distribution is observed in the draft tube region of the bioreactor with MORS.

The presence of the local circular regions of gas holdup above the sparger also indicates that for the given flow rate not all the pores in the MORS open up. At a higher gas flow rate $\left(0.082 \times 10^{-3} \mathrm{~m}^{3} / \mathrm{s}\right)$, more open pores are observed in the tomogram as shown in Fig.4. There is insufficient pressure drop across the sparger at lower flow rates to open all the pores in the sparger. The gas holdup tomograms do not have resolution high enough to judge the exact number of open pores in the MORS. It must be noted that even with a few pores open at low gas flow rates, the MORS still gives a better gas holdup distribution than the SOS.

An alternative, to ensure all pores open, could be to reduce the diameter of the pores further (and there by reduce the open area) with out compromising the number of pores. However, given the nature of the bovine manure slurry and its inconsistency in terms of the characteristic of the solids present in it, the chances of pore clogging would be high during operation for very small pores. The other alternative could be to reduce the number of pores in the sparger such that the minimal gas flow rate covered in the study would open all the pores. This in turn could reduce the number if pores drastically (depending on the gas flow rate it would be designed for). When this design ( with reduced number of pores) is used for higher flow rates the hold up distribution would be affected as the gas would be confined to limited number of pores versus a scenario where it could have been introduced through a higher number of pores, there by giving better gas holdup distribution. For this study, having a different sparger for each of the gas flow rates with specific number of pores (such that all are open during operation) would have been impractical. 
Fig.5 and Fig.6 depict the azimuthally averaged mean gas holdup profiles in the reactor at levels 1 and 2 respectively, where the gas holdup is plotted versus radius. The values for all the flow conditions covered in Table I are shown for both SOS and MORS systems. It is clearly visible that at level 1 (Fig.5) for the case of the SOS there are certain local spots where the gas holdup is higher than that of the MORS for a given gas flow rate. The local peak (at a radius of $1 \times 10^{-2} \mathrm{~m}$ ) in the gas holdup profiles of the MORS is due to the fact that the location of level 1 is just above the MORS; hence the gas coming out the orifice is responsible for this peak. At level 2 (Fig.6), the gas holdup distribution is more radially uniform in the MORS system. In contrast, all the profiles for the SOS system show a distinct peak near the center of the reactor. A better understanding can be obtained by observing the mean of the gas holdup profile. Fig.7 shows the mean gas holdup in the draft tube versus the superficial gas flow velocity. The superficial gas velocity is based on the gas flow rate and the cross-sectional area of the draft tube. The mean gas holdup is calculated by Equation 9

$$
\overline{\varepsilon_{g}}=\frac{\sum_{x=1}^{n_{\text {draft }}} \varepsilon_{g}\langle x\rangle}{n_{\text {draft }}} .
$$

Here $x$ represents the pixel index, and $n_{\text {draft }}$ the number of pixels in the draft tube region. Since the area each pixel represented in the domain is equal, $\overline{\varepsilon_{g}}$ in Equation 9 is an area weighted average gas holdup. Fig.7 clearly confirms that the mean gas holdup is higher for the MORS system at both levels 1 and 2. As gas flow rate increases, the gas holdup increases. This rise in gas holdup with gas velocity is more pronounced in the case of the MORS system. The superficial velocities' effect on gas holdup is well known. However, what is highlighted here is that the effect sparger design and its impact on gas holdup distribution. For a fixed superficial gas velocity the system with MORS gives a higher (spatially) average gas holdup in the draft tube. The impact of this enhancement in gas holdup distribution on the liquid velocity and poorly mixed zones outside the draft tube will be discussed in the CARPT results section.

\subsubsection{Characterization of the uniformity of gas holdup distribution}


It is important to quantify the uniformity of the cross-sectional gas holdup distribution in order to properly compare the effects of the design and operation parameters of the bioreactor. In this section the different approaches have been discussed with their merits and demerits.

Maldistribution in flows, particularly those that contain liquids, has been characterized at length in the literature. Some of these approaches divide the domain of flow into sub domains of a given size. Then statistical tools are used to compare the holdup in this sub domain with the global mean (i.e. mean of the entire domain). Mercandelli et al. (2000) calculated the maldistribution factor for liquid flow in gas-liquid packed columns using Equation 10. The flow domain was divided into nine sectors (denoted by N)

$$
M_{f}=\sqrt{\frac{1}{N(N-1)} \sum_{n=1}^{N}\left(\frac{\varepsilon_{l, n}-\overline{\varepsilon_{l}}}{\overline{\varepsilon_{l}}}\right)} \cdot \quad \text { Eq. } 10
$$

Here $M_{f}$ is the maldistibution factor, $\overline{\varepsilon_{l}}$ is the mean liquid holdup (based on the entire domain) and $\varepsilon_{l, n}$ is the sub domain gas holdup. Roy (2006) has used a T-Test based method on liquid saturation data in packed monolith reactors obtained from computed tomography (CT). In this approach the main domain was divided into several sub domains of 6 pixels each. Each of these sub domains was compared with a hypothetical sub domain of the same size (6 pixels)that has the mean holdup values calculated based on the entire domain (global mean), with the aid of the T-test with a 95\% confidence interval. If the sub domain 'passed' the t-test, the null hypothesis was satisfied, the two groups were statistically the same, and the value 1 was assigned to that sub domain group. If it failed then a 0 was assigned. Finally a uniformity factor was determined as a percentage of the sum of domains labeled with 1 . The sub domain T-test has the right conceptual fundamentals and is able to give a quantitative value to degree of uniformity in terms of a percentage, which is convenient for the purpose of comparison.

However, domain based methods in general have the two drawbacks. The first drawback is the strategy one uses for choosing the size and orientation of the sub domain. Experimentalists who use collectors to make liquid distribution measurements in structured columns typically create sub domains in 
the collectors in the order of 16-25 in number, and hence find it convenient to use a sub domain oriented strategy. Since the orientation of the sub domain remains constant in the set up, liquid flow distribution with different packing materials and operational conditions can be compared. However if one were to vary the sub domain size, the results differ. The second drawback is the problem that could evolve in situations where the holdup of the particular phase of interest is small and confined to a small region. While computing the mean holdup of the entire domain in order to carry out the T-test, the weighted contribution of the holdup to all the pixels, from the few that have the phase in them, may be very small. When the T-Test is done there wouldn't be any statically significant difference in the mean of the sub domain and the global mean. Hence the analysis would indicate that the system is very well distributed, which would be counter intuitive to the visual observation in the tomogram.

To take a better look at the effect of the domain size on the gas holdup distribution, the MORS system data at $0.05 \times 10^{-3} \mathrm{~m}^{3} / \mathrm{s}$ gas flow rate at level 2 was subjected to the T-test based uniformity factor analysis using the Roy (2006) approach. There are 436 pixels in the domain representing the cross-section of the draft tube. Sub domains with pixels blocks varying from 5 to 50 were used yielding results shown in Fig.8. Clearly the distribution factor varies as the size of the domain changes. There is no asymptote visible in Fig.8, even where small numbers of pixels are used for a sub domain, which would make the number of sub domains large. However, if the holdup distribution analysis is performed for different flow conditions in a given experimental setup then one fixed sub domain size may be considered as a basis for comparison. This type of analysis would not inspire confidence in characterizing holdup distribution in a system for universal comparison.

The T-test based analysis was carried out for this study, using a sub domain size of 6 pixels. The results are summarized here. For $V_{g}=1.46 \times 10^{-2} \mathrm{~m} / \mathrm{s}\left(Q_{g}=0.017 \times 10^{-3} \mathrm{~m}^{3} / \mathrm{s}\right)$, at both level 1 and level 2 the uniformity factor for the MORS system is a little more than double as that of the SOS (Level 1: MORS $=61.0 \%$, SOS $=27.8 \%$; Level 2 : MORS $=53.2 \%$, SOS $=30.5 \%$ ) system. There is a similar trend for $V_{g}=4.41 \times 10^{-2} \mathrm{~m} / \mathrm{s}\left(Q_{g}=0.05 \times 10^{-3} \mathrm{~m}^{3} / \mathrm{s}\right)$ at both levels (Level 1: MORS $=42.5 \%, \mathrm{SOS}=21.1 \%$; Level 
2: MORS $=51.0 \%$, SOS $=33.3 \%$ ). However, when compared to the previous flow rate, the uniformity factor values appear to be lowered. Finally for $V_{g}=7.35 \times 10^{-2} \mathrm{~m} / \mathrm{s}\left(Q_{g}=0.083 \times 10^{-3} \mathrm{~m}^{3} / \mathrm{s}\right)$, the values for distribution at levels 1 and 2 are almost comparable for the MORS and SOS systems (Level 1: MORS = $36.2 \%$, SOS $=23.2 \% ;$ Level 2: MORS $=21.0 \%$, SOS $=21.3 \%$. These observations are contrary to tomograms for these levels (not shown) and the azimuthally averaged gas holdup profiles shown in Fig.5 and Fig.6.

A simpler statistical method is developed in this work where the variance of the holdup distribution of the entire domain is calculated based on the gas holdup in each pixel in the domain. The variance is normalized by the mean gas holdup for that domain. This non-dimensional variance (Equation 11) can be called a mal-distribution factor. This equation bears resemblance to the ones used by Marcandelli (2000) and Jiang (2000) to characterize maldistribution $\sigma=N_{\text {Mal }}=\sqrt{\frac{1}{n} \sum_{j=1}^{n}\left(\frac{\varepsilon_{g, j}\langle x\rangle-\overline{\varepsilon_{g}}}{\overline{\varepsilon_{g}}}\right)^{2}}$. Eq. 11

If the variance of the holdup data is zero, this would indicate that that holdup profile is absolutely uniform and even (mal-distribution is zero). If non-dimensional variance tends towards unity, it is mal-distributed. One must be aware that this value could be greater than unity in severely mal-distributed cases.

It is therefore better to call this ratio a 'mal-distribution number $\left(N_{M a l}\right)$ ', just like any nondimensional number, as factors or coefficients tend to be between zero and unity. This method is more simple and fundamental and not dependent on the size or geometrical orientation of the sub domains involved. Also, as the number of pixels ( $n$ in Equation 11) increases, by statistical principles the accuracy of $N_{\text {Mal }}$ will also increase.

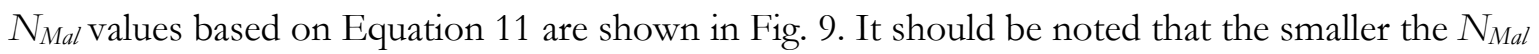
value, the better the gas holdup distribution. Clearly, for $V_{g}=1.46 \times 10^{-2} \mathrm{~m} / \mathrm{s}\left(Q_{g}=0.083 \times 10^{-3} \mathrm{~m}^{3} / \mathrm{s}\right)$ there is difference in $N_{\text {Mal }}$ by a factor of 2 (over 100\% difference) at Level 2 for the SOS and MORS systems. This difference is also seen at $V_{g}=4.41 \times 10^{-2} \mathrm{~m} / \mathrm{s}\left(Q_{g}=0.05 \times 10^{-3} \mathrm{~m}^{3} / \mathrm{s}\right)$. At higher $V g$ values, 
this difference narrows a bit, but still differs by a factor of $70-80 \%$ between the SOS and MORS systems. All the $N_{M a l}$ values for SOS are above unity, this indicates severe maldistribution of the gas in the draft tube region for the system with SOS. Hence it can be concluded that the MORS, in spite of partially opened pores (fig.4), gives a better gas holdup distribution than the SOS for a given gas flow rate.

\subsection{Results from computer automated particle tracking (CARPT)}

\subsubsection{Liquid velocity and flow pattern in digester with MORS and SOS}

Selected results of the azimuthally average mean liquid velocity values computed from the CARPT data are represented in the quiver plots in Fig.10. This figure depicts the azimuthally averaged velocity vectors in the system formed due to its axial and radial components, hence depicting the flow pattern in the digester with the MORS and SOS systems (for $Q_{g}=0.05 \times 10^{-3} \mathrm{~m}^{3} / \mathrm{s}$ and $V_{g}=4.4 \times 10^{-2} \mathrm{~m} / \mathrm{s}$ in draft tube). As mentioned earlier, the system is divided into small cells for data processing; the vectors in this figure are shown for each such cell. It can be observed that two circulation loops exist. The liquid tends to rush into the bottom part of the draft tube in the region where the gas is introduced through the sparger, as is evident for both the MORS and the SOS system. It can be observed in this region that the velocity vectors are more prominent in the SOS system (Fig.10(b)). By virtue of the design of the SOS system, the gas is introduced at one single location into the system, which creates a local low density spot which sucks the liquid into this region. The liquid then is carried up the draft tube and gets released in the upper section of the reactor. The streamlines plot shown in Fig. 13 depict the direction of flow liquid in the system, these confirm the results shown in Fig.10 for the SOS and MORS system.

Similar observations were made by Karim et al. (2004). Gas in the draft tube facilitates this process. With the momentum gained, the liquid is then pushed to the periphery of the reactor, as evident from the high radial component of the velocity vector in the upper part of the bioreactor (Fig.10). The liquid is then pulled back into the region around the exterior of the draft tube after it comes in contact with the wall of the reactor. Hence a recirculation eye is formed in the upper part of the reactor around the draft tube 
region, which suggests that the entire peripheral region of the reactor does not act as a downcomer. The velocity vectors are more uniform in the draft tube region for the MORS system, as they have a stronger axial component than in the SOS system. This suggests that the stream of gas bubbles tends to spiral around in the region between the SOS and the draft tube; hence there is a significant radial component which prevents the arrows from being vertically oriented (Fig.10(b)). This phenomenon does not arise in the MORS system, as a better gas holdup distribution essentially reduces the radial components in the velocity vectors. Hence the flow is uniform in MORS as the arrows appear pointed upwards (Fig.10(a)). The flow pattern trends observed in the quiver plots are similar for both spargers to those observed in Fig.10 for other flow conditions (listed in Table I), and hence they are not shown here.

\subsubsection{Axial liquid velocity}

Fig.11 and Fig.12 shows the plot of the mean azimuthally averaged axial liquid velocity versus the radius of the system. These figures help visualize the values in quantitative terms. It can be seen that the axial velocities in the system with MORS are higher than that with SOS system for any given gas flow rate at both level 1 and level 2. This is attributed to the higher mean gas holdup, as seen in Fig.7, and better gas distribution in the draft tube due to the lower values of $N_{\text {Mal }}$ (Fig. 9) for the MORS system. The liquid velocities are higher at level 2 for any given sparger and gas flow rate. This is attributed to the fact that the gas distribution is better at level 2 for any given gas flow rate and sparger. The higher liquid velocity in the draft tube creates more circulation in the downcomer region, as can be seen in the increase in the negative axial velocity in the down comer region of the reactor for higher gas flow rates. This increase is an indication of better circulation in the system in the system.

\subsubsection{Determining poorly mixed zones and root mean square (RMS) liquid velocity}

To better understand the effect of the sparger configuration on the mixing in the bioreactor a contour plot of root mean square (RMS) values of the radial, azimuthal and axial time averaged components of the velocity was prepared. The velocity contours help identify the zones in the system where the liquid velocity 
is such that it would cause the solids or bio flocs in the slurry to settle. Unfortunately, since the data for bio flocs settling characteristics of the slurry used in this study was not gathered during the experiments, the settling values available in literature were used to mark these contours. The terminal settling velocities for flocs from similar systems are reported in the range of $0.2 \times 10^{-3} \mathrm{~m} / \mathrm{s}$ to $20 \times 10^{-3} \mathrm{~m} / \mathrm{s}$ (Lee et al. 1996; Li and Yuan 2002). Based on Li and Yuan (2002) Karim and Thoma (2006) have used a settling velocity of $0.32 \times 10^{-2} \mathrm{~m} / \mathrm{s}$ based on this as a criteria for determining poorly mixed zones in gas-lift digesters. This same liquid velocity value has been used here to develop a slice of the velocity contours along the radial and axial direction of the bioreactor as shown in Fig.13 for one of the gas flow rate condition studied. The regions of the reactor that has liquid velocity below the contour with a velocity $0.32 \times 10^{-2} \mathrm{~m} / \mathrm{s}$ have been indicated in the darkest shade. This figure also depicts the streamline in the system generated using the streamlineslice function available with Matlab® software, using the velocity data obtained from the CARPT experiments. The streamline clearly depicts the circulation patterns observed in the bioreactor. The bottom part of the system with SOS shows zones with no streamlines indicating negligible liquid velocity. Some breaks appear in the streamlines in Fig. 13, which could be due to experimental error associated with the CAPRT technique and the fact that a two dimensional plane (slice) from of the three dimensional streamlines have been shown. The countour plots show greater fraction of the volume of the reactor under the velocity contour for $0.32 \times 10^{-2} \mathrm{~m} / \mathrm{s}$ in the system with SOS. Table II summarizes the volumetric percentage of poorly mixed zones in the reactor for all the gas flow rates covered for both the system based on the contour plots developed (not shown). These results clearly show that the poorly mixed zones double when a SOS system is used for a given gas flow rate. For the higher superficial gas velocity covered, the MORS reduces the poorly mixed zones by a factor of three. This is because the MORS system has lower $N_{M a l}$ value in the drat tube region for a given flow rate, this creates a higher density gradient between the draft tube region and the outside region within the reactor that increases the liquid circulation. The presence of poorly mixed zones would over a period of time encourage the settling of solids in the reactor which could lead to the drop in performance of the system. Since these types of reactors have a high hydraulic retention 
time, over a period of time the system with SOS is likely to accumulate a lot of solids as compared to MORS.

The mean per cell, time averaged RMS velocity values obtained from all the cells in the draft tube region and the all the cells in the reactor (including draft tube region) for the reactor with MORS and SOS give an idea of the impact of gas flow rate on the liquid velocity. The mean time averaged RMS velocity for the draft tube region alone, as well as the entire reactor, is shown for the different superficial gas velocities in Fig.14. For a given gas flow rate, the MORS system gives higher RMS liquid velocities than the SOS for the entire reactor region and the draft tube. The difference is more pronounced in the draft tube region. This is again attributed to the lower values of $N_{M a l}$ obtained with the MORS system for a given gas flow rate.

\section{Conclusions}

The viability of anaerobic bioreactors used for a source of energy generation from organic waste is strongly dependent on their energy economy. The net process energy consumed for proper operation should not exceed the energy available from the biogas (methane) generated. For optimal performance, mixing should be as efficient as possible. CARPT and CT were successfully implemented on a surrogate system to measure gas holdup distribution and liquid velocity and poorly mixed zones in a gas recirculated anaerobic bioreactor.

For a given power input, fixed by the gas flow rate (based on Equation 1), the Multi Orifice Ring Sparger (MORS) was found to gives better gas phase distribution and higher mean gas holdup in the draft tube when compared to Single Orifice Sparger (SOS). All pores in the MORS do not open, conducting this study at gas flow rates that ensure all the MORS pores are open would have a power input rage that would exceed the recommended range for anaerobic systems (US EPA, 1979). The normalized standard deviation, $N_{M a l}$, for the gas holdup distribution was put in perspective as a parameter for characterization. Lower values of $N_{M a l}$ show better performance of the MORS in terms of gas holdup characteristics. 
There were two loops in the circulation patterns observed in the system. It was also determined by CARPT that the there are poorly mixed zones in the downcomer region of the reactor. Higher liquid velocity values were observed in the draft tube region for the bioreactor with MORS for a fixed gas flow rate. The poorly mixed zones are drastically reduced in the reactor when a MORS system is used as the lower values of gas holdup $N_{M a l}$ ensures better liquid circulation. The same trend was observed with the RMS liquid velocity in the entire reactor. Hence the MORS system is considerably more efficient for mixing the reactor than the SOS system. The reduction in the poorly mixed zones would make a larger impact when the bioreactors based on the configurations discussed here are scaled up for pilot plant operations.

Recirculation of the liquid is facilitated by the density gradient between the material in the draft tube and periphery area of the draft tube. This difference triggers the buoyancy forces that enable the liquid to circulate. High gas holdup and better distribution are therefore desirable in the draft tube region to create an effective density gradient for better mixing in the reactor by reducing the fraction of the poorly mixed zones. This objective could be easily achieved with an increase in the superficial gas velocity. However, the energy constraints in such systems give limited flexibility in manipulating the superficial gas velocity as a operation parameter. Hence this necessitates the careful consideration of the sparger design for introducing gas into the system. The impact of increased uniform gas holdup distribution in the draft tube, and the reduction in the poor mixing zones in the bioreactor on the production of methane from bovine waste could the subject of a performance study. This paper confirms that for a given power input, efficiency in mixing can be obtained by appropriate sparger design.

\section{Nomenclature:}

$D_{p}=$ Diameter of composite radioactive particle $(\mathrm{m})$

$G_{r}=$ Specific biogas recirculation rate $\left(\mathrm{m}^{3} / \mathrm{dm}^{3}\right)$

$g=$ Acceleration due to gravity $(\mathrm{m} / \mathrm{s})$

$n_{\text {dratt }}=$ Number of pixels in the draft tube region

$$
P=\text { Power (Watts } / \mathrm{m}^{3} \text { ) }
$$


$P_{1}=$ Pressure at the injection point $\left(\mathrm{N} / \mathrm{m}^{2}\right)\left(P_{2}+\right.$ Static head of slurry $)$

$P_{2}=$ Head space pressure $\left(101,416.83 \mathrm{~N} / \mathrm{m}^{2}\right)$

$U_{t}=$ Terminal settling velocity $(\mathrm{m} / \mathrm{s})$

$V=$ Volume of slurry in the system $\left(\mathrm{m}^{3}\right)$

$\langle x\rangle=$ Notation for image space (Pixel index)

$\varepsilon_{g}=$ Holdup fraction of gas (dimension less)

$\varepsilon_{L}=$ Holdup fraction of liquid (dimension less)

$\bar{\varepsilon}_{g}=$ Average gas holdup distribution

$\varepsilon^{o}{ }_{g}=$ Holdup fraction of gas in reactor filled with gas alone (dimension less)

$\varepsilon^{0}{ }_{L}=$ Holdup fraction of gas in reactor filled with slurry alone (dimension less)

$\lambda=1.03$

$\mu_{w}=$ Viscosity of water in Equation 2. $(\mathrm{kg} / \mathrm{m}-\mathrm{s})$

$\mu_{L}=$ Ideal mass attenuation of slurry $\left(\mathrm{m}^{-1}\right)$

$\mu_{g}=$ Ideal mass attenuation of gas $\left(\mathrm{m}^{-1}\right)$

$\mu_{E M, g}=$ Mass attenuation computed by EM- algorithm for gas $\left(\mathrm{m}^{-1}\right)$

$\mu_{E M, L}=$ Mass attenuation computed by EM- algorithm for slurry $\left(\mathrm{m}^{-1}\right)$

$\mu_{E M, I-g}=$ Mass attenuation computed by EM- algorithm for gas-slurry system ( $\left.\mathrm{m}^{-1}\right)$

$\rho_{p}=$ Density of composite radioactive particle $\left(\mathrm{kg} / \mathrm{m}^{3}\right)$

$\rho_{w}=$ Density of water $\left(\mathrm{kg} / \mathrm{m}^{3}\right)$ 


\section{Acknowledgements}

The authors would like to acknowledge the United States Department of Energy for sponsoring the research project (Identification number: DE-FC-36-01GO11054)

\section{Reference:}

Borole AP, Klasson. TK. Ridenour W, Justin H., Karim K, Al-Dahhan MH. 2006. Methane production in a 100-L upflow bioreactor by anaerobic digestion of farm waste. Applied Biochemistry and Biotechnology. 131(1-3): 887-89.

Casey TJ. 1986. Requirements and methods for mixing in anaerobic digesters. In: Anaerobic digestion of sewage sludge and organic agricultural wastes. Elsevier Applied Science Publication, Amsterdam, 90-103.

Chisti Y. 1998. Pneumatically agitated bioreactors in industrial and environmental bioprocessing: Hydrodynamics, hydraulics and transport phenomena. Appl. Mech. Rev. Vol. 51:33-112.

Devanathan N. 1991. Investigation of liquid hydrodynamics in bubble column via a computer computer automated radioactive particle tracking (CARPT). D.Sc. Thesis, Washington University, St. Louis.

Degaleesan S.1977. Fluid dynamics measurement and modeling of liquid mixing in bubble columns. D.Sc. Thesis, Washington University, St. Louis.

George DL, Shollenberger K A, Torczynski J R, O’Hern T J, S L Ceccio. 2001. Three phase material distribution measurements in a vertical flow using gamma-densitometry tomography and electricalimpedance tomography. International Journal of Multiphase Flow. 27:1903-1930.

Gosh S. 1997. Anaerobic digestion for renewable energy and environmental restoration. In: Proceedings of the Eight International Conference on Anaerobic Digestion. Ministry of Education Japan, Sendai International Center, Sendai, Japan.

Jiang Y. 2000. Flow distribution and it's impact on performance of packed bed reactors. D.Sc. Thesis, Washington University, St. Louis, MO.

Karim K, Hoffmann R, Klasson TK, Al-Dahhan MH. 2005. Anaerobic digestion of animal waste: Effect of mode of mixing. Water Research , 39(15): 3597-3606.

Karim K, Varma R,Vesvikar V, Al-Dahhan MH. 2004. Flow pattern visualization of a simulated digester. Water Research. 38: 3659-3670.

Karim K, Thoma GJ. 2006. Gas-Lift digester configuration effects on mixing effectiveness. Submitted to Water Research. 
Lee DJ, Chen GW, Liao YC, Hsieh CC. 1996. On the free-settling test for estimating activated sludge floc density. Water Research 30(3):541-550.

Li X-y, Yuan Y. 2002. Settling velocities and permeabilities of microbial aggregates. Water Research 36(12):3110-3120.

Sheffield J. Financial approaches to animal manure managment; 2002; Joint Institute for Energy and Environment. 314 UT Conference Center Building Knoxville, TN 37996-4138. Report Number: JIEE 2002-04.

Kojima H, Saawai J, Uchina, H, Ichige T. 1999. Liquid circulation and Critical gas velocity in slurry bubble column with short size draft tube. Chem. Eng. Sci. 54:5181-5187.

Kumar SB. 1994. Computer tomography measurements of void fraction and modeling of the flow in bubble columns. Ph.D. Thesis, Florida Atlantic University, Boca Raton.

Lange K, Carson R. 1984. EM reconstruction algorithm for emission and transmission tomography. J. Computer Assisted Tomography. 8(2):306-316.

Lee DJ, Chen GW, Liao YC, Hsieh CC. 1996. On the free-settling test for estimating activated sludge floc density. Water Research 30(3):541-550.

Li X-y, Yuan Y. 2002. Settling velocities and permeabilities of microbial aggregates. Water Research 36(12):3110-3120.

Luo H-P., 2005. Analyzing and modeling of airlift photobioreactors for microalgal and cyanobacteria cultures D.Sc. Thesis, Washington University, St. Louis, MO.

Lusk P. 1998. Methane Recovery from animal manures: A current opportunities casebook. 3rd edition, NREL/SR-580-25145. National Reneable Energy Laboratory, Golden, CO. Work performed by Resource Development Associates, Washington, DC.

Marcandelli C, Lamine AS, Bernard JR, Wild G. 2000. Liquid Distribution in Trickle-bed Reactor. Oil \& Gas Science and Technology-Review. IFP. 55(4): 407-415

Ong B., 2003. Experimental investigation of the bubble column hydrodynamics : effect of elevated pressure and superficial gas velocity. D.Sc. Thesis, Washington University, St. Louis, MO.

Pironti FF, Medina VR, Calvo R, Seaza AE. 1995. Effect of draft tune position on the hydrodynamics of a draft tube slurry bybble column. Chemical Engineering Journal. 60(1-3): 155-160.

Rados N. 2003. Slurry bubble column hydrodynamics. D.Sc. Thesis, Washington University, St. Louis, MO.

Reinhold G, Markl H. 1997. Model-based scale-up and performance of the biogas tower reactor for anaerobic wastewater treatment. Water Research 31(8):2057-2065. 
Roy S. 2006. Phase distribution and performance studies of gas-liquid monolith reactors. D.Sc. Thesis, Washington University, St. Louis, MO.

Roy S, Kemoun A, Al-Dahhan MH, Dudukovic' MP, Skourlis TB, Dautzenberg FM. 2004. Countercurrent flow distribution in structured packing via computed tomography. Chemical Engineering and Processing. 44:59-69,

Roy S. 2000 Quantification of two-phase flow in liquid-solid risers. D.Sc. Thesis, Washington University St. Louis.

Speece R.E. 1996. Anaerobic biotechnology for industrial waste waters. Vaderbuilt University, Archae Press.

Stroot S, McMahon KD, Mackie RR, Raskin L. 2001. Anaerobic co-digestion of municipal solid waste and biosolids under various mixing conditions-I. Digester performance. Water Res. 35(7). 1804-1816.

US EPA, 1979. Process Design Manual for Sludge Treatment and Disposal. EPA 625/1-79-011 Cincinnati, $\mathrm{OH}$.

Vesvikar MS, Al-Dahhan MH., 2005. Flow pattern visualization in a mimic anaerobic digester using CFD. Biotechnology and bioengineering. 89(6), 719-32.

Whitmore T N, Lloyd D, Jones G, Willams TN. 1987. Hydrogen-dependant control of continuous anaerobic digestion process. Appl. Microbiol. Biotechnology. 26: 383-388. 
Table I: $\quad$ Flow rates conditions used and Error in calibration process for anaerobic bioreactor with SOS and MORS systems

\begin{tabular}{|c|c|c|c|c|c|}
\hline $\begin{array}{c}\text { Gas flow } \\
\text { rate } \\
\begin{array}{c}Q_{g} \times 10^{3} \\
\left(\mathrm{~m}^{3} / \mathrm{s}\right)\end{array}\end{array}$ & $\begin{array}{c}\text { Superficial gas } \\
\text { velocity based } \\
\text { on draft tube } \\
\text { diameter } \times 10^{3} \\
(\mathrm{~m} / \mathrm{s})\end{array}$ & $\begin{array}{c}\text { Superficial gas } \\
\text { velocity based } \\
\text { on reactor } \\
\text { diameter } \times 10^{3} \\
(\mathrm{~m} / \mathrm{s})\end{array}$ & $\begin{array}{c}\text { Power } \\
\text { Input } \\
\left(\mathrm{W} / \mathrm{m}^{3}\right)\end{array}$ & $\begin{array}{c}\text { Average Calibration } \\
\text { Error for reactor } \\
\text { with MORS } 10^{3} \\
\left(\begin{array}{c}\text { Standard Deviation } \\
\left.\times 10^{3}\right) \\
\mathrm{m} .\end{array}\right.\end{array}$ & $\begin{array}{c}\text { Average } \\
\text { Calibration Error } \\
\text { for reactor with } \\
\text { SOS } 10^{3} \\
\text { (Standard } \\
\left.\text { Deviation } \times 10^{3}\right) \\
\mathrm{m}\end{array}$ \\
\hline 0.017 & 1.47 & 0.09 & 8.71 & $0.26(0.14)$ & $0.37(0.21)$ \\
0.050 & 4.41 & 0.27 & 26.15 & $0.37(0.24)$ & $0.38(0.19)$ \\
0.083 & 7.35 & 0.45 & 43.58 & $0.31(0.17)$ & $0.39(0.23)$ \\
\hline
\end{tabular}

Table II: $\quad$ Percentage of poorly mixed zone in bioreactor for the superficial gas velocities conditions studied for the bioreactor with MORS and SOS.

\begin{tabular}{|c|c|c|}
\hline $\begin{array}{c}\text { Superficial gas } \\
\text { velocity based on } \\
\text { draft tube diameter } x \\
10^{3}(\mathrm{~m} / \mathrm{s})\end{array}$ & Percentage dead volume in bioreactor \\
\cline { 2 - 3 } & MORS & SOS \\
\hline 1.47 & $11.52 \%$ & $24.91 \%$ \\
\hline 4.41 & $6.46 \%$ & $13.27 \%$ \\
\hline 7.35 & $3.48 \%$ & $11.37 \%$ \\
\hline
\end{tabular}




\section{List of Figures:}

Figure 1: Cross section of the surrogate anaerobic bioreactor used with a single orifice sparger (SOS).

Figure 2: (a) Cross section of the surrogate anaerobic bioreactor used a multi orifice ring

Figure 3: $\quad$ Three dimensional representation of tomograms showing cross sectional time averaged gas holdup distribution in the draft tube region for (a) system with SOS and (b) system with MORS at level 1 (5x10-2 m) and level 2 (15x10-2 m) at Qg $=0.05 \times 10^{-3} \mathrm{~m} 3 / \mathrm{s}$ ( superficial draft tube gas velocity $=4.41 \times 10-2 \mathrm{~m} / \mathrm{s}$ ). Color bar represents the holdup value.

Figure 4: $\quad$ Tomograms of system with MORS at level 1 with gas flow rates (a)0.017x10-3 $\mathrm{m} 3 / \mathrm{s}$, (b) $0.05 \times 10-3 \mathrm{~m} 3 / \mathrm{s}$ and (c) $0.083 \times 10-3 \mathrm{~m} 3 / \mathrm{s}$. Tomograms of system with MORS at level 1 with gas flow rates (a) $0.017 \times 10^{-3} \mathrm{~m}^{3} / \mathrm{s}$, (b) $0.05 \times 10^{-3} \mathrm{~m}^{3} / \mathrm{s}$ and (c) $0.083 \times 10^{-3} \mathrm{~m}^{3} / \mathrm{s}$.

Figure 5: $\quad$ Comparison of azimuthally averaged gas holdup profiles for system with SOS and system with MORS at level 1

Figure 7: $\quad$ Mean gas holdup in the draft tube region of the SOS and MORS systems at levels 1 and 2.

Figure 8: $\quad$ Plot showing dependency of distribution factor on number of pixel per block used for computing distribution factor based on T-Test. Gas holdup distribution data from the scan at level 2 for the MORS system with $Q g=0.05 \times 10^{-3} \mathrm{~m} 3 / \mathrm{s}$ was used.

Figure 9: $\quad$ Normalized standard deviation or maldistribution number (NMal) based comparison for gas phase distribution in the draft tube region for a SOS system and MORS system. 
Figure 10: $\quad$ Time averaged velocity vector plots for digester with 5\% (TS) solid loading slurry at for gas flow rate $\mathrm{Qg}=0.05 \times 10-3 \mathrm{~m} 3 / \mathrm{s}(\mathrm{Vg}=4.4 \times 10-2 \mathrm{~m} / \mathrm{s}):(\mathrm{a})$ MORS, (b) SOS. The dark lines indicate the location of the wall of the reactor and the draft tube.

Figure 11: Time averaged axial velocity comparison for level $1(5 \times 10-2 \mathrm{~m})$ from CARPT data. The gas flow rates have been indicated in parenthesis $(\mathrm{Q} 1=0.017 \mathrm{x} 10-3$ $\mathrm{m} 3 / \mathrm{s}, \mathrm{Q} 2=0.050 \times 10-3 \mathrm{~m} 3 / \mathrm{s}$ and $\mathrm{Q} 3=0.083 \times 10-3 \mathrm{~m} 3 / \mathrm{s})$.

Figure 12: $\quad$ Time averaged axial velocity comparison for Level 2 (150x10-2 m) from CARPT data. The gas flow rates have been indicated in parenthesis $(\mathrm{Q} 1=0.017 \mathrm{x} 10-3$ $\mathrm{m} 3 / \mathrm{s}, \mathrm{Q} 2=0.050 \times 10-3 \mathrm{~m} 3 / \mathrm{s}$ and $\mathrm{Q} 3=0.083 \times 10-3 \mathrm{~m} 3 / \mathrm{s})$.

Figure 13: Contour plots showing the time averaged values of the magnitude of the liquid circulation velocity for the bioreactor with 5\% (TS) solid loading slurry at for gas flow rate $\mathrm{Qg}=0.05 \times 10^{-3} \mathrm{~m} 3 / \mathrm{s}\left(\mathrm{Vg}=4.4 \times 10^{-2} \mathrm{~m} / \mathrm{s}\right):(\mathrm{a})$ MORS, (b) SOS. The dark lines indicate the location of the wall of the reactor and the draft tube; the fine lines with arrows indicate the stream lines. Numeric values in the box indicate velocity x102 (m/s) values the contour represents. The black colored zones indicate poorly mixed regions.

Figure 14: Plot of superficial gas velocity is the draft tube versus time averaged average RMS liquid velocity in the draft tube for the MORS and SOS systems 


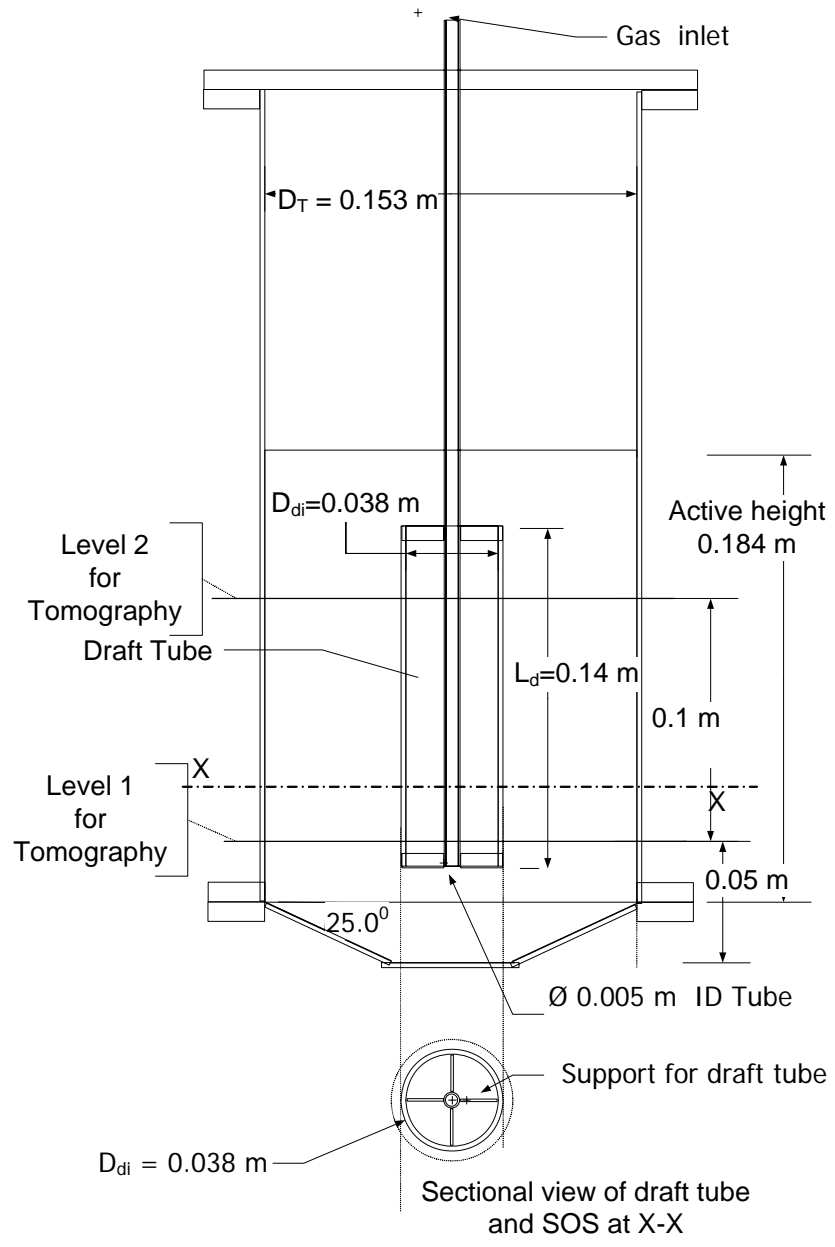

Figure 1: $\quad$ Cross section of the surrogate anaerobic bioreactor used with a single orifice sparger (SOS). 


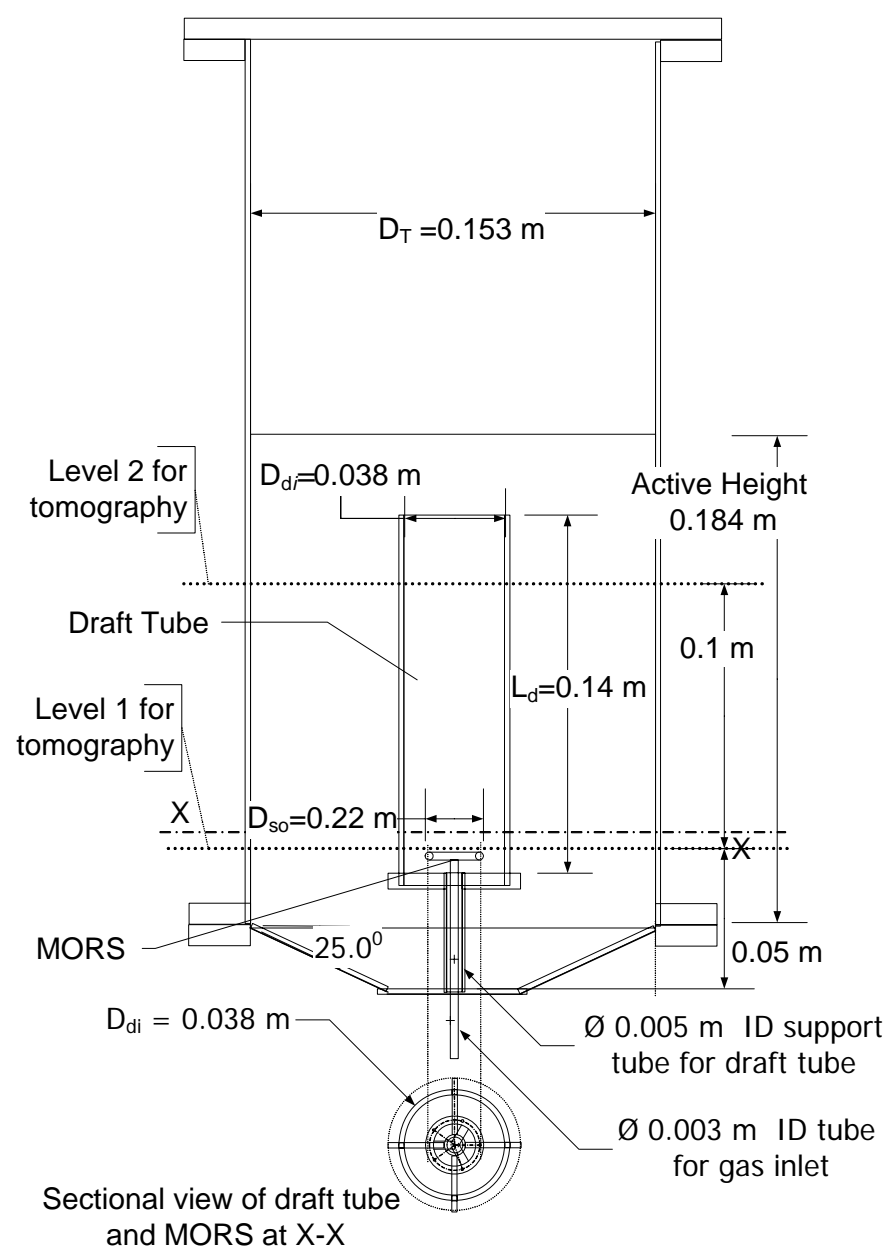

(a)

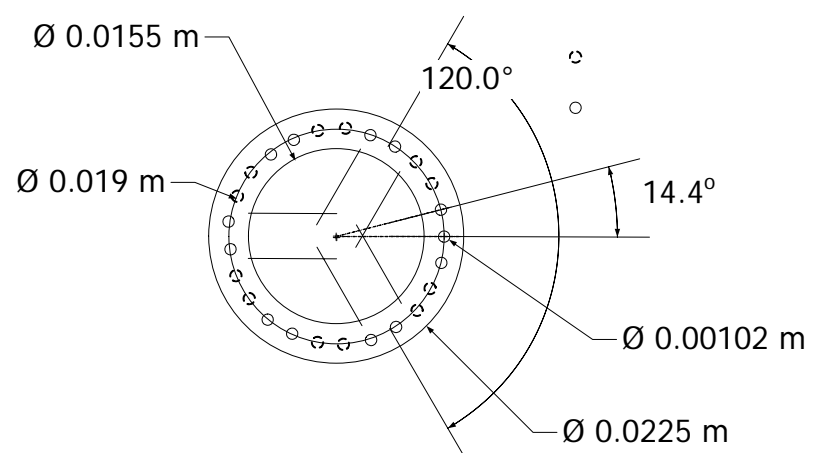

24 holes $(\phi 0.00102 \mathrm{~m})$ are equidistantly $\left(14.4^{\circ}\right.$ apart) opened outward and inward on the lower face of the ring pipe at a angle of $30^{\circ}$.

(b)

Figure 2: (a) Cross section of the surrogate anaerobic bioreactor used a multi orifice ring sparger (MORS). (b) Details of the multi orifice ring sparger (MORS) used. 


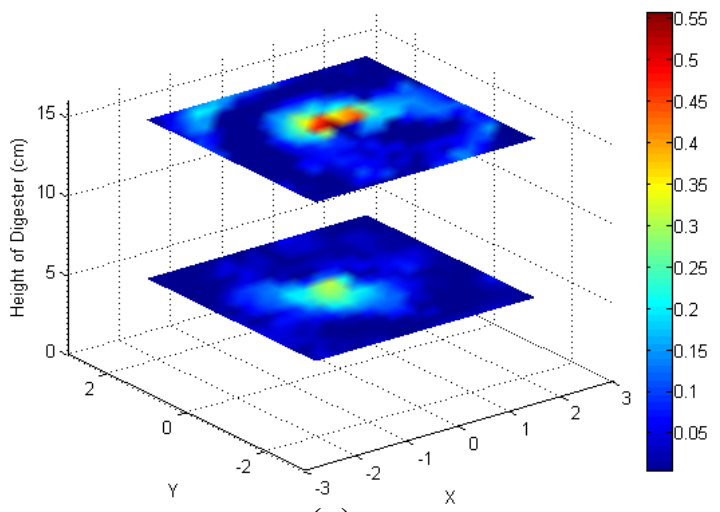

(a)

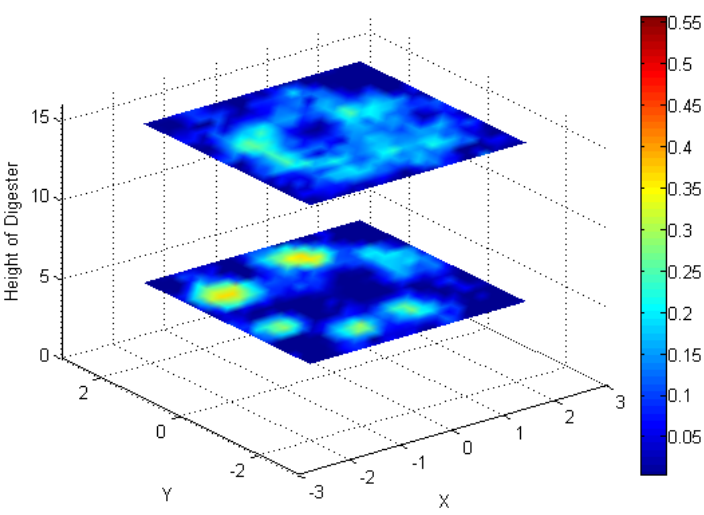

(b)

Figure 3: $\quad$ Three dimensional representation of tomograms showing cross sectional time averaged gas holdup distribution in the draft tube region for (a) system with SOS and (b) system with MORS at level $1\left(5 \times 10^{-2} \mathrm{~m}\right)$ and level $2\left(15 \times 10^{-2} \mathrm{~m}\right)$ at $\mathrm{Q}_{\mathrm{g}}=$ $0.05 \times 10^{-3} \mathrm{~m}^{3} / \mathrm{s}$ ( superficial draft tube gas velocity $=4.41 \times 10^{-2} \mathrm{~m} / \mathrm{s}$ ). Color bar represents the holdup value. 


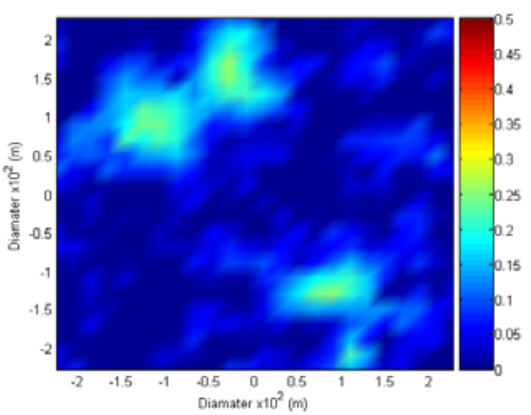

(a)

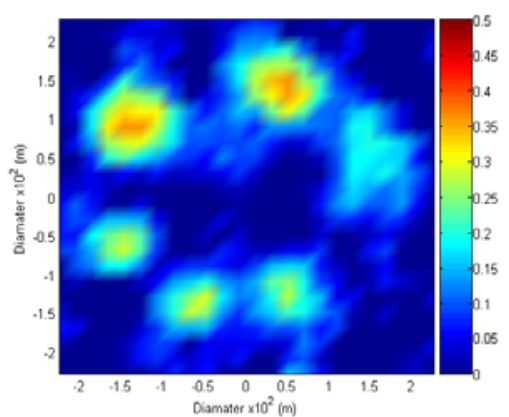

(b)

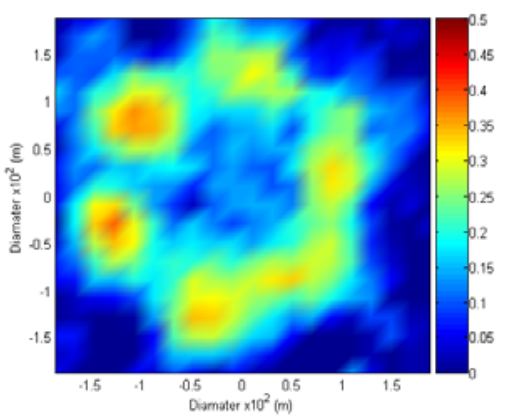

(c)

Figure 4: $\quad$ Tomograms of system with MORS at level 1 with gas flow rates (a) $0.017 \times 10^{-3}$ $\mathrm{m}^{3} / \mathrm{s}$, (b) $0.05 \times 10^{-3} \mathrm{~m}^{3} / \mathrm{s}$ and (c) $0.083 \times 10^{-3} \mathrm{~m}^{3} / \mathrm{s}$. 


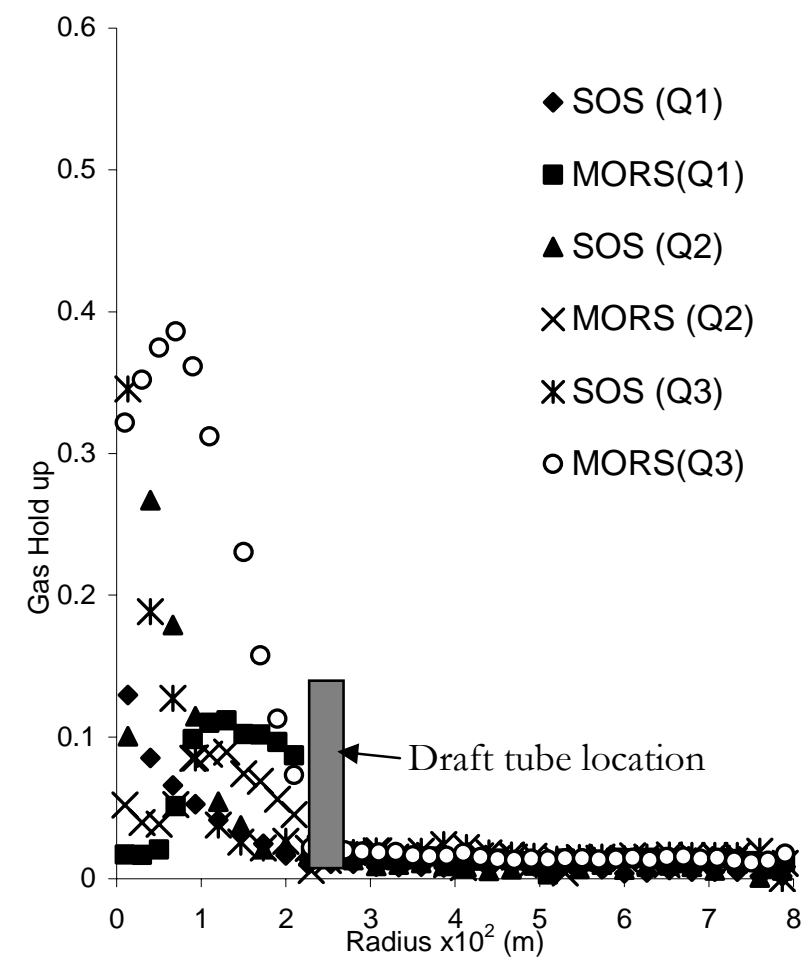

Figure 5: $\quad$ Comparison of azimuthally averaged gas holdup profiles for system with SOS and system with MORS at level 1 for different flow rates (indicated in parenthesis Q1 $=0.017 \times 10^{-3} \mathrm{~m}^{3} / \mathrm{s}, \mathrm{Q} 2=0.050 \times 10^{-3} \mathrm{~m}^{3} / \mathrm{s}$ and $\mathrm{Q} 3=0.083 \times 10^{-3}$ $\mathrm{m}^{3} / \mathrm{s}$ ) 


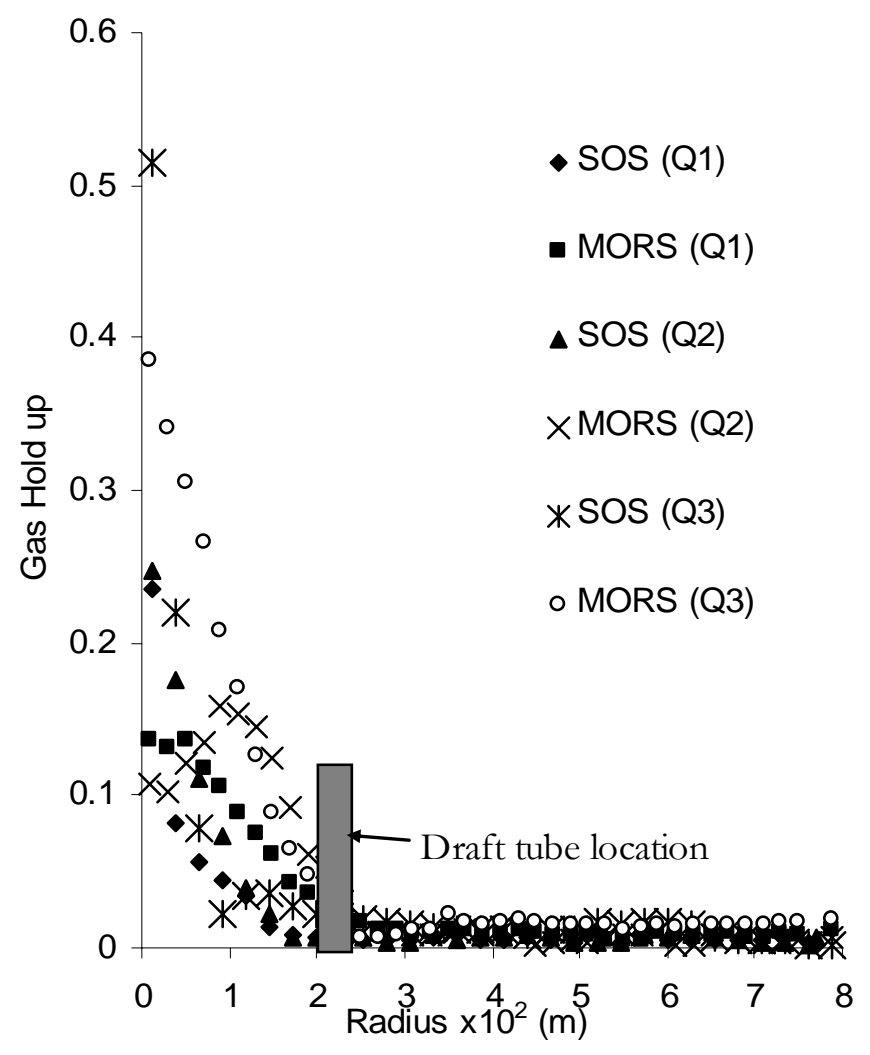

Figure 6: Comparison of azimuthally averaged gas holdup profiles for system with SOS and system with MORS at level 2 for different flow rates (indicated in parenthesis $\mathrm{Q} 1=0.017 \times 10^{-3} \mathrm{~m}^{3} / \mathrm{s}, \mathrm{Q} 2=0.050 \times 10^{-3} \mathrm{~m}^{3} / \mathrm{s}$ and $\mathrm{Q} 3=0.083 \times 10^{-3}$ $\left.\mathrm{m}^{3} / \mathrm{s}\right)$. 


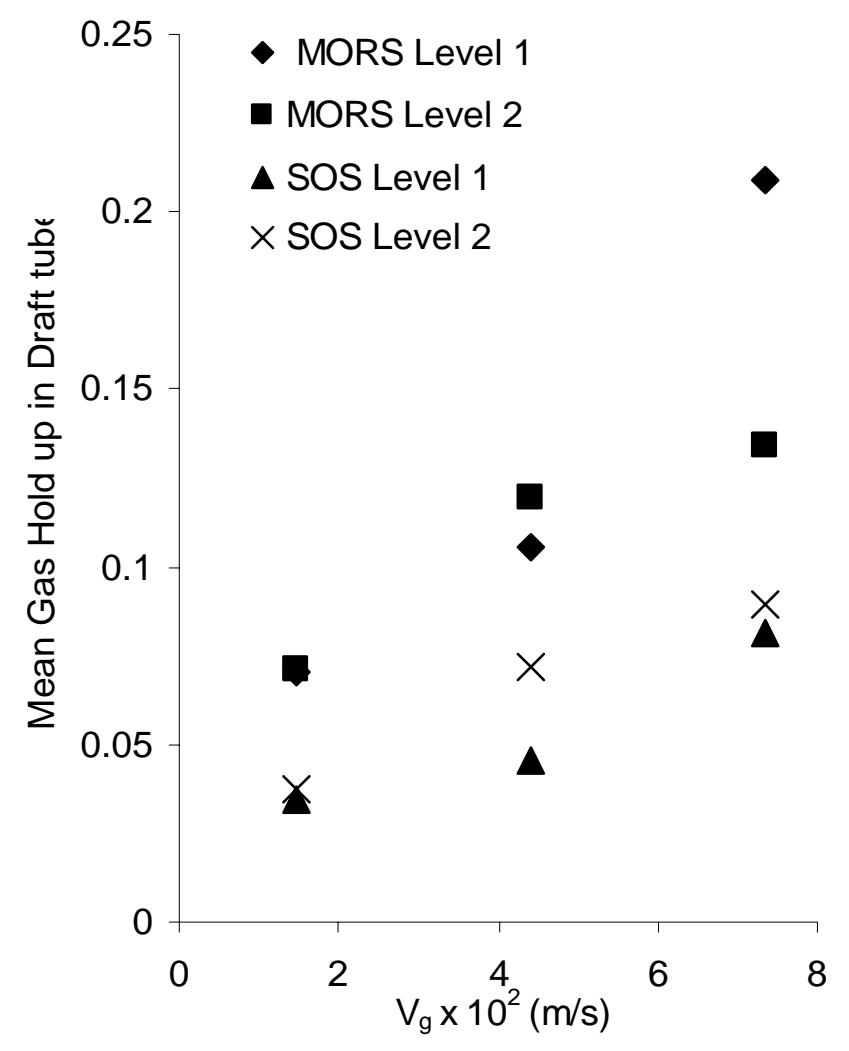

Figure 7: Mean gas holdup in the draft tube region of the SOS and MORS systems at levels 1 and 2 . 


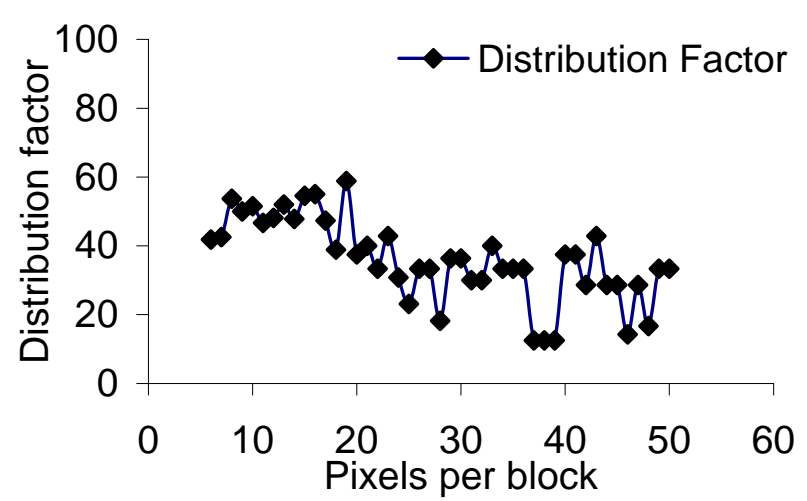

Figure 8: $\quad$ Plot showing dependency of distribution factor on number of pixel per block used for computing distribution factor based on T-Test. Gas holdup distribution data from the scan at level 2 for the MORS system with $Q_{g}=0.05 \times 10^{-3} \mathrm{~m}^{3} / \mathrm{s}$ was used. 


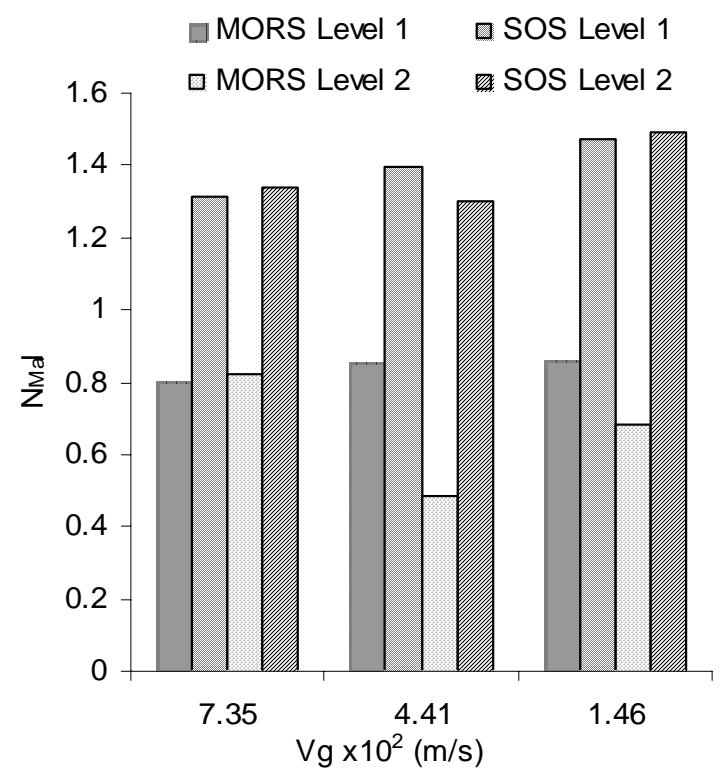

Figure 9: $\quad$ Normalized standard deviation or maldistribution number ( $\left.N_{M a l}\right)$ based comparison for gas phase distribution in the draft tube region for a SOS system and MORS system. 


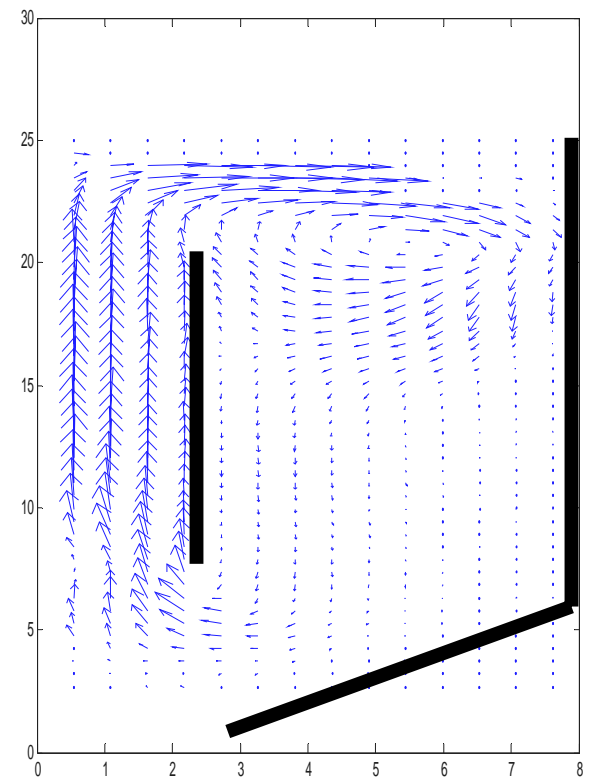

(a)

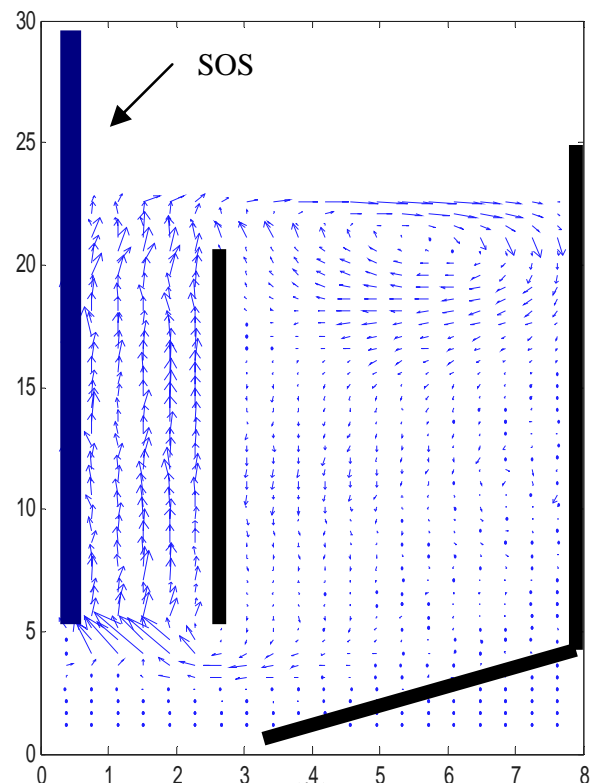

(b)

Figure 10: $\quad$ Time averaged velocity vector plots for digester with $5 \%$ (TS) solid loading slurry at for gas flow rate $Q_{g}=0.05 \times 10^{-3} \mathrm{~m}^{3} / \mathrm{s}\left(V_{g}=4.4 \times 10^{-2} \mathrm{~m} / \mathrm{s}\right)$ : (a) MORS, (b) SOS. The dark lines indicate the location of the wall of the reactor and the draft tube. 


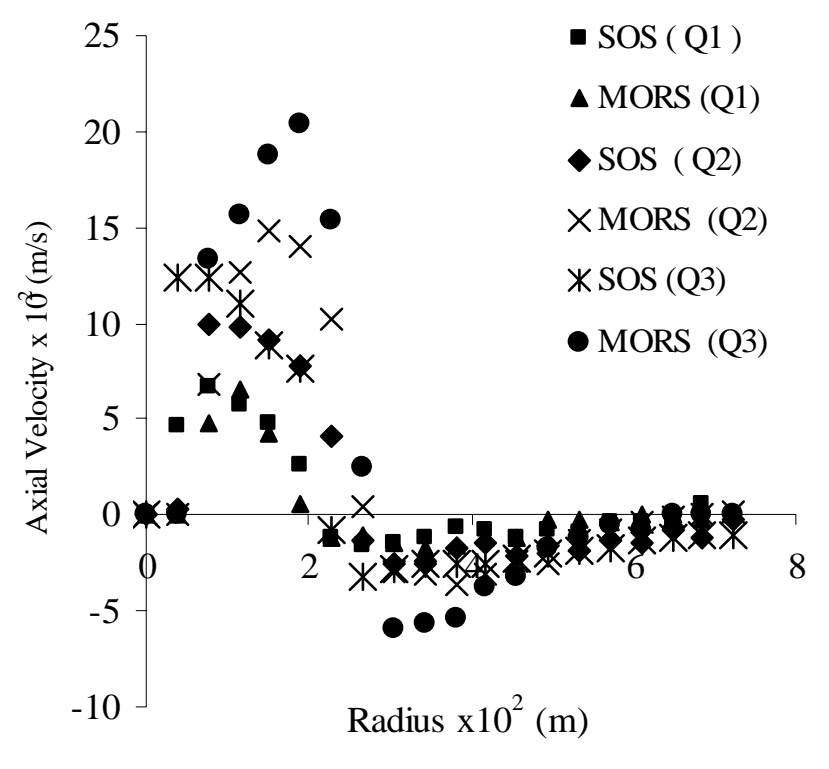

Figure 11: Time averaged axial velocity comparison for level $1\left(5 \times 10^{-2} \mathrm{~m}\right)$ from CARPT data. The gas flow rates have been indicated in parenthesis $\left(\mathrm{Q} 1=0.017 \times 10^{-3}\right.$ $\mathrm{m}^{3} / \mathrm{s}, \mathrm{Q} 2=0.050 \times 10^{-3} \mathrm{~m}^{3} / \mathrm{s}$ and $\mathrm{Q} 3=0.083 \times 10^{-3} \mathrm{~m}^{3} / \mathrm{s}$ ). 


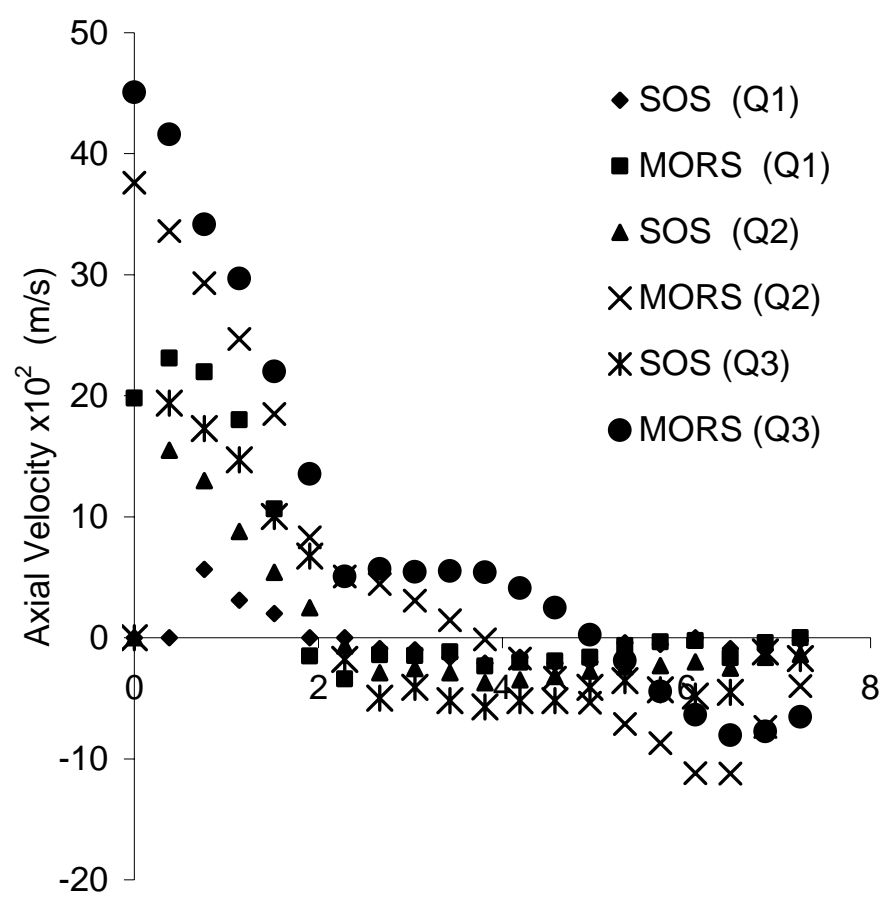

Radius $\times 10^{2}(\mathrm{~m})$

Figure 12: Time averaged axial velocity comparison for Level $2\left(150 \times 10^{-2} \mathrm{~m}\right)$ from CARPT data. The gas flow rates have been indicated in parenthesis $\left(\mathrm{Q} 1=0.017 \times 10^{-3}\right.$ $\mathrm{m}^{3} / \mathrm{s}, \mathrm{Q} 2=0.050 \times 10^{-3} \mathrm{~m}^{3} / \mathrm{s}$ and $\mathrm{Q} 3=0.083 \times 10^{-3} \mathrm{~m}^{3} / \mathrm{s}$ ). 


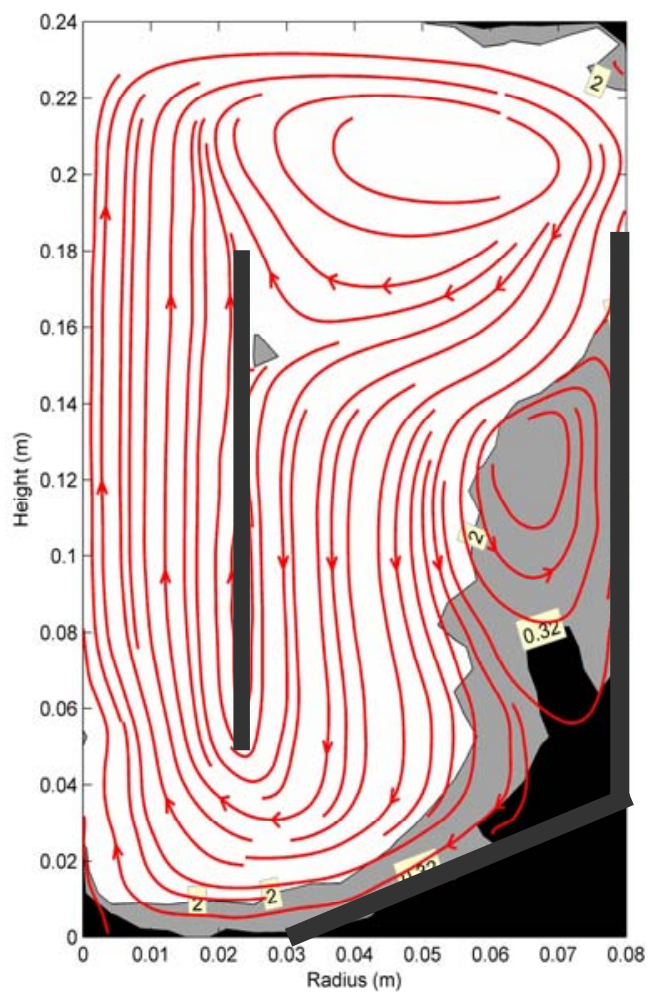

(a)

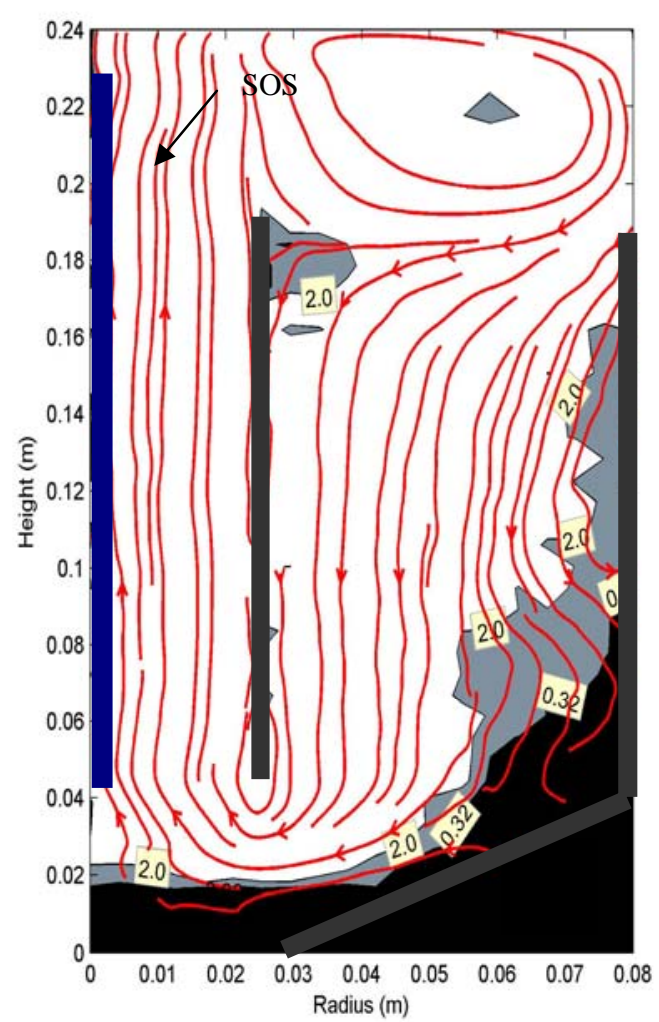

(b)

Figure 13: $\quad$ Contour plots showing the time averaged values of the magnitude of the liquid circulation velocity for the bioreactor with $5 \%$ (TS) solid loading slurry at for gas flow rate $\mathrm{Q}_{\mathrm{g}}=0.05 \times 10^{-3} \mathrm{~m}^{3} / \mathrm{s}\left(\mathrm{V}_{\mathrm{g}}=4.4 \times 10^{-2} \mathrm{~m} / \mathrm{s}\right):$ (a) MORS, (b) SOS. The dark lines indicate the location of the wall of the reactor and the draft tube; the fine lines with arrows indicate the stream lines. Numeric values in the box indicate velocity $\times 10^{2}(\mathrm{~m} / \mathrm{s})$ values the contour represents. The black colored zones indicate poorly mixed regions. 


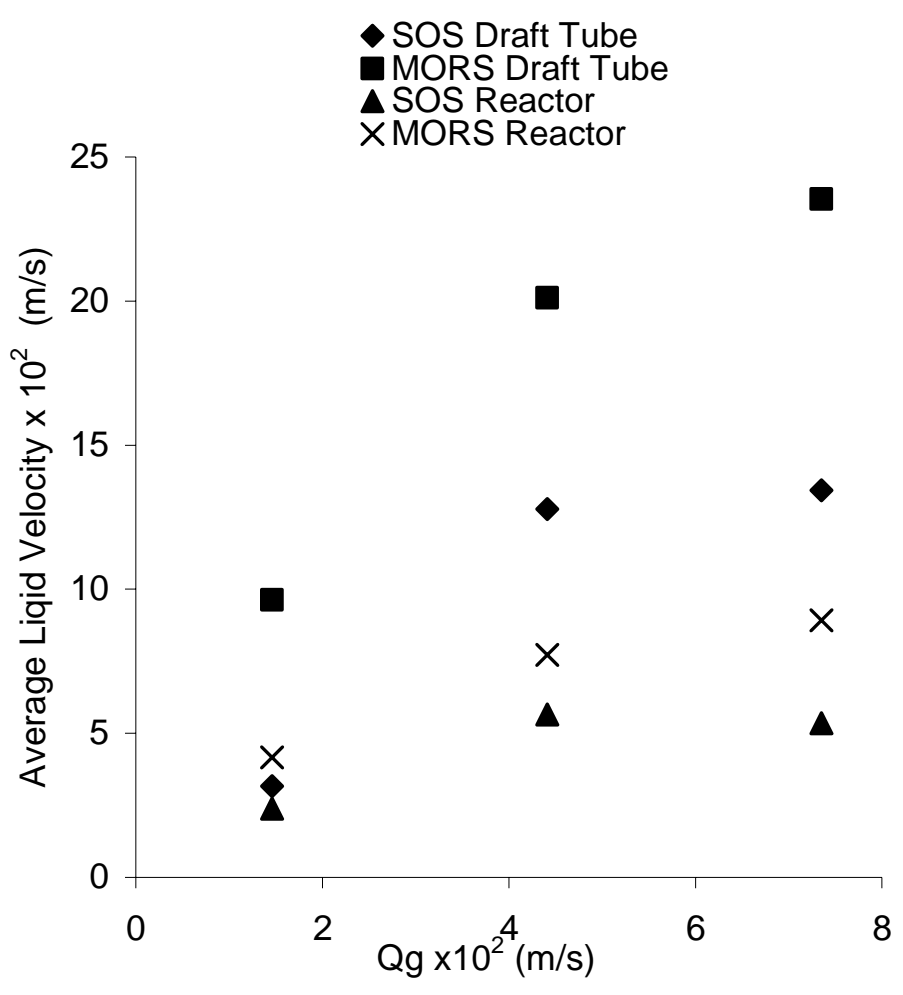

Figure 14: Plot of superficial gas velocity is the draft tube versus time averaged average RMS liquid velocity in the draft tube for the MORS and SOS systems 


\section{Appendix 3.4}

\subsection{Selection of the gamma ray sources for DSCT}

The objective of this experiment is to determine the suitability of the selected sources ${ }^{60} \mathrm{Co}$ and ${ }^{137} \mathrm{Cs}$. This was achieved by using a system designed in such manner where the over all holdups of the individual phases are well defined a priori based on the physical geometry. The details of the set-up for such experimental evaluation is shown in Figure 3.13. The line average densitometry was used to estimate the overall holdups in the three phase system.

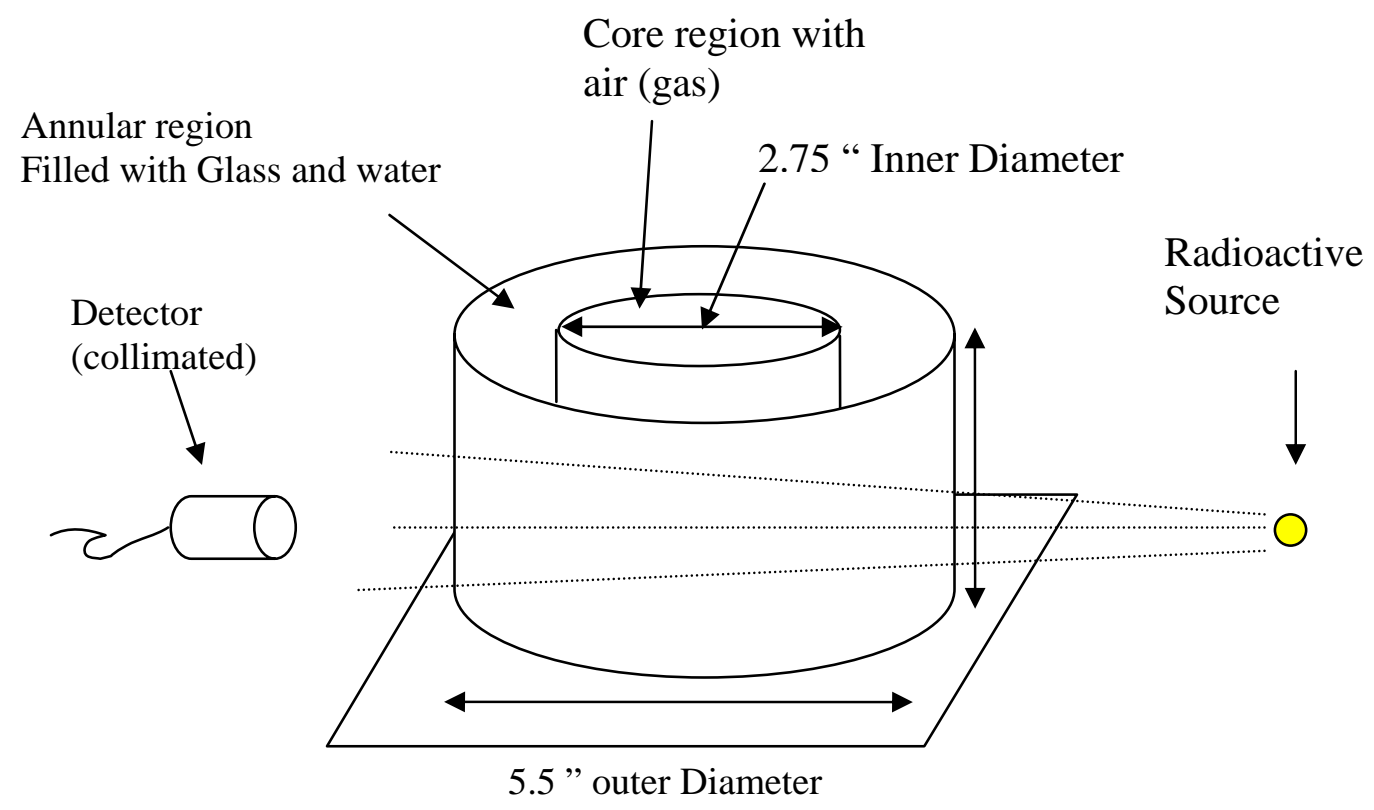

Figure: 3.13 Details of the experimental set up used to evaluate the gamma ray sources.

\subsubsection{Equations used}

Equation 1 is an attenuation equation for three phase system, as explained in the Fourth quarterly report. The three phase system is considered to be consisting of a gas, liquid and solid.

$A_{g-l-s}=A_{g, i j} \varepsilon_{g, i j}+A_{l, i j}\left(1-\varepsilon_{g, i j}-\varepsilon_{s, i j}\right)+\left[\rho_{s} \mu_{s} \varepsilon_{s, i j}\right] L_{i j}$

This is arrived at after doing the basic back ground scans which yield

$$
\begin{aligned}
& A_{g, i j}=\left[\rho_{g} \mu_{g}\right] L_{i j} \\
& A_{l, i j}=\left[\rho_{l} \mu_{l}\right] L_{i j}
\end{aligned}
$$


Since the solids are small particles and don't form a monolith, when the reactor is filled with solids (both the inner core and the outer annular region) the attenuation coefficient can be represented by the following equation

$(\mu \rho)_{g-s}=(\mu \rho)_{s} \varepsilon_{s}^{o}-(\mu \rho)_{g}\left(1-\varepsilon_{s}^{o}\right)$

Where $\varepsilon_{s}^{o}$ is the bed voidage. Since these equation are now used for line averaged densitometry, the suffix $i j$ representing the $i^{\text {th }}$ projection through the $j^{\text {th }}$ pixel is no more used.

For mathematical convenience, we define a new term, $R_{k}$, as follows:

$R_{K}=A_{K}-A_{g}$

where $K$ represents any scan that has been carried with a gas phase i.e. a Gas-liquid-solid system (GLS), gas-liquid system(GL) or a gas-solid system(GS).

Substituting equations 2, 3 and 4 in Equation 1 we obtain the following expression:

$\varepsilon_{g}=R_{g-s} \frac{\varepsilon_{s}}{\varepsilon_{s}^{o}}+R_{L}\left(1-\varepsilon_{s}\right)-R_{g-l-s}$

Also the fact that sum of the three phases adds to unity is used to arrive at Equation $5 \mathrm{~b}$.

$\varepsilon_{g}+\varepsilon_{s}+\varepsilon_{l}=1$

$5 b$

Equation 5 still has two unknowns: $\varepsilon_{s}$ and $\varepsilon_{g}$, that represent the holdups of the gas and solid phases, respectively.

To circumvent this problem of equation closure we repeat the above set of experiments with another radioactive source who's gamma ray photon have another energy peak. Since different energy photons have different attenuation coefficients for the same material we can get another equation in the form of Equation 5.

${ }^{60} \mathrm{Co}$ and ${ }^{137} \mathrm{Cs}$ were used for this purpose as they were found suitable for Dual Source CT application based on the experimental study reported in the section 3.0 of the $6^{\text {th }}$ quarterly report.

The two equations for the Dual Source CT are as follows:

$\varepsilon_{g}=R_{g-s}^{(I)} \frac{\varepsilon_{s}}{\varepsilon_{s}^{o}}+R_{L}^{(I)}\left(1-\varepsilon_{s}\right)-R_{g-l-s}^{(I)}$

$\varepsilon_{g}=R_{g-s}^{(I I)} \frac{\varepsilon_{s}}{\varepsilon_{s}^{o}}+R_{L}^{(I I)}\left(1-\varepsilon_{s}\right)-R_{g-l-s}^{(I I)}$

Where

$\mathrm{I} \rightarrow$ Represents ${ }^{60} \mathrm{Co}$

$\mathrm{II} \rightarrow$ represents ${ }^{137} \mathrm{Cs}$

Equations 6 and 7 are two equations with two unknowns in them, hence, they can be solved for the holdup values of the solids and the gas. 
From this the solids hold up is:

$$
\varepsilon_{s}=\frac{\left(R_{G-L-S}^{(I)}-R_{G-L-S}^{(I)}\right)-\left(R_{L}^{(I)}-R_{L}^{(I)}\right)}{\left(\frac{\left(R_{G-S}^{(I)}-R_{G-S}^{(I)}\right)}{\varepsilon_{s}^{o}}-\left(R_{L}^{(I)}-R_{L}^{(I I)}\right)\right)}
$$

Using the value of $\varepsilon_{s}$ obtained from the above expression we can obtain the value of $\varepsilon_{g}$ from Equations 5b.

\subsubsection{Experimental Procedure}

The line average densitometry experiments were carried out with ${ }^{60} \mathrm{Co}(10 \mathrm{mci})$ and ${ }^{137} \mathrm{Cs}$ (70 mci) in the setup illustrated in Figure 3.13. The background scans were carried out with system filled with water and glass beads alone. The glass beads of $150 \mu \mathrm{m}$ size used are the same as those reported in the experiments that have been described in section 6.2 of the Sixth quarterly report.

A three phase system was obtained by having glass and water in the annular region and air alone in the central (core) region. This way the line averaged densitometry was performed for the above system would have all the three phases i.e. gas-liquid-solid with known volume fractions of each.

\subsubsection{Calculations of the overall holdup of the solids based on the geometry}

The over all voidage of the packed bed was obtained by adding a measured quantity of water to the system packed with the glass beads. The volume of the different compartments (the core and the annulus) was calculated based on the geometry of the system given in Table 3.1

Volume of water added to the system to determine the total voidage $\quad=650 \mathrm{ml}$

Total volume of the system (annular and central core) $\quad=1579 \mathrm{ml}$

Hence the void fraction of the bed

$=650 / 1579$

$=0.405$

Solids fraction $\left(\varepsilon_{s}^{o}\right)$ of the bed

$=1-0.405$

$=0.595$

This value is close to the data that is

available for the packed bed of the glass beads

$=0.59$.

\subsubsection{Hold up of the solids in the annular region calculated theoretically based on the information obtained above}

The volume fraction of the annular region based on geometry as reported in Table 3.1.

Using the value of $\varepsilon_{s}^{o}$ calculated above, the solids holdup in the annular region $\varepsilon_{s}$ (Estimated).

$=0.595 * 0.725$

$=0.431$ 
Table 3.1. Observations based on the geometry of the system illustrated in Figure 3.13.

\begin{tabular}{|l|l|l|l|}
\hline & Volume (sq inches) & Volume (sq cm) & Fraction \\
\hline Annular Region & 70.75 & 1159 & 0.725 \\
\hline Central (core) region & 26.75 & 438 & 0.274 \\
\hline Total & 97.48 & 1597 & 1.0 \\
\hline
\end{tabular}

\subsubsection{Dual source Densitometry observation}

The counts of the gamma ray photons are collected for one minute duration at a sampling frequency of $20 \mathrm{~Hz}$. The needed terms required to compute the solids holdup in the annular region experimentally by Equation 8 are listed in Table 3.2.

Table 3.2: Dual source densitometry

\begin{tabular}{|c|c|c|c|c|c|c|}
\hline & \multicolumn{3}{|l|}{${ }^{60} \mathrm{Co}$} & \multicolumn{3}{|l|}{${ }^{137} \mathrm{Cs}$} \\
\hline & $\begin{array}{c}\text { Average } \\
\text { Counts } \\
\text { (at } 20 \mathrm{~Hz} \text { ) }\end{array}$ & $A^{(I)}$ & $R^{(I)}$ & $\begin{array}{l}\text { Average } \\
\text { Counts } \\
\text { (at } 20 \mathrm{~Hz} \text { ) }\end{array}$ & $A^{(I I)}$ & $R^{(I I)}$ \\
\hline $\begin{array}{c}\mathrm{I}_{0} \\
\text { Incident } \\
\text { counts }\end{array}$ & 160.2 & & & 72.5 & & \\
\hline Empty system & 140.2 & $A_{g}=0.135$ & & 57.03 & $A_{g}=0.24$ & \\
\hline $\begin{array}{l}\text { System with } \\
\text { water in center } \\
\text { and annulus }\end{array}$ & 50.5 & $A_{l}=1.095$ & $R_{L}=0.9631$ & 19.9 & $A_{l}=1.289$ & $R_{L}=1.0492$ \\
\hline $\begin{array}{l}\text { System with } \\
\text { glass beads in } \\
\text { center and } \\
\text { annulus }\end{array}$ & 41.1 & $A_{s}=0.855$ & $R_{G-S}=1.226$ & 13.9 & $A_{s}=1.651$ & $R_{G-S}=1.4066$ \\
\hline $\begin{array}{l}\text { System with } \\
\text { water and } \\
\text { glass beads in } \\
\text { annulus and air } \\
\text { in center }\end{array}$ & 72 & $A_{g-l-s}=0.798$ & $R_{G-S-L}=0.665$ & 24.6 & $A_{g-l-s}=1.08$ & $R_{G-S-L}=0.841$ \\
\hline
\end{tabular}

The values of $R_{L}, R_{G-S}$ and $R_{G-S-L}$ for both the sources ( ${ }^{60} \mathrm{Co}$ and ${ }^{137} \mathrm{Cs}$ ), from Table 3.2, are substituted into Equation 8 with the priori measured value of $\varepsilon_{s}^{\circ}$ calculated in section 3.4.3.

The solids holdup $\varepsilon_{s}$ obtained experimentally (in the annular region) using Equation $8=$ 0.422

Hence the relative error

$$
=\left|\frac{0.422-0.431}{0.431}\right| \times 100=2.08 \%
$$




\subsubsection{Conclusions}

The proposed experiment was successfully carried out and the solids holdup within the system was experimentally determined by dual source densitometry to be $\left(\varepsilon_{s}\right) 0.422$, this is close to the approximated solids hold up based on the geometry of the system and other data available which is 0.431 . The relative error was found to be $2.08 \%$, which is small and can be attributed to experimental error. Hence, the above mentioned system of equations and those developed in the Fourth quarterly report can be used to estimate the holdups in a three phase system, also the sources selected $\left({ }^{60} \mathrm{Co}\right.$ and $\left.{ }^{137} \mathrm{Cs}\right)$ are suitable to do a dual source CT. 


\section{Appendix 3.5 \\ 3.5. Validation of the DSCT operation as a single source CT with a two phase phantom.}

To confirm if the developed Dual Source Computer Tomography system is able to first accomplish what the single source tomography scanners are able to, a two phase phantom was scanned. The idea is to have the two phases static such that the hold up calculated on the basis of the tomography scan can be compared to the actual hold up based on the geometry of the system.

This phantom consisted of two concentric cylinders. The inner cylinder was left empty (hence consisted of air), and the outer annular region was filled with water. This way the system consists of two phases and the hold up of the gaseous phase in the center of the system is one ( $100 \%$ ) and the hold up of the liquid phase in the annular region is one $(100 \%)$. Figure 3.14 shows the phantom details.

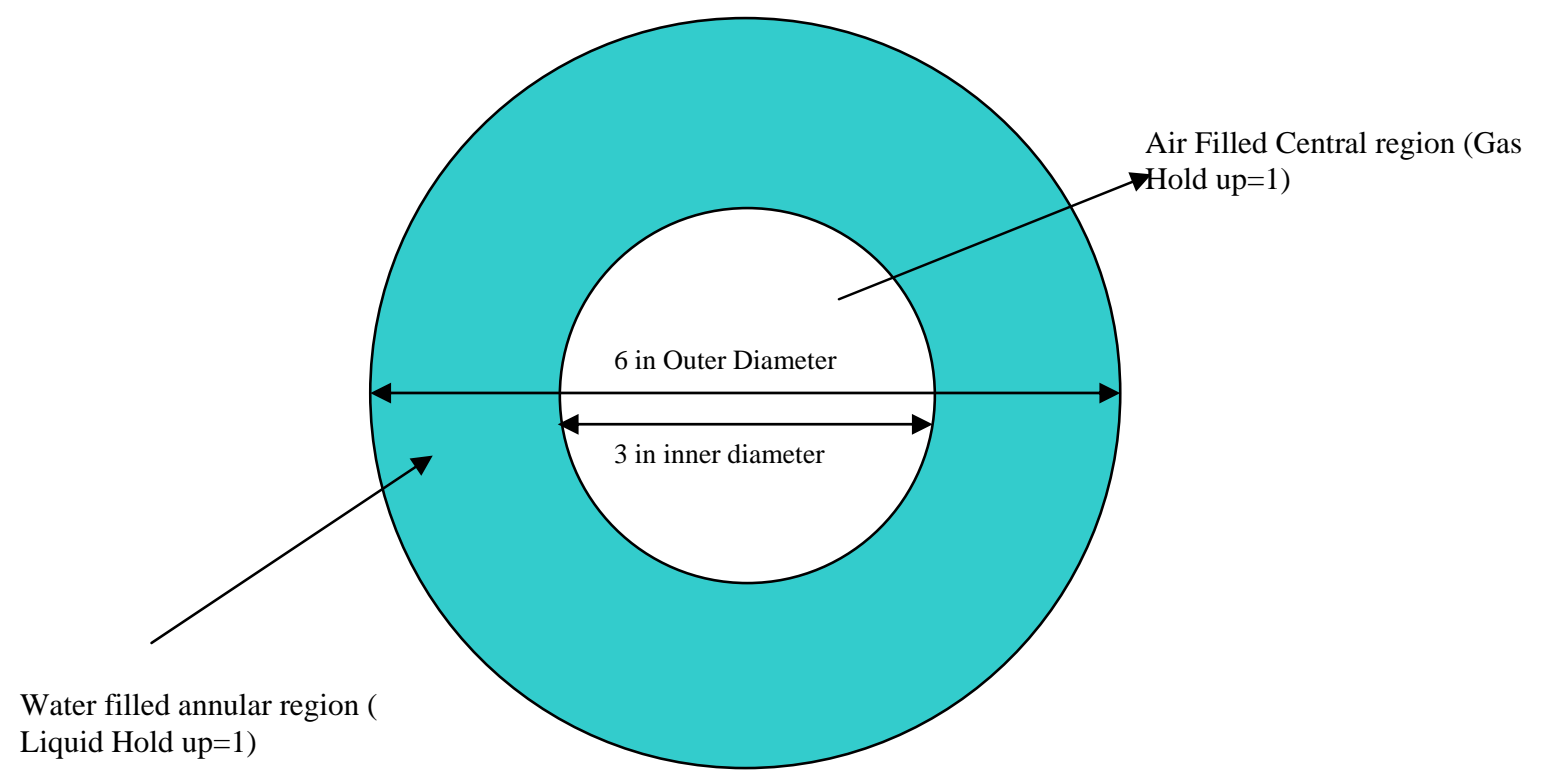

Figure 3.14 Cross sectional schematic of the phantom. The Annular region is filled with water.

\subsubsection{Image reconstruction}

The scan was carried out with 9 detectors as fan beam created with the vertex angle of the detectors subtended with the source would encompass the 6 inch diameter phantom. The data was collected with ${ }^{137} \mathrm{Cs}$ and ${ }^{60} \mathrm{Co}$ gamma ray sources. The details of the algorithm used in the reconstruction process have been discussed in the $7^{\text {th }}$ quarterly report section 2.0. Some of the key equations have been reproduced here. 
The Data collected represents the ration of I/Io where I is the photon count of the attenuated photon beam and the Io is the incident photon count. Hence the left hand side of equation 9 represents the transmission ratio. The term in the exponent represents the line integral of the attenuation of the domain.

$$
\frac{I}{I_{0}}=\exp \left(-\int_{l(x, y)=0} \mu_{e f f}(x, y) d x\right)
$$

The pixel wise image is reconstructed using CREL EM algorithm. The attenuation data $\mu_{e f f}(x)$ for each pixel is computed. This data is further processed with the aid of background scans of the system filled with the pure phases (in this case water and air) to arrive at the hold up values. The computed attenuation coefficient for each pixel is represented by the equation given below.

$$
\left\langle\mu_{E M}(x)\right\rangle=\mu_{l}\left\langle\varepsilon_{l}(x)\right\rangle+\mu_{g}\left\langle\varepsilon_{g}(x)\right\rangle
$$

This quantity is linear sum of the fractions of the individual attenuations by the two pure phases (gas and liquid). To determine the fractions of the individual phases the scans are repeated with the system being filled with the pure phases (air and water). This way the attenuation of the pure phases is determined. Also the fact that the sum of the individual holdup fractions adds up to unity is used.

$$
\begin{array}{lr}
\varepsilon_{l}+\varepsilon_{g}=1 & 11 \\
\left\langle\mu_{l, E M}(x)\right\rangle=\mu_{l}\left\langle\varepsilon^{\circ}{ }_{l}(x)\right\rangle & 12 \\
\left\langle\mu_{g, E M}(x)\right\rangle=\mu_{g}\left\langle\varepsilon^{\circ}{ }_{g}(x)\right\rangle & 13 \\
\varepsilon_{l}^{0}=\varepsilon_{g}^{o}=1 & 14
\end{array}
$$

Equations 11-14 are substituted in Equation 10 to arrive at Equations 15 and 16 that represent the gas phase hold up and liquid phase holdup, respectively.

$$
\begin{aligned}
\left\langle\varepsilon_{g}(x)\right\rangle & =\frac{\left\langle\mu_{E M}(x)\right\rangle-\mu_{l, E M}}{\mu_{g, E M}-\mu_{l, E M}} \\
\left.\left\langle\varepsilon_{l}(x)\right)\right\rangle & =1-\frac{\left\langle\mu_{E M}(x)\right\rangle-\mu_{l, E M}}{\mu_{g, E M}-\mu_{l, E M}}
\end{aligned}
$$

\subsubsection{Results and Discussion}

Figure 3.15 depicts the attenuation profile of the system when filled with water and air, respectively. The walls of the system can be seen clearly when the system is filled with air. 

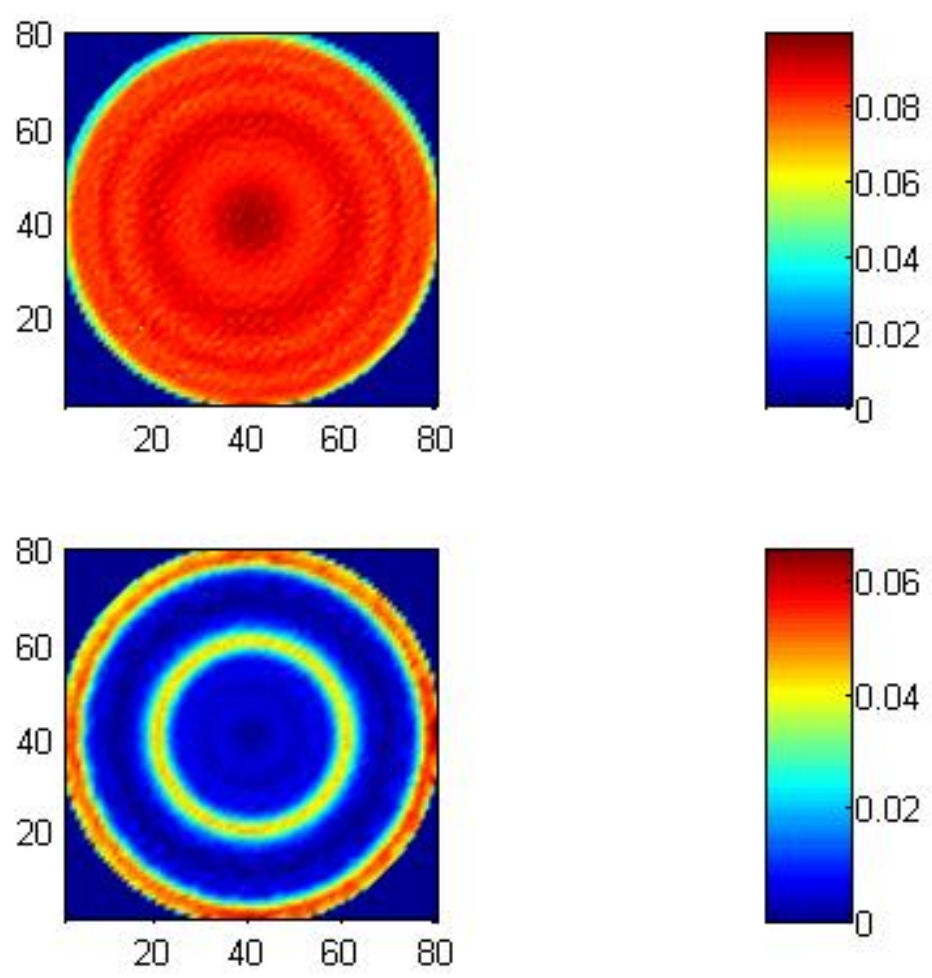

Figure 3.15 Attenuation profiles of the system with pure phases when scanned with ${ }^{137}$ Cs. Profile with water is represented above and the profile with just air is given below. The wall of the cylinder can clearly be seen in the system.

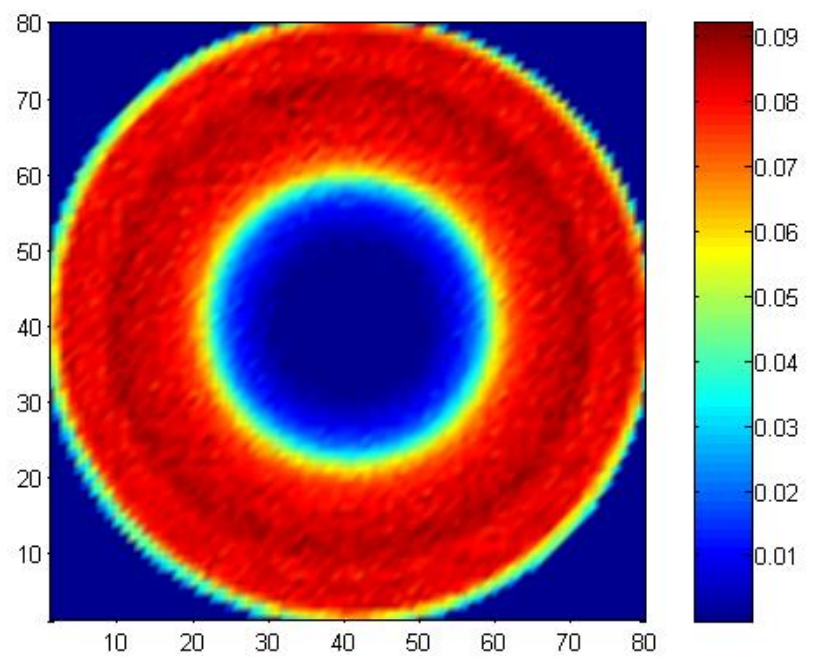

Figure 3.16 Attenuation profiles of the system filled with water (liquid phase) in the annular region. This image is based on data from ${ }^{137} \mathrm{Cs}$ 
The data of Figure 3.15 represent the attenuations obtained by Equations 12 and 13 . The result of the scan carried out with the system of both air and water is shown in Figure 3.16. This image is represented by Equation 10. These images are processed to determine the hold up of the gas and the liquid phases based on the Equations 15 and 16. Figure 3.17 shows that both the phases can be clearly seen in the system and they resemble the phantom. Similar experiments were repeated with the ${ }^{60}$ Co sources. The results are shown below.
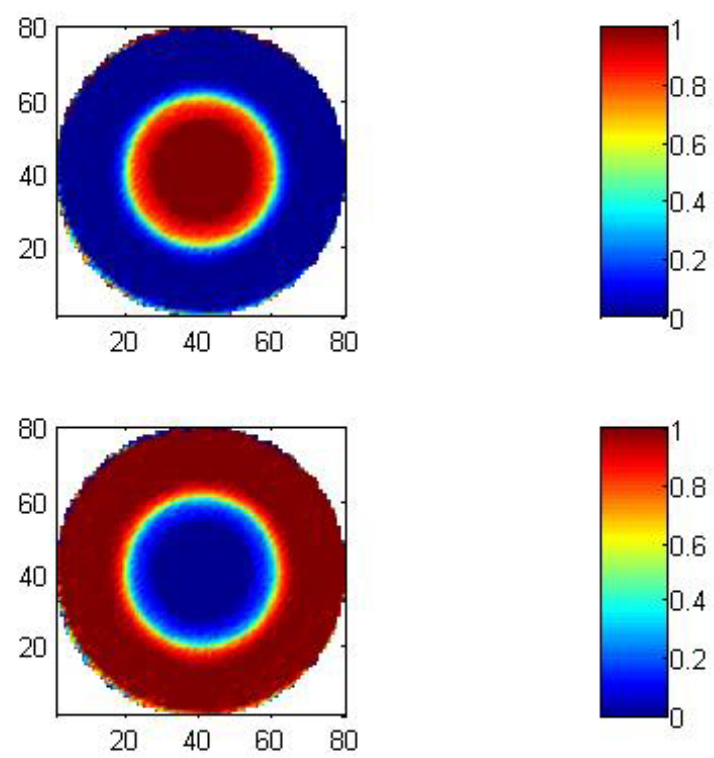

Figure 3.17 Hold up profile of gas phase (air) above and Hold up profile of liquid phase (below) based on data from ${ }^{137} \mathrm{Cs}$.
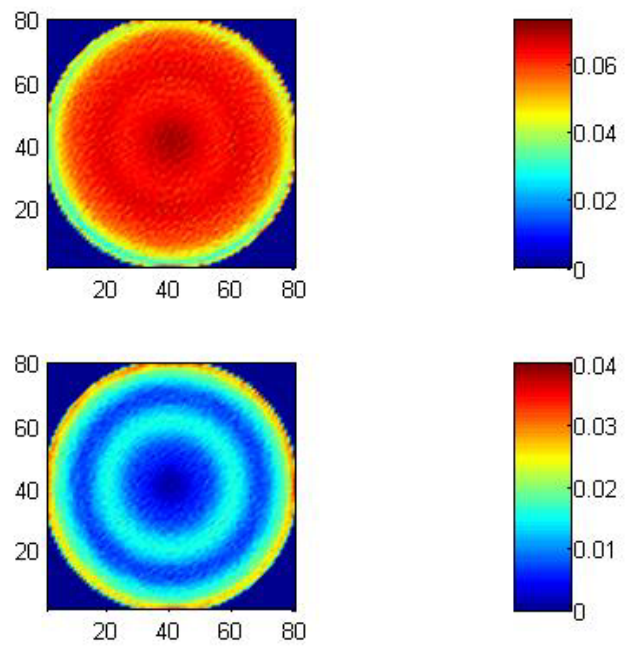

Figure 3.18 Attenuation profiles of the system with pure phases when scanned with ${ }^{60} \mathrm{Co}$. Profile with water is represented above and the profile with just air is given below. 


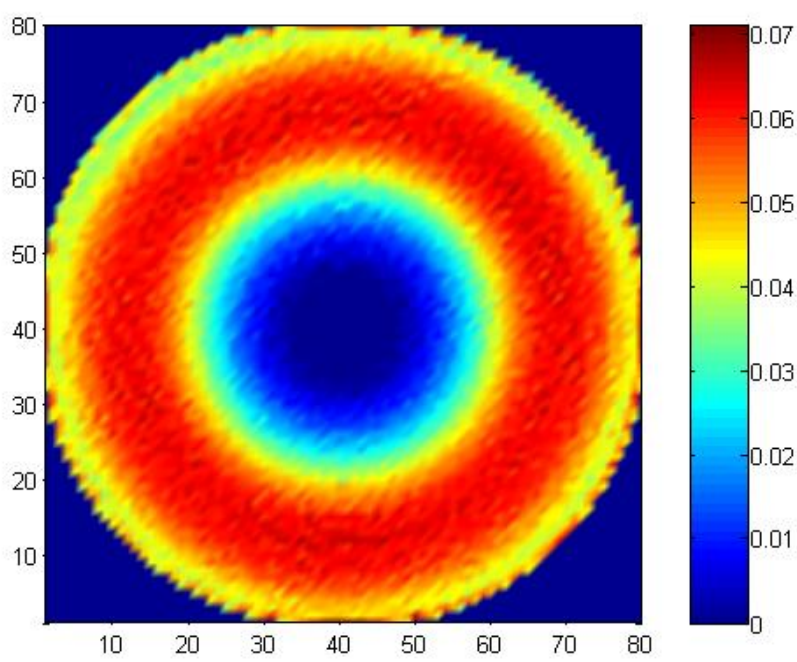

Figure 3.19 Attenuation profiles of the system filled with water (liquid phase) in the annular region. This image is based on data from ${ }^{60} \mathrm{Co}$.
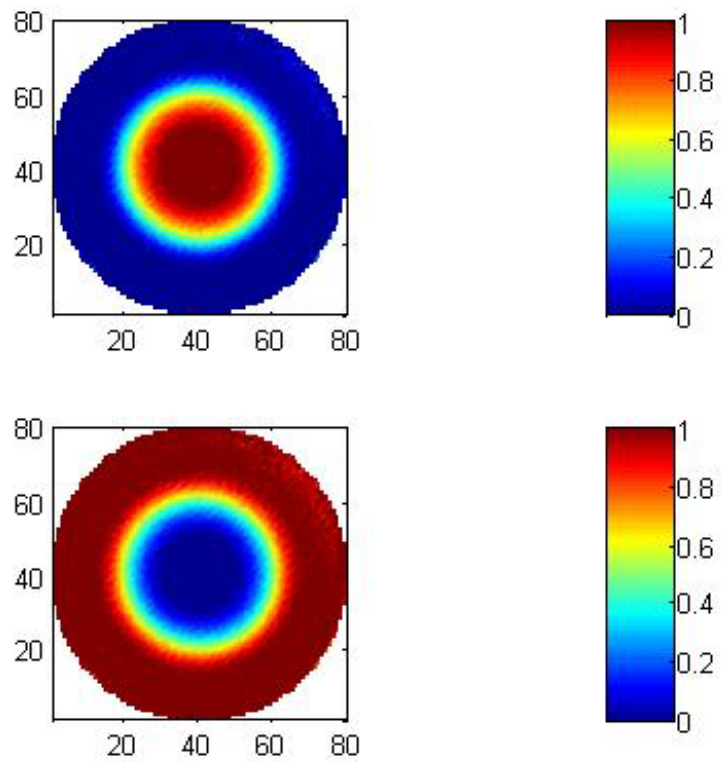

Figure 3.20 Hold up profile of gas phase (air) above and Hold up profile of liquid phase (below) based on data from ${ }^{60} \mathrm{Co}$.

\subsubsection{Conclusion}

The results of the scans as seen in Figures 3.17 and 3.20 clearly show that both the ${ }^{137} \mathrm{Cs}$ and the ${ }^{60}$ Co sources are able to determine the holdup distribution of both phases clearly. Hence, the DSCT setup can be used to image two phase systems with the Cobalt or 
Cesium sources. The liquid hold up profile clearly matches what we see based on the geometry in Figure 3.14. 


\section{Appendix 3.6}

Manuscript: Varma R, Bhusarapu S, O’Sullivan J A and Al-Dahhan M H. (2007). Comparison of alternating minimization and expectation maximization algorithms for single source gamma ray tomography.

In review Measurement Science and Technology. 


\title{
Comparison of alternating minimization and expectation maximization algorithms for single source gamma ray tomography
}

\author{
R Varma', S Bhusarapu², J A O’Sullivan³ and M H Al-Dahhan ${ }^{1,12}$ \\ ${ }^{1}$ Chemical Reaction Engineering Laboratory, Department of Energy, Environment and Chemical Engineering, \\ Campus Box 1198, 1 Brookings Drive, Washington University, St. Louis, MO 63130 USA \\ ${ }^{2}$ Harper International Corporation, West Drullard Avenue, Lancaster, NY 14086 USA. \\ ${ }^{3}$ Electronic Systems and Signals Research Laboratory, Department of Electrical and Systems Engineering, 1 \\ Brookings Drive, Washington University, St. Louis, MO 63130-4899 USA \\ Email: varma@wustl.edu, sbhusarapu@harperintl.com,jao@wustl.edu and muthanna@che.wustl.edu
}

\begin{abstract}
Lange and Carson (1984) defined the image reconstruction for tomography as a maximum likelihood estimation problem and derived an expectation-maximization (EM) algorithm to obtain the maximum likelihood image estimate. However, in the maximization step or M-step of the EM algorithm, an approximation is made in the solution which can affect the image quality, particularly in the case of domains with high attenuating material. O'Sullivan and Benac (2007) reformulated the maximum likelihood problem as a double minimization of an Idivergence to obtain a family of image reconstruction algorithms, called the alternating minimization algorithm (AM). The AM algorithm increases the log-likelihood function while minimizing the I-divergence. In this work we implement the AM algorithm for image reconstruction in gamma ray tomography for industrial applications. Experimental gamma ray transmission data obtained with a fan beam geometry gamma ray scanner, and simulated transmission data based on a synthetic phantom, with two phases' (water and air) were considered in this study. Image reconstruction was carried out with these data using the AM and the EM algorithms to determine and quantitatively compare the holdup distribution images of the two phases in the phantoms. When compared to the EM algorithm, the AM algorithm shows qualitative and quantitative improvement in the holdup distribution images of the two phases for both the experimental and simulated gamma ray transmission data.
\end{abstract}

Keywords: Alternating Minimization, Expectation Maximization, Computed Tomography, I-divergence, Image Reconstruction, Log likelihood, Multiphase Systems, Two Phase Flow

\footnotetext{
${ }^{1}$ Corresponding author

Contract grant sponsor US Department of Energy (DE-FC36-01GO11054)
} 


\subsection{Introduction:}

Computed Tomography has been successfully used in the medical field for radiology diagnostics. Recently, its use has been expanded to process engineering for industrial applications, in particular for visualizing the flow field in multsiphase flow systems (Kumar and Duduković 1997). One key area of research addresses the improvement of image reconstruction algorithms. Several authors have made contributions to the theory of reconstructive tomography and an early overview of such algorithms was presented by Snyder and Cox (1977).

Algebraic algorithms such as Fourier/convolution techniques when applied to determine the phase holdup distribution in two phase systems either assume the systems to be azimuthally symmetric in distribution or consider the gamma ray transmission process to be deterministic there by completely ignore the stochastic nature of the data. Expectation maximization (Lange and Carson 1984) and Alternating minimization (O'Sullivan and Benac 2007) algorithms account for the stochastic nature of the gamma ray transmission across the domain of interest. This makes these algorithms more favorable for image reconstruction to determine the phase holdup distribution.

The EM algorithm has used extensively to process gamma ray tomography data to image holdup distribution in various two phase systems. Some of its applications include imaging, gas holdup in gas liquid bubble column (Kumar et al. 1997), solids holdup in liquid-solid riser (Roy et al. 1997), liquid flow distribution in structured packing (Roy et al. 2004) and monolith reactor (Roy and Al-Dahhan 2005), and gas holdup in stirred tanks (Khopkar et al. 2005). This study applies the Alternating Minimization algorithm proposed for transmission tomography by O'Sullivan and Benac (2007) for the case of single energy gamma ray tomography for imaging phase holdup distribution in two phase systems. Phantoms used for this study are designed to represent multiphase systems studied with EM. Both the AM and EM algorithms are applied to the phantoms. The holdup distribution results obtained with the AM algorithm are compared with those obtained with the Expectation Maximization (EM) algorithm as proposed by Lange and Carson (1984), for simulated and experimental gamma ray transmission data from phantoms containing two phases.

\subsection{The Algorithms:}

Since the objective of this study is to compare the AM and the EM algorithms, these algorithms have been briefly discussed and outlined for the purpose of continuity and for the benefit of the uninitiated reader. For further information the reader is encouraged to read the relevant references cited. 


\subsection{Expectation Maximization Algorithm}

Lange and Carson (1984) defined the image reconstruction for tomography as a maximum likelihood estimation problem and derived an estimation-maximization (EM) algorithm to obtain the maximum likelihood image estimate. In experiments where gamma ray counts statistics is high $(\sim 500$ counts/projection), ignoring the true statistical nature of the data may not be a serious limitation because Poisson counting noise is only a component of the total system noise (Lange and Carson 1984). It is precisely in the low counts experiments ( $<100$ counts/projection) that the EM algorithm is expected to provide the greatest improvement in the reconstruction quality. Usually in the transmission tomography experiments with the amount of shielding provided at the detectors end (to increase the spatial resolution), the counts recorded are bound to be small ( $\sim 100$ or less counts/projection), which forces one to use an EM algorithm. Superiority of the EM algorithms over Fourier techniques and on iterative algebraic methods such as the incorporation of non-negativity constraints and objective measure of quality of reconstruction (e.g. log-likelihood, least squares, maximum entropy) is discussed in detail by Lange and Carson (1984).

The EM algorithm is a general iterative technique for computing maximum likelihood estimates in any general scenario of measurement of statistical quantities. Application to image reconstruction in transmission tomography is only a specific application. Each iteration of the EM algorithm consists of two steps expectation (E step) and maximization (M step). The derivation of the $\mathrm{E}$ and $\mathrm{M}$ steps for transmission tomography are discussed in detail by Lange and Carson (1984). Some of the key elements are highlighted here.

In the expectation step (E-step), conditional expectation of 'entire' or 'complete' data set is estimated on the basis of the measured data (counts from the detector) and the parameter set (attenuation values). The 'complete' data set represents intensity of the photons that enter and leave each pixel in the domain for all the projections. If for a given pixel $x$ along a projection $y$ (Figure 1), we define $X(y \mid x)$ as the random number of photons (or counts) that enter $x$ and $X(y \mid(x+1))$ the random number that survive and leave $x$, then this process can be modeled as a binomial distribution with two out comes: survival of the photon $\exp (-h(y \mid x) \mu(x))$ and absorption of the photons $[1-\exp (-h(y \mid x) \mu(x))]$. Where $h(y \mid x)$ is the segment of projection $y$ in pixel $x$. The probability of this binomial process can be represented by (1), in other words (1) represents the probability of the 'complete' data set for pixel $x$.

$$
p_{X(y \mid(x+1))}=\left(\begin{array}{c}
X(y \mid x) \\
X(y \mid(x+1))
\end{array}\right)[\exp (-h(y \mid x) \mu(x))]^{X(y \mid(x+1))}[1-\exp (-h(y \mid x) \mu(x))]^{X(y \mid x)-X(y \mid(x+1))}
$$




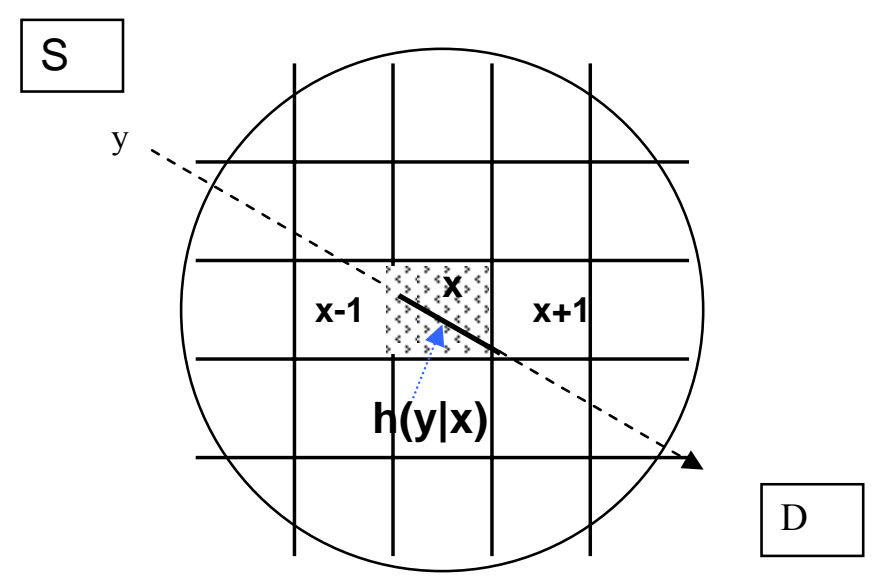

Figure 1: Schematic of the representation of a transmission tomography domain, the boxes represent the pixels. Here S: source, D: detector, $y$ : projection index, $x:$ pixel index and $h(y \mid x)$ is the segment of projection $y$ in pixel $x$.

The first pixel along any projection $y$ receives the photons emitted from the gamma source. This is a Poisson process with mean $\lambda$ which represents the intensity of the source, its probability is given by $p_{(X(y \mid 1))}=\frac{\lambda^{N(y \mid 1)} \exp (-\lambda)}{X(y \mid 1) !}$.

Since the pixels are independent, the likelihood function of the entire 'complete' data (for all pixels along projection $y$ ) set can be represented as the product of the individual likelihood functions of each pixel that lies along a projection $y$. This likelihood function is represented as (3).

$$
f(N(y): \mu)=\frac{\lambda^{N(y \mid)} \exp (-\lambda)}{X(y \mid 1) !} \prod_{x \in X}\left(\begin{array}{c}
X(y \mid x) \\
X(y \mid(x+1))
\end{array}\right)\left[\operatorname { e x p } ( - h ( y | x ) \mu ( x ) ] ^ { X ( y | ( x + 1 ) ) } \left[1-\exp (-h(y \mid x) \mu(x)]^{[(X(y \mid x)-X(y \mid(x+1)))}\right.\right.
$$

If the complete data set was known then computing the attenuation values $\mu(x)$ for every pixel would be straight forward. However, this is not the case since only the counts data observed by the detectors representing the random number of photons that have survived all the pixels along a projection is available. The expectation step involves the computing the expectation of the 'complete' data set for a given pixel, conditional to the observed data $d(y)$ (measured by detector D) given the current estimate of the attenuation function $\hat{\mu}^{(k)}(x)$ as given by (4).

$E\left\lfloor X(y \mid x) \mid d(y), \hat{\mu}^{(k)}(x)\right\rfloor$

Lange and Carson (1984) show that (4) can be represented as

$$
E[X(y \mid x) \mid d(y)]=d(y)+E[X(y \mid x)]-E[d(y)] .
$$


Equation (5) is used to compute $M(y \mid x)$ and $N(y \mid x)$, the expected values of the photons entering and leaving given pixel $x$ respectively along projection $y$. The entities $M(y \mid x)$ and $N(y \mid x)$ are the conditional expatiations related to $X(y \mid x)$ and $X(y \mid(x+1))$ respectively by (4).

In the M-step, this conditional expectation is maximized with respect to a parameter set. In the case of gamma ray transmission tomography, the parameter set is the attenuation values $\mu(x)$ of the pixels in the scanned domain and the measured data set $d(y)$ is the detector signal obtained for each projection $y$. Equation (5) is used to determine the conditional expectation values $M(y \mid x)$ and $N(y \mid x)$ for each pixel in the image which is then summed over all projection and then substituted in the likelihood function. The natural logarithm of this equation is given by (6) which represents the log likelihood of the 'complete' data set over all projections $y$.

$$
\ln [f(N(y): \mu)]=\sum_{y \in Y} \sum_{x \in X}\{N(y \mid x) \ln [\exp (-h(y \mid x) \mu(x)]+(M(y \mid x)-N(y \mid x)) \ln [1-\exp (-h(y \mid x) \mu(x)]\}+R
$$

$R$ represents all the terms that are not dependant on $\mu(x)$. Maximizing (6) by setting the value of the partial derivative with respect to $\mu(x)$ to zero yields a transcendental equation:

$$
\sum_{y \in Y}-N(y \mid x) h(y \mid x)+\sum_{y \in Y}[M(y \mid x)-N(y \mid x)] \frac{h(y \mid x)}{\exp [h(y \mid x) \mu(x)]-1}=0 .
$$

Since (7) cannot be solved exactly due to the exponential term, Lange and Carson (1984) suggest the Taylor series based approximation to simplify the solution:

$$
\frac{1}{\exp [h(y \mid x) \mu(x)]-1}=\frac{1}{h(y \mid x) \mu(x)}-\frac{1}{2}+\frac{h(y \mid x) \mu(x)}{12}+O\left\{[h(y \mid x) \mu(x)]^{3}\right\} \text {. }
$$

The authors recommend using the first three terms of (8) to arrive at (9) in order to make a good approximation of (7):

$$
0=\mu(x)^{2} \cdot \sum_{y \in Y}[M(y \mid x)-N(y \mid x)] \frac{h(y \mid x)^{2}}{12}-\mu(x) \cdot \sum_{y \in Y}[M(y \mid x)+N(y \mid x)] \frac{h(y \mid x)}{2}+\sum_{y \in Y}[M(y \mid x)-N(y \mid x)] .
$$

Equation (9) is a quadratic equation which has a solution given by (10)

$$
\hat{\mu}(x)^{(k+1)}=B / 2 A-\sqrt{B^{2}-4 A C} / 2 A
$$

Where, $A=\sum_{y \in Y}[M(y \mid x)-N(y \mid x)] \frac{h(y \mid x)^{2}}{12}, B=\sum_{y \in Y}[M(y \mid x)-N(y \mid x)] \frac{h(y \mid x)}{2}$, and

$$
C=\sum_{y \in Y}[M(y \mid x)-N(y \mid x)] .
$$


The iterative process starts with an initial guess for $\hat{\mu}(x)^{k}(k=0)$, this is used to compute the conditional expectation values of $M(y \mid x)$ and $N(y \mid x)$ using $d(y)$ based on (5). These values are then used to determine the next estimate of attenuation $\hat{\mu}(x)^{k+1}$ based on (10). By setting $k=k+1$ this process is repeated starting from (5) till the value of $\hat{\mu}(x)^{k+1}$ which maximizes the log likelihood of the complete data set is reached.

The approximation for (7) given by (8) as discussed in the previous paragraph, is valid for small values of $(h(y \mid x) \mu(x))$ and is not accurate for application involving regions of high density, high mass attenuation $\mu(x) / \rho\left(\mathrm{cm}^{2} / \mathrm{g}\right)$, or for coarse pixel resolution where the values of $h(y \mid x)$ are large. This in turn may lead to a decrease in the log-likelihood function from one iteration to the other instead of an increase. This is a major short coming for imaging applications involving industrial multiphase flow systems as they are usually constructed with metals or have internal structures that are also made of metal. To improve accuracy other authors have used modified approaches to derive the M-step (Ollinger 1994) or the E-step (Browne and Holmes 1992) of the EM algorithm in the context of its application to positron emission tomography (PET). Although these approaches can be adapted to image reconstruction in gamma ray transmission tomography for further improvement the work by Lange and Carson (1984) has been compared here to the AM algorithm as there is a precedence of its use for determining phase holdup distribution in multiphase systems using gamma ray tomography.

\subsection{Alternating minimization Algorithm}

Expectation -maximization algorithm is a special case of Alternating-minimization (O'Sullivan and Benac 2007) algorithm, which has been studied extensively in the applications involving x-ray based medical imaging (Benac 2005). O'Sullivan and Benac (2007) reformulated the maximum likelihood problem as a double minimization of an I-divergence to obtain a family of image reconstruction algorithms. Idivergence, introduced by Csiszár (1991), is a measure of discrepancy between two functions $a(y)$ and $b(y)$, which is given as:

$$
I(a \| b)=\sum_{y \in Y}\left\{a(y) \ln \left[\frac{a(y)}{b(y)}\right]-[a(y)-b(y)]\right\}
$$

where $y$ is a finite dimensional space.

Csiszár (1991) examined a wide variety of discrepancy measures (e.g. least squares, entropy) including the I-divergence measure (11) between two functions $a(y)$ and $b(y)$ and arrived at the following conclusion which is relevant for the image reconstruction problem subject to non-negativity 
constraints. Csiszár (1991) concluded that if the functions involved are all real valued, having both positive and negative values, then minimizing the least squares is the only consistent choice; whereas, if all the functions are required to be non-negative, then minimizing the I-divergence is the only consistent choice. This I-divergence measure was first employed for image reconstruction by Snyder et al.(1992).

Each step of minimization in the AM algorithm is claimed to be exact process, without any approximation as in the case of EM (8), which represents one of its advantages over the EM algorithm. The alternating minimization algorithm formulated by O'Sullivan and Benac (2007) is guaranteed to monotonically increase the log-likelihood function at every iteration. In this work the AM algorithm is applied for the first time to image phase hold up distribution using gamma ray tomography to seek improvements in the quality of hold up distribution images for industrial multiphase flow sytems.

The image reconstruction algorithm is based on a statistical model for the measured data, Beer's law and a realistic model for the known point spread function (O'Sullivan and Benac 2007). The reconstruction problem is formulated as an optimization (maximum likelihood) problem in statistical estimation theory.

A brief outline of the algorithm is described below. For more details the reader is encouraged to refer to O'Sullivan and Benac(2007). If we define $g(y: \mu)$ as the mean of the counts $d(y)$ received by the detector (a Poisson random number) per Beer Lambert's law, then

$$
g(y: \mu)=\lambda(y) \exp \left[-\sum_{x \in X} h(y \mid x) \mu(x)\right] .
$$

In the expression for the I-divergence, the function $a(y)$ is taken to be the measured data represented by $d(y)$ and $b(y)$ is taken to be a nonlinear model $q(y)$ representing the transmission of the photons. The term $q(y)$ includes the parameter set $\hat{\mu}(x)$, which is to be estimated.

$$
q(y)=\lambda(y) \exp \left[-\sum_{x \in X} h(y \mid x) \hat{\mu}(x)\right]
$$

If (11) can be written for this case as

$$
I(d \| q)=\sum_{y \in Y}\left\{d(y) \ln \left[\frac{d(y)}{q(y)}\right]-[d(y)-q(y)]\right\} .
$$

The terms $g(y: \mu)$ and $\mathrm{q}$ are related by the following expression

$$
I(d \| g(y: \mu))=\min _{q \in \mu} I(d \| q) .
$$

When $\mu=\hat{\mu}$, the quantities $q$ is equal to $g$. Hence the objective of the algorithm is to find the minima of $I(d \| q)$ with respect to $\hat{\mu}(x)$. The terms in the log likelihood function that depend on the parameter set 
(attenuation values to be estimated) are negative of the corresponding terms in the I-divergence. Thus, minimizing the I-divergence over the parameter set $\hat{\mu}$ is equivalent to maximizing the log-likelihood function. Minimizing the I-divergence offer the unique advantage as it has a know lower bound (equal to zero), for projections data processed from any system. This is not the case with maximizing the log likelihood as the upper bound is not known. The derivation of the iterative algorithm and its proof is discussed by O'Sullivan and Benac (2007).

Minimizing (14) as per (15) yields (16), the expression for updating the parameter set (attenuation values):

$$
\hat{\mu}^{(k+1)}(x)=\hat{\mu}^{(k)}(x)-\frac{1}{Z(x)} \ln \left(\frac{\tilde{b}(x)}{\hat{b}^{(k)}(x)}\right) .
$$

The terms $\tilde{b}(x)$ and $\hat{b}^{(k)}(x)$ are the back projections of $d(y)$ and the current estimates of $\hat{q}^{(k)}(y)$ respectively. In other words, they are the back projections of the measured data and the nonlinear model employed (based on Beer-lamberts law). Their expressions are given by:

$$
\begin{aligned}
& \tilde{b}(x)=\sum_{y \in Y} h(y \mid x) d(y) \\
& \hat{b}^{(k)}(x)=\sum_{y \in Y} h(y \mid x) \hat{q}(y) \\
& \hat{q}^{(k)}(y)=\lambda(y) \exp \left[-\sum_{x \in X} h(y \mid x) \hat{\mu}^{(k)}(x)\right] .
\end{aligned}
$$

The iterative process for computing the image goes backwards starting from (19) to (16). An initial guess for $\mu^{(k=0)}(x)$ is chosen to calculate $\hat{q}(x)$ in (19), then $\hat{q}(x)$ is used to calculate $\hat{b}(x)$ in (18). The back projection $\tilde{b}(x)$ is computed just once based on $d(y) \cdot \mu^{(k+1)}(x)$ is updated based on (16), and the process is started again setting $k=k+1$ using the updated values of attenuation. A non-negativity constraint is applied on the valued of attenuation. Hence at any iteration, if $\hat{\mu}^{(k+1)}(x)<0$ it is over written as $\hat{\mu}^{(k+1)}(x)=0 . Z(x)$ in (16) is an appropriate scaling function chosen for the $x^{\text {th }}$ pixel such that the following criteria is satisfied (O'Sullivan and Benac 2007):

$$
\sum_{x \in X}\left(\frac{h(y \mid x)}{Z(x)}\right) \leq 1
$$


For every pixel $x$ the length of the longest projection $y$ passing through it was chosen as the value of $Z(x)$ such that (20) was satisfied. The values of $Z(x)$ was found to be insensitive to the final convergence values.

\subsection{Data generation methodology}

Transmission data from three different types of phantoms were processed by the algorithms for comparison. The first two cases of the phantom considered were synthetic, as in simulated gamma ray transmission data was generated on the basis of these phantoms and processed. In the third case experimental transmission data was gathered with a second generation fan beam tomography setup and processed. The holdup distribution images of the liquid and the gaseous phases in the three cases of the phantom are determined and the results are compared.

The first phantom has regions of gas (air) and liquid (water) alone. The second phantom has regions with solid (metal) in addition to gas (air) and liquid (water). The objective of using the second phantom is to test for applications where the solid phase in the system is stationary, like in case of gamma ray tomography applied to columns with unavoidable internal structures, or those with structured packing or fixed bed (Roy and Al-Dahhan 2005). In such applications the background scans, or calibration scans, of the column include the solid phase, there by only the liquid and gaseous phases are accounted for when the dynamic system is imaged. In the third case experimental data was collected for a phantom with the gaseous phase (air) and liquid phase (air). Although the gas and liquid phase are stationary in all the phantoms, they are processed assuming them to be dynamic. Hence, the holdup images obtained are meant to provide a time averaged holdup distribution information.

\subsection{Details of the phantom}

3.1.1 Phantom for simulation (cases I and II). In this study a synthetic phantom was considered with dimensions as indicated in Figure 2. 


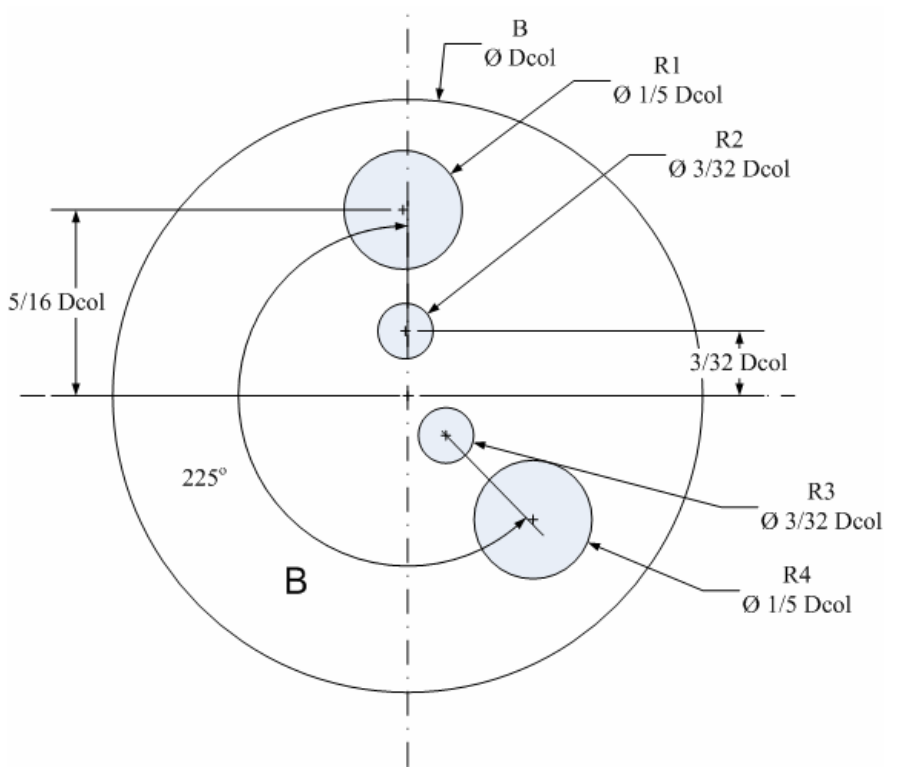

Figure 2:Schematic of phantom for which the data was generated by simulation. Dcol=8 in. The details of the material in regions $\mathrm{R} 1, \mathrm{R} 2, \mathrm{R} 3$, and $\mathrm{R} 4$ are given in table 1 .

The diameter of the phantom, $\mathrm{D}_{\mathrm{col}}$ was set to 8 in., the other region in the phantom have dimensions that are in proportion to the diameter. The proportionality ratios are indicated in Figure 2. In case I, the regions R1 though R4 are filled with air and the background region B is filled with water (table 1).

Table 1: Materials used in the phantom and the attenuation values

Region in

Phantom

(Figure 2)
Material used.

Attenuation values indicated in parenthesis

\section{Case I}

\section{Case II}

\begin{tabular}{ccc}
\hline B & Water $\left(0.0863 \mathrm{~cm}^{-1}\right)$ & Water $\left(0.0863 \mathrm{~cm}^{-1}\right)$ \\
R1 & Air $\left(0.0 \mathrm{~cm}^{-1}\right)$ & Air $\left(0.0 \mathrm{~cm}^{-1}\right)$ \\
R2 & Air $\left(0.0 \mathrm{~cm}^{-1}\right)$ & Iron $\left(0.2197 \mathrm{~cm}^{-1}\right)$ \\
R3 & Air $\left(0.0 \mathrm{~cm}^{-1}\right)$ & Iron $\left(0.2197 \mathrm{~cm}^{-1}\right)$ \\
R4 & Air $\left(0.0 \mathrm{~cm}^{-1}\right)$ & Air $\left(0.0 \mathrm{~cm}^{-1}\right)$ \\
\hline
\end{tabular}

This represents an ideal two phase arrangement consisting of liquid and gas. In case II, the regions R1 and R4 are filled with air, regions R2 and R3 are filled with iron, and the background region is filled with water (Table 1). The shape of the background region is the same as Case I. Case II represents situation where columns have internal structures that are static and are made of metal which usually have high attenuation values. The holdup images are determined only for the gas and liquid present in the case II phantom. 


\subsubsection{Phantom for experimental data (case III): Phantom made of Perspex (acrylic glass) with the} dimension as indicated in Figure 3 is used for case III. This phantom consists of two concentric pipes glued on a flat plate (not shown in the figure). The inner R1 chamber is empty (filled with air) and the outer annular chamber R2 consists of water. This phantom is similar to the phantom in case II (Figure2), as in solid material (walls) is present in the domain; however the attenuation of the Perspex material is far lower than the iron or any other metal. Hence, in essence it is a two phase phantom (like case I) with gas and liquid with a different spatial configuration than case I.

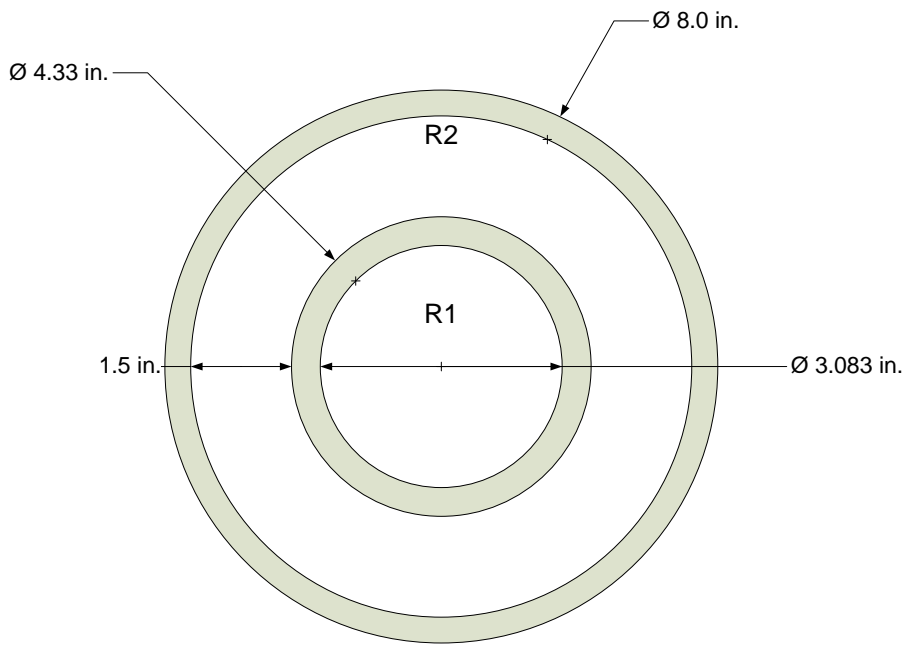

Figure 3 : Schematic cross section of the phantom for which experimental transmission data was gathered.

The walls are made of Perspex. Region R1 is air and region R2 is filled with water

\subsection{Detains of the gamma ray computer tomography (CT) scanner}




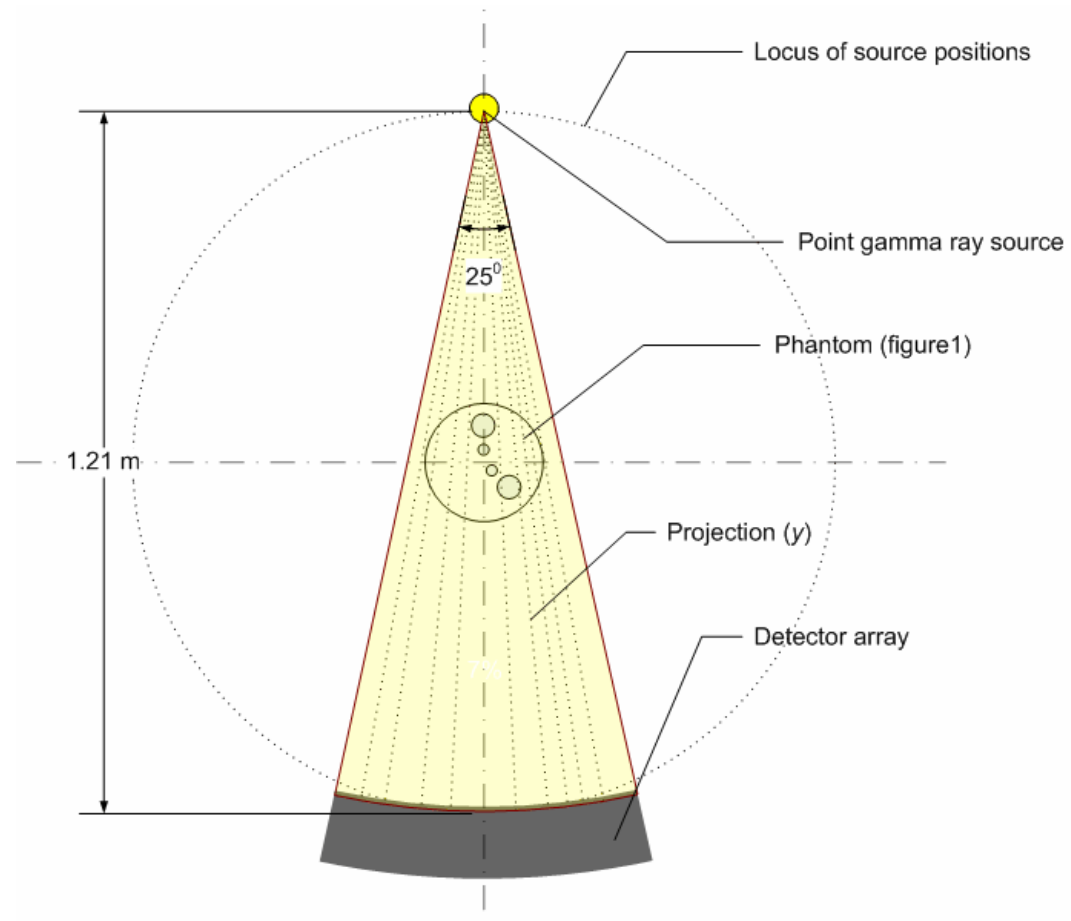

Figure 4: $\quad$ Schematic of the scanner arrangement with phantom in the center. A gamma ray fan beam with a $25^{\circ}$ angle and a detector array with 9 detectors are used (not shown). These detectors are moved through 21 positions effectively creating 189 projections. The region occupied by the detectors is indicated as detector array. The source, and the detector array, is moved along the locus as indicated for 197 positions and projection data for each location is gathered.

Figure 4 shows the schematic of the CT scanner used in this study for gathering the experimental data for case III of the phantom. The dimension of this same scanner was used to generate the simulated data for the phantom (case I and II) in Figure 2. The scanner consists of a point ${ }^{137} \mathrm{Cs}$ gamma ray source that has a photo peak at $660 \mathrm{keV}$. The attenuation data shown in table 1 is based on this energy of the gamma ray photons. An equiangular fan beam arrangement of the source and the detectors is used. The source is at the pointed end of the fan and the detectors are at the curved end of the fan placed $120 \mathrm{~cm}$ apart. The detector arrangement consists if nine 2 in. diameter $\mathrm{NaI}(\mathrm{Tl})$ detectors of 2 in. thickness. Each of these detectors is collimated with a lead collimator that is about $2.5 \mathrm{in}$. thick and has open aperture of dimension 1/16 in. $\mathrm{x} 3 / 16$ in. This aperture reduces the effective exposed area of the crystal to a rectangular region of dimension 1/16 in. $\mathrm{x}$ 3/16 in. The counts received by the detectors is limited to what is incident on this aperture. This detector array is moved with a motor 21 times at an angle of $0.13^{\circ}$ from the source there by creating 189 detector positions effectively for each source position. The projection is modeled as fine line between the source and the detector as the open area of the detectors is very small. A total of 197 source positions (also called views) are considered, hence gamma ray counts data for $189 \mathrm{x}$ 
197 (total 37233) projections passing through domain are collected. The simulated data for the phantom is generated for 189 projections per view for 197 views, based on this same geometry as the scanner described above. An 80x80 pixels resolution is used to reconstruct the image. Hence each pixel represents an area of $2.54 \mathrm{~mm} \times 2.54 \mathrm{~mm}$ of the phantom. Details on the data acquisition hardware, collimators, and detectors used to collect the photons counts data is available with Roy (2006).

\subsection{Gamma ray counts data generation}

Gamma ray counts data was generated by simulation for Case I and II of the phantom. Schaffler's (1970) algorithm is used to generate the counts $I$ that are Poisson random numbers with probability $P_{I}$ given by (21), and mean $g\left(y: \mu_{\text {phantom }}\right)$ given by (21).

$$
\begin{aligned}
& P_{I}=\frac{\left[g\left(y: \mu_{\text {phantom }}\right)\right]^{I}}{I !} \exp \left[g\left(y: \mu_{\text {phantom }}\right)\right] \\
& g\left(y: \mu_{\text {phantom }}\right)=\lambda(y) \exp \left[-\sum_{x \in X} h(x \mid y) \mu_{\text {phantom }}(x)\right]
\end{aligned}
$$

This Poisson number generated for each $y$ is set equal to $d(y)$, the counts received by the detector. The attenuation value $\mu_{\text {Phantom }}(x)$ used to generate the counts data is the attenuation of the $x^{\text {th }}$ pixel in the phantom (Figure 1) along projection $y$. The algorithm (AM and EM) determines these values with out any prior information about the phantom and based purely on the Poisson numbers. To reduce the effect of noise and uncertainty in the data and to get a better quality of the images, the mean value of the counts based on multiple samples or readings for a given projection $y$ is often used for processing the data, as represented by (23). Here $n$ represents the number of data sample of counts for a given projection $y$,

$d(y)=\frac{1}{n} \sum_{i=1}^{n} I_{i}$.

If an infinite number of samples are collected then (24) would give an accurate value of the mean $g\left(y: \mu_{\text {phantom }}\right)$,

$$
\lim _{n \rightarrow \infty} \frac{1}{n} \sum_{i=1}^{n} I_{i}=g\left(y: \mu_{\text {phantom }}\right) \text {. }
$$

Hence when (23) is used with some small finite values of $n$ then it can be said that an approximate estimate of the mean $g\left(y: \mu_{\text {phantom }}\right)$ is used to process the data. To see how the number of data sample used to arrive at the mean value of the counts effects the AM and EM algorithms performance, the mean of 
multiple samples $(n)$ of $I$ (ranging from 1 to 100) are used as estimates of $g\left(y: \mu_{\text {phantom }}\right)$ for $d(y)$ as per (23). It can be seen as: higher the number of samples lesser the noise in the data. This procedure was also followed with the experimental data collected with the scanner for the case III of the phantom. The maximum values of $n=100$ is a optimum choice as the results discussed in section 5.0 show that the maximum difference in reduction of noise, and by extension error, in the holdup images occurs for values lower values of $n$. Hence the reduction in error from $n=1$ to $n=5$ is far greater than the reduction in error from $n=50$ to $n=100$. The value for $n=100$ is also practical when data is collected with the scanner, as higher values would dramatically increase the time required to execute the scan without an increase in accuracy that is commensurate with it.

\subsection{Calculation of holdup distribution}

The counts data generated by simulation is processed by the algorithms (AM and EM) which reconstruct an image that depicts attenuation image, $\hat{\mu}_{l-g}(x)$, of the scanned domain. This attenuation value is a liner sum of the product of the holdup fractions of the phases and their pure attenuation values as given by (25).

$\hat{\mu}_{l-g}(x)=\hat{\mu}_{l}(x) \varepsilon_{l}(x)+\hat{\mu}_{g}(x) \varepsilon_{g}(x)$.

The subscripts $l$ and $g$ represent liquid and gas respectively, $\varepsilon(x)$ represents the holdup fraction and $\hat{\mu}(x)$ the attenuation of the pure phase. The objective of using CT is to determine the values of $\varepsilon(x)$.

The $\hat{\mu}(x)$ values for liquid and gas are usually determined by a background scan where the domain purely consists of only one of the phases. In addition it is also known that the sum of the holdup fraction of the two phases add up to unity (26).

$$
\varepsilon_{l}(x)+\varepsilon_{g}(x)=1 \text {. }
$$

To determine the gas hold up for the case I phantom, equations (25) and (26) are combined with the assumption that $\hat{\mu}_{g}(x)$ (representing calibration attenuation image of the system filled with gas) to be equal to zero, to give (27). This assumption is reasonable as air has negligible contribution to the attenuation of a gamma ray photon with $661 \mathrm{keV}$ energy. Hence gas holdup distribution image is determined using (27), and by extension the liquid holdup distribution image by using (28).

$$
\begin{aligned}
& \varepsilon_{g}(x)=\frac{\hat{\mu}_{l}(x)-\hat{\mu}_{l=g}(x)}{\hat{\mu}_{l}(x)} \\
& \varepsilon_{l}(x)=1-\frac{\hat{\mu}_{l}(x)-\hat{\mu}_{l=g}(x)}{\hat{\mu}_{l}(x)}
\end{aligned}
$$


In (27) and (28), $\hat{\mu}(x)_{l-g}$ represents the attenuation image of the phantom with region B filled with water and regions $\mathrm{R} 1$ through $\mathrm{R} 4$ filled with air, and $\hat{\mu}(x)$, represents the attenuation image of the phantom with region B and R1 through R4 filled with water.

For the cases II and III of phantom which has solid internals, attenuation images $\hat{\mu}(x)_{l-g}$ and $\mu(x)_{l}$ in (25) are replaced with $\hat{\mu}(x)_{l-g-s}$ and $\hat{\mu}(x)_{l-s}$, respectively to give (29).

$\varepsilon_{g}(x)=\frac{\hat{\mu}_{l-s}(x)-\hat{\mu}_{l-g-s}(x)}{\hat{\mu}_{l-s}(x)}$

The image $\hat{\mu}(x)_{l-g-s}$ has the liquid in region $\mathrm{B}$, the gas in regions $\mathrm{R} 1$ and $\mathrm{R} 4$, and the solid in regions $\mathrm{R} 2$ and R3 of the phantom (Figure 2), and region R1 with gas and region R2 with liquid in case III phantom. The background scan image $\hat{\mu}(x)_{l-s}$ has the liquid in regions $\mathrm{B}, \mathrm{R} 1$ and $\mathrm{R} 4$, and solid in regions $\mathrm{R} 2$ and R3 of the case II phantom (Figure 2) and liquid in R1 and R2 of case III phantom (Figure 3).

Similarly, to determine the liquid holdup image for case II of the phantom, (28) is modified substituting $\hat{\mu}(x)$, with $\left[\hat{\mu}_{L-S}(x)-\hat{\mu}_{G-S}(x)\right]$ to give (30). The image $\hat{\mu}(x)_{g-s}$ has gas in regions $\mathrm{B}, \mathrm{R} 1$ and $\mathrm{R} 4$, and solid in regions R2 and R3 of the case II phantom (Figure 2) and gas in R1 and R2 of the case III phantom (Figure 3).

$\varepsilon_{l}(x)=1-\frac{\left[\hat{\mu}_{L-S}(x)-\hat{\mu}_{G-S}(x)\right]-\hat{\mu}_{l=g}(x)}{\left[\hat{\mu}_{L-S}(x)-\hat{\mu}_{G-S}(x)\right]}$

The attenuation image $\mu(x)$ for different configurations as indicated above are calculated using the AM and EM algorithms. The holdup distribution images are determined post attenuation image reconstruction based on (27) through (30), for the liquid and the gaseous phases for all three cases of the phantom. Although the algorithm reconstructs the attenuation images, the holdup distribution images obtained on its basis are analyzed to evaluate the AM and EM algorithms. This is done as ultimately the phase holdup images are of interest to obtain quantitative information about the system being scanned.

\subsection{Results and discussion}

The holdup mages reconstructed based on simulated transmission data for case I and case II phantoms and experimental transmission data from case III phantom, are analyzed in the section. The mean percentage error and the standard deviation of all the pixels in a given domain are used as parameters to qualitatively asses the images. The error values used in this discussion is calculated by comparing the ideal values of holdup with the values of holdup from the reconstructed images. 
4.1.1 Gas holdup images By using (27) and (29) the gas holdup images were successfully obtained.

Figures 5 and 6 show the gas holdup images using AM and EM algorithms respectively.

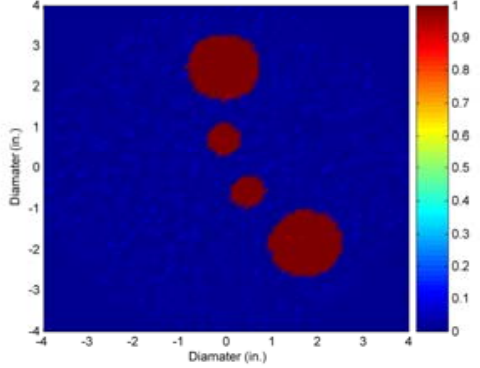

(a)

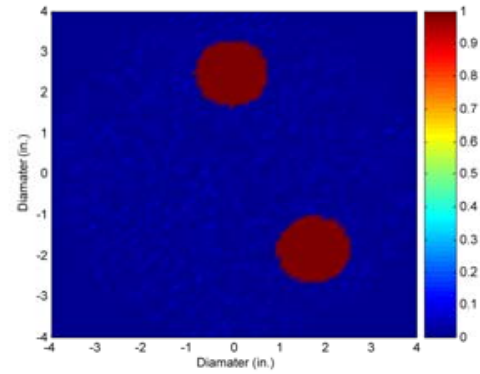

(b)

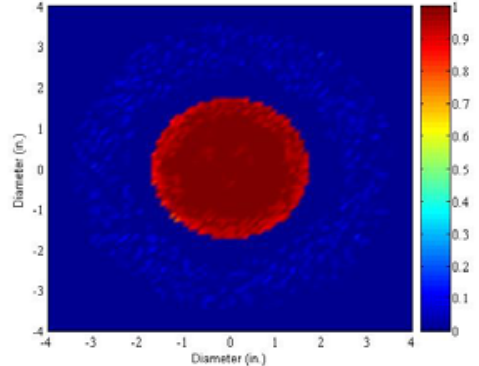

(c)

Figure 5: Gas holdup images obtained with AM algorithm (after 1000 iterations with $n=50$ ). The color bar represents the color code used for representing gas holdup values in the images: (a) Gas holdup based on (27) for case I phantom (Figure2), (b) gas holdup based on (29) for case II of the phantom(Figure 2) and (c) gas holdup based on (29) for case III of the phantom (Figure 3).

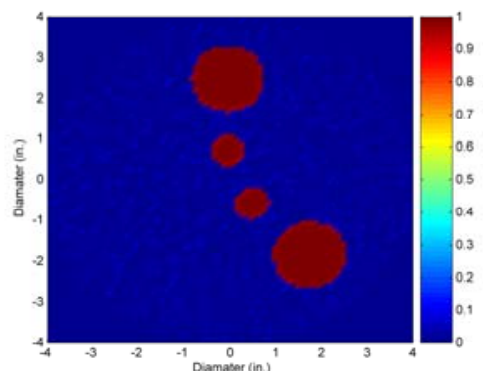

(a)

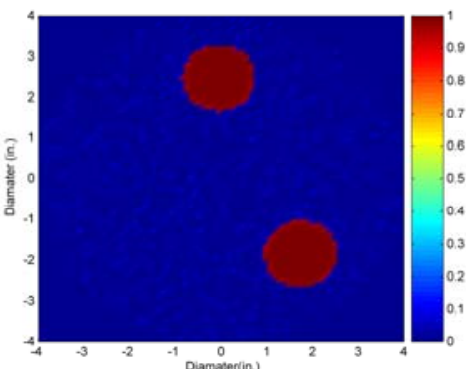

(b)

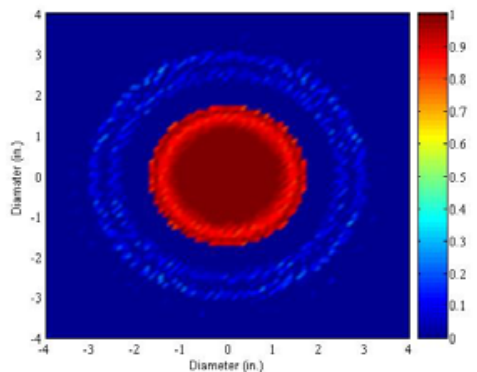

(c)

Figure 6: Gas holdup images obtained with EM algorithm (after 1000 iterations with $n=50$ ). The color bar represents the color code used for representing liquid holdup values in the images. : (a) Gas holdup based on (27) for case I phantom (Figure 2), (b) gas holdup based on (28) for case II of the phantom (Figure 2) and (c) gas holdup based on (29) for case III of the phantom (Figure 3).

Clearly both the algorithms are able to successfully image the gas phase distribution in the all the three phantoms as the gas filled regions are captured in the image with a holdup values close to unity. There is no distortion in the reconstructed images, the geometric shape of the domains (R1 through R4) for caseI, $\mathrm{R} 1$ and R2 for case II and R1 of case III are maintained and appear just as in the phantom. The transition in the gas holdup values at the edge of the domain is sharper in images generated by AM algorithm (Figure5) as compared to those by the EM algorithm (Figure 6). The AM algorithm provides gas holdup mages with uniformity in the domains of the phantom (for all cases of the phantom) where the gas in present. These images also confirm the (29) is correctly formulated as complicated arrangements that have parts of the domain covered by the solid material (Figure 6(b) and (c)) in the phantoms are not misrepresented as part of the gas holdup. 
4.1.2 Quantitative analysis of gas holdup. A quantitative analysis of the images is possible only when the holdup values of all pixels in a given part of the image is analyzed for its error and standard deviation.

Standard deviation indicates the level of noise in the image. If the standard deviation of the pixels is high, it means the image is noisy and a lot of freckles or grains can be seen. The most desirable situation is the one where both the standard deviation and the error are nil. When the standard deviation is small and the error is high then there is a bias in the image.

Figure 7 shows the mean percentage error and Figure 8 shows the standard deviation in the gas holdup values for the three cases of the phantom obtained using both, the AM and the EM algorithms. Clearly the error values in Figure 7 shows that the AM- algorithm performs better than the EM algorithm for any condition of number of data samples or iterations covered in all the phantoms. When transmission data with low levels of noise ( $\mathrm{n}=100$ in (22)) is used the EM algorithm still gives higher error than the AM algorithm, this is more prominent in the case III phantom. For case II of the phantom (Figure 7(b)) the difference in error between the two algorithms is almost an order of magnitude. This indicates that in the presence of high attenuation material the AM algorithm gives a more accurate estimate of gas holdup.

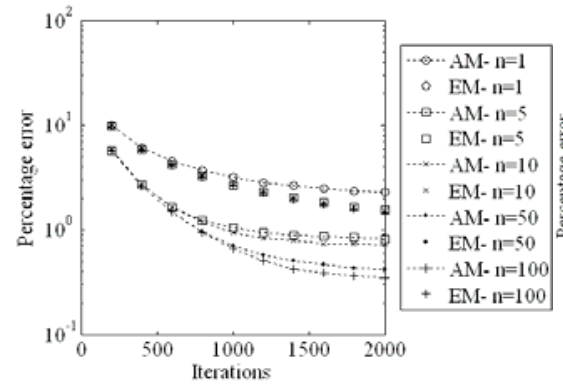

(a)

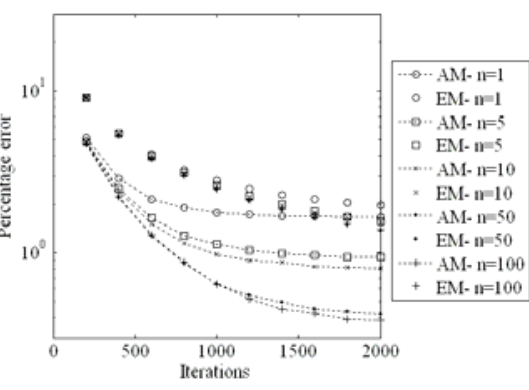

(b)

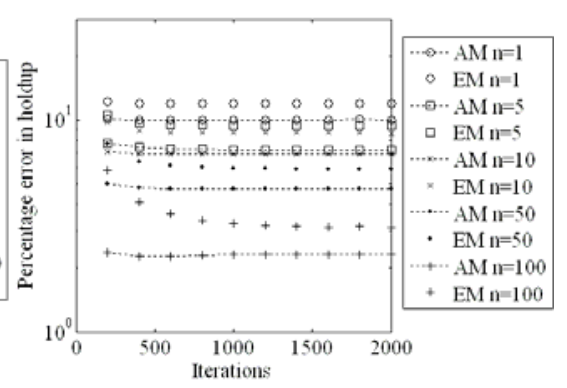

(c)

Figure 7 : Mean percentage error in gas holdup for results obtained with AM and EM algorithms for projection data with $n=1$ through $n=100$ (in (23)). (a) Error in gas hold up for pixels of regions R1, R2, R3 and R4 (Figure 2) of the case I phantom, (b) Error in gas holdup value for pixels in region R1 and R2 for case II(Figure 2) of the phantom and (c)Error in gas holdup value for pixels from region R1 of the case III (Figure 3) phantom.

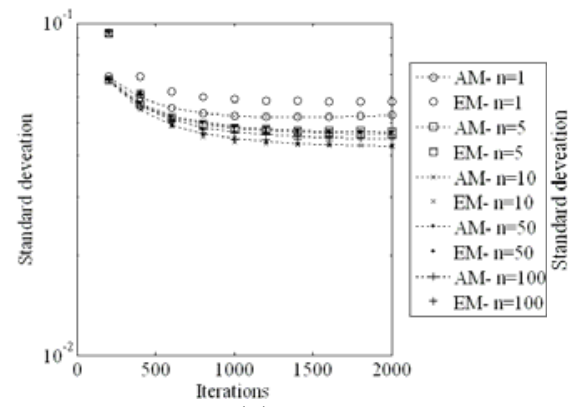

(a)

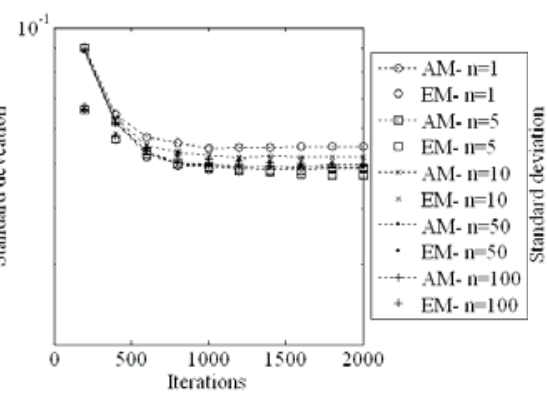

(b)

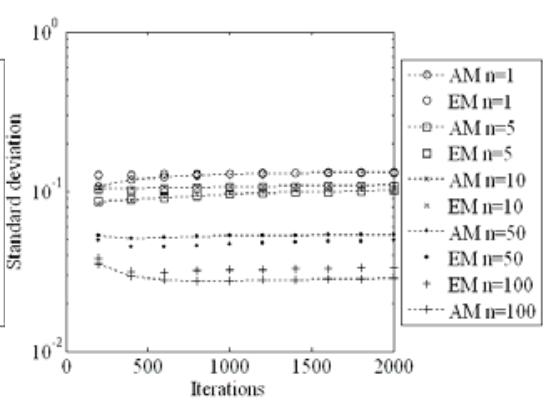

(c)

Figure 8: Standard deviation of gas holdup for results obtained with AM and EM algorithms for projection data with $n=1$ through $n=100$ (from (23)). (a) Standard deviation of gas holdup in pixels of regions R1, R2, R3 and R4 (Figure 2) of the case I phantom, (b) Standard deviation of gas holdup in pixels of regions R1 and R2 
for case II (Figure 2) of the phantom and (c) Standard deviation of gas holdup in pixels of region R1 of the case III (Figure 3) phantom.

The standard deviation of the pixels from the regions of the phantom where gas is present has trends similar to the error values for any given number of iteration and number of transmission data samples for the projections (based on (23)). The values are lower for the results obtained from AM algorithm as compared to the EM algorithm. As the iterations progress the standard deviation values stabilize. This indicates the gas holdup images have less noise in the image. At this point it is important to determine the standard deviation of the gas holdup in the pixels from the region of the phantom that don't have gas. Ideally the holdup values and standard deviation in this region should be zero. However small values are observed.

Figure 9 shows the standard deviation values of the gas hold up in these regions. Again, for all the cases the AM algorithm gives values that are smaller than the EM, hence the noise in the image in these regions is much less.

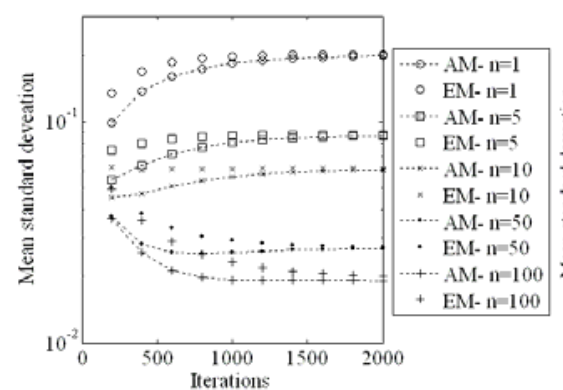

(a)

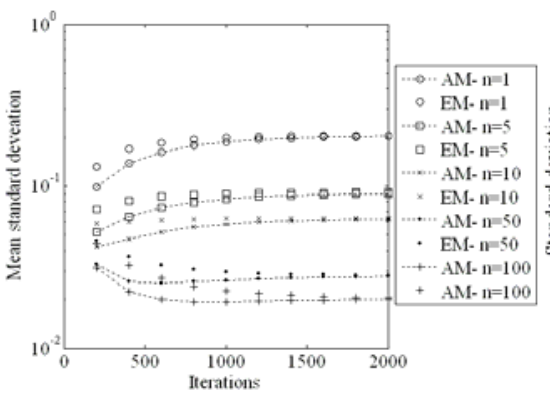

(b)

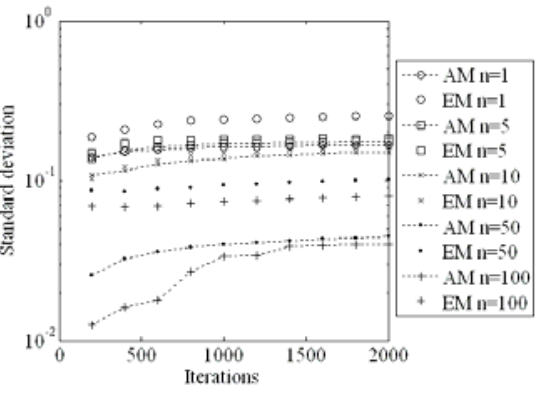

(c)

Figure 9: Standard deviation values of gas holdup from the results obtained in regions of the phantom where ideally the gas holdup is zero. The values obtained with AM and EM algorithms for projections data with $n=1$ through $n$ $=100$ are shown. (a) Standard deviation of gas holdup in pixels of region B (Figure2) of the case I phantom, (b) Standard deviation of gas holdup in pixels of region B, R3 and R4 for case II (Figure 2) of the phantom and (c) Standard deviation of gas holdup in pixels of region R2 of the case III (Figure3) phantom.

4.2.1 Liquid Hold up. The liquid holdup images were successfully obtained by using (28) for case I of the phantom and (30) for case II and III of the phantom. Figure 10 shows the liquid holdup images, obtained using AM algorithm, and Figure 11 shows the images obtained using the EM algorithm. Clearly the liquid phase is separated successfully. There is no distortion in the images, the shapes of the region with liquid in the phantoms is successfully captured. The regions of the cases II and III phantom that have the solids are not misrepresented as liquid holdup in images. As in the case of the gas holdup images the liquid holdup images have sharper transitions at the edge of the domain when AM algorithm is used. The effectiveness of the AM algorithm in this aspect can clearly be seen in the case III phantom where actual 
experimental transmission data is used, by comparing Figure 10(c) and Figure 11(c).

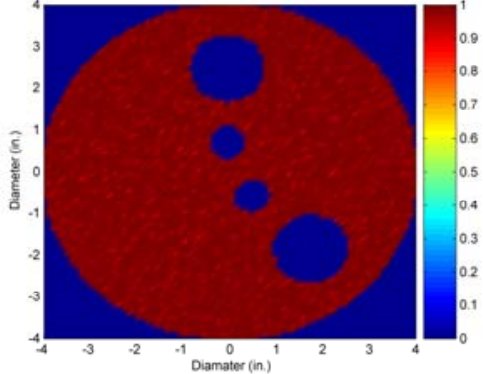

(a)

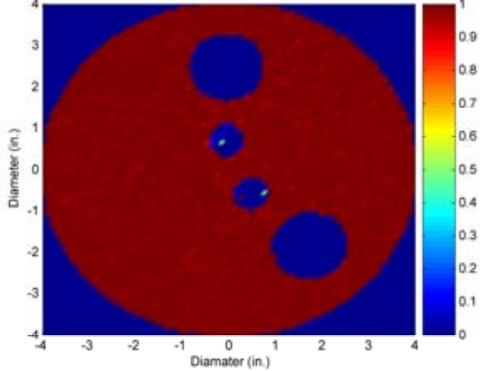

(b)

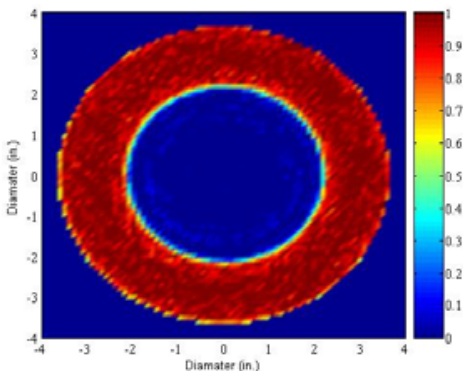

(c)

Figure 10: Liquid holdup images obtained with AM algorithm (after 1000 iterations with $n=50$ ). The color bar represents the color code used for representing liquid holdup values in the images: (a) Liquid holdup based on (28) for case I phantom (Figure 2), (b) liquid holdup based on (30) for case II of the phantom (Figure 2) and (c) liquid holdup based on (30) for case III of the phantom (Figure 3).

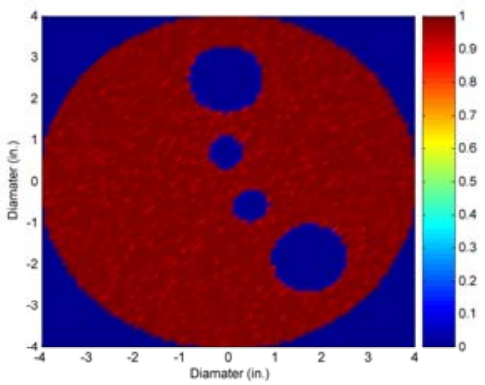

(a)

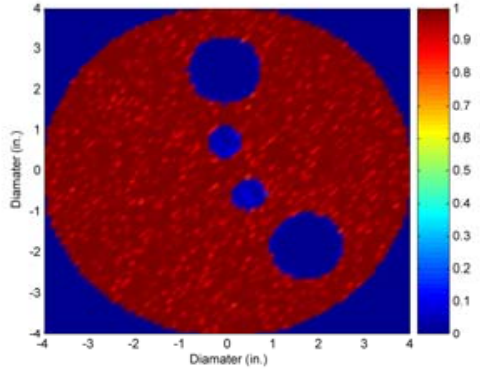

(b)

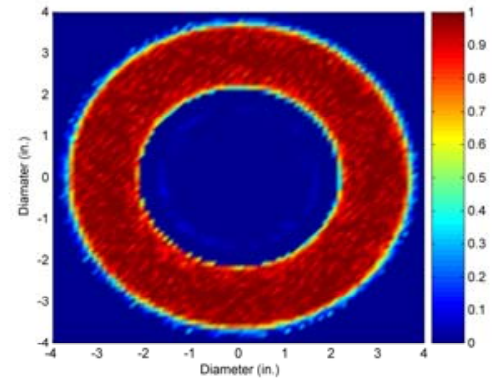

(c)

Figure 11: Liquid holdup images obtained with EM algorithm (after 1000 iterations with $n=50$ ). The color bar represents the color code used for representing liquid holdup values in the images: (a) Liquid holdup based on (28) for case I phantom (Figure 2), (b) liquid holdup based on (30) for case II of the phantom and (c) liquid holdup based on (30) for case III of the phantom.

In cases I and II the phantom is pixilated, as in only one of the two phases are present in any pixel. In case III of the phantom it is not possible to control this and the pixel along the curved boundary of the R2 domain (Figure 3) tend to smudged. In spite of this the AM algorithm gives better results than the EM as the edges of the liquid region appear to be blurred. For the case II phantom, the liquid holdup image obtained with the EM algorithm (Figure 11(b)) has freckles, hence has more noise, as compared to the one obtained with AM algorithm Figure 10(b). Also the holdup in the region occupied by the high attenuation material (R3 and R4 in Figure 3) in the EM algorithm image is slightly above zero. Both these aspects are attributed to the presence of the high attenuation material present in the domain.

\subsubsection{Quantitative analysis of liquid holdup}


Figure 12 shows the mean percentage error in estimating the liquid holdup values in region of the phantoms filled with the liquid. Clearly for all three cases of the phantom the AM algorithm performs better universally than the EM algorithm. For the case II of the phantom there is an order of magnitude difference in the error between the holdups values results from both the algorithms.

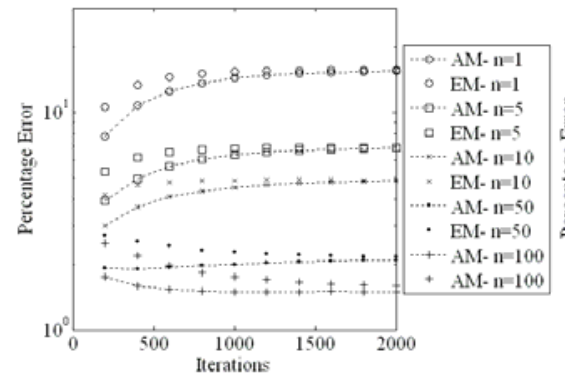

(a)

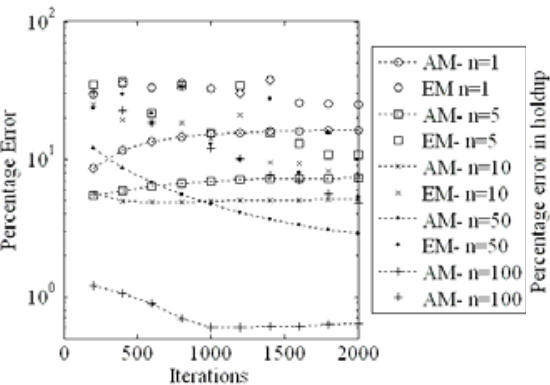

(b)

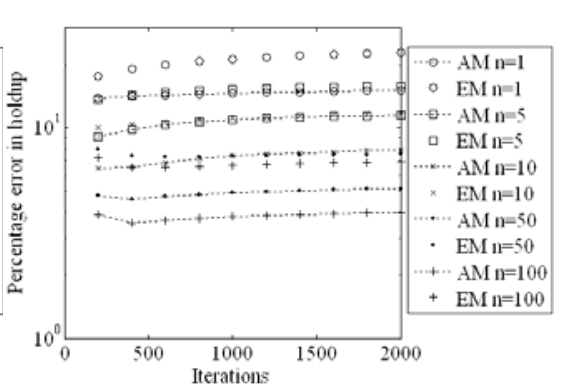

(c)

Figure 12: Mean percentage error in liquid holdup for results obtained with AM and EM algorithms for projection data with $n=1$ through $n=100$. (a) Error in liquid hold up for pixels of region $\mathrm{B}$ (Figure 2) of the case I phantom, (b) Error in liquid holdup value for region B for case II (Figure2) of the phantom and (c)Error in liquid holdup value in region R2 of the case III (Figure 3) phantom.

Also, the error doesn't stabilize or reduce for data with higher noise levels (lower values of $\mathrm{n}$ as per (23)) when the EM algorithm is used for this case of the phantom. It should be noted that the liquid domain in the phantoms of cases I and II are physically similar. This clearly shows that holdup image of a phase that doesn't have a high attenuation is effected by parts of the domain that have the high attenuating material, or phase. The AM algorithm provides images with better quality in such applications as compared to the EM algorithm.

Figure13 shows the standard deviation of the liquid holdup from the pixels that are from the region of the phantom that have the liquid phase.

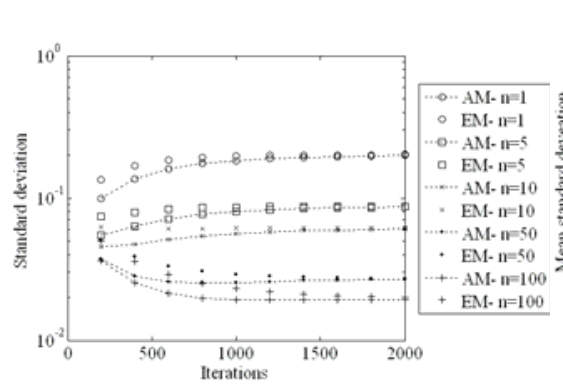

(a)

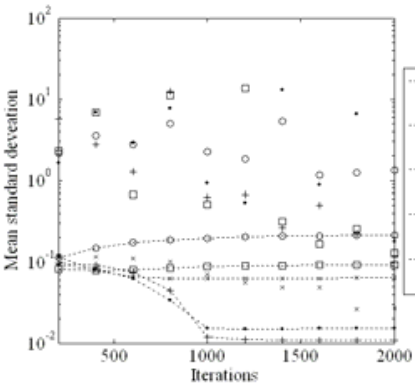

(b)

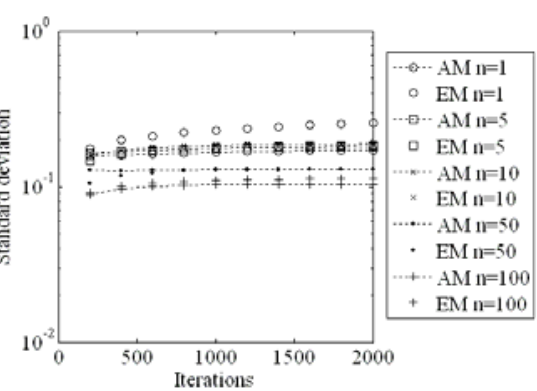

(c)

Figure 13: Standard deviation in liquid holdup for results obtained with AM and EM algorithms for projection data with $n=1$ through $n=100$. (a) Standard deviation in liquid holdup of pixels of region B (Figure 2) of the case I phantom, (b) Standard deviation in liquid holdup of region B for case II (Figure2) of the phantom and (c) Standard deviation in liquid holdup of in region R2 of the case III (Figure3) phantom. 
The results from AM algorithm have a lower standard deviation than compared to the EM algorithm for any value of the iterations or the number of data samples per projection (as per (23)). This trend is true for all the phantoms studied. This indicates that the noise levels in the images obtained using the AM algorithm are much lower than what is seen in results from the EM algorithm. For case II of the phantom, the standard deviation values of the results from the EM algorithm are an order of magnitude different as compared to the AM, reconfirming what was seen for the same case in Figure 12.

The standard deviation and percentage error for transmission data with lower $n$ values is found to increase with the increase in the number of iterations for both the liquid and gas holdup images. This phenomenon is known to occur with EM (Snyder et al, 1987) and AM (Benac 2005) algorithms due to the random nature of the data. This happens even as the solution converges towards a maximum log likelihood estimate. Snyder et al (1987) have suggested strategies that overcome this problem which have not been implemented here as it is out of the scope of this study. The images reconstructed using AM algorithm exhibit this behavior to a far less extent at lower iterations as compared to EM algorithm.

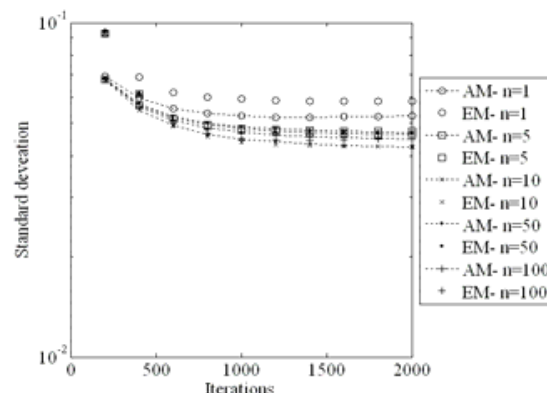

(a)

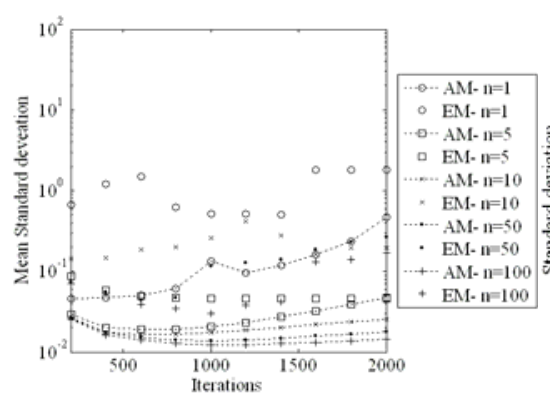

(b)

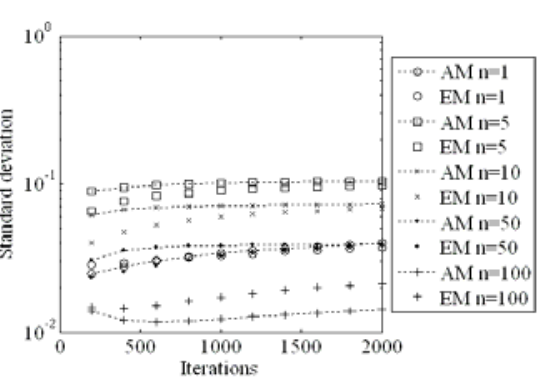

(c)

Figure 14: Standard deviation values of liquid holdup results obtained in regions of the phantom where liquid holdup is ideally zero. The values are obtained with AM and EM algorithms for projections data with $n=1$ through $n=100$ are shown. (a) Standard deviation of liquid holdup in pixels of regions R1, R2, R3 and R4 (Figure 2) of the case I phantom, (b) standard deviation of liquid holdup in pixels of region R1, R2, R3 and R4 for case II (Figure2) of the phantom and (c) standard deviation of gas holdup in pixels of region R1 of the case III (Figure3) phantom.

Figure 14 shows the standard deviation values of liquid holdups from pixels from the regions of the phantom that doesn't have the liquid in them. Ideally these values should be zero and by extension the standard deviation of this should be zero. However some small finite values of holdup are obtained. Here again the results from the AM algorithm shows values of standard derivations that are much lower than the EM algorithm for all cases of the phantom, as the noise level in the images are lower with the use of 
$\mathrm{AM}$ algorithm. The reduction in values of standard deviation by the AM algorithm is more prominent in the case II of the phantom where a high attenuation material (solid) is present in the domain.

\subsection{Conclusions}

The AM algorithm was successfully implemented for the case of single energy gamma ray tomography to determine phase holdup images for two phase systems for phantoms with different configurations. The performance of the algorithm was compared by putting the effect of some critical parameters such as, the noise level in the data and the number of iterations used to reconstruct the image, in perspective. A systematic analysis of the holdup distribution images generated using the AM and EM algorithm were carried out for the three cases of the phantom. The AM algorithm proposed by O'Sullivan and Benac (2007) performs better than the EM algorithm proposed by Lange and Carson (1984) when used for gamma ray tomography to determine holdup images in two phase systems. The results show an over all improvement in the quality of the image in terms of the noise and accuracy of the estimated values when the AM algorithm is used. For data with high noise the AM algorithm gives lesser error than the EM algorithm, and it requires less number of iterations to reach a given level of accuracy. Applications that involve the use of high attenuation material the AM algorithm is more stable and produces holdup images that have a greater degree of accuracy and lower levels of noise. This is true for both, parts of the domain that have the high attenuation material (metals) and parts that don't have it, like the surrounding areas (liquid and gas).

The differences in the performance of the two algorithms could be attributed to the simplification introduced in the M-step of the EM algorithm as indicated in (8) to estimate (7). In spite of using three terms of (8) (shown in (9)) for EM algorithm, as recommended by (Lange and Carson 1984) for maximum accuracy in the results, it doesn't match the performance of the AM algorithm the AM algorithm performs better. Hence the AM algorithm is a better choice for image reconstruction for determining the hold up distribution images in multiphase systems involving two phase flow.

\section{Notation used:}

$d(y)=$ Photon counts received by detector $y$

$E[\bullet]=$ Mean of the given function

$g(y: \mu)=$ Mean value of photon counts received for a given $y$ and $\mu$

$h(y \mid x)=$ Length of the segment of projection $y$ in pixel $x(\mathrm{~cm})$

$I=$ Number of photon counts generated by simulation

$I(a \| b)=$ I-divergence of quantities $a$ and $b$

$M(y \mid x)=$ Conditioned expected values of photons that enter pixel $x$ along projection $y$ 
$n=$ Number of samples of counts data used to estimate the mean counts for each projection

$N(y \mid x)=$ Conditioned expected values of photons that survive and leave pixel $x$ along projection $y$

$p_{x(y \mid x)}=$ Probability of complete data set

$x=$ Index for pixel in image space

$X(y \mid x)=$ Random number of photons entering a pixel $x$ along projection $y$

$y=$ Index for projection, or source detector pair

\section{Greek:}

$\varepsilon(x)=$ Holdup fraction of any given phase

$\lambda=$ Source intensity (counts)

$\hat{\mu}(x)=$ Attenuation values estimated by algorithm for a given pixel $x\left(\mathrm{~cm}^{-1}\right)$

$\mu_{\text {Phantom }}(x)=$ Attenuation value of simulated phantom for which transmission data is generated $\left(\mathrm{cm}^{-1}\right)$

\section{Subscripts:}

$l=$ System filled with liquid

$g=$ System filled with gas

$l-g=$ System filled with liquid and gas

$l-g-s=$ System filled with liquid, gas, and solid

\section{Acknowledgements}

The authors would like to acknowledge the United States Department of Energy for sponsoring the research project (Identification number: DE-FC-36-01GO11054)

\section{References:}

Benac J. 2005. Alternating minimization algorithms for X-ray computed tomography : multigrid acceleration and dual energy application [D.Sc.]: Washington University- St. Louis.

Browne JA, Holmes TJ. 1992. Developments with maximum likelihood X-ray computed tomography. Medical Imaging, IEEE Transactions on 11(1):40-52.

Csiszár I. 1991. Why Least Squares and Maximum Entropy? An Axiomatic Approach to Inference for Linear Inverse Problems. Annals of Statistics 19:2033-2066.

Khopkar AR, Rammohan AR, Ranade VV, Dudukovic MP. 2005. Gas-liquid flow generated by a Rushton turbine in stirred vessel: CARPT/CT measurements and CFD simulations. Chemical Engineering Science 60(8-9):2215-2229. 
Kumar S, Duduković MP. 1997. Computer Assisted Gamma and X-ray Tomography: Applications to Multiphase Flow Systems. In: J. Chaouki FL, M.P.Duduković, editor. Non-Inavsive Monitoring of Multiphase Flows: Elsevier. p Chapter 2.

Kumar SB, Moslemian D, Dudukovic MP. 1997. Gas-holdup measurements in bubble columns using computed tomography. AIChE Journal 43(6):1414-1425.

Lange K, Carson R. 1984. EM Reconstruction Algorithims for Emission and Transmission Tomography. Journal of Computer Assisted Tomography 8(2):306-316.

O'Sullivan JA, Benac J. 2007. Alternating Minimization Algorithms for Transmission Tomography. Medical Imaging, IEEE Transaction on 26 (3):283-297.

Ollinger JM. 1994. Maximum-likelihood reconstruction of transmission images in emission computed tomography via the EM algorithm. Medical Imaging, IEEE Transactions on 13(1):89-101.

Roy S. 2006. Phase distribution and performance studies of gas-liquid monolith reactor. [D.Sc.]: Washington University - St. Louis. 203 p.

Roy S, Al-Dahhan M. 2005. Flow distribution characteristics of a gas-liquid monolith reactor. Catalysis Today 105(3-4):396-400.

Roy S, Chen J, Kumar SB, Al-Dahhan MH, Dudukovic MP. 1997. Tomographic and Particle Tracking Studies in a Liquid-Solid Riser. Ind. Eng. Chem. Res. 36(11):4666-4669.

Roy S, Kemoun A, Al-Dahhan MH, Dudukovic MP, Skourlis TB, Dautzenberg FM. 2004. Countercurrent flow distribution in structured packing via computed tomography. Chemical Engineering and Processing 44(1):59-69.

Schaffer HE. 1970. Algorithms 369: Generation of random numbers satisfying the poission distribution Communication of the assocations for computing machinery 13(1).

Snyder DL, Cox J, J.R. 1977. An Overview of Reconstructive Tomography and Limitations Imposed by a Finite Number of Projections. In: Ter-Pogossian MM, editor. Reconstruction Tomography in Diagnostic Radiology and Nuclear Medicine: Baltimore : University Park Press.

Snyder DL, Schulz TJ, O'Sullivan JA. 1992. Deblurring Subject to Nonnegativity Constraints. IEEE transactions on signal processing 40(5):1143-1150. 